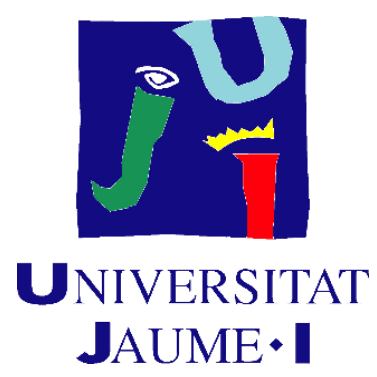

Programa de Doctorat en Ciències

Escola de Doctorat de la Universitat Jaume I

\title{
SYNTHESIS OF COMBRETASTATIN ANALOGUES WITH ANTINEOPLASTIC PROPERTIES
}

\section{Síntesi d'anàlegs de combretastatina amb propietats} antineoplàsiques

Memòria presentada per Laura Conesa Milián per a optar al grau de doctora per la Universitat Jaume I

Laura Conesa Milián

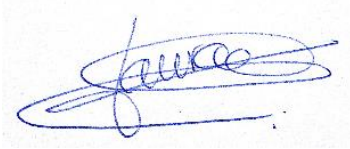

Miguel Carda Usó

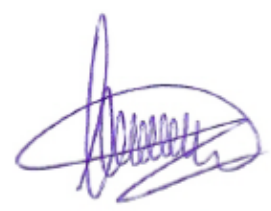

Eva Falomir Ventura

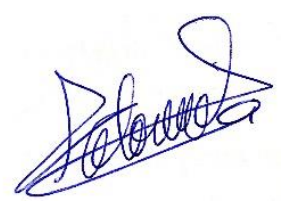

Castelló de la Plana, maig 2019 



\section{FINANÇAMENT REBUT}

Agències finançadores de la doctoranda:

- Contracte predoctoral FPU14/00878 (Miniteri d'Educació, Cultura i Esport).

Agències finançadores del projecte de recerca o dels recursos materials específics del grup de recerca:

- Projecte CT2014-52949-P (Ministeri d'Economia i Competitivitat).

- Projecte PROMETEO 2013/027 (Conselleria d'Educació, Investigació, Cultura i Esport de la Generalitat Valenciana).

- Projectes PI-1B2015-75 i UJI-B2018-38 (Universitat Jaume I). 



\section{AGRAIIMENTS}

Aquesta Tesi Doctoral ha estat realitzada al Departament de Química Inorgànica i Orgànica de l'Escola Superior de Tecnologia i Ciències Experimentals de la Universitat Jaume I de Castelló, sota la direcció del Dr. Miguel Carda Usó i el Dr. Juan Murga Clausell. A ells m'agradaria agrair-los l'oportunitat que m'han donat de formar part del seu grup de treball i iniciar-me en el món de la investigació. Gràcies pels consells, les aportacions i tota la dedicació mostrada. També, al Dr. J. Alberto Marco Ventura per l'experiència proporcionada, així com la seua exigència en el treball realitzat.

També vull expressar el meu agraïment a la Dra. Eva Falomir Ventura, ella ha estat un exemple a seguir des del primer moment que vaig entrar al grup. Gràcies per haver confiat en mi i per tot el recolzament que m'has donat. M'has sabut transmetre la teua passió per la ciència i eixe esperit de superació i valentia per afrontar els moments més difícils.

Gràcies als tècnics de Serveis Centrals, a les tècniques de laboratori i a tots els professors del departament, per ajudar-me en la realització de les tasques al llarg d'aquests quatre anys. Vull fer extensiu el meu agraïment a la Dra. Sandra Liekens de la Universitat de Lovaina i als membres del seu grup, per acceptar-me durant l'estada, per tots els coneixements transmesos i per la càlida acollida que em vau donar.

Per suposat, gràcies a tots els companys del meu laboratori. Als que em vau ajudar durant els meus inicis en la investigació, Maria, Rosa, Conxín, Steven i Ana M; als que heu passat amb mi la major part de les hores de treball, Raül, Celia i Pilar; i als que he tingut l'oportunitat d'ensenyar i ajudar, Raquel, Inés i Alberto. Tampoc m'oblide dels companys de la planta zero, gràcies sobretot a Ana T. i Carles pel saber compartit i per la bona companyia durant els ratets de dinar. 
També vull compartir aquest treball amb les meues amigues químiques, sé que enteneu de bona mà tot el gran esforç que açò m'ha suposat. Marta, ets una de les raons per les quals estic on estic. Són ja 12 anys d'amistat, sempre brillant, decidida i valenta. Ana B., ets una de les persones que més m'ha comprés durant aquesta etapa, m'he sentit molt identificada amb tu en tot moment. Eixa empatia i capacitat de treball que tens et farà arribar lluny. Maria, gràcies per la teua alegria i optimisme, m'has transmès molta força i motivació en els moments on tot pareixia que no tenia sortida. També, Alba i Olga moltes gràcies per enrecorda-vos de mi i trobar sempre un ratet per compartir durant les vostres visites "express" a Castelló.

També gràcies a la resta d'amics, sé que alguns mai heu tingut molt clar de què va açò del món de la investigació però sempre he pogut comptar amb vosaltres i disfrutar de la vida que hi ha més enllà de la UJI. Anna F. i Silvia, vos vaig conèixer en els inicis d'aquesta etapa i estic molt agraïda per tota la preocupació que heu mostrat al llarg d'aquest temps. Alba, Coral, Núria... i totes les del poble en general, cadascuna seguint projectes de vida diferents però amb eixa gran complicitat que ens acompanya. Elia, amiga i confident desde menudetes, sempre ahí per escoltar i aconsellar, moltes gràcies per eixos ratets de desconnexió, han estat totalment necessaris.

Per últim, un agraïment molt especial a la meua família, als "auelos", "tios" i cosins. Sobretot als meus pares i a Enric, vosaltres sou els que heu permés que tot açò haja estat possible. M'heu ajudat a portar de la millor manera els patiments, les frustracions i el mal humor... La vostra paciència i recolzament incondicional ha fet que mai perguera la confiança en mi. Gràcies de tot cor! 
Science is a jouney, never an arrival.

(Karl Popper) 



\section{ABBREVIATIONS AND ACRONYMS}

A549: human lung adenocarcinoma cell line

$\mathrm{Ac}_{2} \mathrm{O}$ : acetic anhydride

AcOH: acetic acid

ADP: adenosine diphosphate

AmCA-4: aminocombretastatin

APCl: atmospheric pressure chemical ionization

app: signal with apparent multiplicity

ATP: adenosine triphosphate

BLI: bioluminescence imaging

BMS: Bristol-Myers Squibb

BSA: bovine serum albumin

br: broad signal

CA-4: combretastatin A-4

CDI: carbonyldiimidazole

CDK: cyclin dependent kinase

CLSM: confocal laser scanning microscope

c-Myc: avian myelocytomatosis viral oncogene homolog

CrC: critical concentration

CTLA-4: cytotoxic T-lymphocyte antigen 4

d: doublet

DMEM: dubelcco's modified eagle's medium

DMF: dimethylformamide

DMSO: dimethylsulfoxide

DNA: deoxyribonucleic acid

EBI: $N, N$-ethylene-bis(iodoacetamide)

EC: endothelial cell

ECM: extracellular matrix

EGM-2MV: microvascular endothelial cell growth medium-2

EGTA: ethylene glycol tetraacetic acid ELISA: enzyme-linked immunosorbent assay
Erk1/2: extracellular signal-regulated protein kinases 1 and 2

ESI: electrospray ionization

$\mathrm{Et}_{3} \mathrm{~N}$ : triethylamine

$\mathrm{Et}_{2} \mathrm{O}$ : diethyl ether

EtOAc: ethyl acetate

EtOH: ethanol

FBS: fetal bovine serum

FDA: Food and Drug Administration

FGF: fibroblast growth factor

FITC: fluorescein isothiocyanate

GAB: glycerol-assembling buffer

GDP: guanosine diphosphate

GTP: guanosine triphosphate

HeLa: epitheloid cervix carcinoma cell line

HEK-293: human embryonic kidney cell line

HL-60: human promyelocytic leukemia cells

HMEC-1: human microvascular endothelial cell line

HR-ESMS: high resolution electrospray mass spectrometry

HRP: horseradish peroxidase

HT-29: human colorectal adenocarcinoma cell line

hTERT: human telomerase reverse transcriptase

IC $_{50}$ : half maximal inhibitory

concentration

i.p.: intraperitoneally

IR: infrared radiation

It ${ }_{50}$ : time to reach $50 \%$ polymerization

J: coupling constant

$\mathbf{K}_{\mathrm{i}}$ : inhibitory constant

LD $_{50}$ : median lethal dose 
LM2: fibroblastoid mammary carcinoma cell line

Log P: partition coefficient

m: multiplet

MAP: microtubule-associated protein

MCF-7: human breast adenocarcinoma cell line

MDA-MB-231: human breast adenocarcinoma cell line

MMP: matrix metalloproteinase

m. p.: melting point

MTT: 3-(4,5-dimethylthiazol-2-yl)-2,5diphenyltetrazolium bromide

NMR: nuclear magnetic resonance

p: calculated probability

PBS: phosphate-buffered saline

PEM: buffer composed by Pipes, EGTA and $\mathrm{MgCl}_{2}$

PEMP: PEM with polyethylene glycol

PEMT: PEM with Triton $X-100$

p-Erk1/2: phospho-Erk1/2

PD-1: programmed death-protein 1

PD-L1: programmed death-ligand 1

PI: propidium iodide

PVDF: polyvinylidene fluoride

p-VEGFR-2: phospho-VEGFR-2

q: quadruplet

Q-TOF: quadrupole-time of flight

RNA: ribonucleic acid

r. t.: room temperature

s: singlet

SCID: severe combined immunodeficiency

SD: standard deviation

SDS-PAGE: sodium dodecyl sulphate polyacrylamide gel electrophoresis

SI: selectivity index

t: triplet

TBS: tris-buffered saline

TBST: tris-buffer saline with Tween 20
THF: tetrahydrofuran

TLC: thin layer chromatography

TMB: tetramethylbenzidine

VEGF: vascular endothelial growth

factor

VEGFR: vascular endothelial growth factor receptor

VDA: vascular disrupting agent

WB: western blot 


\section{INDEX}

1. Introduction 1

1.1. Medicinal chemistry 1

1.2. Cancer 3

1.2.1. Hallmarks of cancer 5

1.2.2. Apoptosis 1

1.2.3. Metastasis 8

1.2.4. Resistance 9

1.3. Antineoplastic treatments 11

1.3.1. Antimitotic strategy 11

$\begin{array}{ll}\text { 1.3.2. Antiangiogenic strategy } & 28\end{array}$

1.3.3. Immunotherapy 39

2. Objectives $\quad 47$

3. Synthesis of aminocombretastatin derivatives 49

3.1. Synthesis of aminocombretastatin (AmCA-4) 49

3.2. Synthesis of family 1 compounds 49

3.3. Synthesis of family 2 compounds 50

3.4. Synthesis of family 3 compounds 51

3.5. Experimental section 54

3.5.1. General techniques $\quad 54$

3.5.2. Synthesis of aminocombretastatin (AmCA-4) 56

3.5.3. Synthesis of carbamates derived from AmCA-4 58

3.5.4. Synthesis of ureas derived from AmCA-4 74

3.5.5. Synthesis of family 3 derivatives 89

4. Biological evaluation 105

4.1. General techniques 105

4.2. Preliminary bioavailability prediction study 117 
4.3. Biological evaluation of family 1 as VDAs 120

4.3.1. Inhibition of cell proliferation 120

4.3.2. Tubulin polymerization inhibition 122

4.3.3. Tubulin interaction evaluation 123

4.3.4. Mitotic arrest and inhibition of interphase microtubules 124

4.3.5. Induction of apoptosis 126

4.3.6. Tube destruction evaluation 127

4.3.7. Conclusions of biological evaluation of family 1

4.4. Biological evaluation of family 2 as antiangiogenics 131

4.4.1. Inhibition of cell proliferation 131

4.4.2. Docking studies 133

4.4.3. Mitotic arrest and inhibition of interphase microtubules $\quad 137$

4.4.4. Induction of apoptosis 138

4.4.5. Tube formation inhibition 139

4.4.6. Ligand-VEGFR-2 direct interaction 142

4.4.7. Effect on cellular VEGFR-2 143

4.4.8. Inhibition of VEGFR-2 kinase activity 146

4.4.9. Conclusions of biological evaluation of family 2

4.5. Biological evaluation of family 3 as multitarget inhibitors 149

4.5.1. Preliminary docking studies $\quad 149$

4.5.2. Biological evaluation of triazolyl-ureas T.1-T.8 151

4.5.2.1. Inhibition of cell proliferation 151

4.5.2.2. Effect on cellular VEGFR-2 151

4.5.2.3. Inhibition of VEGFR-2 kinase activity 152

4.5.2.4. Tube formation inhibition 154

4.5.2.5. Effect on PD-L1 and C-Myc proteins 155

4.5.2.6. Cell proliferation evaluation in co-cultures 156 
4.5.3. Biological evaluation of ureas S.1-S.9 158

4.5.3.1. Inhibition of cell proliferation 158

4.5.3.2. Induction of apoptosis 159

4.5.3.3. Effect of on cellular VEGFR-2 160

4.5.3.4. Inhibition of VEGFR-2 kinase activity 162

4.5.3.5. Tube formation inhibition 162

4.5.3.6. Effect on PD-L1 and C-Myc proteins 164

4.5.3.7. Cell proliferation evaluation in co-cultures 165

4.5.4. Conclusions of biological evaluation of family 3

4.6. In vivo experiments 168

4.6.1. Summary of preliminary in vitro studies 168

4.6.2. In vivo evaluation $\quad 170$

4.7. Experimental section 174

5. General conclusions 183

6. Productions derived from doctoral thesis 185

6.1. Congress communications 185

6.2. Publications 186

$\begin{array}{ll}\text { 7. Selected spectra } & 187\end{array}$ 




\section{INTRODUCTION}

\subsection{Medicinal chemistry}

The origin of medicinal chemistry resides in the obtention of therapeutic products from natural sources. It was between 1815 and 1820 that the first active principles were isolated from plants. During the first half of $19^{\text {th }}$ century, the study of drugs was included within the traditional term Materia Medica. However, gradually, the word pharmacology was used with more frequency. The first one considers a static view of drugs, their production and composition, whereas the second one contemplates drugs from a more dynamic point of view studying their mechanism of action. ${ }^{1}$

In 1973, the IUPAC $^{2}$ gave a definition of Medicinal Chemistry as the branch of chemistry focussed on the discovery, development, identification and interpretation of the mode of action of biologically active compounds at molecular level. Therefore, medicinal chemistry is an interdisciplinary discipline that encompasses organic chemistry, biochemistry, physical and computational chemistry, pharmacology and molecular biology.

Most of the first drugs were discovered rather than developed and advances were often made as a result of trial and error. Large number of analogues were synthetized based on the structure of a known active compound (lead), but little was known about their detailed mechanism of action or the interaction with the target structure. ${ }^{3}$ Nowadays, drug development sequence consists in 3 steps. Firstly, the discovery, which involves the choice of the therapeutic target and the identification of a substance interacting with it. Secondly, the optimization, in which drug potency and selectivity are improved. And finally, the formulation,

\footnotetext{
${ }^{1}$ Camille G. Wermuth. The Practice of Medicinal Chemistry. Academic Press, London. 2003. ISBN 0-12-744481-5.

2 IUPAC, Information Bulletin, Tech. Rep. 1974, 13.

3 G. L. Patrick. An Introduction to Medicinal Chemistry. Oxford University Press, 2001. ISBN 9780198505334.
} 
where the pharmaceutical properties are tuned to turn the drug suitable for clinical use.

When designing a new drug both pharmacodynamics and pharmacokinetics must be considered. The first is referred to the action mechanism of the drug and the biological effect that is derived, while the second determines the temporal variation of drug concentration within the organism. The latter is also related to ADMET properties ${ }^{4}$ (absorption, distribution, metabolism, excretion and toxicity) and bioavailability, a measure of the amount of an administered dose that reaches the bloodstream. Concretely, in 1997, Lipinski ${ }^{5}$ described "the rule of five", which tries to predict the bioavailability of a drug candidate according to the following criteria:

- Molecular weight (Mw) must be lower than 500.

- Partition coefficient logarithm $(\log P)$ must be lower than 5.

- $\quad$ Number of $\mathrm{H}$-bond donor groups must be lower than 5.

- Number of H-bond acceptor groups must be lower than 10.

Figure 1.1 shows the whole process involved in drug discovery. After target validation, different hits are identified and optimized with the help of different techniques such as High Throughput Screening (HTS), computational chemistry or Quantitative Structure-Activity Relationship (QSAR) in order to find a lead. In preclinical studies, drugs are tested in vitro and in vivo to study their toxicity, pharmacokinetics and formulation. As regards clinical assays, these consist in 3 phases in which the experimental drug is examined in terms of efficacy and security before its commercialization. In some cases, it is possible to detect adverse effects after drug approval, thus, there is a phase IV of vigilance that

\footnotetext{
${ }^{4}$ Norinder, U.; Bergstroem, C. A. S. ChemMedChem. 2006, 1, 920-937.

${ }^{5}$ Lipinski, C. A.; Lombardo, F.; Dominy, B. W.; Feeney, P. J. Adv. Drug Delivery Rev.1997, $23,3-$ 25.
} 
could imply from a dose readjustment to removal from the market, if it is required. ${ }^{6}$

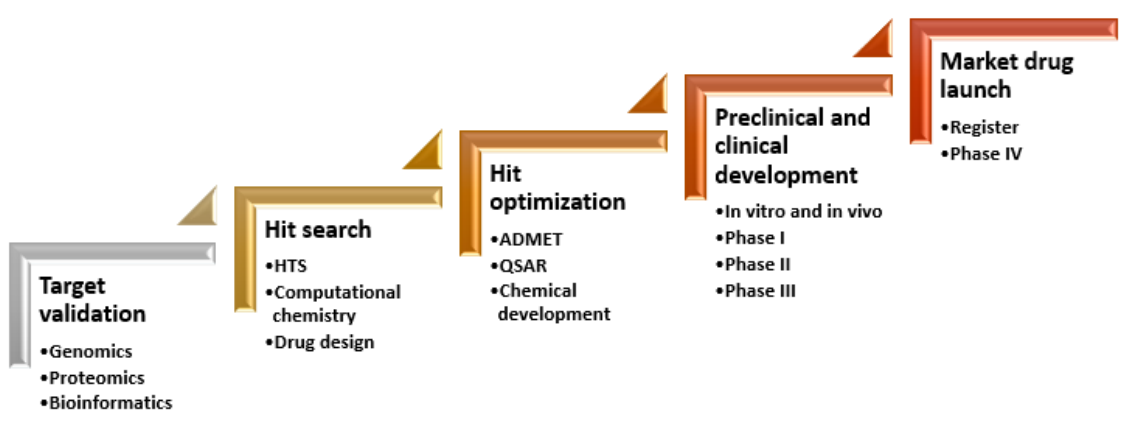

Figure 1.1. Steps in drug discovery process.

\subsection{Cancer}

Cancer is the second leading cause of mortality all over the world and was responsible for 18.1 million new cases and 9.6 million cancer deaths in 2018. About 1 in 6 deaths is due to cancer. Concretely, Europe accounts for $20.3 \%$ of the global cancer deaths and this number is stimated to increase from 1.9 to 2.5 million of deaths by 2040. As it is shown in figure 1.2, lung, breast and colorectum cancers are the top three types in terms of incidence. ${ }^{7}$
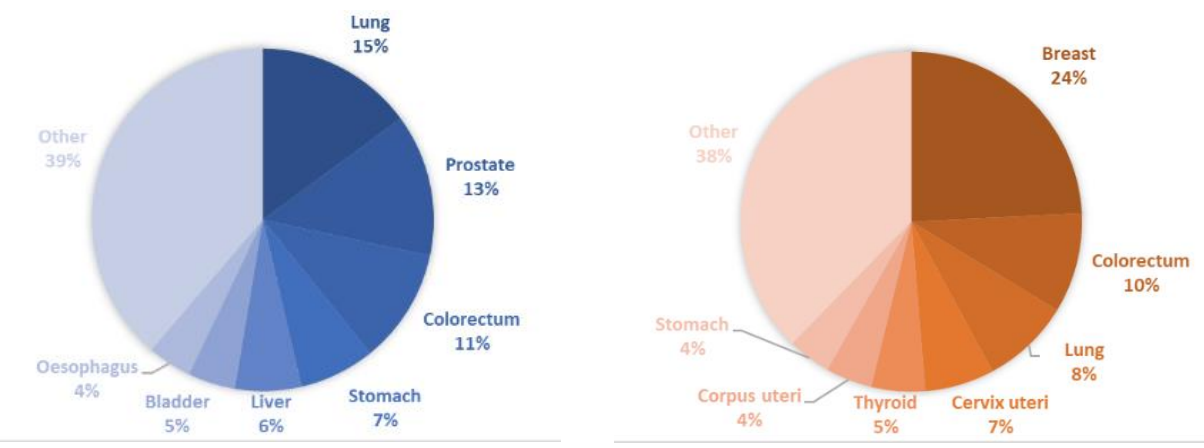

Figure 1.2. Cases of cancer in 2018 worldwide (left: males, right: females). ${ }^{8}$

\footnotetext{
${ }^{6}$ Rang, H. P.; Dale, M. M.; Ritter, J. M.; Flower, R. J.; Henderson, G. Pharmacology, 7th ed. Elsevier. 2012.

${ }^{7}$ IARC Global Cancer Observatory, WHO, press release nำ263, 12 September 2018.

${ }^{8}$ Data source: Globocan 2018, http://gco.iarc.fr.
} 
Cancer is a generic term to describe a large group of diseases. Other terms used are malignant tumors or neoplasms. One defining feature of cancer is the rapid creation of abnormal cells that grow beyond their usual boundaries invading adjoining parts of the body and spreading to other organs, process known as metastasis.

Malignant tumors are created in a multistage process that generally progresses from a pre-cancerous lesion. This is the result of the interaction between a person's genetic predisposition and external agents classified in physical (UV and ionizing radiation), chemical (asbestos, components of tobacco smoke, aflatoxins and arsenic) and biological carcinogens (infections from certain viruses, bacteria or parasites). Ageing is another fundamental factor for the development of cancer since cellular repair mechanisms lose effectiveness as a person grows older.

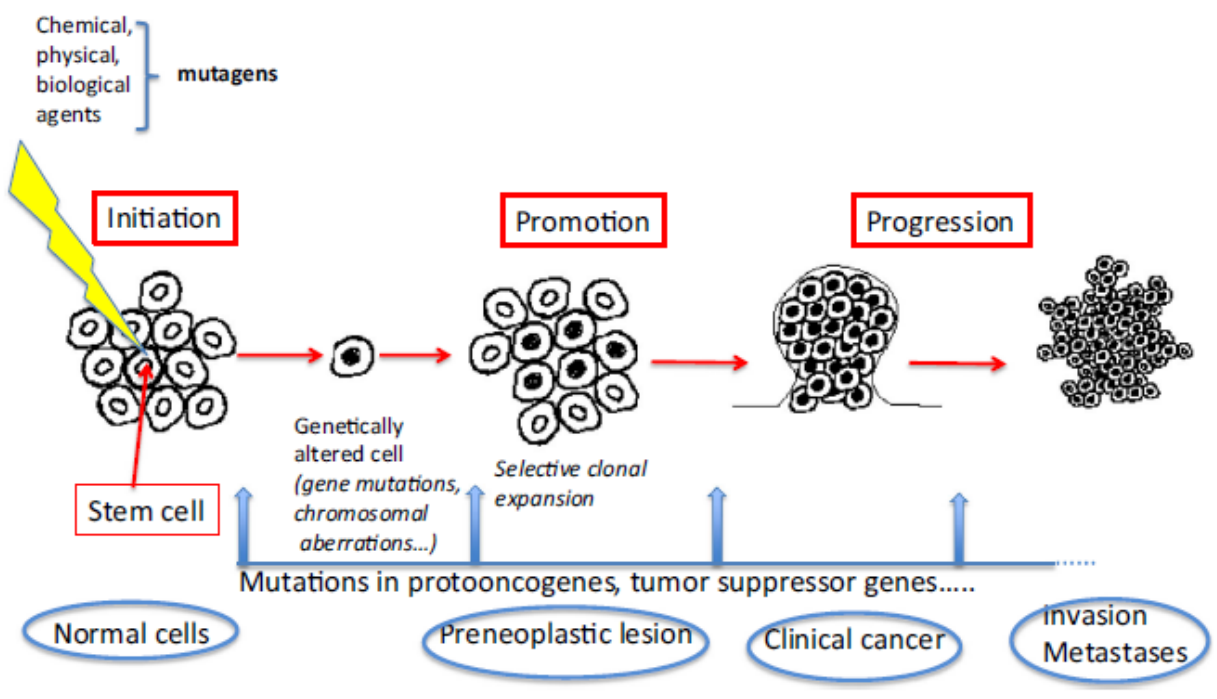

Figure 1.3. Carcinogenesis process. ${ }^{9}$

${ }^{9}$ Burgio, E.; Migliore, L. Mol. Biol. Rep. 2015, 42, 777-790. 
As it can be observed in figure 1.3, there are different genetic modifications responsible for cancer promotion: ${ }^{10}$

- Inactivation of tumor suppressor genes: tumor cells contain genes able to supress and repair mutations. An example of this is p53 gene, which is responsible for DNA reparation, cell cycle detention or apoptosis induction. The loss of function of these genes can generate cancer disease.

- Oncogene activation: protooncogenes are genes implied in the regulation of cell division, differentiation and apoptosis. These genes can become oncogenes if their sequence suffers any modification, causing thus a malign cell transformation. c-Myc is one of the most studied protooncogenes, since it is involved in telomerase activity, cell cycle progression, metabolism, apoptosis and cell motility. ${ }^{11}$

Achieving an efficient targeting of tumor pathway requires a detailed understanding of molecular lesions and abnormal mutations. A potential anticancer drug must be highly selective for tumor cells, choosing as biological targets specific features which are not present in healthy cells. Alterations in genes that control cell proliferation and differentiation have proved to be the first step in malignization process. Therefore, these constitute interesting therapeutic targets in treatments against cancer.

\subsubsection{Hallmarks of cancer}

In 2000, Hanahan and Weinberg published an article entitled The Hallmarks of Cancer ${ }^{12}$ which comprises 6 common biological capabilities acquired during

\footnotetext{
${ }^{10}$ Rang, H. P.; Dale, M. M.; Ritter, J. M.; Flower, R. J.; Henderson, G. Pharmacology, 7th ed. Elsevier, 2012.

${ }^{11}$ Hoffman, B.; Lieberman, D. A. Oncogene 2008, 27, 6462-6472.

12 Hanahan, D.; Weinberg, R. A. Cell 2000, 100, 57-70.
} 
the development of human tumors. Later, in 2011 these features were extended with 4 additional more constituting the following hallmarks: ${ }^{13}$

1) Sustaining proliferative signaling by the activation of growth factors or oncogenes such as Ras or c-Myc.

2) Evasion of growth suppressor signals, most of which are dependent of tumor suppressor genes.

3) High capacity to invade tissues and generate metastasis.

4) Replicative immortality, which is consequence of telomerase overexpression.

5) Angiogenesis induction to obtain nutrients and oxygen needed for tumor development.

6) Cell death resistance by the inactivation of proapoptotic factors or activation of antiapoptotic factors.

7) Evasion of immune destruction by T cells, B cells, macrophages or NT (Natural Killer) cells.

8) Modification or reprogramming of cellular energetics to keep cell growing and proliferation.

9) Inflammation processes to stimulate tumor progression.

10) Development of genome instability and mutations.

${ }^{13}$ Hanahan, D.; Weinberg, R. A. Cell 2011, 144, 646-674. 


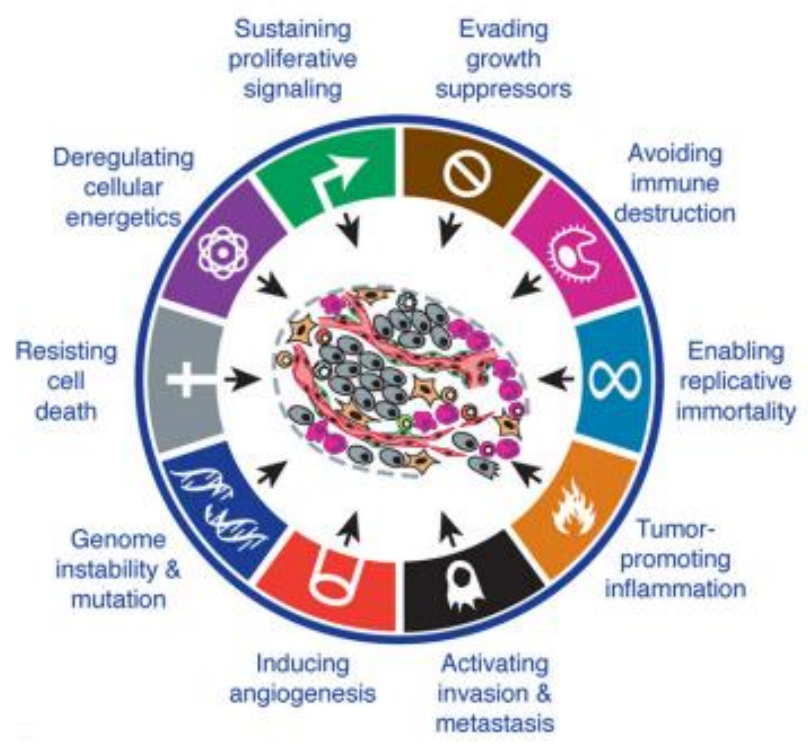

Figure 1.4. Hallmarks of cancer.

\subsubsection{Apoptosis}

Among the different mechanisms that cells undergo in order to dye, apoptosis has been hardly studied. This is a natural and programmed mechanism to eliminate unwanted cells and is characterized by cytoplasmic shrinkage exhibition, chromatin condensation, nuclear fragmentation and plasma membrane blebbing, culminating with the formation of small vesicles that are efficiently taken up by neighbouring cells with phagocytic activity and degraded within lysosomes. ${ }^{14}$ Apoptosis is a highly regulated process that can be activated by both intracellular and extracellular signals. Apoptotic process is carried out by caspases, which are a class of cysteine proteases that break target proteins. Intrinsic apoptosis is initiated by a variety of microenvironmental perturbations including growth factor withdrawal, DNA damage, endoplasmic reticulum stress, reactive oxygen species (ROS) overload, replication stress, microtubular alterations or mitotic defects.

\footnotetext{
${ }^{14}$ Galluzzi, L. et al, Cell Death Differ. 2018, 25, 486-541.
} 
The use of cell's own mechanism in order to get apoptosis is a highly effective method in cancer therapy. The most common strategies are stimulation of proapoptotic molecules or inhibition of the antiapoptotic ones. Some of the targets that have been studied include ligands for death-receptors, inhibitors for BCL-2 antiapoptotic protein, XIAP inhibition and alkylphospholipid analogs (APL) which act as apoptotic signals. ${ }^{15}$ Concretely, venetoclax (ABT-199) has recently gained FDA approval. ${ }^{16}$

\subsubsection{Metastasis}

Metastasis is defined as the dissemination of cancer cells from a primary tumor and the consequent seeding in distant tissues and organs. ${ }^{17}$ Generally, cells have adhesion molecules in their surface that allow the attachment to other similar cells and to the extracellular matrix (ECM), which is constituted by a network of insoluble proteins that fill interstitial space and keep cells together. Tumor cells have less adhesion capacity and are able to pervade the ECM of endothelial cells by the secretion of MMPs (matrix metalloproteinases), facilitating the entrance of tumor cells to the blood stream ${ }^{18}$. Consequently, tumor cells lose their differentiation, evade the immune system and expand through blood and lymphatic vessels to remote organs constituting micrometastatic colonies that become secondary tumors. ${ }^{19}$ Metastasis usually takes place in high vascularized organs such as lung, kidney or brain. It is the principal cause of mortality in most cancers and constitutes a serious problem in antineoplastic treatments. ${ }^{20}$

\footnotetext{
${ }^{15}$ Pfeffer, C. M.; Singh A. T. K. Int. J. Mol. Sci. 2018, 19, 448.

16 Letai, A. Annu. Rev. Cancer Biol. 2017, 1, 275-94.

17 Editorial team, Cell 2017, 168, 545-546.

18 Angosto. M. C.; Álvarez-Gómez J. A. An. R. Acad. Nac. Farm. 2010, 76, 59-84.

${ }^{19}$ Alizadeh, A. M.; Shiri, S.; Farsinejad, S. Tumor Biol. 2014, 35, 8483-8523

${ }^{20}$ Martin, T. A.; Ye. L.; Sanders, A. J.; Jiang, W. G. Bioscience, 2013, 135-168.
} 


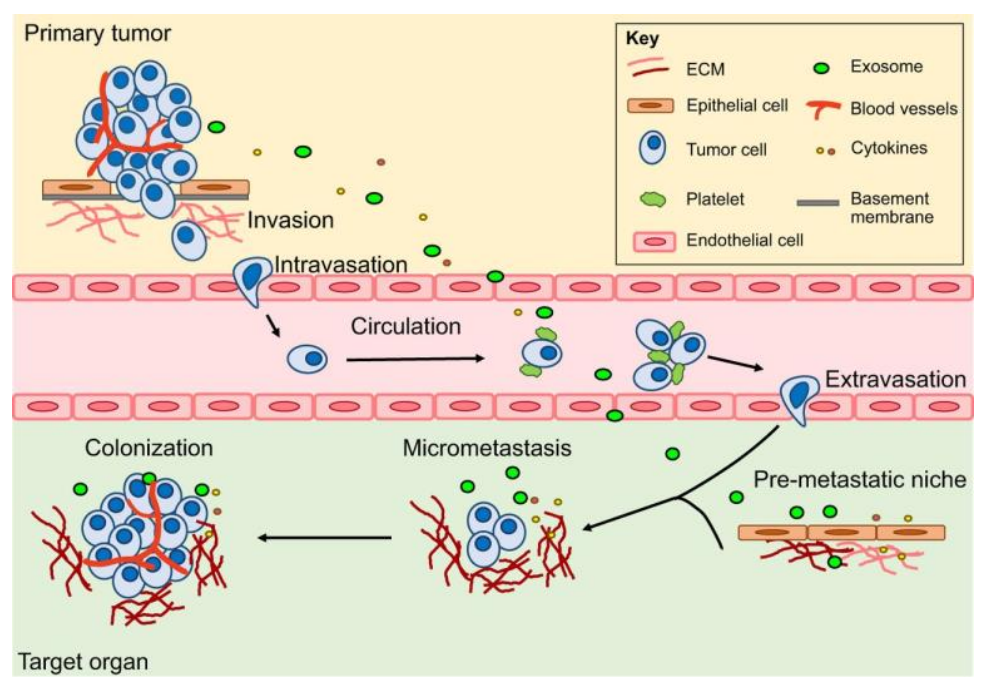

Figure 1.5. Metastasis process. ${ }^{21}$

\subsubsection{Resistance}

There are different mechanisms that make tumors to develop resistance: drug target modification, drug pump expression, detoxification mechanisms, reduced apoptosis susceptibility, increased ability to repair DNA damage or altered proliferation. These often occur together, making the combat more complicated $^{22}$ and limiting the effectiveness of both classical chemotherapy and targeted therapy. Resistance can be intrinsic, if it is already present before treatment, or acquired, if it is consequence of various adaptive responses induced by therapy. Single-agent resistance is limited to the drugs to which patients are exposed, while multidrug resistance (MDR) refers to multiple failures when responding to drugs with different mechanisms of action. ${ }^{23}$ The latter is a common resistance mechanism in tumors and is related to an overexpression of MDR1 gene, which codifies P-glycoprotein.

${ }^{21}$ Gómez-Cuadrado, L.; Tracey, N.; Ma, R.; Qian, B.; Brunton, V. G. DMM 2017, 10, 1061-1074.

22 Holohan, C.; Schaeybroeck, S. V.; Longley, D. B.; Johnston, P. G. Nat. Rev. Cancer 2013, 13, 714-726.

${ }^{23}$ Gottesman, M. M.; Lavi, O.; Hall, M. D.; Gillet, J.-P. Annu. Rev. Pharmacol. Toxicol. 2016, 56, 85-102. 
P-glycoprotein is a transport protein involved in the complex ABC (ATPbinding cassette) and present in cell surface. It is usually responsible for toxin removal in non-tumor cells. However, in mutated cells it is overexpressed and it expulses a wide variety of molecules. One of the strategies to overcome drug resistance proposes the use of P-glycoprotein inhibitors, such as tariquidar, biricodar or elacridar. ${ }^{24}$

Figure 1.6 depicts the mechanism of action of P-glycoprotein. This acts as an ATP-dependent extrusion pump and takes advantage of the energy delivered in ATP hydrolysis to change its conformation and remove the drug. Pglycoprotein inhibitors act as competitors and avoid the expulsion blocking the exit channel of the protein.

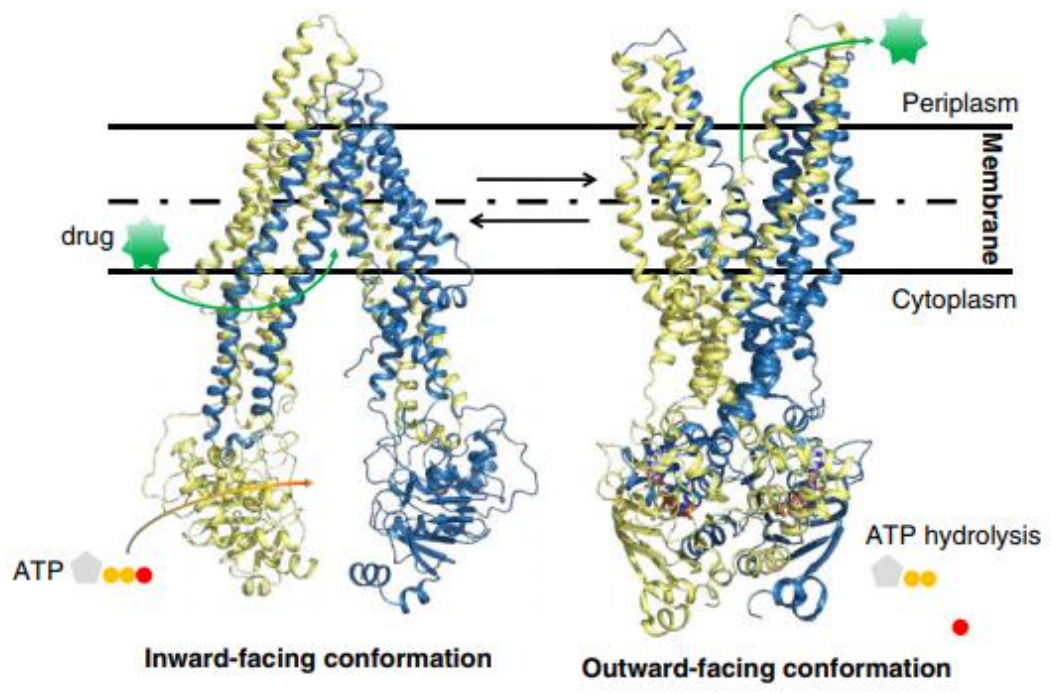

Figure 1.6. Conformational changes of $A B C$ exporters. ${ }^{25}$

\footnotetext{
${ }^{24}$ a) Gottesman, M. M.; Fojo, T.; Bates, S. E. Nat. Rev. Cancer 2002, 2, 48-58. b) Ozben, T. FEBS Lett. 2006, 580, 2903-2909.

${ }^{25}$ Martinez, L.; Falson, P. Advances in Cellular and Molecular Otolaryngology 2014, 2, 23955.
} 


\subsection{Antineoplastic treatments}

The possibilities offered by cancer treatment have changed over the last decades. Conventional methods include surgery, radiotherapy, chemotherapy or endocrine therapy, which can be used isolated or in combination. However, the lack of selectivity and specificity, the appearance of drug resistance and the inability to penetrate aggressive solid tumors explain the research of new therapies able to overcome the disadvantages mentioned. ${ }^{26}$ Among the upcoming therapies, ${ }^{27}$ more selective methods can be found such as monoclonal antibodies, small molecules inhibitors, antiangiogenics, immunotoxins and viral therapy.

Anticancer drugs development has been strongly focused on the action against a specific target with high potency and selectivity. Clinical experience in cancer chemotherapy has disclosed that single targeting might not always produce the desired biological effect, even if the target is inactivated or inhibited. $^{28}$ Thus, multi-targeting therapeutics arises with two contrasting strategies. The first is referred to combination drug therapy by creating an additive or synergistic effect of multiple drugs acting on separate targets. The second strategy involves discovering a single agent that can act on two or more targets simultaneously. ${ }^{29}$

\subsubsection{Antimitotic strategy}

\section{Cell cycle}

The cell cycle is an ordered and regulated process that allow cells to grow and divide into two daughter cells. In eukaryotic cells, two general stages are

\footnotetext{
${ }^{26}$ Urruticoechea, A.; Alemany, R.; Balart, J.; Villanueva, A.; Vinals, F.; Capella, G. Curr. Pharm. Des. 2010, 16, 3-10.

${ }^{27}$ Baudino, T. A. Curr. Drug Discov. Tech. 2015, 12, 3-20.

${ }^{28}$ Boran, A. D.; lyengar, R. Curr. Opin. Drug Discov. Devel. 2010, 13, 297-309.

${ }^{29}$ Raghavendra, N. M. et al. Eur. J. Med. Chem. 2018, 143, 1277-1300.
} 
found: the interphase, where cells grow and replicate their DNA; and M phase or cell division, which comprises mitosis and cytokinesis.

The cycle starts with $\mathrm{G}_{1}$ (gap 1) phase, in which cell synthesizes RNA and proteins. Then, during $S$ (synthesis) phase, DNA and centrosomes are replicated. In $\mathrm{G}_{2}$ (gap 2) phase, cell continues growing and acquires energy in terms of ATP in order to posteriorly afford cell division.

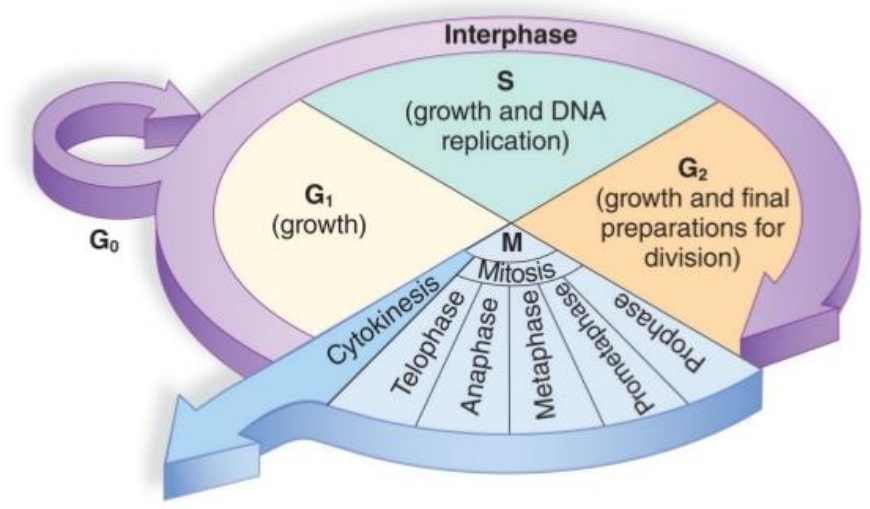

Figure 1.7. Cell cycle stages. ${ }^{30}$

The cycle ends with $M$ phase, constituted by mitosis, where the genetic material is distributed, and cytokinesis or cytoplasm division. At the same time, mitosis is divided into 5 steps: prophase, prometaphase, metaphase, anaphase and telophase, which are represented in figure 1.8.

In prophase, chromosomes are individualized and differentiated within the nucleus and centrosomes start moving to the opposite poles of the cell. In prometaphase, nuclear membrane disappears, and microtubules form the mitotic spindle which attaches to centromeres of chromosomes. During metaphase, sister chromatids are aligned and located in the equatorial plane of the cell. In anaphase, the mitotic spindle pulls each sister chromatid to one pole. Finally, in telophase, the mitotic spindle disappears and nuclear membrane is reconstituted forming two different nuclei.

\footnotetext{
${ }^{30}$ Copyright (C) McGraw-Hill Education.
} 


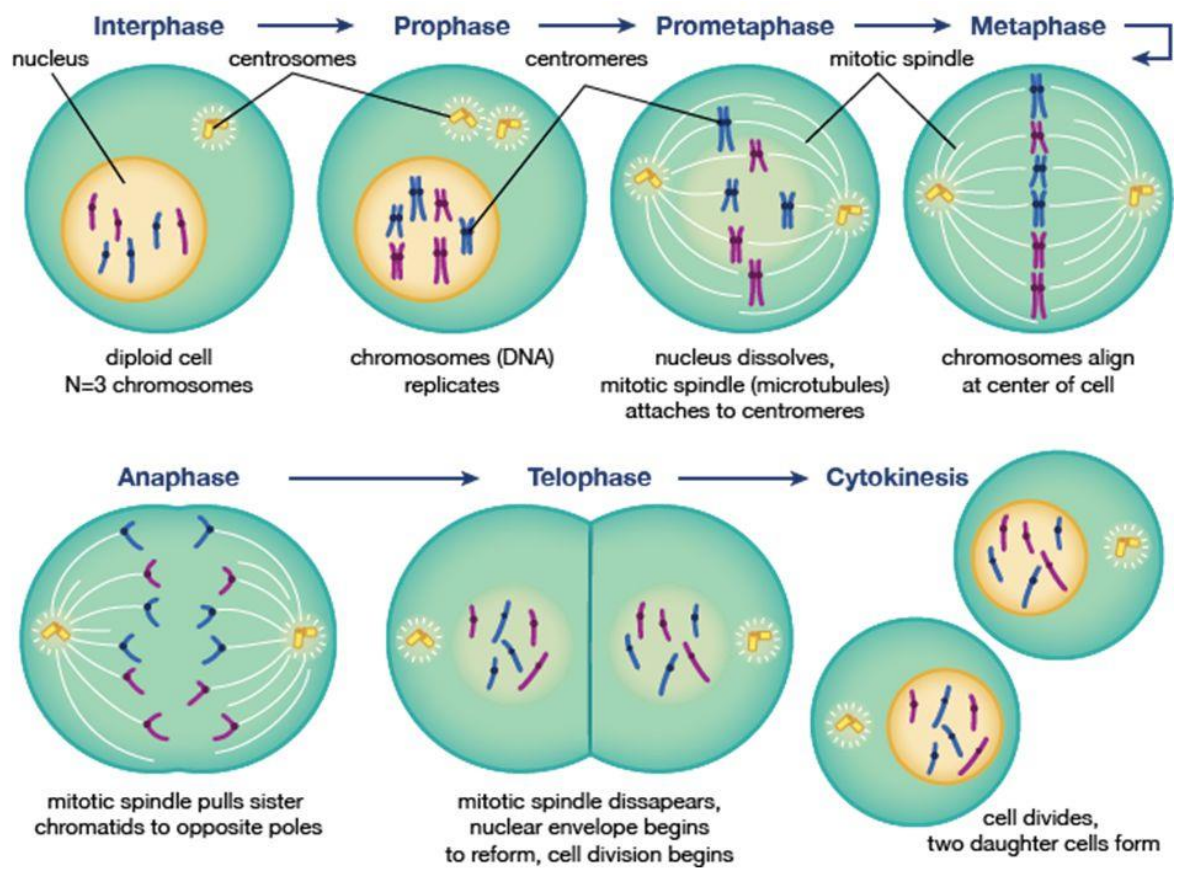

Figure 1.8. Mitosis phases in eukaryotic cell. ${ }^{31}$

The absence of mitogenic signalling factors provokes the cessation of cell proliferation and cells enter in a resting state known as $\mathrm{G}_{0}$. As an example, neurons remain permanently in this stage of non-proliferation.

Throughout the cell cycle there are mechanisms of control known as checkpoints that can arrest the process if the conditions required by the division are not appropriate. ${ }^{32}$ Concretely, this regulation is driven by CDK proteins (Cyclin-Dependent Kinases), which are capable of recognizing the defects in DNA replication or in the chromosome scission and repair them or stop the division process. ${ }^{33}$ In figure 1.9, three internal checkpoints are represented.

\footnotetext{
${ }^{31} \mathrm{https}: / /$ www.thinglink.com/scene/596109759946424320

32 De Maria, E.; Fages, F.; Rizk, A.; Soliman S. Theor. Comput. Sci. 2011, 21, 2108-2127.

${ }^{33}$ Malumbres, M.; Barbacid, M. Nat. Rev. Cancer 2009, 9, 153-166.
} 


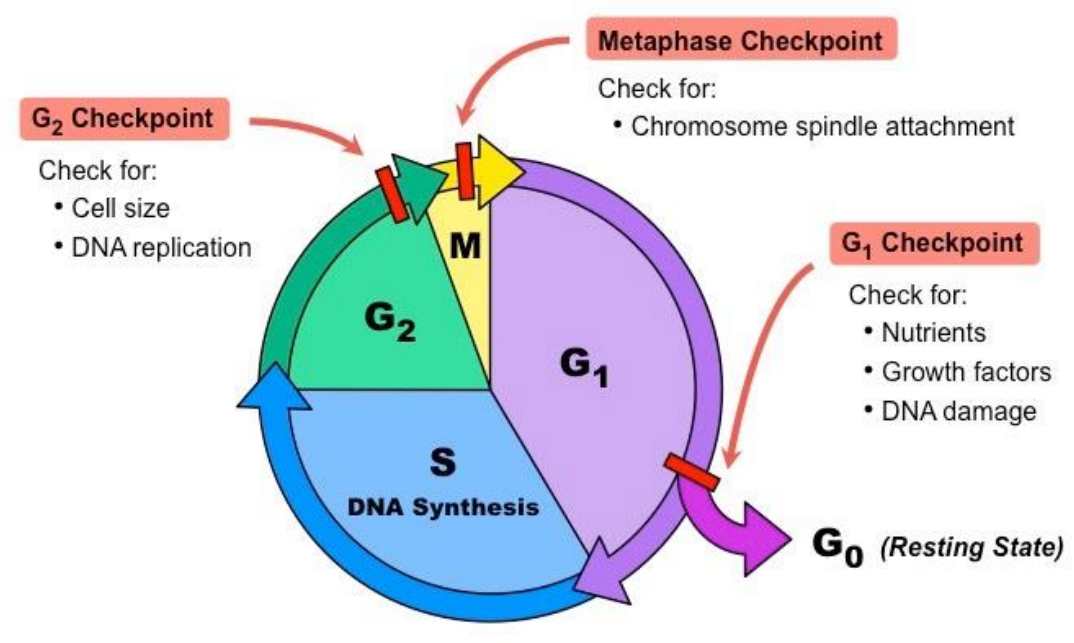

Figure 1.9. Cell cycle checkpoints. ${ }^{34}$

Cancer is considered a cell cycle-dependent disease, since the dysregulation of the cycle is a frequent alteration during tumor development. Both overexpression of cycle activators and suppression of negative regulators can originate cancer. ${ }^{35}$

\section{Tubulin and microtubules}

Several chemotherapeutic agents exert their cytotoxic effect over the mitotic spindle. This is constituted by microtubules which are, at the same time, formed by tubulin.

Tubulin is a heterodimeric protein composed by two subunits ( $\alpha$ and $\beta$ ) with obout 450 amino acids, non-covalently connected. ${ }^{36}$ The most remarkable difference between them resides in the loop S9-S10, which contains eight amino acids more in a subunit than in $\beta$. Both subunits have one GTP molecule associated. In $\alpha$, it is fixed and is not exchangeable, while in $\beta$, it can be

\footnotetext{
${ }^{34}$ Image Credits: Ginsnet.

${ }^{35}$ Park, M.-T.; Lee, S.-J. J. Biochem. Mol. Biol. 2003, 36, 60-65.

${ }^{36}$ Ducki, S.; Mackenzie, G.; Greedy, B.; Armitage, S.; Fournier Dit Chabert, J.; Bennett E.; Nettles, J.; Snyder, J.; Lawrence, Nicholas J., Bioorg. Med. Chem. Lett. 2009, $17,7711$.
} 
hydrolysed to GDP and posteriorly exchanged by GTP. ${ }^{37}$ Figure 1.10 represents the three-dimensional structure of a tubulin heterodimer.

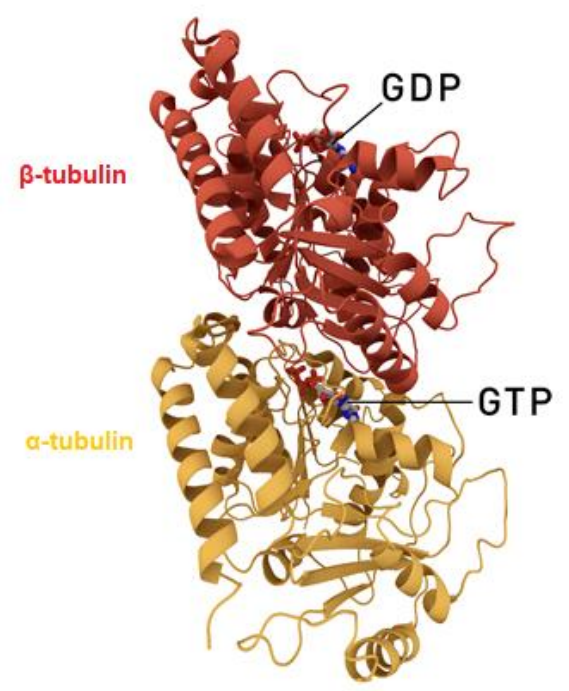

Figure 1.10. Tubulin heterodimer. ${ }^{38}$

Polymerization of tubulin dimers originates protofilaments, and the connection of 13 protofilaments generates the microtubules. These are cylindric structures with a diameter of $25 \mathrm{~nm}$ and a variable length of 25-200 $\mu \mathrm{m}$. Microtubules can be classified in stable microtubules, which constitute cilia and flagella; and dynamic microtubules, located in the cytoplasm. The latter assemble the mitotic spindle and are responsible of chromosome separation during mitosis. They are also involved in the movement of organelles and in the organization of vesicular traffic. ${ }^{39}$

Each protofilament has its structural polarity. Thus, a-tubulin is placed in the minus end (-) and $\beta$-tubulin in the plus end (+). The new tubulin dimers are joined to $\beta$-tubulin with greater efficiency than to $\alpha$-tubulin, so the plus end is the

${ }^{37}$ (a) Nogales, E.; Wolf, S. G.; Downing, K. H. Nature 1998, 391, 199-203. (b) Janke, C. J. Cell Biol. 2014, 206, 461-472.

38 By Thomas Splettstoesser (www.scistyle.com) - Own work (rendered with Maxon Cinema 4D), CC BY-SA 4.0, https://commons. wikimedia.org/w/index.php?curid=41014713

39 Jordan, M. A.; Wilson L. Nat. Rev. Cancer 2004, 4, 253-265. 
preferential site for microtubule growth. Longitudinal contacts between protofilaments are established by polar hydrophobic interactions.

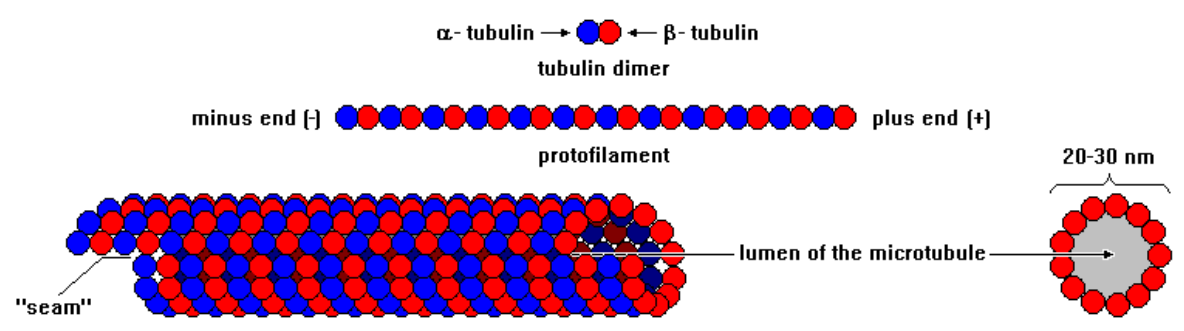

Figure 1.11. Protofilament and microtubule structures. ${ }^{40}$

Microtubules are arranged placing their plus end (+) free in the cytoplasm while the minus end (-) is associated with the centrosome or MicroTubuleOrganizing Center (MTOC).$^{41}$ Centrosome is constituted by two centrioles and the Pericentriolar Material (PCM), which is composed by $\mathrm{y}$-tubulin and forms a ring-shape matrix that allows microtubule growth. ${ }^{42}$

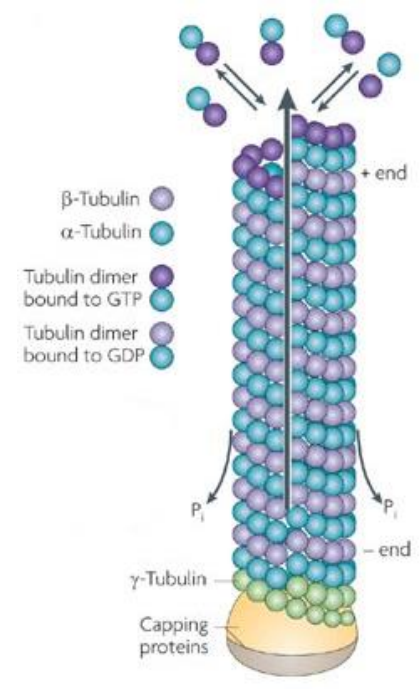

Figure 1.12. Microtubule assembly. ${ }^{43}$

\footnotetext{
40 https://ca.m.wikipedia.org/wiki/Fitxer:Mikrotubula007_en.png

${ }^{41}$ Orr, G.; Verdier-Pinard, P. Oncogene 2003, 22, 7280-7295.

42 Warner, S. L.; Gray, P. J.; Von Hoff, D. D. Sem. Oncol. 2006, 33, 436-448.

${ }^{43}$ Conde, C.; Cáceres, A. Nat. Rev. Neurosci. 2009, 10, 319-332.
} 
Microtubule polymerization dynamics plays a crucial role in cell function. When a new tubulin dimer is added to the plus end (+) of the growing microtubule, the catalytic domain of a-tubulin contacts with the GTP of the previously incorporated $\beta$-tubulin hydrolysing it to GDP. As a result, microtubules are mostly composed by $\beta$-tubulin GDP, except in the plus end (+), where there is a GTP cap. The loss of this cap provokes microtubule depolymerization and release of $\alpha, \beta$-tubulin dimers to cell medium. The alternance between polymerization and depolymerization processes is known as dynamic instability of microtubules. ${ }^{44}$ As it is shown in figure 1.13, the growth step is known as rescue while the shrinking is called catastrophe. ${ }^{45}$ These dynamic properties are regulated by Microtubule Associated Proteins (MAP) and are the responsible of chromosome segregation during cell division. ${ }^{46}$

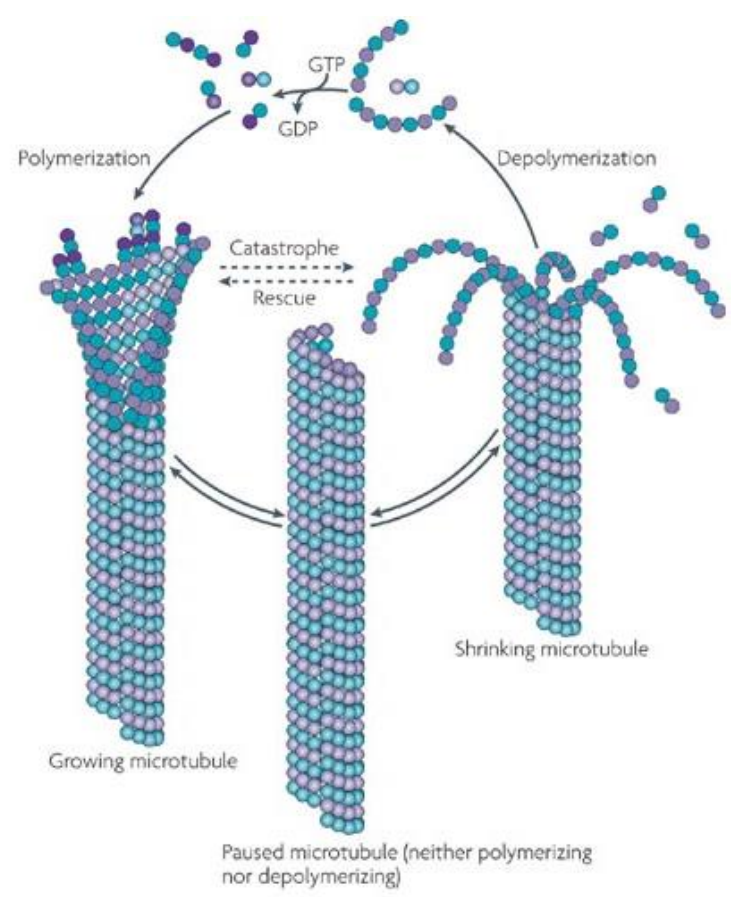

Figure 1.13. Dynamics of microtubules..$^{43}$

\footnotetext{
44 Jordan, M. A.; Wilson, L. Nat. Rev. Cancer 2004, 4, 253-265.

45 Nogales, E. Annu. Rev. Biophys. Biomol. Struct. 2001, 30, 397-420.

${ }^{46}$ Honore, S.; Pasquier, E.; Braguer, D. Cell. Mol. Life Sci. 2005, 62, 3039-3056.
} 


\section{Antimitotic agents}

Compounds that bind to tubulin can stabilize or destabilize microtubule formation causing mitotic arrest and cell death. A lot of antitumoral drugs whose biological target is the tubulin are natural products or derivatives thereof. ${ }^{47}$ The most of these drugs interact with $\beta$-tubulin stabilizing or destabilizing the microtubules. Compounds that interact with a-tubulin only develop their pharmacological action destabilizing the microtubules. ${ }^{48}$ Figure 1.14 represents the classification of these agents and their binding sites on tubulin.

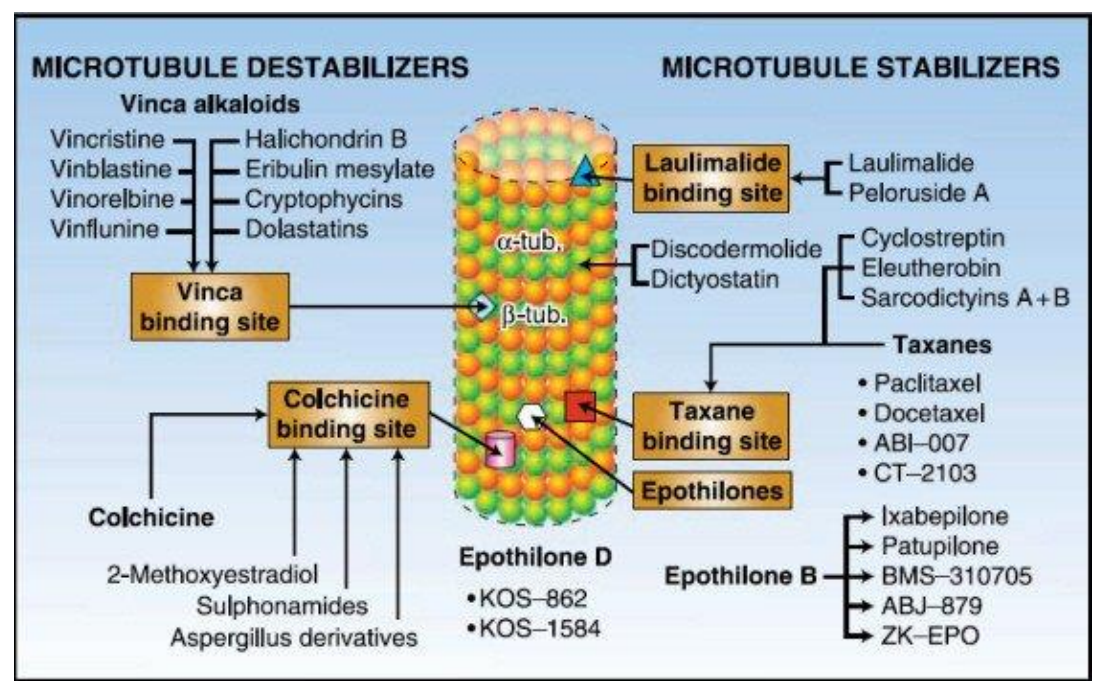

Figure 1.14. Microtubule destabilizers and stabilizers. ${ }^{49}$

\footnotetext{
${ }^{47}$ Mani, S.; Macapinlac, M. Jr.; Goel, S.; Verdier-Pinard, D.; Fojo, T.; Rothenberg, M.; Colevas, D. Anticancer Drugs 2004, 15, 553-558.

48 (a) Sánchez-Pedregal, V. M.; Griesinger, C. Top. Curr. Chem. 2009, 286, 151-208. (b) Nettles, J. H.; Downing, K. H. Top. Curr. Chem. 2009, 286, 209-257. (c) Botta, M.; Forli, S.; Magnani, M.; Manetti, F. Top. Curr. Chem. 2009, 286, 279-328. (d) Dumontet, C.; Jordan, M. A. Nat Rev Drug Discov. 2010, 9, 790-803.

${ }^{49}$ Morris, P. G.; Fornier, M. N. Clin. Cancer Res. 2008, 14, 7167-7172.
} 


\section{Microtubule stabilizing agents}

Microtubule stabilizing agents stop cell division by blocking the depolymerization process. These include taxanes, epothilones, eleutherobin, laulimalide, peroluside, discodermolide or sarcodictyins. ${ }^{50}$ Figure 1.15 represents some of these structures.

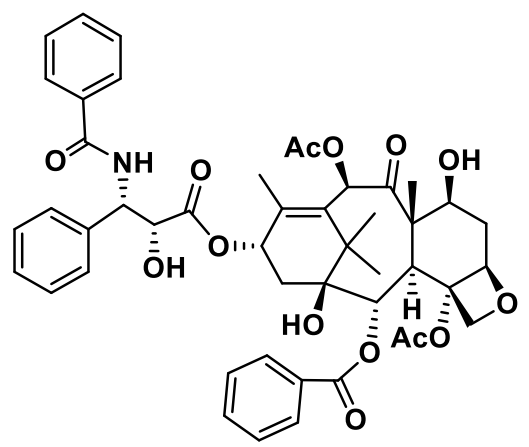

Paclitaxel (Taxol®)<smiles>CO[C@@H](C[C@@H]1O[C@]1(C)CCCC[C@@H](C)[C@@H](O)[C@@H](C)C(=O)C(C)(C)[C@H](O)CC(=O)O)/C(C)=C/c1csc(C)n1</smiles>

Epothilone B

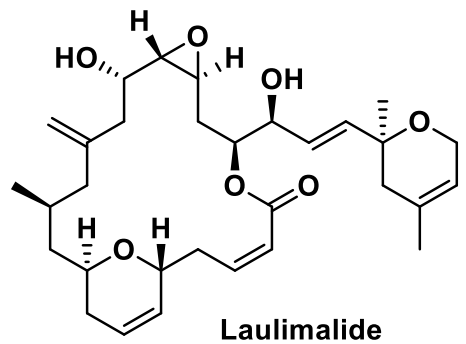

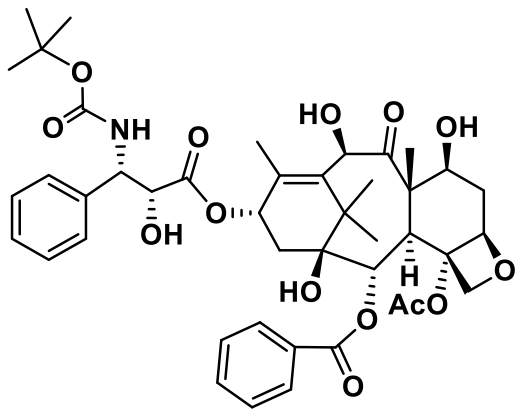

Docetaxel (Taxotere $®)$<smiles>CC(C)=CC[C@H]1C[C@@](C)(C(=O)[C@H](C)[C@@H](O)[C@@H](C)CCC/C(C)=C/c2csc(C)n2)[C@H](O)CC(=O)O1</smiles>

Epothilone D

Figure 1.15. Structure of some microtubule stabilizing agents.

${ }^{50}$ Prota, A. E.; Bargsten, K.; Northcote, P. T.; Marsh, M.; Altmann, K-H.; Miller, J. H.; Diaz, J. F.; Steinmetz, M. O. Angew. Chem. Int. Ed. 2014, 53, 1621-1625. 
Paclitaxel $($ Taxol®) is a diterpenic metabolite isolated in the 60 s by Wall and Wani from the bark of Taxus brevifolia tree. It was approved by the FDA in 1992 to treat breast and refractory ovarian cancer. Its success generated a great interest in searching better analogues, such as docetaxel, which is more water soluble than its predecessor and is used in the treatment of breast, lung, head and neck, stomach and prostate cancers. ${ }^{51}$ Figure 1.16 represents the binding site of paclitaxel in $\beta$-tubulin subunit. This interaction changes microtubule conformation and increases polymerization process. ${ }^{52}$

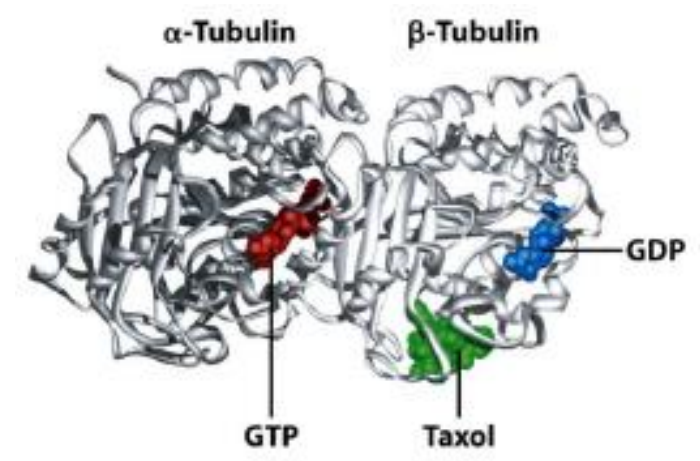

Figure 1.16. Paclitaxel binding site.

Epothilones are isolated from gram-negative bacteria Sorangium cellulosum. ${ }^{53}$ They present some advantages over taxanes, since they maintain their activity in cells expressing P-glycoprotein ${ }^{54}$ and they are able to cross the blood-brain barrier (BBB). ${ }^{55}$

Laulimalide is extracted from marine sponges in Pacific Ocean ${ }^{56}$ and pelorudise $\mathrm{A}$ is obtained from Mycale hentscheli, a marine sponge of New

51 Kingston, D. G. I. J. Nat. Prod. 2009, 72, 507-515.

52 Schiff, P. B., Fant, J. Horwitz, S. B. Nature 1979, 277, 665-667.

53 Gerth, K.; Bedorf, N.; Hoefle, G.; et al. J. Antibiot. 1996, 49, 560-563.

${ }^{54}$ Fojo, T.; Menefee, M. Ann. Oncol. 2007, 18 (Suppl 5), v3-8.

55 Hoffmann, J.; Fichtner, I.; Lemm, M.; Lienau, P.; et al. Neuro. Oncol. 2009, 11,158-166.

56 (a) Quiñoa, E.; Kakou, Y.; Crews, P. J. Org. Chem. 1988, 53, 3642-3644. (b) Corley, D. G.; Herb, R.; Moore, R. E.; Sheuer, P. J.; Paul, V. J. J. Org. Chem. 1988, 53, 3644-3646. 
Zealand. Both are macrolides that have shown potent antiproliferative activity in cell cultures and tumor growth inhibition in mice. ${ }^{57}$

\section{Microtubule destabilizing agents}

Microtubule destabilizing agents stop cell division by facilitating depolymerization process. These include vinca alcaloids such as vinblastine or vincristine and other compounds such as colchicine, combretastatin A-4, 2methoxyestradiol or podophilotoxin. In figure 1.17, the corresponding structures are represented.

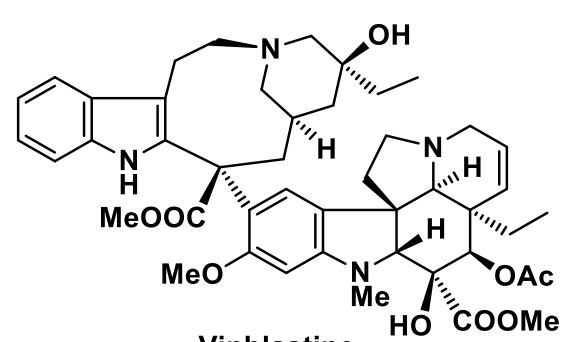

Vinblastine

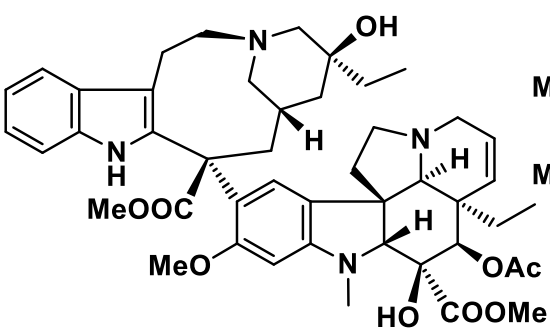

Vincristine<smiles>COc1ccc(/C=C\c2cc(OC)c(OC)c(OC)c2)cc1O</smiles>

Combretastatin A-4

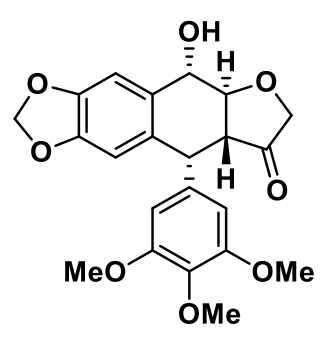

Podophilotoxin

Figure 1.17. Structure of microtubule destabilizing agents.

Vinblastine and vincristine are isolated from Catharanthus roseus commonly named as Madagascar periwinkle. The first one is administrated in non-Hodgkin lymphoma, testicular, breast, lung, head and neck, bladder cancers and

\footnotetext{
${ }^{57}$ Kanakkanthara, A.; Northcote, P. T.; Miller, J. H. Nat. Prod. Rep. 2016, 33, 549-561.
} 
choriocarcinoma. ${ }^{58}$ The second one is employed in treatment of acute leukemia, Ewing sarcoma, multiple myeloma, thyroid cancer and brain tumors. ${ }^{59}$

Colchicine is an alkaloid obtained from Colchicum autumnale that binds to $\beta$-tubulin near GTP subunit of $\alpha$-tubulin (see figure 1.18). ${ }^{60}$ Colchicine interacts with free tubulin forming tubulin-colchicine (T-C) complexes, which block microtubule growth when T-C complexes are attached to the plus end $(+){ }^{61}$

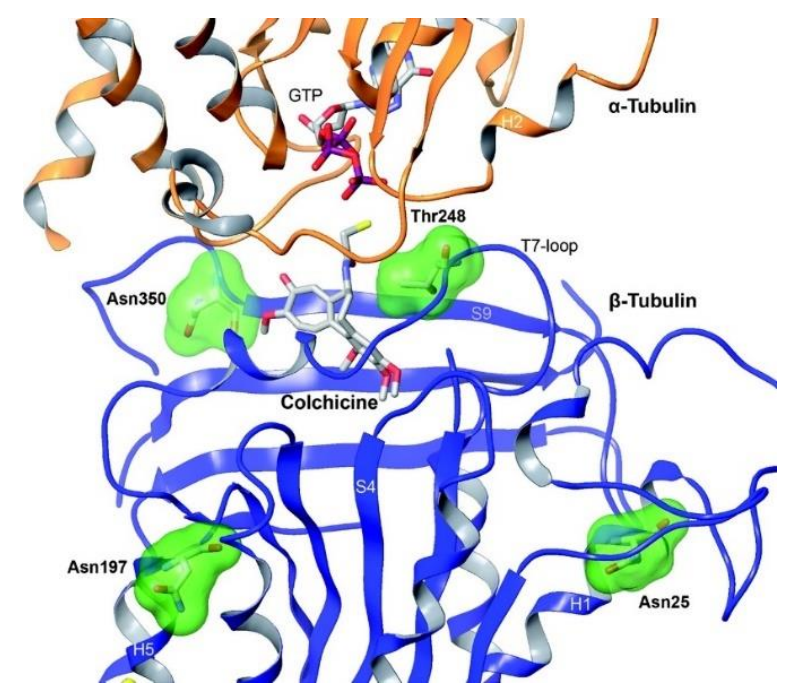

Figure 1.18. Colchicine binding site.

As it has been explained above, there is a high number of antimitotic compounds that interact with $\beta$-tubulin. However, the number of compounds that interact with a-tubulin is relatively low. Some examples are pironetin or hemiasterlin. ${ }^{62}$

58 (a) Klement, G.; Baruchel, S.; Rak, J.; Man, S.; Clark, K.; Hicklin, D. J.; Bohlen, P.; Kerbel, R. S. J. Clin. Invest. 2000, 105, R15-24. (b) Bates, D. J. P.; Salerni, B. L.; Lowrey C. H.; Eastman. A. Cancer. Biol. Ther. 2011, 12, 314-325.

59 (a) Gidding, C. E.; Kellie, S. J.; Kamps, W. A.; et al. Crit. Rev. Oncol. Hematol. 1999, 29, $267-$ 287. (b) Groth-Pedersen, L; Stampe Ostenfeld, M. S.; Høyer- Hansen, M.; Nylandsted, J.; Jäättelä, M. Cancer Res. 2007, 67, 2217-2225.

${ }^{60}$ Liaw, T.; Salam, N.; McKay, M.; Cunningham, A.; Hibbs, D.; Kavallaris, M. Mol. Cancer Ther. 2008, 7, 3150-3159.

61 Keates, R. A.; Mason, G. B. Can. J. Biochem. 1981, 59, 361-370.

62 Sarabia, F.; García-Castro, M.; Sánchez-Ruiz, A. Curr. Bioact. Comp. 2006, 2, 269-299. 

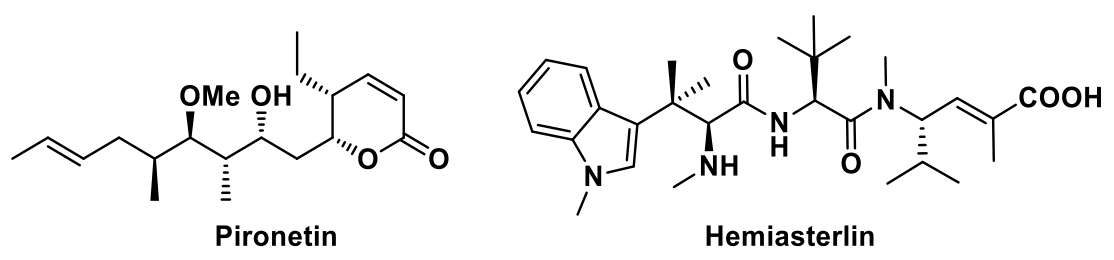

Figure 1.19. Antimitotic compounds interacting with $\alpha$-tubulin.

\section{Combretastatins}

Combretastatins are a family of natural products that exhibit significant antimitotic properties. Combretastatins A-1, A-2 and A-3 were isolated in 1987 from the bark of Combretum caffrum tree in South Africa and India by Petit and collaborators. ${ }^{63}$ Combretastatin A-4 was isolated by the same team in $1989 .{ }^{64}$<smiles>COc1ccc(/C=C\c2cc(OC)c(OC)c(OC)c2)c(O)c1O</smiles><smiles>COc1ccc(/C=C\c2cc(OC)c3c(c2)OCO3)cc1O</smiles><smiles>COc1ccc(/C=C\c2cc(O)c(OC)c(OC)c2)cc1O</smiles><smiles>COc1ccc(/C=C\c2ccc(OC)c(OC)c2)cc1OC</smiles>

Figure 1.20. Combretastatins family.

Combretastatins induce cell death by selectively binding to tubulin blocking cell cycle in the transition from metaphase to anaphase. ${ }^{65}$ Concretely, combretastatin A-4 is the most potent antitumoral agent in the combretastatins family. Structure-activity relationship studies have proven that cis-stilbene

\footnotetext{
${ }^{63}$ (a) Pettit, G. R.; Cragg, G. M.; Singh, S. B. J. Nat. Prod. 1987, 50, 386-391. (b) Pettit, G. R.; Singh, S. B. Can. J. Chem. 1987, 65, 2390-2396.

64 Pettit, G. R.; Singh, S. B.; Hamel, E.; Lin, C. M.; Alberts, D. S.; Garcia-Kendall, D. Experientia 1989, 45, 209-211.

${ }^{65}$ Fürst, R.; Zupkó, I.; Berényi, A.; Ecker, G.; Rinner, U. Bioorg. Med. Chem. Lett. 2009, 19, 69486951.
} 
system of combretastatin A-4 places the aromatic rings with an inclination of $50^{\circ}-$ $60^{\circ}$ between them, which is essential for the interaction with the colchicine binding site. ${ }^{66}$ Trimethoxy phenyl ring $(A)$ of combretastatin A-4 is fundamental in its cytotoxic ability and tubulin polymerization inhibition. It has been demonstrated that substitutions in this ring originate derivatives with less activity (see figure 1.21). ${ }^{67}$

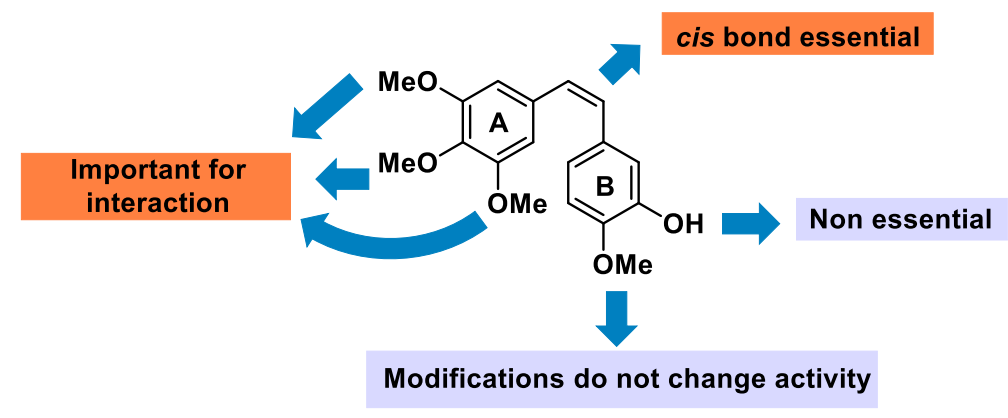

Figure 1.21. Structure-activity relationship in combretastatin A-4.

Functional modifications in ring $B$ exert less influence in biological activity of combretastatin A-4. The replacement of methoxy group does not generate better compounds. The substitution of hydroxy group by bromo maintains antimitotic potency and the introduction of amino groups improves water solubility. ${ }^{68}$ The exchange of ring $B$ by rings with less than 6 members provokes a loss in activity. However, its replacement by bicyclic systems do not decrease potency. ${ }^{69}$

Recently, Steinmetz and co-workers have confirmed, by the resolution of the crystalline structure of a tubulin-combretastatin A-4 complex, that the binding

\footnotetext{
${ }^{66}$ Woods, J. A.; Hadfield, J. A.; Pettit, G. R.; Fox, B. W.; McGown, A. T. Br. J. Cancer, 1995, 71, 705-711.

67 (a) Gaukroger, K.; Hadfield, J.; Lawrence, N. J.; Nolan, S.; McGown, A. T. Org. Biomol. Chem. 2003, 1, 3033-3037. (b) Álvarez-Lozano. Antimitotics related to combretastatins and phenstatins. Doctoral Thesis. 2009. University of Salmanca.

68 Tron, G.; Pirali, T.; Sorba, G.; Pagliai, F.; Busacca, S.; Genazzani, A. J. Med. Chem. 2006, 49, 3033-3044.

${ }^{69}$ Maya, A. B.; Pérez-Melero, C.; Mateo, C.; Alonso, D.; Fernández, J. L.; Gajate, C.; Mollinde, F.; Pélaez, R.; Caballero, E.; Medarde, M. J. Med. Chem. 2005, 48, 556-558.
} 
site of combretastatin A-4 to $\beta$-tubulin is the colchicine site. In figure 1.22 , it is represented the structure of the quaternary complex formed by combretastatin A-4 bound to two heterodimers of tubulin, which are stabilized by tubulin-tyrosine ligase (TTL) and RB3 protein. ${ }^{70}$

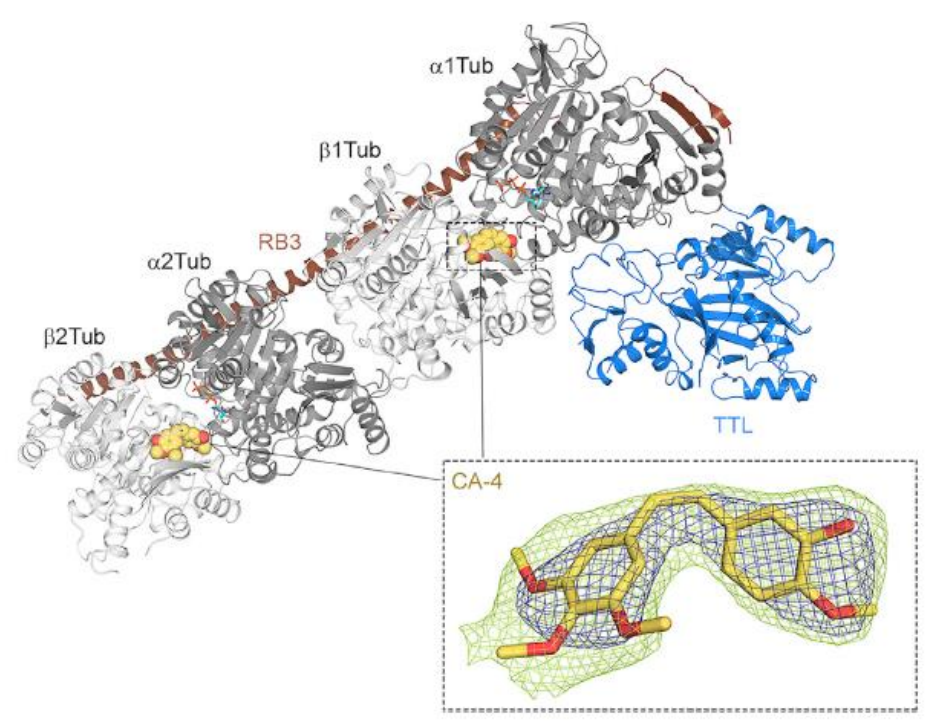

Figure 1.22. Tubulin-combretastatin A-4 complex.

Tubulin dimers adopt a straight structure when form microtubules but a curved structure when remain free. Combretastatin A-4 exerts its action inhibiting the curved-straight structural transition.

Computational calculations ${ }^{70}$ have proven that trans isomer cannot be adapted to colchicine site and in vitro studies have shown that it barely presents antimitotic activity. However, it is convenient to indicate that combretastatins easily suffer isomerization from cis to trans configuration during their storage, administration or solution in protic solvents. ${ }^{71}$

${ }^{70}$ Gaspari, R.; Prota, A. E.; Bargsten, K.; Cavalli, A.; Steinmetz, M. O. Chem. 2017, 2, 102-113.

${ }^{71}$ Schobert, R.; Effenberg-Neidnicht, K.; Biersack, B. Int. J. Clin. Pharmacol. Ther. 2011, 49, 71. 
Consequently, this configurational instability has led to the development of synthetic strategies to prepare more stable analogues. ${ }^{72} \mathrm{~A}$ huge variety of rigid combretastatin A-4 analogues that keep both aromatic rings in cis position has been synthetized and evaluated. Moreover, $Z$ geometry can be also maintained by introducing functional groups such as ketone, ether, nitrile or sulfonate. ${ }^{73}$

Combretastatin A-4 shows potent cytotoxic activity against a wide variety of human cancer cells including some MDR cancer cell lines. ${ }^{74}$ However, its high lipophilicity and low water-solubility have limited in vivo antitumoral efficacy. ${ }^{75}$ Thus, the corresponding phosphate ester (CA-4P) has been synthetized. This is currently in phase II clinical trials in the United States and United Kingdom for the treatment of solid tumors. ${ }^{76}$

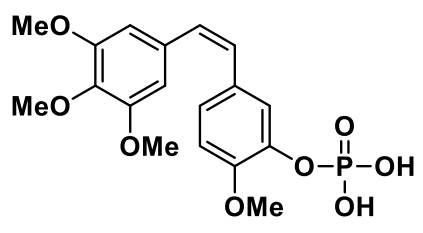

Figure 1.23. CA-4P structure.

In 1998, K. Ohsumi and collaborators prepared a series of azo-derivatives of combretastatin and evaluated their effect in tubulin polymerization and their cytotoxicity against murine Colon 26 adenocarcinoma. Among all the evaluated

72 (a) Nam, N. H. Curr Med Chem. 2003, 10, 1697-1672. (b) Chaudhary, A.; Pandeya, S. N.; Kumar, P.; Sharma, P. P.; Gupta, S.; Soni, N.; Verma, K. K.; Bhardwaj, G. Mini Rev. Med. Chem. 2007, 7, 1186-205.

${ }^{73}$ Chaudhary, V.; Venghateri, J. B.; Dhaked, H. P. S.; Bhoyar, A. S.; Guchhait, S. K.; Panda, D. J. Med. Chem. 2016, 59, 3439-3451.

74 (a) McGown, A. T.; Fox, B. W. Cancer Chemother. Pharmacol. 1990, 26, 79-81. (b) El-Zayat, A. A. E.; Degen, D.; Drabek, S.; Clark, G. M.; Pettit, G. R.; Von Hoff, D. D. Anti-Cancer Drugs 1993, 4, 19-25.

${ }^{75}$ Dorr, R. T.; Dvorakova, K.; Snead, K.; Alberts, D. S.; Salmon, S. E.; Pettit, G. R. Invest. New Drugs 1996, 14, 131-137.

${ }^{76}$ (a) https://www.clinicaltrials.gov/ct2/show/NCT01305213?term=Zybrestat\&rank=1

(b) https://www.clinicaltrials.gov/ct2/show/NCT00653939?term=Zybrestat\&rank=4 
compounds, aminocombretastatin (AmCA-4) showed the best $\mathrm{IC}_{50}$ value (5.1 $\mathrm{nM})$ in the tumoral cell line indicated above. ${ }^{77}$

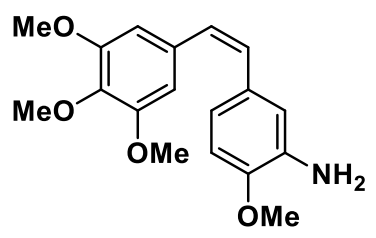

Figure 1.24. AmCA-4 structure.

Apart from its antimitotic properties, combretastatin A-4 acts as a Vascular Disrupting Agent (VDA) causing modifications in cytoskeleton and, therefore, in the morphology of endothelial cells. These alterations explain the antiangiogenic activity of combretastatin A-4, since vascular permeability is increased and the tumor blood flow is disrupted provoking ischemic necrosis. ${ }^{78}$

Several VDAs are in clinical phase, among which derivatives of combretastatin A-4 or plinabulin deserve to be highlighted. ${ }^{79}$ Although all these compounds bind to colchicine binding site of tubulin, its mechanism of action differs from that of other compounds that also bind to tubulin, such as taxanes or vinca alkaloids. While they mainly exert a cytotoxic action against a wide range of cells, the compounds indicated in figure 1.25 mainly exert an antivascular effect. Although they have a certain cytotoxic activity, it is not as marked as in the rest of antimitotic drugs, which entails less adverse effects.

77 Ohsumi, K.; Nakagawa, R.; Fukuda, Y.; Hatanaka, T.; et al. J. Med. Chem. 1998, 41, 30223032.

78 (a) Hori, K.; Saito, S.; Kubota K. Br. J. Cancer 2002, 86, 1604-1614. (b) West, C. M. L.; Price, P. Anti-Cancer Drugs 2004, 3, 179-187. (c) Kim, T. J., Ravoori, M., Landen, C. N.; Kamat, A. A.; et al. Cancer Res. 2007, 67, 9337-9345. (d) Cai, S. X. Recent Pat. Anticancer Drug Discov. 2007, 2, 79-101. (e) Su, M.; Huang, J.; Liu, S. et al. Sci. Rep. 2016, 6, 1-11.

${ }^{79}$ (a) Tozer, G. M.; Kanthou, C.; Parkins, C. S.; Hill, S. A. Int. J. Exper. Pathol. 2002, 83, 21-38. (b) Kanthou, C.; Tozer, G. M. Anticancer Drugs 1993, 4, 3-17. 
<smiles>COc1ccc(/C=C\c2cc(OC)c(OC)c(OC)c2)cc1O</smiles>

Combretastatin A-4 (CA-4)<smiles>COc1ccc(/C=C\c2cc(OC)c(OC)c(OC)c2)cc1NC(=O)[C@H](N)CO</smiles>

Ombrabulin<smiles>COc1ccc(/C=C\c2ccc(OC)c(OP(=O)(O)O)c2)cc1OC</smiles>

Fosbretabulin (CA-4P)<smiles>CC(C)(C)c1[nH]cnc1/C=C1\NC(=O)C(Cc2ccccc2)NC1=O</smiles>

Plinabulin

Figure 1.25. Structures of the most relevant VDAs.

\subsubsection{Antiangiogenic strategy}

\section{Angiogenesis}

Angiogenesis process involves the creation of new blood vessels from preexisting ones and is essential for wound healing and embryonic development. Vasculogenesis is a similar process that involves de novo formation of blood vessels from progenitor endothelial cells (ECs). ${ }^{80}$ Generally, angiogenesis is deactivated, but ECs start to proliferate when the organism needs it. ${ }^{81}$ Thus, after an angiogenic stimulus, proteolytic enzymes degrade the extracellular matrix and the basement membrane allowing ECs to proliferate and migrate into the perivascular area. Subsequent lumenation of the primary sprouts leads to formation of capillary loops. Finally, a new basement membrane is synthetized, and blood vessels mature to complete tube-like structures (see figure 1.26). ${ }^{82}$

\footnotetext{
${ }^{80}$ Alba, E.; Rodríguez-Quesada, A. Angiogénesis y Cáncer. TACTICS MD, S.L. 2009.

81 Carmeliet, P. Nature 2005, 438, 932-936.

82 Rajabi, M.; Mousa, S. A. Biomedicines 2017, 5, 34.
} 


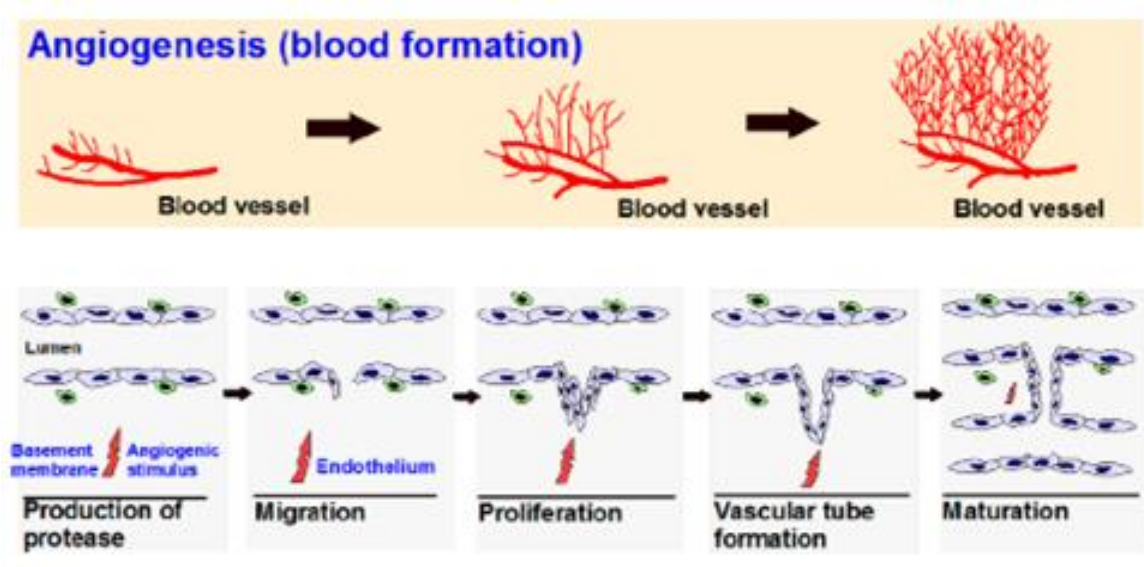

Figure 1.26. Angiogenesis steps.

This process is controlled by an angiogenic switch mechanism, which suggests that changes in the relative balance of inducers and inhibitors can activate or deactivare the switch. In this sense, two types of angiogenesis can be distinguishing: physiological or pathological. ${ }^{83}$ The first one occurs during embryogenesis and continues in the post-natal development. The second one is driven by an irregular persistency of activator signals and is associated with various disorders, such as rheumatoid arthritis, psoriasis, diabetic retinopathy, endometriosis or tumoral growth. ${ }^{84}$

${ }^{83}$ Martínez-Ezquerro, J. D.; Herrera, L. A. Cancerología 2006, 1, 83-88.

${ }^{84}$ Folkman, J.; Shing, Y. J. Biol. Chem. 1992, 267, 10931-10934. 


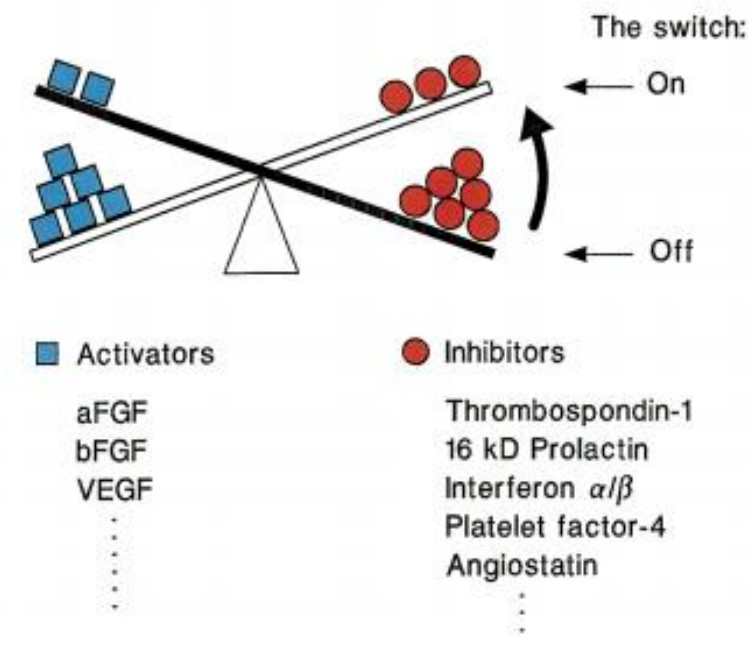

Figure 1.27. The balance hypothesis for the angiogenic switch. ${ }^{85}$

Angiogenesis is switched on if activator molecules (FGF, VEGF...) dominate in the balance. This is associated with obesity, diabetes, cirrhosis, endometriosis, AIDS, bacterial infections or auto-immune diseases. However, if antiangiogenic factors predominate (thrombospondin-1, interferon, platelet factor-4, angiostatin...), angiogenesis is inhibited preventing cicatrisation and regeneration of blood vessels.

Tumor growth is an angiogenesis-dependent process, since tumor cells need a constant supply of nutrients and oxygen to develop and replicate. ${ }^{86}$ When a tumor grows more than 1-2 mm of diameter, cells which are located in the inner side of the tumor do not recibe enough nutrients and are under hypoxic conditions. ${ }^{87}$ This lack of oxygen activates the hypoxia inducible factor (HIF$1 \alpha)^{88}$, which overexpresses different pro-angiogenic factors as the vascular

\footnotetext{
${ }^{85}$ Hanahan, D.; Folkman, J. Cell 1996, 86, 353-364.

${ }^{86}$ Crivellato, E.; Ribatti, D. Recent Advances in Angiogenesis and Antiangiogenesis. Chapter 4: Cross-Linked Between Inflamation and Angiogenesis 2009, 30-39.

${ }^{87}$ Folkman, J. Curr. Mol. Med. 2003, 3, 643-651.

88 (a) Kachs, G. U.; Patterson, A. V. et al. Nat. Med. 1997, 3, 515-520. (b) Fruehauf, J. P.; Meyskens, F. L. Jr. Clin. Cancer Res. 2007, 13, 789-794.
} 
endothelial growth factor (VEGF). ${ }^{89}$ This protein was identified in 1989 by Ferrara and Henzel as the principal factor involved in angiogensis. ${ }^{90}$ It stimulates migration of ECs to hypoxic tumor regions, activating the signaling pathway of angiogenesis. ${ }^{91}$

J. Folkman states that angiogenesis inhibition would stop tumor growth, reducing the size until reaching an inactivity status. ${ }^{92}$ One characteristic of tumoral angiogenesis is the creation of an aberrant vasculature as consequence of a chaotic and bad-regulated expression of proangiogenic factors. Tumor ECs do not develop regular monolayers nor possess a normal endothelial barrier-like function, which leads to porous and disorganized vessels that allow tumor cells move to other tissues and provoke metastasis. ${ }^{93}$

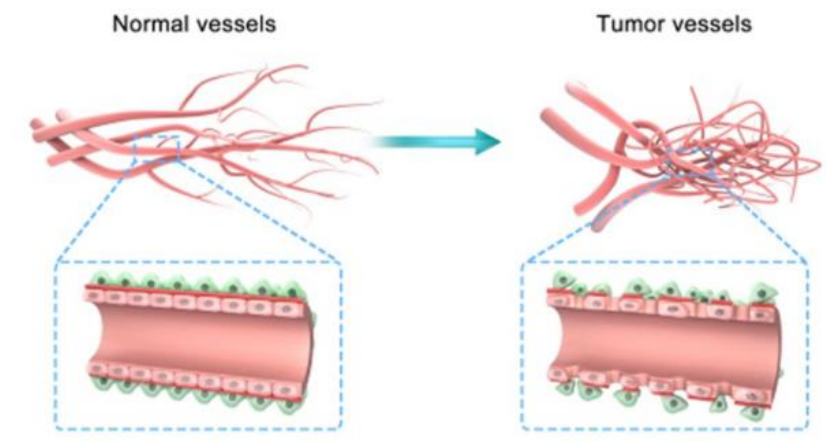

Figure 1.28. Comparison between normal and tumor vessels. ${ }^{94}$

Cancer treatment based on antiangiogenic therapy offers some theoretical advantages over conventional chemotherapy or radiotherapy. Thus, it can be applied to any solid tumor, since angiogenesis takes place in all of them and it

89 (a) Mazure, N. M.; Chen, E. Y.; Laderote, K. R.; Giaccia, A. J. Blood 1997, 90, 3322-3331. (b) Folkman, J. Semin. Oncol. 2002, 29, 15-18. (c) Beppu, K.; Nakamura, K.; Linehan, W. M.; Rapisarda, A.; Thiele, C. J. Cancer Res. 2005, 65, 4775-4781.

90 (a) Ferrara, N.; Henzel, W. J. Biochem. Biophys. Res. Commun. 1989, 161, 851. (b) Kim, K. J.; Li, B.; Winer, J.; Armanini, M.; Gillet, N.; Phillips, H. S.; Ferrara, N. Nature 1993, 362, 841.

91 (a) Darland, D. C.; D'Amore, P. A. J. Clin. Invest. 1999, 103, 157-158. (b) Neufeld, G.; Cohen, T.; Gengrinovitch, S.; Poltorak, Z. FASEB J. 1999, 13, 9-22.

${ }^{92}$ Folkman, J. N. Engl. J. Med. 1971, 285, 1182-1186.

${ }^{93}$ Carmeliet, P.; Jain, R. K. Nature 2000, 407, 249-257.

${ }^{94}$ Abdalla, A.; Xiao, L.; Ullah, M. W.; Yu, M.; Ouyang, C.; Yang, G. Theranostics 2018, 8, 533548. 
can be also utilized in situations in which chemotherapy is not effective, such as in metastatic cancer. Additionally, side effects are reduced since antiangiogenic drugs operate only in activated ECs and development of resistance mechanisms is limited due to the genomic stability presented by ECs. ${ }^{95}$

Nonetheless, it is important to note that angiogenesis inhibitors are more effective when combined with traditional therapies, especially chemotherapy. In fact, the action of antitumor drugs is favored by the use of antiangiogenic agents, which avoid the formation of imperfect capillaries and allow the drugs to circulate through non-porous blood vessels and reach their target. ${ }^{96}$ In specific cases, antiangiogenic treatments are chronically administered to patients since an interruption could accelerate the revascularization and tumor could reappear. ${ }^{97}$

\section{VEGF and VEGFR}

Vascular endothelial growth factors (VEGFs) are a family of homodimeric proteins bound by disulfide bonds, that includes VEGF-A, VEGF-B, VEGF-C, VEGF-D, VEGF-E, VEGF-F and also placental growth factors (PIGF). VEGF-A is the most studied member and is simply denominated by VEGF. Six VEGF-A human isoforms have been described with a different number of amino acids (VEGF 121, 145, 165, 183, 189 and 206), generated as result of the alternative processing of mRNA. ${ }^{98}$ All the isoforms share the same $\mathrm{N}$-terminal fragment and six C-terminal residues. VEGF-121 and VEGF-165 are the most abundant isoforms. The first one is the responsible for increased vascular permeability while the second one is an important stimulator of EC proliferation.

\footnotetext{
95 Mauriz, J. L.; Linares, P.; González, P.; Culebras, J. M. Cir. Esp. 2005, 78, 3-11.

96 https://www.cancer.gov/about-cancer/treatment/types/immunotherapy/angiogenesis-inhibitorsfact-sheet

97 (a) Folkman, J. Nat. Rev. Drug Discov. 2007, 6, 273-286. (b) Ebos, J. M.; Lee, C. R.; Christensen, J. G.; Mutsaers, A. J.; Kerbel, R. S. Proc. Natl. Acad. Sci. U. S. A. 2007, 104, 17069 17074. (c) Mancuso, M. R.; Davis, R.; Norberg, S. M.; O`Brien, S.; Sennino, B.; Nakahara, T.; Yao, V. J.; Inai, T.; Brooks, P.; Freimark, B.; Shalinsky, D. R.; Hu-Lowe, D. D.; McDonald, D. M. J. Clin. Invest. 2006, 116, 2610-2621.

98 Park, J. E.; Keller, G. A.; N. Mol. Biol. Cell. 1993, 4, 1317-1326.
} 
VEGF expression is mediated by tyrosine-kinase receptors known as VEGFRs, which are located in the surface of vascular ECs and cell derived from the bone marrow. These receptors carry an extracellular domain for ligand binding (immunoglobulin-like domain), a transmembrane section, and a cytoplasmic region that contains the tyrosine kinase domains. Figure 1.29 schematically depicts the different members of VEGF family and their relationship with the associated receptors (VEGFRs).

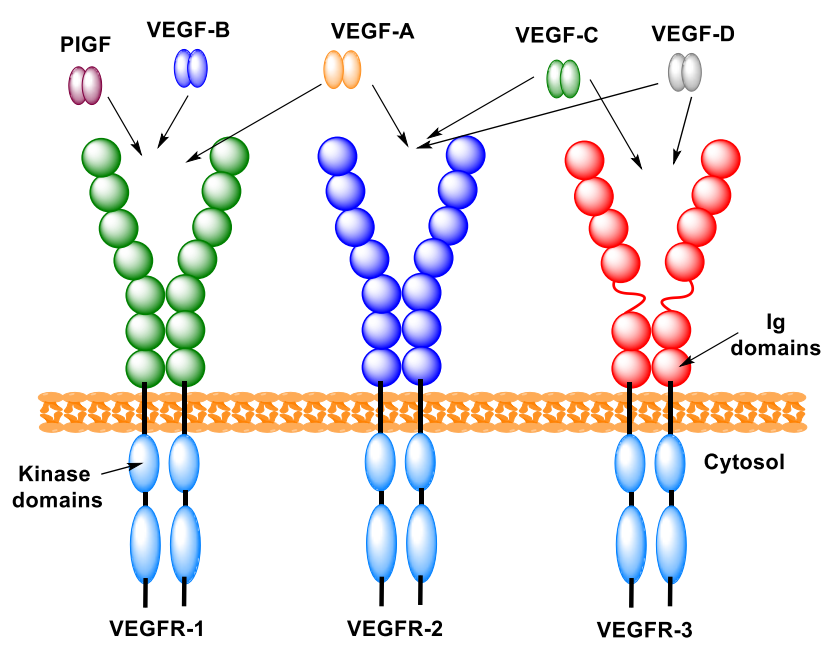

Figure 1.29. VEGF family members and associated receptors. ${ }^{99}$

VEGF-A employs symmetric binding sites in each pole of the dimer to bind to VEGFR-1 or VEGFR-2 and can also introduce heterodimers between both receptors. ${ }^{100}$ VEGF-B binds to VEGFR-1 modulating VEGF-A activity in ECs during embryonic development and pathological angiogenesis. ${ }^{101}$ VEGF-C and VEGF-D bind to VEGFR-2 in ECs of blood vessels and to VEGFR-3 in lymphatic tissues promoting lymphangiogenesis. VEGF-E binds specifically to VEGFR-2

\footnotetext{
${ }^{99}$ Schmidt, T.; Carmeliet, P. Hematology 2011, 1, 1-8.

100 (a) Veikkola, T.; Karkkainen, M.; Cleasson-Welsh, L.; Alitalo, K. Cancer Res. 2000, 60, 203212. (b) Dvorak H. F. Thromb. Haemost. 2005, 3, 1835-1842.

101 Partanen, T. A.; Paavonen, K. Microsc. Res. Tech. 2001, 55, 108-121.
} 
developing a mitogenic effect and promoting vascular permeability, similar to VEGF-A. ${ }^{102}$ VEGF-F function is still under characterization.

The interaction between VEGF and VEGFR induces receptor dimerization and changes its conformation leading to exposure of the ATP-binding site in the intracellular domain, subsequent kinase activation and auto- or transphosphorylation of tyrosine residues on the receptor dimer. The latter step creates binding sites for intracellular signaling mediators allowing the activation of biological responses such as cell proliferation, migration, differentiation and transformation (see figure 1.30). ${ }^{103}$

VEGF is highly expressed in colorectal, liver, lung, thyroid, breast, bladder, ovary and uterine cancers, angiosarcomas, germ cell tumors and intracranial tumors, among others. ${ }^{104}$ It has been observed that a high VEGF value in serum correlates with low patient survival. Likewise, VEGFR-2 can be expressed in non-endothelial neoplastic cells so that VEGF can present autocrine activity in tumor cells causing constitutively VEGFR-2 phosphorylation. Thus, VEGFR-2 has become a central focus of molecularly targeted cancer therapy. ${ }^{105}$

\footnotetext{
102 Shibuya, M. Cell Struct. Funct. 2001, 26, 25-35.

103 Cébe-Suarez, S.; Zehnder-Fjällman, A.; Ballmer-Hofer, K. Cell. Mol. Life Sci. 2006, 63, 601615.

104 Homsi, J.; Daud, A. Cancer Control 2007 14, 285-294.

105 Takahashi S. Biol. Pharm. Bull. 2011, 34, 1785-1788.
} 


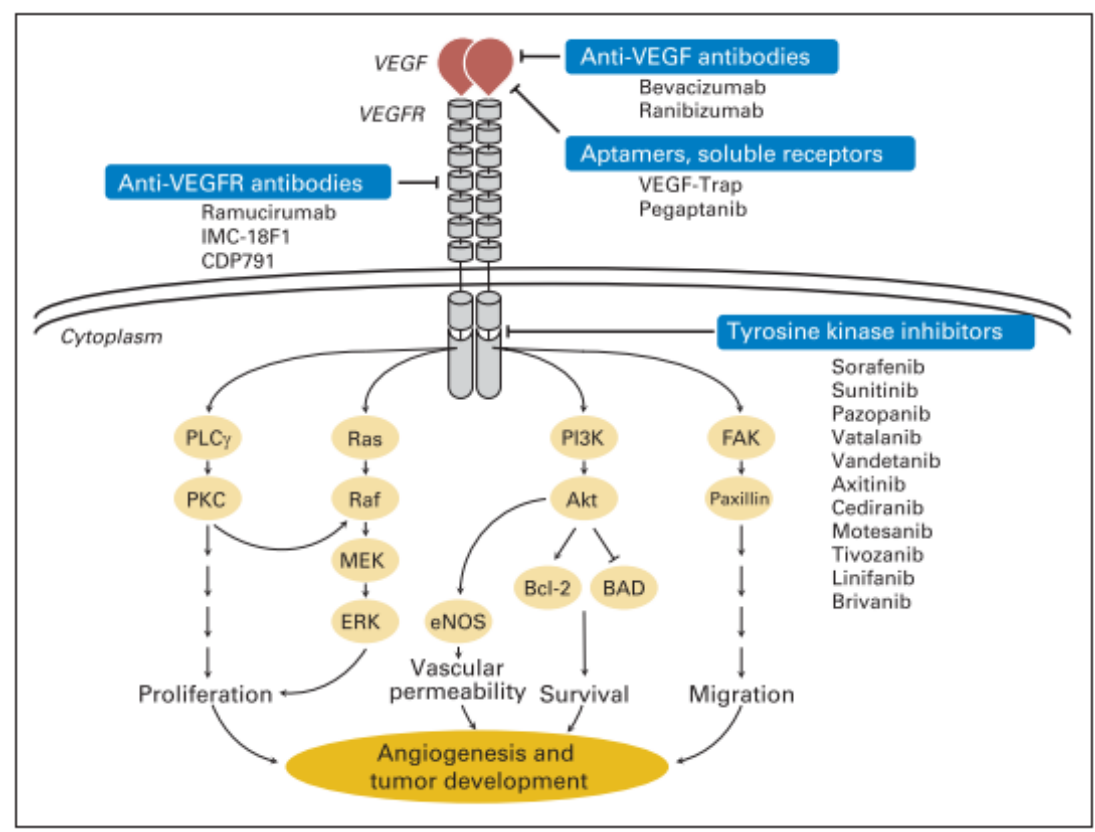

Figure 1.30. Processes mediated by VEGF/VEGFR and inhibitory strategies. ${ }^{106}$

\section{Antiangiogenic drugs}

There are several antiangiogenic drugs that have received approval for the treatment of highly prevalent tumors such as colorectal, gastrointestinal, lung, breast, glioblastoma and renal cell cancer. Currently, more than 200 clinical trials of different angiogenesis inhibitors are being conducted in the United States, some already in phase III. ${ }^{107}$

Bevacizumab (Avastin $\AA$ ) is a humanized monoclonal antibody employed in the treatment of colorectal, breast, non-microcytic lung and metastatic renal cancers. ${ }^{108}$ Bevacizumab is bound to VEGF-A preventing its union to VEGFR-1 and VEGFR-2. ${ }^{109}$ Pegaptanib (Macugen $\AA$ ) is an oligonucleotide with high affinity

106 Koo, P. J.; Morgensztern, D.; Boyer, J. L.; Herbst, R. S. J. Clin. Oncol. 2012, 30, 1137-1139.

107 www.clinicaltrials.gov

108 European Medicines Agency, document EMA/487898/2016.

109 (a) Hurwitz, H.; Fehrenbacher, L.; Novotny, W.; Cartwright, T.; Hainsworth, J.; Heim, W.; Berlin, J.; Baron, A.; Griffing, S.; Holmgren, E.; Ferrara, N.; Fyfe, G.; Rogers, B.; Ross, R.; Kabbinavar, F. N. Engl. J. Med. 2004, 350, 2335-2342. (b) Cao, Y. Exp. Cell Res. 2010, 316, 1304-1308. 
to VEGF-165, and ranibizumab (Lucentis $\AA$ ) is a fragment derived from bevacizumab. ${ }^{110}$

Other antiangiogenic strategies are focussed on the administration of small molecules that block tyrosine-kinase activity of VEGFR. This approach possibilities an oral administration, while the previous antibodies can only be administered intravenously. To date, four different inhibitors have been approved by the FDA: sunitinib (Sutent $\AA$ ) and pazopanib (Votrient $\AA$ ) for gastrointestinal stromal tumors and metastatic renal-cell carcinoma (RCC), sorafenib (Nexavar®) for unresectable hepatocellular carcinoma and metastatic $\mathrm{RCC}$, and vandetanib (Zactima®) for medullary thyroid cancer. ${ }^{94}$<smiles>CNC(=O)c1cc(Oc2ccc(NC(=O)Nc3ccc(Cl)c(C(F)(F)F)c3)cc2)ccn1</smiles><smiles>CCN(CC)CCNC(=O)c1c(C)[nH]c(/C=C2\C(=O)Nc3ccccc32)c1C</smiles><smiles>COc1cc2c(Nc3ccc(Br)cc3F)ncnc2cc1OCC1CCN(C)CC1</smiles>

Vandetanib<smiles>Cc1ccc(Nc2nccc(N(C)c3ccc4c(C)n(C)nc4c3)n2)cc1S(N)(=O)=O</smiles>

Figure 1.31. VEGFR inhibitors.

Different types of tyrosine kinase inhibitors have been described. Type I inhibitors recognize the active conformation of a kinase. They bind to the ATPbinding site by presenting one to three hydrogen bonds which mimic the hydrogen bonds normally formed by ATP. Sunitinib is classified as a type I inhibitor. Type II inhibitors recognize the inactive conformation of a kinase and

110 (a) Ng, E. W.; Shima, D. T.; Calias, P.; Cunningham, E. T. Jr.; Guyer, D. R.; Adamis, A. P. Nat. Rev. Drug Discov. 2006, 5, 123-132. (b) Wolf, S.; Balciuniene, V. J.; Laganovska, G.; Menchini, U.; Ohno-Matsui, K.; Sharma, T.; Wong, T. Y.; Silva, R.; Pilz, S.; Gekkieva, M. Ophthalmology 2014, 121, 682-692. 
indirectly compete with ATP by occupying the hydrophobic pocket which is directly adjacent to the ATP-binding site. These inhibitors can modulate kinase activity in an allosteric way. Sorafenib is an example of a type II inhibitor (see figure 1.32). ${ }^{111}$

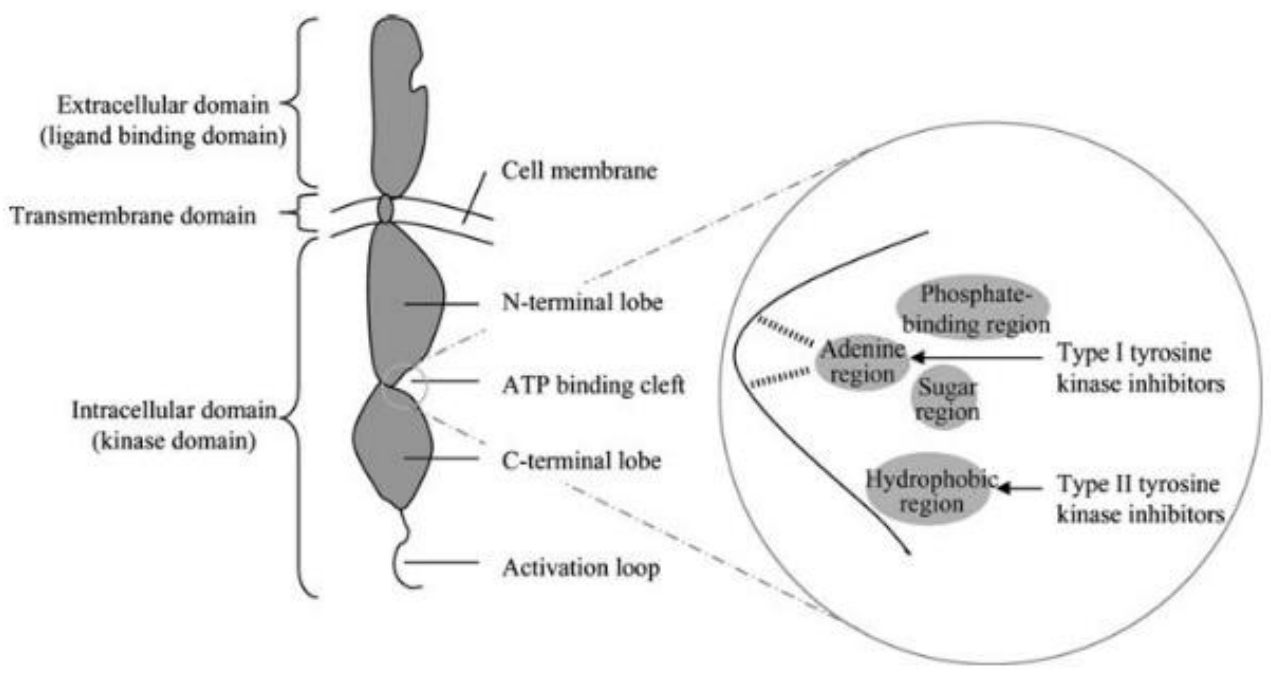

Figure 1.32. Structure of a tyrosine kinase receptor.

\section{Sorafenib}

Sorafenib is a multiple kinase inhibitor and a novel oral bis-aryl urea compound that exhibits strong anticancer effects. ${ }^{112}$ It binds to receptors on the surface of cells or to other proteins in the downstream signaling pathways, blocking their activities (see figure 1.33). ${ }^{113}$

111 Gotink, K. J.; Verheul, H. M. Angiogenesis 2010, 13, 1-14.

112 Wei-Feng, M.; Min-Hua, S.; Pin-Ting, G.; Ji, M.; Hui-juan, L.; Gai-ling, L.; Bao-hui, H.; Chonggang, Y. Acta Pharmacol. Sin. 2012, 33, 1311-1318.

113 Liu, L.; Cao, Y.; Chen, C.; Xiaomei Zhang, X.; et al. Cancer Res. 2006, 66, 11851-11858. 


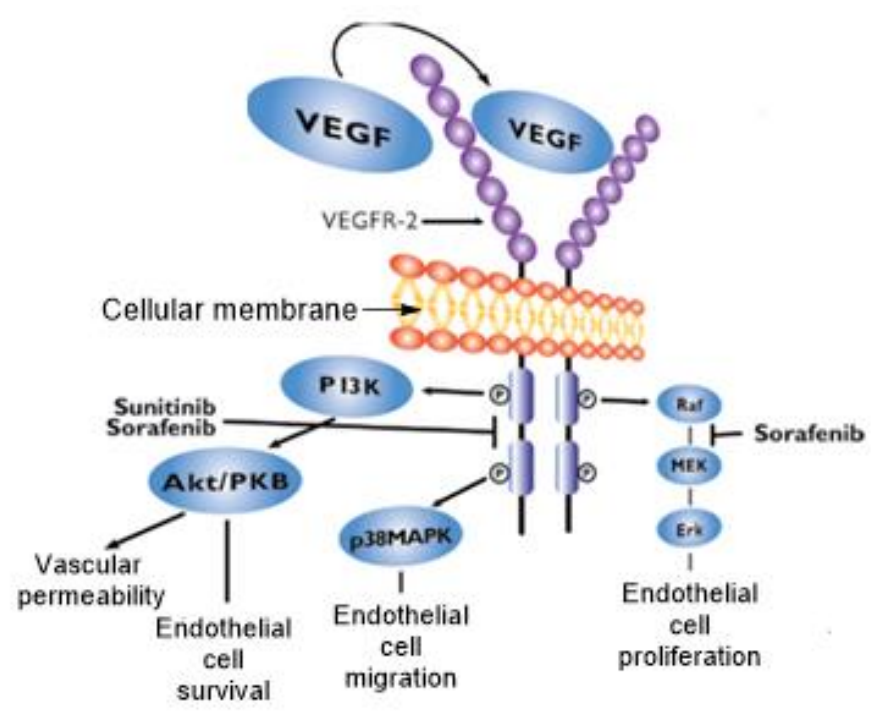

Figure 1.33. Interaction points of sorafenib and sunitinib on VEGFR-2.

Sorafenib bears a urea-like aromatic fragment that has shown, both through X-ray diffraction studies ${ }^{114}$ and through computational studies ${ }^{115}$, to be determinant in its interaction with the kinase domain. Docking studies have revealed that sorafenib can form 5 hydrogen bonds with the kinase domain of VEGFR-2: two with Glu885, two weak links with Cys919 and one with Asp1046 (see figure 1.34). The other interactions with the kinase domain are of the hydrophobic type. It has also been established that the strongest link is formed with the Asp1046.

\footnotetext{
${ }^{114}$ McTigue, M.; Murray, B. W.; Chen, J. H.; Deng, Y. L.; Solowiej, J.; Kania, R. S. Proc. Natl. Acad. Sci. USA 2012, 109, 18281-18289.

115 (a) Meng, F. Journal of Theoretical Chemistry 2013, http://dx.doi.org/10.1155/2013/739574 (b) Wein, T.; Tu, W.; Zhao, B.; Lan, Y.; Bao, J.; Dai, Z. Sci. Rep. 4, article 3982, doi:10.1038/srep03982.
} 


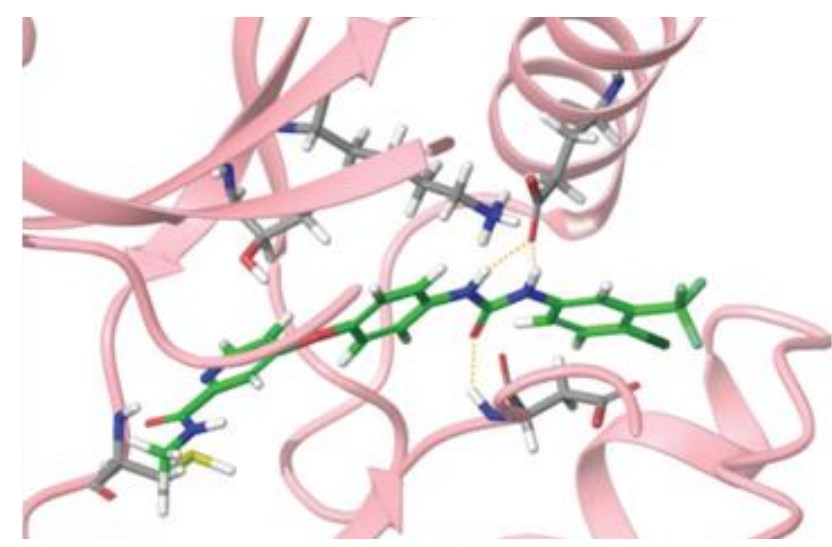

Figure 1.34. Interactions between sorafenib and VEGFR-2.

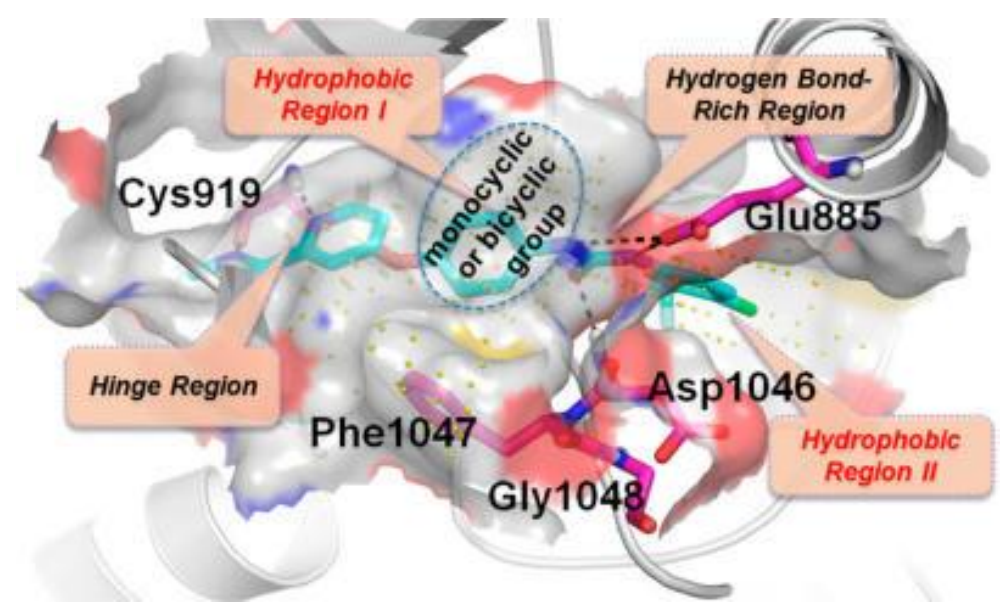

Figure 1.35. Binding site of VEGFR-2 in complex with sorafenib. ${ }^{116}$

\subsubsection{Immunotherapy}

Immunotherapy is another promising strategy to overcome cancer drug resistance. This uses the ability of an individual's immune system to fight cancer, offering durable and complete remissions and fewer side effects than traditional treatments. ${ }^{117}$ Biological modifiers such as cytokines or vaccines, adoptive cell therapies, oncolytic viruses and antibodies against immune checkpoint inhibitors

\footnotetext{
116 Zhang, Y.; Chen, Y.; Zhang, D.; Wang, L.; Lu, T.; Jiao, Y. J. Med. Chem. 2018, 61, 140-157.
}

117 Moore, C. D.; Chen, I. US Pharm. 2018, 43, 27-31. 
are some of the approaches that are arising. ${ }^{118}$ Targeting the tumor microenvironment may provide a novel strategy for immunotherapy towards personalized medicine. ${ }^{119}$ The heterogeneity that tumors present requires the need of predictive biomarkers to clinically differentiate patients and demands for individualised treatment strategies. ${ }^{120}$

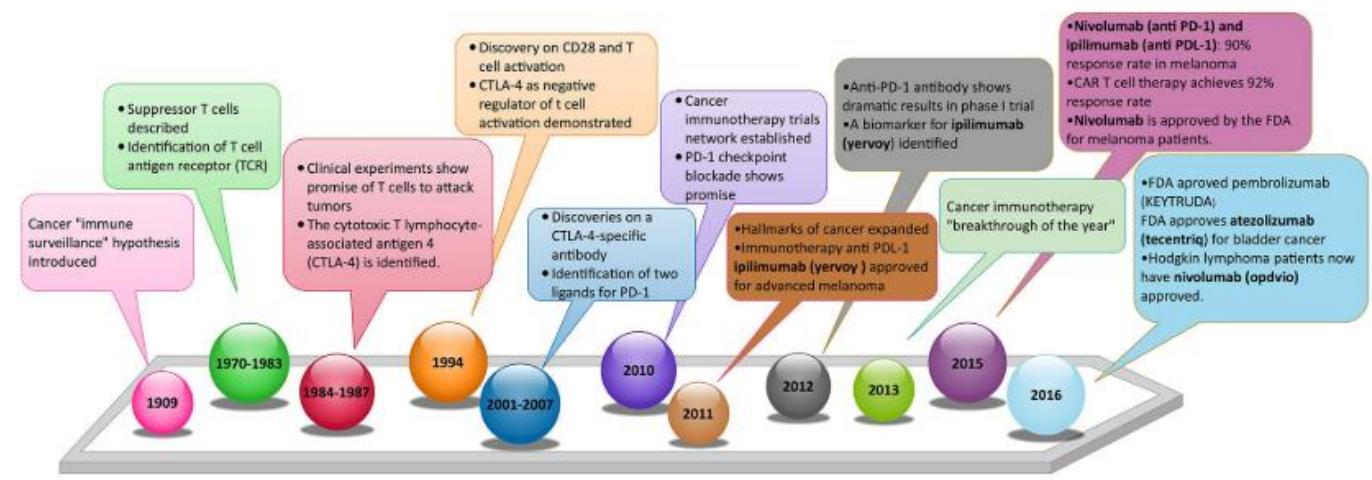

Figure 1.36. Immunotherapy timeline. ${ }^{126}$

Recently, in 2018, James P. Allison and Tasuko Honjo received the Nobel Prize in Physiology or Medicine for their discovery of cancer therapy by inhibition of negative immune regulation. During the 1990s, in his laboratory at the University of California, James P. Allison studied the T-cell protein CTLA-4. ${ }^{121} \mathrm{~A}$ few years before Allison's discovery, Tasuku Honjo discovered PD-1, another protein expressed on the surface of T-cells. ${ }^{122}$ Of the two treatment strategies, checkpoint therapy against PD-1 has proven to be more effective and positive results are being observed in several types of cancer, including lung cancer, renal cancer, lymphoma and melanoma. New clinical studies indicate that combination therapy, targeting both CTLA-4 and PD-1, can be even more effective, as demonstrated in patients with melanoma. Thus, Allison and Honjo

\footnotetext{
118 Oiseth S. J. et al. J. Cancer Metastasis Treat 2017, 3, 250-261.

119 Yu, Y.; Cui, J. Oncology Letters, 2018, 16, 4105-4113.

120 Cree, I. A.; Charlton, P. BMC Cancer 2017, 17, 10.

121 Leach, D. R.; Krummel, M. F.; Allison, J. P. Science, 1996, 271, 1734-1736.

122 Ishida, Y.; Agata, Y.; Shibahara, K.; Honjo, T. EMBO J. 1992, 11, 3887-3895.
} 
have inspired efforts to combine different strategies to release the brakes on the immune system with the aim of eliminating tumor cells even more efficiently. A large number of checkpoint therapy trials are currently underway against most types of cancer, and new checkpoint proteins are being tested as targets. ${ }^{123}$

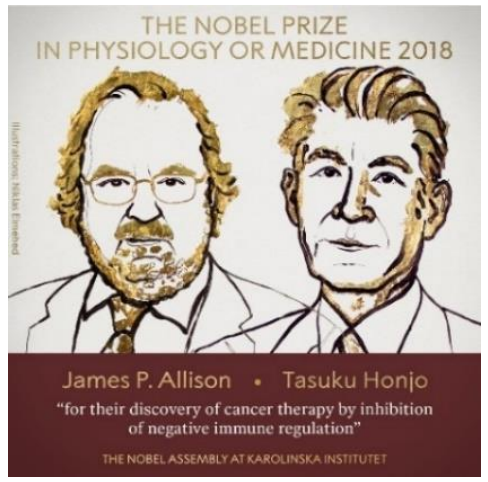

Figure 1.37. The Nobel Prize in Physiology or Medicine 2018. ${ }^{124}$

\section{PD-1/PD-L1 checkpoint}

The capacity of tumor cells to avoid immune destruction constitutes an important obstacle to the satisfactory treatment of cancer. However, the study of these protective mechanisms has provided new therapeutic strategies to deal with cancer, such as the immune checkpoint antibodies, that reverse the action exerted by the negative regulators of $\mathrm{T}$ cells. ${ }^{125} \mathrm{~A}$ good example of that is the interaction between the Programmed Death-Ligand 1 (PD-L1), located on the surface of tumor cells, with the Programmed Death-1 receptor (PD-1), placed on cytotoxic $T$ lymphocytes, which inactivates these immune effectors and, consequently, generates immune escape.

Physiologically, the PD-1/PD-L1 pathway has emerged because of the need to control the inflammatory processes and protect normal tissue from damage. ${ }^{126}$ Programmed death-ligand 1 (PD-L1) is a $40 \mathrm{kDa}$ type 1 transmembrane protein

\footnotetext{
123 Iwai, Y.; Terawaki, S.; Honjo, T. Int. Immunol. 2005, 17, 133-144.

124 Illustration: Niklas Elmehed.

125 Gotwals, P. et al. Nat. Rev. Cancer 2017, 17, 286-301.

${ }^{126}$ Alsaab, H.; Sau, S.; Alzhrani, R. et al. Frontiers in pharmacology, 2017, 8, 1-15.
} 
that plays a major role in suppressing the immune system during particular events such as pregnancy, tissue allografts, autoimmune diseases and other disease states such as hepatitis. PD-L1 binds to its receptor PD-1, found on activated T cells, B cells and myeloid cells, to modulate activation or inhibition.

Cancer cells drive high expression levels of PD-L1 on their surface, allowing activation of PD-1 receptor on any $T$ cells that infiltrate the tumor microenvironment, switching those cells off. ${ }^{127}$ Upregulation of PD-L1 expression levels has been demonstrated in many cancers such as melanoma, non-small cell lung cancer and multiple myeloma ${ }^{128}$ allowing them to evade the host immune system. ${ }^{129}$ PD-L1 tumor expression might be suggestive of an immune response suppression, and this could serve as a potential biomarker for clinical benefit. PD-1/PD-L1 inhibitors prevent PD-1/PD-L1 interaction, thus facilitating a positive immune response to kill the tumor (see figure 1.38).
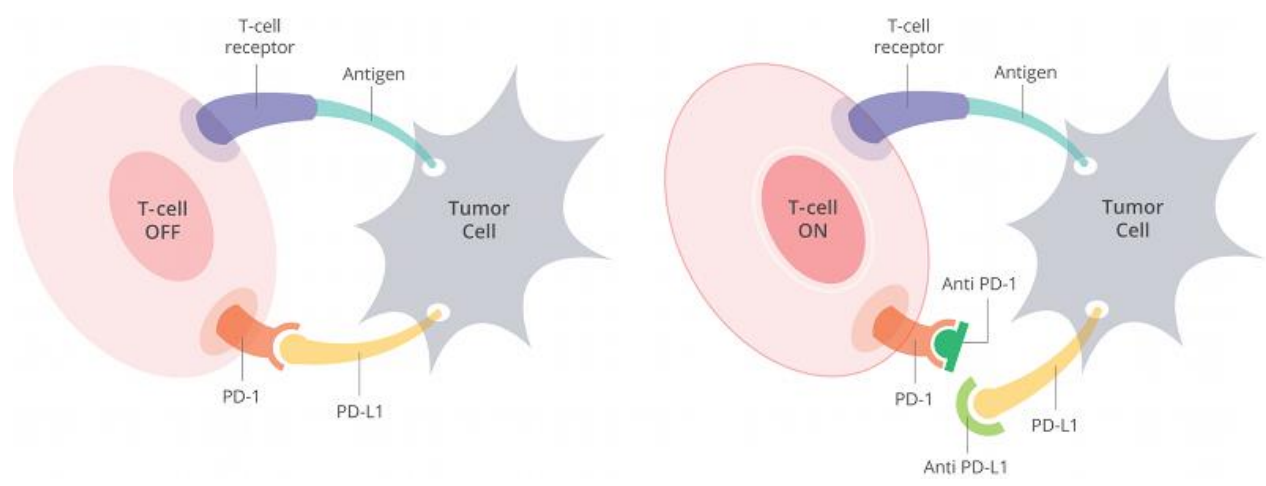

Figure 1.38. PD-1/PD-L1 interaction. ${ }^{130}$

Antibodies targeting the PD-1/PD-L1 immune checkpoint achieved spectacular success in the recent years. Many PD-L1 inhibitors are in development as immuno-oncology therapies and are showing good results in

\footnotetext{
${ }^{127}$ Butte, M. J.; Keir, M. E.; Phamduy, T. B.; Freeman, G. J.; Sharpe, A. H. Immunity 2007, 27, 111-122.

128 Liu, J.; Hamrouni, A.; Wolowiec, D.; Coiteux, V.; Kuliczkowski, K.; Hetuin D. Blood 2007, 110, 296-304.

${ }_{129}$ Shi, L.; Chen, S.; Yang, L.; Li, Y. J. Hematol. Oncol. 2013, 6, 74.

$130 \mathrm{https}: / / \mathrm{www}$. smartpatients.com/targets/pd-1
} 
clinical trials. ${ }^{131}$ Clinically available examples include nivolumab and pidilizumab. However, their high immunogenicity and low stability have led to the research for new non-peptidic molecules. ${ }^{132}$

In 2015, Brystol-Myers Squibb reported the discovery of the first non-peptidic molecules able to inhibit the formation of the PD-1/PD-L1 complex. ${ }^{133}$ The mode of interaction of these compounds, indicated as BMS-8, BMS-37, BMS-202 and BMS-242 in figure 1.40, was established in 2016. ${ }^{134}$ Using differential scanning fluorimetry (DSF) techniques, it was determined that these compounds induce thermal stabilization of PD-L1. Finally, the protein bound to the substrates was crystallized and the binding sites were determined. These compounds are linked in a hydrophobic groove formed by the amino acids Tyr56, Met115, lle116, Ala121 and Tyr123 and promote the dimerization of PD-L1 protein (see figure 1.39). Thus, it is possible to inhibit the formation of the PD-1/PD-L1 complex by means of a double pathway: the inhibitors occupy part of the area involved in the PD-1/PD-L1 interaction and, in addition, when the dimer is formed between two PD-L1 molecules, one of them has the opposite orientation to the one necessary to interact with PD-1. Consequently, the interaction between PD-1 and PD-L1 is disabled.

Checkpoint therapy has now revolutionized cancer treatment and has fundamentally changed the way we view how cancer can be managed.

\footnotetext{
131 Velcheti, V.; Schalper, K. A.; Carvajal, D. E. et al. Lab. Invest. 2014, 94, 107-116.

132 Skalniak, L.; Zak, K. M. Oncotarget, 2017, 8, 72167-72181.

133 Chupak, L. S.; Zheng X. Bristol-Myers Squibb Company 2015, WO2015034820 A1.

134 Zak, K. M.; Grudnik, P.; Guzik, K.; Zieba, B.J.; Musielak, B.; Domling, A.; Dubin, G.; Holak, T.A. Oncotarget, 2016, 7, 30323-30335.
} 


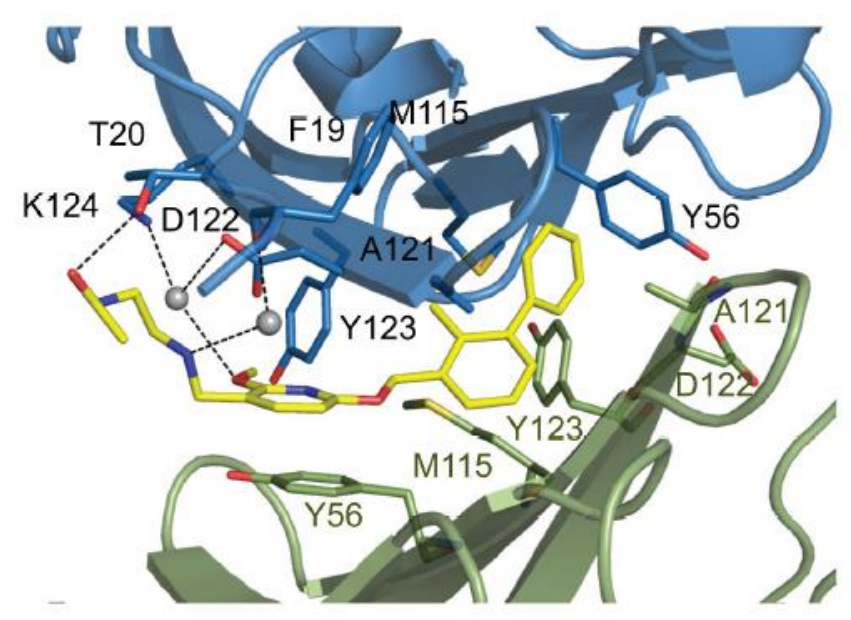

Figure 1.39. Interaction of BMS-202 (in yellow) with dimer PD-L1/PD-L1.<smiles>COc1nc(OCc2cccc(-c3ccccc3)c2C)ccc1CNCCNC(C)=O</smiles>

BMS-202<smiles>COc1cc(OCc2cccc(-c3ccccc3)c2C)nc(OC)c1CNCCNC(C)=O</smiles><smiles>Cc1c(COc2ccc(CN3CCCCC3C(=O)O)cc2Br)cccc1-c1ccccc1</smiles>

BMS-8<smiles>COc1cc(OCc2cccc(-c3ccccc3)c2C)nc(OC)c1CNC(CO)C(C)C</smiles>

Figure 1.40. Non-peptidic inhibitors of PD-L1.

\section{c-Myc oncogene}

Myc family consists of three related human genes: c-Myc, I-Myc and n-Myc. c-Myc (or MYC) was the first gene to be discovered in this family, due to the homology with the viral gene v-Myc (myelocytomatosis virus). c-Myc is a regulator gene that codes for a transcription factor, a nuclear phosphoprotein that is involved in cell cycle progression, apoptosis and cellular transformation. As shown in figure 1.41, the encoded protein dimerizes with the related transcription factor Max and binds target DNA sequences or E boxes (with the 
sequence 5'-CANNTG-3', where $\mathrm{N}$ can be any nucleotide) to regulate transcription of genes involved in cell growth and proliferation. ${ }^{135} \mathrm{c}-\mathrm{Myc}$ overexpression is related to tumorigenesis, so this has become a relevant biological target to fight cancer.
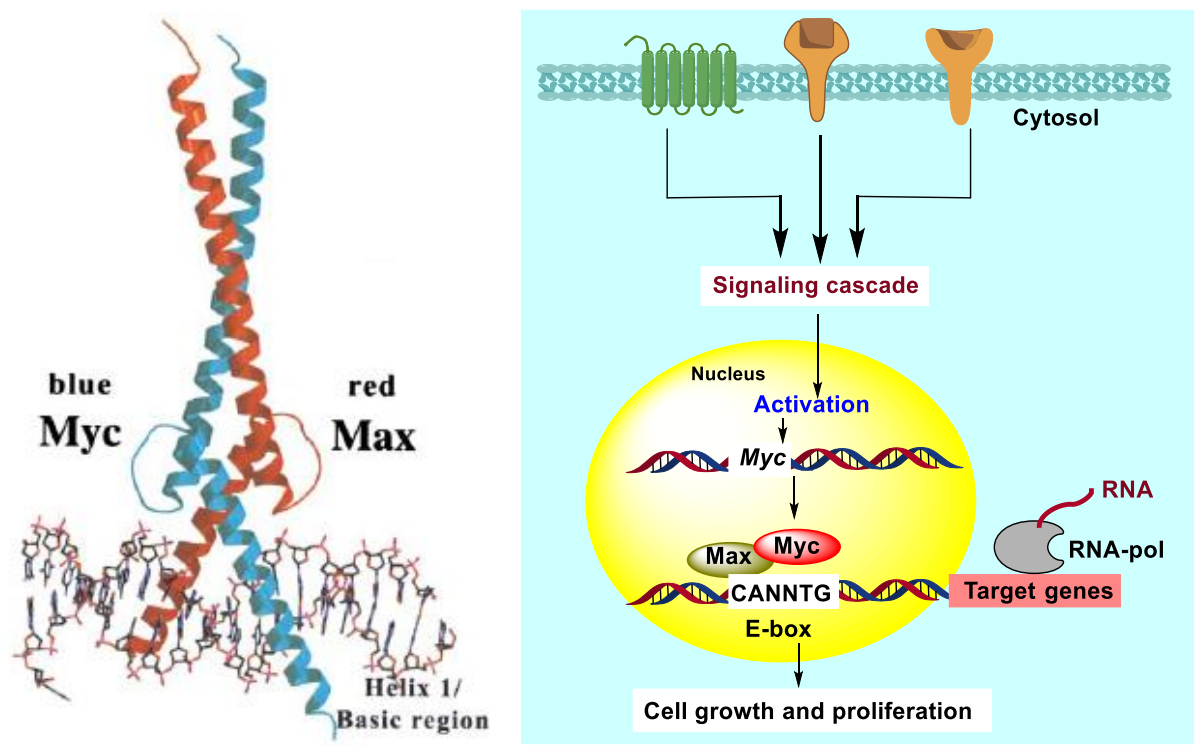

Figure 1.41. c-Myc activation. ${ }^{136}$

It has been reported that c-Myc oncogene has a direct role in preventing immune cells from efficiently attacking tumor cells. c-Myc fosters tumor growth by increasing the levels of two immune checkpoint proteins, CD47 and PD-L1, which help thwart the host immune response. ${ }^{137}$ When c-Myc overexpression is turned off, the levels of CD47 and PD-L1 decrease and tumor regression takes place. Thus, c-Myc inactivation in tumors appears to engage the immune system to elicit cellular senescence in tumor cells and to collapse the vascular endothelial cells. ${ }^{138}$

\footnotetext{
135 Dang, C. V. Cell 2012, 149, 22-35.

136 Nair, A. K.; Burley, S. K. Cell 2003, 112, 193-205.

137 Casey, S. C.; Tong, L.; Li, Y.; Do, R.; Walz, S.; Fitzgerald, K. N.; Gouw, A. M.; Baylot, V.; Gütgemann, I.; Eilers, M. Science, 2016, 352, 227-231.

138 Casey, S. C.; Baylot, V.; Dean W.; Felsher, D. W. Trends Immunol. 2017, 38, 298-305.
} 
In relation to angiogenesis process, there is a c-Myc requirement for the expression of angiogenic factors such as VEGF or angiopoietin-2. Therefore, cMyc function is also necessary for the angiogenic switch which is indispensable for the progression and metastasis of tumors. ${ }^{139}$

The integration of all these potentially complementary research fields provides new opportunities to improve cancer treatments. Combination of chemotherapy, antiangiogenics and immunotherapy may limit chemoresistance and progression to metastatic disease.

139 Baudino, T. A. et al. Genes \& development 2002, 16, 2530-2543. 
2. OBJECTIVES 



\section{OBJECTIVES}

As it has been explained in the introduction section, aminocombretastatin (AmCA-4) exhibits antimitotic and antiangiogenic properties, so that the objectives of this thesis are focussed on the synthesis and biological evaluation of three families of aminocombretastatin derivatives.

Compounds of family 1 contain a carbamate group in their structure. These derivatives have been studied as antimitotic and vascular disrupting agents (VDAs). Carbamate functionality confers chemical and proteolytic stability and a great capacity to pass through cell membranes which explains the increasing use of carbamates in medicinal chemistry. ${ }^{140}$

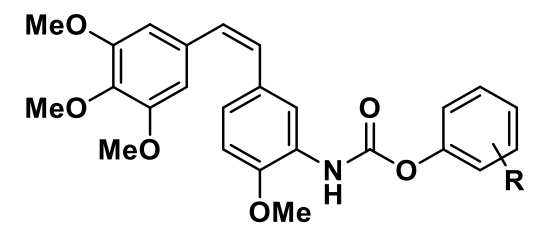

Figure 2.1. General structure of family 1 compounds.

Compounds of family 2 have been designed considering the structure of the antiangiogenic drug sorafenib. Thus, a fragment of urea has replaced the carbamate moiety in order to study these derivatives as antiangiogenic drugs.

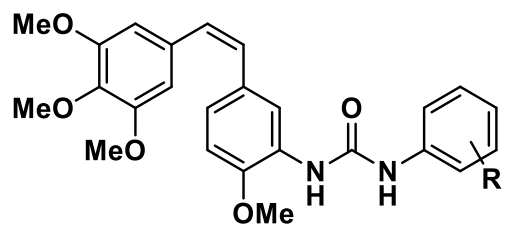

Figure 2.2. General structure of family 2 compounds.

Compounds of family $\mathbf{3}$ have been designed as multitarget agents, both as antiangiogenic and immunomodulator. Their structure keeps the urea fragment, responsible for VEGFR-2 interaction, and also would allow the interaction with PD-L1. Both the binding site of sorafenib in the kinase domain of VEGFR-2 and

${ }^{140}$ Ghosh, A. K.; Brindisi, M. J. Med. Chem. 2015, 58, 2895-2940. 
the binding site in PD-L1, possess a hydrophobic groove in which aromatic rings can be introduced. Our goal is the design of compounds able to interact with the mentioned binding sites acting on both targets.<smiles>[R]c1cccc(NC(=O)Nc2cccc(/C=C\c3ccc(OC)cc3)c2)c1</smiles>

F3.A<smiles>[R]c1cccc(NC(=O)Nc2cccc(CCc3ccc(OC)cc3)c2)c1</smiles>

F3.C<smiles>[R]c1cccc(NC(=O)Nc2cccc(/C=C/c3ccc(OC)cc3)c2)c1</smiles>

F3.B

F3.D

Figure 2.3. General structures of family 3 compounds.

Finally, with the purpose of knowing all the perspectives of a biological evaluation process, an in vivo study has been developed in a SCID mice model. 


\section{SYNTHESIS OF AMINOCOMBRETASTATIN DERIVATIVES}





\section{SYNTHESIS OF AMINOCOMBRETASTATIN DERIVATIVES}

\subsection{Synthesis of aminocombretastatin (AmCA-4)}

The preparation of compounds belonging to familiy 1 and 2 required the previous synthesis of aminocombretastatin (AmCA-4), which is indicated in scheme 3.1. It was initiated by the Perkin condensation between 2-(3,4,5trimethoxyphenyl)acetic acid $\mathbf{1}$ and 4-methoxy-3-nitrobenzaldehyde 2, which was carried out by refluxing a mixture of $\mathbf{1}$ and $\mathbf{2}$ for $3 \mathrm{~h}$ in the presence of acetic anhydride and triethylamine. After reaction processing with $\mathrm{HCl}$, neutralization and ethanol crystallization, the acid 3 , with $E$ configuration, was achieved. Decarboxylation of 3, upon Cu-quinoline treatment at reflux, led to compound $\mathbf{4}$, whose nitro group was reduced with zinc in acetic acid in order to give AmCA4. ${ }^{141}$

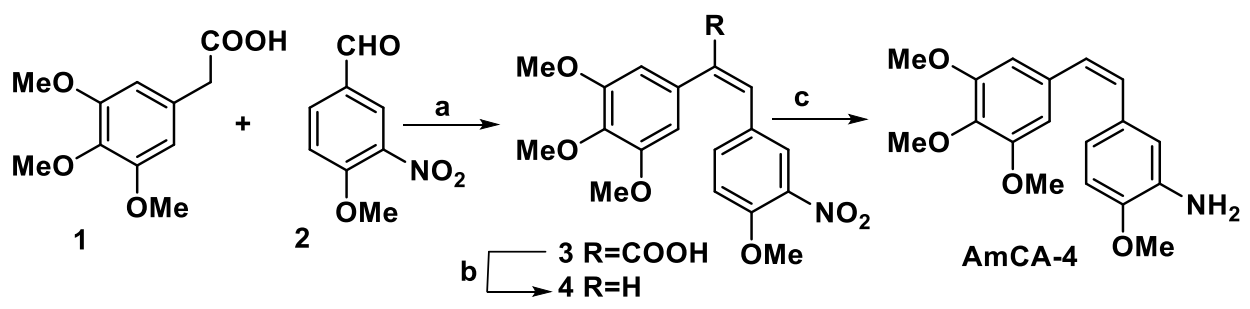

\section{Scheme 3.1}

Reagents and conditions: (a) $\mathrm{Ac}_{2} \mathrm{O}, \mathrm{Et}_{3} \mathrm{~N}$, reflux, $3 \mathrm{~h}$, then $\mathrm{HCl}$ conc. $49 \%$; (b) $\mathrm{Cu}$, quinoline, reflux, $3 \mathrm{~h}, 47 \%$ (Z/E 98:2); (c) $\mathrm{Zn}, \mathrm{AcOH}$, r. t., $4 \mathrm{~h}, 87 \%$.

\subsection{Synthesis of family 1 compounds}

For the synthesis of carbamate derivatives two different procedures were applied. In the indicated as Method a in scheme 3.2, carbamates were directly achieved upon reaction of AmCA-4 with commercially available aryl chloroformates in THF in the presence of pyridine. ${ }^{142}$ The low commercial availability of aryl chloroformates allowed only the synthesis of carbamates $\mathbf{C . 1}$,

141 Ying-Qian, L.; Xiao-Jing, L.; Chun-Yan, Z.; Xiang, N.; Jing, T.; Morris-Natschke, S.L.; Zhi-Jun, Z.; Xiao-Ming, Y.; Liu, Y.; Lin-Hai, L.; Xing-Wen, Z.; Kuo-Hsiung, L. Bioorg. Med. Chem., 2013, 21, 1248-1256.

142 Zhang, L.; Xia, W.; Wang, B.; Luo, Y.; Lu, W. Synth. Commun. 2011, 41, 3140-3146. 
C.4 and C.7. The remaining carbamates were prepared by a one-pot procedure, named as Method $b$, in which AmCA-4 was converted into the corresponding trichloromethylcarbamate upon reaction with triphosgene and $\mathrm{Et}_{3} \mathrm{~N}$, which in turn was transformed into carbamates by reaction with a range of phenols (see scheme 3.2). ${ }^{143}$

$$
\begin{array}{ll}
\text { AmCA-4 Method a or b } & \\
&
\end{array}
$$

\section{Scheme 3.2}

Reagents and conditions: Method a: $\mathrm{ArOCOCl}$, pyridine, THF, $0^{\circ} \mathrm{C} 30 \mathrm{~min}$, then r.t. 1 h. Method b: (i) thiphosgene, Et ${ }_{3} \mathrm{~N}$, THF, r. t., $10 \mathrm{~min}$; (ii) $\mathrm{ArOH}$, THF, 45으, $1 \mathrm{~h}$.

\subsection{Synthesis of family 2 compounds}

Ureas belonging to family 2 were obtained by the synthetic route denoted in scheme 3.3. Thus, reaction of AmCA-4 N-phenyl carbamate (C.1) with a range of anilines in $\mathrm{THF}$, in the presence of $\mathrm{Et}_{3} \mathrm{~N}$, provided the corresponding urea derivatives.

${ }^{143}$ Yang, L.; Li, G.; Ma, S.; Zou, C.; Zhou, S.; Sun, Q.; Cheng, C.; Chen, X.; Wang, L.; Feng, S.; Li, L.; Yang, S. J. Med. Chem. 2013, 56, 1641-1655. 


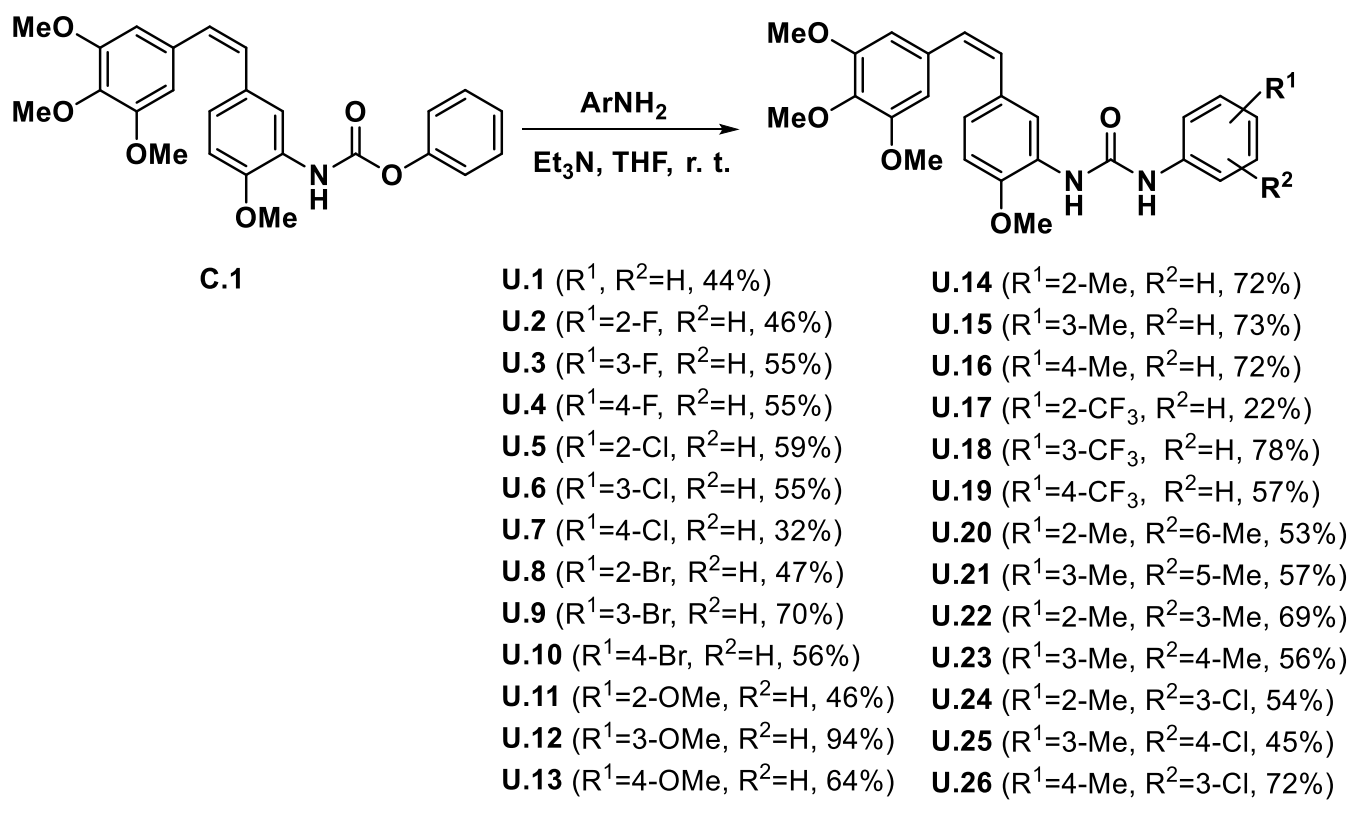

\section{Scheme 3.3}

\subsection{Synthesis of family 3 compounds}

First, it was decided to synthesize the derivatives with the general structure F3.D (see figure 2.3) due to its easy synthetic accessibility. Thus, triazoles T.1T.8 indicated in scheme 3.5 were prepared by alkyne-azide cycloaddition. The alkyne required for this reaction was 1-methoxy-4-(prop-2-yn-1-yl)benzene 7 which was prepared from (4-methoxyphenyl)methanol 5 as indicated in scheme 3.4 .<smiles>C#CCc1ccc(OC)cc1</smiles>

\section{Scheme 3.4}

Reagents and conditions: (a) $\mathrm{PBr}_{3}, \mathrm{CH}_{2} \mathrm{Cl}_{2}, 0^{\circ} \mathrm{C}, 3 \mathrm{~h}$; (b) ethynylmagnesium bromide, $\mathrm{CuCl}, \mathrm{THF}$, reflux, $2 \mathrm{~h}$. 
Scheme 3.5 depicts the synthetic sequence that led to triazoles T.1-T.8. Thus, 2-azidoethan-1-amine $\mathbf{8}^{144}$ was converted into ureas A.1-A.8 which were submitted to cycloaddition reaction with alkyne 7 upon heating in a mixture $\mathrm{DMF} / \mathrm{H}_{2} \mathrm{O}$ in the presence of $\mathrm{CuSO}_{4} \cdot 5 \mathrm{H}_{2} \mathrm{O}$ and sodium ascorbate. ${ }^{145}$ Under these conditions triazoles T.1-T.8 were achieved in moderate yields. Unfortunately, all attempts to prepare the ortho-halogenated isomers failed due probably to the steric hindrance caused by the halogen in ortho position.

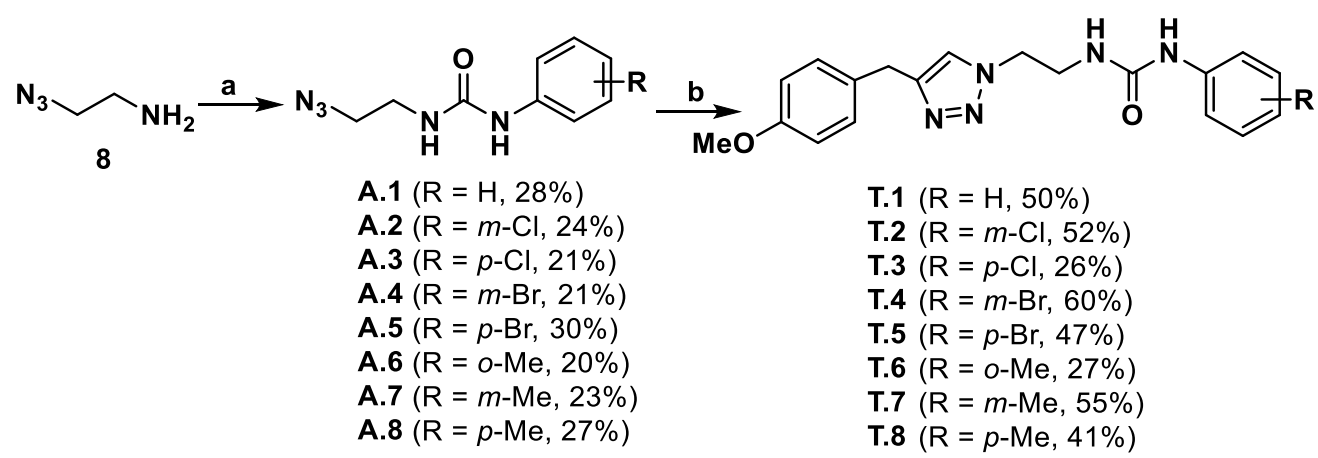

Scheme 3.5

Reagents and conditions: (a) (i) $\mathrm{CDI}, \mathrm{Et}_{3} \mathrm{~N}, \mathrm{DMF}, 3 \mathrm{~h}$, r. t.; (ii) aniline, $50^{\circ} \mathrm{C}$, overnight; (b) $7, \mathrm{CuSO}_{4} \cdot 5 \mathrm{H}_{2} \mathrm{O}$, sodium ascorbate, $\mathrm{DMF} / \mathrm{H}_{2} \mathrm{O}(9 / 1), 60^{\circ} \mathrm{C}, 2 \mathrm{~h}$.

The triazolyl-ureas that contain a chlorine atom in their structure were the ones that combined the best activities in the biological tests (see chapter 4). Therefore, it was decided to prepare new compounds with general structure F3.A, F3.B and F3.C (see figure 2.3) containing a chlorine atom in the phenyl ring. Concretely, ureas S.1-S.9 were prepared as indicated in scheme 3.6. Thus, Wittig reaction of the phosphonium salt 10 with 4-methoxybenzaldehyde afforded 1-(4-methoxystyryl)-3-nitrobenzene $\mathbf{1 1}$ as a mixture of $E / Z$ isomers. Reduction of this mixture gave rise to a mixture of $E / Z$ anilines which was

\footnotetext{
${ }^{144}$ Aminoazide 8 was prepared according to: Loison, S; Cottet, M.; Orcel, H.; Adihou, H.; Rahmeh, R.; Lamarque, L.; Trinquet, E.; Kellenberger, E.; Hibert, M.; Durroux, T.; Mouillac, B.; Bonnet, D. J. Med. Chem. 2012, 55, 8588-8602.

${ }^{145}$ Rostovtsev, V. V.; Green, L. G.; Fokin, V. V.; Sharpless, K. B. Angew. Chem. Int. Ed. 2002, 41, 2596-2599.
} 
separated by column chromatography to afford (Z)-3-(4-methoxystyryl)aniline 12 and $(E)$-3-(4-methoxystyryl)aniline 13. On the other hand, hydrogenation of 11 led to 3-(4-methoxyphenethyl)aniline 14. Compounds 12, 13 and 14 were converted into carbamates 15, 16 and 17, which upon reaction with chloroanilines gave rise to ureas S.1-S.9.

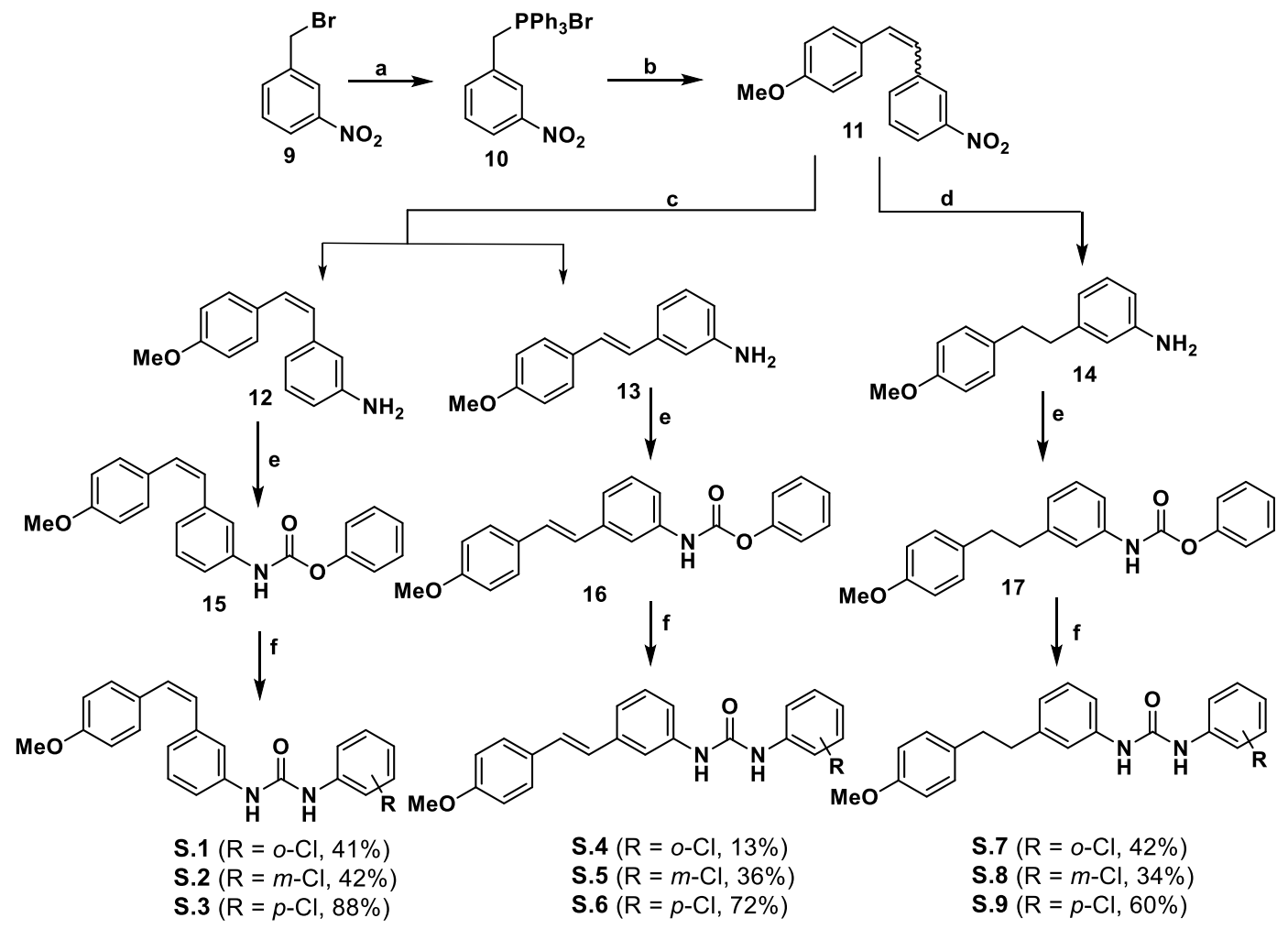

Scheme 3.6

Reagents and conditions: (a) $\mathrm{PPh}_{3}, \mathrm{CH}_{2} \mathrm{Cl}_{2}, 3$ h, r. t., 71\%; (b) 4methoxybenzaldehyde, $\mathrm{K}_{2} \mathrm{CO}_{3}$, 18-crown-6, $\mathrm{CH}_{2} \mathrm{Cl}_{2}$, reflux, overnight, $73 \%$; (c) $\mathrm{Zn}$, $\mathrm{AcOH}, 1$ h, r. t., (Z/E 6:4); (d) $\mathrm{H}_{2}, \mathrm{Pd} / \mathrm{C}$, EtOAc, 2 h, r. t., 66\%; (e) phenyl chloroformate, pyridine, THF, $0^{\circ} \mathrm{C} 30 \mathrm{~min}$, then $1 \mathrm{~h}$ r. t.; (f) chloroaniline, $\mathrm{Et}_{3} \mathrm{~N}$, THF, $40-50^{\circ} \mathrm{C}$. 


\subsection{Experimental section}

\subsubsection{General techniques}

NMR spectra were registered at $25^{\circ} \mathrm{C}$ with Varian Unity spectrometer (operation frequencies, $500 \mathrm{MHz}$ for ${ }^{1} \mathrm{H} ; 125 \mathrm{MHz}$ for ${ }^{13} \mathrm{C}$ ). Complete signal assignments in ${ }^{1} \mathrm{H}$ and ${ }^{13} \mathrm{C}$ NMR spectra were made with the aid of $2 \mathrm{D}$ homoand heteronuclear pulse sequences (COSY, HSQC). Spectra were measured in $\mathrm{CDCl}_{3}, \mathrm{CD}_{3} \mathrm{OD}$, acetone- $d_{6}$ or DMSO- $d_{6}$ solvents. Chemical shifts $(\delta)$ are indicated in ppm taking residual signals of deuterated solvents as reference. In ${ }^{1} \mathrm{H}, \delta=7.27 \mathrm{ppm}$ for $\mathrm{CDCl}_{3}, \delta=3.31 \mathrm{ppm}$ for $\mathrm{CD}_{3} \mathrm{OD}, \delta=2.05$ for acetone- $d_{6}$ and $\delta=2.50$ ppm for DMSO- $d_{6} \cdot \ln { }^{13} \mathrm{C}, \delta=77.0 \mathrm{ppm}$ for $\mathrm{CDCl}_{3}, \delta=49.0 \mathrm{ppm}$ for $\mathrm{CD}_{3} \mathrm{OD}$, $\delta=29.8 \mathrm{ppm}$ for acetone $-d_{6}$ and $\delta=39.5 \mathrm{ppm}$ for DMSO- $d_{6}$. Multiplicity assignments in ${ }^{1} \mathrm{H}-\mathrm{RMN}$ consider $s$ for singlet, $d$ for doublet, $t$ for triplet, $q$ for quadruplet, $m$ for multiplet, $b r$ for broad signals and app for signals with apparent multiplicity.

IR spectra were obtained using $\mathrm{KBr}$ plates in Jasco FT/IR-6200 spectrometer, covering the region $4000-600 \mathrm{~cm}^{-1}$. Only most relevant signals are indicated $\left(\mathrm{C}=\mathrm{O}, \mathrm{O}-\mathrm{H}, \mathrm{N}-\mathrm{H}, \mathrm{N}_{3}\right)$.

Mass spectra were measured with a mass spectrometer Q-TOF (Waters, Manchester, UK), with a combined ionization source ESI and APCI with Z-spray design; capillary voltage of $3.5 \mathrm{KV}$ was applied in positive sense and cone voltage was stablished at $20 \mathrm{~V}$.

Melting points were meaured in a melting point apparatus, Digital, SMP10 (Stuart Scientific).

Thin Layer Chromatography (TLC) was developed in silica gel coated chromatoplaques, Merck 5554.

Commercially available reagents were used as received without preliminary treatment, from commercial properties Sigma-Aldrich, Fluka, Scharlab or Acros. 
Triethylamine was distilled over potassium hydroxide. DMF was distilled and stored in $3 \AA$ sieves.

Air-sensitive reagents were handled under dry $\mathrm{N}_{2}$ atmosphere avoiding contact with air or moisture.

Chemical compounds containing cis isomery were synthetized protected from light to avoid isomerization. 


\subsubsection{Synthesis of amicocombretastatin (AmCA-4)}

a) (E)-3-(4-methoxy-3-nitrophenyl)-2-(3,4,5-trimethoxyphenyl)acrylic acid<smiles>COc1ccc(/C=C(/C(=O)O)c2cc(OC)c(OC)c(OC)c2)cc1[N+](=O)[O-]</smiles>

A mixture of 1 (25 mmol) and 2 (12.5 mmol) in $\mathrm{Ac}_{2} \mathrm{O}(12 \mathrm{~mL})$ and $\mathrm{Et}_{3} \mathrm{~N}(6 \mathrm{~mL})$ was refluxed for $3 \mathrm{~h}$. Then, the resulting solution was acidified with concentrated $\mathrm{HCl}(6 \mathrm{~mL})$ and filtered in order to precipitate crude 3 , which was subsequently recrystallized from $\mathrm{EtOH}$.

49\% yield, yellowish solid, m. p. 229-231ํㅡ.

${ }^{1}$ H NMR (500 MHz, DMSO-d $)$ ס 12.70 (br s, 1H), 7.71 (s, 1H), 7.54 (s, 1H), 7.40 (d, $J=8.3 \mathrm{~Hz}, 1 \mathrm{H}), 7.26(\mathrm{~d}, J=8.8 \mathrm{~Hz}, 1 \mathrm{H}), 6.48(\mathrm{~s}, 2 \mathrm{H}), 3.89(\mathrm{~s}, 3 \mathrm{H}), 3.70$ (s, $9 \mathrm{H})$.

${ }^{13} \mathrm{C}$ NMR (125 MHz, DMSO-d $\left.)\right) \delta(\mathrm{C}) 168.4,153.9$ (x 2), 152.7, 139.2, 137.9, 134.0, 131.7, 127.3, (CH) 136.7, 136.6, 127.0, 114.7, 107.1 (x 2), $\left(\mathrm{CH}_{3}\right) 60.6$, 57.3, $56.5(\times 2)$.

HR ESMS $m / z$ 388.1031 [M-H]. Calc. for $\mathrm{C}_{19} \mathrm{H}_{18} \mathrm{NO}_{8}, 388.1032$.

b) (Z)-1,2,3-trimethoxy-5-(4-methoxy-3-nitrostyryl)benzene<smiles>COc1ccc(/C=C\c2cc(OC)c(OC)c(OC)c2)cc1[N+](=O)[O-]</smiles>

Acid 3 (2.6 mmol) was added to a suspensión of Cu (13.4 mmol) in quinoline (10 $\mathrm{mL}$ ) and the resulting mixture was refluxed for $3 \mathrm{~h}$ under $\mathrm{N}_{2}$ atmosphere. After cooling down, $\mathrm{Et}_{2} \mathrm{O}(10 \mathrm{~mL})$ was added and $\mathrm{Cu}$ was filtered through Celite. The filtrate was washed with $\mathrm{HCl} 1 \mathrm{M}$ and the aqueous phase was extracted with $\mathrm{Et}_{2} \mathrm{O}$. Organic extracts were collected, washed with aqueous saturated $\mathrm{NaHCO}_{3}$ solution and dried with $\mathrm{Na}_{2} \mathrm{SO}_{4}$. Finally, solvent was removed under reduced 
pressure and the residue was subjected to column chromatography on silica-gel (Hexane-EtOAc mixtures as eluant) to afford compound 4.

$47 \%$ yield, yellowish solid, m. p. 121-123ㄷ.

${ }^{1} \mathrm{H}$ NMR $\left(500 \mathrm{MHz}, \mathrm{CDCl}_{3}\right) \delta 7.80(\operatorname{app} \mathrm{d}, J=2.0 \mathrm{~Hz}, 1 \mathrm{H}), 7.44$ (dd, $J=8.8,2.0$ $\mathrm{Hz}, 1 \mathrm{H}), 6.95(\mathrm{~d}, J=8.3 \mathrm{~Hz}, 1 \mathrm{H}), 6.59(\mathrm{~d}, J=12.2 \mathrm{~Hz}, 1 \mathrm{H}), 6.48(\mathrm{~s}, 2 \mathrm{H}), 6.46$ (d, $J=12.2$ Hz. 1H), 3.94 (s, 3H), 3.86 (s, 3H), $3.72(\mathrm{~s}, 6 \mathrm{H})$.

${ }^{13} \mathrm{C}$ NMR (125 MHz, $\left.\mathrm{CDCl}_{3}\right) \delta(\mathrm{C}) 153.2$ (x 2), 151.7, 139.5, 137.8, 131.8, 129.8, (CH) 134.6, 131.3, 126.8, 125.9, 113.1, 105.9 (x 2), $\left(\mathrm{CH}_{3}\right)$ 60.9, 56.6, 56.0 (x 2);

IR $v_{\max }\left(\mathrm{cm}^{-1}\right)$ 1529-1280 (N-O).

HR ESMS $m / z 368.1109[\mathrm{M}+\mathrm{Na}]^{+}$. Calc. for $\mathrm{C}_{18} \mathrm{H}_{19} \mathrm{NNaO}_{6} 368.1110$.

c) (Z)-2-methoxy-5-(3,4,5-trimethoxystyryl)aniline (AmCA-4)<smiles>COc1ccc(/C=C\c2cc(OC)c(OC)c(OC)c2)cc1N</smiles>

Zinc powder (218 mmol) was added to a solution of compound $4(1.5 \mathrm{mmol})$ in acetic acid $(50 \mathrm{~mL})$ and the resulting mixture was stirred at $r$. t. for $6 \mathrm{~h}$. Then, it was filtered through celite, neutralized with aqueous $\mathrm{NaHCO}_{3}$ and extracted with AcOEt. Organic fractions were collected and dried with $\mathrm{Na}_{2} \mathrm{SO}_{4}$. Finally, solvent was removed under reduced pressure and the residue was subjected to column chromatography on silica-gel (Hexane-EtOAc mixtures as eluant) affording AmCA-4.

$87 \%$ yield, orange oil.

${ }^{1} \mathrm{H}$ NMR $\left(500 \mathrm{MHz}, \mathrm{CDCl}_{3}\right) \delta 6.71(\mathrm{~s}, 1 \mathrm{H}), 6.69(\mathrm{~s}, 2 \mathrm{H}), 6.56(\mathrm{~s}, 2 \mathrm{H}), 6.46(\mathrm{~d}, \mathrm{~J}$ $=12.2 \mathrm{~Hz}, 1 \mathrm{H}), 6.38(\mathrm{~d}, J=12.2 \mathrm{~Hz}, 1 \mathrm{H}), 3.85(\mathrm{~s}, 3 \mathrm{H}), 3.84(\mathrm{~s}, 3 \mathrm{H}), 3.71(\mathrm{~s}, 6 \mathrm{H})$.

${ }^{13} \mathrm{C} \mathrm{NMR}\left(125 \mathrm{MHz}, \mathrm{CDCl}_{3}\right) \delta(\mathrm{C}) 152.8$ (x 2), 146.7, 137.1, 135.8, 133.0, 130.1, (CH) 130.0, 128.4, 119.5, 115.2, 110.1, 106.1 (x 2), $\left(\mathrm{CH}_{3}\right)$ 60.9, 55.9 (x 2), 55.6. IR $v_{\max }\left(\mathrm{cm}^{-1}\right) 3471-3371 \mathrm{~cm}^{-1}(\mathrm{~N}-\mathrm{H})$.

HR ESMS $m / z 316.1547[\mathrm{M}+\mathrm{H}]^{+}$. Calc. for $\mathrm{C}_{18} \mathrm{H}_{22} \mathrm{NO}_{4} 316.1549$. 


\subsubsection{Synthesis of carbamates derived from AmCA-4}

a) General procedure for the synthesis of carbamates by method a

A solution of AmCA-4 $(0.7 \mathrm{mmol})$ in THF $(3.5 \mathrm{~mL})$ was cooled at $0^{\circ} \mathrm{C}$ and anhydrous pyridine $(1.7 \mathrm{mmol})$ and the corresponding phenyl chloroformate (1.0 $\mathrm{mmol}$ ) were added under inert atmosphere. The resulting mixture was stirred in the dark for $20 \mathrm{~min}$ at $0^{\circ} \mathrm{C}$ and for $1 \mathrm{~h}$ at r. t. After this time, $\mathrm{H}_{2} \mathrm{O}(3.4 \mathrm{~mL})$ and $\mathrm{HCl} 1 \mathrm{M}(1.7 \mathrm{~mL})$ were added to the reaction mixture, which was then extracted with $\mathrm{CH}_{2} \mathrm{Cl}_{2}(3 \times 20 \mathrm{~mL})$. The organic layer was washed with brine, and then dried on anhydrous $\mathrm{Na}_{2} \mathrm{SO}_{4}$. Removal of volatiles under reduced pressure afforded an oily residue which was subjected to column chromatography on silica-gel (Hexane-EtOAc mixtures as eluant) to afford the desired products with the yields indicated below.

Phenyl (Z)-(2-methoxy-5-(3,4,5-trimethoxystyryl)phenyl)carbamate C.1<smiles>COc1ccc(/C=C\c2cc(OC)c(OC)c(OC)c2)cc1NC(=O)Oc1ccccc1</smiles>

$89 \%$ yield, off-white solid, m. p. 124-126ㄷ.

${ }^{1} \mathrm{H}$ NMR $\left(500 \mathrm{MHz}, \mathrm{CDCl}_{3}\right) \delta 8.11$ (br s, 1H), 7.52 (br s, 1H, NH), 7.39 (t, J 7.5 $\mathrm{Hz}, 2 \mathrm{H}), 7.24(\mathrm{t}, J \sim 7.5 \mathrm{~Hz}, 1 \mathrm{H}), 7.19(\mathrm{~d}, \sim 7.5 \mathrm{~Hz}, 2 \mathrm{H}), 7.00(\mathrm{~d}, J=8.5 \mathrm{~Hz}, 1 \mathrm{H})$, $6.76(\mathrm{~d}, J=8.5 \mathrm{~Hz}, 1 \mathrm{H}), 6.52(\mathrm{~s}, 2 \mathrm{H}), 6.51(\mathrm{~d}, J=12 \mathrm{~Hz}, 1 \mathrm{H}), 6.45(\mathrm{~d}, J=12 \mathrm{~Hz}$, $1 \mathrm{H}), 3.89(\mathrm{~s}, 3 \mathrm{H}), 3.81(\mathrm{~s}, 3 \mathrm{H}), 3.70(\mathrm{~s}, 6 \mathrm{H})$.

${ }^{13} \mathrm{C}$ NMR $\left(125 \mathrm{MHz}, \mathrm{CDCl}_{3}\right) \delta(\mathrm{C}) 152.9$ (x 2), 151.3, 150.6, 146.9, 137.2, 132.7, 130.3, 126.9, (CH) 129.5, 129.3 (x 2), 129.2, 125.5, 123.9, 121.6 (x 2), 119.3, 109.6, 106.1 (x 2), $\left(\mathrm{CH}_{3}\right)$ 60.8, 55.9 (x 2), 55.8.

IR $v_{\max }\left(\mathrm{cm}^{-1}\right) 3415(\mathrm{~N}-\mathrm{H}), 1750(\mathrm{C}=\mathrm{O})$.

HR ESMS $m / z 458.1588[\mathrm{M}+\mathrm{Na}]^{+}$. Calc. for $\mathrm{C}_{25} \mathrm{H}_{25} \mathrm{NNaO}_{6}, 458.1580$. 
4-Fluorophenyl (Z)-(2-methoxy-5-(3,4,5-trimethoxystyryl)phenyl)carbamate C.4

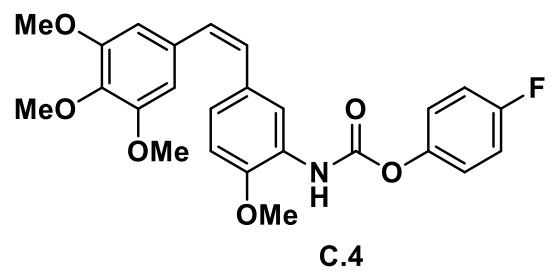

$64 \%$ yield, yellowish solid, m. p. 138-142 $\mathrm{C}$.

${ }^{1} \mathrm{H}$ NMR $\left(500 \mathrm{MHz}, \mathrm{CDCl}_{3}\right) \delta 8.10(\mathrm{~s}, 1 \mathrm{H}), 7.50(\mathrm{~s}, 1 \mathrm{H}), 7.14(\mathrm{dd}, J=9,4.5 \mathrm{~Hz}$, $2 \mathrm{H}), 7.07$ (t, $J=9 \mathrm{~Hz}, 2 \mathrm{H}), 7.01(\mathrm{dd}, J=8.5,2 \mathrm{~Hz}, 1 \mathrm{H}), 6.76(\mathrm{~d}, J=8.5 \mathrm{~Hz}, 1 \mathrm{H})$, 6.52 (s, 2H), 6.50 (d, J=12 Hz, 1H), 6.45 (d, J=12 Hz, 1H), 3.90 (s, 3H), 3.82 (s, 3H), $3.70(\mathrm{~s}, 6 \mathrm{H})$.

${ }^{13} \mathrm{C}$ NMR (125 MHz, $\mathrm{CDCl}_{3}$ ) $\delta(\mathrm{C}) 160.1$ (d, $\left.{ }^{1} \mathrm{~J}_{\mathrm{C}-\mathrm{F}} \sim 242 \mathrm{~Hz}\right), 152.9$ (x 2), 146.9, 146.5, 146.4, 137.2, 132.7, 130.3, 126.7, (CH) 129.5, 129.3, 124.1, 123.1 (x 2, d, $\left.{ }^{3} J_{C-F} \sim 7.5 \mathrm{~Hz}\right), 119.3,115.9\left(x 2, d,{ }^{2} J_{C-F} \sim 23 \mathrm{~Hz}\right), 109.7,106.1(x 2),\left(\mathrm{CH}_{3}\right)$ $60.8,55.9$ (x 2), 55.8.

IR $v_{\max }\left(\mathrm{cm}^{-1}\right) 3416(\mathrm{~N}-\mathrm{H}), 1747(\mathrm{C}=\mathrm{O})$.

HR ESMS $m / z 476.1484[\mathrm{M}+\mathrm{Na}]^{+}$. Calc. for $\mathrm{C}_{25} \mathrm{H}_{24} \mathrm{FNNaO}_{6}, 476.1485$.

\section{4-Chlorophenyl (Z)-(2-methoxy-5-(3,4,5-trimethoxystyryl)phenyl)carbamate}

C.7

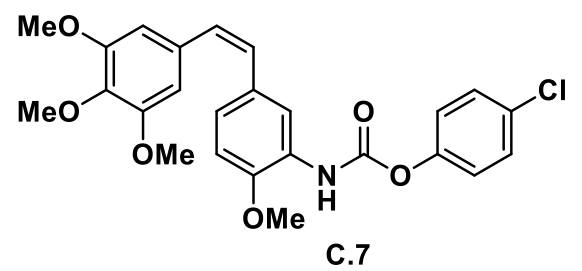

$62 \%$ yield, off-white solid, m. p. $138-140^{\circ} \mathrm{C}$.

${ }^{1} \mathrm{H}$ NMR $\left(500 \mathrm{MHz}, \mathrm{CDCl}_{3}\right.$ ) $\delta 8.06$ (br s, 1H), 7.50 (br s, 1H, NH), 7.35 (app d, J $=8.8 \mathrm{~Hz}, 2 \mathrm{H}), 7.13(\operatorname{app} \mathrm{d}, J=8.8 \mathrm{~Hz}, 2 \mathrm{H}), 7.01(\mathrm{dd}, J=8.5,2.5 \mathrm{~Hz}, 1 \mathrm{H}), 6.76$ $(\mathrm{d}, J=8.5 \mathrm{~Hz}, 1 \mathrm{H}), 6.51(\mathrm{~s}, 2 \mathrm{H}), 6.50(\mathrm{~d}, J=12 \mathrm{~Hz}, 1 \mathrm{H}), 6.46(\mathrm{~d}, J=12 \mathrm{~Hz}, 1 \mathrm{H})$, $3.89(\mathrm{~s}, 3 \mathrm{H}), 3.81(\mathrm{~s}, 3 \mathrm{H}), 3.70(\mathrm{~s}, 6 \mathrm{H})$.

${ }^{13} \mathrm{C}$ NMR $\left(125 \mathrm{MHz}, \mathrm{CDCl}_{3}\right) \delta(\mathrm{C}) 152.8$ (x 2), 150.9, 149.1, 146.9, 137.2, 132.6, 130.8, 130.3, 126.6, (CH) 129.4, 129.3 (x 2), 124.1, 122.9 (x 2), 119.3, 109.7, 106.1 (x 2), $\left(\mathrm{CH}_{3}\right) 60.7,55.8,55.8$ (x 2). 
IR $v_{\max }\left(\mathrm{cm}^{-1}\right) 3414(\mathrm{~N}-\mathrm{H}), 1749(\mathrm{C}=\mathrm{O})$.

HR ESMS m/z 492.1189 [M+Na] ${ }^{+}$. Calc. for $\mathrm{C}_{25} \mathrm{H}_{24} \mathrm{CINNaO}_{6}, 492.1190$.

\section{b) General procedure for the synthesis of carbamates by method b}

A solution of AmCA-4 $(0.14 \mathrm{mmol})$ in THF $(0.6 \mathrm{~mL})$ was added to a solution of triphosgene $(0.3 \mathrm{mmol})$ in THF $(0.2 \mathrm{~mL})$. The resulting mixture was stirred in the dark for 10 min at r. t. Then $\mathrm{Et}_{3} \mathrm{~N}(4.3 \mathrm{mmol})$ was slowly added and the resulting residue was resuspended in THF $(0.8 \mathrm{~mL})$. The corresponding phenol $(0.8$ $\mathrm{mmol}$ ) was added to the mixture which was stirred in the dark for $1 \mathrm{~h}$ at $45^{\circ} \mathrm{C}$. After this time, volatiles were removed under reduced pressure and the remaining residue was dissolved in acetone. The precipitated which remained without dissolving was discarded by simple filtration, and then the filtrate was concentrated under reduced pressure to give an oily residue which was subjected to column chromatography on silica-gel (Hexane-EtOAc mixtures as eluant) affording the desired products with the yields indicated below.

\section{2-Fluorophenyl (Z)-(2-methoxy-5-(3,4,5-trimethoxystyryl)phenyl)carbamate C.2}

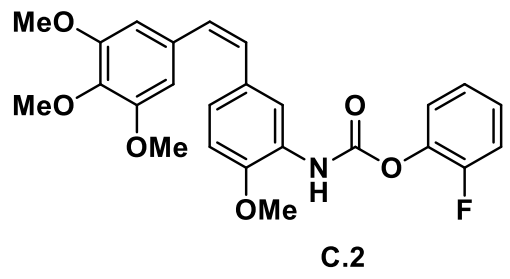

$73 \%$ yield, yellowish solid, m. p. $138-142^{\circ} \mathrm{C}$.

${ }^{1} \mathrm{H}$ NMR $\left(500 \mathrm{MHz}, \mathrm{CDCl}_{3}\right) \delta 8.10(\mathrm{~s}, 1 \mathrm{H}), 7.60(\mathrm{~s}, 1 \mathrm{H}), 7.30-7.15$ (br m, 4H), 7.00 (dd, $J=8.5,2 \mathrm{~Hz}, 1 \mathrm{H}$ ), 6.76 (d, $J=8.5 \mathrm{~Hz}, 1 \mathrm{H}), 6.52(\mathrm{~s}, 2 \mathrm{H}), 6.50$ (d, $J=$ $12 \mathrm{~Hz}, 1 \mathrm{H}), 6.45(\mathrm{~d}, J=12 \mathrm{~Hz}, 1 \mathrm{H}), 3.90(\mathrm{~s}, 3 \mathrm{H}), 3.78(\mathrm{~s}, 3 \mathrm{H}), 3.70(\mathrm{~s}, 6 \mathrm{H})$.

${ }^{13} \mathrm{C}$ NMR $\left(125 \mathrm{MHz}, \mathrm{CDCl}_{3}\right) \delta$ (C) 154.6 (d, $\left.{ }^{1} \mathrm{~J}_{\mathrm{C}-\mathrm{F}} \sim 248 \mathrm{~Hz}\right), 152.9$ (x 2), 150.2, $146.9,138.0$ (d, ${ }^{2} J_{C-F} \sim 11.5 \mathrm{~Hz}$ ), 137.2, 132.7, 130.3, 126.6, (CH) 129.5, 129.3, $126.8\left(\mathrm{~d},{ }^{3} \mathrm{~J}_{\mathrm{C}-\mathrm{F}} \sim 7.5 \mathrm{~Hz}\right), 124.3\left(\mathrm{~d},{ }^{3} \mathrm{~J}_{\mathrm{C}-\mathrm{F}} \sim 3.8 \mathrm{~Hz}\right), 124.2,124.1,119.2,116.6$ (doublet with ${ }^{2} J_{C-F} \sim 18.5 \mathrm{~Hz}$ ), 109.7, 106.1 (x 2), $\left(\mathrm{CH}_{3}\right) 60.8,55.9$ (x 2), 55.8.

IR $v_{\max }\left(\mathrm{cm}^{-1}\right) 3416(\mathrm{~N}-\mathrm{H}), 1753(\mathrm{C}=\mathrm{O})$.

HR ESMS $m / z 476.1484[\mathrm{M}+\mathrm{Na}]^{+}$. Calc. for $\mathrm{C}_{25} \mathrm{H}_{24} \mathrm{FNNaO}_{6}, 476.1485$. 
3-Fluorophenyl (Z)-(2-methoxy-5-(3,4,5-trimethoxystyryl)phenyl)carbamate C.3

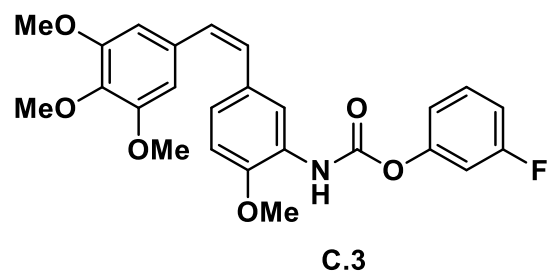

$33 \%$ yield, off-white solid, m. p. 127-130ㄷ.

${ }^{1} \mathbf{H}$ NMR $\left(500 \mathrm{MHz}, \mathrm{CDCl}_{3}\right) \delta 8.07$ (br s, 1H), 7.50 (br s, 1H, NH), 7.34 (app q, J $=7.7 \mathrm{~Hz}, 1 \mathrm{H}), 7.02(\mathrm{dd}, J=8.5,1.5 \mathrm{~Hz}, 1 \mathrm{H}), 7.00-6.95(\mathrm{~m}, 3 \mathrm{H}), 6.76(\mathrm{~d}, J=8.5$ $\mathrm{Hz}, 1 \mathrm{H}), 6.52(\mathrm{~s}, 2 \mathrm{H}), 6.51(\mathrm{~d}, J=12 \mathrm{~Hz}, 1 \mathrm{H}), 6.46$ (d, J = $12 \mathrm{~Hz}, 1 \mathrm{H}), 3.90$ (s, $3 \mathrm{H}), 3.81(\mathrm{~s}, 3 \mathrm{H}), 3.70(\mathrm{~s}, 6 \mathrm{H})$.

${ }^{13} \mathrm{C}$ NMR (125 MHz, $\left.\mathrm{CDCl}_{3}\right) \delta(\mathrm{C}) 162.8$ (d, $\left.{ }^{1} \mathrm{~J}_{\mathrm{C}-\mathrm{F}} \sim 246 \mathrm{~Hz}\right), 152.9$ (x 2), 151.5 $\left(\mathrm{d},{ }^{3} \mathrm{~J}_{\mathrm{C}-\mathrm{F}} \sim 10 \mathrm{~Hz}\right), 150.7,146.9,137.2,132.7,130.4,126.6,(\mathrm{CH}) 130.0\left(\mathrm{~d},{ }^{3} \mathrm{~J}_{\mathrm{C}-\mathrm{F}}\right.$ $\sim 8.5 \mathrm{~Hz}$ ), 129.5, 129.4, 124.2, 119.3, $117.3\left(\mathrm{~d},{ }^{4} \mathrm{~J}_{\mathrm{C}-\mathrm{F}} \sim 3 \mathrm{~Hz}\right), 112.5\left(\mathrm{~d},{ }^{2} \mathrm{~J}_{\mathrm{C}-\mathrm{F}} \sim 10\right.$ $\mathrm{Hz}), 109.7$ (d, ${ }^{2} J_{\mathrm{C}-\mathrm{F}} \sim 5 \mathrm{~Hz}$ ), 109.6, 106.1 (x 2), $\left(\mathrm{CH}_{3}\right)$ 60.8, 55.9 (x 2), 55.8.

IR $v_{\max }\left(\mathrm{cm}^{-1}\right) 3413(\mathrm{~N}-\mathrm{H}), 1753(\mathrm{C}=\mathrm{O})$.

HR ESMS $m / z$ 476.1482 [M+Na] $]^{+}$. Calc. for $\mathrm{C}_{25} \mathrm{H}_{24} \mathrm{FNNaO}_{6}, 476.1485$.

\section{2-Chlorophenyl (Z)-(2-methoxy-5-(3,4,5-trimethoxystyryl)phenyl)carbamate} C.5

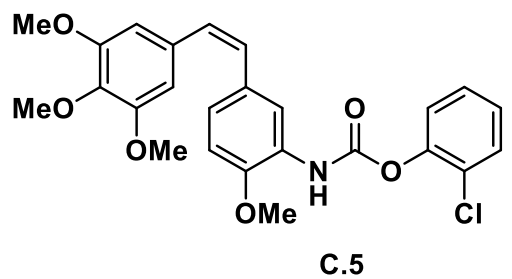

$35 \%$ yield, off-white solid, m. p. $130-131 \stackrel{\circ}{ } \mathrm{C}$.

${ }^{1} \mathrm{H}$ NMR $\left(500 \mathrm{MHz} \mathrm{CDCl}_{3}\right) \delta 8.11$ (br s, 1H), 7.65 (br s, 1H, NH), 7.45 (dd, $J=$ 7.8, $1.5 \mathrm{~Hz}, 1 \mathrm{H}$ ), 7.30-7.20 (m, 3H), 7.01 (dd, $J=8.5,2 \mathrm{~Hz}, 1 \mathrm{H}), 6.76$ (d, J=8.5 $\mathrm{Hz}, 1 \mathrm{H}), 6.52(\mathrm{~s}, 2 \mathrm{H}), 6.49(\mathrm{~d}, J=12 \mathrm{~Hz}, 1 \mathrm{H}), 6.44(\mathrm{~d}, J=12 \mathrm{~Hz}, 1 \mathrm{H}), 3.90$ (s, $3 \mathrm{H}), 3.78(\mathrm{~s}, 3 \mathrm{H}), 3.70(\mathrm{~s}, 6 \mathrm{H})$.

${ }^{13} \mathrm{C}$ NMR (125 MHz, $\left.\mathrm{CDCl}_{3}\right) \delta(\mathrm{C}) 152.9$ (x 2), 146.9, 146.7, 137.2, 132.7, 130.3, 130.0, 127.4, 126.7, (CH) 130.3, 129.5, 129.3, 127.6, 126.9, 124.2, 124.1, 119.2, 109.7, 106.1 (x 2), $\left(\mathrm{CH}_{3}\right) 60.8,55.9$ (x 2), 55.8. 
IR $v_{\max }\left(\mathrm{cm}^{-1}\right) 3413(\mathrm{~N}-\mathrm{H}), 1755(\mathrm{C}=\mathrm{O})$.

HR ESMS $m / z 492.1188[\mathrm{M}+\mathrm{Na}]^{+}$. Calc. for $\mathrm{C}_{25} \mathrm{H}_{24} \mathrm{CINNaO}_{6}, 492.1190$.

3-Chlorophenyl (Z)-(2-methoxy-5-(3,4,5-trimethoxystyryl)phenyl)carbamate C.6

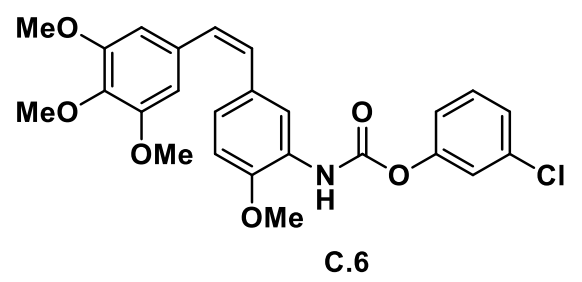

$33 \%$ yield, off-white solid, m. p. $128-130^{\circ} \mathrm{C}$.

${ }^{1} \mathrm{H}$ NMR $\left(500 \mathrm{MHz}, \mathrm{CDCl}_{3}\right) \delta 8.07$ (br s, 1H), 7.50 (br s, 1H, NH), 7.31 (t, J = 8.3 $\mathrm{Hz}, 1 \mathrm{H}), 7.22(\mathrm{~m}, 2 \mathrm{H}), 7.09$ (dd, $J=8.3,1.5 \mathrm{~Hz}, 1 \mathrm{H}), 7.01$ (dd, $J=8.5,2 \mathrm{~Hz}$, $1 \mathrm{H}), 6.76(\mathrm{~d}, J=8.5 \mathrm{~Hz}, 1 \mathrm{H}), 6.52(\mathrm{~s}, 2 \mathrm{H}), 6.51(\mathrm{~d}, J=12 \mathrm{~Hz}, 1 \mathrm{H}), 6.46(\mathrm{~d}, J=$ $12 \mathrm{~Hz}, 1 \mathrm{H}), 3.89(\mathrm{~s}, 3 \mathrm{H}), 3.81(\mathrm{~s}, 3 \mathrm{H}), 3.70(\mathrm{~s}, 6 \mathrm{H})$.

${ }^{13} \mathrm{C}$ NMR $\left(125 \mathrm{MHz}, \mathrm{CDCl}_{3}\right) \delta(\mathrm{C}) 152.9$ (x 2), 151.1, 150.7, 146.9, 137.2, 134.5, 132.7, 130.3, 126.5, (CH) 130.0, 129.5, 129.4, 125.8, 124.2, 122.2, 119.9, 119.3, 109.7, 106.1 (x 2), $\left(\mathrm{CH}_{3}\right)$ 60.8, 55.9 (x 2), 55.8.

IR $v_{\max }\left(\mathrm{cm}^{-1}\right) 3413(\mathrm{~N}-\mathrm{H}), 1754(\mathrm{C}=\mathrm{O})$.

HR ESMS m/z 492.1189 [M+Na] ${ }^{+}$. Calc. for $\mathrm{C}_{25} \mathrm{H}_{24} \mathrm{CINNaO}_{6}, 492.1190$.

\section{2-Bromophenyl (Z)-(2-methoxy-5-(3,4,5-trimethoxystyryl)phenyl)carbamate}

C.8

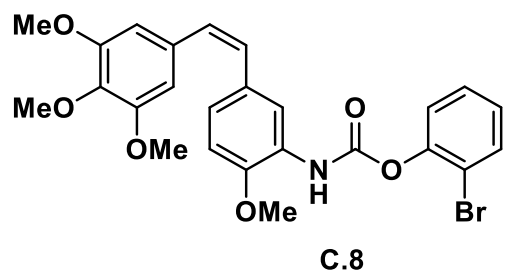

$36 \%$ yield, off-white solid, m. p. 130-132으.

${ }^{1} \mathrm{H}$ NMR $\left(500 \mathrm{MHz}, \mathrm{CDCl}_{3}\right) \delta 8.11$ (br s, 1H), 7.60 (br s, 1H, NH), 7.61 (dd, $J=$ 7.8, $1.5 \mathrm{~Hz}, 1 \mathrm{H}), 7.34$ (td, $J=7.8,1.5 \mathrm{~Hz}, 1 \mathrm{H}), 7.24(\mathrm{dd}, J=7.8,1.5 \mathrm{~Hz}, 1 \mathrm{H}$ ), 7.13 (td, $J=7.8,1.5 \mathrm{~Hz}, 1 \mathrm{H}$ ), 7.01 (dd, $J=8.5,2 \mathrm{~Hz}, 1 \mathrm{H}$ ), $6.76(\mathrm{~d}, J=8.5 \mathrm{~Hz}$, 
$1 \mathrm{H}), 6.52(\mathrm{~s}, 2 \mathrm{H}), 6.49(\mathrm{~d}, J=12 \mathrm{~Hz}, 1 \mathrm{H}), 6.45(\mathrm{~d}, J=12 \mathrm{~Hz}, 1 \mathrm{H}), 3.90(\mathrm{~s}, 3 \mathrm{H})$, $3.78(\mathrm{~s}, 3 \mathrm{H}), 3.70(\mathrm{~s}, 6 \mathrm{H})$.

${ }^{13} \mathrm{C}$ NMR (125 MHz, $\left.\mathrm{CDCl}_{3}\right) \delta(\mathrm{C}) 152.9$ (x 2), 150.2, 147.9, 146.9, 137.2, 132.7, 130.3, 126.6, 116.6, (CH) 133.2, 129.4, 129.2, 128.3, 127.2, 124.2, 124.1, 119.2, 109.7, 106.1 (x 2), $\left(\mathrm{CH}_{3}\right)$ 60.8, 55.9 (x 2), 55.8.

IR $v_{\max }\left(\mathrm{cm}^{-1}\right) 3413(\mathrm{~N}-\mathrm{H}), 1754(\mathrm{C}=\mathrm{O})$.

HR ESMS $m / z 536.0685[\mathrm{M}+\mathrm{Na}]^{+}$. Calc. for $\mathrm{C}_{25} \mathrm{H}_{24} \mathrm{BrNNaO}_{6}, 536.0685$.

\section{3-Bromophenyl (Z)-(2-methoxy-5-(3,4,5-trimethoxystyryl)phenyl)carbamate}

C.9

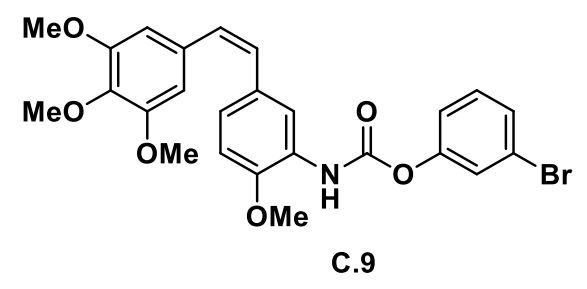

$58 \%$ yield, off-white solid, m. p. 102-105ㄷ.

${ }^{1} \mathrm{H}$ NMR $\left(500 \mathrm{MHz} \mathrm{CDCl}_{3}\right) \delta 8.07$ (br s, 1H), $7.50(\mathrm{br} \mathrm{s}, 1 \mathrm{H}, \mathrm{NH}), 7.40-7.35(\mathrm{~m}$, $2 \mathrm{H}), 7.25$ (t, $J=8 \mathrm{~Hz}, 1 \mathrm{H}), 7.14(\mathrm{br} d, J=8 \mathrm{~Hz}, 1 \mathrm{H}), 7.01(\mathrm{dd}, J=8.5,2 \mathrm{~Hz}, 1 \mathrm{H})$, $6.76(\mathrm{~d}, J=8.5 \mathrm{~Hz}, 1 \mathrm{H}), 6.51(\mathrm{~s}, 2 \mathrm{H}), 6.50(\mathrm{~d}, J=12 \mathrm{~Hz}, 1 \mathrm{H}), 6.46(\mathrm{~d}, J=12 \mathrm{~Hz}$, $1 \mathrm{H}), 3.89(\mathrm{~s}, 3 \mathrm{H}), 3.81(\mathrm{~s}, 3 \mathrm{H}), 3.70(\mathrm{~s}, 6 \mathrm{H})$.

${ }^{13} \mathrm{C}$ NMR $\left(125 \mathrm{MHz}, \mathrm{CDCl}_{3}\right) \delta(\mathrm{C}) 152.9$ (x 2), 151.1, 150.7, 146.9, 137.2, 132.7, 130.3, 126.5, 122.2, (CH) 130.3, 129.4, 129.3, 128.7, 125.1, 124.2, 120.4, 119.3, 109.7, 106.1 (x 2), $\left(\mathrm{CH}_{3}\right)$ 60.8, 55.9 (x 2), 55.8.

IR $v_{\max }\left(\mathrm{cm}^{-1}\right) 3412(\mathrm{~N}-\mathrm{H}), 1753(\mathrm{C}=\mathrm{O})$.

HR ESMS $m / z 536.0687[\mathrm{M}+\mathrm{Na}]^{+}$. Calc. for $\mathrm{C}_{25} \mathrm{H}_{24} \mathrm{BrNNaO}_{6}, 536.0685$. 


\section{4-Bromophenyl (Z)-(2-methoxy-5-(3,4,5-trimethoxystyryl)phenyl)carbamate \\ C.10}

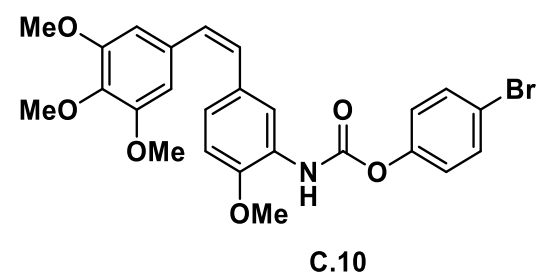

$61 \%$ yield, off-white solid, m. p. 146-149ㄷ․

${ }^{1} \mathrm{H}$ NMR $\left(500 \mathrm{MHz}, \mathrm{CDCl}_{3}\right.$ ) $\delta 8.06$ (br s, 1H), 7.50 (br s, 1H, NH), 7.48 (app d, J $=8.8 \mathrm{~Hz}, 2 \mathrm{H}), 7.07(\operatorname{app~d}, J=8.8 \mathrm{~Hz}, 2 \mathrm{H}), 7.01(\mathrm{dd}, J=8.5,2.0 \mathrm{~Hz}, 1 \mathrm{H}), 6.76$ (d, J=8.5 Hz, 1H), $6.51(\mathrm{~s}, 2 \mathrm{H}), 6.50(\mathrm{~d}, J=12 \mathrm{~Hz}, 1 \mathrm{H}), 6.45(\mathrm{~d}, J=12 \mathrm{~Hz}, 1 \mathrm{H})$, $3.89(\mathrm{~s}, 3 \mathrm{H}), 3.81(\mathrm{~s}, 3 \mathrm{H}), 3.70(\mathrm{~s}, 6 \mathrm{H})$.

${ }^{13} \mathrm{C}$ NMR $\left(125 \mathrm{MHz}, \mathrm{CDCl}_{3}\right) \delta(\mathrm{C}) 152.9$ (x 2), 150.8, 149.7, 146.9, 137.2, 132.7, 130.3, 126.6, 118.5, (CH) 132.3 (x 2), 129.4, 129.3, 124.1, 123.3 (x 2), 119.3, 109.7, 106.1 (x 2), $\left(\mathrm{CH}_{3}\right)$ 60.8, 55.9 (x 2), 55.8.

IR $v_{\max }\left(\mathrm{cm}^{-1}\right) 3415(\mathrm{~N}-\mathrm{H}), 1749(\mathrm{C}=\mathrm{O})$.

HR ESMS $m / z 536.0690[\mathrm{M}+\mathrm{Na}]^{+}$. Calc. for $\mathrm{C}_{25} \mathrm{H}_{24} \mathrm{BrNNaO}_{6}, 536.0685$.

\section{2-Methoxyphenyl (Z)-(2-methoxy-5-(3,4,5-trimethoxystyryl)phenyl)carbamate}

C.11

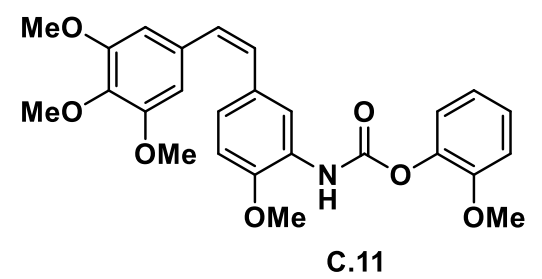

41\% yield, off-white solid, m. p. 110-113ㅇ․

${ }^{1} \mathrm{H}$ NMR $\left(500 \mathrm{MHz}, \mathrm{CDCl}_{3}\right) \delta 8.13(\mathrm{br} \mathrm{s}, 1 \mathrm{H}), 7.60(\mathrm{br} \mathrm{s}, 1 \mathrm{H}, \mathrm{NH}), 7.22$ (td, $J=$ 8.3, $2 \mathrm{~Hz}, 1 \mathrm{H}$ ), 7.14 (dd, J=7.8, $1.5 \mathrm{~Hz}, 1 \mathrm{H}), 7.00-6.95$ (br m, 3H), 6.75 (d, J= $8.5 \mathrm{~Hz}, 1 \mathrm{H}), 6.52(\mathrm{~s}, 2 \mathrm{H}), 6.50(\mathrm{~d}, J=12 \mathrm{~Hz}, 1 \mathrm{H}), 6.43(\mathrm{~d}, J=12 \mathrm{~Hz}, 1 \mathrm{H}), 3.89$ (s, 3H), $3.86(\mathrm{~s}, 3 \mathrm{H}), 3.79(\mathrm{~s}, 3 \mathrm{H}), 3.70(\mathrm{~s}, 6 \mathrm{H})$.

${ }^{13} \mathrm{C}$ NMR $\left(125 \mathrm{MHz}, \mathrm{CDCl}_{3}\right) \delta(\mathrm{C}) 152.8(x 2), 151.7,151.0,146.8,139.5,137.2$, 132.7, 130.3, 127.1, (CH) 129.6, 129.1, 126.7, 123.7, 123.3, 120.7, 119.1, 112.4, 109.6, 106.1 (x 2), $\left(\mathrm{CH}_{3}\right) 60.8,55.9$ (x 3), 55.8. 
IR $v_{\max }\left(\mathrm{cm}^{-1}\right) 3413(\mathrm{~N}-\mathrm{H}), 1751(\mathrm{C}=\mathrm{O})$.

HR ESMS $m / z 488.1684[\mathrm{M}+\mathrm{Na}]^{+}$. Calc. for $\mathrm{C}_{26} \mathrm{H}_{27} \mathrm{NNaO}_{7}, 488.1685$.

3-Methoxyphenyl (Z)-(2-methoxy-5-(3,4,5-trimethoxystyryl)phenyl)carbamate C.12

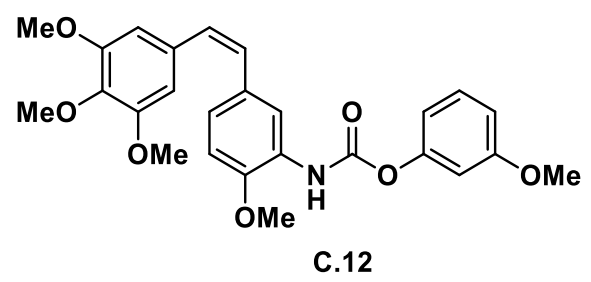

91\% yield, off-white solid, m. p. 105-108ㄷ.

${ }^{1} \mathrm{H}$ NMR $\left(500 \mathrm{MHz} \mathrm{CDCl}_{3}\right) \delta 8.10(\mathrm{br} \mathrm{s}, 1 \mathrm{H}), 7.50$ (br s, $\left.1 \mathrm{H}, \mathrm{NH}\right), 7.28(\mathrm{t}, J=8$ $\mathrm{Hz}, 1 \mathrm{H}), 7.00$ (dd, J=8.3, $2 \mathrm{~Hz}, 1 \mathrm{H}), 6.79(\mathrm{dt}, J=8,2 \mathrm{~Hz}, 2 \mathrm{H}), 6.75(\mathrm{~m}, 2 \mathrm{H})$, $6.52(\mathrm{~s}, 2 \mathrm{H}), 6.51(\mathrm{~d}, J=12 \mathrm{~Hz}, 1 \mathrm{H}), 6.45(\mathrm{~d}, J=12 \mathrm{~Hz}, 1 \mathrm{H}), 3.88(\mathrm{~s}, 3 \mathrm{H}), 3.80$ $(\mathrm{s}, 6 \mathrm{H}), 3.69(\mathrm{~s}, 6 \mathrm{H})$.

${ }^{13} \mathrm{C}$ NMR (125 MHz, $\left.\mathrm{CDCl}_{3}\right) \delta(\mathrm{C})$ 160.4, 152.9 (x 2), 151.6, 151.2, 146.9, 137.2, 132.7, 130.3, 126.8, (CH) 129.6, 129.5, 129.2, 123.9, 119.3, 113.8, 111.5, 109.6, 107.6, 106.1 ( × 2), $\left(\mathrm{CH}_{3}\right) 60.8,55.9$ (x 2), 55.8, 55.3.

IR $v_{\max }\left(\mathrm{cm}^{-1}\right) 3421(\mathrm{~N}-\mathrm{H}), 1751(\mathrm{C}=\mathrm{O})$.

HR ESMS $m / z 488.1683[\mathrm{M}+\mathrm{Na}]^{+}$. Calc. for $\mathrm{C}_{26} \mathrm{H}_{27} \mathrm{NNaO}_{7}, 488.1685$.

4-Methoxyphenyl (Z)-(2-methoxy-5-(3,4,5-trimethoxystyryl)phenyl)carbamate C.13<smiles>COc1ccc(OC(=O)Nc2cc(/C=C\c3cc(OC)c(OC)c(OC)c3)ccc2OC)cc1</smiles>

$73 \%$ yield, off-white solid, m. p. 136-138ㄷ.

${ }^{1} \mathrm{H}$ NMR (500 MHz, CDCl $\left.)_{3}\right) \delta 8.10$ (br s, 1H), 7.50 (br s, 1H, NH), 7.09 (app d, J $=9 \mathrm{~Hz}, 2 \mathrm{H}), 6.99(\mathrm{dd}, J=8.5,2 \mathrm{~Hz}, 1 \mathrm{H}), 6.89(\operatorname{app~d}, J=9 \mathrm{~Hz}, 2 \mathrm{H}), 6.75(\mathrm{~d}, J=$ $8.5 \mathrm{~Hz}, 1 \mathrm{H}), 6.52(\mathrm{~s}, 2 \mathrm{H}), 6.50(\mathrm{~d}, J=12 \mathrm{~Hz}, 1 \mathrm{H}), 6.44$ (d, J=12 Hz, 1H), 3.88 (s, 3H), $3.81(\mathrm{~s}, 3 \mathrm{H}), 3.80(\mathrm{~s}, 3 \mathrm{H}), 3.70(\mathrm{~s}, 6 \mathrm{H})$. 
${ }^{13} \mathrm{C}$ NMR $\left(125 \mathrm{MHz}, \mathrm{CDCl}_{3}\right) \delta(\mathrm{C})$ 157.1, 152.8 (x 2), 151.8, 146.8, 144.1, 137.2, 132.7, 130.3, 126.9, (CH) 129.5, 129.2, 123.8, 122.4 (x 2), 119.2, 114.3 (x 2), 109.6, 106.1 (x 2), $\left(\mathrm{CH}_{3}\right)$ 60.8, 55.8 (x 3), 55.5.

IR $v_{\max }\left(\mathrm{cm}^{-1}\right) 3419(\mathrm{~N}-\mathrm{H}), 1748(\mathrm{C}=\mathrm{O})$.

HR ESMS $m / z 488.1686[\mathrm{M}+\mathrm{Na}]^{+}$. Calc. for $\mathrm{C}_{26} \mathrm{H}_{27} \mathrm{NNaO}_{7}, 488.1685$.

o-Tolyl (Z)-(2-methoxy-5-(3,4,5-trimethoxystyryl)phenyl)carbamate C.14

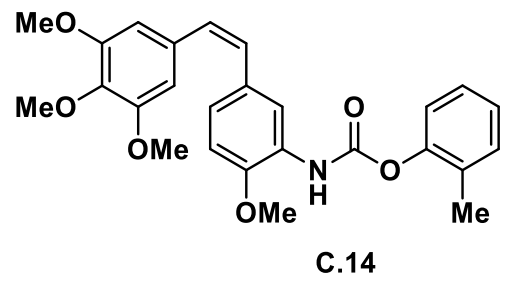

$27 \%$ yield, off-white solid, m. p. 146-148ํ․

${ }^{1} \mathrm{H}$ NMR $\left(500 \mathrm{MHz}, \mathrm{CDCl}_{3}\right) \delta 8.12$ (br s, $\left.1 \mathrm{H}\right), 7.55$ (br s, $\left.1 \mathrm{H}, \mathrm{NH}\right), 7.25-7.20(\mathrm{~m}$, 2H), $7.16(\operatorname{app~t}, J=7.5 \mathrm{~Hz}, 1 \mathrm{H}), 7.10(\mathrm{~d}, J=7.5 \mathrm{~Hz}, 1 \mathrm{H}), 7.01$ (dd, $J=8.5,1.5$ $\mathrm{Hz}, 1 \mathrm{H}), 6.76(\mathrm{~d}, J=8.5 \mathrm{~Hz}, 1 \mathrm{H}), 6.52(\mathrm{~s}, 2 \mathrm{H}), 6.50(\mathrm{~d}, J=12 \mathrm{~Hz}, 1 \mathrm{H}), 6.44(\mathrm{~d}$, $J=12 \mathrm{~Hz}, 1 \mathrm{H}), 3.91(\mathrm{~s}, 3 \mathrm{H}), 3.79(\mathrm{~s}, 3 \mathrm{H}), 3.70(\mathrm{~s}, 6 \mathrm{H}), 2.27(\mathrm{~s}, 3 \mathrm{H})$.

${ }^{13} \mathrm{C}$ NMR $\left(125 \mathrm{MHz}, \mathrm{CDCl}_{3}\right) \delta(\mathrm{C}) 152.9$ (x 2), 151.2, 149.1, 146.8, 137.2, 132.7, 130.7, 130.3, 127.0, (CH) 131.1, 129.6, 129.2, 126.8, 125.9, 123.9, 122.2, 119.2, 109.6, 106.1 (x 2), $\left(\mathrm{CH}_{3}\right)$ 60.8, 55.9 (x 2), 55.8, 16.1 .

IR $v_{\max }\left(\mathrm{cm}^{-1}\right) 3424(\mathrm{~N}-\mathrm{H}), 1750(\mathrm{C}=\mathrm{O})$.

HR ESMS $m / z 472.1727[\mathrm{M}+\mathrm{Na}]^{+}$. Calc. for $\mathrm{C}_{26} \mathrm{H}_{27} \mathrm{NNaO}_{6}, 472.1736$.

m-Tolyl (Z)-(2-methoxy-5-(3,4,5-trimethoxystyryl)phenyl)carbamate C.15

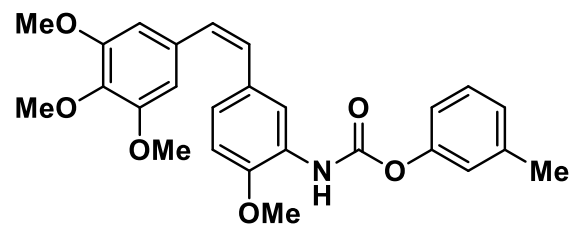

C.15

45\% yield, yellowish solid, m. p. 113-115으.

${ }^{1} \mathbf{H}$ NMR $\left(500 \mathrm{MHz}, \mathrm{CDCl}_{3}\right) \delta 8.10$ (br s, 1H), 7.50 (br s, 1H, NH), 7.28 (app t, J $=7.5 \mathrm{~Hz}, 1 \mathrm{H}), 7.10-7.00(\mathrm{~m}, 4 \mathrm{H}), 6.76(\mathrm{~d}, J=8.5 \mathrm{~Hz}, 1 \mathrm{H}), 6.52(\mathrm{~s}, 2 \mathrm{H}), 6.51(\mathrm{~d}$, 
$J=12 \mathrm{~Hz}, 1 \mathrm{H}), 6.45(\mathrm{~d}, J=12 \mathrm{~Hz}, 1 \mathrm{H}), 3.90(\mathrm{~s}, 3 \mathrm{H}), 3.81(\mathrm{~s}, 3 \mathrm{H}), 3.71(\mathrm{~s}, 6 \mathrm{H})$, $2.38(\mathrm{~s}, 3 \mathrm{H})$.

${ }^{13} \mathrm{C}$ NMR $\left(125 \mathrm{MHz}, \mathrm{CDCl}_{3}\right) \delta(\mathrm{C}) 152.8$ (x 2), 151.5, 150.5, 146.8, 139.4, 137.2, 132.7, 130.3, 126.9, (CH) 129.5, 129.2, 129.0, 126.3, 123.8, 122.2, 119.3, 118.5, 109.6, 106.1 (x 2), $\left(\mathrm{CH}_{3}\right) 60.8,55.9$ (x 2), 55.8, 21.2.

IR $v_{\max }\left(\mathrm{cm}^{-1}\right) 3426(\mathrm{~N}-\mathrm{H}), 1750(\mathrm{C}=\mathrm{O})$.

HR ESMS $m / z 472.1738[\mathrm{M}+\mathrm{Na}]^{+}$. Calc. for $\mathrm{C}_{26} \mathrm{H}_{27} \mathrm{NNaO}_{6}, 472.1736$.

p-Tolyl (Z)-(2-methoxy-5-(3,4,5-trimethoxystyryl)phenyl)carbamate C.16

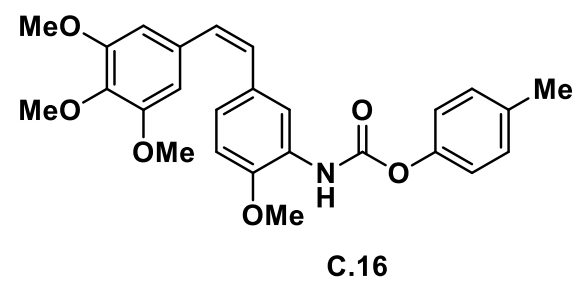

49\% yield, off-white solid, m. p. 162-164ํㅡ.

${ }^{1} \mathbf{H}$ NMR $\left(500 \mathrm{MHz}, \mathrm{CDCl}_{3}\right) \delta 8.10$ (br s, 1H), 7.50 (br s, 1H, NH), 7.18 (app d, J $=8 \mathrm{~Hz}, 2 \mathrm{H}), 7.06(\operatorname{app} \mathrm{d}, J=8, \mathrm{~Hz}, 2 \mathrm{H}), 7.00(\mathrm{dd}, J=8.5,2 \mathrm{~Hz}, 1 \mathrm{H}), 6.75(\mathrm{~d}, J$ $=8.5 \mathrm{~Hz}, 1 \mathrm{H}), 6.52(\mathrm{~s}, 2 \mathrm{H}), 6.50(\mathrm{~d}, J=12 \mathrm{~Hz}, 1 \mathrm{H}), 6.44(\mathrm{~d}, J=12 \mathrm{~Hz}, 1 \mathrm{H}), 3.89$ (s, 3H), $3.81(\mathrm{~s}, 3 \mathrm{H}), 3.70(\mathrm{~s}, 6 \mathrm{H}), 2.36(\mathrm{~s}, 3 \mathrm{H})$.

${ }^{13} \mathrm{C}$ NMR $\left(125 \mathrm{MHz}, \mathrm{CDCl}_{3}\right) \delta(\mathrm{C}) 152.9$ (x 2), 151.6, 148.4, 146.9, 137.2, 135.2, 132.7, 130.3, 127.0, (CH) 129.8 (x 2), 129.6, 129.2, 123.8, 121.3 (x 2), 119.3, 109.6, 106.1 (x 2), $\left(\mathrm{CH}_{3}\right)$ 60.8, 55.9 (x 2), 55.8, 20.8.

IR $v_{\max }\left(\mathrm{cm}^{-1}\right) 3425(\mathrm{~N}-\mathrm{H}), 1750(\mathrm{C}=\mathrm{O})$.

HR ESMS $m / z 472.1728[\mathrm{M}+\mathrm{Na}]^{+}$. Calc. for $\mathrm{C}_{26} \mathrm{H}_{27} \mathrm{NNaO}_{6}, 472.1736$. 
2-(Trifluoromethyl)phenyl (Z)-(2-methoxy-5-(3,4,5-trimethoxystyryl)phenyl)carbamate C.17<smiles>COc1ccc(/C=C\c2cc(OC)c(OC)c(OC)c2)cc1NC(=O)Oc1ccccc1C(F)(F)F</smiles>

$65 \%$ yield, off-white solid, m. p. 132-134으.

${ }^{1} \mathrm{H}$ NMR $\left(500 \mathrm{MHz} \mathrm{CDCl}_{3}\right) \delta 8.09(\mathrm{br} \mathrm{s}, 1 \mathrm{H}), 7.68(\mathrm{~d}, J=7.8 \mathrm{~Hz}, 1 \mathrm{H}), 7.60-7.55$ $(\mathrm{m}, 2 \mathrm{H}), 7.35(\mathrm{~m}, 2 \mathrm{H}), 7.01(\mathrm{~d}, J=8.5 \mathrm{~Hz}, 1 \mathrm{H}), 6.77(\mathrm{~d}, J=8.5 \mathrm{~Hz}, 1 \mathrm{H}), 6.52(\mathrm{~s}$, $2 \mathrm{H}), 6.50(\mathrm{~d}, J=12 \mathrm{~Hz}, 1 \mathrm{H}), 6.45(\mathrm{~d}, J=12 \mathrm{~Hz}, 1 \mathrm{H}), 3.90(\mathrm{~s}, 3 \mathrm{H}), 3.77(\mathrm{~s}, 3 \mathrm{H})$, $3.70(\mathrm{~s}, 6 \mathrm{H})$.

${ }^{13} \mathrm{C}$ NMR $\left(125 \mathrm{MHz}, \mathrm{CDCl}_{3}\right) \delta(\mathrm{C}) 152.9(\mathrm{x} 2), 150.4,148.0,147.0,137.2,130.3$, 126.5, 123.1 (q, $\left.{ }^{2} J_{\mathrm{C}-\mathrm{F}} \sim 31 \mathrm{~Hz}\right), 121.9,(\mathrm{CH}) 132.8,129.4,129.3,126.8\left(\mathrm{q},{ }^{3} \mathrm{~J}_{\mathrm{C}-\mathrm{F}}\right.$ $\sim 4 \mathrm{~Hz}$ ), 125.7, 124.6, 124.3, 119.2, 109.7, 106.0 (x 2), $\left(\mathrm{CH}_{3}\right) 60.8,55.9$ (x 3).

IR $v_{\max }\left(\mathrm{cm}^{-1}\right) 3425(\mathrm{~N}-\mathrm{H}), 1762(\mathrm{C}=\mathrm{O})$.

HR ESMS $m / z$ 526.1448 [M+Na] $]^{+}$. Calc. for $\mathrm{C}_{26} \mathrm{H}_{24} \mathrm{~F}_{3} \mathrm{NNaO}_{6}, 526.1453$.

3-(Trifluoromethyl)phenyl (Z)-(2-methoxy-5-(3,4,5-trimethoxystyryl)phenyl)carbamate $\mathbf{C . 1 8}$

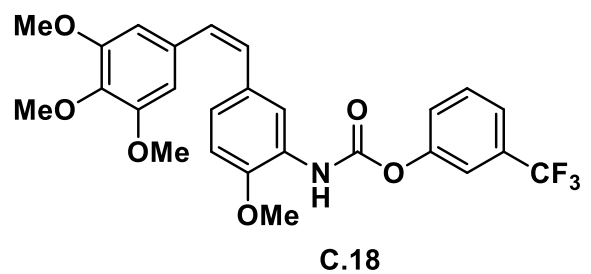

$50 \%$ yield, viscous oil.

${ }^{1} \mathrm{H}$ NMR $\left(500 \mathrm{MHz}, \mathrm{CDCl}_{3}\right) \delta 8.06(\mathrm{br} \mathrm{s}, 1 \mathrm{H}), 7.55-7.45(\mathrm{~m}, 4 \mathrm{H}), 7.40$ (br s, $\left.1 \mathrm{H}\right)$, 7.02 (dd, $J=8.5,2 \mathrm{~Hz}, 1 \mathrm{H}), 6.77$ (d, $J=8.5 \mathrm{~Hz}, 1 \mathrm{H}), 6.51(\mathrm{~s}, 2 \mathrm{H}), 6.51$ (d, J = $12 \mathrm{~Hz}, 1 \mathrm{H}), 6.46(\mathrm{~d}, J=12 \mathrm{~Hz}, 1 \mathrm{H}), 3.91(\mathrm{~s}, 3 \mathrm{H}), 3.80(\mathrm{~s}, 3 \mathrm{H}), 3.70(\mathrm{~s}, 6 \mathrm{H})$.

${ }^{13} \mathrm{C}$ NMR $\left(125 \mathrm{MHz}, \mathrm{CDCl}_{3}\right) \delta(\mathrm{C}) 152.9$ (x 2), 150.7, 150.6, 147.0, 137.2, 132.7, $131.8\left(\mathrm{q},{ }^{2} J_{\mathrm{C}-\mathrm{F}} \sim 33 \mathrm{~Hz}\right), 130.4,126.4,123.5$ (q, $\left.{ }^{1} J_{\mathrm{C}-\mathrm{F}} \sim 270 \mathrm{~Hz}\right),(\mathrm{CH}) 129.9$, 129.4, 129.3, 125.1, 124.3, 122.2 (q, $\left.{ }^{3} \mathrm{~J}_{\mathrm{C}-\mathrm{F}} \sim 4 \mathrm{~Hz}\right), 119.3,118.8$ (q, ${ }^{3} \mathrm{~J}_{\mathrm{C}-\mathrm{F}} \sim 4 \mathrm{~Hz}$ ), 109.7, 106.1 (x 2), $\left(\mathrm{CH}_{3}\right) 60.8,55.9$ (x 2), 55.8. 
IR $v_{\max }\left(\mathrm{cm}^{-1}\right) 3425(\mathrm{~N}-\mathrm{H}), 1753(\mathrm{C}=\mathrm{O})$.

HR ESMS $m / z 526.1452[\mathrm{M}+\mathrm{Na}]^{+}$. Calc. for $\mathrm{C}_{26} \mathrm{H}_{24} \mathrm{~F}_{3} \mathrm{NNaO}_{6}, 526.1453$.

4-(Trifluoromethyl)phenyl (Z)-(2-methoxy-5-(3,4,5-trimethoxystyryl)phenyl)carbamate C.19<smiles>COc1ccc(/C=C\c2cc(OC)c(OC)c(OC)c2)cc1NC(=O)Oc1ccc(C(F)(F)F)cc1</smiles>

$75 \%$ yield, off-white solid, m. p. $138-141^{\circ} \mathrm{C}$.

${ }^{1} \mathrm{H}$ NMR $\left(500 \mathrm{MHz}, \mathrm{CDCl}_{3}\right) \delta 8.07$ (br s, 1H), 7.66 (app d, J=7.5 Hz, 2H), 7.55 (br s, 1H, NH), 7.32 (app d, J=7.5 Hz, 2H), $7.03(\mathrm{~d}, J=8.5 \mathrm{~Hz}, 1 \mathrm{H}), 6.78(\mathrm{~d}, J$ $=8.5 \mathrm{~Hz}, 1 \mathrm{H}), 6.52(\mathrm{~s}, 2 \mathrm{H}), 6.51(\mathrm{~d}, J=12 \mathrm{~Hz}, 1 \mathrm{H}), 6.46(\mathrm{~d}, J=12 \mathrm{~Hz}, 1 \mathrm{H}), 3.91$ (s, 3H), $3.81(\mathrm{~s}, 3 \mathrm{H}), 3.71(\mathrm{~s}, 6 \mathrm{H})$.

${ }^{13} \mathrm{C}$ NMR (125 MHz, $\left.\mathrm{CDCl}_{3}\right) \delta(\mathrm{C})$ 153.2, 152.9 (x 2), 150.5, 147.0, 137.3, 132.7, 130.4, 127.7 (q, $\left.{ }^{2} J_{\mathrm{C}-\mathrm{F}} \sim 32 \mathrm{~Hz}\right), 126.4,123.8$ (q, $\left.{ }^{1} J_{\mathrm{C}-\mathrm{F}} \sim 270 \mathrm{~Hz}\right),(\mathrm{CH}) 129.4$ (x 2), 126.6 (x 2) (q, $\left.{ }^{3} \mathrm{~J}_{\mathrm{C}-\mathrm{F}} \sim 4 \mathrm{~Hz}\right), 124.3,121.9$ (x 2), 119.4, 109.7, 106.1 (x 2), $\left(\mathrm{CH}_{3}\right)$ 60.8, $55.9(\times 2), 55.8$.

IR $v_{\max }\left(\mathrm{cm}^{-1}\right) 3426(\mathrm{~N}-\mathrm{H}), 1751(\mathrm{C}=\mathrm{O})$.

HR ESMS $m / z$ 526.1448 [M+Na] $]^{+}$. Calc. for $\mathrm{C}_{26} \mathrm{H}_{24} \mathrm{~F}_{3} \mathrm{NNaO}_{6}, 526.1453$.

\section{2,6-Dimethylphenyl (Z)-(2-methoxy-5-(3,4,5-trimethoxystyryl)phenyl)-} carbamate C.20

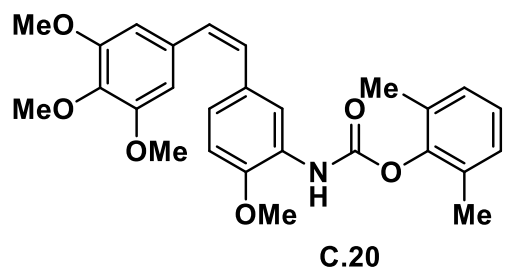

$24 \%$ yield, off-white solid, m. p. 109-111 $\stackrel{\circ}{ } \mathrm{C}$.

${ }^{1} \mathrm{H}$ NMR $\left(500 \mathrm{MHz}, \mathrm{CDCl}_{3}\right) \delta 8.15$ (br s, 1H), 7.60 (br s, 1H, NH), 7.07 (br s, 3H), 7.00 (dd, $J=8.5,1.5 \mathrm{~Hz}, 1 \mathrm{H}$ ), 6.77 (d, $J=8.5 \mathrm{~Hz}, 1 \mathrm{H}$ ), 6.53 (s, 2H), 6.49 (d, J= 
$12 \mathrm{~Hz}, 1 \mathrm{H}), 6.44(\mathrm{~d}, J=12 \mathrm{~Hz}, 1 \mathrm{H}), 3.91$ (s, 3H), 3.78 (s, 3H), 3.70 (s, 6H), 2.23 $(\mathrm{s}, 6 \mathrm{H})$.

${ }^{13} \mathrm{C}$ NMR $\left(125 \mathrm{MHz}, \mathrm{CDCl}_{3}\right) \delta(\mathrm{C}) 152.9$ (x 2), 150.8, 147.7, 146.8, 137.2, 132.7, 130.9 (x 2), 130.3, 127.0, (CH) 129.5, 129.2, 128.5 (x 2), 125.8, 123.8, 119.0, 109.6, 106.1 (x 2), $\left(\mathrm{CH}_{3}\right) 60.8,55.9$ (x 2), 55.8, 16.2 (x 2).

IR $v_{\max }\left(\mathrm{cm}^{-1}\right) 3426(\mathrm{~N}-\mathrm{H}), 1749(\mathrm{C}=\mathrm{O})$.

HR ESMS $m / z$ 486.1891 [M+Na] $]^{+}$. Calc. for $\mathrm{C}_{27} \mathrm{H}_{29} \mathrm{NNaO}_{6}, 486.1893$.

\section{3,5-Dimethylphenyl (Z)-(2-methoxy-5-(3,4,5-trimethoxystyryl)phenyl)-} carbamate C.21

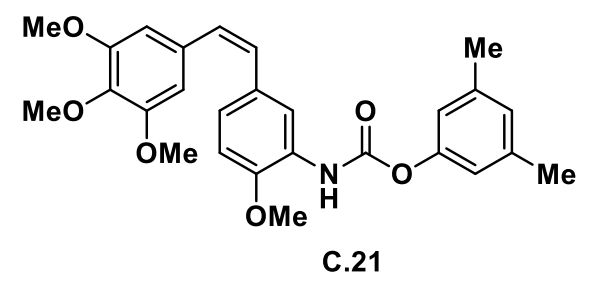

$18 \%$ yield, viscous oil.

${ }^{1} \mathrm{H}$ NMR $\left(500 \mathrm{MHz}, \mathrm{CDCl}_{3}\right) \delta 8.10$ (br s, 1H), 7.50 (br s, 1H, NH), 7.00 (dd, J = 8.5, $1.5 \mathrm{~Hz}, 1 \mathrm{H}), 6.88$ (br s, 1H), 6.80 (br s, 2H), $6.75(\mathrm{~d}, J=8.5 \mathrm{~Hz}, 1 \mathrm{H}), 6.52$ (s, 2H), 6.50 (d, J=12 Hz, 1H), 6.44 (d, J = $12 \mathrm{~Hz}, 1 \mathrm{H}), 3.89$ (s, 3H), 3.81 (s, $3 \mathrm{H}), 3.70(\mathrm{~s}, 6 \mathrm{H}), 2.33(\mathrm{~s}, 6 \mathrm{H})$.

${ }^{13} \mathrm{C}$ NMR (125 MHz, $\left.\mathrm{CDCl}_{3}\right) \delta(\mathrm{C}) 152.9$ (x 2), 151.6, 150.5, 146.8, $139.2(\mathrm{x} 2)$, 137.2* 132.8, 130.4, 127.0, (CH) 129.6, 129.2, 127.3, 123.8, 119.3, 119.2 (x 2), 109.6, 106.1 (x 2), $\left(\mathrm{CH}_{3}\right)$ 60.8, 55.9 (x 2), 55.8, 21.2 (x 2).

IR $v_{\max }\left(\mathrm{cm}^{-1}\right) 3427(\mathrm{~N}-\mathrm{H}), 1750(\mathrm{C}=\mathrm{O})$.

HR ESMS $m / z$ 486.1898 $[\mathrm{M}+\mathrm{Na}]^{+}$. Calc. for $\mathrm{C}_{27} \mathrm{H}_{29} \mathrm{NNaO}_{6}, 486.1893$. 
2,3-Dimethylphenyl (Z)-(2-methoxy-5-(3,4,5-trimethoxystyryl)phenyl)carbamate C.22

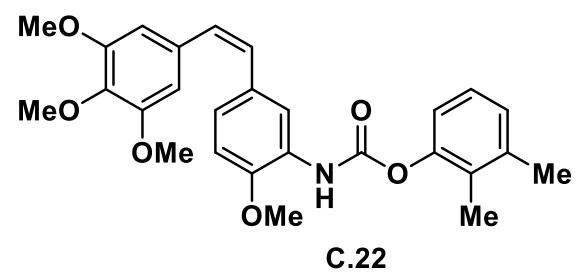

$28 \%$ yield, yellowish solid, m. p. 136-138ㄷ․

${ }^{1} \mathrm{H}$ NMR $\left(500 \mathrm{MHz}_{\mathrm{CDCl}}\right) \delta 8.13$ (br s, 1H), 7.56 (br s, 1H, NH), 7.11 (br t, J= $7.6 \mathrm{~Hz}, 1 \mathrm{H}), 7.06$ (br d, $J=7.6, \mathrm{~Hz}, 1 \mathrm{H}), 7.00(\mathrm{~d}, J=8.5 \mathrm{~Hz}, 1 \mathrm{H}), 6.95(\mathrm{~d}, J=$ $7.6 \mathrm{~Hz}, 1 \mathrm{H}), 6.76(\mathrm{~d}, J=8.5 \mathrm{~Hz}, 1 \mathrm{H}), 6.52(\mathrm{~s}, 2 \mathrm{H}), 6.49(\mathrm{~d}, J=12 \mathrm{~Hz}, 1 \mathrm{H}), 6.44$ (d, J=12 Hz, 1H), 3.91 (s, 3H), 3.80 (s, 3H), 3.70 (s, 6H), 2.32 (s, 3H), 2.16 (s, $3 \mathrm{H})$.

${ }^{13} \mathrm{C}$ NMR $\left(125 \mathrm{MHz}, \mathrm{CDCl}_{3}\right) \delta(\mathrm{C}) 152.9$ (x 2), 151.5, 148.9, 146.8, 138.4, 137.2, 132.7, 130.3, 129.3, 127.1, (CH) 129.6, 129.2, 127.4, 126.0, 123.8, 119.7, 119.2, 109.6, 106.1 (x 2), $\left(\mathrm{CH}_{3}\right)$ 60.8, 55.9 (x 2), 55.8, 20.0, 12.3.

IR $v_{\max }\left(\mathrm{cm}^{-1}\right) 3424(\mathrm{~N}-\mathrm{H}), 1750(\mathrm{C}=\mathrm{O})$.

HR ESMS $m / z 486.1889[\mathrm{M}+\mathrm{Na}]^{+}$. Calc. for $\mathrm{C}_{27} \mathrm{H}_{29} \mathrm{NNaO}_{6}, 486.1893$.

\section{3,4-Dimethylphenyl (Z)-(2-methoxy-5-(3,4,5-trimethoxystyryl)phenyl)-} carbamate $\mathbf{C . 2 3}$

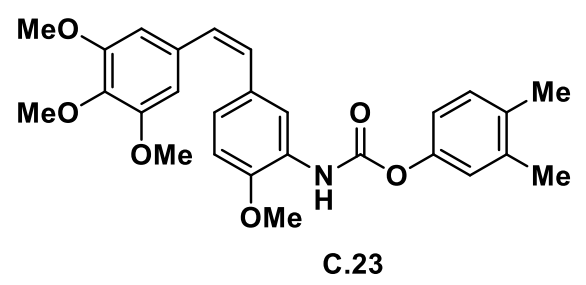

$27 \%$ yield, viscous oil.

${ }^{1} \mathrm{H}$ NMR $\left(500 \mathrm{MHz}, \mathrm{CDCl}_{3}\right) \delta 8.10$ (br s, 1H), 7.50 (br s, 1H, NH), 7.13 (d, J=8 $\mathrm{Hz}, 1 \mathrm{H}), 6.99$ (d, J = 8.5 Hz, 1H), 6.96 (br s, 1H), 6.90 (d, J = 8 Hz, 1H), 6.75 (d, $J=8.5 \mathrm{~Hz}, 1 \mathrm{H}), 6.52(\mathrm{~s}, 2 \mathrm{H}), 6.50(\mathrm{~d}, J=12 \mathrm{~Hz}, 1 \mathrm{H}), 6.44(\mathrm{~d}, J=12 \mathrm{~Hz}, 1 \mathrm{H})$, $3.89(\mathrm{~s}, 3 \mathrm{H}), 3.81(\mathrm{~s}, 3 \mathrm{H}), 3.70(\mathrm{~s}, 6 \mathrm{H}), 2.27(\mathrm{~s}, 3 \mathrm{H}), 2.25(\mathrm{~s}, 3 \mathrm{H})$. 
${ }^{13} \mathrm{C}$ NMR $\left(125 \mathrm{MHz}, \mathrm{CDCl}_{3}\right) \delta(\mathrm{C}) 152.9$ (x 2), 151.8, 148.5, 146.8, 137.7, 137.2, 133.9, 132.8, 130.4, 127.0, (CH) 130.2, 129.7, 129.2, 123.8, 122.6, 119.3, 118.7, 109.6, 106.1 (x 2), $\left(\mathrm{CH}_{3}\right)$ 60.8, 55.9 (x 2), 55.8, 19.8, 19.1.

IR $v_{\max }\left(\mathrm{cm}^{-1}\right) 3427(\mathrm{~N}-\mathrm{H}), 1750(\mathrm{C}=\mathrm{O})$.

HR ESMS $m / z$ 486.1896 $[\mathrm{M}+\mathrm{Na}]^{+}$. Calc. for $\mathrm{C}_{27} \mathrm{H}_{29} \mathrm{NNaO}_{6}, 486.1893$.

3-Chloro-2-methylphenyl (Z)-(2-methoxy-5-(3,4,5-trimethoxystyryl)phenyl)carbamate C.24

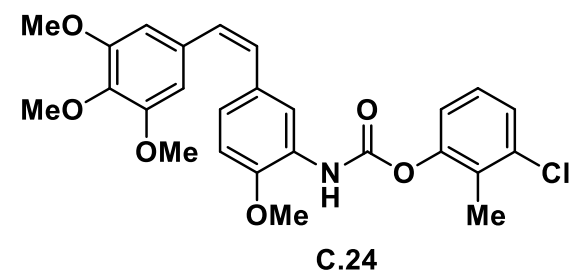

$55 \%$ yield, off-white solid, m. p. $130-132^{\circ} \mathrm{C}$.

${ }^{1} \mathrm{H}$ NMR $\left(500 \mathrm{MHz}, \mathrm{CDCl}_{3}\right) \delta 8.10(\mathrm{br} \mathrm{s}, 1 \mathrm{H}), 7.60(\mathrm{br} \mathrm{s}, 1 \mathrm{H}, \mathrm{NH}), 7.27(\mathrm{~d}, J=8$ $\mathrm{Hz}, 1 \mathrm{H}), 7.15(\mathrm{t}, J=8 \mathrm{~Hz}, 1 \mathrm{H}), 7.05-7.00(\mathrm{~m}, 2 \mathrm{H}), 6.77(\mathrm{~d}, J=8.5 \mathrm{~Hz}, 1 \mathrm{H}), 6.52$ (s, 2H), $6.50(\mathrm{~d}, J=12 \mathrm{~Hz}, 1 \mathrm{H}), 6.45(\mathrm{~d}, J=12 \mathrm{~Hz}, 1 \mathrm{H}), 3.90(\mathrm{~s}, 3 \mathrm{H}), 3.79$ (s, $3 \mathrm{H}), 3.70(\mathrm{~s}, 6 \mathrm{H}), 2.30(\mathrm{~s}, 3 \mathrm{H})$.

${ }^{13} \mathrm{C}$ NMR $\left(125 \mathrm{MHz}, \mathrm{CDCl}_{3}\right) \delta(\mathrm{C}) 152.9$ (x 2), 150.8, 149.6, 146.8, 137.2, 135.3, 132.6, 130.3, 129.7, 126.6, (CH) 129.4, 129.3, 126.7, 126.6, 124.1, 120.8, 119.2, 109.6, 106.1 (x 2), $\left(\mathrm{CH}_{3}\right)$ 60.8, 55.9 (x 2), 55.8, 13.3.

IR $v_{\max }\left(\mathrm{cm}^{-1}\right) 3424(\mathrm{~N}-\mathrm{H}), 1751(\mathrm{C}=\mathrm{O})$.

HR ESMS $m / z$ 506.1354 [M+Na] $]^{+}$. Calc. for $\mathrm{C}_{26} \mathrm{H}_{26} \mathrm{O}_{6} \mathrm{NNaCl}, 506.1346$.

4-Chloro-3-methylphenyl (Z)-(2-methoxy-5-(3,4,5-trimethoxystyryl)phenyl)carbamate C.25

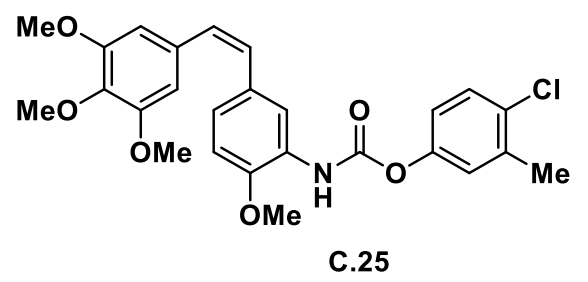


$31 \%$ yield, viscous oil.

${ }^{1} \mathrm{H}$ NMR $\left(500 \mathrm{MHz}, \mathrm{CDCl}_{3}\right) \delta 8.06$ (br s, 1H), 7.50 (br s, 1H, NH), 7.33 (d, J=8.8 $\mathrm{Hz}, 1 \mathrm{H}), 7.08(\mathrm{~s}, 1 \mathrm{H}), 7.01(\mathrm{~d}, J=8.8 \mathrm{~Hz}, 1 \mathrm{H}), 6.96(\mathrm{~d}, J=8.5 \mathrm{~Hz}, 1 \mathrm{H}), 6.76(\mathrm{~d}$, $J=8.5 \mathrm{~Hz}, 1 \mathrm{H}), 6.51(\mathrm{~s}, 2 \mathrm{H}), 6.50(\mathrm{~d}, J=12 \mathrm{~Hz}, 1 \mathrm{H}), 6.45(\mathrm{~d}, J=12 \mathrm{~Hz}, 1 \mathrm{H})$, $3.89(\mathrm{~s}, 3 \mathrm{H}), 3.81(\mathrm{~s}, 3 \mathrm{H}), 3.70(\mathrm{~s}, 6 \mathrm{H}), 2.38(\mathrm{~s}, 3 \mathrm{H})$.

${ }^{13} \mathrm{C} \mathrm{NMR}\left(125 \mathrm{MHz}, \mathrm{CDCl}_{3}\right) \delta(\mathrm{C}) 152.9$ (x 2), 151.2, 148.9, 146.9, 137.3, 137.2, 132.7, 131.1, 130.4, 126.7, (CH) 129.6, 129.5, 129.3, 124.1, 124.0, 120.3, 119.3, 109.7, 106.1 (x 2), $\left(\mathrm{CH}_{3}\right) 60.8,55.9$ (x 2), 55.8, 20.1.

IR $v_{\max }\left(\mathrm{cm}^{-1}\right) 3424(\mathrm{~N}-\mathrm{H}), 1751(\mathrm{C}=\mathrm{O})$.

HR ESMS $m / z 506.1340[\mathrm{M}+\mathrm{Na}]^{+}$. Calc. for $\mathrm{C}_{26} \mathrm{H}_{26} \mathrm{O}_{6} \mathrm{NNaCl}, 506.1346$.

3-Chloro-4-methylphenyl (Z)-(2-methoxy-5-(3,4,5-trimethoxystyryl)phenyl)carbamate C.26

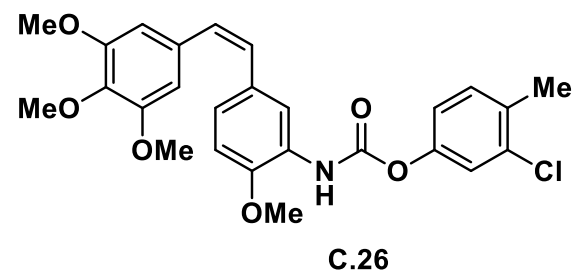

$24 \%$ yield, viscous oil.

${ }^{1} \mathrm{H}$ NMR $\left(500 \mathrm{MHz}, \mathrm{CDCl}_{3}\right) \delta 8.06$ (br s, $\left.1 \mathrm{H}\right), 7.50$ (br s, $\left.1 \mathrm{H}, \mathrm{NH}\right), 7.22(\mathrm{~m}, 2 \mathrm{H})$, $7.00(\mathrm{~m}, 2 \mathrm{H}), 6.75(\mathrm{~d}, J=8.5 \mathrm{~Hz}, 1 \mathrm{H}), 6.51(\mathrm{~s}, 2 \mathrm{H}), 6.50(\mathrm{~d}, J=12 \mathrm{~Hz}, 1 \mathrm{H}), 6.46$ (d, $J=12 \mathrm{~Hz}, 1 \mathrm{H}), 3.90(\mathrm{~s}, 3 \mathrm{H}), 3.81(\mathrm{~s}, 3 \mathrm{H}), 3.70(\mathrm{~s}, 6 \mathrm{H}), 2.37(\mathrm{~s}, 3 \mathrm{H})$.

${ }^{13} \mathrm{C} \mathrm{NMR}\left(125 \mathrm{MHz}, \mathrm{CDCl}_{3}\right) \delta(\mathrm{C}) 152.9$ (x 2), 151.1, 148.9, 146.9, 136.3, 134.4, 133.4, 132.7, 130.4, 126.7, (CH) 131.0, 129.5, 129.3, 124.1, 122.4, 119.9, 119.4, 109.7, 106.1 (x 2), $\left(\mathrm{CH}_{3}\right) 60.9,55.9$ (x 2), 55.8, 19.4 .

IR $v_{\max }\left(\mathrm{cm}^{-1}\right) 3425(\mathrm{~N}-\mathrm{H}), 1752(\mathrm{C}=\mathrm{O})$.

HR ESMS $m / z 506.1350[\mathrm{M}+\mathrm{Na}]^{+}$. Calc. for $\mathrm{C}_{26} \mathrm{H}_{26} \mathrm{O}_{6} \mathrm{NNaCl}, 506.1346$. 


\subsubsection{Synthesis of ureas derived from AmCA-4}

\section{General procedure for the synthesis of ureas}

A solution of the corresponding aniline $(0.5 \mathrm{mmol})$ in dry THF $(2 \mathrm{~mL})$ was treated with $\mathrm{Et}_{3} \mathrm{~N}(5.4 \mathrm{mmol})$ under inert atmosphere. After stirring the mixture for $5 \mathrm{~min}$, a solution of $\mathbf{C . 1}(0.5 \mathrm{mmol})$ in THF $(5 \mathrm{~mL})$ was added. The resulting mixture was then stirred in the dark at $40-50^{\circ} \mathrm{C}$ for $24-72 \mathrm{~h}$ (TLC monitoring). After this time, $\mathrm{CH}_{2} \mathrm{Cl}_{2}(15 \mathrm{ml})$ and $\mathrm{HCl} 1 \mathrm{M}$ were added to the reaction mixture, which was then extracted with $\mathrm{CH}_{2} \mathrm{Cl}_{2}(2 \times 10 \mathrm{~mL})$. The organic layer was washed with brine and then dried on anhydrous $\mathrm{Na}_{2} \mathrm{SO}_{4}$. Removal of volatiles under reduced pressure afforded an oily residue which was subjected to column chromatography on silica-gel (Hexane-EtOAc mixtures as eluent) to afford the desired products with the yields indicated below.

(Z)-1-(2-methoxy-5-(3,4,5-trimethoxystyryl)phenyl)-3-phenylurea U.1

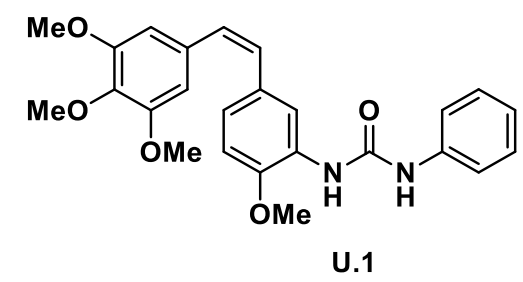

$44 \%$ yield, off-white solid, m. p. 58-62 ${ }^{\circ} \mathrm{C}$.

${ }^{1} \mathbf{H}$ NMR $\left(500 \mathrm{MHz}, \mathrm{CDCl}_{3}\right) \delta 8.03$ (app s, $\left.J=2.0 \mathrm{~Hz}, 1 \mathrm{H}\right), 7.32(\mathrm{~s}, 1 \mathrm{H}), 7.28(\mathrm{~d}$, $J=8.3 \mathrm{~Hz}, 2 \mathrm{H}), 7.20($ app t, $J=8.3,7.8 \mathrm{~Hz}, 2 \mathrm{H}), 7.15(\mathrm{~s}, 1 \mathrm{H}), 6.97(\mathrm{t}, J=7.8$ $\mathrm{Hz}, 1 \mathrm{H}), 6.85(\mathrm{dd}, J=8.3,2.0 \mathrm{~Hz}, 1 \mathrm{H}), 6.58(\mathrm{~d}, J=8.8 \mathrm{~Hz}, 1 \mathrm{H}), 6.44(\mathrm{~s}, 2 \mathrm{H})$, $6.40(\mathrm{~d}, J=12.2 \mathrm{~Hz}, 1 \mathrm{H}), 6.32(\mathrm{~d}, J=12.2 \mathrm{~Hz}, 1 \mathrm{H}), 3.75(\mathrm{~s}, 3 \mathrm{H}), 3.64(\mathrm{~s}, 3 \mathrm{H})$, $3.59(\mathrm{~s}, 6 \mathrm{H})$.

${ }^{13} \mathrm{C}$ NMR (125 MHz, $\left.\mathrm{CDCl}_{3}\right) \delta(\mathrm{C})$ 153.0, 152.8 (x 2), 147.4, 138.4, 137.0, 133.0, 130.3, 128.0, (CH) 129.9, 129.1 (x 2), 129.0, 123.7, 123.4, 120.8, 120.5 (x 2), 109.8, 106.1 (x 2), $\left(\mathrm{CH}_{3}\right)$ 60.9, 55.9 (x 2), 55.8.

IR $\boldsymbol{V}_{\max }\left(\mathrm{cm}^{-1}\right) 3354(\mathrm{~N}-\mathrm{H}), 1541(\mathrm{C}=\mathrm{O})$.

HR ESMS $m / z 457.1733[\mathrm{M}+\mathrm{Na}]^{+}$. Calc. for $\mathrm{C}_{25} \mathrm{H}_{26} \mathrm{~N}_{2} \mathrm{O}_{5} \mathrm{Na}, 457.1739$. 
(Z)-1-(2-fluorophenyl)-3-(2-methoxy-5-(3,4,5-trimethoxystyryl)phenyl)urea U.2

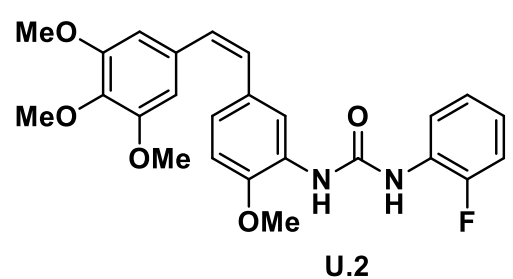

46\% yield, off-white solid, m. p. 69-72ㄷ.

${ }^{1} \mathrm{H}$ NMR $\left(500 \mathrm{MHz}, \mathrm{CDCl}_{3}\right) \delta 8.11$ (app t, $\left.J=8.3 \mathrm{~Hz}, 1 \mathrm{H}\right), 8.10$ (app s, $J=1.5$ $\mathrm{Hz}, 1 \mathrm{H}), 7.49$ (s, 1H), $7.34(\mathrm{~s}, 1 \mathrm{H}), 7.05(\mathrm{~m}, 3 \mathrm{H}), 6.96(\mathrm{dd}, J=8.3,2.5 \mathrm{~Hz}, 1 \mathrm{H})$, $6.68(\mathrm{~d}, \mathrm{~J}=8.3 \mathrm{~Hz}, 1 \mathrm{H}), 6.53(\mathrm{~s}, 2 \mathrm{H}), 6.48(\mathrm{~d}, J=12.2 \mathrm{~Hz}, 1 \mathrm{H}), 6.41(\mathrm{~d}, J=12.2$ $\mathrm{Hz}, 1 \mathrm{H}), 3.84(\mathrm{~s}, 3 \mathrm{H}), 3.74(\mathrm{~s}, 3 \mathrm{H}), 3.68(\mathrm{~s}, 6 \mathrm{H})$.

${ }^{13} \mathrm{C}$ NMR (125 MHz, $\left.\mathrm{CDCl}_{3}\right) \delta(\mathrm{C})$ 152.9, 152.4, 152.8 (x 2), 147.6, 137.0, 132.9, 130.2, 127.7, 126.9, (CH) 129.7, 129.0, 124.4, 120.8, 123.6, 123.3, 121.8, 121.0, 114.8, 109.7, 106.1 (x 2), $\left(\mathrm{CH}_{3}\right) 60.8,55.8$ (x 2), 55.7.

IR $\boldsymbol{V}_{\max }\left(\mathrm{cm}^{-1}\right) 3349(\mathrm{~N}-\mathrm{H}), 1541(\mathrm{C}=\mathrm{O})$.

HR ESMS $m / z 475.1648[\mathrm{M}+\mathrm{Na}]^{+}$. Calc. for $\mathrm{C}_{25} \mathrm{H}_{25} \mathrm{~N}_{2} \mathrm{O}_{5} \mathrm{NaF}, 475.1645$.

(Z)-1-(3-fluorophenyl)-3-(2-methoxy-5-(3,4,5-trimethoxystyryl)phenyl)urea U.3

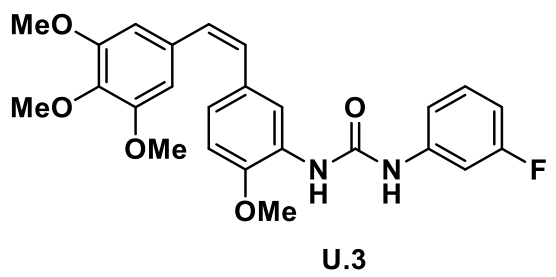

$55 \%$ yield, off-white solid, m. p. 68-70ํㅡ.

${ }^{1} \mathrm{H}$ NMR $\left(500 \mathrm{MHz}, \mathrm{CDCl}_{3}\right) \delta 8.07(\operatorname{app~s}, J=2.0 \mathrm{~Hz}, 1 \mathrm{H}), 7.81(\mathrm{~s}, 1 \mathrm{H}), 7.58(\mathrm{~s}$, $1 \mathrm{H}), 7.28$ (dt, $J=11.3,2.0 \mathrm{~Hz}, 1 \mathrm{H}), 7.14(\mathrm{q}, J=7.8 \mathrm{~Hz}, 1 \mathrm{H}), 7.04(\mathrm{~d}, J=7.8$, $1.0 \mathrm{~Hz}, 1 \mathrm{H}$ ), 6.93 (dd, $J=8.3,2.0 \mathrm{~Hz}, 1 \mathrm{H}), 6.68(\mathrm{td}, J=8.3,2.0 \mathrm{~Hz}, 1 \mathrm{H}), 6.64$ $(\mathrm{d}, J=8.3 \mathrm{~Hz}, 1 \mathrm{H}), 6.52(\mathrm{~s}, 2 \mathrm{H}), 6.44(\mathrm{~d}, J=12.2 \mathrm{~Hz}, 1 \mathrm{H}), 6.38(\mathrm{~d}, J=12.2 \mathrm{~Hz}$, $1 \mathrm{H}), 3.86(\mathrm{~s}, 3 \mathrm{H}), 3.68(\mathrm{~s}, 3 \mathrm{H}), 3.67(\mathrm{~s}, 6 \mathrm{H})$.

${ }^{13} \mathrm{C}$ NMR $\left(125 \mathrm{MHz}, \mathrm{CDCl}_{3}\right) \delta(\mathrm{C})$ 163.0, 152.8, 152.7 (x 2), 147.7, 140.5, 136.7, 133.1, 130.1, 129.9, (CH) 129.8, 128.9, 127.7, 123.6, 121.0, 114.7, 109.7, 109.5, 106.7, 106.1 (x 2), $\left(\mathrm{CH}_{3}\right)$ 60.9, 55.8 (x 2), 55.6.

IR $\boldsymbol{v}_{\max }\left(\mathrm{cm}^{-1}\right) 3353(\mathrm{~N}-\mathrm{H}), 1540(\mathrm{C}=\mathrm{O})$.

HR ESMS $m / z 475.1648[\mathrm{M}+\mathrm{Na}]^{+}$. Calc. for $\mathrm{C}_{25} \mathrm{H}_{25} \mathrm{~N}_{2} \mathrm{O}_{5} \mathrm{NaF}, 475.1645$. 
(Z)-1-(4-fluorophenyl)-3-(2-methoxy-5-(3,4,5-trimethoxystyryl)phenyl)urea U.4

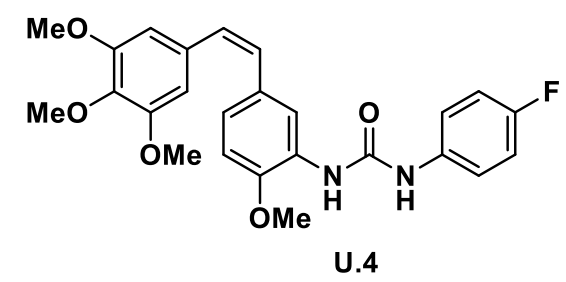

$55 \%$ yield, off-white solid, m. p. $73-76^{\circ} \mathrm{C}$.

${ }^{1} \mathrm{H}$ NMR $\left(500 \mathrm{MHz}, \mathrm{CDCl}_{3}\right) \delta 8.08(\mathrm{~s}, 1 \mathrm{H}), 7.42(\mathrm{~s}, 2 \mathrm{H}), 7.30(\mathrm{~m}, 2 \mathrm{H}), 6.95(\mathrm{~d}, J$ $=8.3 \mathrm{~Hz}, 1 \mathrm{H}), 6.94(\mathrm{~m}, 2 \mathrm{H}), 6.66(\mathrm{~d}, J=8.3 \mathrm{~Hz}, 1 \mathrm{H}), 6.52(\mathrm{~s}, 2 \mathrm{H}), 6.46(\mathrm{~d}, J=$ $12.2 \mathrm{~Hz}, 1 \mathrm{H}), 6.40$ (d, J=12.2 Hz, 1H), $3.84(\mathrm{~s}, 3 \mathrm{H}), 3.70(\mathrm{~s}, 3 \mathrm{H}), 3.67(\mathrm{~s}, 6 \mathrm{H})$.

${ }^{13} \mathrm{C}$ NMR $\left(125 \mathrm{MHz}, \mathrm{CDCl}_{3}\right) \delta(\mathrm{C}) 159.1,153.2,152.7$ (x 2), 147.5, 136.9, 134.4, 133.0, 130.3, 129.8, (CH) 129.0, 127.9, 123.4, 122.3 (x 2), 120.8, 115.6 (x 2), 109.8, 106.1 (x 2), $\left(\mathrm{CH}_{3}\right)$ 60.9, 55.8 (x 2), 55.7.

IR $\boldsymbol{V}_{\max }\left(\mathrm{cm}^{-1}\right) 3353(\mathrm{~N}-\mathrm{H}), 1508(\mathrm{C}=\mathrm{O})$.

HR ESMS $m / z 475.1644[\mathrm{M}+\mathrm{Na}]^{+}$. Calc. for $\mathrm{C}_{25} \mathrm{H}_{25} \mathrm{~N}_{2} \mathrm{O}_{5} \mathrm{NaF}, 475.1645$.

(Z)-1-(2-chlorophenyl)-3-(2-methoxy-5-(3,4,5-trimethoxystyryl)phenyl)urea U.5

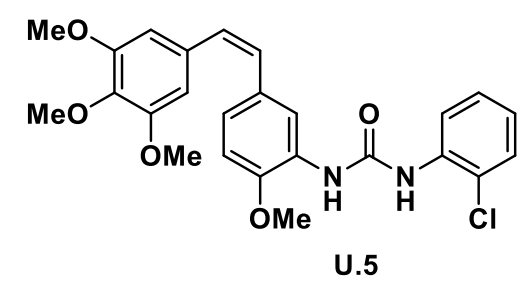

$59 \%$ yield, yellowish solid, m. p. $182-186^{\circ} \mathrm{C}$.

${ }^{1} \mathrm{H}$ NMR $\left(500 \mathrm{MHz}, \mathrm{CDCl}_{3}\right) \delta 8.18(\mathrm{~d}, J=7.8 \mathrm{~Hz}, 1 \mathrm{H}), 8.02(\mathrm{~s}, 1 \mathrm{H}), 7.35(\mathrm{~d}, J=$ $7.8 \mathrm{~Hz}, 1 \mathrm{H}), 7.26(\mathrm{~m}, 1 \mathrm{H}), 7.14(\mathrm{~s}, 1 \mathrm{H}), 7.12(\mathrm{~s}, 1 \mathrm{H}), 6.99(\mathrm{~m}, 2 \mathrm{H}), 6.74(\mathrm{~d}, J=$ $8.3 \mathrm{~Hz}, 1 \mathrm{H}), 6.53(\mathrm{~s}, 2 \mathrm{H}), 6.52(\mathrm{~d}, J=13.2 \mathrm{~Hz}, 1 \mathrm{H}), 6.45(\mathrm{~d}, J=11.7 \mathrm{~Hz}, 1 \mathrm{H})$, $3.84(\mathrm{~s}, 3 \mathrm{H}), 3.84(\mathrm{~s}, 3 \mathrm{H}), 3.70(\mathrm{~s}, 6 \mathrm{H})$.

${ }^{13} \mathrm{C}$ NMR $\left(125 \mathrm{MHz}, \mathrm{CDCl}_{3}\right) \delta(\mathrm{C}) 152.9$ (x 2), 152.0, 147.9, 137.2, 135.2, 132.8, 130.4, 127.3, 122.9, (CH) 129.5, 129.2, 129.0, 127.7, 124.3, 123.8, 121.5, 121.4, 109.9, 106.1 (x 2), $\left(\mathrm{CH}_{3}\right)$ 60.9, 55.9 (x 2), 55.8.

IR $\boldsymbol{V}_{\max }\left(\mathrm{cm}^{-1}\right) 3338(\mathrm{~N}-\mathrm{H}), 1535(\mathrm{C}=\mathrm{O})$.

HR ESMS $m / z 469.1531[\mathrm{M}+\mathrm{H}]^{+}$. Calc. for $\mathrm{C}_{25} \mathrm{H}_{26} \mathrm{CIN}_{2} \mathrm{O}_{5}, 469.1530$. 
(Z)-1-(3-chlorophenyl)-3-(2-methoxy-5-(3,4,5-trimethoxystyryl)phenyl)urea U.6

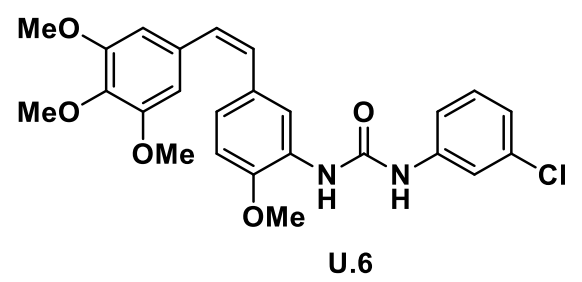

$55 \%$ yield, off-white solid, m. p. 68-70ํㅡ.

${ }^{1} \mathrm{H}$ NMR $\left(500 \mathrm{MHz}, \mathrm{CDCl}_{3}\right) \delta 8.06(\operatorname{app~s}, J=1.8 \mathrm{~Hz}, 1 \mathrm{H}), 7.63(\mathrm{~s}, 1 \mathrm{H}), 7.53(\mathrm{~s}$, $1 \mathrm{H}), 7.45(\mathrm{~s}, 1 \mathrm{H}), 7.22(\mathrm{dd}, J=8.3,1.0 \mathrm{~Hz}, 1 \mathrm{H}), 7.13(\mathrm{t}, J=8.1 \mathrm{~Hz}, 1 \mathrm{H}), 6.97$ (dd, $J=7.8,1.0 \mathrm{~Hz}, 1 \mathrm{H}), 6.94(\mathrm{dd}, J=8.8,2.0 \mathrm{~Hz}, 1 \mathrm{H}), 6.65(\mathrm{~d}, J=8.3 \mathrm{~Hz}, 1 \mathrm{H})$, $6.52(\mathrm{~s}, 2 \mathrm{H}), 6.46(\mathrm{~d}, J=12.2 \mathrm{~Hz}, 1 \mathrm{H}), 6.40(\mathrm{~d}, J=12.2 \mathrm{~Hz}, 1 \mathrm{H}), 3.86(\mathrm{~s}, 3 \mathrm{H})$, $3.70(\mathrm{~s}, 3 \mathrm{H}), 3.67(\mathrm{~s}, 6 \mathrm{H})$.

${ }^{13} \mathrm{C}$ NMR $\left(125 \mathrm{MHz}, \mathrm{CDCl}_{3}\right) \delta(\mathrm{C})$ 152.9, 152.6 (x 2), 147.7, 140.1, 136.7, 134.4, 133.1, 130.1, 129.8, (CH) 129.7, 128.8, 127.7, 123.6, 122.8, 121.0, 119.4, 117.4, 109.7, 106.1 (x 2), $\left(\mathrm{CH}_{3}\right) 60.9,55.8$ (x 2), 55.6.

IR $\boldsymbol{V}_{\max }\left(\mathrm{cm}^{-1}\right) 3352(\mathrm{~N}-\mathrm{H}), 1538(\mathrm{C}=\mathrm{O})$.

HR ESMS $\mathrm{m} / z 491.1351[\mathrm{M}+\mathrm{Na}]^{+}$. Calc. for $\mathrm{C}_{25} \mathrm{H}_{25} \mathrm{~N}_{2} \mathrm{O}_{5} \mathrm{NaCl}, 491.1350$.

(Z)-1-(4-chlorophenyl)-3-(2-methoxy-5-(3,4,5-trimethoxystyryl)phenyl)urea U.7<smiles>COc1ccc(/C=C\c2ccc(OC)c(OC)c2)cc1NC(=O)Nc1ccc(Cl)cc1</smiles>

U.7

$32 \%$ yield, off-white solid, m. p. $71-73^{\circ} \mathrm{C}$.

${ }^{1} \mathbf{H}$ NMR $\left(500 \mathrm{MHz}, \mathrm{CDCl}_{3}\right) \delta 8.06(\mathrm{app} \mathrm{s}, J=2.0 \mathrm{~Hz}, 1 \mathrm{H}), 7.56(\mathrm{~s}, 1 \mathrm{H}), 7.44(\mathrm{~s}$, $1 \mathrm{H}), 7.30(\mathrm{~d}, J=8.3 \mathrm{~Hz}, 2 \mathrm{H}), 7.19(\mathrm{~d}, J=8.8 \mathrm{~Hz}, 2 \mathrm{H}), 6.94(\mathrm{dd}, J=8.3,2.0 \mathrm{~Hz}$, $1 \mathrm{H}), 6.66(\mathrm{~d}, J=8.3 \mathrm{~Hz}, 1 \mathrm{H}), 6.52(\mathrm{~s}, 2 \mathrm{H}), 6.46(\mathrm{~d}, J=12.2 \mathrm{~Hz}, 1 \mathrm{H}), 6.40(\mathrm{~d}, J$ $=11.7 \mathrm{~Hz}, 1 \mathrm{H}), 3.84(\mathrm{~s}, 3 \mathrm{H}), 3.71(\mathrm{~s}, 3 \mathrm{H}), 3.67(\mathrm{~s}, 6 \mathrm{H})$.

${ }^{13} \mathrm{C}$ NMR (125 MHz, $\left.\mathrm{CDCl}_{3}\right) \delta(\mathrm{C})$ 152.8, 152.7 (x 2), 147.5, 137.3, 136.9, 133.0, 130.2, 128.2, 127.7, (CH) 129.8, 129.0, 128.9 (x 2), 123.5, 121.0 (x 2), 120.9, 109.8, 106.1 (x 2), $\left(\mathrm{CH}_{3}\right) 60.9,55.8$ (x 2), 55.7.

IR $\boldsymbol{V}_{\max }\left(\mathrm{cm}^{-1}\right) 3351(\mathrm{~N}-\mathrm{H}), 1540(\mathrm{C}=\mathrm{O})$. 
HR ESMS $m / z 491.1356[\mathrm{M}+\mathrm{Na}]^{+}$. Calc. for $\mathrm{C}_{25} \mathrm{H}_{25} \mathrm{~N}_{2} \mathrm{O}_{5} \mathrm{NaCl}, 491.1350$.

(Z)-1-(2-bromophenyl)-3-(2-methoxy-5-(3,4,5-trimethoxystyryl)phenyl)urea U.8

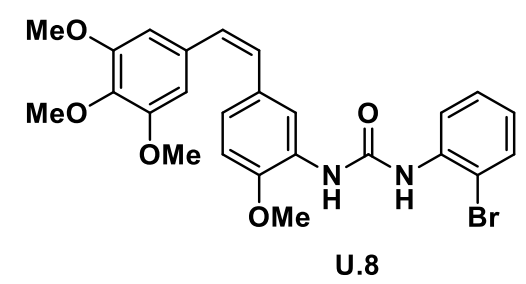

47\% yield, brownish solid, m. p. 163-164\% $\mathrm{C}$.

${ }^{1} \mathrm{H}$ NMR $\left(500 \mathrm{MHz}, \mathrm{CDCl}_{3}\right) \delta 8.14(\mathrm{dd}, J=8.3,1.8 \mathrm{~Hz}, 1 \mathrm{H}), 8.01(\mathrm{~s}, 1 \mathrm{H}), 7.51$ (dd, $J=7.8,1.5 \mathrm{~Hz}, 1 \mathrm{H}), 7.29$ (td, $J=8.3,1.0 \mathrm{~Hz}, 1 \mathrm{H}), 7.18(\mathrm{~s}, 2 \mathrm{H}), 7.01$ (dd, $J$ $=8.3,2.0 \mathrm{~Hz}, 1 \mathrm{H}), 6.93(\mathrm{td}, J=7.3,1.5 \mathrm{~Hz}, 1 \mathrm{H}), 6.73(\mathrm{~d}, J=8.3 \mathrm{~Hz}, 1 \mathrm{H}), 6.53$ $(\mathrm{s}, 2 \mathrm{H}), 6.51(\mathrm{~d}, J=12.2 \mathrm{~Hz}, 1 \mathrm{H}), 6.44(\mathrm{~d}, J=11.7 \mathrm{~Hz}, 1 \mathrm{H}), 3.84(\mathrm{~s}, 3 \mathrm{H}), 3.83$ (s, 3H), $3.70(\mathrm{~s}, 6 \mathrm{H})$.

${ }^{13} \mathrm{C}$ NMR $\left(125 \mathrm{MHz}, \mathrm{CDCl}_{3}\right) \delta(\mathrm{C}) 152.9$ (x 2), 152.2, 148.0, 137.2, 136.4, 132.8, 130.3, 127.2, 113.8, (CH) 132.3, 129.5, 129.2, 128.2, 124.4, 124.4, 122.0, 121.7, 110.0, 106.1 ( × 2), $\left(\mathrm{CH}_{3}\right) 60.9,55.9$ (x 2), 55.8.

IR $\boldsymbol{V}_{\max }\left(\mathrm{cm}^{-1}\right) 3340(\mathrm{~N}-\mathrm{H}), 1530(\mathrm{C}=\mathrm{O})$.

HR ESMS $m / z 513.1022[\mathrm{M}+\mathrm{H}]^{+}$. Calc. for $\mathrm{C}_{25} \mathrm{H}_{26} \mathrm{BrN}_{2} \mathrm{O}_{5}, 513.1025$.

(Z)-1-(3-bromophenyl)-3-(2-methoxy-5-(3,4,5-trimethoxystyryl)phenyl)urea U.9

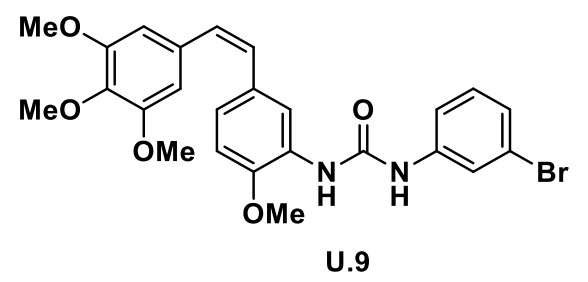

$70 \%$ yield, yellowish solid, m. p. 85-90ㄷ․

${ }^{1} \mathrm{H}$ NMR $\left(500 \mathrm{MHz}, \mathrm{CDCl}_{3}\right) \delta 8.06(\mathrm{~s}, 1 \mathrm{H}), 7.60$ (s, 1H), 7.58 (s, 1H), 7.50 (s, $1 \mathrm{H}), 7.28(\mathrm{~d}, J=7.3 \mathrm{~Hz}, 1 \mathrm{H}), 7.13(\mathrm{~d}, J=7.8 \mathrm{~Hz}, 1 \mathrm{H}), 7.08(\mathrm{t}, J=8.1 \mathrm{~Hz}, 1 \mathrm{H})$, $6.94(\mathrm{~d}, J=8.3 \mathrm{~Hz}, 1 \mathrm{H}), 6.66(\mathrm{~d}, J=8.3 \mathrm{~Hz}, 1 \mathrm{H}), 6.52(\mathrm{~s}, 2 \mathrm{H}), 6.47(\mathrm{~d}, J=12.2$ $\mathrm{Hz}, 1 \mathrm{H}), 6.40$ (d, J=12.2 Hz, 1H), 3.85 (s, 3H), 3.71 (s, 3H), 3.67 (s, 6H). 
${ }^{13} \mathrm{C}$ NMR $\left(125 \mathrm{MHz}, \mathrm{CDCl}_{3}\right) \delta(\mathrm{C})$ 152.8, 152.7 (x 2), 147.7, 140.2, 136.7, 133.1, 130.1, 127.7, 122.5, (CH) 130.1, 129.8, 128.9, 125.9, 123.6, 122.3, 121.0, 118.0, 109.7, 106.1 (x 2), $\left(\mathrm{CH}_{3}\right) 60.9,55.8$ (x 2), 55.6.

IR $\boldsymbol{V}_{\max }\left(\mathrm{cm}^{-1}\right) 3350(\mathrm{~N}-\mathrm{H}), 1536(\mathrm{C}=\mathrm{O})$.

HR ESMS $m / z$ 513.1021 $[\mathrm{M}+\mathrm{H}]^{+}$. Calc. for $\mathrm{C}_{25} \mathrm{H}_{26} \mathrm{BrN}_{2} \mathrm{O}_{5}, 513.1025$.

(Z)-1-(4-bromophenyl)-3-(2-methoxy-5-(3,4,5-trimethoxystyryl)phenyl)urea U.10

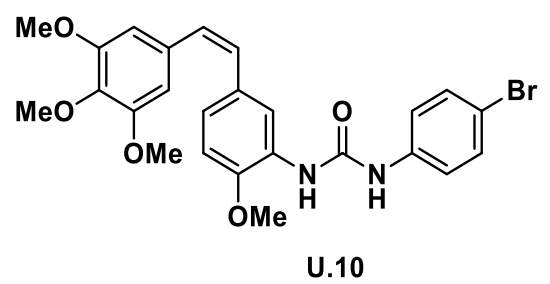

$56 \%$ yield, yellowish solid, m. p. 162-164ํㅡ.

${ }^{1} \mathrm{H}$ NMR $\left(500 \mathrm{MHz}, \mathrm{CDCl}_{3}\right) \delta 8.05(\mathrm{~s}, 1 \mathrm{H}), 7.37(\mathrm{~d}, J=8.3 \mathrm{~Hz}, 2 \mathrm{H}), 7.31(\mathrm{~s}, 1 \mathrm{H})$, $7.27(\mathrm{~s}, 1 \mathrm{H}), 7.24(\mathrm{~d}, J=8.3 \mathrm{~Hz}, 2 \mathrm{H}), 6.96(\mathrm{~d}, J=8.3 \mathrm{~Hz}, 1 \mathrm{H}), 6.69(\mathrm{~d}, J=8.8$ $\mathrm{Hz}, 1 \mathrm{H}), 6.52$ (s, 2H), 6.49 (d, J=12.2 Hz, 1H), $6.42(\mathrm{~d}, J=11.7 \mathrm{~Hz}, 1 \mathrm{H}), 3.85$ (s, 3H), $3.75(\mathrm{~s}, 3 \mathrm{H}), 3.69(\mathrm{~s}, 6 \mathrm{H})$.

${ }^{13} \mathrm{C}$ NMR $\left(125 \mathrm{MHz}, \mathrm{CDCl}_{3}\right) \delta(\mathrm{C}) 152.8(x 2), 152.6,147.5,137.7,137.0,133.0$, 130.4, 127.7, 115.9, (CH) 131.9 (x 2), 129.8, 129.1, 123.7, 121.5 (x 2), 120.9, 109.8, 106.2 (x 2), $\left(\mathrm{CH}_{3}\right)$ 60.9, 55.9 (x 2), 55.8.

IR $\boldsymbol{V}_{\max }\left(\mathrm{cm}^{-1}\right) 3347(\mathrm{~N}-\mathrm{H}), 1538(\mathrm{C}=\mathrm{O})$.

HR ESMS $m / z 513.1021[\mathrm{M}+\mathrm{H}]^{+}$. Calc. for $\mathrm{C}_{25} \mathrm{H}_{26} \mathrm{BrN}_{2} \mathrm{O}_{5}, 513.1025$.

(Z)-1-(2-methoxy-5-(3,4,5-trimethoxystyryl)phenyl)-3-(2-methoxyphenyl)urea U.11<smiles>COc1ccccc1NC(=O)Nc1cc(/C=C\c2cc(OC)c(OC)c(OC)c2)ccc1OC</smiles> 
$46 \%$ yield, off-white solid, m. p. 84-86으.

${ }^{1} \mathrm{H}$ NMR $\left(500 \mathrm{MHz}, \mathrm{CDCl}_{3}\right) \delta 8.12(\mathrm{~m}, 2 \mathrm{H}), 7.20(\mathrm{~s}, 1 \mathrm{H}), 7.15(\mathrm{~s}, 1 \mathrm{H}), 6.99(\mathrm{~m}$, $3 \mathrm{H}), 6.88$ (dd, $J=8.3,1.5 \mathrm{~Hz}, 1 \mathrm{H}), 6.71(\mathrm{~d}, J=8.3 \mathrm{~Hz}, 1 \mathrm{H}), 6.54(\mathrm{~s}, 2 \mathrm{H}), 6.53$ (d, $J=12.2 \mathrm{~Hz}, 1 \mathrm{H}), 6.44(\mathrm{~d}, J=11.7 \mathrm{~Hz}, 1 \mathrm{H}), 3.86(\mathrm{~s}, 3 \mathrm{H}), 3.84(\mathrm{~s}, 3 \mathrm{H}), 3.83$ (s, 3H), $3.70(\mathrm{~s}, 6 \mathrm{H})$.

${ }^{13} \mathrm{C}$ NMR $\left(125 \mathrm{MHz}, \mathrm{CDCl}_{3}\right) \delta(\mathrm{C}) 152.8$ (x 2), 152.3, 148.2, 147.3, 137.1, 132.9, 130.4, 128.1, 128.0, (CH) 129.8, 129.0, 123.4, 122.9, 121.2, 120.7, 119.7, 110.1, 109.7, 106.1 (x 2), $\left(\mathrm{CH}_{3}\right) 60.9,55.9$ (x 2), 55.8, 55.6.

IR $\boldsymbol{V}_{\max }\left(\mathrm{cm}^{-1}\right) 3344(\mathrm{~N}-\mathrm{H}), 1536(\mathrm{C}=\mathrm{O})$.

HR ESMS $m / z$ 465.2024 [M+H] . Calc. for $\mathrm{C}_{26} \mathrm{H}_{29} \mathrm{~N}_{2} \mathrm{O}_{6}, 465.2026$.

\section{(Z)-1-(2-methoxy-5-(3,4,5-trimethoxystyryl)phenyl)-3-(3-methoxyphenyl)urea}

U.12

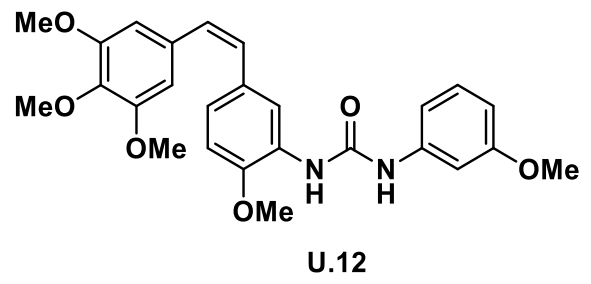

94\% yield, yellowish solid, m. p. $70-72^{\circ} \mathrm{C}$.

${ }^{1} \mathrm{H}$ NMR $\left(500 \mathrm{MHz}, \mathrm{CDCl}_{3}\right) \delta 8.10(\mathrm{~s}, 1 \mathrm{H}), 7.36(\mathrm{~s}, 1 \mathrm{H}), 7.19(\mathrm{t}, J=8.3 \mathrm{~Hz}, 1 \mathrm{H})$, $7.09(\mathrm{~s}, 1 \mathrm{H}), 7.03(\mathrm{~s}, 1 \mathrm{H}), 6.95$ (dd, $J=8.3,2.0 \mathrm{~Hz}, 1 \mathrm{H}), 6.87(\mathrm{~d}, J=8.3 \mathrm{~Hz}$, $1 \mathrm{H}), 6.68(\mathrm{~d}, J=8.8 \mathrm{~Hz}, 1 \mathrm{H}), 6.64(\mathrm{dd}, J=8.3,2.5 \mathrm{~Hz}, 1 \mathrm{H}), 6.53(\mathrm{~s}, 2 \mathrm{H}), 6.51$ (d, $J=12.2 \mathrm{~Hz}, 1 \mathrm{H}), 6.42(\mathrm{~d}, J=12.2 \mathrm{~Hz}, 1 \mathrm{H}), 3.84(\mathrm{~s}, 3 \mathrm{H}), 3.79(\mathrm{~s}, 3 \mathrm{H}), 3.76$ (s, 3H), $3.69(\mathrm{~s}, 6 \mathrm{H})$.

${ }^{13} \mathrm{C}$ NMR $\left(125 \mathrm{MHz}, \mathrm{CDCl}_{3}\right) \delta(\mathrm{C})$ 160.4, 152.8 (x 2), 152.7, 147.4, 139.6, 137.0, 132.9, 130.4, 127.9, (CH) 129.8, 129.0, 123.5, 120.8, 112.7, 109.7, 109.7, 106.3, 106.1 (x 2), $\left(\mathrm{CH}_{3}\right) 60.9,55.9$ (x 2), 55.8, 55.3.

IR $\boldsymbol{v}_{\max }\left(\mathrm{cm}^{-1}\right) 3353(\mathrm{~N}-\mathrm{H}), 1537(\mathrm{C}=\mathrm{O})$.

HR ESMS $m / z 465.2027[\mathrm{M}+\mathrm{H}]^{+}$. Calc. for $\mathrm{C}_{26} \mathrm{H}_{29} \mathrm{~N}_{2} \mathrm{O}_{6}, 465.2026$. 
(Z)-1-(2-methoxy-5-(3,4,5-trimethoxystyryl)phenyl)-3-(4-methoxyphenyl)urea

U.13<smiles>COc1ccc(NC(=O)Nc2cc(/C=C\c3cc(OC)c(OC)c(OC)c3)ccc2OC)cc1</smiles>

$64 \%$ yield, off-white solid, m. p. $170-171^{\circ} \mathrm{C}$.

${ }^{1} \mathrm{H}$ NMR $\left(500 \mathrm{MHz}, \mathrm{CDCl}_{3}\right) \delta 8.12(\mathrm{~s}, 1 \mathrm{H}), 7.28(\mathrm{~s}, 1 \mathrm{H}), 7.26(\mathrm{~d}, J=8.8 \mathrm{~Hz}, 2 \mathrm{H})$, $6.94(\mathrm{dd}, J=8.3,2.0 \mathrm{~Hz}, 1 \mathrm{H}), 6.90(\mathrm{~s}, 1 \mathrm{H}), 6.86(\mathrm{~d}, J=8.8 \mathrm{~Hz}, 2 \mathrm{H}), 6.66(\mathrm{~d}, J$ $=8.3 \mathrm{~Hz}, 1 \mathrm{H}), 6.53(\mathrm{~s}, 2 \mathrm{H}), 6.50(\mathrm{~d}, J=12.2 \mathrm{~Hz}, 1 \mathrm{H}), 6.41(\mathrm{~d}, J=12.2 \mathrm{~Hz}, 1 \mathrm{H})$, $3.84(\mathrm{~s}, 3 \mathrm{H}), 3.79(\mathrm{~s}, 3 \mathrm{H}), 3.73(\mathrm{~s}, 3 \mathrm{H}), 3.68(\mathrm{~s}, 6 \mathrm{H})$.

${ }^{13} \mathrm{C}$ NMR $\left(125 \mathrm{MHz}, \mathrm{CDCl}_{3}\right) \delta(\mathrm{C})$ 156.8, 153.6, 152.8 (x 2), 147.3, 137.0, 132.9, 130.8, 130.3, 128.1, (CH) 129.9, 128.9, 123.9 (x 2), 123.2, 120.6, 114.5 (x 2), 109.7, 106.1 (x 2), $\left(\mathrm{CH}_{3}\right) 60.9,55.9(\times 2), 55.8,55.5$.

IR $\boldsymbol{V}_{\max }\left(\mathrm{cm}^{-1}\right) 3352(\mathrm{~N}-\mathrm{H}), 1510(\mathrm{C}=\mathrm{O})$.

HR ESMS $m / z 465.2021[\mathrm{M}+\mathrm{H}]^{+}$. Calc. for $\mathrm{C}_{26} \mathrm{H}_{29} \mathrm{~N}_{2} \mathrm{O}_{6}, 465.2026$.

(Z)-1-(2-methoxy-5-(3,4,5-trimethoxystyryl)phenyl)-3-(o-tolyl)urea U.14

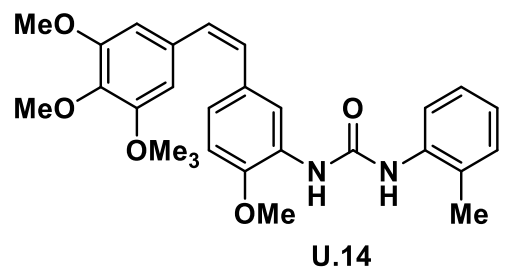

$72 \%$ yield, brownish solid, m. p. 160-164으.

${ }^{1} \mathbf{H}$ NMR $\left(500 \mathrm{MHz}, \mathrm{CDCl}_{3}\right) \delta 8.12(\mathrm{~s}, 1 \mathrm{H}), 7.51(\mathrm{~d}, J=7.3 \mathrm{~Hz}, 1 \mathrm{H}), 7.27(\mathrm{~s}, 1 \mathrm{H})$, 7.22 (app s, 2H), $7.13(\operatorname{app~t}, J=7.3 \mathrm{~Hz}, 1 \mathrm{H}), 6.94(\mathrm{~d}, J=8.3 \mathrm{~Hz}, 1 \mathrm{H}), 6.65$ (s app, 2H), $6.52(\mathrm{~s}, 2 \mathrm{H}), 6.49(\mathrm{~d}, J=11.7 \mathrm{~Hz}, 1 \mathrm{H}), 6.41(\mathrm{~d}, J=12.2 \mathrm{~Hz}, 1 \mathrm{H}), 3.83$ (s, 3H), $3.71(\mathrm{~s}, 3 \mathrm{H}), 3.68(\mathrm{~s}, 6 \mathrm{H}), 2.27(\mathrm{~s}, 3 \mathrm{H})$.

${ }^{13} \mathrm{C}$ NMR (125 MHz, $\mathrm{CDCl}_{3}$ ) $\delta(\mathrm{C})$ 153.6, 152.8 (x 2), 147.3, 137.1, 135.7, 132.9, 132.2, 130.3, 128.0, (CH) 130.9, 129.8, 128.9, 126.9, 125.9, 125.2, 123.3, 120.7, 109.7, 106.1 (x 2), $\left(\mathrm{CH}_{3}\right) 60.8,55.9$ (x 2), 55.8, 17.8.

IR $\boldsymbol{V}_{\max }\left(\mathrm{cm}^{-1}\right) 3342(\mathrm{~N}-\mathrm{H}), 1540(\mathrm{C}=\mathrm{O})$.

HR ESMS $m / z 449.2070[\mathrm{M}+\mathrm{H}]^{+}$. Calc. for $\mathrm{C}_{26} \mathrm{H}_{29} \mathrm{~N}_{2} \mathrm{O}_{5}, 449.2076$. 


\section{(Z)-1-(2-methoxy-5-(3,4,5-trimethoxystyryl)phenyl)-3-(m-tolyl)urea U.15}

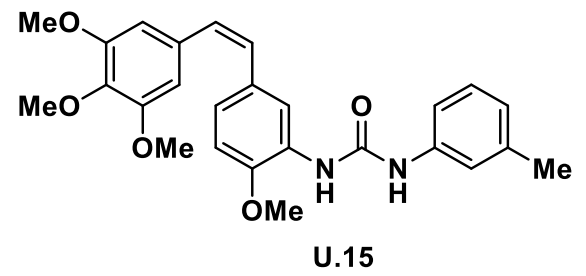

$73 \%$ yield, off-white solid, m. p. 149-151ํㅡ.

${ }^{1} \mathrm{H}$ NMR (500 MHz, $\left.\mathrm{CDCl}_{3}\right) \delta 8.14(\mathrm{~s}, 1 \mathrm{H}), 7.48(\mathrm{~s}, 1 \mathrm{H}), 7.23(\mathrm{~s}, 2 \mathrm{H}), 7.15(\mathrm{~m}$, $2 \mathrm{H}), 6.94(\mathrm{~d}, J=8.3 \mathrm{~Hz}, 1 \mathrm{H}), 6.88(\mathrm{~d}, J=7.3 \mathrm{~Hz}, 1 \mathrm{H}), 6.66(\mathrm{~d}, J=8.3 \mathrm{~Hz}, 1 \mathrm{H})$, $6.53(\mathrm{~s}, 2 \mathrm{H}), 6.49(\mathrm{~d}, J=12.2 \mathrm{~Hz}, 1 \mathrm{H}), 6.41(\mathrm{~d}, J=12.2 \mathrm{~Hz}, 1 \mathrm{H}), 3.85(\mathrm{~s}, 3 \mathrm{H})$, $3.73(\mathrm{~s}, 3 \mathrm{H}), 3.68(\mathrm{~s}, 6 \mathrm{H}), 2.30(\mathrm{~s}, 3 \mathrm{H})$.

${ }^{13} \mathrm{C}$ NMR $\left(125 \mathrm{MHz}, \mathrm{CDCl}_{3}\right) \delta(\mathrm{C})$ 153.0, 152.8 (x 2), 147.4, 139.0, 138.3, 137.0, 133.0, 130.3, 128.1, (CH) 129.9, 128.9, 128.9, 124.5, 123.2, 121.2, 120.7, 117.6, 109.7, 106.1 (x 2), $\left(\mathrm{CH}_{3}\right) 60.8,55.9$ (x 2), 55.7, 21.4.

IR $\boldsymbol{V}_{\max }\left(\mathrm{cm}^{-1}\right) 3353(\mathrm{~N}-\mathrm{H}), 1539(\mathrm{C}=\mathrm{O})$.

HR ESMS $m / z 449.2073[\mathrm{M}+\mathrm{H}]^{+}$. Calc. for $\mathrm{C}_{26} \mathrm{H}_{29} \mathrm{~N}_{2} \mathrm{O}_{5}, 449.2076$.

(Z)-1-(2-methoxy-5-(3,4,5-trimethoxystyryl)phenyl)-3-(p-tolyl)urea U.16

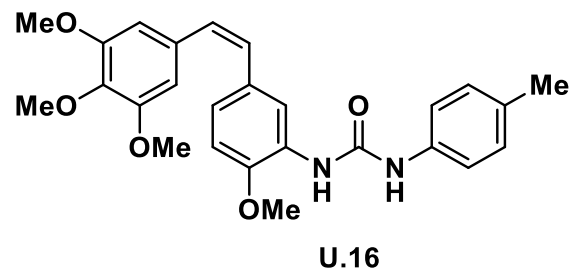

$72 \%$ yield, off-white solid, m. p. 181-183ำ.

${ }^{1} \mathrm{H}$ NMR $\left(500 \mathrm{MHz}, \mathrm{CDCl}_{3}\right) \delta 8.14(\mathrm{~s}, 1 \mathrm{H}), 7.46(\mathrm{~s}, 1 \mathrm{H}), 7.24(\mathrm{app} \mathrm{d}, 3 \mathrm{H}), 7.08$ $(\mathrm{d}, J=7.8 \mathrm{~Hz}, 2 \mathrm{H}), 6.93(\mathrm{~d}, J=8.3 \mathrm{~Hz}, 1 \mathrm{H}), 6.65(\mathrm{~d}, J=8.3 \mathrm{~Hz}, 1 \mathrm{H}), 6.53(\mathrm{~s}$, 2H), 6.48 (d, $J=11.7 \mathrm{~Hz}, 1 \mathrm{H}), 6.40$ (d, $J=12.2 \mathrm{~Hz}, 1 \mathrm{H}), 3.84$ (s, 3H), 3.71 (s, $3 \mathrm{H}), 3.68(\mathrm{~s}, 6 \mathrm{H}), 2.30(\mathrm{~s}, 3 \mathrm{H})$.

${ }^{13} \mathrm{C}$ NMR (125 MHz, $\left.\mathrm{CDCl}_{3}\right) \delta(\mathrm{C})$ 153.3, 152.7 (x 2), 147.4, 136.9, 135.7, 133.4, 133.0, 130.3, 128.1, (CH) 129.9, 129.6 (x 2), 128.9, 123.1, 121.0 (x 2), 120.7, 109.7, 106.1 (x 2), $\left(\mathrm{CH}_{3}\right)$ 60.8, 55.8 (x 2), 55.7, 20.7.

IR $\boldsymbol{V}_{\max }\left(\mathrm{cm}^{-1}\right) 3354(\mathrm{~N}-\mathrm{H}), 1541(\mathrm{C}=\mathrm{O})$.

HR ESMS $m / z 449.2076[\mathrm{M}+\mathrm{H}]^{+}$. Calc. for $\mathrm{C}_{26} \mathrm{H}_{29} \mathrm{~N}_{2} \mathrm{O}_{5}, 449.2076$. 
(Z)-1-(2-methoxy-5-(3,4,5-trimethoxystyryl)phenyl)-3-(2-(trifluoromethyl)phenyl)urea U.17

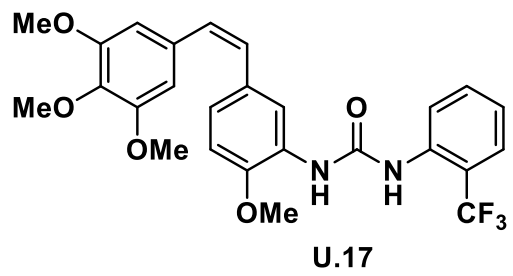

$22 \%$ yield, viscous oil.

${ }^{1} \mathrm{H}$ NMR $\left(500 \mathrm{MHz}, \mathrm{CDCl}_{3}\right) \delta 8.07(\mathrm{~d}, J=8.3 \mathrm{~Hz}, 1 \mathrm{H}), 7.96(\mathrm{~s}, 1 \mathrm{H}), 7.61(\mathrm{~d}, J=$ $7.8 \mathrm{~Hz}, 1 \mathrm{H}), 7.56(\mathrm{t}, J=7.8 \mathrm{~Hz}, 1 \mathrm{H}), 7.21(\mathrm{t}, J=7.6 \mathrm{~Hz}, 1 \mathrm{H}), 7.04(\mathrm{~d}, J=8.3 \mathrm{~Hz}$, $1 \mathrm{H}), 6.91(\mathrm{~s}, 1 \mathrm{H}), 6.80(\mathrm{~s}, 1 \mathrm{H}), 6.75(\mathrm{~d}, J=8.3 \mathrm{~Hz}, 1 \mathrm{H}), 6.54(\mathrm{~s}, 2 \mathrm{H}), 6.52(\mathrm{~d}, J$ $\approx 13.7 \mathrm{~Hz}, 1 \mathrm{H}), 6.46(\mathrm{~d}, J=12.2 \mathrm{~Hz}, 1 \mathrm{H}), 3.86(\mathrm{~s}, 3 \mathrm{H}), 3.84(\mathrm{~s}, 3 \mathrm{H}), 3.71(\mathrm{~s}, 6 \mathrm{H})$. ${ }^{13} \mathrm{C}$ NMR $\left(125 \mathrm{MHz}, \mathrm{CDCl}_{3}\right) \delta(\mathrm{C}) 152.9$ (x 2), 150.4, 148.0, 147.0, 137.3, 130.3, 126.5, 123.1, 122.0, (CH) 132.8, 129.4, 129.3, 126.8, 125.7, 124.6, 124.4, 119.2, 109.8, 106.1 (x 2), $\left(\mathrm{CH}_{3}\right) 60.8,55.9$ (x 3).

IR $\boldsymbol{V}_{\max }\left(\mathrm{cm}^{-1}\right) 3352(\mathrm{~N}-\mathrm{H}), 1540(\mathrm{C}=\mathrm{O})$.

HR ESMS $m / z 525.1612[\mathrm{M}+\mathrm{Na}]^{+}$. Calc. for $\mathrm{C}_{26} \mathrm{H}_{25} \mathrm{~F}_{3} \mathrm{~N}_{2} \mathrm{O}_{5} \mathrm{Na}, 525.1613$.

(Z)-1-(2-methoxy-5-(3,4,5-trimethoxystyryl)phenyl)-3-(3-(trifluoromethyl)phenyl)urea U.18

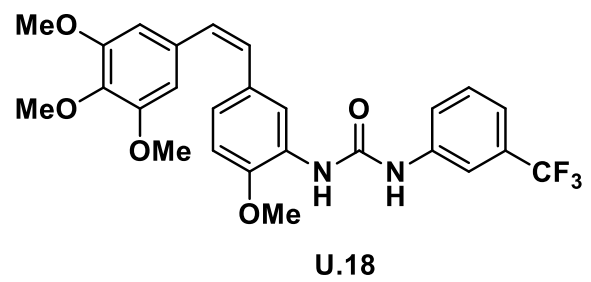

$78 \%$ yield, viscous oil.

${ }^{1} \mathrm{H}$ NMR $\left(500 \mathrm{MHz}, \mathrm{CDCl}_{3}\right) \delta 8.08(\mathrm{~s}, 1 \mathrm{H}), 7.96(\mathrm{~s}, 1 \mathrm{H}), 7.66(\mathrm{~s}, 1 \mathrm{H}), 7.63(\mathrm{~s}$, $1 \mathrm{H}), 7.55(\mathrm{~d}, J=8.3 \mathrm{~Hz}, 1 \mathrm{H}), 7.30(\mathrm{t}, J=7.8 \mathrm{~Hz}, 1 \mathrm{H}), 7.22(\mathrm{~d}, J=7.3 \mathrm{~Hz}, 1 \mathrm{H})$, $6.93(\mathrm{~d}, J=8.3 \mathrm{~Hz}, 1 \mathrm{H}), 6.64(\mathrm{~d}, J=8.3 \mathrm{~Hz}, 1 \mathrm{H}), 6.52(\mathrm{~s}, 2 \mathrm{H}), 6.44(\mathrm{~d}, J=12.2$ $\mathrm{Hz}, 1 \mathrm{H}), 6.38$ (d, J=12.2 Hz, 1H), $3.86(\mathrm{~s}, 3 \mathrm{H}), 3.67$ (s, 3H), $3.66(\mathrm{~s}, 6 \mathrm{H})$.

${ }^{13} \mathrm{C}$ NMR (125 MHz, $\left.\mathrm{CDCl}_{3}\right) \delta(\mathrm{C})$ 152.9, 152.7 (x 2), 147.8, 139.5, 136.7, 133.2, 131.1, 130.1, 127.6, 123.9, (CH) 129.8, 129.3, 128.9, 123.7, 122.4, 121.1, 119.3, 115.9, 109.7, 106.1 (x 2), $\left(\mathrm{CH}_{3}\right) 60.9,55.8$ (x 2), 55.5. 
IR $\boldsymbol{v}_{\max }\left(\mathrm{cm}^{-1}\right) 3352(\mathrm{~N}-\mathrm{H}), 1540(\mathrm{C}=\mathrm{O})$.

HR ESMS $m / z 503.1796[\mathrm{M}+\mathrm{H}]^{+}$. Calc. for $\mathrm{C}_{26} \mathrm{H}_{26} \mathrm{~F}_{3} \mathrm{~N}_{2} \mathrm{O}_{5}, 503.1794$.

(Z)-1-(2-methoxy-5-(3,4,5-trimethoxystyryl)phenyl)-3-(4-(trifluoromethyl)phenyl)urea U.19<smiles>COc1ccc(/C=C\c2cc(OC)c(OC)c(OC)c2)cc1NC(=O)Nc1ccc(C(F)(F)F)cc1</smiles>

$57 \%$ yield, yellowish solid, m. p. 186-188ㄷ.

${ }^{1} \mathrm{H}$ NMR $\left(500 \mathrm{MHz}, \mathrm{CDCl}_{3}\right) \delta 8.05(\mathrm{~s}, 1 \mathrm{H}), 7.50(\mathrm{~s}, 4 \mathrm{H}), 7.41(\mathrm{~s}, 1 \mathrm{H}), 7.34(\mathrm{~s}$, $1 \mathrm{H}), 6.97(\mathrm{~d}, J=8.4 \mathrm{~Hz}, 1 \mathrm{H}), 6.69(\mathrm{~d}, J=8.5 \mathrm{~Hz}, 1 \mathrm{H}), 6.53(\mathrm{~s}, 2 \mathrm{H}), 6.49(\mathrm{~d}, J=$ $12.3 \mathrm{~Hz}, 1 \mathrm{H}), 6.43(\mathrm{~d}, J=12.2 \mathrm{~Hz}, 1 \mathrm{H}), 3.85(\mathrm{~s}, 3 \mathrm{H}), 3.75(\mathrm{~s}, 3 \mathrm{H}), 3.68(\mathrm{~s}, 6 \mathrm{H})$.

${ }^{13} \mathrm{C}$ NMR $\left(125 \mathrm{MHz}, \mathrm{CDCl}_{3}\right) \delta$ (C) 152.8 (x 2), 152.4, 147.7, 142.0, 136.9, 133.1, 130.3, 127.5, 124.9, (CH) 129.8, 129.1, 126.1 (x 2), 123.9, 121.1, 118.7 (x 2), 109.8, 106.2 (x 2), $\left(\mathrm{CH}_{3}\right) 60.9,55.9$ (x 2), 55.7.

IR $\boldsymbol{v}_{\max }\left(\mathrm{cm}^{-1}\right) 3353(\mathrm{~N}-\mathrm{H}), 1540(\mathrm{C}=\mathrm{O})$.

HR ESMS $m / z$ 503.1791 [M+H]+. Calc. for $\mathrm{C}_{26} \mathrm{H}_{26} \mathrm{~F}_{3} \mathrm{~N}_{2} \mathrm{O}_{5}, 503.1794$.

(Z)-1-(2,6-dimethylphenyl)-3-(2-methoxy-5-(3,4,5-trimethoxystyryl)phenyl)urea U.20

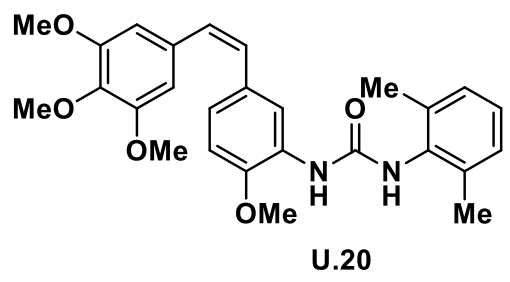

$53 \%$ yield, off-white solid, m. p. 192-194C.

${ }^{1} \mathrm{H}$ NMR $\left(500 \mathrm{MHz}, \mathrm{CDCl}_{3}\right) \delta 8.15(\operatorname{app~d}, J=2.0 \mathrm{~Hz}, 1 \mathrm{H}), 7.16(\mathrm{~m}, 3 \mathrm{H}), 6.93$ (dd, $J=8.3,2.0 \mathrm{~Hz}, 1 \mathrm{H}), 6.87(\mathrm{~s}, 1 \mathrm{H}), 6.62(\mathrm{~d}, J=8.3 \mathrm{~Hz}, 1 \mathrm{H}), 6.53(\mathrm{~s}, 2 \mathrm{H})$, $6.51(\mathrm{~d}, J=12.2 \mathrm{~Hz}, 1 \mathrm{H}), 6.42(\mathrm{~d}, J=12.2 \mathrm{~Hz}, 1 \mathrm{H}), 6.12(\mathrm{~s}, 1 \mathrm{H}), 3.83(\mathrm{~s}, 3 \mathrm{H})$, $3.69(\mathrm{~s}, 6 \mathrm{H}), 3.66(\mathrm{~s}, 3 \mathrm{H}), 2.33(\mathrm{~s}, 6 \mathrm{H})$. 
${ }^{13} \mathrm{C}$ NMR $\left(125 \mathrm{MHz}, \mathrm{CDCl}_{3}\right) \delta(\mathrm{C})$ 153.9, $152.8(x 2), 147.2,137.1,133.7,132.9$ (x 2), $130.3(x 2), 128.1,(\mathrm{CH}) 129.9,128.9,128.7(x)$ 2), 127.9, 123.1, 120.5, 109.6, $106.1(x 2),\left(\mathrm{CH}_{3}\right) 60.9,55.9(x 2), 55.8,18.2(x 2)$.

IR $\boldsymbol{V}_{\max }\left(\mathrm{cm}^{-1}\right) 3341(\mathrm{~N}-\mathrm{H}), 1541(\mathrm{C}=\mathrm{O})$.

HR ESMS $m / z 463.2237[\mathrm{M}+\mathrm{H}]^{+}$. Calc. for $\mathrm{C}_{27} \mathrm{H}_{31} \mathrm{~N}_{2} \mathrm{O}_{5}, 463.2233$.

(Z)-1-(3,5-dimethylphenyl)-3-(2-methoxy-5-(3,4,5-trimethoxystyryl)phenyl)urea U.21

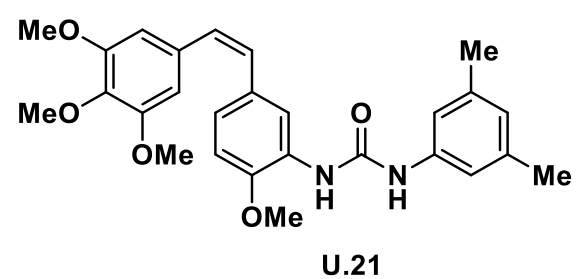

$57 \%$ yield, viscous solid, m. p. $65-68^{\circ} \mathrm{C}$.

${ }^{1} \mathrm{H}$ NMR $\left(500 \mathrm{MHz}, \mathrm{CDCl}_{3}\right) \delta 8.17$ (s, 1H), 7.61 (s, 1H), 7.37 (s, 1H), 6.99 (s, $2 \mathrm{H}), 6.93(\mathrm{~d}, J=8.3 \mathrm{~Hz}, 1 \mathrm{H}), 6.70(\mathrm{~s}, 1 \mathrm{H}), 6.65(\mathrm{~d}, J=8.3 \mathrm{~Hz}, 1 \mathrm{H}), 6.54(\mathrm{~s}, 2 \mathrm{H})$, $6.48(\mathrm{~d}, J=12.2 \mathrm{~Hz}, 1 \mathrm{H}), 6.39(\mathrm{~d}, J=12.2 \mathrm{~Hz}, 1 \mathrm{H}), 3.85(\mathrm{~s}, 3 \mathrm{H}), 3.71(\mathrm{~s}, 3 \mathrm{H})$, $3.68(\mathrm{~s}, 6 \mathrm{H}), 2.25(\mathrm{~s}, 6 \mathrm{H})$.

${ }^{13} \mathrm{C}$ NMR (125 MHz, $\left.\mathrm{CDCl}_{3}\right) \delta(\mathrm{C})$ 153.2, 152.7 (x 2), 147.3, 138.7 (x 2), 138.2, 136.9, 133.0, 130.2, 128.2, (CH) 129.9, 128.8, 125.3, 123.0, 120.6, $118.3(x 2)$, 109.6, 106.1 (x 2), $\left(\mathrm{CH}_{3}\right)$ 60.8, 55.8 (x 2), 55.7, 21.2 (x 2).

IR $\boldsymbol{V}_{\max }\left(\mathrm{cm}^{-1}\right) 3350(\mathrm{~N}-\mathrm{H}), 1537(\mathrm{C}=\mathrm{O})$.

HR ESMS $m / z 463.2226[\mathrm{M}+\mathrm{H}]^{+}$. Calc. for $\mathrm{C}_{27} \mathrm{H}_{31} \mathrm{~N}_{2} \mathrm{O}_{5}, 463.2233$.

(Z)-1-(2,3-dimethylphenyl)-3-(2-methoxy-5-(3,4,5-trimethoxystyryl)phenyl)urea U.22

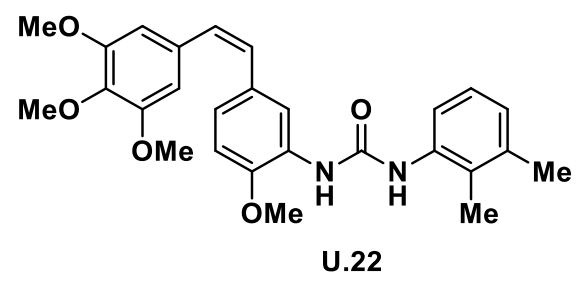


$69 \%$ yield, off-white solid, m. p. 150-153ㅇ․

${ }^{1} \mathrm{H}$ NMR $\left(500 \mathrm{MHz}, \mathrm{CDCl}_{3}\right) \delta 8.15(\mathrm{~s}, 1 \mathrm{H}), 7.27(\mathrm{~d}, J=7.3 \mathrm{~Hz}, 1 \mathrm{H}), 7.24(\mathrm{~s}, 1 \mathrm{H})$, $7.13(\operatorname{app~t}, J=7.8,7.3 \mathrm{~Hz} 1 \mathrm{H}), 7.08(\mathrm{~d}, J=7.3 \mathrm{~Hz}, 1 \mathrm{H}), 6.94(\mathrm{~d}, J=8.3 \mathrm{~Hz}$, $1 \mathrm{H}), 6.65(\mathrm{~d}, J=8.8 \mathrm{~Hz}, 1 \mathrm{H}), 6.63(\mathrm{~s}, 1 \mathrm{H}), 6.53(\mathrm{~s}, 2 \mathrm{H}), 6.49(\mathrm{~d}, J=12.2 \mathrm{~Hz}$, $1 \mathrm{H}), 6.41(\mathrm{~d}, J=12.2 \mathrm{~Hz}, 1 \mathrm{H}), 3.84(\mathrm{~s}, 3 \mathrm{H}), 3.70(\mathrm{~s}, 3 \mathrm{H}), 3.68(\mathrm{~s}, 6 \mathrm{H}), 2.31$ (s, $3 \mathrm{H}), 2.20(\mathrm{~s}, 3 \mathrm{H})$.

${ }^{13} \mathrm{C}$ NMR $\left(125 \mathrm{MHz}, \mathrm{CDCl}_{3}\right) \delta(\mathrm{C})$ 153.9, 152.8 (x 2), 147.2, 138.1, 137.0, 135.4, 132.9, 132.3, 130.3, 128.0, (CH) 129.9, 128.9, 128.1, 126.1, 124.0, 123.1, 120.6, 109.6, 106.1 (x 2), $\left(\mathrm{CH}_{3}\right) 60.8,55.9$ (x 2), 55.8, 20.5, 13.9.

IR $\boldsymbol{V}_{\max }\left(\mathrm{cm}^{-1}\right) 3342(\mathrm{~N}-\mathrm{H}), 1542(\mathrm{C}=\mathrm{O})$.

HR ESMS $m / z 463.2229[\mathrm{M}+\mathrm{H}]^{+}$. Calc. for $\mathrm{C}_{27} \mathrm{H}_{31} \mathrm{~N}_{2} \mathrm{O}_{5}, 463.2233$.

(Z)-1-(3,4-dimethylphenyl)-3-(2-methoxy-5-(3,4,5-trimethoxystyryl)phenyl)urea U.23

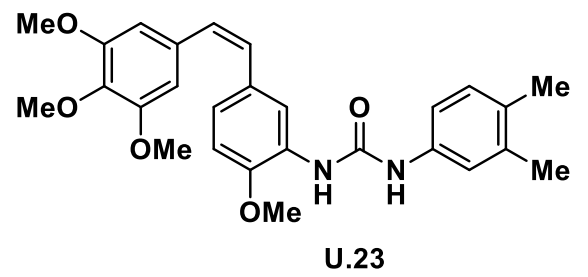

$56 \%$ yield, off-white solid, m. p. 63-64ํㅡ.

${ }^{1} \mathrm{H}$ NMR $\left(500 \mathrm{MHz}, \mathrm{CDCl}_{3}\right) \delta 8.15(\mathrm{~s}, 1 \mathrm{H}), 7.47(\mathrm{~s}, 1 \mathrm{H}), 7.16(\mathrm{~s}, 1 \mathrm{H}), 7.13(\mathrm{~s}$, $1 \mathrm{H}), 7.05(\mathrm{~m}, 2 \mathrm{H}), 6.93(\mathrm{~d}, J=8.3 \mathrm{~Hz}, 1 \mathrm{H}), 6.66(\mathrm{~d}, J=8.3 \mathrm{~Hz}, 1 \mathrm{H}), 6.53(\mathrm{~s}$, 2H), 6.49 (d, $J=12.2 \mathrm{~Hz}, 1 \mathrm{H}), 6.40$ (d, $J=12.2 \mathrm{~Hz}, 1 \mathrm{H}), 3.84(\mathrm{~s}, 3 \mathrm{H}), 3.73$ (s, $3 \mathrm{H}), 3.68(\mathrm{~s}, 6 \mathrm{H}), 2.21(\mathrm{~s}, 6 \mathrm{H})$.

${ }^{13} \mathrm{C}$ NMR $\left(125 \mathrm{MHz}, \mathrm{CDCl}_{3}\right) \delta(\mathrm{C})$ 153.3, 152.8 (x 2), 147.3, 137.4, 137.0, 135.8, 133.0, 132.3, 130.3, 128.2, (CH) 130.1, 129.9, 128.9, 123.1, 122.5, 120.6, 118.6, 109.7, 106.1 (x 2), $\left(\mathrm{CH}_{3}\right) 60.8,55.8$ (x 2), 55.7, 19.8, 19.0.

IR $\boldsymbol{V}_{\max }\left(\mathrm{cm}^{-1}\right) 3354(\mathrm{~N}-\mathrm{H}), 1541(\mathrm{C}=\mathrm{O})$.

HR ESMS $m / z 463.2229[\mathrm{M}+\mathrm{H}]^{+}$. Calc. for $\mathrm{C}_{27} \mathrm{H}_{31} \mathrm{~N}_{2} \mathrm{O}_{5}, 463.2233$. 
(Z)-1-(3-chloro-2-methylphenyl)-3-(2-methoxy-5-(3,4,5-trimethoxystyryl)phenyl)urea U.24

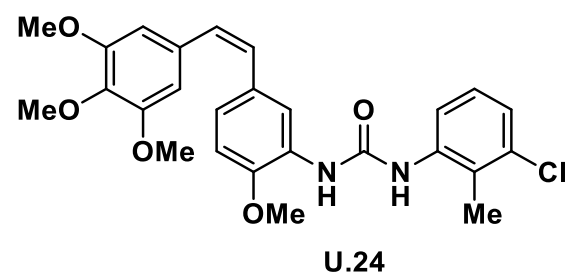

$54 \%$ yield, yellowish solid, m. p. 188-190C.

${ }^{1} \mathrm{H}$ NMR $\left(500 \mathrm{MHz}, \mathrm{CDCl}_{3}\right) \delta 8.08(\mathrm{~s}, 1 \mathrm{H}), 7.43(\mathrm{~d}, J=7.8 \mathrm{~Hz}, 1 \mathrm{H}), 7.26$ (app t, $J=7.8,7.8 \mathrm{~Hz}, 1 \mathrm{H}), 7.19(\mathrm{~s}, 1 \mathrm{H}), 7.15(\mathrm{t}, J=7.8,7.8 \mathrm{~Hz}, 1 \mathrm{H}), 6.97(\mathrm{~d}, J=8.3$ $\mathrm{Hz}, 1 \mathrm{H}), 6.68(\mathrm{~d}, J=8.3 \mathrm{~Hz}, 1 \mathrm{H}), 6.65(\mathrm{~s}, 1 \mathrm{H}), 6.52(\mathrm{~s}, 2 \mathrm{H}), 6.49(\mathrm{~d}, J=12.2$ $\mathrm{Hz}, 1 \mathrm{H}), 6.43(\mathrm{~d}, J=12.2 \mathrm{~Hz}, 1 \mathrm{H}), 3.84(\mathrm{~s}, 3 \mathrm{H}), 3.76(\mathrm{~s}, 3 \mathrm{H}), 3.69(\mathrm{~s}, 6 \mathrm{H}), 2.32$ (s, 3H).

${ }^{13} \mathrm{C}$ NMR $\left(125 \mathrm{MHz}, \mathrm{CDCl}_{3}\right) \delta(\mathrm{C})$ 153.2, 152.8 (x 2), 147.5, 137.1, 137.1, 135.3, 132.9, 130.8, 130.4, 127.7, (CH) 129.7, 129.1, 127.0, 126.8, 123.8, 123.7, 120.9, 109.8, 106.1 (x 2), $\left(\mathrm{CH}_{3}\right) 60.9,55.9$ (x 2), 55.8, 14.8.

IR $\boldsymbol{v}_{\max }\left(\mathrm{cm}^{-1}\right) 3343(\mathrm{~N}-\mathrm{H}), 1543(\mathrm{C}=\mathrm{O})$.

HR ESMS $m / z 483.1682[\mathrm{M}+\mathrm{H}]^{+}$. Calc. for $\mathrm{C}_{26} \mathrm{H}_{28} \mathrm{CIN}_{2} \mathrm{O}_{5}, 483.1687$.

(Z)-1-(4-chloro-3-methylphenyl)-3-(2-methoxy-5-(3,4,5-trimethoxystyryl)phenyl)urea U.25

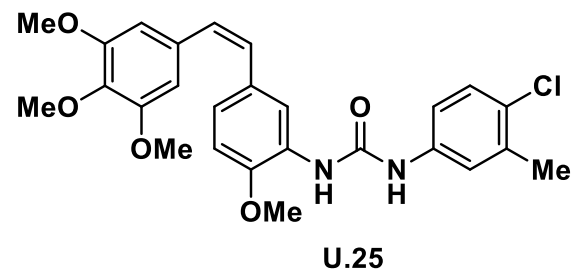

$45 \%$ yield, viscous oil.

${ }^{1} \mathrm{H}$ NMR (500 MHz, $\left.\mathrm{CDCl}_{3}\right) \delta 8.06(\mathrm{~s}, 1 \mathrm{H}), 7.29(\mathrm{~s}, 1 \mathrm{H}), 7.23$ (app d, J = 7.3 Hz, $2 \mathrm{H}), 7.10(\mathrm{~d}, J=8.8 \mathrm{~Hz}, 1 \mathrm{H}), 6.96(\mathrm{~d}, J=8.3 \mathrm{~Hz}, 1 \mathrm{H}), 6.92(\mathrm{~s}, 1 \mathrm{H}), 6.69(\mathrm{~d}, J=$ $8.3 \mathrm{~Hz}, 1 \mathrm{H}), 6.52(\mathrm{~s}, 2 \mathrm{H}), 6.50(\mathrm{~d}, J=12.2 \mathrm{~Hz}, 1 \mathrm{H}), 6.43(\mathrm{~d}, J=12.2 \mathrm{~Hz}, 1 \mathrm{H}$ ), $3.84(\mathrm{~s}, 3 \mathrm{H}), 3.78(\mathrm{~s}, 3 \mathrm{H}), 3.69(\mathrm{~s}, 6 \mathrm{H}), 2.33(\mathrm{~s}, 3 \mathrm{H})$.

${ }^{13} \mathrm{C}$ NMR $\left(125 \mathrm{MHz}, \mathrm{CDCl}_{3}\right) \delta(\mathrm{C}) 152.8$ (x 3), 147.5, 137.0, 136.7, $133.0(\mathrm{x} 2)$, 130.3, 128.8, 127.8, (CH) 129.8, 129.3, 129.0, 123.5, 122.6, 120.9, 118.9, 109.8, 106.1 (x 2), $\left(\mathrm{CH}_{3}\right) 60.9,55.9$ (x 2), 55.7, 20.1. 
IR $\boldsymbol{V}_{\max }\left(\mathrm{cm}^{-1}\right) 3352(\mathrm{~N}-\mathrm{H}), 1541(\mathrm{C}=\mathrm{O})$.

HR ESMS $m / z 483.1683[\mathrm{M}+\mathrm{H}]^{+}$. Calc. for $\mathrm{C}_{26} \mathrm{H}_{28} \mathrm{CIN}_{2} \mathrm{O}_{5}, 483.1687$.

(Z)-1-(3-chloro-4-methylphenyl)-3-(2-methoxy-5-(3,4,5-trimethoxystyryl)phenyl)urea U.26

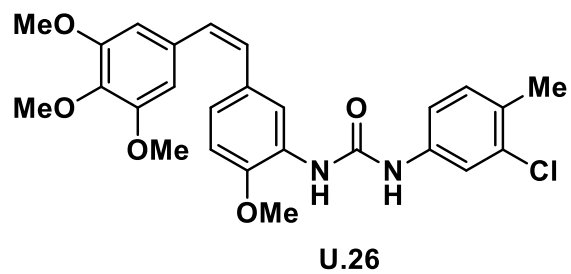

$72 \%$ yield, off-white solid, m. p. $80-84^{\circ}{ }^{\circ} \mathrm{C}$.

${ }^{1} \mathrm{H}$ NMR $\left(500 \mathrm{MHz}, \mathrm{CDCl}_{3}\right) \delta 8.08(\mathrm{~s}, 1 \mathrm{H}), 7.45$ (s, 1H), 7.41 (s, 1H), 7.37 (s, $1 \mathrm{H}), 7.14(\mathrm{~d}, J=8.3 \mathrm{~Hz}, 1 \mathrm{H}), 7.07(\mathrm{~d}, J=8.3 \mathrm{~Hz}, 1 \mathrm{H}), 6.94(\mathrm{~d}, J=8.3 \mathrm{~Hz}, 1 \mathrm{H})$, $6.66(\mathrm{~d}, J=8.3 \mathrm{~Hz}, 1 \mathrm{H}), 6.53(\mathrm{~s}, 2 \mathrm{H}), 6.47(\mathrm{~d}, J=11.7 \mathrm{~Hz}, 1 \mathrm{H}), 6.40(\mathrm{~d}, J=12.2$ $\mathrm{Hz}, 1 \mathrm{H}), 3.85$ (s, 3H), $3.72(\mathrm{~s}, 3 \mathrm{H}), 3.69(\mathrm{~s}, 6 \mathrm{H}), 2.29(\mathrm{~s}, 6 \mathrm{H})$.

${ }^{13} \mathrm{C}$ NMR $\left(125 \mathrm{MHz}, \mathrm{CDCl}_{3}\right) \delta(\mathrm{C})$ 152.8, 152.8 (x 2), 147.6, 137.4, 136.9, 134.4, 133.0, 130.8, 130.2, 127.8, (CH) 131.0, 129.8, 129.0, 123.5, 120.9, 120.6, $118.5,109.8,106.1$ ( $\times 2),\left(\mathrm{CH}_{3}\right) 60.9,55.9$ (x 2), 55.7, 19.3.

IR $\boldsymbol{V}_{\max }\left(\mathrm{cm}^{-1}\right) 3352(\mathrm{~N}-\mathrm{H}), 1537(\mathrm{C}=\mathrm{O})$.

HR ESMS $m / z 483.1683[\mathrm{M}+\mathrm{H}]^{+}$. Calc. for $\mathrm{C}_{26} \mathrm{H}_{28} \mathrm{CIN}_{2} \mathrm{O}_{5}, 483.1687$. 


\subsubsection{Synthesis of family 3 derivatives}

\section{a) Experimental procedure for the synthesis of alkyne 7}

The alcohol $5(0.1 \mathrm{~mol})$ was dissolved in $\mathrm{CH}_{2} \mathrm{Cl}_{2}(150 \mathrm{~mL})$ and cooled at $0^{\circ} \mathrm{C}$. Then, $\mathrm{PBr}_{3}(0.1 \mathrm{~mol})$ was slowly added and the mixture was stirred at $0^{\circ} \mathrm{C}$ under inert atmosphere. Three hours later, the mixture was poured over a saturated aqueous solution of $\mathrm{NaHCO}_{3}$ and extracted with $\mathrm{CH}_{2} \mathrm{Cl}_{2}$. The organic solvent was evaporated under reduced pressure and the crude was purified by flash silica gel chromatography (Hexane as eluent) to afford compound 6. $\mathrm{Next}, \mathrm{CuCl}$ (5 mmol) and compound $6(10 \mathrm{mmol})$ were added to an ethynylmagnesium bromide $(20 \mathrm{mmol})$ solution $0.5 \mathrm{M}$ in THF. The mixture was stirred at reflux $\left(70^{\circ} \mathrm{C}\right)$ for $2 \mathrm{~h}$. After cooling down the reaction mixture, it was poured over a saturated aqueous solution of $\mathrm{NH}_{4} \mathrm{Cl}$ and extracted with $\mathrm{Et}_{2} \mathrm{O}$. The organic phase was washed with $\mathrm{H}_{2} \mathrm{O}$ and brine and dried over anhydrous $\mathrm{MgSO}_{4}$. Finally, the organic solvent was evaporated under reduced pressure and the crude was purified by flash silica gel chromatography (Hexane:EtOAc mixtures as eluent) to afford compound 7.

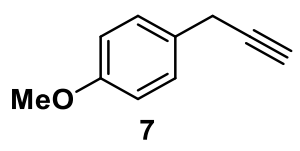

$57 \%$ yield, yellow liquid.

${ }^{1} \mathbf{H}$ NMR $\left(500 \mathrm{MHz}, \mathrm{CDCl}_{3}\right) \delta 7.30(\mathrm{~d}, J=8.8 \mathrm{~Hz}, 2 \mathrm{H}), 6.90(\mathrm{~d}, J=8.3 \mathrm{~Hz}, 2 \mathrm{H})$, $3.82(\mathrm{~s}, 3 \mathrm{H}), 3.58(\mathrm{~d}, J=2.4 \mathrm{~Hz}, 2 \mathrm{H}), 2.21$ (t, $J=2.5 \mathrm{~Hz}, 1 \mathrm{H})$.

${ }^{13} \mathrm{C}$ NMR $\left(125 \mathrm{MHz}, \mathrm{CDCl}_{3}\right) \delta(\mathrm{C}) 158.4,128.1,82.4,(\mathrm{CH}) 128.8(\mathrm{x} 2), 113.9$ (x 2), 70.1, $\left(\mathrm{CH}_{2}\right)$ 23.9, $\left(\mathrm{CH}_{3}\right) 55.2$.

IR $\boldsymbol{V}_{\max }\left(\mathrm{cm}^{-1}\right) 3292(\mathrm{Csp}-\mathrm{H})$.

\section{b) General procedure for the synthesis of ureas A.1-A.8}

A solution of 2-azidoethanamine $(1.2 \mathrm{mmol})$ in DMF was treated with $\mathrm{Et}_{3} \mathrm{~N}(2.4$ mmol). The solution was stirred for $10 \mathrm{~min}$ at $\mathrm{r}$. t. and then CDI $(2.4 \mathrm{mmol})$ was added. The resulting mixture was stirred for $20 \mathrm{~min}$ at $r$. $t$. Then, the corresponding aniline $(2.4 \mathrm{mmol})$ was added to the reaction mixture and stirred overnight at $50^{\circ} \mathrm{C}$. After that time, the solvent was evaporated under reduced pressure and the crude was purified by flash silica gel chromatography (Hexane:EtOAc mixtures as eluent) to afford the desired products with the yields indicated below. 
1-(2-azidoethyl)-3-phenylurea A.1<smiles>N#CCNC(=O)Nc1ccccc1</smiles>

A.1

$28 \%$ yield, off-white solid, m. p. $98-101^{\circ} \mathrm{C}$

${ }^{1} \mathrm{H}$ NMR $\left(500 \mathrm{MHz}, \mathrm{CDCl}_{3}\right) \delta 7.87$ (s, 1H), 7.29 (s, 4H), 7.06 (app t, 1H), 6.16 (s, $1 \mathrm{H}), 3.33$ (s, 4H).

${ }^{13} \mathrm{C} \mathrm{NMR}\left(125 \mathrm{MHz}, \mathrm{CDCl}_{3}\right) \delta(\mathrm{C})$ 156.7, 138.5, (CH) $129.0(x$ 2), 123.4, 120.5 (x 2), $\left(\mathrm{CH}_{2}\right)$ 51.2, 39.4 .

IR V $\max \left(\mathrm{cm}^{-1}\right) 3336(\mathrm{~N}-\mathrm{H}), 2100\left(\mathrm{~N}_{3}\right), 1556(\mathrm{C}=\mathrm{O})$.

HR ESMS $m / z$ 206.1041 [M+H] $]^{+}$. Calc. for $\mathrm{C}_{9} \mathrm{H}_{12} \mathrm{~N}_{5} \mathrm{O}, 206.1042$.

1-(2-azidoethyl)-3-(3-chlorophenyl)urea A.2<smiles>N#CCCNC(=O)Nc1cccc(Cl)c1</smiles>

A.2

$24 \%$ yield, off-white solid, decomposes at $213^{\circ} \mathrm{C}$.

${ }^{1} \mathrm{H}$ NMR $\left(500 \mathrm{MHz}\right.$, acetone- $\left.\mathrm{d}_{6}\right) \delta 8.34(\mathrm{~s}, 1 \mathrm{H}), 7.73(\mathrm{~s}, 1 \mathrm{H}), 7.25(\mathrm{~d}, J=7.3 \mathrm{~Hz}$, $1 \mathrm{H}), 7.18(\mathrm{t}, J=7.2 \mathrm{~Hz}, 1 \mathrm{H}), 6.91(\mathrm{~d}, J=6.9 \mathrm{~Hz}, 1 \mathrm{H}), 6.23(\mathrm{~s}, 1 \mathrm{H}), 3.43(\mathrm{~m}, 4 \mathrm{H})$.

${ }^{13} \mathrm{C}$ NMR (125 MHz, acetone- $\left.\mathrm{d}_{6}\right) \delta(\mathrm{C}) 155.2,141.9,134.1,(\mathrm{CH}) 129.9,121.4$, 118.1, 116.4, $\left(\mathrm{CH}_{2}\right) 51.1,39.2$.

IR V $\max \left(\mathrm{cm}^{-1}\right) 3335(\mathrm{~N}-\mathrm{H}), 2102\left(\mathrm{~N}_{3}\right), 1654(\mathrm{C}=\mathrm{O})$.

HR ESMS m/z $240.0652[\mathrm{M}+\mathrm{H}]^{+}$. Calc. for $\mathrm{C}_{9} \mathrm{H}_{11} \mathrm{CIN}_{5} \mathrm{O}, 240.0652$.

1-(2-azidoethyl)-3-(4-chlorophenyl)urea A.3<smiles>N#CCNC(=O)Nc1ccc(Cl)cc1</smiles>

A.3

$21 \%$ yield, off-white solid, m. p. $127-130^{\circ} \mathrm{C}$.

${ }^{1} \mathrm{H}$ NMR $\left(500 \mathrm{MHz}\right.$, acetone- $\left.\mathrm{d}_{6}\right) \delta 8.18(\mathrm{~s}, 1 \mathrm{H}), 7.47(\mathrm{~d}, J=7.5 \mathrm{~Hz}, 2 \mathrm{H}), 7.21$ (d, $J=7.2 \mathrm{~Hz}, 2 \mathrm{H}), 6.11(\mathrm{~s}, 1 \mathrm{H}), 3.41(\mathrm{~m}, 4 \mathrm{H})$. 
${ }^{13} \mathrm{C}$ NMR (125 MHz, acetone- $\left.\mathrm{d}_{6}\right) \delta(\mathrm{C})$ 156.0, 140.3, $126.6(\mathrm{CH}) 129.0(\mathrm{x} \mathrm{2})$, $120.6(\times 2),\left(\mathrm{CH}_{2}\right) 52.1,39.7$.

IR Vmax $\left(\mathrm{cm}^{-1}\right) 3321(\mathrm{~N}-\mathrm{H}), 2101\left(\mathrm{~N}_{3}\right), 1634(\mathrm{C}=\mathrm{O})$.

HR ESMS m/z 240.0651 [M+H] $]^{+}$. Calc. for $\mathrm{C}_{9} \mathrm{H}_{11} \mathrm{CIN}_{5} \mathrm{O}, 240.0652$.

\section{1-(2-azidoethyl)-3-(3-bromophenyl)urea A.4}<smiles>N#CCNC(=O)Nc1cccc(Br)c1</smiles>

A. 4

$21 \%$ yield, off-white solid, m. p. $104-106^{\circ} \mathrm{C}$.

${ }^{1} \mathrm{H}$ NMR (500 MHz, acetone- $\left.\mathrm{d}_{6}\right) \delta 8.21(\mathrm{~s}, 1 \mathrm{H}), 7.88(\mathrm{~s}, 1 \mathrm{H}), 7.30(\mathrm{~d}, J=8.1 \mathrm{~Hz}$, $1 \mathrm{H}), 7.14(\mathrm{t}, J=8.0 \mathrm{~Hz}, 1 \mathrm{H}), 7.07(\mathrm{~d}, J=7.9 \mathrm{~Hz}, 1 \mathrm{H}), 6.16(\mathrm{~s}, 1 \mathrm{H}), 3.44(\mathrm{~m}, 4 \mathrm{H})$.

${ }^{13} \mathrm{C}$ NMR $\left(125 \mathrm{MHz}\right.$, acetone- $\left.\mathrm{d}_{6}\right) \delta(\mathrm{C})$ 155.1, 142.0, 122.0, (CH) 130.3, 124.3, 120.9, 116.9, $\left(\mathrm{CH}_{2}\right)$ 51.1, 39.2.

IR V $\max \left(\mathrm{cm}^{-1}\right) 3330(\mathrm{~N}-\mathrm{H}), 2102\left(\mathrm{~N}_{3}\right), 1653(\mathrm{C}=\mathrm{O})$.

HR ESMS $m / z 284.0141[\mathrm{M}+\mathrm{H}]^{+}$. Calc. for $\mathrm{C}_{9} \mathrm{H}_{11} \mathrm{BrN}_{5} \mathrm{O}, 284.0147$.

\section{1-(2-azidoethyl)-3-(4-bromophenyl)urea A.5}<smiles>N#CCNC(=O)Nc1ccc(Br)cc1</smiles>

A.5

$30 \%$ yield, off-white solid, m. p. $162-164^{\circ} \mathrm{C}$.

${ }^{1} \mathrm{H}$ NMR $\left(500 \mathrm{MHz}\right.$, acetone- $\left.\mathrm{d}_{6}\right) \delta 8.17(\mathrm{~s}, 1 \mathrm{H}), 7.42(\mathrm{~d}, J=9.0 \mathrm{~Hz}, 2 \mathrm{H}), 7.36(\mathrm{~d}$, $J=9.0 \mathrm{~Hz}, 2 \mathrm{H}), 6.13(\mathrm{~s}, 1 \mathrm{H}), 3.42(\mathrm{~m}, 4 \mathrm{H})$.

${ }^{13} \mathrm{C}$ NMR (125 MHz, acetone- $\left.\mathrm{d}_{6}\right) \delta(\mathrm{C})$ 155.2, 139.8, $113.4(\mathrm{CH}) 131.5(\mathrm{x} 2)$, 120.2 (x 2), $\left(\mathrm{CH}_{2}\right)$ 51.2, 39.2.

IR Vmax $\left(\mathrm{cm}^{-1}\right) 3320(\mathrm{~N}-\mathrm{H}), 2102\left(\mathrm{~N}_{3}\right), 1633(\mathrm{C}=\mathrm{O})$.

HR ESMS $m / z 284.0145[\mathrm{M}+\mathrm{H}]^{+}$. Calc. for $\mathrm{C}_{9} \mathrm{H}_{11} \mathrm{BrN}_{5} \mathrm{O}, 284.0147$. 
1-(2-azidoethyl)-3-(o-tolyl)urea A.6

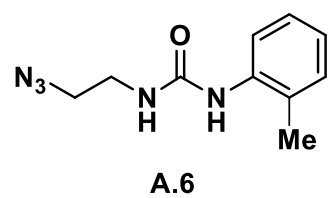

$20 \%$ yield, off-white solid, m. p. $115-116^{\circ} \mathrm{C}$.

${ }^{1} \mathrm{H}$ NMR $\left(500 \mathrm{MHz}, \mathrm{CDCl}_{3}\right) \delta 7.41(\mathrm{~d}, J=7.8 \mathrm{~Hz}, 1 \mathrm{H}), 7.13(\mathrm{~m}, 3 \mathrm{H}), 7.02(\mathrm{t}, J=$ $7.3 \mathrm{~Hz}, 1 \mathrm{H}), 5.99(\mathrm{~s}, 1 \mathrm{H}), 3.28(\mathrm{~s}, 4 \mathrm{H}), 2.18(\mathrm{~s}, 3 \mathrm{H})$.

${ }^{13} \mathrm{C}$ NMR $\left(125 \mathrm{MHz}, \mathrm{CDCl}_{3}\right) \delta(\mathrm{C}) 156.9,136.1,131.6,(\mathrm{CH}) 130.6,126.6,125.1$, 124.7, $\left(\mathrm{CH}_{2}\right)$ 51.3, 39.5, $\left(\mathrm{CH}_{3}\right)$ 17.6.

IR V $\max \left(\mathrm{cm}^{-1}\right) 3314(\mathrm{~N}-\mathrm{H}), 2103\left(\mathrm{~N}_{3}\right), 1635(\mathrm{C}=\mathrm{O})$.

HR ESMS $m / z 220.1197[\mathrm{M}+\mathrm{H}]^{+}$. Calc. for $\mathrm{C}_{10} \mathrm{H}_{14} \mathrm{~N}_{5} \mathrm{O}, 220.1198$.

1-(2-azidoethyl)-3-(m-tolyl)urea A.7

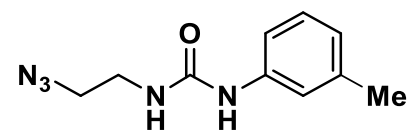

A.7

$23 \%$ yield, yellowish liquid.

${ }^{1} \mathrm{H}$ NMR $\left(500 \mathrm{MHz}, \mathrm{CDCl}_{3}\right) \delta 7.88(\mathrm{~s}, 1 \mathrm{H}), 7.12(\mathrm{~m}, 3 \mathrm{H}), 6.84(\mathrm{~s}, 1 \mathrm{H}), 6.23(\mathrm{~s}$, $1 \mathrm{H}), 3.32(\mathrm{~s}, 4 \mathrm{H}), 2.25(\mathrm{~s}, 3 \mathrm{H})$.

${ }^{13} \mathrm{C}$ NMR $\left(125 \mathrm{MHz}, \mathrm{CDCl}_{3}\right) \delta(\mathrm{C})$ 156.7, 138.8, 138.5, (CH) 128.7, 124.0, 121.0, 117.4, $\left(\mathrm{CH}_{2}\right)$ 51.2, 39.3, $\left(\mathrm{CH}_{3}\right) 21.2$.

IR V $\max \left(\mathrm{cm}^{-1}\right) 3329(\mathrm{~N}-\mathrm{H}), 2101\left(\mathrm{~N}_{3}\right), 1561(\mathrm{C}=\mathrm{O})$.

HR ESMS m/z 220.1195 [M+H] $]^{+}$. Calc. for $\mathrm{C}_{10} \mathrm{H}_{14} \mathrm{~N}_{5} \mathrm{O}, 220.1198$.

1-(2-azidoethyl)-3-(p-tolyl)urea A.8<smiles></smiles>

A.8

$27 \%$ yield, off-white solid, m. p. $109-111^{\circ} \mathrm{C}$.

${ }^{1} \mathrm{H}$ NMR (500 MHz, $\left.\mathrm{CDCl}_{3}\right) \delta 7.57(\mathrm{~s}, 1 \mathrm{H}), 7.14(\mathrm{~d}, J=7.8 \mathrm{~Hz}, 2 \mathrm{H}), 7.06$ (d, J = $7.8 \mathrm{~Hz}, 2 \mathrm{H}), 5.96(\mathrm{~s}, 1 \mathrm{H}), 3.33(\mathrm{~s}, 4 \mathrm{H}), 2.28(\mathrm{~s}, 3 \mathrm{H})$. 
${ }^{13} \mathrm{C} \mathrm{NMR}\left(125 \mathrm{MHz}, \mathrm{CDCl}_{3}\right) \delta(\mathrm{C})$ 156.7, 135.7, 133.3, (CH) 129.6 (x 2), 121.2 (x 2), $\left(\mathrm{CH}_{2}\right)$ 51.3, 39.5, $\left(\mathrm{CH}_{3}\right) 20.7$.

IR Vmax $\left(\mathrm{cm}^{-1}\right) 3326(\mathrm{~N}-\mathrm{H}), 2104\left(\mathrm{~N}_{3}\right), 1641(\mathrm{C}=\mathrm{O})$.

HR ESMS $m / z 220.1198[\mathrm{M}+\mathrm{H}]^{+}$. Calc. for $\mathrm{C}_{10} \mathrm{H}_{14} \mathrm{~N}_{5} \mathrm{O}, 220.1198$.

\section{c) General procedure for the synthesis of triazoles T.1-T.8}

A solution of the alkyne $7(0.2 \mathrm{mmol})$ in DMF was treated with the corresponding previously prepared azido-urea $(0.23 \mathrm{mmol})$. Then, a mixture of $\mathrm{CuSO}_{4} \cdot 5 \mathrm{H}_{2} \mathrm{O}$ $(0.02 \mathrm{mmol})$ and sodium ascorbate $(0.02 \mathrm{mmol})$ in $\mathrm{DMF} / \mathrm{H}_{2} \mathrm{O}(9: 1)$ was added to the reaction mixture which was stirred for $2 \mathrm{~h}$ at $60^{\circ} \mathrm{C}$. After that time, the mixture was concentrated, redissolved in EtOAc and washed with brine repeated times. Finally, the organic solvent was evaporated under reduced pressure and the crude was purified by flash chromatography on silica gel (Hexane:EtOAc mixtures as eluent) to afford the desired products with the yields indicated below.

\section{1-(2-(4-(4-methoxybenzyl)-1H-1,2,3-triazol-1-yl)ethyl)-3-phenylurea T.1}<smiles>COc1ccc(Cc2cn(CCNC(=O)Nc3ccccc3)nn2)cc1</smiles>

$50 \%$ yield, yellowish solid, m. p. $128-131^{\circ} \mathrm{C}$.

${ }^{1} \mathrm{H}$ NMR $\left(500 \mathrm{MHz}, \mathrm{CDCl}_{3}\right) \delta 7.90$ (s, 1H), 7.27 (app s, 3H), 7.21 (t, J = 6.9 Hz, 2H), $7.06(\operatorname{app~d}, J=7.8 \mathrm{~Hz}, 2 \mathrm{H}), 6.98(\mathrm{t}, J=6.4 \mathrm{~Hz}, 1 \mathrm{H}), 6.74(\operatorname{app} \mathrm{d}, J=7.8$ $\mathrm{Hz}, 2 \mathrm{H}$ ), 6.01 (s, 1H), 4.38 (d, J=4.9 Hz, 2H), 3.91 (d, J=4.9 Hz, 2H), 3.70 (app $\mathrm{d}, J=4.9 \mathrm{~Hz}, 3 \mathrm{H}), 3.67$ (t, $J=5.4 \mathrm{~Hz}, 2 \mathrm{H})$.

${ }^{13}$ C NMR $\left(125 \mathrm{MHz}, \mathrm{CDCl}_{3}\right) \delta(\mathrm{C})$ 158.3, 156.0, 139.1, 130.5, 129.8, (CH) 129.6 (x 2), 129.0 (x 2), 122.9, 121.5, 119.5 (x 2), 114.1 (x 2), $\left(\mathrm{CH}_{2}\right)$ 50.2, 39.6, 31.1, $\left(\mathrm{CH}_{3}\right) 55.2$.

IR $v_{\max }\left(\mathrm{cm}^{-1}\right) 3342(\mathrm{~N}-\mathrm{H}), 1678(\mathrm{C}=\mathrm{O})$.

HR ESMS $m / z 352.1771[\mathrm{M}+\mathrm{H}]^{+}$. Calc. for $\mathrm{C}_{19} \mathrm{H}_{22} \mathrm{~N}_{5} \mathrm{O}_{2}, 352.1769$. 
1-(3-chlorophenyl)-3-(2-(4-(4-methoxybenzyl)-1H-1,2,3-triazol-1yl)ethyl)urea T.2<smiles>[R]Oc1ccc(Cc2cn(CCNC(=O)Nc3cccc(Cl)c3)nn2)cc1</smiles>

$52 \%$ yield, off-white solid, m. p. 171-172 $\mathrm{C}$.

${ }^{1} \mathbf{H}$ NMR (500 MHz, DMSO-d 6 ) $\delta 8.81$ (s, 1H), 7.80 (s, 1H), 7.67 (app t, J = 2.1 $\mathrm{Hz}, 1 \mathrm{H}), 7.20(\mathrm{~m}, 4 \mathrm{H}), 6.95(\mathrm{~d}, J=8.8 \mathrm{~Hz}, 1 \mathrm{H}), 6.81(\mathrm{~d}, J=8.2 \mathrm{~Hz}, 2 \mathrm{H}), 6.32(\mathrm{t}$, $J=5.3 \mathrm{~Hz}, 1 \mathrm{H}), 4.41(\mathrm{t}, J=5.3 \mathrm{~Hz}, 2 \mathrm{H}), 3.92(\mathrm{~s}, 2 \mathrm{H}), 3.71(\mathrm{~s}, 3 \mathrm{H}), 3.56$ (app t, $J=5.3 \mathrm{~Hz}, 2 \mathrm{H})$.

${ }^{13} \mathrm{C}$ NMR (125 MHz, DMSO-d 6 ) $\delta(C)$ 158.1, 155.4, 146.9, 142.4, 133.6, 131.9, (CH) $130.7,130.0$ (x 2), 123.1, 121.2, 117.5, 116.5, 114.2 (x 2), $\left(\mathrm{CH}_{2}\right)$ 49.8, 39.8, 30.9, $\left(\mathrm{CH}_{3}\right) 55.5$.

IR $v_{\max }\left(\mathrm{cm}^{-1}\right) 3318(\mathrm{~N}-\mathrm{H}), 1546(\mathrm{C}=\mathrm{O})$.

HR ESMS $m / z$ 386.1379 $[\mathrm{M}+\mathrm{H}]^{+}$. Calc. for $\mathrm{C}_{19} \mathrm{H}_{21} \mathrm{CIN}_{5} \mathrm{O}_{2}, 386.1384$.

1-(4-chlorophenyl)-3-(2-(4-(4-methoxybenzyl)-1H-1,2,3-triazol-1yl)ethyl)urea T.3

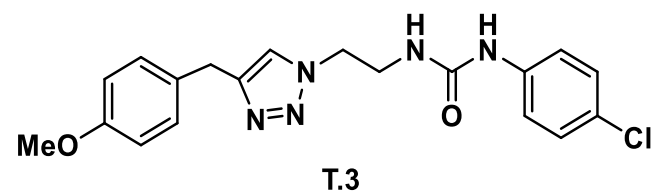

26\% yield, off-white solid, m. p. 141-144ํㅡ.

${ }^{1} \mathrm{H}$ NMR (500 MHz, DMSO-d 6 ) $\delta 8.73(\mathrm{~s}, 1 \mathrm{H}), 7.78(\mathrm{~s}, 1 \mathrm{H}), 7.40(\mathrm{~d}, J=9.1 \mathrm{~Hz}$, $2 \mathrm{H}), 7.24(\mathrm{~d}, J=8.6 \mathrm{~Hz}, 2 \mathrm{H}), 7.13(\mathrm{~d}, J=9.6 \mathrm{~Hz}, 2 \mathrm{H}), 6.79(\mathrm{~d}, J=8.3 \mathrm{~Hz}, 2 \mathrm{H})$, $6.28(\mathrm{t}, J=6.4 \mathrm{~Hz}, 1 \mathrm{H}), 3.39(\mathrm{t}, J=6.7 \mathrm{~Hz}, 2 \mathrm{H}), 3.90(\mathrm{~s}, 2 \mathrm{H}), 3.70(\mathrm{~s}, 3 \mathrm{H}), 3.52$ (app t, $J=6.4 \mathrm{~Hz}, 2 \mathrm{H}$ ).

${ }^{13}$ C NMR (125 MHz, DMSO-d 6 ) $\delta(C) ~ 158.1,155.4,146.9,139.8,131.9,125.1$, (CH) 130.0 (x 2), 128.9 (x 2), 123.1, 119.6 (x 2), 114.2 (x 2), $\left(\mathrm{CH}_{2}\right)$ 49.9, 39.6, 30.9, $\left(\mathrm{CH}_{3}\right) 55.5$.

IR $v_{\max }\left(\mathrm{cm}^{-1}\right) 3326(\mathrm{~N}-\mathrm{H}), 1633(\mathrm{C}=\mathrm{O})$.

HR ESMS $m / z$ 386.1383 $[\mathrm{M}+\mathrm{H}]^{+}$. Calc. for $\mathrm{C}_{19} \mathrm{H}_{21} \mathrm{CIN}_{5} \mathrm{O}_{2}, 386.1384$. 
1-(3-bromophenyl)-3-(2-(4-(4-methoxybenzyl)-1H-1,2,3-triazol-1yl)ethyl)urea T.4

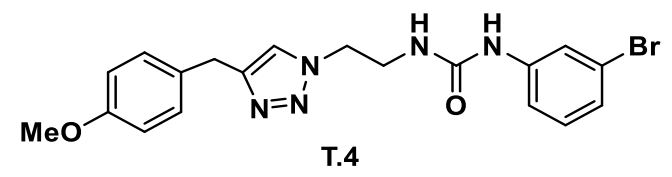

$60 \%$ yield, off-white solid, m. p. 185-187으.

${ }^{1} \mathrm{H}$ NMR $\left(500 \mathrm{MHz}, \mathrm{DMSO}-\mathrm{d}_{6}\right) \delta 8.80(\mathrm{~s}, 1 \mathrm{H}), 7.79(\operatorname{app} \mathrm{t}, J=1.8 \mathrm{~Hz}, 1 \mathrm{H}), 7.77$ (s, 1H), $7.18(\mathrm{~m}, 4 \mathrm{H}), 7.05(\mathrm{~d}, J=7.6 \mathrm{~Hz}, 1 \mathrm{H}), 6.79(\mathrm{~d}, J=8.6 \mathrm{~Hz}, 2 \mathrm{H}), 6.33(\mathrm{t}$, $J=5.8 \mathrm{~Hz}, 1 \mathrm{H}), 4.39(\mathrm{t}, J=5.8 \mathrm{~Hz}, 2 \mathrm{H}), 3.90(\mathrm{~s}, 2 \mathrm{H}), 3.68(\mathrm{~s}, 3 \mathrm{H}), 3.52(\mathrm{q}, J=$ $5.9 \mathrm{~Hz}, 2 \mathrm{H})$.

${ }^{13} \mathrm{C}$ NMR (125 MHz, DMSO-d $\left.\mathrm{d}_{6}\right) \delta(\mathrm{C})$ 158.1, 155.3, 146.9, 142.5, 131.9, 122.1 (CH) $131.0,130.0$ (x 2), 124.1, 123.1, 120.4, 116.9, $114.2(x 2),\left(\mathrm{CH}_{2}\right)$ 49.8, 39.9, 30.9, $\left(\mathrm{CH}_{3}\right) 55.5$.

IR $v_{\max }\left(\mathrm{cm}^{-1}\right) 3361(\mathrm{~N}-\mathrm{H}), 1683(\mathrm{C}=\mathrm{O})$.

HR ESMS $m / z 430.0875[\mathrm{M}+\mathrm{H}]^{+}$. Calc. for $\mathrm{C}_{19} \mathrm{H}_{21} \mathrm{BrN}_{5} \mathrm{O}_{2}, 430.0879$.

\section{1-(4-bromophenyl)-3-(2-(4-(4-methoxybenzyl)-1H-1,2,3-triazol-1-} yl)ethyl)urea T.5<smiles>COc1ccc(Cc2cn(CCNC(=O)Nc3ccc(Br)cc3)nn2)cc1</smiles>

$47 \%$ yield, off-white solid, m. p. 154-156ㅇ․

${ }^{1} \mathbf{H}$ NMR $\left(500 \mathrm{MHz}\right.$, DMSO- $\left.\mathrm{d}_{6}\right) \delta 8.72(\mathrm{~s}, 1 \mathrm{H}), 7.78(\mathrm{~s}, 1 \mathrm{H}), 7.37(\mathrm{~m}, 4 \mathrm{H}), 7.14$ $(\mathrm{d}, J=8.7 \mathrm{~Hz}, 2 \mathrm{H}), 6.80(\mathrm{~d}, J=8.7 \mathrm{~Hz}, 2 \mathrm{H}), 6.27(\mathrm{t}, J=5.7 \mathrm{~Hz}, 1 \mathrm{H}), 4.39(\mathrm{t}, J=$ $5.7 \mathrm{~Hz}, 2 \mathrm{H}), 3.91(\mathrm{~s}, 2 \mathrm{H}), 3.69(\mathrm{~s}, 3 \mathrm{H}), 3.54(\mathrm{q}, J=6.0 \mathrm{~Hz}, 2 \mathrm{H})$.

${ }^{13} \mathrm{C}$ NMR (125 MHz, DMSO-d $) \delta(C)$ 157.2, 154.5, 146.0, 139.4, 131.1, 112.0, (CH) 130.9 (x 2), 129.1 (x 2), 122.2, 119.2 (x 2), 113.4 (x 2), $\left(\mathrm{CH}_{2}\right)$ 49.0, 39.7, 30.0, $\left(\mathrm{CH}_{3}\right)$ 54.6.

IR $v_{\max }\left(\mathrm{cm}^{-1}\right) 3331(\mathrm{~N}-\mathrm{H}), 1633(\mathrm{C}=\mathrm{O})$.

HR ESMS $m / z 430.0880[\mathrm{M}+\mathrm{H}]^{+}$. Calc. for $\mathrm{C}_{19} \mathrm{H}_{21} \mathrm{BrN}_{5} \mathrm{O}_{2}, 430.0879$. 
1-(2-(4-(4-methoxybenzyl)-1H-1,2,3-triazol-1-yl)ethyl)-3-(o-tolyl)urea T.6<smiles>COc1ccc(Cc2cn(CCNC(=O)Nc3ccccc3C(C)(F)F)nn2)cc1</smiles>

$27 \%$ yield, off-white solid, m. p. 164-166으.

${ }^{1} \mathrm{H}$ NMR (500 MHz, DMSO-d 6 ) $\delta 7.78(\mathrm{~s}, 1 \mathrm{H}), 7.75(\mathrm{~d}, J=7.8 \mathrm{~Hz}, 1 \mathrm{H}), 7.69$ (s, $1 \mathrm{H}), 7.15(\mathrm{~d}, J=8.3 \mathrm{~Hz}, 2 \mathrm{H}), 7.08(\mathrm{~m}, 2 \mathrm{H}), 6.88(\mathrm{t}, J=7.8 \mathrm{~Hz}, 1 \mathrm{H}), 6.80(\mathrm{~d}, J=$ $8.3 \mathrm{~Hz}, 2 \mathrm{H}$ ), 6.61 (t, $J=5.4 \mathrm{~Hz}, 1 \mathrm{H}), 4.40$ (t, $J=5.4 \mathrm{~Hz}, 2 \mathrm{H}), 3.92(\mathrm{~s}, 2 \mathrm{H}), 3.70$ (s, 3H), 3.55 (app t, $J=5.9 \mathrm{~Hz}, 2 \mathrm{H}$ ), 2.14 (s, 3H).

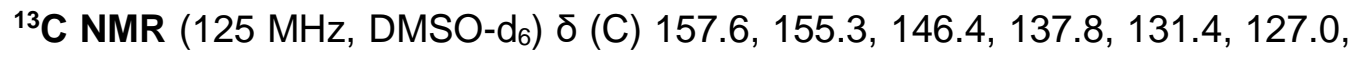
$(\mathrm{CH}) 129.9,129.4$ (x 2), 125.9, 122.0, 120.7, 113.6 (x 2), $\left(\mathrm{CH}_{2}\right)$ 49.5, 39.2, 30.4, $\left(\mathrm{CH}_{3}\right) 54.9,17.8$.

IR $v_{\max }\left(\mathrm{cm}^{-1}\right) 3314(\mathrm{~N}-\mathrm{H}), 1542(\mathrm{C}=\mathrm{O})$.

HR ESMS $m / z$ 366.1924 $[\mathrm{M}+\mathrm{H}]^{+}$. Calc. for $\mathrm{C}_{20} \mathrm{H}_{24} \mathrm{~N}_{5} \mathrm{O}_{2}, 366.1939$.

1-(2-(4-(4-methoxybenzyl)-1H-1,2,3-triazol-1-yl)ethyl)-3-(m-tolyl)urea T.7<smiles>COc1ccc(Cc2cn(CCNC(=O)Nc3cccc(C)c3)nn2)cc1</smiles>

$55 \%$ yield, off-white solid, m. p. 150-151ํㅡ.

${ }^{1} \mathrm{H}$ NMR $(500 \mathrm{MHz}$, DMSO-d 6 ) $\delta 8.45(\mathrm{~s}, 1 \mathrm{H}), 7.78(\mathrm{~s}, 1 \mathrm{H}), 7.21(\mathrm{~s}, 1 \mathrm{H}), 7.14(\mathrm{~m}$, $3 \mathrm{H}), 7.08$ (t, $J=7.8 \mathrm{~Hz}, 1 \mathrm{H}), 6.80(\mathrm{~d}, J=8.8 \mathrm{~Hz}, 2 \mathrm{H}), 6.71(\mathrm{~d}, J=6.9 \mathrm{~Hz}, 1 \mathrm{H})$, $6.19(\mathrm{t}, J=5.9 \mathrm{~Hz}, 1 \mathrm{H}), 4.39(\mathrm{t}, J=5.9 \mathrm{~Hz}, 2 \mathrm{H}), 3.92(\mathrm{~s}, 2 \mathrm{H}), 3.69(\mathrm{~s}, 3 \mathrm{H}), 3.54$ (app t, $J=5.4 \mathrm{~Hz}, 2 \mathrm{H}$ ), $2.24(\mathrm{~s}, 3 \mathrm{H})$.

${ }^{13}$ C NMR (125 MHz, DMSO-d 6 ) $\delta(C) ~ 157.6,155.3,146.4,137.8,131.3,127.0$, (CH) 129.9, 129.4 (x 2), 125.9, 122.0, 120.7, 113.6 (x 2), $\left(\mathrm{CH}_{2}\right)$ 49.5, 39.2, 30.4, $\left(\mathrm{CH}_{3}\right)$ 54.9, 17.8 .

IR $v_{\max }\left(\mathrm{cm}^{-1}\right) 3331(\mathrm{~N}-\mathrm{H}), 1511(\mathrm{C}=\mathrm{O})$.

HR ESMS $m / z 366.1930[\mathrm{M}+\mathrm{H}]^{+}$. Calc. for $\mathrm{C}_{20} \mathrm{H}_{24} \mathrm{~N}_{5} \mathrm{O}_{2}, 366.1930$. 
1-(2-(4-(4-methoxybenzyl)-1H-1,2,3-triazol-1-yl)ethyl)-3-(p-tolyl)urea T.8

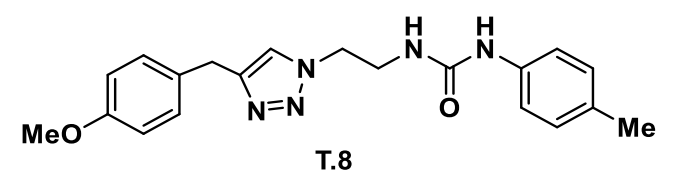

$41 \%$ yield, off-white solid, m. p. 146-149ㅇ.

${ }^{1} \mathrm{H}$ NMR $\left(500 \mathrm{MHz}, \mathrm{CDCl}_{3}\right) \delta 7.72(\mathrm{~s}, 1 \mathrm{H}), 7.28(\mathrm{~s}, 1 \mathrm{H}), 7.15(\mathrm{~d}, J=8.3 \mathrm{~Hz}, 2 \mathrm{H})$, 7.09 (d, J = 8.3 Hz, 2H), 7.03 (d, J=7.8 Hz, 2H), 6.77 (d, J=8.8 Hz, 2H), 6.02 (t, $J=5.4 \mathrm{~Hz}, 1 \mathrm{H}), 4.39$ (t, $J=5.4 \mathrm{~Hz}, 2 \mathrm{H}), 3.93(\mathrm{~s}, 2 \mathrm{H}), 3.73(\mathrm{~s}, 3 \mathrm{H}), 3.67$ (t, J $=5.4 \mathrm{~Hz}, 2 \mathrm{H}), 2.28(\mathrm{~s}, 3 \mathrm{H})$.

${ }^{13} \mathrm{C}$ NMR $\left(125 \mathrm{MHz}, \mathrm{CDCl}_{3}\right) \delta(\mathrm{C})$ 158.3, 156.1, 136.2, 132.6 130.6, 129.7, (CH) 129.5 (x 2), 129.4 (x 2), 122.9, 120.0 (x 2), 114.0 (x 2), $\left(\mathrm{CH}_{2}\right) 50.1,39.6,31.0$ $\left(\mathrm{CH}_{3}\right)$ 55.2, 20.7 .

IR $v_{\max }\left(\mathrm{cm}^{-1}\right) 3346(\mathrm{~N}-\mathrm{H}), 1510(\mathrm{C}=\mathrm{O})$.

HR ESMS $m / z$ 366.1933 $[\mathrm{M}+\mathrm{H}]^{+}$. Calc. for $\mathrm{C}_{20} \mathrm{H}_{24} \mathrm{~N}_{5} \mathrm{O}_{2}, 366.1930$.

\section{d) Experimental procedure for synthesis of phosphonium salt $\mathbf{1 0}$}

A solution of 3-nitrobenzyl bromide $(4.63 \mathrm{mmol})$ and $\mathrm{PPh}_{3}(4.63 \mathrm{mmol})$ in dry $\mathrm{CH}_{2} \mathrm{Cl}_{2}(20 \mathrm{~mL})$ was stirred for $3 \mathrm{~h}$ at $\mathrm{r}$. t. The white precipitate was filtered and sequentially washed with $\mathrm{CH}_{2} \mathrm{Cl}_{2}$ and hexane affording compound $\mathbf{1 0}$ as a white solid (1.56 g, $71 \%$, m. p. $\left.273^{\circ} \mathrm{C}\right)$.

\section{e) Experimental procedure for Wittig reaction (scheme 3.6, b)}

4-methoxybenzaldehyde (3.26 mmol) was dissolved in $\mathrm{CH}_{2} \mathrm{Cl}_{2}(20 \mathrm{~mL})$ and then, the phosphonium salt 10 (3.26 mmol), $\mathrm{K}_{2} \mathrm{CO}_{3}(3.6 \mathrm{mmol})$ and 18-crown-6 (0.6 $\mathrm{mmol}$ ) were added to the solution. The mixture was refluxed overnight and then filtered and concentrated to afford compound 11 (73\% yield) as an $E / Z$ stereoisomeric mixture.

\section{f) Experimental procedure for nitro group reduction (scheme 3.6, c)}

Zn powder $(0.6 \mathrm{~mol})$ was added to a solution of compound $11(4.08 \mathrm{mmol})$ in $\mathrm{AcOH}(133 \mathrm{~mL})$. The resulting mixture was vigorously stirred at $\mathrm{r}$. $\mathrm{t}$. for $1 \mathrm{~h}$ in a flask that was protected from the light. Then, the solids were filtered over Celite to remove the $\mathrm{Zn}$, and were thoroughly washed with EtOAc. The filtrate was 
neutralized with saturated aqueous $\mathrm{NaHCO}_{3}$ and the organic phase was separated and dried over anhydrous $\mathrm{MgSO}_{4}$. After filtration and solvent evaporation, column chromatography on silica gel (Hexane-EtOAc mixtures as eluent) was used to isolate the stereoisomers 12 and 13 (Z/E 6:4).

\section{g) Experimental procedure for hydrogenation reaction (scheme 3.6, d)}

Compound $11(2.35 \mathrm{mmol})$ was dissolved in EtOAc $(30 \mathrm{~mL})$ and hydrogenated with $10 \% \mathrm{Pd} / \mathrm{C}(300 \mathrm{mg})$ for $2 \mathrm{~h}$ at $\mathrm{r}$. t. The resulting mixture was filtered through a pad of Celite and the filtrate was evaporated under reduced pressure. Then, the residue was purified by column chromatography on silica gel (Hexane-EtOAc mixtures as eluent) to give the compound $\mathbf{1 4}$ as a brown solid (66\%, m. p. 81$\left.85^{\circ} \mathrm{C}\right)$.

\section{h) General procedure for the synthesis of carbamates 15-17}

A solution of 12,13 or $14(0.2 \mathrm{mmol})$ in THF $(1 \mathrm{~mL})$ was cooled at $0^{\circ} \mathrm{C}$, then anhydrous pyridine $(0.5 \mathrm{mmol})$ and phenyl chloroformate $(0.3 \mathrm{mmol})$ were added under inert atmosphere. The resulting mixture was stirred in the dark for $20 \mathrm{~min}$ at $0^{\circ} \mathrm{C}$ and for $1 \mathrm{~h}$ at $\mathrm{r}$. t. After this time, $\mathrm{H}_{2} \mathrm{O}(1 \mathrm{~mL})$ and $\mathrm{HCl} 1 \mathrm{M}(0.5 \mathrm{~mL})$ were added to the reaction mixture, which was then extracted with $\mathrm{CH}_{2} \mathrm{Cl}_{2}(3 \times 20 \mathrm{~mL})$. The organic layer was washed with brine and then dried on anhydrous $\mathrm{Na}_{2} \mathrm{SO}_{4}$. Removal of volatiles under reduced pressure afforded an oily residue which was subjected to column chromatography on silica-gel (Hexane-EtOAc mixtures as eluant) obtaining the desired products with the yields indicated below.

\section{Phenyl (Z)-(3-(4-methoxystyryl)phenyl)carbamate 15}

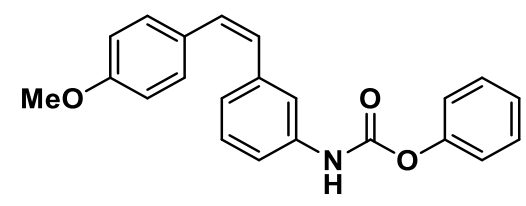

15

$85 \%$ yield, yellow oil.

${ }^{1} \mathrm{H}$ NMR $\left(500 \mathrm{MHz}, \mathrm{CDCl}_{3}\right) \delta 7.43(\mathrm{~s}, 1 \mathrm{H}), 7.38$ (t, $\left.J=16.1 \mathrm{~Hz}, 2 \mathrm{H}\right), 7.20(\mathrm{~m}$, $7 \mathrm{H}), 7.02(\mathrm{~d}, J=7.8 \mathrm{~Hz}, 1 \mathrm{H}), 6.92(\mathrm{br} \mathrm{s}, 1 \mathrm{H}), 6.77(\mathrm{~d}, J=8.8 \mathrm{~Hz}, 2 \mathrm{H}), 6.55(\mathrm{~d}, J$ $=12.2 \mathrm{~Hz}, 1 \mathrm{H}), 6.47(\mathrm{~d}, J=12.2 \mathrm{~Hz}, 1 \mathrm{H}), 3.78(\mathrm{~s}, 3 \mathrm{H})$. 
${ }^{13} \mathrm{C} \mathrm{NMR}\left(125 \mathrm{MHz}, \mathrm{CDCl}_{3}\right) \delta(\mathrm{C}) 158.7,151.6,150.5,138.6,137.4,129.3,(\mathrm{CH})$ 130.3, 130.1 (x 2), 129.3 (x 2), 128.9, 128.1, 125.5, 124.3, 121.5 (x 2), 119.1, 117.4, $113.6(\times 2),\left(\mathrm{CH}_{3}\right) 55.1$.

IR $\boldsymbol{V}_{\max }\left(\mathrm{cm}^{-1}\right) 3381-3320(\mathrm{~N}-\mathrm{H}), 1747(\mathrm{C}=\mathrm{O})$.

HR ESMS $m / z$ 368.1261 [M+Na] $]^{+}$. Calc. for $\mathrm{C}_{22} \mathrm{H}_{19} \mathrm{O}_{3} \mathrm{NNa}$, 368.1263.

\section{Phenyl (E)-(3-(4-methoxystyryl)phenyl)carbamate 16}

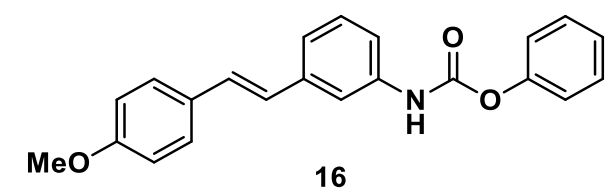

$86 \%$ yield, off-white solid, m. p. 159-161ํㅡ.

${ }^{1} \mathrm{H}$ NMR $\left(500 \mathrm{MHz}, \mathrm{CDCl}_{3}\right) \delta 7.73(\mathrm{br} \mathrm{s}, 1 \mathrm{H}), 7.47(\mathrm{~m}, 4 \mathrm{H}), 7.34(\mathrm{~m}, 1 \mathrm{H}), 7.28$ $(\mathrm{m}, 5 \mathrm{H}), 7.14$ (d, J=16.1 Hz, 1H), 7.03 (br s, 1H), 7.00 (s, 1H), 6.98 (d, J=16.1 $\mathrm{Hz}, 1 \mathrm{H}), 6.94(\mathrm{~m}, 2 \mathrm{H}), 3.88(\mathrm{~s}, 3 \mathrm{H})$.

${ }^{13} \mathrm{C} \mathrm{NMR}\left(125 \mathrm{MHz}, \mathrm{CDCl}_{3}\right) \delta(\mathrm{C}) 159.4,151.6,150.6,138.8,137.7,129.9,(\mathrm{CH})$ 129.4 (x 2), 129.3, 129.0, 127.8 (x 2), 126.0, 125.7, 122.1, 121.6 (x 2), 117.5, 116.2, $114.2(\times 2),\left(\mathrm{CH}_{3}\right)$ 55.3.

IR $\boldsymbol{v}_{\max }\left(\mathrm{cm}^{-1}\right) 3428(\mathrm{~N}-\mathrm{H}), 1752(\mathrm{C}=\mathrm{O})$.

HR ESMS $m / z 368.1263[\mathrm{M}+\mathrm{Na}]^{+}$. Calc. for $\mathrm{C}_{22} \mathrm{H}_{19} \mathrm{NO}_{3} \mathrm{Na}$, 368.1365.

\section{Phenyl (3-(4-methoxyphenethyl)phenyl)carbamate 17}

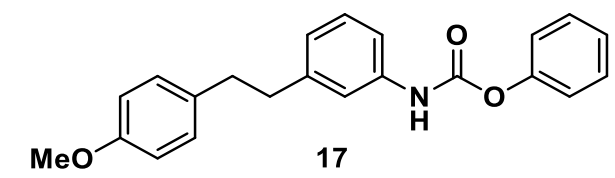

$66 \%$ yield, off-white solid, m. p. 106-114으.

${ }^{1} \mathbf{H}$ NMR $\left(500 \mathrm{MHz}, \mathrm{CDCl}_{3}\right) \delta 7.42(\mathrm{t}, J=8.0 \mathrm{~Hz}, 2 \mathrm{H}), 7.36(\mathrm{~s}, 1 \mathrm{H}), 7.27$ (t, $J=$ $8.0 \mathrm{~Hz}, 2 \mathrm{H}), 7.26(\mathrm{~d}, J=7.5 \mathrm{~Hz}, 1 \mathrm{H}), 7.23(\mathrm{~d}, J=8.0 \mathrm{~Hz}, 2 \mathrm{H}), 7.12(\mathrm{~d}, J=8.5$ $\mathrm{Hz}, 1 \mathrm{H}), 7.04$ (s, 1H), 6.87 (d, J=8.5 Hz, 2H), 3.82 (s, 3H), 2.90 (s, $4 \mathrm{H})$.

${ }^{13} \mathrm{C}$ NMR $\left(125 \mathrm{MHz}, \mathrm{CDCl}_{3}\right) \delta(\mathrm{C}) 157.8,152.5,150.6,143.0,137.3,133.7,(\mathrm{CH})$ 129.3 (x 2), 129.3, 129.0, 125.6, 124.1, 121.6 (x 2), 113.7 (x 2), $\left(\mathrm{CH}_{2}\right)$ 38.1, 36.8, $\left(\mathrm{CH}_{3}\right) 55.2$.

IR $\boldsymbol{v}_{\max }\left(\mathrm{cm}^{-1}\right) 3380(\mathrm{~N}-\mathrm{H}), 1746(\mathrm{C}=\mathrm{O})$.

ESI-MS m/z 370.1415 [M+Na] $]^{+}$. Calc. for $\mathrm{C}_{22} \mathrm{H}_{21} \mathrm{NO}_{3} \mathrm{Na}, 370.1415$. 


\section{i) General procedure for the synthesis of ureas S.1-S.9}

A solution of the corresponding chloroaniline $(0.5 \mathrm{mmol})$ in dry THF $(2 \mathrm{~mL})$ was treated with $\mathrm{Et}_{3} \mathrm{~N}(5.4 \mathrm{mmol})$ under inert atmosphere. After stirring the mixture for $5 \mathrm{~min}$, the corresponding carbamate $(0.5 \mathrm{mmol})$, previously prepared, was added dissolved in THF $(5 \mathrm{~mL})$. The resulting mixture was then stirred in the dark at $40-50 \stackrel{\circ}{\circ}$ for $24-72 \mathrm{~h}$ (TLC monitoring). After this time, $\mathrm{CH}_{2} \mathrm{Cl}_{2}$ (15 mL) and $\mathrm{HCl} 1 \mathrm{M}(10 \mathrm{~mL})$ were added to the reaction mixture, which was then extracted with $\mathrm{CH}_{2} \mathrm{Cl}_{2}(2 \times 10 \mathrm{~mL})$. The organic layer was washed with brine and then dried on anhydrous $\mathrm{Na}_{2} \mathrm{SO}_{4}$. Removal of volatiles under reduced pressure afforded an oily residue which was subjected to column chromatography on silica-gel (Hexane-EtOAc mixtures as eluent) to afford the desired products with the yields indicated below.

(Z)-1-(2-chlorophenyl)-3-(3-(4-methoxystyryl)phenyl)urea S.1<smiles>COc1ccc(/C=C\c2cccc(NC(=O)Nc3ccccc3Cl)c2)cc1</smiles>

S.1

$41 \%$ yield, off-white solid, m. p. 146-147으.

${ }^{1} \mathrm{H}$ NMR $\left(500 \mathrm{MHz}, \mathrm{CDCl}_{3}\right) \delta 7.72(\mathrm{~s}, 1 \mathrm{H}), 7.64(\mathrm{~s}, 1 \mathrm{H}), 7.20(\mathrm{~s}, 1 \mathrm{H}), 7,11(\mathrm{~m}$, $3 \mathrm{H}), 7.05(\mathrm{~m}, 4 \mathrm{H}), 6.94(\mathrm{~m}, 2 \mathrm{H}), 6.69(\mathrm{~d}, J=6.9 \mathrm{~Hz}, 2 \mathrm{H}), 6.46(\mathrm{~d}, J=12.2 \mathrm{~Hz}$, $1 \mathrm{H}), 6.32(\mathrm{~d}, J=12.2 \mathrm{~Hz}, 1 \mathrm{H}), 3.70(\mathrm{~s}, 3 \mathrm{H})$.

${ }^{13} \mathrm{C}$ NMR (125 MHz, $\left.\mathrm{CDCl}_{3}\right) \delta(\mathrm{C}) 158.7,154.0,139.3,138.6,137.7,134.5$, 129.4, (CH) 130.2, 130.1 (x 2), 129.9, 128.9, 128.1, 124.6, 123.6, 121.3, 120.4, 119.5, 118.4, 113.6 (x 2), $\left(\mathrm{CH}_{3}\right) 55.1$.

IR $\boldsymbol{V}_{\max }\left(\mathrm{cm}^{-1}\right) 3332(\mathrm{~N}-\mathrm{H}), 1555(\mathrm{C}=\mathrm{O})$.

HR ESMS $m / z 379.1215[\mathrm{M}+\mathrm{Na}]^{+}$. Calc. for $\mathrm{C}_{22} \mathrm{H}_{19} \mathrm{O}_{2} \mathrm{~N}_{2} \mathrm{NaCl}, 379.1213$. 
(Z)-1-(3-chlorophenyl)-3-(3-(4-methoxystyryl)phenyl)urea S.2

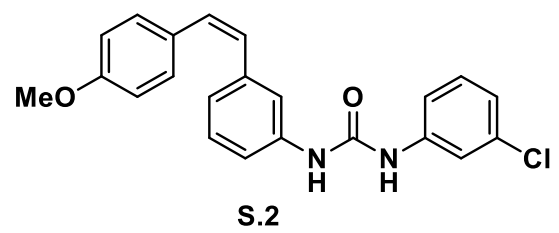

$42 \%$ yield, off-white solid, m. p. $174^{\circ} \mathrm{C}$.

${ }^{1} \mathrm{H}$ NMR $\left(500 \mathrm{MHz}, \mathrm{CDCl}_{3}\right) \delta 8.19(\mathrm{~s}, 1 \mathrm{H}), 8.18(\mathrm{~s}, 1 \mathrm{H}), 7.33(\mathrm{~m}, 2 \mathrm{H}), 7.27(\mathrm{~m}$, $3 \mathrm{H}), 7.19(\mathrm{~m}, 2 \mathrm{H}), 7.11(\mathrm{~d}, J=7.8 \mathrm{~Hz}, 1 \mathrm{H}), 7.07(\mathrm{~s}, 1 \mathrm{H}), 7.00(\mathrm{t}, J=7.3 \mathrm{~Hz}, 1 \mathrm{H})$, $6.74(\mathrm{~d}, J=6.8 \mathrm{~Hz}, 2 \mathrm{H}), 6.58(\mathrm{~d}, J=12.2 \mathrm{~Hz}, 1 \mathrm{H}), 6.49(\mathrm{~d}, J=12.2 \mathrm{~Hz}, 1 \mathrm{H})$, $3.75(\mathrm{~s}, 3 \mathrm{H})$.

${ }^{13} \mathrm{C} \mathrm{NMR}\left(125 \mathrm{MHz}, \mathrm{CDCl}_{3}\right) \delta(\mathrm{C})$ 158.9, 152.5, 139.2, 137.3, 135.1 129.3, 125.8 (CH) $130.7,130.2,130.2$ (x 2), 129.5, 129.0, 127.8, 127.8, 123.9, 123.0, 122.6, 121.0, $113.7(\mathrm{x} \mathrm{2}),\left(\mathrm{CH}_{3}\right) 55.2$.

IR $\boldsymbol{V}_{\max }\left(\mathrm{cm}^{-1}\right) 3287(\mathrm{~N}-\mathrm{H}), 1649(\mathrm{C}=\mathrm{O})$.

HR ESMS $m / z 379.1213[\mathrm{M}+\mathrm{H}]^{+}$. Calc. for $\mathrm{C}_{22} \mathrm{H}_{20} \mathrm{O}_{2} \mathrm{~N}_{2} \mathrm{Cl}, 379.1213$.

(Z)-1-(4-chlorophenyl)-3-(3-(4-methoxystyryl)phenyl)urea S.3

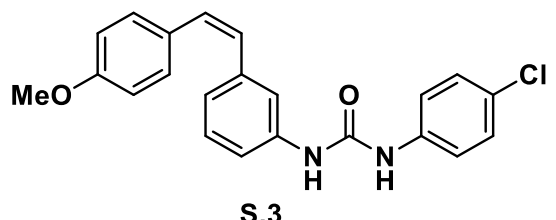

$88 \%$ yield, off-white solid, m. p. 173ㄷ․

${ }^{1} \mathbf{H}$ NMR (500 MHz, DMSO-d 6 ) $\delta 9.11(\mathrm{~s}, 1 \mathrm{H}), 8.98(\mathrm{~s}, 1 \mathrm{H}), 7.48(\mathrm{~d}, J=8.8 \mathrm{~Hz}$, $2 \mathrm{H}), 7.40(\mathrm{~d}, J=8.8 \mathrm{~Hz}, 1 \mathrm{H}), 7.31(\mathrm{~m}, 3 \mathrm{H}), 7.17(\mathrm{~m}, 3 \mathrm{H}), 6.82(\mathrm{~m}, 3 \mathrm{H}), 6.53(\mathrm{~d}$, $J=12.2 \mathrm{~Hz}, 1 \mathrm{H}), 6.49$ (d, $J=12.2 \mathrm{~Hz}, 1 \mathrm{H}), 3.72(\mathrm{~s}, 3 \mathrm{H})$.

${ }^{13} \mathrm{C}$ NMR (125 MHz, DMSO-d $\left.\mathrm{d}_{6}\right) \delta(\mathrm{C}) 158.4,152.4,139.8,138.8,137.8,128.9$, 128.3, (CH) 129.9 (x 2), 129.6, 128.7, 128.5 (x 2), 125.1, 121.9, 119.6 (x 2), 118.2, 117.1, 113.7 (x 2), $\left(\mathrm{CH}_{3}\right)$ 55.0.

IR $\boldsymbol{V}_{\max }\left(\mathrm{cm}^{-1}\right) 3291(\mathrm{~N}-\mathrm{H}), 1633(\mathrm{C}=\mathrm{O})$.

HR ESMS $m / z 379.1207[\mathrm{M}+\mathrm{H}]^{+}$. Calc. for $\mathrm{C}_{22} \mathrm{H}_{20} \mathrm{O}_{2} \mathrm{~N}_{2} \mathrm{Cl}, 379.1213$. 
(E)-1-(2-chlorophenyl)-3-(3-(4-methoxystyryl)phenyl)urea S.4

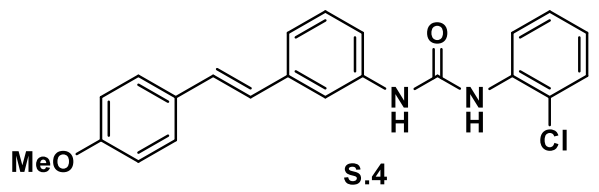

$13 \%$ yield, off-white solid, m. p. 230-232으.

${ }^{1} \mathrm{H}$ NMR (500 MHz, DMSO-d 6 ) $\delta 9.43(\mathrm{~s}, 1 \mathrm{H}), 8.33(\mathrm{~s}, 1 \mathrm{H}), 8.17$ (d, J = 6.7 Hz, $1 \mathrm{H}), 7.67(\mathrm{~s}, 1 \mathrm{H}), 7.56(\mathrm{~d}, J=8.8 \mathrm{~Hz}, 2 \mathrm{H}), 7.46(\mathrm{~d}, J=9.3 \mathrm{~Hz}, 1 \mathrm{H}), 7.3(\mathrm{~m}, 3 \mathrm{H})$, $7.2(\mathrm{~d}, J=6.9 \mathrm{~Hz}, 1 \mathrm{H}), 7.14(\mathrm{~d}, J=16.0 \mathrm{~Hz}, 1 \mathrm{H}), 7.06(\mathrm{~d}, J=16.0 \mathrm{~Hz}, 1 \mathrm{H}) 7.05$ (t, $J=7.3 \mathrm{~Hz}, 1 \mathrm{H}), 6.95$ (d, $J=8.3 \mathrm{~Hz}), 3.79(\mathrm{~s}, 3 \mathrm{H})$.

${ }^{13}$ C NMR (500 MHz, DMSO-d 6 ) $\delta(C) ~ 159.0,152.2,139.8,138.0,135.9,129.5$, 129.1, (CH) 129.1, 128.1, 127.8 (x 2), 127.5, 126.2, 123.3, 121.9, 121.3, 120.1, 117.3, 115.9, $114.2(x 2),\left(\mathrm{CH}_{3}\right) 55.0$.

IR $\boldsymbol{V}_{\max }\left(\mathrm{cm}^{-1}\right) 3690(\mathrm{~N}-\mathrm{H}), 1421(\mathrm{C}=\mathrm{O})$.

HR ESMS $m / z 379.1213[\mathrm{M}+\mathrm{H}]^{+}$. Calc. for $\mathrm{C}_{22} \mathrm{H}_{20} \mathrm{~N}_{2} \mathrm{O}_{2}, 379.1205$.

(E)-1-(3-chlorophenyl)-3-(3-(4-methoxystyryl)phenyl)urea S.5<smiles>COc1ccc(/C=C/c2cccc(NC(=O)Nc3cccc(Cl)c3)c2)cc1</smiles>

$36 \%$ yield, off-white solid, m. p. $235-237^{\circ} \mathrm{C}$.

${ }^{1}$ H NMR (500 MHz, DMSO-d 6 ) $\delta 8.92(\mathrm{~s}, 1 \mathrm{H}), 8.77(\mathrm{~s}, 1 \mathrm{H}), 7.75(\mathrm{t}, J=2.0 \mathrm{~Hz}$, $1 \mathrm{H}), 7.68(\mathrm{~s}, 1 \mathrm{H}), 7.55(\mathrm{~d}, J=8.8 \mathrm{~Hz}, 2 \mathrm{H}), 7.27(\mathrm{~m}, 4 \mathrm{H}), 7.22(\mathrm{~m}, 1 \mathrm{H}), 7.13(\mathrm{~d}, J$ $=16.0 \mathrm{~Hz}, 1 \mathrm{H}), 7.07(\mathrm{~d}, J=16.0 \mathrm{~Hz}, 1 \mathrm{H}), 7.05(\mathrm{~m}, 1 \mathrm{H}), 6.95(\mathrm{~d}, J=8.3 \mathrm{~Hz}, 2 \mathrm{H})$ $3.78(\mathrm{~s}, 3 \mathrm{H})$.

${ }^{13} \mathrm{C}$ NMR $\left(500 \mathrm{MHz}\right.$, DMSO-d $\left.\mathrm{d}_{6}\right) \delta(\mathrm{C})$ 159.0, 152.39, 141.25, 140.0, 137.9, 133.2, (CH) 139.7 (x 2), 130.3, 129.5, 129.0, 128.1, 127.8, 126.2, 121.4, 120.1, 117.6, 116.6, 116.1, $114.1(\mathrm{x} 2),\left(\mathrm{CH}_{3}\right) 55.1$.

IR $\boldsymbol{V}_{\max }\left(\mathrm{cm}^{-1}\right) 3542(\mathrm{~N}-\mathrm{H}), 1421(\mathrm{C}=\mathrm{O})$.

HR ESMS $m / z 401.1033[\mathrm{M}+\mathrm{Na}]^{+}$. Calc for $\mathrm{C}_{22} \mathrm{H}_{19} \mathrm{~N}_{2} \mathrm{O}_{2} \mathrm{Na} 401.1042$. 
(E)-1-(4-chlorophenyl)-3-(3-(4-methoxystyryl)phenyl)urea S.6<smiles>COc1ccc(/C=C/c2cccc(NC(=O)Nc3ccc(Cl)cc3)c2)cc1</smiles>

$72 \%$ yield, off-white solid, m. p. 240-243으.

${ }^{1} \mathrm{H}$ NMR (500 MHz, DMSO-d 6 ) $88.51(\mathrm{~s}, 1 \mathrm{H}), 7.26(\mathrm{~s}, 1 \mathrm{H}), 7.66(\mathrm{~s}, 1 \mathrm{H}), 7.5$ (d, $J=8.3 \mathrm{~Hz}, 2 \mathrm{H}), 7.51(\mathrm{~d}, J=8.3 \mathrm{~Hz}, 2 \mathrm{H}), 7.31(\mathrm{~d}, J=8.3 \mathrm{~Hz}, 2 \mathrm{H}), 7.29(\mathrm{~m}, 2 \mathrm{H})$, $7.21(\mathrm{~d}, J=6.8 \mathrm{~Hz}, 1 \mathrm{H}), 7.12(\mathrm{~d}, J=16.6 \mathrm{~Hz}, 1 \mathrm{H}), 7.08(\mathrm{~d}, J=16.6 \mathrm{~Hz}, 1 \mathrm{H})$, $6.94(\mathrm{~d}, J=8.3 \mathrm{~Hz}, 2 \mathrm{H}), 3.77(\mathrm{~s}, 3 \mathrm{H})$.

${ }^{13} \mathrm{C}$ NMR (500 MHz, DMSO-d $\left.\mathrm{d}_{6}\right) \delta(\mathrm{C})$ 159.0, 152.4, 141.2, 140.0, 137.9, 133.2, (CH) 139.7 (x 2), 130.3, 129.5, 129.0, 128.1, 127.8, 126.2, 121.4, 120.1, 117.5, 116.6, 116.2, $114.1(\mathrm{x} 2),\left(\mathrm{CH}_{3}\right) 55.1$.

IR $\boldsymbol{V}_{\max }\left(\mathrm{cm}^{-1}\right) 3691(\mathrm{~N}-\mathrm{H}), 1421(\mathrm{C}=\mathrm{O})$.

HR ESMS $m / z 401.1033[\mathrm{M}+\mathrm{Na}]^{+}$. Calc. for $\mathrm{C}_{22} \mathrm{H}_{19} \mathrm{~N}_{2} \mathrm{O}_{2} \mathrm{Na} 401.1027$.

1-(2-chlorophenyl)-3-(3-(4-methoxyphenethyl)phenyl)urea S.7<smiles>COc1ccc(CCc2cccc(NC(=O)Nc3ccccc3Cl)c2)cc1</smiles>

$42 \%$ yield, off-white solid, m. p. 175-178ㅇ.

${ }^{1} \mathrm{H}$ NMR (500 MHz, DMSO-d $)$ ס $9.34(\mathrm{~s}, 1 \mathrm{H}), 8.27(\mathrm{~s}, 1 \mathrm{H}), 8.16(\mathrm{~d}, J=8.3 \mathrm{~Hz}$ $1 \mathrm{H}), 7.44$ (d, J = 8.3 Hz 1H), $7.33(\mathrm{~s}, 1 \mathrm{H}), 7.29$ (t, $J=7.8 \mathrm{~Hz}, 1 \mathrm{H}), 7.28(\mathrm{~d}, J=$ $7.8 \mathrm{~Hz}, 1 \mathrm{H}$ ), $7.18(\mathrm{t}, J=7.8 \mathrm{~Hz}, 1 \mathrm{H}), 7.13(\mathrm{~d}, J=8.3 \mathrm{~Hz}, 2 \mathrm{H}), 7.02$ (t, $J=8.3 \mathrm{~Hz}$, $1 \mathrm{H}), 6.84(\mathrm{~d}, J=7.8 \mathrm{~Hz}, 1 \mathrm{H}), 6.82(\mathrm{~d}, J=8.3 \mathrm{~Hz}, 2 \mathrm{H}), 3.70(\mathrm{~s}, 3 \mathrm{H}), 2.81(\mathrm{~s}, 4 \mathrm{H})$.

${ }^{13} \mathrm{C}$ NMR (125 MHz, DMSO-d $\left.\mathrm{d}_{6}\right) \delta(\mathrm{C})$ 157.5, 152.2, 142.3, 139.4, 136.0, 133.4, $121.4(\mathrm{CH}) 129.4,127.7,123.3,122.4,122.0,118.2,116.0,113.7(\mathrm{x} 2),\left(\mathrm{CH}_{2}\right)$ 37.5, 36.1, $\left(\mathrm{CH}_{3}\right) 55.1$.

IR $\boldsymbol{V}_{\max }\left(\mathrm{cm}^{-1}\right) 3301(\mathrm{~N}-\mathrm{H}), 1645(\mathrm{C}=\mathrm{O})$.

HR ESMS $m / z 381.1368[\mathrm{M}+\mathrm{H}]^{+}$. Calc. for $\mathrm{C}_{22} \mathrm{H}_{22} \mathrm{CIN}_{2} \mathrm{O}_{2}, 381.1370$. 
1-(3-chlorophenyl)-3-(3-(4-methoxyphenethyl)phenyl)urea S.8<smiles>COc1ccc(CCc2cccc(NC(=O)Nc3cccc(Cl)c3)c2)cc1</smiles>

$34 \%$ yield, brownish solid, m. p. 180-182ํㅡ.

${ }^{1} \mathrm{H}$ NMR $\left(500 \mathrm{MHz}, \mathrm{DMSO}-\mathrm{d}_{6}\right) \delta 8.83(\mathrm{~s}, 1 \mathrm{H}), 8.65$ (s, 1H), 7.72 (br s, 1H), 7.33 (s, 1H), 7.29 (t, J=8.0 Hz, 1H), $7.27(\mathrm{t}, J=8.5 \mathrm{~Hz}, 1 \mathrm{H}), 7.27(\mathrm{~d}, J=8.0 \mathrm{~Hz}, 1 \mathrm{H})$, $7.18(\mathrm{~d}, J=7.0 \mathrm{~Hz}, 2 \mathrm{H}), 7.14(\mathrm{~d}, J=8.5 \mathrm{~Hz}, 1 \mathrm{H}), 7.01(\mathrm{~d}, J=8.0 \mathrm{~Hz}, 1 \mathrm{H}), 6.85$ (d, $J=7.0 \mathrm{~Hz}, 2 \mathrm{H}), 6.83(\mathrm{~d}, J=8.5 \mathrm{~Hz}, 1 \mathrm{H}), 3.71(\mathrm{~s}, 3 \mathrm{H}), 2.81(\mathrm{~s}, 4 \mathrm{H})$.

${ }^{13} \mathrm{C}$ NMR (125 MHz, DMSO-d $\left.\mathrm{d}_{6}\right) \delta(\mathrm{C}) 157.4,152.4,142.2,141.3,139.3,133.3$, 133.2, (CH) 129.4, 129.1, 122.3, 121.3, 118.45 118.2, 117.6, 117.4, 116.7, 113.6 (x 2), $\left(\mathrm{CH}_{2}\right) 37.4,36.1,\left(\mathrm{CH}_{3}\right)$ 55.0.

IR $\boldsymbol{v}_{\max }\left(\mathrm{cm}^{-1}\right) 3304(\mathrm{~N}-\mathrm{H}), 1633(\mathrm{C}=\mathrm{O})$.

HR ESMS $m / z$ 403.1188 [M+Na] $]^{+}$. Calc. for $\mathrm{C}_{22} \mathrm{H}_{21} \mathrm{CIN}_{2} \mathrm{O}_{2} \mathrm{Na}, 403.1189$.

1-(4-chlorophenyl)-3-(3-(4-methoxyphenethyl)phenyl)urea S.9<smiles>COc1ccc(CCc2cccc(NC(=O)Nc3ccc(Cl)cc3)c2)cc1</smiles>

$60 \%$ yield, brownish solid, m. p. 192-193ํ․

${ }^{1} \mathbf{H}$ NMR (500 MHz, DMSO-d 6 ) $\delta 8.77(\mathrm{~s}, 1 \mathrm{H}), 8.61(\mathrm{~s}, 1 \mathrm{H}), 7.48(\mathrm{~d}, J=8.5 \mathrm{~Hz}$, 2H), 7.31 (d, $J=9.0,2 \mathrm{H}), 7.31(\mathrm{t}, J=7.5 \mathrm{~Hz}, 1 \mathrm{H}), 7.26(\mathrm{~d}, J=7.5 \mathrm{~Hz}, 1 \mathrm{H}), 7.18$ $(\mathrm{d}, J=7.5 \mathrm{~Hz}, 1 \mathrm{H}), 7.14(\mathrm{~d}, J=9 \mathrm{~Hz}, 2 \mathrm{H}), 6.83(\mathrm{~d}, J=8.5 \mathrm{~Hz}, 2 \mathrm{H}), 6.83(\mathrm{~s}, 1 \mathrm{H})$, $3.71(\mathrm{~s}, 3 \mathrm{H}), 2.81(\mathrm{~s}, 4 \mathrm{H})$.

${ }^{13} \mathrm{C}$ NMR $\left(125 \mathrm{MHz}\right.$, DMSO-d $\left.\mathrm{d}_{6}\right) \delta(\mathrm{C}) 157.4,152.4,142.2,139.4,138.7,133.1$, $125.2(\mathrm{CH}) 129.2$ (x 2), 128.6 (x 2), 122.1, 119.6 (x 2), 118.2 (x 2), $115.9(\mathrm{CH})$, $113.6(x 2),\left(\mathrm{CH}_{2}\right) 37.4,36.0,\left(\mathrm{CH}_{3}\right)$ 54.9.

IR $\boldsymbol{V}_{\max }\left(\mathrm{cm}^{-1}\right) 3290(\mathrm{~N}-\mathrm{H}), 1638(\mathrm{C}=\mathrm{O})$.

HR ESMS m/z 379.1208 [M-H]'. Calc. for $\mathrm{C}_{22} \mathrm{H}_{20} \mathrm{CIN}_{2} \mathrm{O}_{2}, 379.1213$. 
4. BIOLOGICAL EVALUATION 



\section{BIOLOGICAL EVALUATION}

\subsection{General techniques}

\subsubsection{Molecular docking}

Molecular docking approach is used to predict the best interaction between a ligand and a macromolecule in the atomic level, which allows the determination of the biochemical behaviour of small molecules in the binding site of target proteins. It consists in the generation of a number of possible orientations (poses) of the ligand within the protein. Thus, the availability of the threedimensional structure of the molecular target is a necessary condition. Moreover, knowing the location of the binding site before docking processes significantly increases the efficiency. ${ }^{146}$

The identification of the most likely conformations requires two steps: firstly, the exploration of a large conformational space representing various potential binding modes and, secondly, the accurate assessment of the binding affinity associated with each of the predicted binding conformations. As it is shown in figure 4.1, the ligand is docked into the binding cavity of the receptor and the presumed conformations are explored until the best intermolecular interactions are identified. This process is carried out repeatedly until converging to a solution of minimum energy.

146 (a) Meng, X-Y.; Zhang, H-X.; Mezei, M.; Cui, M. Curr. Comput. Aided Drug Des. 2011, 7, 146157. (b) Salmaso, V.; Moro, S. Font. Pharmacol. 2018, 9, 923. 
A

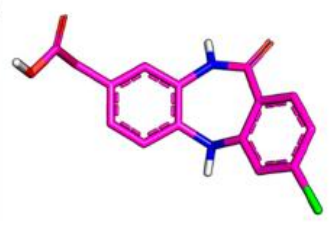

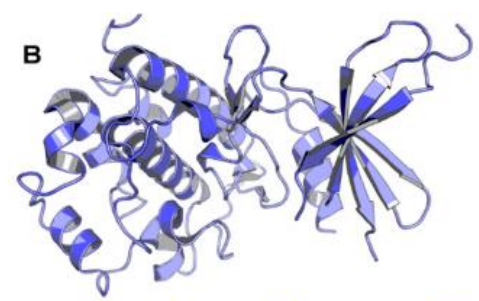
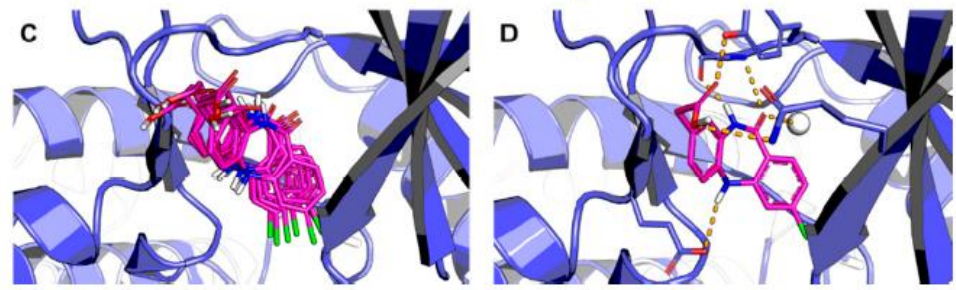

Figure 4.1. Molecular docking process. ${ }^{147}$

\subsubsection{Cell proliferation assay}

The ability of compounds to inhibit cell proliferation was determined by $\mathrm{IC}_{50}$ value, which is defined as the concentration of compound needed to inhibit $50 \%$ of cell proliferation. This parameter is calculated by the colorimetric assay of the tetrazolium dye MTT (3-(4,5-dimethylthiazol-2-yl)-2,5-diphenyltetrazolium bromide), which is based on the reduction of yellow MTT to its purple and insoluble formazan by mitochondrial reductase enzyme in living cells (see scheme 4.1). ${ }^{148}$ This method allows cell proliferation measurement since the amount of living cells is proportional to the amount of formazan that is formed.

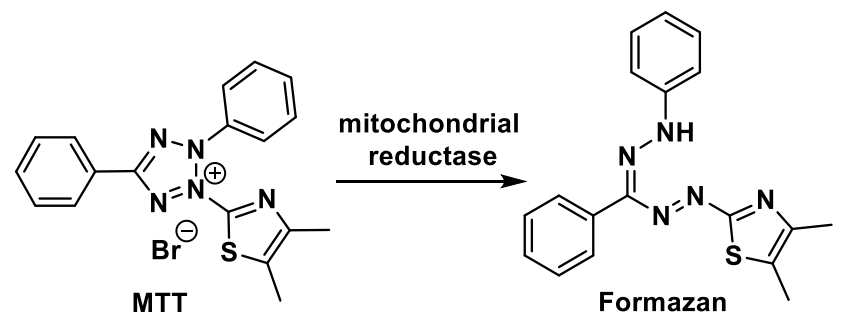

Scheme 4.1. MTT reduction to formazan.

147 Ferreira, L. G.; dos Santos, R. N.; Oliva, G.; Andricopulo, A. D. Molecules, 2015, 20, 1338413421.

148 a) Mosmann, T. J. Immunol. Methods 1983, 65, 55-63. b) Denizot, F.; Lang, R. J. Immunol. Methods 1986, 89, 271-277. 
After formazan solubilization with DMSO, the absorbance of the colored solution is quantified by measuring at 450-600 $\mathrm{nm}$ wavelength with a spectrophotometer. Then, GraphPad Prim 4 software is employed to adjust the results to a sinusoidal regression whose inflextion point corresponds to the $\mathrm{IC}_{50}$ value (see figure 4.2).
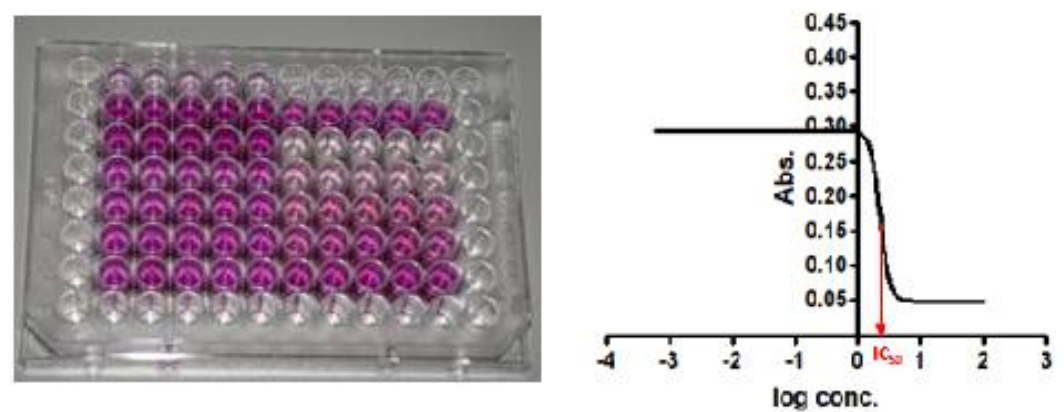

Figure 4.2. MTT assay and $\mathrm{IC}_{50}$ calculation.

\subsubsection{Cell cycle assay}

Cell cycle studies allow the evaluation of cell distribution after treatment with the corresponding compounds. Flow cytometry instrumentation enables cell counting and classification depending on morphologic characteristics. Cells suspended in a fluid pass through a thin transparent tube in which a laser light strikes. The light is transmitted and dispersed when passing through the tube and is collected by a detector allowing to discern the size and complexity of the cells.

Additionally, fluorescent tags are generally used, such as propidium iodide (PI) which is able to intercalate in the DNA and, thus, provides information about the cell distribution in the different phases of the cell cyle depending on the DNA amount they contain. Figure 4.3 shows a typical histogram representing the DNA content of different analyzed cells. 


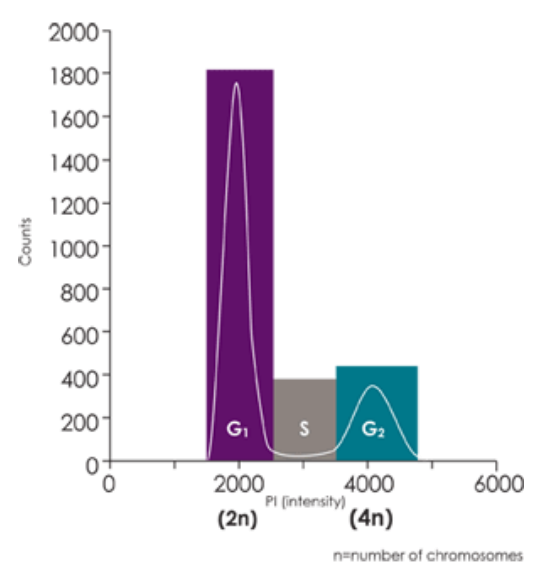

Figure 4.3. Cell cycle histogram. ${ }^{149}$

Three differentiated phases can be distinguished in the histogram:

- Resting and presynthesis $\left(G_{0} / G_{1}\right)$, in which cells have a fixed diploid DNA content corresponding to 23 pairs of chromosomes.

- Synthesis (S), in which cells synthesize genetic material until DNA is duplicated.

- Division $\left(\mathrm{G}_{2} / \mathrm{M}\right)$, in which genetic material remains duplicated with respect to the $\mathrm{G}_{0} / \mathrm{G}_{1}$ phase.

Generally, compounds that interact with tubulin accumulate cells in $G_{2} / M$ phase.

\subsubsection{Immunofluorescence}

Immunofluorescence is based on the use of specific antibodies which have been chemically conjugated to fluorescent dyes. These are subjected to high energy light (blue or UV) selected with a monochromator and, consequently, they emit light of lower intensity that can be observed with a fluorescence microscope.

149 Source: Nexcelom Bioscience website. 
This technique allows for visualization of the presence as well as the distribution of different target molecules in a sample (see figure 4.4).
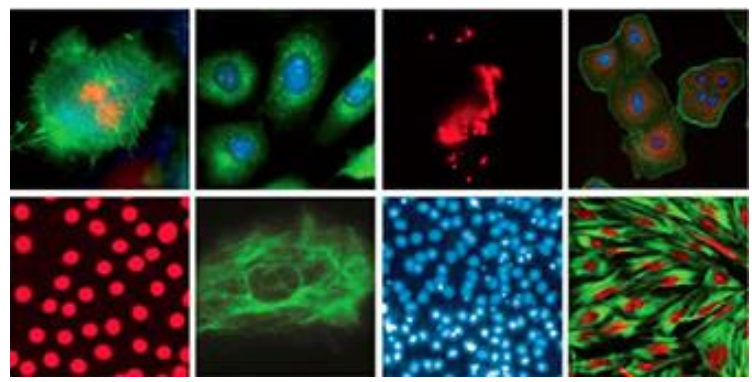

Figure 4.4. Immunofluorescence images. ${ }^{150}$

\subsubsection{In vitro tubulin self-assembly}

Microtubules polymerization is a dynamic process composed by three phases (see figure 4.5): nucleation, when tubulin oligomers are assembled; elongation, in which tubulin subunits are added to the end of growing microtubule; and steady state, when microtubules remain in a dynamic equilibrium and tubulin subunits are constantaly coming on and off. In the last phase, the concentration value of tubulin subunits that remain in solution is known as critical concentration ( $\mathrm{CrC})$.

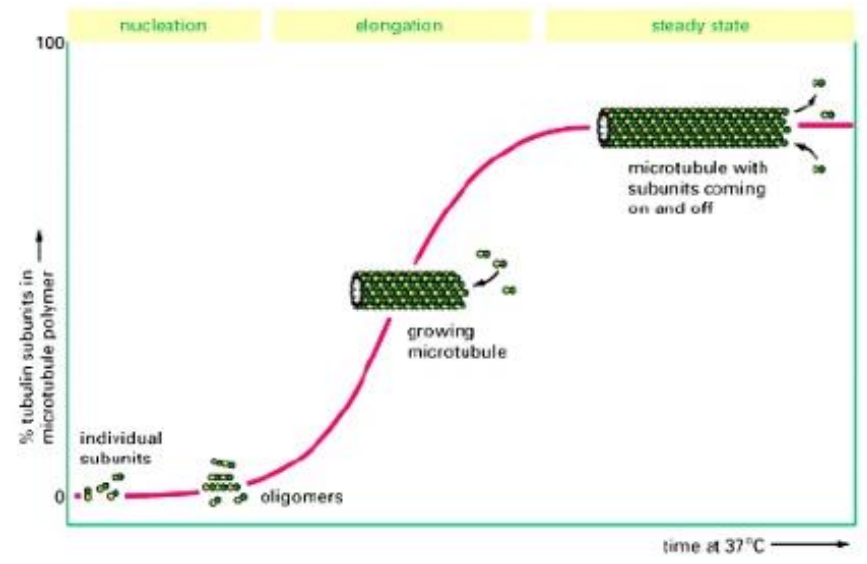

Figure 4.5. Tubulin polymerization. ${ }^{151}$

\footnotetext{
150 Source: Ibidi website.

${ }^{151}$ Alberts, B.; Bray, D.; Lewis, J.; Raff, M.; Roberts, K.; Watson, J. D. Molecular Biology of the Cell, 3rd edition, 1994.
} 
Concretely, tubulin critical concentration is defined as the minimum concentration needed to allow the reversible self-assembly of microtubules. This value depends on different conditions such as temperature, $\mathrm{pH}$ and concentration of compounds present in the medium. Microtubule-stabilizing compounds present lower $\mathrm{CrC}$ values than the one offered by the control, whereas microtubule-destabilizing agents show higher $\mathrm{CrC}$ values.

As microtubules are not water soluble, turbidimetry measurements are developed in order to study the effect of different compounds over tubulin polymerization.

\subsubsection{Apoptosis assay}

Apoptotic cells can be identified based on the alteration of cellular morphology. When apoptosis is initiated, phosphatidylserine usually present in the inner side of cell membrane migrates to the external side. Annexin $\mathrm{V}$ specifically binds to phosphatidylserine in the presence of protein-dependent $\mathrm{Ca}$ ion. Thus, by using fluorescent-labeled Annexin V, apoptotic cells can be easily quantified by flow cytometry (see figure 4.6).

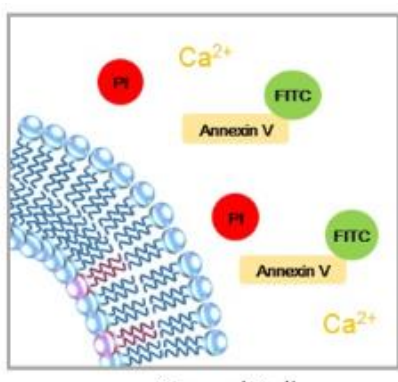

Normal Cell

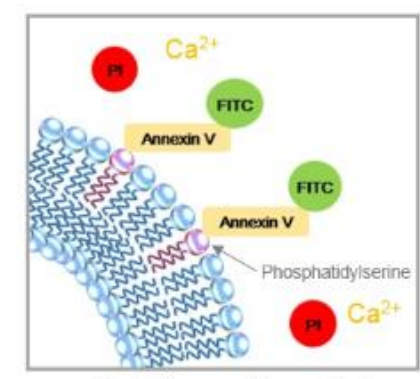

Early stages of apoptosis

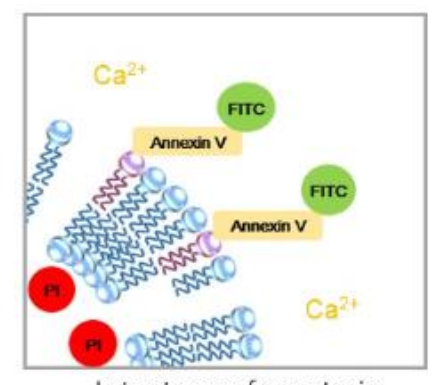

Late stages of apoptosis

Figure 4.6. Apoptosis measurement. ${ }^{152}$

\subsubsection{Tubulogenesis assay}

Tubulogenesis evaluation is a commonly used in vitro experiment to model the reorganization stage of angiogenesis. The assay involves plating endothelial

152 Source: Dojindo website. 
cells onto a suitable extracellular matrix support where the cells form capillarylike structures within 6 to $20 \mathrm{~h}$. Quantification is performed by measuring the number, length or area of these structures in two-dimensional microscope images (see figure 4.7).
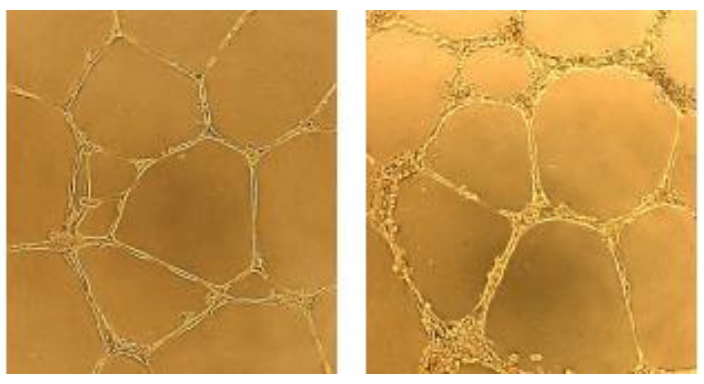

Figure 4.7. Tubulogenesis visualization. ${ }^{153}$

This assay is usually performed over a Matrigel® coating (see figure 4.8), which is constituted by a mixture of proteins that mimics the in vivo environment providing a three-dimensional surface suitable to endothelial cells adhesion and tubular structure formation.
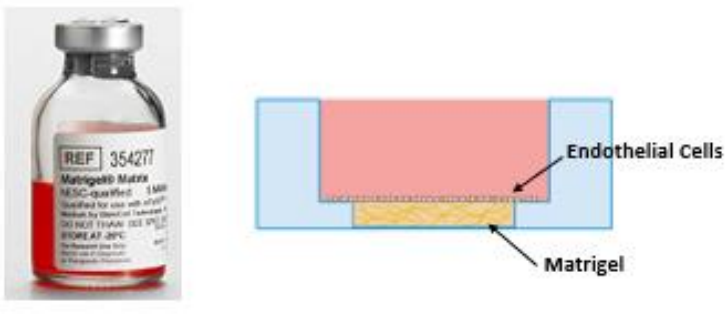

Figure 4.8. Matrigel coating.

Tubulogenesis assay can be used to evaluate both the effect of antiangiogenic compounds and the effect of VDAs. In the first case, compounds are added at the beginning of the process, thus tube formation inhibition is evaluated. In the second case, compounds are added once tubes are already formed, so tube destruction effect is measured.

153 Source: PromoCell website. 


\subsubsection{Protein quantification by flow cytometry}

Flow cytometry is a powerful technique to measure intracellular proteins on a single-cell level. It allows to distinguish the various cell populations in a sample if the appropriate antibodies are used. The most common way to detect the complex between a monoclonal antibody and an antigen is to covalently bind the antibody to different fluorescent molecules (fluorophores). After exposure to radiation from an excitation source, these fluorophores emit photons with longer wavelengths. Thus, fluorescence intensity can be correlated to the amount of protein on cell surface. Nowadays, there is a wide range of commercially available fluorophores such as fluorescein isothiocyanate (FITC), cyanine or Alexa series dyes (see figure 4.9).

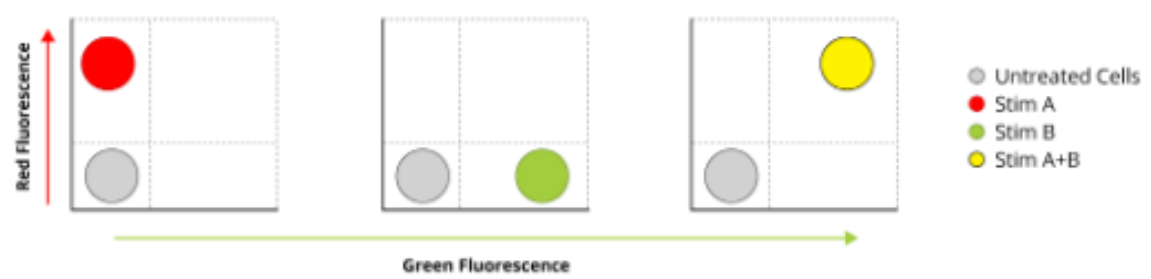

Figure 4.9. Multi-detection by flow cytometry.

In contrast to conventional cell surface labelling methods, intracellular flow cytometry requires fixation and permeabilization of the cells before staining the intracellular antigens. Formaldehyde is a crosslinking reagent typically used for fixation, and detergents such as Triton X-100 or saponin are usually used for permeabilization. ${ }^{154}$

\subsubsection{Protein quantification by ELISA}

The Enzyme-Linked Immunosorbent Assay (ELISA) is an immunological assay commonly used to measure antibodies, antigens or proteins in biological

154 Schmid, I. Flow Cytometry- Recent Perspectives, IntechOpen, 2012, ISBN: 978-953-51-06265 . 
samples. It is typically performed in 96-well polystyrene plates, allowing multiple samples to be measured in a single experiment. These plates need to be special adsorbent plates to ensure the antibody or antigen sticks to the surface. Each ELISA measures a specific antigen, and kits for a variety of antigens are widely available.

The key step of the assay is the immobilization of the antigen of interest, which can be accomplished by direct adsorption to the assay plate or via a capture antibody that has been previously attached to the plate. The antigen is then detected either directly (labelled primary antibody) or indirectly (labelled secondary antibody). The most powerful ELISA format is the sandwich assay, in which the analyte to be measured is bound between two primary antibodies (see figure 4.10).

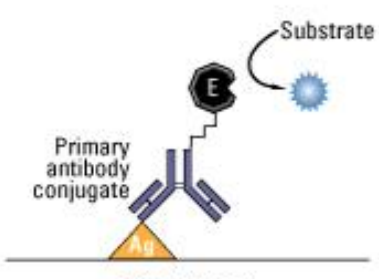

Direct Assay

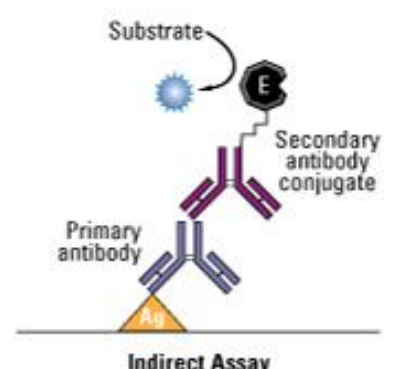

Indirect Assay

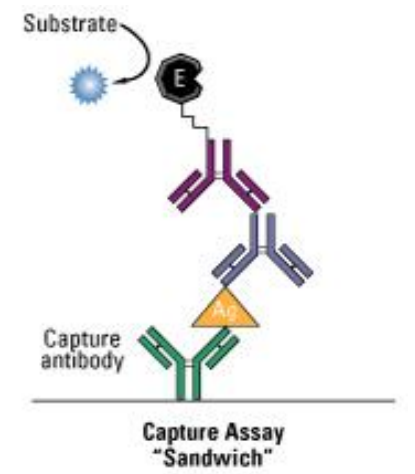

Figure 4.10. Common ELISA formats. ${ }^{155}$

Horseradish peroxidase (HRP) is a commonly used enzyme label, which converts tetramethylbenzidine (TMB) substrate into its blue-coloured diimine oxidation product. Then, the reaction can be halted by addition of a stop reagent. Concretely, sulphuric acid turns the blue solution into a yellow one, whose absorbance can be measured on a spectrophotometer at $450 \mathrm{~nm}$ (see scheme $4.2)$.

155 Source: ThermoFisher Scientific website. 
<smiles>CC1=CC(=C2C=C(C)C(=N[18O]C(C)C)C(C)=C2)C=C(C)C1=N</smiles>

Scheme 4.2. TMB oxidation by HRP.

\subsubsection{Protein quantification by Western blot (WB)}

Western blot, or protein immunoblot, is an analytical technique widely used in molecular biology research to identify specific proteins. Concretely, it involves a preliminary sample treatment to protein denaturation followed by gel electrophoresis (SDS-PAGE), which allows protein separation based on molecular weight. Then, the results are transferred to polyvinylidene difluoride (PVDF) membranes, which are sequentially incubated with primary antibodies, specific to the protein of interest, and with HRP secondary antibodies that generate a recordable signal in the presence of suitable substrates (see figure 4.11). ${ }^{156}$

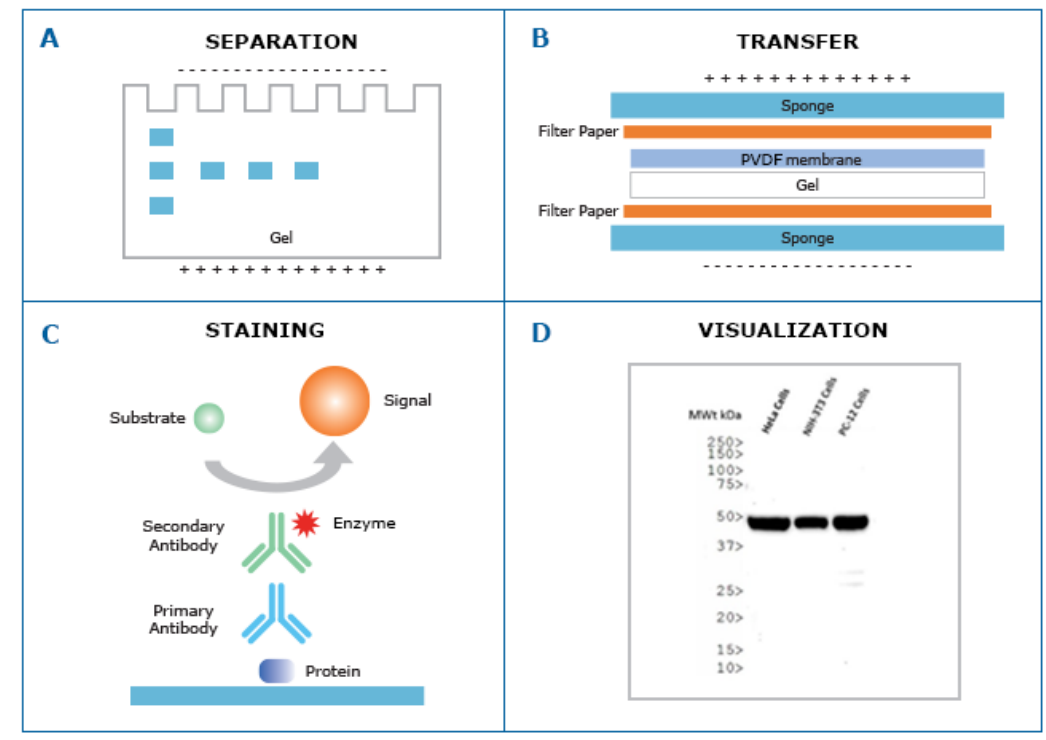

Figure 4.11. Western blot steps. ${ }^{157}$

156 Mahmood, T.; Yang, P-C. N. Am. J. Med. Sci. 2012, 4, 429-434.

157 Source: Novus Biologicals website. 
The most popular chemiluminescent substrates are luminol-based ones. In the presence of HRP and peroxide buffer, luminol oxidizes and forms an excited product that emits light at $425 \mathrm{~nm}$ as it returns to the ground state. Emission occurs only during the reaction, therefore, once the substrate is exhausted, signal output ceases (see scheme 4.3).

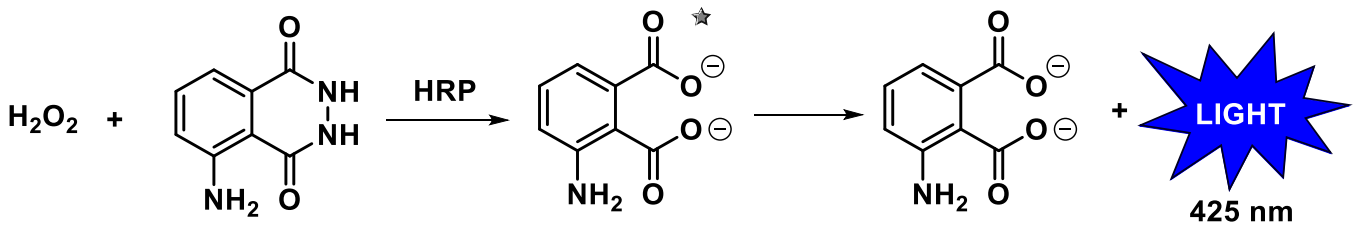

Scheme 4.3. Chemiluminescent reaction of luminol. ${ }^{158}$

\subsubsection{In vivo bioluminiscence imaging (BLI)}

In vivo bioluminescent imaging (BLI) is a versatile and sensitive tool based on detection of light produced by living organisms after a biochemical reaction. Luciferase enzymes are usually used, which catalyze the oxidation of luciferin substrate (see scheme 4.4).

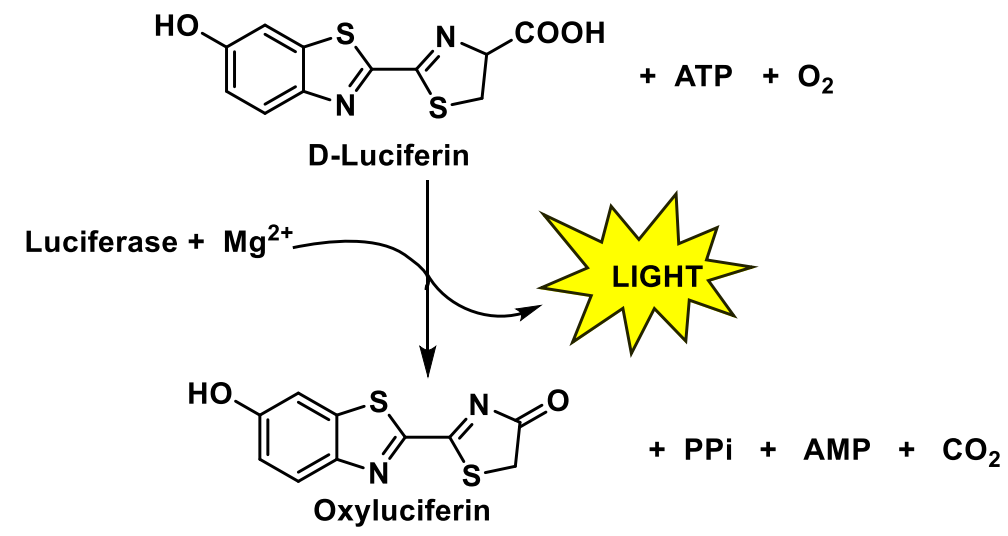

Scheme 4.4. Luciferin oxidation by luciferase enzyme..$^{159}$

158 Source: ThermoFisher Scientific website.

159 Source: BRS Bioscience website. 
Bioluminescence allows non-invasive and real-time analysis of disease processes at the molecular level in living organisms. It has the ability to track tumor cells expressing luciferase and, thus, to study tumor growth, metastasis and therapeutic responses in vivo. Figure 4.12 schematically represents the experimental process that is usually followed. Firstly, tumor cells are transfected with luciferase gene and, then, are inoculated into engineered mouse models. Secondly, luciferin substrate is administrated by intravascular or intraperitoneal injection and, finally, data is collected by in vivo imaging systems. ${ }^{160}$

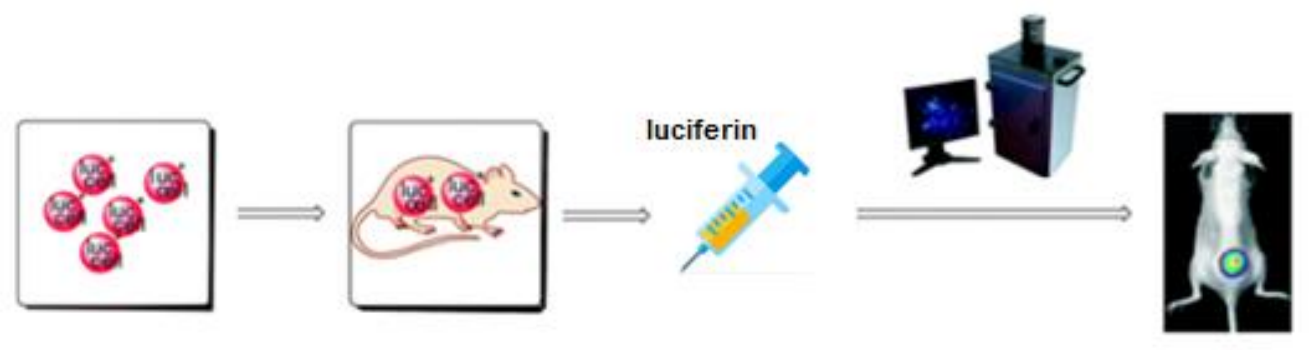

Figure 4.12. Bioluminescence imaging procedure. ${ }^{161}$

160 Sato, A.; Klaunberg, B.; Tolwani, R. Comp. Med. 2004, 54, 631-634.

${ }^{161}$ Figure adapted from: Li, J.; Chen, L.; Dua, L.; Li, M. Chem. Soc. Rev., 2013,42, 662-676. 


\subsection{Preliminary bioavailability prediction study}

SwissADME online tool ${ }^{162}$ was employed to check if synthesized compounds accomplish "Lipinski rule of five", which tries to predict the bioavailability of a drug candidate. ${ }^{163}$ Concretely, the violation of two of the conditions proposed by Lipinski possibly implies a low absorption or permeability.

Table 4.1 shows the results obtained for family 1 compounds. It can be observed that some of the derivatives violate two of the conditions, being C.8, C.9, C.10, C.17, C.18, C.19 bad candidates as therapeutic drugs.

Table 4.1. Lipinski checking for family 1 .

\begin{tabular}{|c|c|c|c|c|c|}
\hline Compound & $\log P$ & Mw (g/mol) & $\begin{array}{l}\text { H-bond } \\
\text { acceptors }\end{array}$ & $\begin{array}{l}\text { H-bond } \\
\text { donors }\end{array}$ & Violations \\
\hline AmCA-4 & 3.14 & 315.36 & 4 & 1 & 0 \\
\hline C. 1 & 4.53 & 435.47 & 6 & 1 & 0 \\
\hline C. 2 & 4.84 & 453.46 & 7 & 1 & 0 \\
\hline C.3 & 4.85 & 453.46 & 7 & 1 & 0 \\
\hline C. 4 & 4.85 & 453.46 & 7 & 1 & 0 \\
\hline C. 5 & 5.07 & 469.91 & 6 & 1 & 1 \\
\hline C. 6 & 5.07 & 469.91 & 6 & 1 & 1 \\
\hline C.7 & 5.07 & 469.91 & 6 & 1 & 1 \\
\hline C. 8 & 5.15 & 514.37 & 6 & 1 & 2 \\
\hline C.9 & 5.15 & 514.37 & 6 & 1 & 2 \\
\hline C.10 & 5.15 & 514.37 & 6 & 1 & 2 \\
\hline C.11 & 4.54 & 465.5 & 7 & 1 & 0 \\
\hline C.12 & 4.54 & 465.5 & 7 & 1 & 0 \\
\hline C.13 & 4.54 & 465.5 & 7 & 1 & 0 \\
\hline C.14 & 4.84 & 449.5 & 6 & 1 & 0 \\
\hline C.15 & 4.86 & 449.5 & 6 & 1 & 0 \\
\hline C.16 & 4.87 & 449.5 & 6 & 1 & 0 \\
\hline C.17 & 5.57 & 503.47 & 9 & 1 & 2 \\
\hline C.18 & 5.58 & 503.47 & 9 & 1 & 2 \\
\hline C.19 & 5.59 & 503.47 & 9 & 1 & 2 \\
\hline C.20 & 5.15 & 463.52 & 6 & 1 & 1 \\
\hline
\end{tabular}

162 Daina, A.; Michielin, O.; Zoete, V. Scientific Reports 2017, 7, 1-13.

163 Lipinski, C. A. Drug Discovery Today: Technol. 2004, 1, 337-341. 


\begin{tabular}{|l|c|c|c|c|c|}
\hline C.21 & 5.1 & 463.52 & 6 & 1 & 1 \\
\hline C.22 & 5.13 & 463.52 & 6 & 1 & 1 \\
\hline C.23 & 5.19 & 463.52 & 6 & 1 & 1 \\
\hline C.24 & 5.4 & 483.94 & 6 & 1 & 1 \\
\hline C.25 & 5.39 & 483.94 & 6 & 1 & 1 \\
\hline C.26 & 5.4 & 483.94 & 6 & 1 & 1 \\
\hline
\end{tabular}

Table 4.2 shows the results obtained for family 2 compounds, being $\mathbf{U} .17$, U.18, U.19 bad candidates as therapeutic drugs.

Table 4.2. Lipinski checking for family 2.

\begin{tabular}{|c|c|c|c|c|c|}
\hline Compound & logP & Mw (g/mol) & $\begin{array}{c}\text { H-bond } \\
\text { acceptors }\end{array}$ & $\begin{array}{c}\text { H-bond } \\
\text { donors }\end{array}$ & Violations \\
\hline U.1 & 4.22 & 434.48 & 5 & 2 & 0 \\
\hline U.2 & 4.47 & 452.47 & 6 & 2 & 0 \\
\hline U.3 & 4.48 & 452.47 & 6 & 2 & 0 \\
\hline U.4 & 4.51 & 452.47 & 6 & 2 & 0 \\
\hline U.5 & 4.68 & 468.93 & 5 & 2 & 0 \\
\hline U.6 & 4.71 & 468.93 & 5 & 2 & 0 \\
\hline U.7 & 4.73 & 468.93 & 5 & 2 & 0 \\
\hline U.8 & 4.76 & 513.38 & 5 & 2 & 1 \\
\hline U.9 & 4.82 & 513.38 & 5 & 2 & 1 \\
\hline U.10 & 4.83 & 513.38 & 5 & 2 & 1 \\
\hline U.11 & 4.2 & 464.51 & 6 & 2 & 0 \\
\hline U.12 & 4.21 & 464.51 & 6 & 2 & 0 \\
\hline U.13 & 4.17 & 464.51 & 6 & 2 & 0 \\
\hline U.14 & 4.47 & 448.51 & 5 & 2 & 0 \\
\hline U.15 & 4.53 & 448.51 & 5 & 2 & 0 \\
\hline U.16 & 4.54 & 448.51 & 5 & 2 & 0 \\
\hline U.17 & 5.21 & 502.48 & 8 & 2 & 2 \\
\hline U.18 & 5.3 & 502.48 & 8 & 2 & 2 \\
\hline U.19 & 5.33 & 502.48 & 8 & 2 & 2 \\
\hline U.20 & 4.93 & 462.54 & 5 & 2 & 0 \\
\hline U.21 & 4.79 & 462.54 & 5 & 2 & 0 \\
\hline U.22 & 4.81 & 462.54 & 5 & 2 & 0 \\
\hline U.23 & 4.82 & 462.54 & 5 & 2 & 0 \\
\hline U.24 & 5.05 & 482.96 & 5 & 2 & 1 \\
\hline & & & & 5 & 2 \\
\hline
\end{tabular}




\begin{tabular}{|l|c|c|c|c|c|}
\hline U.25 & 5.06 & 482.96 & 5 & 2 & 1 \\
\hline U.26 & 5.1 & 482.96 & 5 & 2 & 1 \\
\hline
\end{tabular}

Table 4.3 shows the results obtained for family 3 compounds, where it can be appreciated that none of them violate any condition.

Table 4.3. Lipinski checking for family 3.

\begin{tabular}{|c|c|c|c|c|c|}
\hline Compound & logP & Mw (g/mol) & $\begin{array}{c}\text { H-bond } \\
\text { acceptors }\end{array}$ & $\begin{array}{c}\text { H-bond } \\
\text { donors }\end{array}$ & Violations \\
\hline T.1 & 2.41 & 351.4 & 4 & 2 & 0 \\
\hline T.2 & 2.97 & 385.85 & 4 & 2 & 0 \\
\hline T.3 & 2.93 & 385.85 & 4 & 2 & 0 \\
\hline T.4 & 3.05 & 430.3 & 4 & 2 & 0 \\
\hline T.5 & 3.02 & 430.3 & 4 & 2 & 0 \\
\hline T.6 & 2.73 & 365.43 & 4 & 2 & 0 \\
\hline T.7 & 2.71 & 365.43 & 4 & 2 & 0 \\
\hline T.8 & 2.7 & 365.43 & 4 & 2 & 0 \\
\hline S.1 & 4.73 & 378.85 & 2 & 2 & 0 \\
\hline S.2 & 4.73 & 378.85 & 2 & 2 & 0 \\
\hline S.3 & 4.66 & 378.85 & 2 & 2 & 0 \\
\hline S.4 & 4.73 & 378.85 & 2 & 2 & 0 \\
\hline S.5 & 4.73 & 378.85 & 2 & 2 & 0 \\
\hline S.6 & 4.66 & 378.85 & 2 & 2 & 0 \\
\hline S.7 & 4.72 & 380.87 & 2 & 2 & 0 \\
\hline S.8 & 4.73 & 380.87 & 2 & 2 & 0 \\
\hline S.9 & 4.7 & 380.87 & 2 & 2 & 0 \\
\hline
\end{tabular}




\subsection{Biological evaluation of family 1 as VDAs}

\subsubsection{Inhibition of cell proliferation}

The ability of compounds C.1-C.26 to inhibit cell proliferation was established by means of their $\mathrm{IC}_{50}$ values towards the human tumor cell lines HT-29 (colon adenocarcinoma), MCF-7 (breast adenocarcinoma), HeLa (epithelioid cervix carcinoma), A549 (pulmonary adenocarcinoma), HL-60 (promyelocytic leukemia) and MDA-MB-231 (breast adenocarcinoma) as well as towards the endothelial cell line HMEC-1 (human microvascular endothelial cells) and the non-tumor cell line HEK-293 (human embryonic kidney cells). The $\mathrm{IC}_{50}$ values are presented in table 4.4 along with $\mathrm{IC}_{50}$ values for the reference compounds CA-4 and AmCA-4.

Table 4.4. $I_{50}$ values for $C A-4(\mu M)$, AmCA-4 $(n M)$ and C.1-C.26 (nM). ${ }^{a}$

\begin{tabular}{lllllllll}
\hline Comp. & HT-29 & MCF-7 & HeLa & A549 & HL-60 & $\begin{array}{l}\text { MDA-MB- } \\
\mathbf{2 3 1}\end{array}$ & HMEC-1 & HEK-293 \\
\hline CA-4 & $4.2 \pm 0.5$ & $1 \pm 0.2$ & $2.1 \pm 0.6$ & $0.13 \pm 0.02$ & $4 \pm 1$ & - & $3.4 \pm 0.4$ & $25 \pm 0.3$ \\
AmCA-4 & $22.0 \pm 0.7$ & $8.0 \pm 0.9$ & $2.6 \pm 0.5$ & $117 \pm 7$ & $4.5 \pm 0.9$ & $12 \pm 2$ & $12 \pm 6$ & $7.1 \pm 1.0$ \\
C.1 & $2.9 \pm 0.5$ & $9.8 \pm 0.1$ & $4.8 \pm 0.2$ & $77 \pm 12$ & $4 \pm 2$ & $11.8 \pm 0.2$ & $12 \pm 5$ & $3.3 \pm 0.2$ \\
C.2 & $110 \pm 30$ & $62 \pm 2$ & $17 \pm 2$ & $320 \pm 190$ & $4 \pm 2$ & $71 \pm 3$ & $180 \pm 20$ & $65 \pm 4$ \\
C.3 & $10.6 \pm 0.5$ & $7.0 \pm 0.6$ & $2.7 \pm 1.3$ & $150 \pm 40$ & $60 \pm 20$ & $14.8 \pm 0.3$ & $20 \pm 2$ & $13.2 \pm 0.2$ \\
C.4 & $60 \pm 20$ & $95.4 \pm 1.3$ & $60 \pm 20$ & $400 \pm 200$ & $50 \pm 30$ & $13.8 \pm 0.9$ & $65 \pm 13$ & $185 \pm 2$ \\
C.5 & $17.8 \pm 0.7$ & $10.1 \pm 0.8$ & $5 \pm 2$ & $130 \pm 40$ & $3.7 \pm 0.7$ & $13.8 \pm 1.2$ & $50 \pm 30$ & $41 \pm 2$ \\
C.6 & $9.2 \pm 1.3$ & $4.4 \pm 0.6$ & $4.79 \pm 0.03$ & $100 \pm 20$ & $14 \pm 2$ & $15.1 \pm 0.8$ & $9 \pm 6$ & $14.5 \pm 0.4$ \\
C.7 & $31 \pm 14$ & $82 \pm 13$ & $24 \pm 3$ & $800 \pm 200$ & $770 \pm 170$ & $9.4 \pm 0.1$ & $76 \pm 13$ & $142 \pm 9$ \\
C.8 & $7.5 \pm 0.6$ & $18.9 \pm 0.9$ & $2.6 \pm 1.0$ & $180 \pm 50$ & $3 \pm 2$ & $13.4 \pm 0.6$ & $22 \pm 7$ & $8 \pm 5$ \\
C.9 & $11.3 \pm 0.7$ & $5.9 \pm 0.3$ & $2.6 \pm 0.5$ & $160 \pm 30$ & $5.6 \pm 0.6$ & $13.4 \pm 0.5$ & $12 \pm 4$ & $16 \pm 2$ \\
C.10 & $3.5 \pm 0.2$ & $0.75 \pm 0.05$ & $1.2 \pm 0.2$ & $150 \pm 30$ & $5 \pm 3$ & $4.07 \pm 0.05$ & $7 \pm 4$ & $19 \pm 7$ \\
C.11 & $290 \pm 100$ & $230 \pm 180$ & $348 \pm 13$ & $3000 \pm 1100$ & $4000 \pm 200$ & $>100$ & $400 \pm 15$ & $160 \pm 40$ \\
C.12 & $4.8 \pm 0.5$ & $7.18 \pm 0.07$ & $3.4 \pm 0.4$ & $160 \pm 40$ & $4.9 \pm 0.5$ & $8 \pm 6$ & $5 \pm 2$ & $8.1 \pm 0.2$ \\
C.13 & $350 \pm 20$ & $230 \pm 30$ & $200 \pm 40$ & $550 \pm 120$ & $50 \pm 6$ & $69 \pm 3$ & $120 \pm 50$ & $130 \pm 40$
\end{tabular}




\begin{tabular}{|c|c|c|c|c|c|c|c|c|}
\hline C.14 & $160 \pm 70$ & $380 \pm 50$ & $50 \pm 30$ & $460 \pm 110$ & $222 \pm 7$ & $21 \pm 6$ & $55 \pm 6$ & $60 \pm 20$ \\
\hline C.15 & $46 \pm 3$ & $25 \pm 9$ & $23 \pm 13$ & $390 \pm 180$ & $67 \pm 2$ & $13.6 \pm 0.5$ & $50 \pm 20$ & $40 \pm 20$ \\
\hline C.16 & $270 \pm 80$ & $200 \pm 60$ & $370 \pm 160$ & $1500 \pm 300$ & $52 \pm 2$ & $14 \pm 6$ & $160 \pm 40$ & $144 \pm 10$ \\
\hline C.17 & $16 \pm 7$ & $44 \pm 2$ & $15 \pm 4$ & $110 \pm 40$ & $6.7 \pm 0.6$ & $13.7 \pm 0.2$ & $19 \pm 5$ & $4.1 \pm 1.0$ \\
\hline C.19 & $27.6 \pm 0.6$ & $9.2 \pm 0.3$ & $21 \pm 8$ & $270 \pm 70$ & $40 \pm 30$ & $21.5 \pm 1.0$ & $30 \pm 8$ & $57 \pm 22$ \\
\hline C.20 & $600 \pm 200$ & $440 \pm 14$ & $320 \pm 120$ & $3900 \pm 1200$ & $470 \pm 70$ & $76.0 \pm 1.4$ & $380 \pm 140$ & $490 \pm 80$ \\
\hline C.23 & $130 \pm 20$ & $170 \pm 30$ & $21 \pm 6$ & $1300 \pm 600$ & $69 \pm 11$ & $41 \pm 28$ & $130 \pm 50$ & $97 \pm 9$ \\
\hline C.24 & $47 \pm 2$ & $39 \pm 3$ & $23 \pm 12$ & $140 \pm 30$ & $70 \pm 30$ & $14 \pm 2$ & $20 \pm 2$ & $14 \pm 2$ \\
\hline C.25 & $11.4 \pm 0.3$ & $8.1 \pm 0.9$ & $3.3 \pm 0.5$ & $140 \pm 30$ & $0.4 \pm 0.2$ & $4 \pm 3$ & $7 \pm 1$ & $8.4 \pm 0.6$ \\
\hline C.26 & $23 \pm 2$ & $6.1 \pm 0.8$ & $3.7 \pm 0.3$ & $90 \pm 20$ & $1.8 \pm 0.6$ & $13.70 \pm 0.01$ & $6 \pm 2$ & $7.0 \pm 0.8$ \\
\hline
\end{tabular}

${ }^{\mathrm{a}} \mathrm{C}_{50}$ values are expressed as the compound concentration that inhibits the cell growth by $50 \%$. Data are the average $( \pm S D)$ of three experiments.

Some trends can be deduced from table 4.4. Thus, the majority of compounds show activity in the low nanomolar range in all cell lines except in A549, in which the activity lies in the high nanomolar range. In general, carbamate derivatives show better $\mathrm{IC}_{50}$ values than AmCA-4 in most of the tested tumor cell lines. Moreover, halo- and methoxy-phenyl carbamates substituted at position 3 of the phenyl ring show higher antiproliferative activity than their regioisomeric counterparts, particularly in MCF-7, HT-29, HeLa and A549 cell lines. As regards therapeutic safety, compounds C.4, C.5, C.6, C.9, C.10 and $\mathbf{C} .19$ show greater selectivity since the most of their $\mathrm{IC}_{50}$ values in MCF-7, HT-29, HeLa and HL-60 tumor cell lines are 2- to 11-fold lower than their $\mathrm{IC}_{50}$ values in the non-tumor cell line HEK-293. As regards endothelial cells (HMEC-1), compounds C.6, C.10, C.12, C.25 and C.26 show better antiproliferative activity than AmCA-4. These latter compounds are mainly substituted in meta and para positions with halogen atoms or their bioisosters. 


\subsubsection{Tubulin polymerization inhibition}

In order to evaluate the effect on tubulin self-assembly, the critical concentration ( $\mathrm{CrC})$ was determined. All compounds were tested at a concentration of $27.5 \mu \mathrm{M}$ in glycerol-assembling buffer (GAB) and GTP, to which tubulin was added at a concentration of $25 \mu \mathrm{M}$. The results achieved are shown in table 4.5 and are compared with those attained in the absence of any ligand (control, DMSO) and in the presence of CA-4 and AmCA-4.

Table 4.5. Critical concentration $(\mathrm{CrC})$ values.

\begin{tabular}{lc|ll}
\hline Compound & CrC $(\mu \mathrm{M})$ & Compound & CrC $(\mu \mathrm{M})$ \\
\hline Control & $8 \pm 1$ & C.13 & $23 \pm 2$ \\
CA-4 & $22 \pm 1$ & C.14 & $22 \pm 3$ \\
AmCA-4 & $23 \pm 2$ & C.15 & $20 \pm 3$ \\
C.1 & $20 \pm 2$ & C.16 & $25 \pm 2$ \\
C.2 & $18 \pm 3$ & C.17 & $23 \pm 2$ \\
C.3 & $24 \pm 4$ & C.18 & $14 \pm 2$ \\
C.4 & $22 \pm 2$ & C.19 & $20 \pm 3$ \\
C.5 & $21 \pm 3$ & C.20 & $17 \pm 3$ \\
C.6 & $23 \pm 2$ & C.21 & $24 \pm 2$ \\
C.7 & $24 \pm 2$ & C.22 & $25 \pm 2$ \\
C. 8 & $22 \pm 2$ & C.23 & $21 \pm 2$ \\
C.9 & $25 \pm 3$ & C.24 & $23 \pm 1$ \\
C.10 & $23 \pm 2$ & C.25 & $24 \pm 3$ \\
C.11 & $16 \pm 1$ & C.26 & $24 \pm 3$ \\
C.12 & $20 \pm 2$ & & \\
\hline
\end{tabular}

Data are the average $( \pm S D)$ of three experiments.

All carbamates show $\mathrm{CrC}$ values similar to those achieved in the presence of $\mathrm{CA}-4$ or AmCA-4, which are increased relative to the value measured in the control.

Figure 4.12 shows the effect of some selected ligands on the in vitro tubulin polymerization process studied by turbidimetry time-course measurements at 
$350 \mathrm{~nm}$ and $37^{\circ} \mathrm{C}$. In order to compare, paclitaxel, a microtubule stabilizer, was also evaluated.

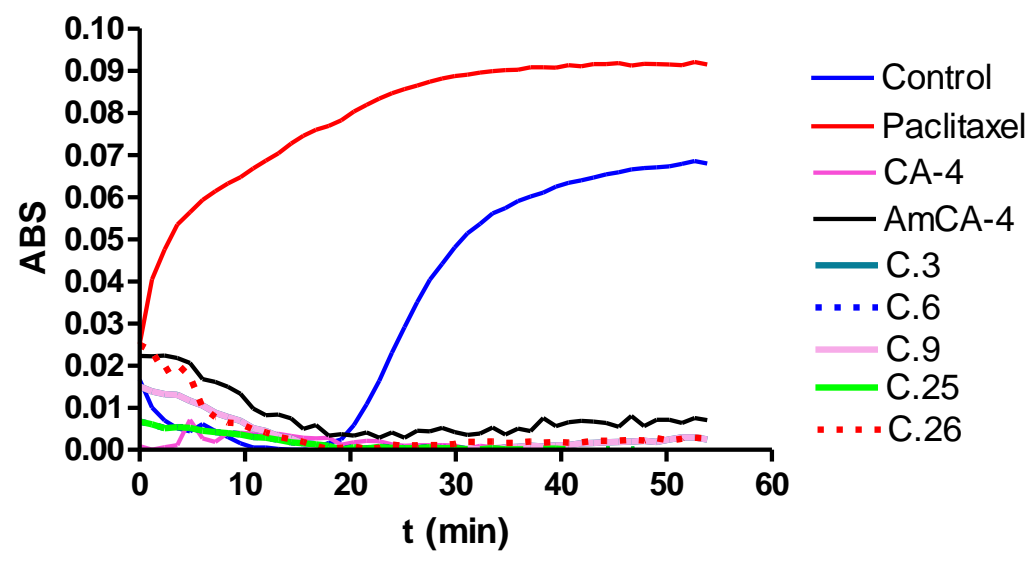

Figure 4.12. Effect on the in vitro tubulin polymerization.

\subsubsection{Tubulin interaction evaluation}

In order to check whether the carbamates interact with tubulin in the colchicine-binding site, the EBI assay was undertaken. This method is based on the property of $N, N^{\prime}$-ethylene-bis(iodoacetamide) (EBI), a homobifunctional thioalkylating agent, to crosslink the Cys-239 and the Cys-354 residues present in the colchicine-binding site of $\beta$-tubulin. The covalent binding of EBI to $\beta$-tubulin forms an adduct that is easily detected by Western blot as a second immunoreacting band of $\beta$-tubulin that migrates faster than the native $\beta$-tubulin band. Consequently, treatment of the cells with a compound that binds to this colchicine-binding site will impair the binding of EBI, resulting in the absence of the second band ${ }^{164}$. The carbamates tested were selected according to their $I_{50}$ value in MDA-MB-231 cell line, thus the three compounds with the lowest $\mathrm{IC}_{50}$ values (C.10, C.12 and C.25) and two additional carbamates with higher $\mathrm{IC}_{50}$ values (C.11 and $\mathbf{C . 1 3}$ ) were chosen for this study. With this selection it was

${ }^{164}$ Fortin, S.; Lacroix, J.; Côté, M.-F.; Petitclerc, É.; Gaudreault, R. C. Biol. Proced. Online 2010, 12, 113-117. 
intended to ascertain if there was any correlation between the antiproliferative activity of the compounds and their capacity to interact with tubulin. The results of this assay are depicted in figure 4.13.

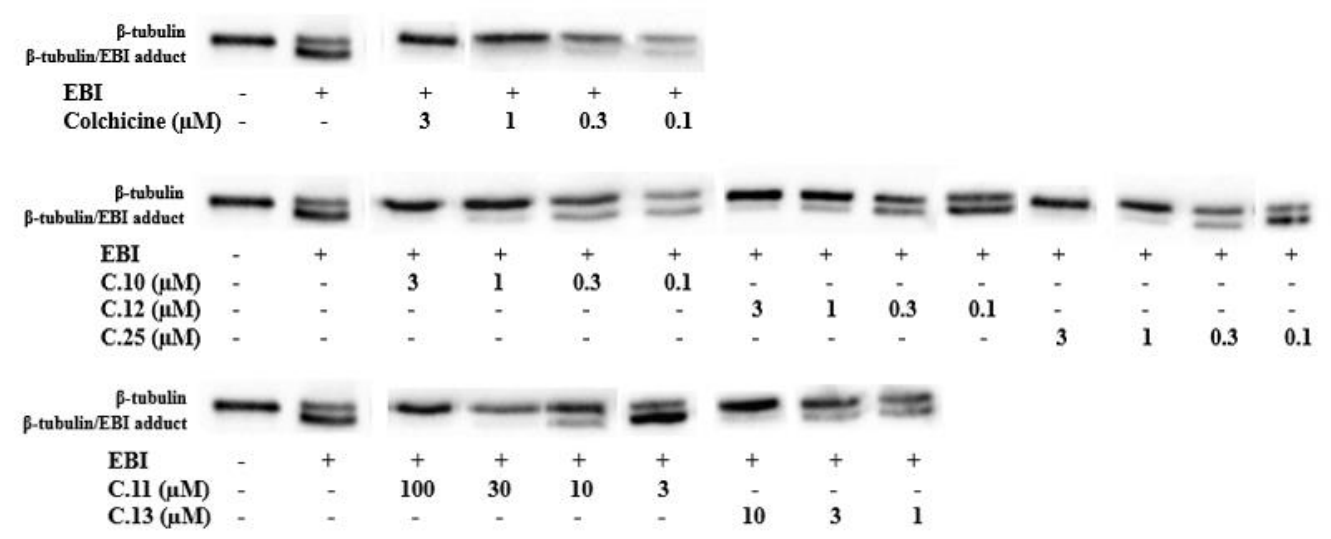

Figure 4.13. Western blot results of EBI assay.

As shown in figure 4.13, there is a dose-response effect for all compounds tested. Concretely, C.10, C.12 and C.25 were able to inhibit the formation of EBI adduct at $3 \mu \mathrm{M}$, showing a similar behaviour to that of colchicine at this concentration. However, C.11 and C.13 were less effective since they required higher concentrations ( $100 \mu \mathrm{M}$ and $10 \mu \mathrm{M}$, respectively) to avoid being displaced by EBI from tubulin. Thus, this experiment demonstrates that carbamates bind tubulin at the colchicine-binding site and that their antiproliferative capacity correlates with tubulin binding.

\subsubsection{Mitotic arrest and inhibition of interphase microtubules}

The effect of carbamates on cell cycle distribution was evaluated in A549 cells. Thus, cells were incubated for $20 \mathrm{~h}$ in the presence of CA-4, AmCA-4 and compounds C.1-C.26 and then, DNA content was measured by flow cytometry (see table 4.6). All the carbamates extensively arrested cells in the $G_{2} / M$ phase at a concentration half of their $\mathrm{IC}_{50}$ value. 
Table 4.6. Cell cycle distribution (\%).

\begin{tabular}{|c|c|c|c|c|c|}
\hline Comp. & Conc. (nM) & SubGo & $\mathrm{G}_{0} / \mathbf{G}_{1}$ & $S$ & $\mathrm{G}_{2} / \mathrm{M}$ \\
\hline Control & - & $6.6 \pm 0.1$ & $66 \pm 1$ & $16 \pm 1$ & $11 \pm 1$ \\
\hline CA-4 & 50 & $15 \pm 1$ & $21 \pm 2$ & $22 \pm 1$ & $43 \pm 1$ \\
\hline AmCA-4 & 60 & $14 \pm 1$ & $21 \pm 1$ & $24 \pm 1$ & $41 \pm 2$ \\
\hline C.1 & 40 & $20 \pm 3$ & $21 \pm 1$ & $15 \pm 1$ & $44 \pm 3$ \\
\hline C.2 & 160 & $17 \pm 1$ & $31 \pm 3$ & $13 \pm 1$ & $39 \pm 2$ \\
\hline C. 3 & 75 & $19 \pm 1$ & $29 \pm 1$ & $10 \pm 1$ & $43 \pm 2$ \\
\hline C. 4 & 200 & $21 \pm 1$ & $26 \pm 5$ & $19 \pm 1$ & $34 \pm 1$ \\
\hline C.5 & 65 & $16 \pm 1$ & $29 \pm 2$ & $12 \pm 2$ & $43 \pm 3$ \\
\hline C. 6 & 50 & $16 \pm 2$ & $30 \pm 1$ & $10 \pm 1$ & $44 \pm 2$ \\
\hline C.7 & 400 & $20 \pm 1$ & $21 \pm 1$ & $18 \pm 1$ & $41 \pm 1$ \\
\hline C. 8 & 90 & $17 \pm 2$ & $29 \pm 1$ & $10 \pm 1$ & $44 \pm 3$ \\
\hline C.9 & 80 & $17 \pm 3$ & $30 \pm 1$ & $12 \pm 1$ & $39 \pm 1$ \\
\hline C.10 & 75 & $19 \pm 1$ & $32 \pm 3$ & $17 \pm 1$ & $33 \pm 1$ \\
\hline C.11 & 1500 & $17 \pm 1$ & $29 \pm 4$ & $12 \pm 3$ & $43 \pm 1$ \\
\hline C.12 & 80 & $17 \pm 1$ & $31 \pm 1$ & $16 \pm 2$ & $36 \pm 3$ \\
\hline C.13 & 275 & $14 \pm 1$ & $25 \pm 1$ & $14 \pm 1$ & $46 \pm 2$ \\
\hline C.14 & 230 & $16 \pm 1$ & $31 \pm 2$ & $13 \pm 2$ & $40 \pm 1$ \\
\hline C.15 & 200 & $22 \pm 3$ & $26 \pm 2$ & $16 \pm 3$ & $36 \pm 1$ \\
\hline C.16 & 750 & $27 \pm 5$ & $24 \pm 1$ & $16 \pm 2$ & $32 \pm 3$ \\
\hline C.17 & 55 & $20 \pm 2$ & $21 \pm 2$ & $15 \pm 1$ & $44 \pm 5$ \\
\hline C.18 & 65 & $16 \pm 3$ & $28 \pm 1$ & $15 \pm 1$ & $41 \pm 1$ \\
\hline C.19 & 135 & $26 \pm 1$ & $22 \pm 1$ & $14 \pm 1$ & $39 \pm 2$ \\
\hline C.20 & 2000 & $23 \pm 2$ & $25 \pm 2$ & $17 \pm 1$ & $35 \pm 1$ \\
\hline C.21 & 750 & $22 \pm 3$ & $24 \pm 3$ & $16 \pm 1$ & $38 \pm 1$ \\
\hline C.22 & 450 & $12 \pm 1$ & $24 \pm 1$ & $22 \pm 3$ & $42 \pm 2$ \\
\hline C.23 & 650 & $17 \pm 1$ & $24 \pm 1$ & $19 \pm 1$ & $41 \pm 1$ \\
\hline C.24 & 70 & $20 \pm 1$ & $24 \pm 1$ & $18 \pm 2$ & $39 \pm 1$ \\
\hline C.25 & 70 & $18 \pm 1$ & $24 \pm 1$ & $22 \pm 9$ & $36 \pm 8$ \\
\hline C.26 & 45 & $29 \pm 4$ & $21 \pm 1$ & $14 \pm 1$ & $36 \pm 3$ \\
\hline
\end{tabular}

Data are the average $( \pm S D)$ of three experiments. 
Next, the effect of carbamates on the microtubule cytoskeleton was studied. Therefore, A549 cells were incubated for $16 \mathrm{~h}$ in the presence of AmCA-4 and C.1-C.26 at concentrations twice their $\mathrm{IC}_{50}$ value. Figure 4.14 depicts some selected results, where it can be appreciated that in the presence of AmCA-4 and carbamates, tubulin appears agregated and nuclei are compressed and fragmented, which is characteristic of cells that have been disturbed during their division process.
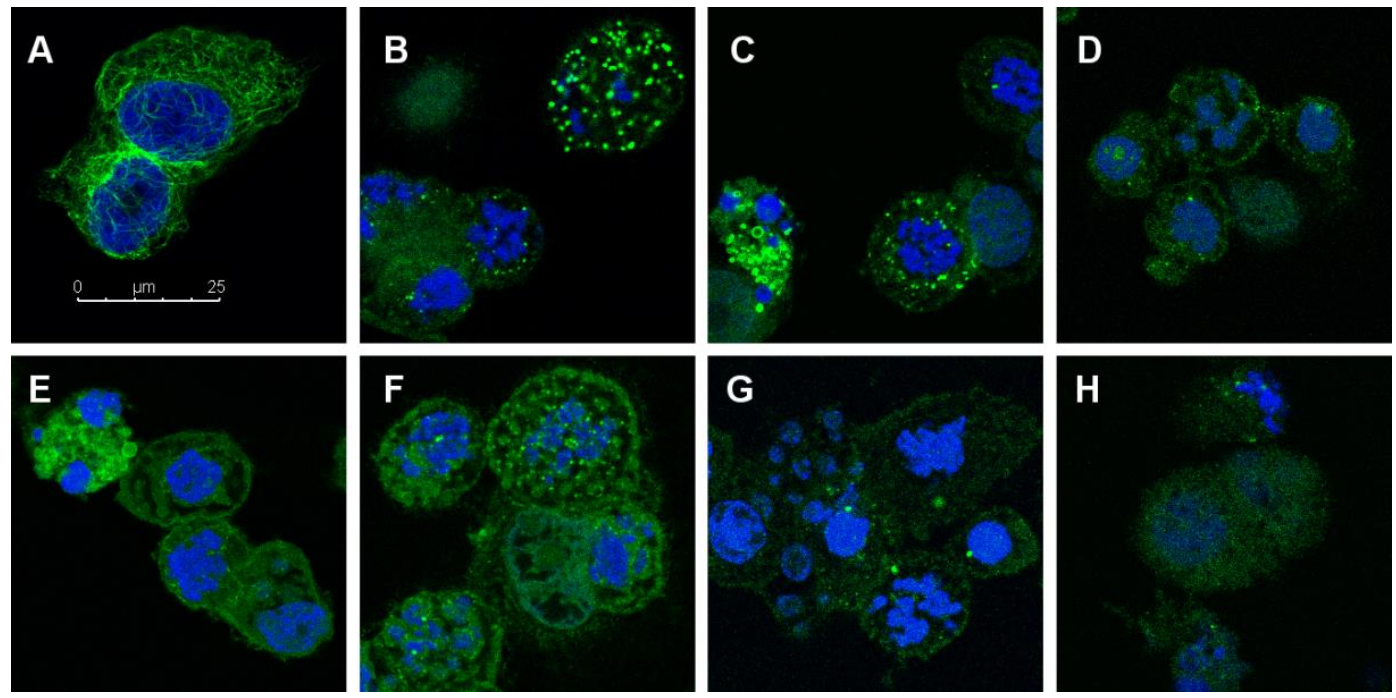

Figure 4.14. Effects of AmCA-4 and some selected carbamates on the microtubule network. A549 cells were treated for $16 \mathrm{~h}$ and processed for immunofluorescence microscopy. (A) DMSO, (B) 230 nM AmCA-4, (C) 150 nM C.1, (D) 300 nM C.3, (E) 260 nM C.5, (F) 200 nM C.6, (G) 220 nM C.17 and (H) 260 nM C.18.

\subsubsection{Induction of apoptosis}

Cell cycle distribution (see table 4.6) clearly shows that the percentage of subG $\mathrm{G}_{0}$ cells is increased in cells treated with CA-4, AmCA-4 and carbamates. This exhibition of a sub-diploid DNA content, together with the high percentage of cells arrested in mitotic phase, could be characteristic of apoptosis. Consequently, induction of apoptosis was studied by measuring the translocation of phosphatidylserine from the cytoplasmic to the extracellular side of the plasma membrane. Thus, A549 cells were incubated for $20 \mathrm{~h}$ in the presence of AmCA-4 and some representative carbamates, after which annexin 
$\mathrm{V}$ content was measured by flow cytometry. Carbamates which displayed the best antiproliferative activity were selected for this assay.

From figure 4.15 it can be concluded that carbamates induce apoptosis at both concentrations tested. With exception of compound C.26, all of them are more active than AmCA-4 at 100 nM. Moreover, compounds C.9 and C.12 show a similar effect to AmCA-4 at $10 \mathrm{nM}$.

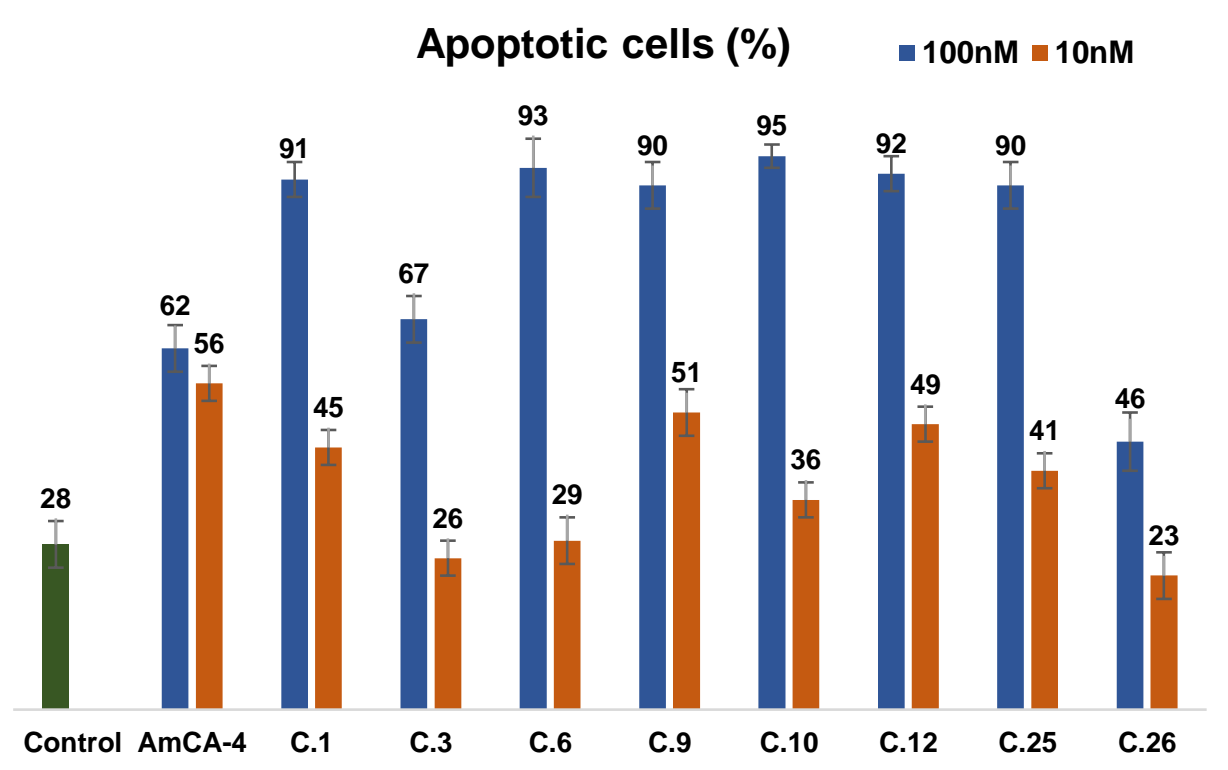

Figure 4.15. Apoptotic effect of AmCA-4 and carbamates. Data are the average ( $\pm S D)$ of three experiments.

\subsubsection{Tube destruction evaluation}

Vascular disrupting agents do not only affect tumor cells but also endothelial cells. Thus, the capacity of synthetic carbamates to destroy a pre-existing vasculature network formed by endothelial cells was evaluated. Therefore, cells were seeded on top of Matrigel $\AA^{\circledR}$, which induced the formation of a network of endothelial tubes. Then, the cultures were treated with different concentrations of AmCA-4 or carbamates and pictures were taken $4 \mathrm{~h}$ later in order to evaluate the tube destruction effect. Carbamates with the best antiproliferative activity in HMEC-1 cells (C.10, C.12 and C.25) were selected for this assay. Additionally, 
two less active carbamates (C.11 and $\mathbf{C . 1 3}$ ) were tested in order to establish a correlation between antiproliferative capacity and tube destruction. As shown in figure 4.16, all tested compounds displayed a vascular disrupting activity in a dose-dependent manner. Concretely, C.10, C.12 and C.25 exhibited this property at concentrations higher than $3 \mathrm{nM}$, improving the effect manifested by AmCA-4 and being about 10-fold more active than C.13 and 100-fold more active than $\mathbf{C . 1 1}$. Therefore, the higher the antiproliferative activity, the greater the capacity for tube destruction.

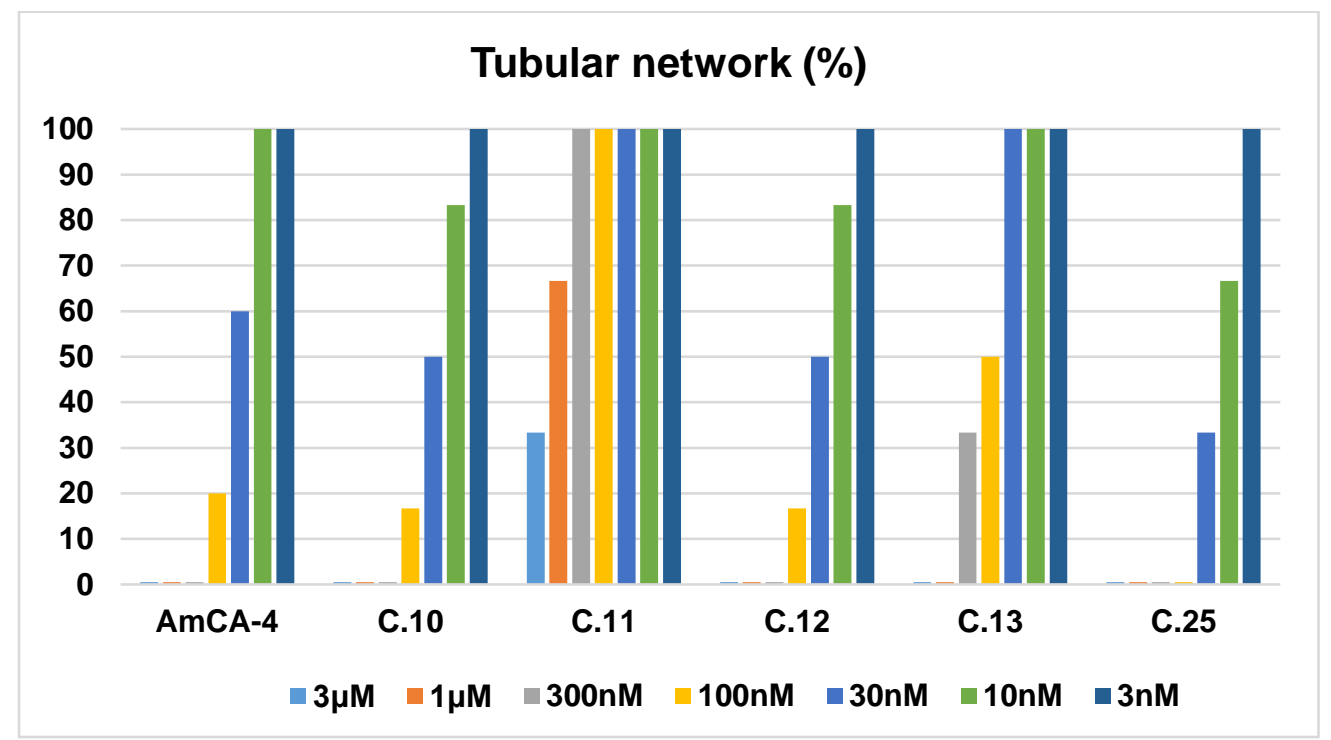

Figure 4.16. Percentage of tubular network in respect to control (DMSO, 100\%).

Images obtained for carbamate C.25 are shown in figure 4.17. This compound was able to totally destroy the vascular network at $100 \mathrm{nM}$, whereas in the presence of AmCA-4, or the other carbamates, part of the vascular network remained still intact. 

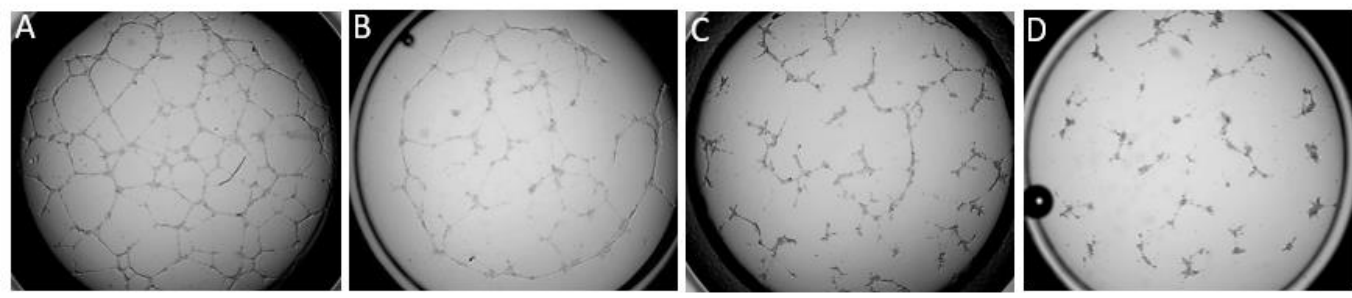

Figure 4.17. Effect of C.25 on the tubular network. (A) $3 \mathrm{nM}$, (B) $10 \mathrm{nM}$, (C) $30 \mathrm{nM}$ and (D) $100 \mathrm{nM}$.

\subsubsection{Conclusions of biological evaluation of family 1}

Carbamate derivatives offer $I \mathrm{C}_{50}$ values in the nanomolar range, improving by far the activity of CA-4 and, in some cases, the activity of AmCA-4. For instance, derivatives C.6 $(m-\mathrm{Cl}), \mathbf{C . 9}(m-\mathrm{Br}), \mathbf{C . 1 0}(p-\mathrm{Br})$ and C.12 ( $m-\mathrm{OMe})$ exceeded the activity of AmCA-4 in HT-29, MCF-7 and HMEC-1 cell lines and also offered a good selectivity over non-tumor cell lines. From the structures of these compounds it can be concluded that the most active carbamates are the ones bearing chloro, bromo or methoxy groups in the meta position of the phenyl ring.

In addition, all the carbamates proved as potent as CA-4 and AmCA-4 in inhibiting in vitro tubulin polymerization. It was also demonstrated that carbamates interact with tubulin at the colchicine-binding site and that the compounds with higher antiproliferative activity (C.10 ( $p-\mathrm{Br}), \mathbf{C . 1 2}(m-\mathrm{OMe})$ and C.25 ( $m$-Me- $p-\mathrm{Cl}))$ also show greater tubulin binding capacity.

From cell cycle analysis and immunofluorescence studies, it can be deduced that carbamates cause a mitotic arrest in A549 cells causing nuclei fragmentation and tubulin aggregation. Moreover, carbamates induced apoptosis in a dose-dependent manner, with more than $90 \%$ of apoptotic cells at $100 \mathrm{nM}$ dose. Compounds $\mathbf{C . 9}(m-\mathrm{Br})$ and $\mathbf{C . 1 2}(m-\mathrm{OMe})$ proved as active as AmCA-4 even at a concentration of $10 \mathrm{nM}$.

Finally, some selected carbamates displayed a vascular disrupting activity of endothelial cells in a dose-dependent manner which correlated with their 
antiproliferative activity. Thus, highly anti-proliferative carbamates C.10 ( $p-\mathrm{Br})$, C.12 ( $m$-OMe) and C.25 ( $m$-Me- $p$-Cl) were able to disrupt tubular network at concentrations higher than $3 \mathrm{nM}$, improving the effect shown by AmCA-4. 


\subsection{Biological evaluation of family 2 as antiangiogenics}

\subsubsection{Inhibition of cell proliferation}

The ability of compounds U.1-U.26 to inhibit cell proliferation was established by means of their $\mathrm{IC}_{50}$ values towards the human tumor cell lines HT-29, MCF7, HeLa, A549 and HL-60, as well as towards the endothelial cell line HMEC-1 and the non-tumor cell line HEK-293. The $\mathrm{IC}_{50}$ values are presented in table 4.7 along with the values for the reference compounds CA-4, AmCA-4, sunitinib and sorafenib ${ }^{165}$.

Table 4.7. I $\mathrm{C}_{50}$ values $(\mu \mathrm{M})$ for $\mathrm{CA}-4$, AmCA-4 $(\mathrm{nM})$, sunitinib, sorafenib and U.1-U.26. ${ }^{a}$

\begin{tabular}{llllllll}
\hline Comp. & HT-29 & MCF-7 & HeLa & A549 & HL-60 & HMEC-1 & HEK-293 \\
\hline CA-4 & $4.2 \pm 0.5$ & $1.0 \pm 0.2$ & $2.1 \pm 0.6$ & $0.13 \pm 0.02$ & $4 \pm 1$ & $3.4 \pm 0.4$ & $25.0 \pm 0.3$ \\
AmCA-4 & $22.0 \pm 0.7$ & $8.0 \pm 0.9$ & $2.6 \pm 0.5$ & $117 \pm 7$ & $4.5 \pm 0.9$ & $12 \pm 6$ & $7.1 \pm 1.0$ \\
Sunitinib & $3.8 \pm 0.5$ & $0.08 \pm 0.02$ & $7.8 \pm 1.0$ & $11 \pm 2$ & $7 \pm 3$ & $7 \pm 4$ & $5.0 \pm 0.8$ \\
Sorafenib & $17 \pm 4$ & $14 \pm 4$ & $6.1 \pm 0.4$ & $27 \pm 2$ & $5 \pm 1$ & $34 \pm 3$ & $5.0 \pm 0.7$ \\
U.1 & $7.0 \pm 1.0$ & $3.5 \pm 0.6$ & $2.15 \pm 0.07$ & $12 \pm 3$ & $10.8 \pm 0.4$ & $80 \pm 30$ & $5.0 \pm 0.1$ \\
U.2 & $9.2 \pm 0.6$ & $9.0 \pm 0.5$ & $1.52 \pm 0.02$ & $5.8 \pm 0.9$ & $4.5 \pm 0.1$ & $1.6 \pm 0.8$ & $1.3 \pm 0.3$ \\
U.3 & $2.7 \pm 0.4$ & $1.6 \pm 0.3$ & $1.08 \pm 0.04$ & $12 \pm 3$ & $11.3 \pm 0.2$ & $40 \pm 20$ & $5.03 \pm 0.03$ \\
U.4 & $5.0 \pm 1.0$ & $1.26 \pm 0.03$ & $0.87 \pm 0.06$ & $7 \pm 3$ & $8 \pm 2$ & $1.4 \pm 0.3$ & $1.61 \pm 0.01$ \\
U.5 & $0.51 \pm 0.06$ & $0.4 \pm 0.2$ & $0.38 \pm 0.01$ & $4.4 \pm 1.0$ & $0.3 \pm 0.1$ & $3 \pm 2$ & $0.3 \pm 0.2$ \\
U.6 & $2.3 \pm 1.0$ & $6.9 \pm 0.2$ & $2.6 \pm 0.4$ & $8 \pm 3$ & $2.0 \pm 0.6$ & $102 \pm 2$ & $2.1 \pm 0.2$ \\
U.7 & $4.0 \pm 0.5$ & $3.2 \pm 0.5$ & $2.9 \pm 0.3$ & $22 \pm 14$ & $9.5 \pm 0.1$ & $13 \pm 9$ & $5.8 \pm 0.1$ \\
U.8 & $0.12 \pm 0.01$ & $0.05 \pm 0.03$ & $0.3 \pm 0.1$ & $1.2 \pm 0.2$ & $0.2 \pm 0.1$ & $0.30 \pm 0.02$ & $0.07 \pm 0.01$ \\
U.9 & $1.0 \pm 0.2$ & $0.67 \pm 0.09$ & $0.45 \pm 0.06$ & $5.4 \pm 0.8$ & $1.2 \pm 0.1$ & $2.0 \pm 1.0$ & $0.9 \pm 0.2$ \\
U.10 & $10.0 \pm 0.5$ & $5.4 \pm 1.6$ & $4.5 \pm 0.7$ & $16 \pm 4$ & $10.5 \pm 0.4$ & $13 \pm 9$ & $9.3 \pm 0.7$ \\
U.11 & $0.6 \pm 0.2$ & $0.33 \pm 0.05$ & $0.26 \pm 0.02$ & $5 \pm 3$ & $0.7 \pm 0.1$ & $1.2 \pm 0.3$ & $0.4 \pm 0.1$ \\
U.12 & $0.9 \pm 0.5$ & $1.56 \pm 0.02$ & $0.55 \pm 0.03$ & $6 \pm 3$ & $1.8 \pm 0.6$ & $3.5 \pm 0.9$ & $1.2 \pm 0.4$
\end{tabular}

165 Sunitinib was commercially available. Sorafenib was synthetized following the experimental procedure described in Bankston, D.; Dumas, J.; Natero, R.; Riedl, B.; Monahan, M-K.; Sibley, R. Org. Proc. Res. Dev. 2002, 6, 777-781. 


\begin{tabular}{llllllll} 
U.13 & $1.78 \pm 0.08$ & $1.5 \pm 0.2$ & $1.4 \pm 0.7$ & $8.3 \pm 1.0$ & $2.4 \pm 0.3$ & $2.2 \pm 1.0$ & $0.7 \pm 0.2$ \\
U.14 & $0.57 \pm 0.01$ & $0.45 \pm 0.06$ & $0.30 \pm 0.01$ & $5.0 \pm 1.0$ & $0.4 \pm 0.1$ & $0.9 \pm 0.3$ & $0.34 \pm 0.02$ \\
U.15 & $1.10 \pm 0.05$ & $0.7 \pm 0.2$ & $0.67 \pm 0.08$ & $9 \pm 2$ & $2.2 \pm 0.5$ & $4 \pm 2$ & $0.24 \pm 0.05$ \\
U.16 & $0.5 \pm 0.2$ & $0.50 \pm 0.01$ & $0.29 \pm 0.01$ & $4.7 \pm 0.7$ & $0.9 \pm 0.2$ & $4 \pm 3$ & $0.30 \pm 0.02$ \\
U.17 & $0.2 \pm 0.1$ & $0.17 \pm 0.01$ & $0.12 \pm 0.01$ & $2.0 \pm 0.2$ & $0.26 \pm 0.01$ & $0.3 \pm 0.1$ & $0.20 \pm 0.3$ \\
U.18 & $4.6 \pm 0.9$ & $4.2 \pm 0.6$ & $3.2 \pm 0.8$ & $28.7 \pm 0.5$ & $8.8 \pm 0.9$ & $13 \pm 7$ & $2.9 \pm 0.3$ \\
U.19 & $5.3 \pm 0.1$ & $3.9 \pm 1.1$ & $4.5 \pm 0.4$ & $27 \pm 2$ & $5.5 \pm 0.4$ & $5.1 \pm 0.1$ & $6 \pm 2$ \\
U.20 & $0.6 \pm 0.2$ & $0.8 \pm 0.2$ & $0.34 \pm 0.09$ & $12 \pm 5$ & $2.8 \pm 0.8$ & $7 \pm 3$ & $0.3 \pm 0.1$ \\
U.21 & $4.4 \pm 0.7$ & $3.3 \pm 0.5$ & $3.4 \pm 0.6$ & $24 \pm 9$ & $4.4 \pm 1.0$ & $60 \pm 20$ & $3.5 \pm 0.3$ \\
U.22 & $0.30 \pm 0.01$ & $0.85 \pm 0.03$ & $0.17 \pm 0.05$ & $9 \pm 2$ & $1.67 \pm 0.03$ & $5 \pm 4$ & $0.21 \pm 0.03$ \\
U.23 & $2.4 \pm 0.3$ & $2.6 \pm 0.5$ & $1.6 \pm 0.2$ & $14 \pm 3$ & $1.8 \pm 0.4$ & $6 \pm 5$ & $1.87 \pm 0.01$ \\
U.24 & $0.54 \pm 0.05$ & $0.32 \pm 0.09$ & $0.30 \pm 0.05$ & $3.3 \pm 0.3$ & $0.7 \pm 0.5$ & $1.1 \pm 0.6$ & $0.25 \pm 0.04$ \\
U.25 & $3.5 \pm 0.2$ & $2.7 \pm 0.5$ & $2.9 \pm 0.3$ & $22 \pm 8$ & $2.4 \pm 1.2$ & $7.4 \pm 1.0$ & $3.4 \pm 0.4$ \\
U.26 & $2.4 \pm 0.3$ & $3.65 \pm 0.09$ & $1.5 \pm 0.4$ & $20 \pm 8$ & $3 \pm 2$ & $9 \pm 2$ & $1.6 \pm 0.1$ \\
\hline
\end{tabular}

${ }^{\text {a }} \mathrm{C}_{50}$ values are expressed as the compound concentration that inhibits the cell growth by $50 \%$. Data are the average $( \pm S D)$ of three experiments.

Some trends can be deduced from table 4.7. The majority of the compounds show activity in the low micromolar range in all cell lines, with U.5, U.8, U.11, U.14, U.16, U.17, U.20, U.22 and U.24 showing $\mathrm{IC}_{50}$ values at submicromolar level. Any synthetic urea exceeds the antiproliferative activity shown by AmCA4, although their antiproliferative action is comparable with that of sunitinib and sorafenib. Interestingly, the most active ureas, that have even higher antiproliferative action than sunitinib, show in all cases ortho substitution. As regards the therapeutic safety margin, U.1, U.3, U.4, U.7, U.9, U.10, U.11, U.12, U.17, U.19 and U.25 show greater selectivity in MCF-7 and HeLa when compared to HEK-293. In endothelial cells (HMEC-1), ureas with the best antiproliferative action were U.5 (o-chloro), U.8 (o-bromo), U.11 (o-methoxy), U.14 (o-methyl), U.17 (o-trifluormethyl) and U.24 (o-methyl-m-chloro), all of them showing ortho substituents. 


\subsubsection{Docking studies}

Before starting the anti-VEGFR-2 evaluation of these urea derivatives, docking studies were carried out in order to find the compounds that bind more strongly to the VEGFR-2 kinase in a similar way to sorafenib. Thus, Autodock $4.2^{166}$ was used to perform molecular docking calculations employing the crystal structure of VEGFR-2 kinase domain (PDB ID: 3EWH) as a template. Sorafenib and compounds U.1-U.26 were modelled (see table 4.8). These docking experiments suggested that most of the derivatives exhibit conformations close to that of sorafenib. As an example, figures 4.18 and 4.19 show superimposition of sorafenib and compounds U.5, U.8, U.21 and U.23. The docking score, based on free energy of binding, is also included in table 4.8. All compounds showed at least three hydrogen bond interactions with at least two of the amino acids Lys868, Glu885, Glu917, Cys919 and Asp1046. As an example, figure 4.20 shows the hydrogen bonds formed by sorafenib and U.5, U.8, U.21 and U.23 at the VEGFR-2 kinase domain. Top ranked 10 compounds (U.5-U.9, U.15, U.21, U.23-U.25), showing at least four hydrogen bonds, were selected to go on further in vitro studies described below.

\footnotetext{
${ }^{166}$ Morris, G. M.; Huey, R.; Lindstrom, W.; Sanner, M. F.; Belew, R. K.; Goodsell, D. S.; Olson, A.
} J. J. Comput Chem. 2009, 30, 2785-2791. 
Table 4.8. Docking analysis of derivatives U.1-U.26.

\begin{tabular}{|c|c|c|c|c|}
\hline Compound & Ranked & $\begin{array}{l}\text { Binding energy } \\
\text { (kcal/mol) }\end{array}$ & $\mathrm{Ki}(\mathrm{nM})$ & H-bonding \\
\hline Sorafenib & - & -10.75 & 13.13 & $\begin{array}{l}\text { Cys919, Asp1046, } \\
\text { Glu885, Glu885 }\end{array}$ \\
\hline U.1 & 17 & -10.66 & 15.31 & $\begin{array}{l}\text { Glu885, Glu885, Lys868, } \\
\text { Asp1046 }\end{array}$ \\
\hline $\mathrm{U} .2$ & 23 & -10.38 & 24.6 & $\begin{array}{l}\text { Glu885, Glu885, Lys868, } \\
\text { Asp1046 }\end{array}$ \\
\hline U.3 & 22 & -10.54 & 18.9 & $\begin{array}{l}\text { Glu885, Glu885, Lys868, } \\
\text { Asp1046 }\end{array}$ \\
\hline U.4 & 19 & -10.62 & 16.36 & $\begin{array}{l}\text { Glu885, Glu885, Lys868, } \\
\text { Asp1046 }\end{array}$ \\
\hline U.5 & 10 & $-11,1$ & 7.24 & $\begin{array}{l}\text { Glu885, Glu885, Lys868, } \\
\text { Asp1046 }\end{array}$ \\
\hline $\mathrm{U} .6$ & 4 & -11.32 & 5.07 & $\begin{array}{l}\text { Glu885, Glu885, Lys868, } \\
\text { Asp1046 }\end{array}$ \\
\hline U.7 & 9 & -11.10 & 7.23 & $\begin{array}{l}\text { Glu885, Glu885, Lys868, } \\
\text { Asp1046 }\end{array}$ \\
\hline U.8 & 8 & -11.10 & 7.26 & $\begin{array}{l}\text { Glu885, Glu885, Lys868, } \\
\text { Asp1046 }\end{array}$ \\
\hline U.9 & 3 & -11.43 & 4.21 & $\begin{array}{l}\text { Glu885, Glu885, Lys868, } \\
\text { Asp1046 }\end{array}$ \\
\hline U.10 & 16 & -10.69 & 14.52 & Glu885, Glu885, Lys868 \\
\hline U.11 & 24 & -10.35 & 26.04 & $\begin{array}{l}\text { Glu885, Glu885, } \\
\text { Cys919, Asp1046 }\end{array}$ \\
\hline U.12 & 15 & -10.87 & 10.79 & $\begin{array}{l}\text { Glu885, Glu885, Lys868, } \\
\text { Asp1046 }\end{array}$ \\
\hline U.13 & 21 & -10.6 & 16.95 & $\begin{array}{l}\text { Glu885, Glu885, Lys868, } \\
\text { Asp1046 }\end{array}$ \\
\hline U.14 & 18 & -10.63 & 16.12 & Glu885, Glu885, Cys919 \\
\hline U.15 & 6 & -11.29 & 5.28 & $\begin{array}{l}\text { Glu885, Glu885, } \\
\text { Cys919, Asp1046 }\end{array}$ \\
\hline U.16 & 11 & -11.09 & 7.39 & Glu885, Glu885, Cys919 \\
\hline U.17 & 20 & -10.62 & 16.33 & Glu885, Glu885, Cys919 \\
\hline U.18 & 25 & -10.13 & 37.27 & Glu885, Glu885, Cys919 \\
\hline U.19 & 26 & -10.06 & 42.24 & Glu885, Glu885, Cys919 \\
\hline
\end{tabular}




\begin{tabular}{|c|c|c|c|c|}
\hline U.20 & 10 & -11.09 & 7.32 & $\begin{array}{l}\text { Glu885, Glu885, } \\
\text { Cys919, Asp1046 }\end{array}$ \\
\hline U.21 & 2 & -11.53 & 3.56 & $\begin{array}{l}\text { Glu885, Glu885 Cys919, } \\
\text { Asp1046 }\end{array}$ \\
\hline U.22 & 13 & -11.03 & 8.28 & Glu885, Glu885, Cys919 \\
\hline U.23 & 1 & -11.90 & 1.89 & $\begin{array}{l}\text { Glu885, Glu885, Lys868, } \\
\text { Cys919, Asp1046 }\end{array}$ \\
\hline U.24 & 7 & -11.19 & 6.31 & $\begin{array}{l}\text { Glu885, Glu885, } \\
\text { Cys919, Asp1046 }\end{array}$ \\
\hline U.25 & 5 & -11.3 & 5.18 & $\begin{array}{l}\text { Glu885, Glu885, } \\
\text { Cys919, Asp1046 }\end{array}$ \\
\hline U.26 & 12 & -11.05 & 7.73 & $\begin{array}{l}\text { Glu885, Glu885, } \\
\text { Cys919, Asp1046 }\end{array}$ \\
\hline
\end{tabular}

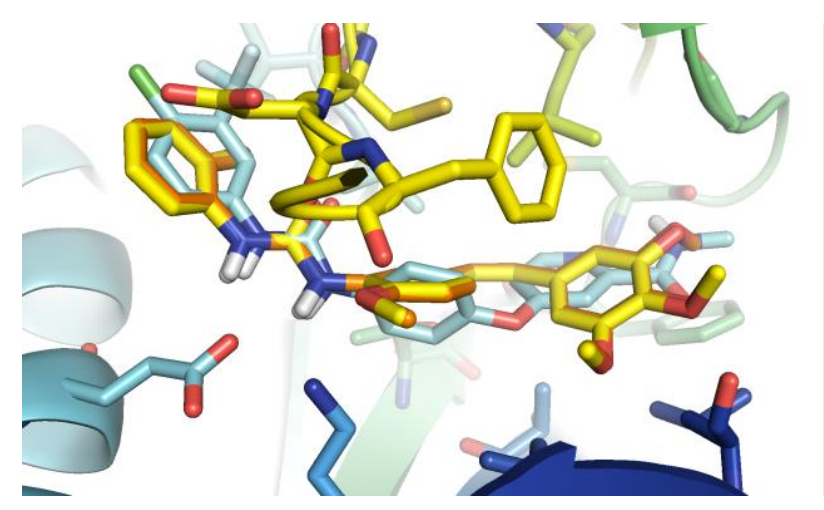

Figure 4.18. Superimposition of modelized sorafenib (light blue), U.5 (orange) and U.8 (yellow) at the VEGFR-2 kinase binding site.

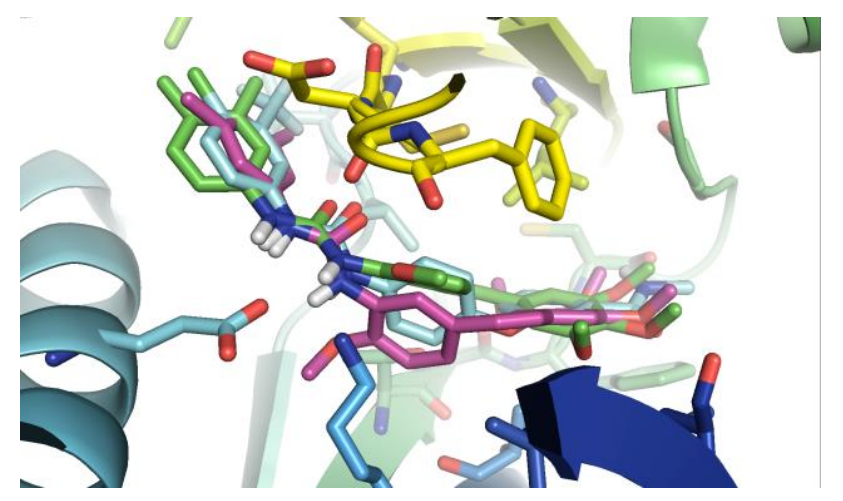

Figure 4.19. Superimposition of modelized sorafenib (light blue), U.21 (purple) and U.23 (green) at the VEGFR-2 kinase binding site. 

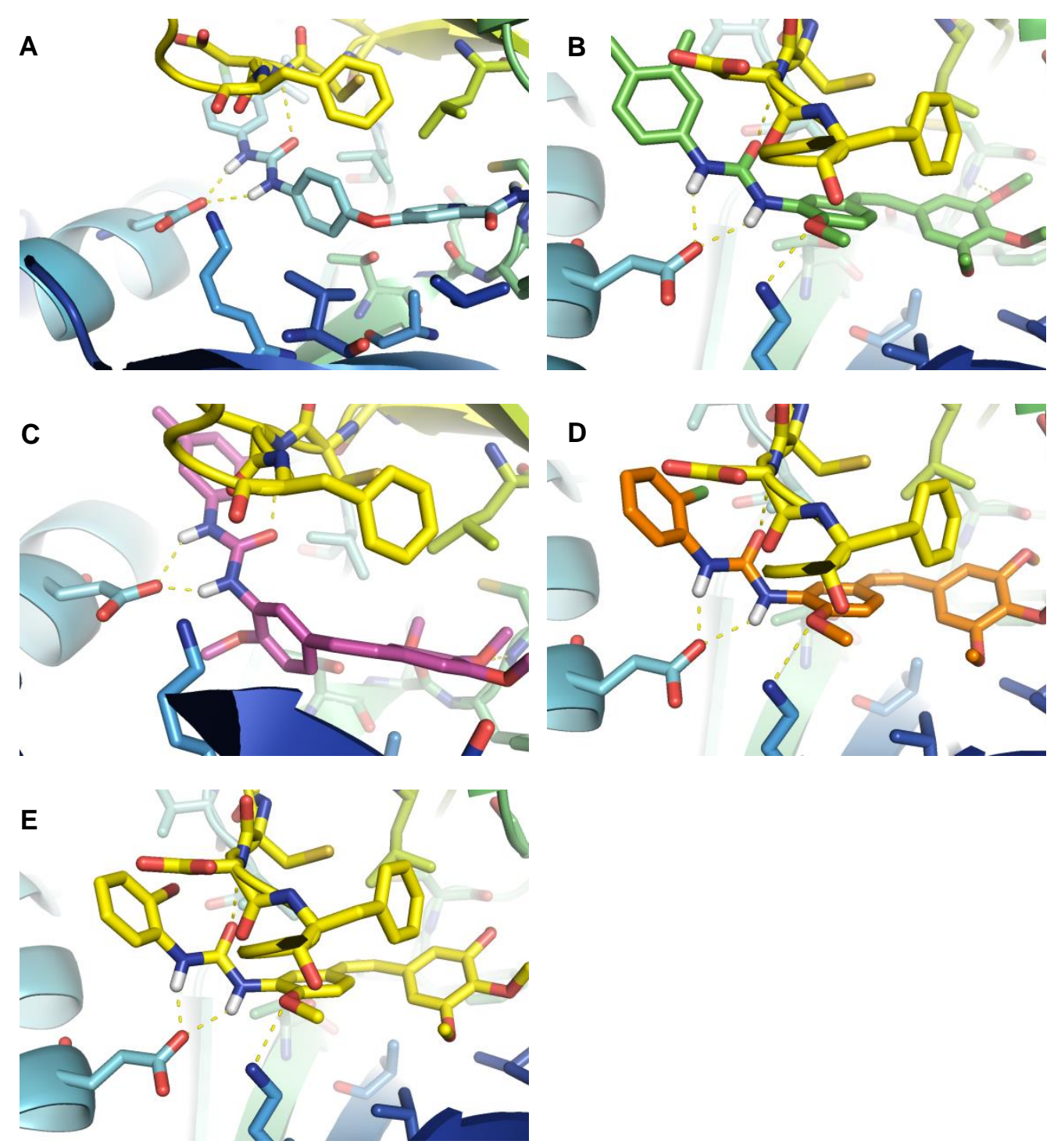

Figure 4.20. Modelized structures at the VEGFR-2 kinase binding site and their $\mathrm{H}$-bond interactions. (A) sorafenib (light blue), (B) U.23 (green), (C) U.21 (purple), (D) U.5 (orange) and (E) U.8 (yellow). 


\subsubsection{Mitotic arrest and inhibition of interphase microtubules}

The effect of ureas on the cell cycle was evaluated on A549 cells. Thus, cells were incubated for $20 \mathrm{~h}$ in the presence of CA-4, AmCA-4, sunitinib, sorafenib and selected ureas, and then, the DNA content was measured by flow cytometry (see table 4.9).

Table 4.9. Cell cycle distribution (\%).

\begin{tabular}{llllll}
\hline Comp. & Conc. $(\mu \mathrm{M})$ & $\mathrm{SubG}_{0}$ & $\mathrm{G}_{0} / \mathrm{G}_{1}$ & $\mathrm{~S}$ & $\mathrm{G}_{2} / \mathrm{M}$ \\
\hline Control & - & $2.0 \pm 0.8$ & $60 \pm 1$ & $28.0 \pm 0.6$ & $10.0 \pm 0.8$ \\
CA-4 & 0.2 & $15.0 \pm 0.3$ & $21 \pm 2$ & $22 \pm 1$ & $43.0 \pm 0.5$ \\
AmCA-4 & 0.23 & $14.0 \pm 0.6$ & $21 \pm 1$ & $24.0 \pm 0.4$ & $41 \pm 2$ \\
Sunitinib & 22 & $2.4 \pm 0.6$ & $47 \pm 1$ & $35.0 \pm 0.4$ & $16 \pm 2$ \\
Sorafenib & 54 & $3.1 \pm 0.3$ & $46.8 \pm 0.4$ & $31 \pm 1$ & $19.4 \pm 0.6$ \\
U.5 & 8.8 & $16 \pm 2$ & $12.0 \pm 0.3$ & $29.0 \pm 0.5$ & $44 \pm 2$ \\
U.6 & 16 & $18 \pm 3$ & $18.0 \pm 1$ & $30.0 \pm 0.5$ & $34.0 \pm 0.6$ \\
U.7 & 44 & $23.0 \pm 0.5$ & $12.0 \pm 0.6$ & $25 \pm 2$ & $40 \pm 3$ \\
U.8 & 2.4 & $46 \pm 3$ & $5 \pm 3$ & $11 \pm 1$ & $37.0 \pm 0.4$ \\
U.9 & 10.8 & $23.0 \pm 0.6$ & $12.0 \pm 0.3$ & $25 \pm 5$ & $41.0 \pm 0.8$ \\
U.15 & 18 & $24 \pm 3$ & $12 \pm 2$ & $24.0 \pm 0.2$ & $40 \pm 5$ \\
U.21 & 48 & $21.0 \pm 0.4$ & $17 \pm 1$ & $25 \pm 3$ & $37 \pm 3$ \\
U.23 & 28 & $33 \pm 3$ & $7 \pm 1$ & $19.0 \pm 0.4$ & $41 \pm 4$ \\
U.24 & 6.6 & $30 \pm 1$ & $10 \pm 1$ & $22 \pm 2$ & $38.0 \pm 0.5$ \\
U.25 & 44 & $20.0 \pm 0.6$ & $15 \pm 2$ & $27.0 \pm 0.4$ & $39 \pm 3$ \\
\hline
\end{tabular}

Data are the average $( \pm S D)$ of three experiments.

Data from table 4.9 show that sunitinib and sorafenib behave in a similar way to control, that is, they do not affect the cell division process. On the contrary, all the urea tested act in the same manner as CA-4 and AmCA-4 causing an extensive arrest of cells in the $\mathrm{G}_{2} / \mathrm{M}$ phase and also an increase in the percentage of cells in subG 0 phase at concentrations twice of their $\mathrm{IC}_{50}$ values. 
Next, the effect of ureas on the microtubule cytoskeleton was studied by immunofluorescence microscopy. Therefore, A549 cells were incubated for 16 $\mathrm{h}$ in the presence of AmCA-4 and ureas at concentration twice their $\mathrm{IC}_{50}$ value. Figure 4.21 shows the effect of some selected compounds, where it can be observed that in the presence of AmCA-4 or ureas tubulin becomes aggregated and nuclei are compressed and fragmented, as usually seen in cells that have been disturbed during their division process. The effect of sunitinib and sorafenib on the microtubule cytoskeleton has not been studied since the previous results of cell cycle distribution showed that both of them act in a similar way to control and do not have antimitotic effect.
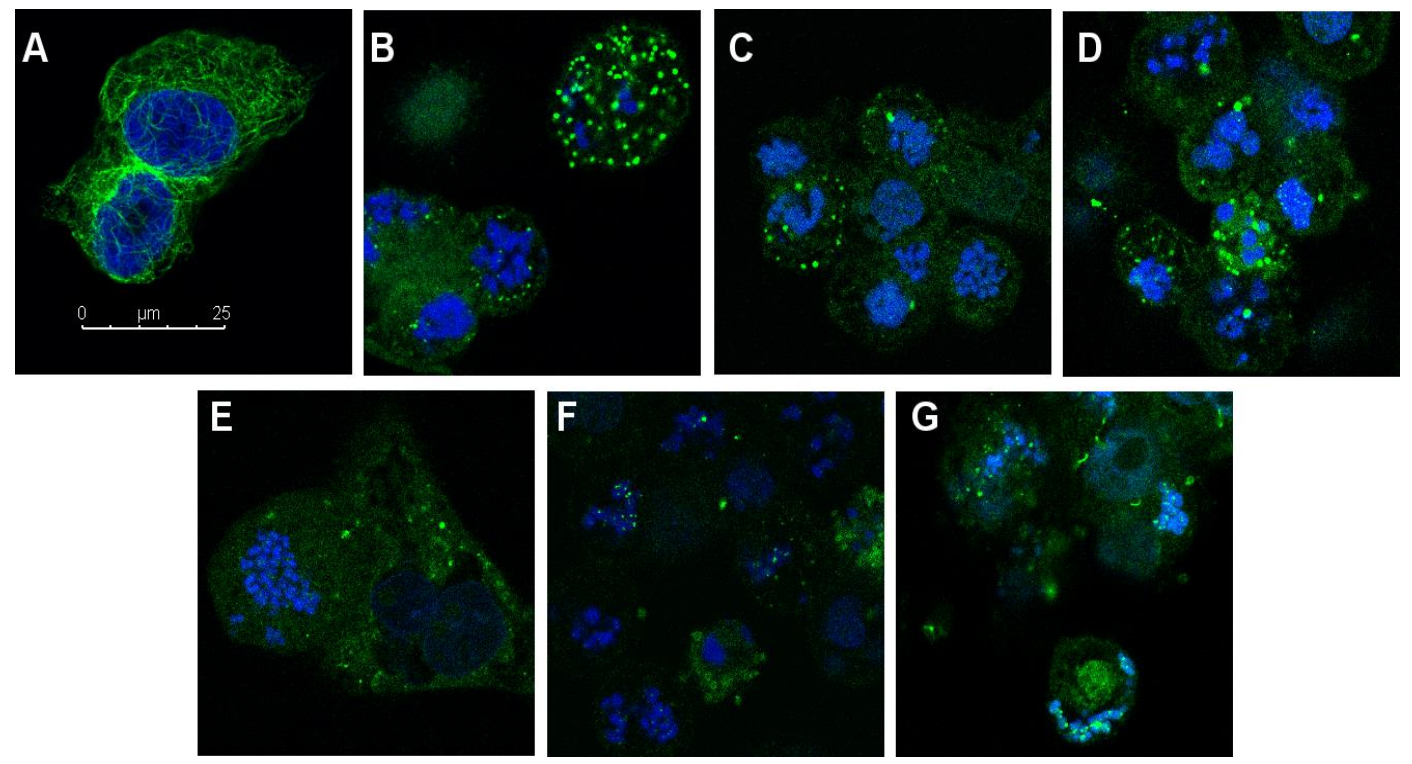

Figure 4.21. Effects of some selected ureas on the microtubule network. (A) DMSO, (B) $0.23 \mu \mathrm{M}$ AmCA-4, (C) $2.4 \mu \mathrm{M}$ U.8, (D) $10.8 \mu \mathrm{M}$ U.9, (E) $18 \mu \mathrm{M}$ U.15, (F) $28 \mu \mathrm{M}$ U.23 and (G) $6.6 \mu \mathrm{M}$ U.24.

\subsubsection{Induction of apoptosis}

Cell cycle distribution (see table 4.9) clearly shows that the percentage of subG $G_{0}$ cells is increased in cells treated with AmCA-4, sunitinib, sorafenib and ureas. Consequently, induction of apoptosis was studied by measuring the translocation of phosphatidylserine from the cytoplasmic to the extracellular side 
of the plasma membrane. Thus, A549 cells were incubated for $20 \mathrm{~h}$ in the presence of sunitinib, sorafenib and ureas, after which annexin $V$ content was measured by flow cytometry. The apoptotic effect is indicated in figure 4.22.

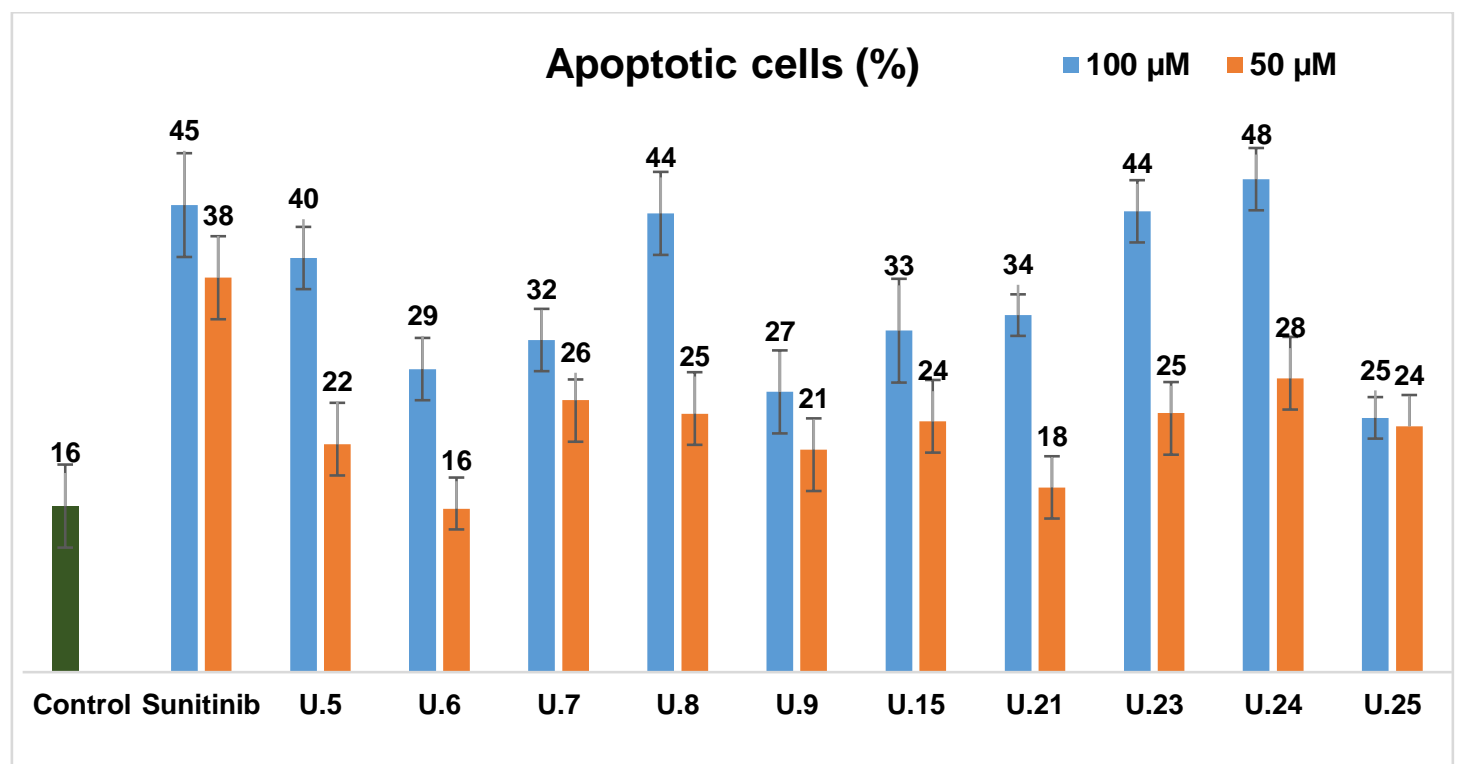

Figure 4.22. Apoptotic effect. Data are the average $( \pm S D)$ of three experiments.

It can be concluded from figure 4.22 that the majority of assayed ureas induce apoptosis at both concentrations tested. Moreover, U.5 (o-chloro), U.8 (o-bromo), U.23 (m,p-dimethyl) and U.24 (o-methyl-m-chloro) show a similar effect to sunitinib at $100 \mu \mathrm{M}$.

\subsubsection{Tube formation inhibition}

Antiangiogenic agents mainly affect endothelial cells, thus the capacity of synthetic ureas to inhibit the formation of new vasculature network formed by endothelial cells was evaluated. Therefore, HMEC-1 cells were seeded on top of Matrige|ß and simultaneously treated with different concentrations of ureas. Pictures were taken $20 \mathrm{~h}$ later in order to evaluate the tube formation inhibition effect. Table 4.10 shows the minimum concentration at which ureas are active and begin to inhibit tube formation. 
Table 4.10. Tube formation inhibition effect.

\begin{tabular}{lll} 
Comp. & $\begin{array}{l}\text { IC } 50 \pm \text { SD } \\
\text { HMEC-1 }(\mu \mathrm{M})\end{array}$ & $\begin{array}{l}\text { Min. active } \\
\text { conc. }(\mu \mathrm{M})\end{array}$ \\
\hline AmCA-4 & $0.012 \pm 0.006$ & 0.003 \\
Sunitinib & $7 \pm 4$ & 3 \\
Sorafenib & $34 \pm 3$ & 10 \\
U.5 & $3 \pm 2$ & 0.1 \\
U.6 & $102 \pm 2$ & 1 \\
U.7 & $13 \pm 9$ & 3 \\
U.8 & $0.30 \pm 0.02$ & 0.1 \\
U.9 & $2.0 \pm 1.0$ & 0.3 \\
U.15 & $4 \pm 2$ & 1 \\
U.21 & $60 \pm 20$ & 3 \\
U.23 & $6 \pm 5$ & 3 \\
U.24 & $1.1 \pm 0.6$ & 0.3 \\
U.25 & $7.4 \pm 1.0$ & 3 \\
\hline
\end{tabular}

Data are representative of three experiments.

Comparing the minimum active concentration values with $\mathrm{IC}_{50}$ values for HMEC-1 cell line it can be concluded that there is a correlation between antiproliferative activity and tube formation inhibition capacity, since compounds with lower $I_{50}$ values exhibit tube formation inhibition activity at lower concentrations. Moreover, it is observed that some of the ureas tested are more active than sunitinib and sorafenib, particularly U.5 (o-chloro) and U.8 (o-bromo), which are 100-fold more active than sorafenib. It can be concluded from this table that these ureas inhibit neovascularization in a dose-dependent manner. Figure 4.23 depicts the percentage of vascular network for U.5 and U.8 at different concentrations. 


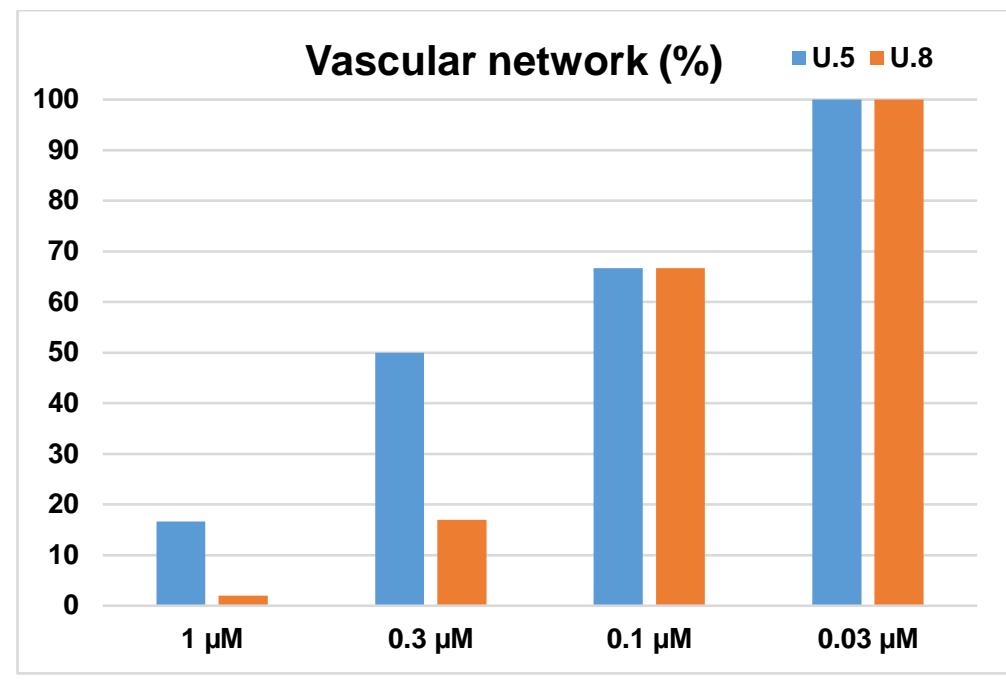

Figure 4.23. Percentage of vascular network in respect to control (DMSO, 100\%).

From figure 4.23 it is also deduced that $\mathbf{U . 8}$ is more active than $\mathbf{U . 5}$, since it is able to completely inhibit tube formation at $1 \mu \mathrm{M}$ concentration, whereas at this dose U.5 only inhibits $80 \%$ of the neovascularization process. Figure 4.24 displays pictures for the inhibition of neovascularization achieved by $\mathbf{U} . \mathbf{8}$ at different concentrations.

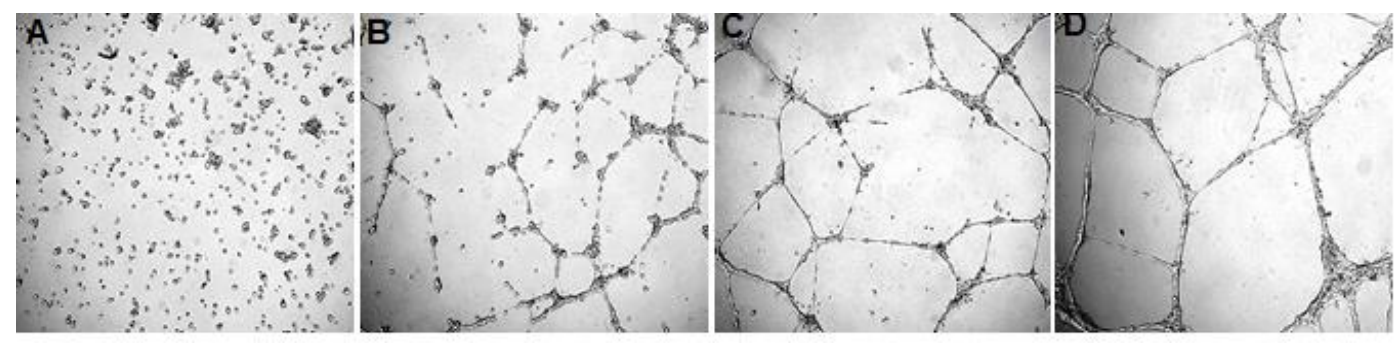

Figure 4.24. Effect of compound U.8 on tubular network. (A) $1 \mu \mathrm{M}$, (B) $0.3 \mu \mathrm{M}$, (C) 0.1 $\mu \mathrm{M}$ and (D) $0.03 \mu \mathrm{M}$. 


\subsubsection{Ligand-VEGFR-2 direct interaction}

In order to study whether the derivatives were able to interact with VEGFR2 , selected ureas were placed in contact with the receptor $(103 \mathrm{pg} / \mathrm{mL})$ at a concentration of $30 \mu \mathrm{M}$ and VEGFR-2 amount was quantified by ELISA test. The results are expressed in table 4.11 as the percentage of free VEGFR-2 detected with respect to control (DMSO, 100\%).

Table 4.11. Percentage of free VEGFR-2 detected.

\begin{tabular}{ll}
\hline Comp. & VEGFR-2 (\%) \\
\hline Sunitinib & $80 \pm 10$ \\
U.5 & $60 \pm 10$ \\
U.7 & $90 \pm 30$ \\
U.8 & $40 \pm 20$ \\
U.9 & $92 \pm 10$ \\
U.15 & $48 \pm 17$ \\
U.21 & $57 \pm 6$ \\
U.23 & $>100$ \\
U.24 & $>100$ \\
U.25 & $80 \pm 6$ \\
\hline
\end{tabular}

Data are the average $( \pm S D)$ of three experiments.

Compounds U.5 (o-chloro), U.8 (o-bromo), U.15 (m-methyl) and disubstituted derivative $\mathbf{U} . \mathbf{2 1}$ ( $m, m$-dimethyl) were the most active ones offering values of detected VEGFR-2 around $50 \%$ with respect to control. Again, ortho monohalogenated and meta methylated derivatives were more active than their corresponding analogues in the direct interaction to the selected target. 


\subsubsection{Effect on cellular VEGFR-2}

The effect of the selected compounds on VEGFR-2 in endothelial cells was determined by both flow cytometry and immunofluorescence technique. For these assays, cells were incubated for $24 \mathrm{~h}$ in the presence of the corresponding compounds at a concentration double their $\mathrm{IC}_{50}$ value. Then, cells were fixed with formaldehyde and treated with anti-VEGFR-2-alexafluor®647 to quantify membrane VEGFR-2 by flow cytometry. On the other hand, permeabilization of cells with Triton $\mathrm{X}-100$ prior to fixation step, allowed the quantification of total VEGFR-2 in cells by flow cytometry and immunofluorescence. Table 4.12 and figure 4.25 show the effect of the selected urea derivatives on VEGFR-2 expression and distribution in endothelial cells. The results in table 4.12 are expressed as the percentage of VEGFR-2 detected with respect to control (DMSO, $100 \%$ ).

Table 4.12. Effect of ureas on VEGFR-2 expression in HMEC-1 cells.

\begin{tabular}{llll}
\hline Comp. & Conc. $(\boldsymbol{\mu M})$ & $\begin{array}{l}\text { Membrane } \\
\text { VEGFR-2 }(\%)\end{array}$ & $\begin{array}{l}\text { Total } \\
\text { VEGFR-2 (\%) }\end{array}$ \\
\hline Sorafenib & 68 & $47 \pm 7$ & $65 \pm 6$ \\
Sunitinib & 14 & $66 \pm 8$ & $135 \pm 13$ \\
U.5 & 6 & $43 \pm 4$ & $46 \pm 7$ \\
U.8 & 0.6 & $48 \pm 3$ & $80 \pm 13$ \\
U.15 & 8 & $71 \pm 11$ & $87 \pm 9$ \\
U.21 & 120 & $62 \pm 10$ & $15 \pm 3$ \\
U.23 & 12 & $61 \pm 11$ & $148 \pm 16$ \\
U.24 & 2.2 & $71 \pm 16$ & $97 \pm 11$ \\
U.25 & 15 & $80 \pm 8$ & $100 \pm 14$ \\
\hline
\end{tabular}

Data are the average $( \pm S D)$ of three experiments.

Results from table 4.12 indicate that sorafenib and compounds U.5 (ochloro) and U.8 (o-bromo) were able to down-regulate the expression of VEGFR-2 to the half of the control and maintained an equilibrated distribution of 
VEGFR-2 in membrane and inside cell. Sunitinib and U.23 (m,p-dimethyl) exerted their regulation only on membrane VEGFR-2 while seemed to promote internalization of the target. On the other hand, disubstituted derivative U.21 (m,m-dimethyl) was able to down-regulate membrane VEGFR-2 almost to the half of the control while inhibiting internalization of the target, reducing the total level of the protein to $15 \%$ of the control. Compound U.15 (m-methyl) was able to moderately down-regulate both membrane and total VEGFR-2 while disubstituted derivatives $\mathbf{U} .24$ and $\mathbf{U} .25$ exerted only a moderate reduction of membrane target.

Immunofluorescence assay correlated well with flow cytometry results. In figure 4.25 it can be observed that in the control assay (A) VEGFR-2 is distributed throughout the cell in granular form. On the other hand, sunitinib (B) promotes the internalization of VEGFR-2 from membrane to the cytoplasm and the nucleus. It can be appreciated how the target accumulates in granules not only inside the nucleus but also near the nuclear membrane and in the nuclear pores. Compounds U.5 (C) and U.8 (D) exerted an overall reduction of the target showing a distribution of VEGFR-2 inside cell similar to sunitinib though, in these cases, the presence of granulose VEGFR-2 inside nucleus is reduced. Compound U.15 (E) causes a significant reduction of membrane VEGFR-2 and accumulates granules of the target in the perinuclear membrane, which shows that this compound is able to down-regulate the internalization of the protein to the nucleus. Compound U.21 (F) also exerted a significant reduction of the target throughout the cell although in this case accumulation of filaments of VEGFR-2 along all the perinuclear membrane and also inside the nucleus is observed. Finally, compounds U.23 $(\mathrm{G})$ and U.25 $(\mathrm{H})$ seem to exhibit similar effect to sunitinib with granules of the target in the cytoplasm and in the nucleus. 

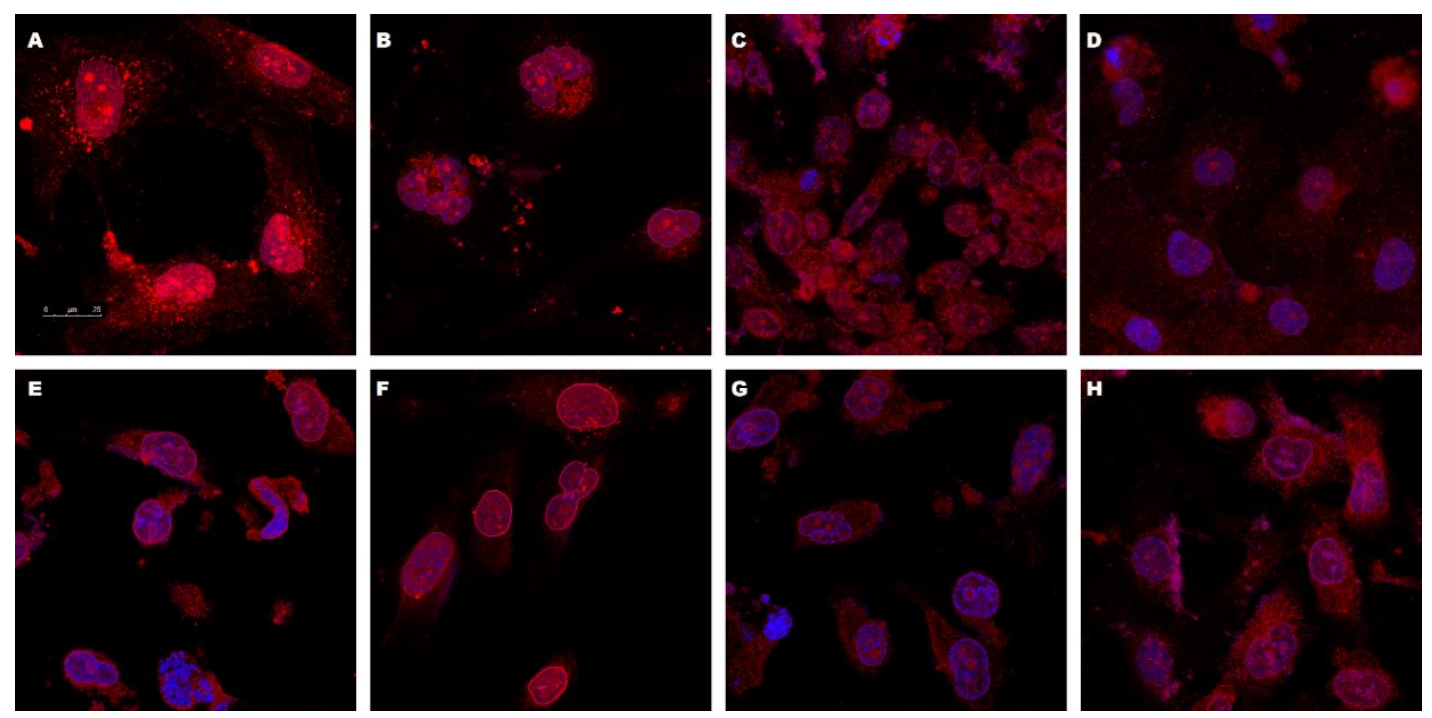

Figure 4.25. Effect of some selected ureas on the VEGFR-2 in HMEC-1 cells. (A) DMSO, (B) $14 \mu \mathrm{M}$ sunitinib, (C) $6 \mu \mathrm{M}$ U.5, (D) $0.6 \mu \mathrm{M}$ U.8, (E) $8 \mu \mathrm{M}$ U.15, (F) $120 \mu \mathrm{M}$ U.21, (G) $12 \mu \mathrm{M}$ U.23 and (H) $15 \mu \mathrm{M}$ U.25.

The effect of the selected compounds on VEGFR-2 in tumor cells was also studied by immunofluorescence (see figure 4.26). In the control assay (A), VEGFR-2 is distributed throughout the cell in granular form. On the other hand, sunitinib (B) provokes a drastic reduction of the target in all the cells. In tumor cells, the effect presented by the tested ureas U.5 (C), U.8 (D), U.15 (E), U.21 $(F)$, U.23 $(\mathrm{G})$ and $\mathbf{U} .25(\mathrm{H})$ was very similar, all of them exerting an overall reduction of the target showing accumulation of granules in the cytoplasm and in the nucleus. 

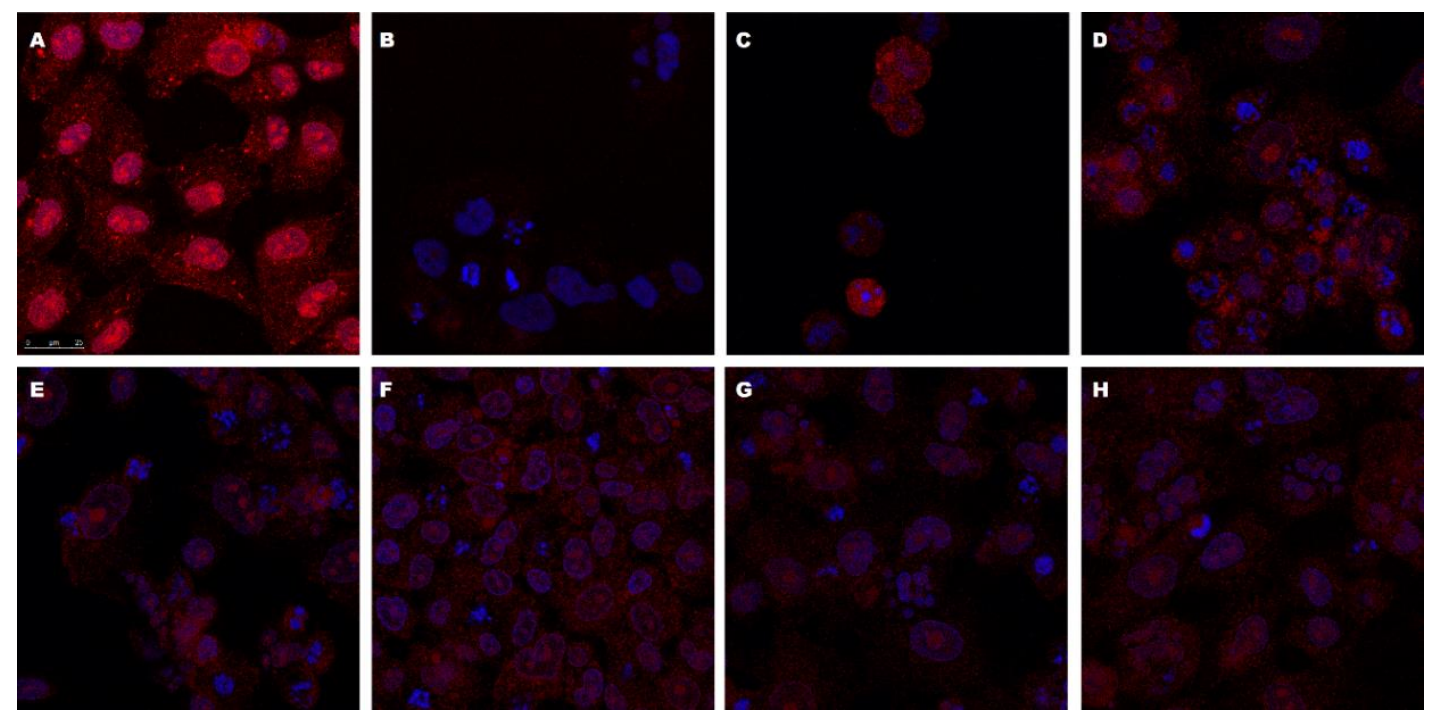

Figure 4.26. Effect of some selected ureas on the VEGFR-2 in A549 cells: (A) DMSO, (B) $22 \mu \mathrm{M}$ sunitinib, (C) $8.8 \mu \mathrm{M}$ U.5, (D) $2.4 \mu \mathrm{M}$ U.8, (E) $18 \mu \mathrm{M}$ U.15, (F) $48 \mu \mathrm{M}$ U.21, (G) $28 \mu \mathrm{M}$ U.23 and $(\mathrm{H}) 44 \mu \mathrm{M}$ U.25.

\subsubsection{Inhibition of VEGFR-2 kinase activity}

VEGF binding to VEGFR-2 causes phosphorylation of the receptor and triggers the signaling cascade that promotes phosphorylation of Erk1/2 (extracellular signal-regulated kinase). This phosphorylation activates endothelial cell proliferation, migration and differentiation ${ }^{167}$. In order to assess the inhibition of VEGFR-2 kinase activity exerted by the ureas, as well as its associated signaling cascade, the amount of phosphorylated receptor and phosphorylated Erk1/2 was measured. Thus, the selected ureas were added to HMEC-1 or A549 cells at a concentration of $10 \mu \mathrm{M}$. After the corresponding treatment, cells were lysed and p-VEGFR-2 was quantified by ELISA analysis from lysates. Western blot analysis of the lysates allowed the relative

167 (a) Svensson, S.; Jirström, K.; Rydén, L.; Roos, G.; Emdin, S.; Ostrowski, M. C.; Landberg, G. Oncogene 2005, 24, 4370-4379. (b) Almalki, S. G.; Agrawal, D. K. Stem Cell Res. Ther. 2017, 8, 113. 
quantification of $p$-Erk1/2. Table 4.13 shows the percentage of $p$-VEGFR-2 and p-Erk for each compound referred to control (DMSO, 100\%).

Table 4.13. $p$-VEGFR-2 and $p$-Erk $1 / 2$ detected in the presence of selected ureas.

\begin{tabular}{|c|c|c|c|c|}
\hline Comp. & $\begin{array}{l}\text { HMEC-1a } \\
\text { p-VEGFR-2 (\%) }\end{array}$ & $\begin{array}{l}\text { HMEC-1 } \\
\text { p-Erk1/2 (\%) }\end{array}$ & $\begin{array}{l}\text { A549b } \\
\text { p-VEGFR-2 (\%) }\end{array}$ & $\begin{array}{l}\text { A549b } \\
\text { p-Erk1/2 (\%) }\end{array}$ \\
\hline Sorafenib & $77 \pm 9$ & $80 \pm 8$ & $90 \pm 6$ & $50 \pm 7$ \\
\hline Sunitinib & $79 \pm 11$ & - & $70 \pm 6$ & - \\
\hline U.5 & $52 \pm 3$ & $36 \pm 3$ & $>100$ & $45 \pm 2$ \\
\hline U.8 & $78 \pm 5$ & $53 \pm 6$ & $60 \pm 8$ & $65 \pm 14$ \\
\hline U.15 & $73 \pm 6$ & $68 \pm 10$ & $70 \pm 12$ & $>100$ \\
\hline U.21 & $69 \pm 7$ & $87 \pm 16$ & $>100$ & $85 \pm 11$ \\
\hline $\mathrm{U} .23$ & $80 \pm 9$ & $>100$ & $70 \pm 11$ & $80 \pm 12$ \\
\hline U.25 & $90 \pm 14$ & $44 \pm 3$ & $>100$ & 27 \\
\hline
\end{tabular}

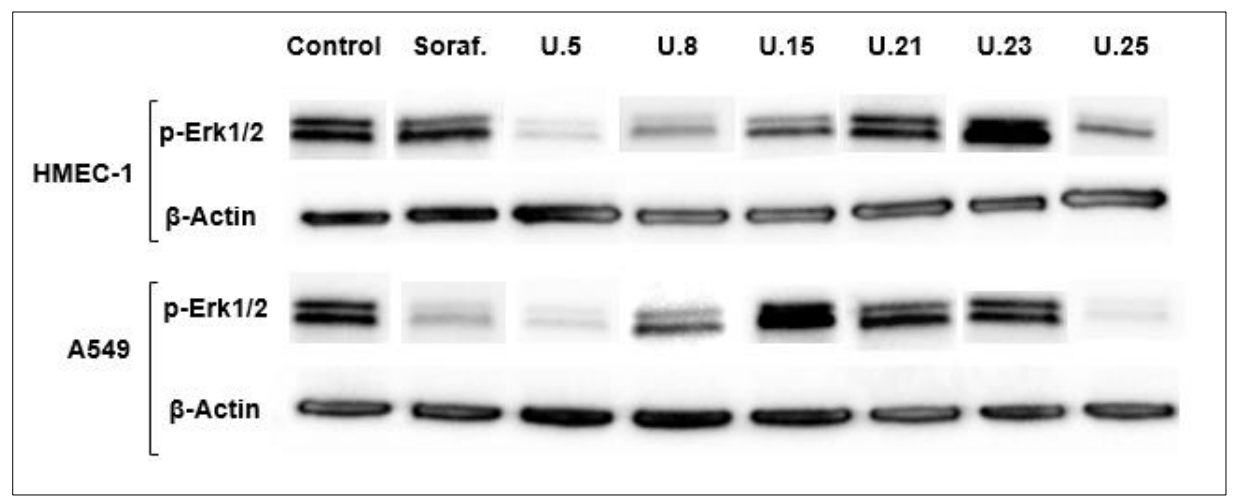

The best inhibitory kinase activity in endothelial cells was achieved by U.5 (o-chloro) which is able to reduce the phosphorylation of VEGFR-2 by half of that measured in the control. Compounds U.8 (o-bromo), U.15 (m-methyl) and U.21 ( $m, m$-dimethyl) exert anti-kinase activity similar to sorafenib or sunitinib. In addition, there is an important inhibition of Erk1/2 phosphorylation by U.5, U.8 and U.25 (m-methyl-p-chloro), thus improving the results obtained with sorafenib. 
As regards the results obtained in A549 cell line, compounds U.8, U.15 and U.23 ( $m, p$-dimethyl) were the most active ones in the inhibition of VEGFR-2 phosphorylation showing similar results to sunitinib. In these tumor cells, again compounds U.5, U.8 and U.25 were able to reduce Erk1/2 phosphorylation at levels similar to sorafenib.

\subsubsection{Conclusions of biological evaluation of family 2}

The majority of the synthetic ureas show antiproliferative activity in the low micromolar range with better results than the reference compounds sorafenib and sunitinib. As regards the substitution pattern, ortho monosubstituted ureas were the most active ones with $\mathrm{IC}_{50}$ values at the submicromolar level.

All the tested ureas extensively arrested $A 549$ cells in the $G_{2} / M$ phase and caused an increase in the percentage of cells in subG 0 phase at concentrations twice of their $\mathrm{IC}_{50}$ values. This behaviour is very different from that shown by sorafenib and sunitinib, which are unable to cause mitotic arrest. Compounds U.5 (o-chloro), U.8 (o-bromo), U.23 ( $m, p$-dimethyl) and U.24 (o-methyl- $m$-chloro) exhibit an important apoptotic effect at $10 \mu \mathrm{M}$ concentration in a dose-dependent manner improving the results obtained with sunitinib.

Ureas inhibit tube formation of endothelial cells at $0.1 \mu \mathrm{M}$, again improving the results observed with sorafenib. As regards inhibition of VEGFR-2 tyrosine kinase activity, ortho substituted $\mathbf{U} .5$ and $\mathbf{U . 8}$ were the most active ones in both down-regulation of VEGFR-2 and inhibition of the kinase activity of this receptor, improving the results obtained with sunitinib and sorafenib. 


\subsection{Biological evaluation of family 3 as multitarget inhibitors}

\subsubsection{Preliminary docking studies}

A docking study using AutoDock 4.2 software was developed on the kinase domain of VEGFR-2 and on the site identified in PD-L1 in order to locate relatively simple structures that could interact with both sites. Several general structures capable of establishing at least 3 of the hydrogen bonds shown by sorafenib and able to interact with the binding site in PD-L1 were identified (see figure 4.27). These structures are characterized by having a urea system (essential to interact with the kinase domain) to which aromatic rings are bound in order to interact with the hydrophobic grooves present in both proteins.

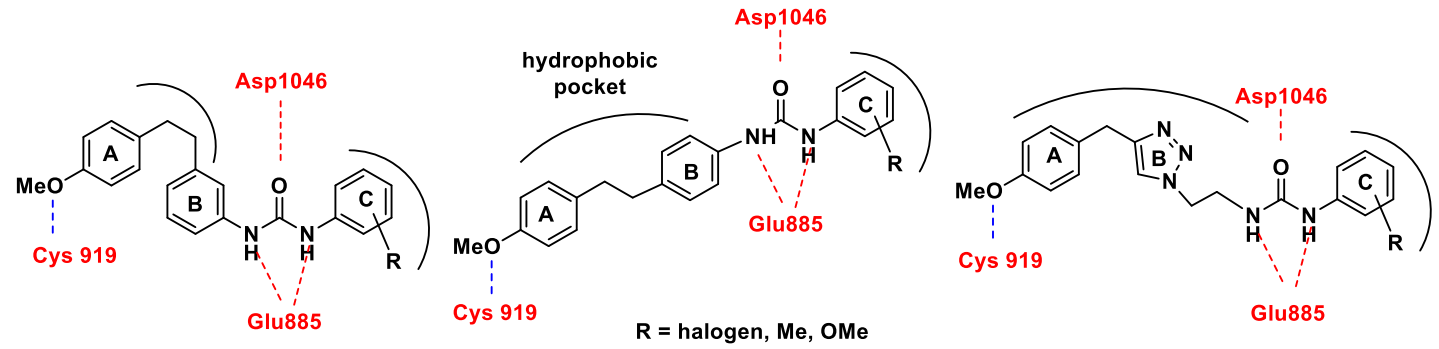

Figure 4.27. Identified general structures able to interact with VEGFR-2 and PD-L1. The hydrogen bonds that can be formed with VEGFR-2 are indicated in red. The zones of the molecules that interact with the hydrophobic pockets of both binding sites are also indicated.

As an example, the docking obtained for three of the analyzed structures is shown in figure 4.28. It can be appreciated in (A) that compounds D.1, D.2 and D.3 occupy the binding site in the kinase domain of VEGFR-2 in much the same way as sorafenib does. These compounds establish hydrogen bonds between the OMe group and Cys919, between the two NH of the urea system and Glu885 and between the $\mathrm{O}$ of urea and Asp1046. The 3 aromatic rings interact with two hydrophobic zones present in the binding site. Additionally, it can be seen in (B) that structures D.1, D.2 and D.3 also occupy the binding site of BMS-202 in PDL1 protein. The aromatic rings are introduced in a hydrophobic groove establishing interactions with Tyr56, Me115, Ala121 and Tyr123. The more polar urea system points towards the outer zone of the groove. 


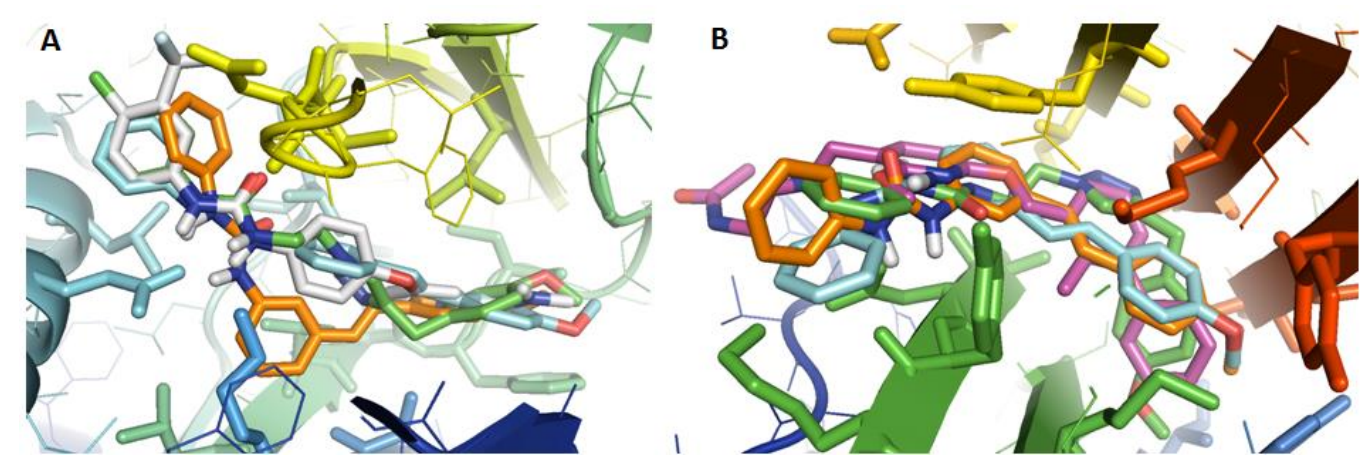<smiles>COc1ccc(/C=C/c2cccc(NC(=O)Nc3ccccc3)c2)cc1</smiles>

Figure 4.28. (A) Superposition of D.1 (orange), D.2 (light blue), D.3 (green) and sorafenib (grey) in the kinase domain of VEGFR-2. (B) Superposition of D.1 (orange), D.2 (light blue), D.3 (green) and BMS-202 (violet) at the PD-L1 binding site.

With the objective to verify these preliminary results obtained from the insilico study, the in vitro biological evaluation of family 3 compounds was carried out as described below. 


\subsubsection{Biological evaluation of triazolyl-ureas T.1-T.8}

\subsubsection{Inhibition of cell proliferation}

The ability of compounds T.1-T.8 to inhibit cell proliferation was evaluated towards the human tumor cell lines HT-29, MCF-7, HeLa and A549 as well as towards the endothelial cell line HMEC-1 and the non-tumor cell line HEK-293. All the $\mathrm{IC}_{50}$ values obtained for triazolyl-ureas were higher than $200 \mu \mathrm{M}$ in all the cell lines tested.

\subsubsection{Effect on cellular VEGFR-2}

The effect of triazolyl-ureas T.1-T.8 on VEGFR-2 in HMEC-1 cell line was determined by flow cytometry. For this assay, cells were incubated for $24 \mathrm{~h}$ in the presence of the corresponding compounds at $100 \mu \mathrm{M}$ concentration. Then, cells were fixed with formaldehyde and treated with anti-VEGFR-2alexafluor ${ }^{8647}$ to quantify membrane VEGFR-2. On the other hand, permeabilization of cells with Triton X-100 prior to fixation step, allowed the quantification of total VEGFR-2 in cells. Table 4.14 shows the effect of the derivatives on VEGFR-2 expression and distribution in HMEC-1 endothelial cell line, referred to control (DMSO, $100 \%)$.

Table 4.14. Effect on VEGFR-2 in HMEC-1 cells.

\begin{tabular}{lll}
\hline Comp. & Membrane VEGFR-2 (\%) & Total VEGFR-2 (\%) \\
\hline Sorafenib & $46 \pm 8$ & $64 \pm 4$ \\
T.1 & $97 \pm 14$ & $105 \pm 10$ \\
T.2 & $74 \pm 6$ & $81 \pm 14$ \\
T.3 & $90 \pm 4$ & $102 \pm 5$ \\
T.4 & $79 \pm 10$ & $78 \pm 1$ \\
T.5 & $40 \pm 10$ & $55 \pm 5$ \\
T.6 & $79 \pm 9$ & $100 \pm 11$ \\
T.7 & $76 \pm 4$ & $91 \pm 9$ \\
T.8 & $97 \pm 15$ & $90 \pm 7$ \\
\hline
\end{tabular}

Data are the average $( \pm S D)$ of three experiments. 
Results from table 4.14 indicate that T.5 $(p-\mathrm{Br})$ is the derivative which better interacts with VEGFR-2 in HMEC-1 cells improving the effect exerted by sorafenib. Compounds T.2 $(m-\mathrm{Cl})$ and T.4 $(m-\mathrm{Br})$ are also able to down-regulate the expression of VEGFR-2 moderately inhibiting the effect of the control in a $20 \%$.

Table 4.15 depicts the effect of T.2, T.4 and T.5 on VEGFR-2 expression and distribution in A549 tumor cell line at $100 \mu \mathrm{M}$ concentration, referred to control (DMSO, 100\%).

Table 4.15. Effect on VEGFR-2 in A549 cells.

\begin{tabular}{lll}
\hline Comp. & Membrane VEGFR-2 (\%) & Total VEGFR-2 (\%) \\
\hline Sorafenib & $92 \pm 3$ & $85 \pm 5$ \\
Sunitinib & $66 \pm 8$ & $135 \pm 14$ \\
T.2 & $95 \pm 3$ & $86 \pm 6$ \\
T.4 & $98 \pm 6$ & $80 \pm 8$ \\
T.5 & $70 \pm 4$ & $61 \pm 11$ \\
\hline
\end{tabular}

Data are the average $( \pm S D)$ of three experiments.

Results from table 4.15 indicate again that T.5 $(p-\mathrm{Br})$ is the derivative which better interacts with VEGFR-2 in A549 cells improving the effect exerted by reference compounds.

\subsubsection{Inhibition of VEGFR-2 kinase activity}

After having proved that triazolyl-ureas T.1-T.8 interact with VEGFR-2 in endothelial cells, their capacity to inhibit kinase activity was also studied. Thus, HMEC-1 cells were treated with the derivatives for $24 \mathrm{~h}$ at $100 \mu \mathrm{M}$, then cells were lysed and phospho-VEGFR-2 was quantified by ELISA analysis. Western blot analysis of the compounds that were active in inhibiting phospho-VEGFR-2 allowed the relative quantification of phospho-Erk1/2. Table 4.16 shows the percentage of $\mathrm{p}$-VEGFR-2 and $\mathrm{p}$-Erk for each compound referred to control (DMSO, 100\%). 
Table 4.16. $p$-VEGFR-2 and $p$-Erk $1 / 2$ detected in HMEC-1 cells.

\begin{tabular}{lll}
\hline Comp. & p-VEGFR-2 (\%) & p-Erk1/2 (\%) \\
\hline Sorafenib & $77 \pm 9$ & $80 \pm 8$ \\
T.1 & $100 \pm 12$ & - \\
T.2 & $70 \pm 16$ & $38 \pm 14$ \\
T.3 & $86 \pm 3$ & $72 \pm 6$ \\
T.4 & $>100$ & - \\
T.5 & $>100$ & - \\
T.6 & $83 \pm 4$ & $82 \pm 11$ \\
T.7 & $90 \pm 10$ & - \\
T.8 & $>100$ & - \\
\hline
\end{tabular}

Data are the average $( \pm S D)$ of three experiments.

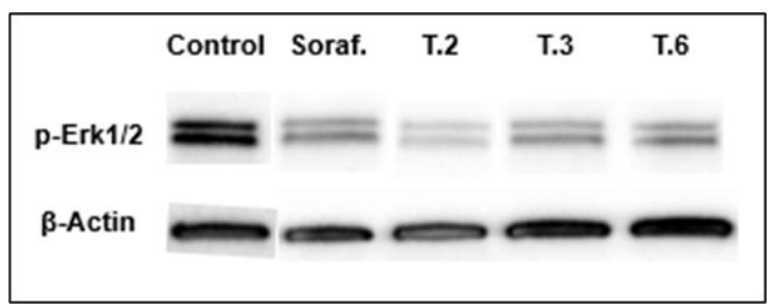

It is deduced from table 4.16 that the best inhibitory kinase activity in endothelial cells is achieved by T.2 ( $m$-chloro), T.3 ( $p$-chloro) and T.6 (o-methyl) which are able to reduce the phosphorylation of VEGFR-2 at similar levels to sorafenib. Interestingly, there is an important inhibition of Erk1/2 phosphorylation by T.2, improving the results obtained with sorafenib.

On the other hand, inhibition of VEGFR-2 kinase activity in A549 cells was also tested. In this case, none of the selected derivatives improved the effect observed for sorafenib. Table 4.17 shows the percentage of $p$-VEGFR-2 and $p$ Erk for each compound referred to control (DMSO, 100\%). 
Table 4.17. $p$-VEGFR-2 and $p-E r k 1 / 2$ detected in A549 cells.

\begin{tabular}{lll}
\hline Comp. & p-VEGFR-2 (\%) & p-Erk1/2 (\%) \\
\hline Sorafenib & $84 \pm 4$ & $50 \pm 7$ \\
T.2 & $121 \pm 11$ & $99 \pm 9$ \\
T.3 & $125 \pm 12$ & $78 \pm 7$ \\
T.6 & $121 \pm 16$ & $119 \pm 8$ \\
T.7 & $112 \pm 10$ & $90 \pm 13$ \\
\hline
\end{tabular}

Data are the average $( \pm S D)$ of three experiments.

\begin{tabular}{|lllllll}
\hline Control Soraf. T.2 & T.3 & T.6 & T.7 \\
p-Erk1/2 & $=$ & $=$ & $=$ & $=$ & $=$ & $=$
\end{tabular}

\subsubsection{Tube formation inhibition}

The capacity of synthetic compounds to inhibit the formation of new vasculature network formed by endothelial cells was evaluated. Therefore, HMEC-1 cells were seeded on top of Matrigel® and simultaneously treated with different concentrations of derivatives. Pictures were taken $24 \mathrm{~h}$ later in order to evaluate the tube formation inhibition effect. Table 4.18 shows the minimum concentration at which compounds are active in the tube formation inhibition. Only T.2, T.3 and T.6 were tested, since they offered the best activity in the inhibition of VEGFR-2 kinase activity (see table 4.16). 
Table 4.18. Tube formation inhibition effect.

\begin{tabular}{lc}
\hline Comp. & Min. active conc. $(\mu \mathrm{M})$ \\
\hline Sunitinib & 3 \\
Sorafenib & 10 \\
T.2 & 50 \\
T.3 & 400 \\
T.6 & 200 \\
\hline Data are representative of three experiments.
\end{tabular}

Data from table 4.18 confirm that triazolyl-ureas are able to inhibit tube formation but at higher doses than sunitinib and sorafenib, being T.2 ( $m$-chloro) the most potent compound.

\subsubsection{Effect on PD-L1 and C-Myc proteins}

In order to evaluate the immunomodulator properties of the synthetic derivatives, the effect on PD-L1 and C-Myc proteins was studied. Thus, compounds were added to A549 cells at $100 \mu \mathrm{M}$ concentration and, after $24 \mathrm{~h}$, cells were lysed and ELISA analysis was performed to determine the relative amount of both proteins compared to DMSO treated cells. Table 4.19 shows the percentage of free PD-L1 detected for each compound referred to control (DMSO). The percentage of c-Myc has been quantified only for the compounds which exerted similar or better action than the reference compound BMS-8 in the inhibition of PD-L1. 
Table 4.19. PD-L1 and c-Myc detection in A549 cells.

\begin{tabular}{lll}
\hline Comp. & PD-L1 (\%) & c-Myc (\%) \\
\hline BMS-8 & $68 \pm 5$ & $60 \pm 7$ \\
T.1 & $83 \pm 9$ & - \\
T.2 & $70 \pm 8$ & $60 \pm 8$ \\
T.3 & $69 \pm 10$ & $73 \pm 5$ \\
T.4 & $80 \pm 12$ & - \\
T.5 & $>100$ & - \\
T.6 & $82 \pm 20$ & - \\
T.7 & $90 \pm 20$ & - \\
T.8 & $66 \pm 6$ & $70 \pm 8$ \\
\hline
\end{tabular}

From table 4.19 it can be concluded that compounds T.2 (m-chloro), T.3 ( $p$ chloro) and T.8 ( $p$-methyl) inhibit PD-L1 and c-Myc proteins in a similar way as BMS-8.

\subsubsection{Cell proliferation evaluation in co-cultures}

Some tumor cell lines such as A549 and HT-29 show high PD-L1 overexpression ${ }^{168}$. Therefore, cell proliferation of these has been studied in presence of PD-1-expressing Jurkat $\mathrm{T}$ cells in order to evaluate whether the observed cell proliferation inhibition is due to the blockage of PD-1/PD-L1 system. Thus, tumor cells were treated for $24 \mathrm{~h}$ with the selected compounds at $200 \mu \mathrm{M}$ in presence of Jurkat T cells and then, living cells were counted using trypan blue in a Neubauer chamber. Figure 4.29 shows the inhibition of tumor cell proliferation exhibited by the derivatives in the presence of Jurkat T cells.

168 Patel, S; Kurzrock, R. Mol. Cancer Ther. 2015, 14, 847-856. 


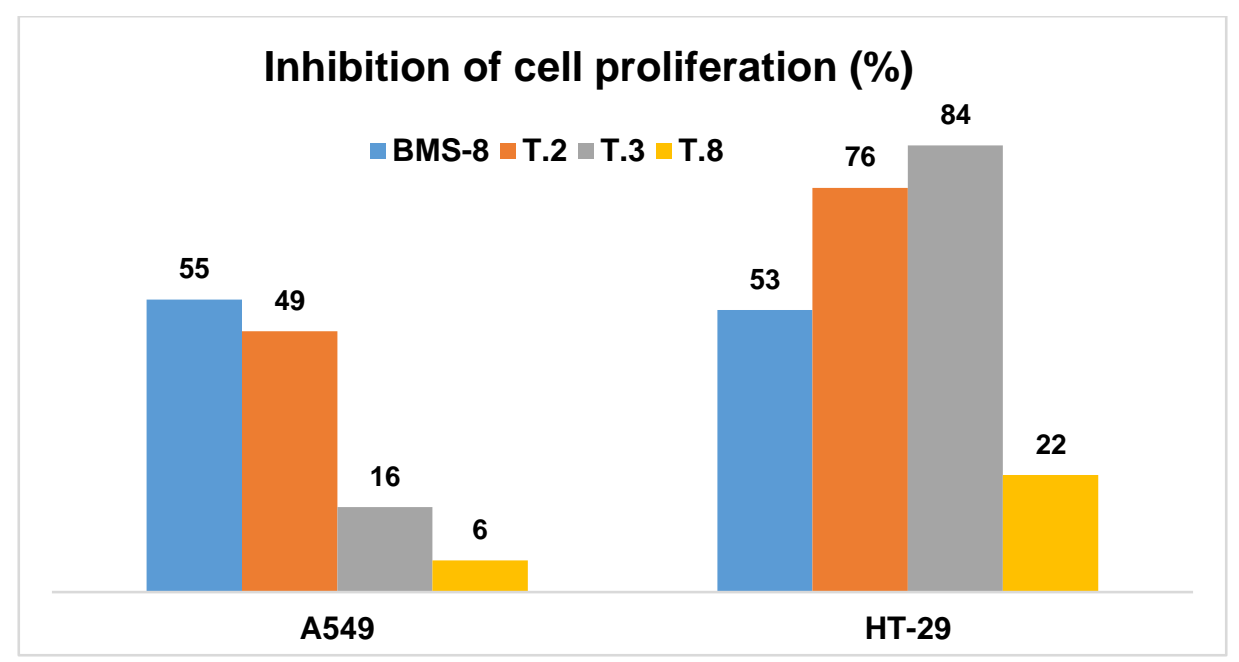

Figure 4.29. Inhibition of cell proliferation (\%) in the presence of Jurkat T cells. Data are representative of three experiments.

From data provided in figure 4.29, it can be suggested that the tested compounds are able to inhibit A549 cell proliferation in co-culture but do no improve the effect exerted by BMS-8. Interestingly, in HT-29 cell line this effect is highly incremented for compounds T.2 ( $m$-chloro) and T.3 ( $p$-chloro), improving the effect exhibited by BMS-8. 


\subsubsection{Biological evaluation of ureas S.1-S.9}

\subsubsection{Inhibition of cell proliferation}

The ability of ureas S.1-S.9 to inhibit cell proliferation was evaluated towards the human tumor cell lines HT-29, MCF-7, HeLa and A549, as well as towards the endothelial cell line HMEC-1 and the non-tumor cell line HEK-293. The corresponding $\mathrm{IC}_{50}$ values are presented in table 4.20 along with $\mathrm{IC}_{50}$ values for the reference compounds sunitinib, sorafenib and BMS-8.

Table 4.20. IC 50 values $(\mu \mathrm{M})$ for sunitinib, sorafenib, BMS-8 and S.1-S.9. ${ }^{a}$

\begin{tabular}{lllllll}
\hline Comp. & HT-29 & MCF-7 & HeLa & A549 & HMEC-1 & HEK-293 \\
\hline Sunitinib & $3.8 \pm 0.5$ & $0.08 \pm 0.02$ & $7.8 \pm 1.0$ & $11 \pm 2$ & $7 \pm 4$ & $5.0 \pm 0.8$ \\
Sorafenib & $17 \pm 4$ & $14 \pm 4$ & $6.1 \pm 0.4$ & $27 \pm 2$ & $34 \pm 3$ & $5.0 \pm 0.7$ \\
BMS-8 & $17 \pm 4$ & $20 \pm 3$ & $>100$ & $6 \pm 1$ & - & $60 \pm 10$ \\
S.1 & $12 \pm 4$ & $19 \pm 2$ & $22 \pm 3$ & $21 \pm 9$ & $33.0 \pm 0.6$ & $6.5 \pm 0.2$ \\
S.2 & $20 \pm 10$ & $37.2 \pm 0.8$ & $10.5 \pm 0.6$ & $38 \pm 3$ & $32.9 \pm 0.5$ & $5 \pm 2$ \\
S.3 & $33 \pm 5$ & $17 \pm 3$ & $8 \pm 1$ & $29 \pm 3$ & $33 \pm 3$ & $20 \pm 10$ \\
S.4 & $47 \pm 4$ & $>100$ & $>100$ & $61 \pm 3$ & $>100$ & $57 \pm 3$ \\
S.5 & $3.5 \pm 0.6$ & $4.3 \pm 0.7$ & $11.3 \pm 0.7$ & $7.7 \pm 0.2$ & $2 \pm 1$ & $2.4 \pm 0.6$ \\
S.6 & $0.52 \pm 0.09$ & $0.40 \pm 0.09$ & $0.7 \pm 0.3$ & $1.5 \pm 0.9$ & $1.8 \pm 0.8$ & $1.0 \pm 0.4$ \\
S.7 & $20 \pm 3$ & $>100$ & $69 \pm 5$ & $24 \pm 5$ & $48 \pm 7$ & $90 \pm 10$ \\
S.8 & $14 \pm 4$ & $8.2 \pm 0.6$ & $16.5 \pm 0.7$ & $>200$ & $50 \pm 20$ & $5.3 \pm 0.9$ \\
S.9 & $1.5 \pm 0.1$ & $15.3 \pm 0.4$ & $21 \pm 1$ & $>200$ & $>200$ & $1.8 \pm 0.7$ \\
\hline
\end{tabular}

${ }^{a} C_{50}$ values are expressed as the compound concentration that inhibits the cell growth by $50 \%$. Data are the average $( \pm S D)$ of three experiments.

Tested ureas show antiproliferative activity in the low micromolar range in all cell lines, comparable to that shown by reference compounds sunitinib, sorafenib and BMS-8. Derivative S.6 (E-p-chloro) is the most active one since it offers $I_{50}$ values at submicromolar level and it exhibits lower $I_{50}$ values in cancer cell lines than in non-tumor ones. This fact allows to study the biological 
activity of this compound without resulting in unaccepatble side-effects or toxicity.

\subsubsection{Induction of apoptosis}

Induction of apoptosis was studied by measuring the translocation of phosphatidylserine from the cytoplasmic to the extracellular side of the plasma membrane. Thus, A549 cells were incubated for $24 \mathrm{~h}$ in the presence of compounds at $100 \mu \mathrm{M}$, after which annexin $\mathrm{V}$ content was measured by flow cytometry. The apoptotic effect of compounds S.1-S.9 is indicated in figure 4.30.

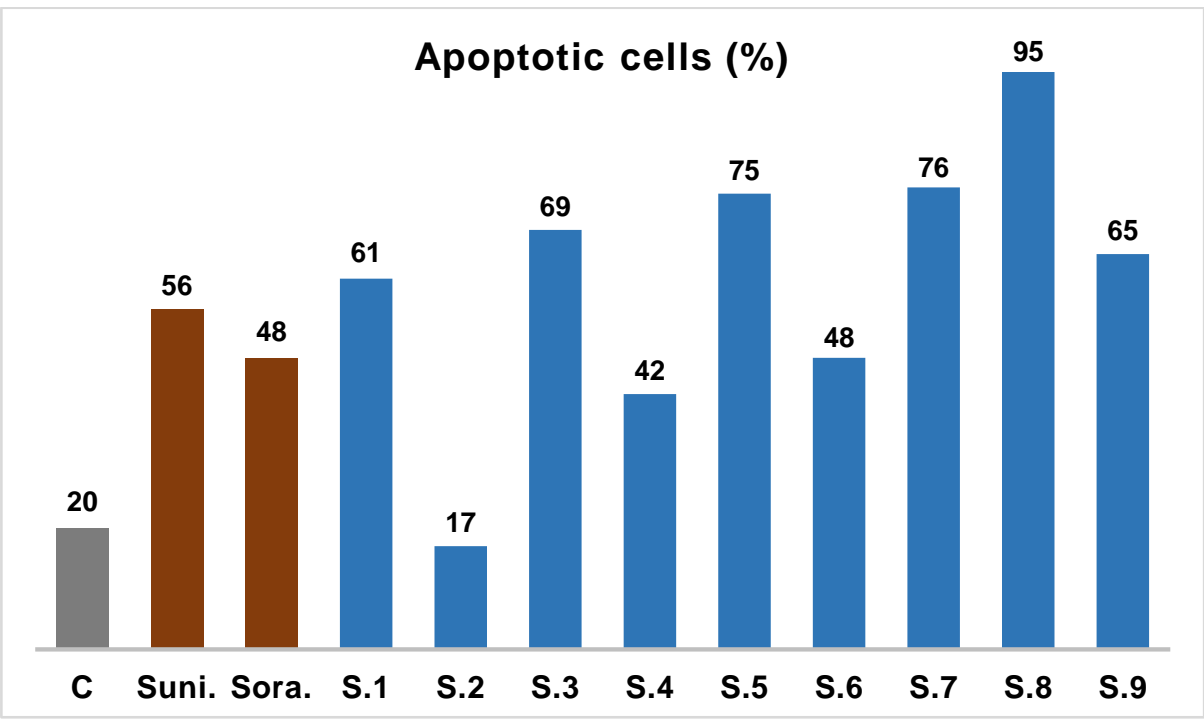

Figure 4.30. Apoptotic effect (\%) in A549 cells. Data are representative of three experiments.

All the tested compounds induce apoptosis at $100 \mu \mathrm{M}$, with the exception of compound S.2, which act as control (DMSO). Moreover, compounds S.3, S.5, S.7, S.8 and S.9 improve the effect depicted by sunitinib and sorafenib. BMS-8 and T.1-T.8 derivatives were also tested but they did not exhibit any effect in the induction of apoptosis. 


\subsubsection{Effect on cellular VEGFR-2}

The effect of ureas S.1-S.9 on VEGFR-2 in A549 tumor cell line was determined by both flow cytometry and immunofluorescence technique. For these assays, cells were incubated for $24 \mathrm{~h}$ in the presence of the corresponding compounds at $10 \mu \mathrm{M}$ concentration. Table 4.21 and figure 4.31 show the results obtained for every compound on VEGFR-2 presence and distribution in A549 cell line, referred to control (DMSO, $100 \%$ ).

Table 4.21. Effect on VEGFR-2 expression in A549 cells.

\begin{tabular}{lll}
\hline Comp. & Membrane VEGFR-2 (\%) & Total VEGFR-2 (\%) \\
\hline Sorafenib & $92 \pm 3$ & $85 \pm 5$ \\
Sunitinib & $66 \pm 8$ & $135 \pm 14$ \\
S.1 & $114 \pm 20$ & $177 \pm 9$ \\
S.2 & $102 \pm 10$ & $186 \pm 10$ \\
S.3 & $118 \pm 3$ & $100 \pm 7$ \\
S.4 & $109 \pm 18$ & $42 \pm 9$ \\
S.5 & $52 \pm 10$ & $32 \pm 4$ \\
S.6 & $56 \pm 11$ & $10 \pm 2$ \\
S.7 & $100 \pm 19$ & $100 \pm 16$ \\
S.8 & $97 \pm 10$ & $98 \pm 3$ \\
S.9 & $97 \pm 11$ & $96 \pm 7$ \\
\hline
\end{tabular}

Data are the average $( \pm S D)$ of three experiments.

Trans derivatives $\mathbf{S . 5}$ ( $m$-chloro) and $\mathbf{S . 6}$ (p-chloro) are the best ones in reducing the presence of VEGFR-2 improving the effect exerted by sorafenib. Concretely, they are able to down-regulate VEGFR-2 to the half of the control inhibiting internalization of the target and reducing the total level of the protein to $10-30 \%$ of the control.

Immunofluorescence assay correlates well with flow cytometry results. It can be observed in figure 4.31 that both compounds $\mathbf{S . 5}(\mathrm{B})$ and $\mathbf{S . 6}(\mathrm{C})$ provoked a 
significant reduction of membrane VEGFR-2, and concretely compound S.6 exerted a higher inhibition of the internalization of the protein to the nucleus.
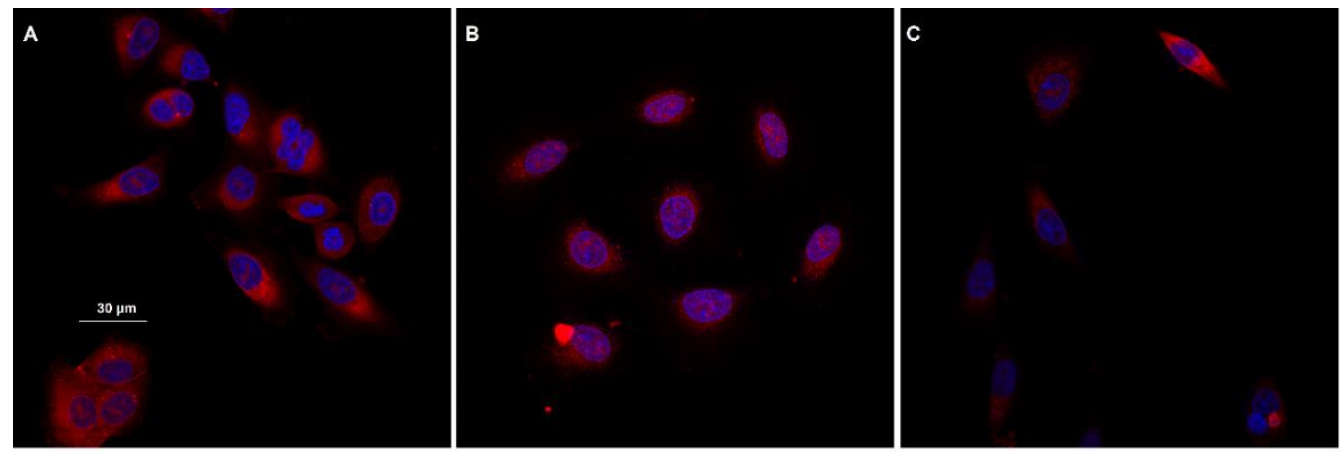

Figure 4.31. Effect on the VEGFR-2 in A549 cells (A) DMSO, (B) S.5 and (C) S.6.

The effect of trans derivatives on VEGFR-2 presence and distribution in endothelial cells was also evaluated (see table 4.22), referred to control (DMSO, $100 \%)$.

Table 4.22. Effect on VEGFR-2 expression in HMEC-1 cells.

\begin{tabular}{lll}
\hline Comp. & Membrane VEGFR-2 (\%) & Total VEGFR-2 (\%) \\
\hline Sorafenib & $46 \pm 8$ & $64 \pm 4$ \\
S.4 & $76 \pm 9$ & $121 \pm 8$ \\
S.5 & $55 \pm 8$ & $106 \pm 16$ \\
S.6 & $56 \pm 8$ & $109 \pm 17$ \\
\hline \multicolumn{3}{r}{ Data are the average ( \pm SD) of three experiments. }
\end{tabular}

Data are the average $( \pm S D)$ of three experiments.

Results from table 4.22 indicate that trans derivatives do not inhibit internalization of VEGFR-2 in HMEC-1 cells but reduce the presence of the target in the membrane. Again, $\mathbf{S . 5}$ and $\mathbf{S . 6}$ exhibit the best activity, similar to sorafenib. 


\subsubsection{Inhibition of VEGFR-2 kinase activity}

The effect on kinase activity of VEGFR-2 exerted by trans derivatives was studied by Western blot on two cell lines, A549 and HMEC-1. In this case, cells were treated for $24 \mathrm{~h}$ at $10 \mu \mathrm{M}$ concentration of the corresponding compounds. Table 4.23 shows the relative amount of $p$-VEGFR-2 and $p$-Erk $1 / 2$ detected on A549 when treated with each compound referred to control (DMSO, 100\%).

Table 4.23. p-VEGFR-2 and p-Erk1/2 detected in A549 cells.

\begin{tabular}{lll}
\hline Comp. & p-VEGFR-2 (\%) & p-Erk1/2 (\%) \\
\hline Sorafenib & $84 \pm 4$ & $50 \pm 7$ \\
S.4 & $76 \pm 3$ & $100 \pm 18$ \\
S.5 & $75 \pm 5$ & $56 \pm 12$ \\
S.6 & $82 \pm 3$ & $66 \pm 8$ \\
\hline Data are the average $( \pm$ SD) of three experiments.
\end{tabular}

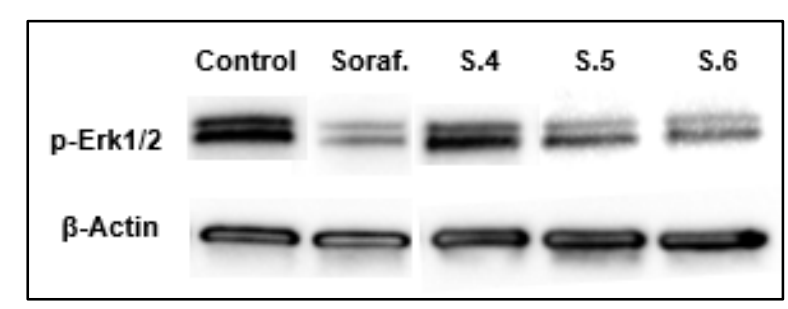

From results obtained in table 4.23, it is deduced that $\mathbf{S . 5}$ and $\mathbf{S . 6}$ reduce VEGFR-2 and Erk1/2 phosphorylation in the tumor cell line in a similar way to sorafenib. However, no kinase activity inhibition of VEGFR-2 on the endothelial cell line HMEC-1 was observed for any of the tested compounds.

\subsubsection{Tube formation inhibition}

The capacity of ureas S.1-S.9 to inhibit the formation of new vasculature network formed by HMEC-1 was evaluated. Table 4.24 shows the minimum concentration at which compounds are active and begin to inhibit the microtube formation. 
Table 4.24. Inhibition effect of tube formation.

\begin{tabular}{lc}
\hline Comp. & Min. active conc. $(\mu \mathrm{M})$ \\
\hline Sunitinib & 3 \\
Sorafenib & 10 \\
S.1 & 10 \\
S.2 & 10 \\
S.3 & 1 \\
S.4 & 10 \\
S.5 & 0.1 \\
S.6 & 0.1 \\
S.7 & 1 \\
S.8 & 10 \\
S.9 & 100 \\
\hline ata are representative of three experiments.
\end{tabular}

Comparing the minimum active concentration values to $\mathrm{IC}_{50}$ values for HMEC-1 cell line (see table 4.20) it can be observed that there is a correlation between antiproliferative activity and tube formation inhibition capacity, since compounds with lower $\mathrm{IC}_{50}$ values exhibit microtube inhibition activity at lower concentrations. Moreover, it is observed that some of the tested compounds are more active than sunitinib and sorafenib, particularly trans derivatives $\mathbf{S . 5}(\mathrm{m}$ chloro) and S.6 (p-chloro), which are 100-fold more active than sorafenib. Pictures for the inhibition of neovascularization achieved by compound S.6, at different concentrations, are displayed in figure 4.32 .
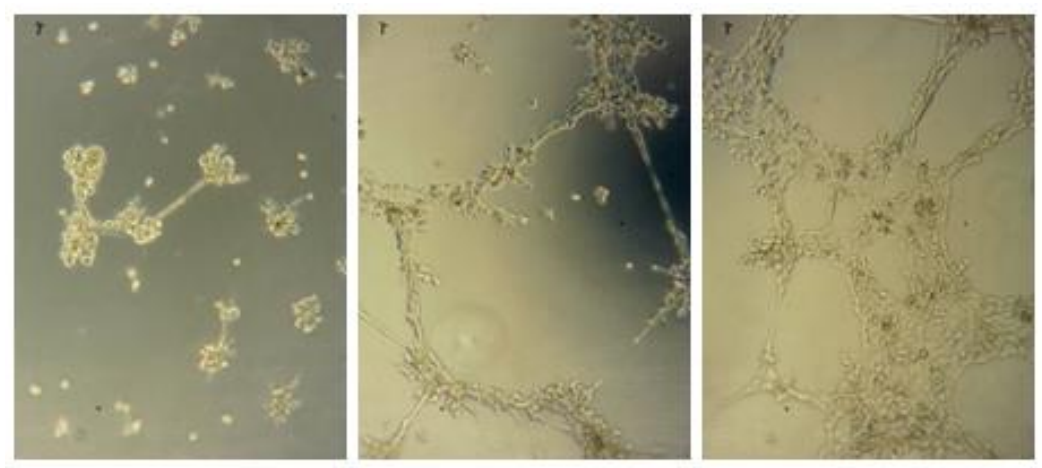

Figure 4.32. Effect of S.6 at 1, 0.1 and $0.01 \mu \mathrm{M}$ (from left to right). 


\subsubsection{Effect on PD-L1 and C-Myc proteins}

The immunomodulator properties of these derivatives were also evaluated in a similar manner as it was done for triazolyl-ureas. Thus, after $24 \mathrm{~h}$ of treatment with $100 \mu \mathrm{M}$ of compounds, A549 cells were lysed and ELISA analysis was performed. Table 4.25 shows the percentage of free PD-L1 detected for each compound referred to control (DMSO, 100\%). Again, the percentage of cMyc has been quantified only for the compounds which exerted similar or better action than the reference compound BMS-8 in the inhibition of PD-L1.

Table 4.25. PD-L1 and C-Myc detection in A549 cells.

\begin{tabular}{|c|c|c|}
\hline Comp. $^{a}$ & PD-L1 (\%) & c-Myc (\%) \\
\hline BMS-8 & $68 \pm 5$ & $60 \pm 7$ \\
\hline S.1 & $83 \pm 3$ & - \\
\hline $\mathrm{S} .2$ & $92 \pm 10$ & - \\
\hline S.3 & $75 \pm 6$ & - \\
\hline S.4 & $74 \pm 7$ & - \\
\hline S.5 & $49 \pm 8$ & $65 \pm 12$ \\
\hline S.6 & $25 \pm 9$ & $37 \pm 5$ \\
\hline S.7 & $61 \pm 6$ & $63 \pm 11$ \\
\hline S.8 & $51 \pm 9$ & $51 \pm 10$ \\
\hline S.9 & $38 \pm 3$ & $44 \pm 6$ \\
\hline
\end{tabular}

a S.1-S.6 were tested at $15 \mu \mathrm{M}$. BMS-8 and S.7-S.9 were tested at $100 \mu \mathrm{M}$.

Data are the average $( \pm S D)$ of three experiments.

From table 4.25 it can be deduced that compounds S.5, S.6, S.8 and S.9 are able to inhibit PD-L1 and C-Myc proteins improving the effect exerted by BMS-8, being $\mathbf{S . 6}$ ( $p$-chloro) the most active in both targets.

Consequently, immunofluorescence was performed for compound S.6. In this assay, cells were incubated for $24 \mathrm{~h}$ with $100 \mu \mathrm{M}$ of treatment. Then, cells were fixed, permeabilized and treated with anti-PD-L1-alexafluor®647 and antic-Myc-FITC. The pictures shown in figure 4.33 correlate well with the results 
obtained by ELISA test (see table 4.25), since compound S.6 (C) inhibits PD-L1 and c-Myc proteins in a higher proportion than BMS-8 (B).
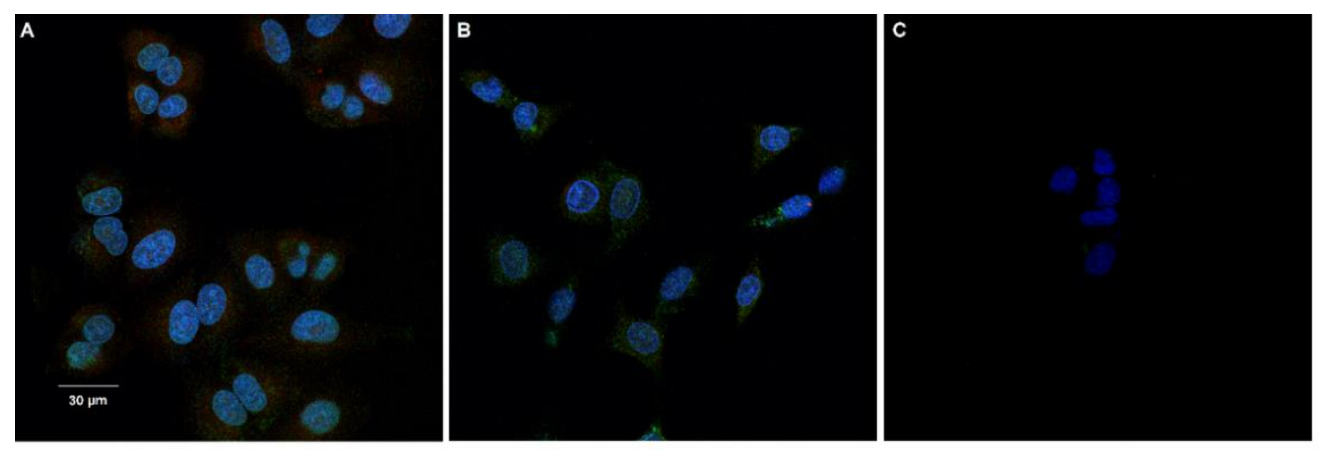

Figure 4.33. Effect on PD-L1 (red) and c-Myc (green) in A549 cells. (A) DMSO, (B) BMS-8 and (C) S.6.

\subsubsection{Cell proliferation evaluation in co-cultures}

The effect of compounds S.5, S.6 and S.9, which have shown good PD-L1 inhibition, was also studied in affecting tumor cell proliferation in the presence of PD-1 expressing Jurkat T-cells. Thus, A549 cells were treated for $24 \mathrm{~h}$ with the selected compounds at $200 \mu \mathrm{M}$ in presence of Jurkat T cells and then, living cells were counted using trypan blue and a Neubauer chamber. Figure 4.34 shows the inhibition of tumor cell proliferation exhibited by the selected compounds in the presence of Jurkat T cells.

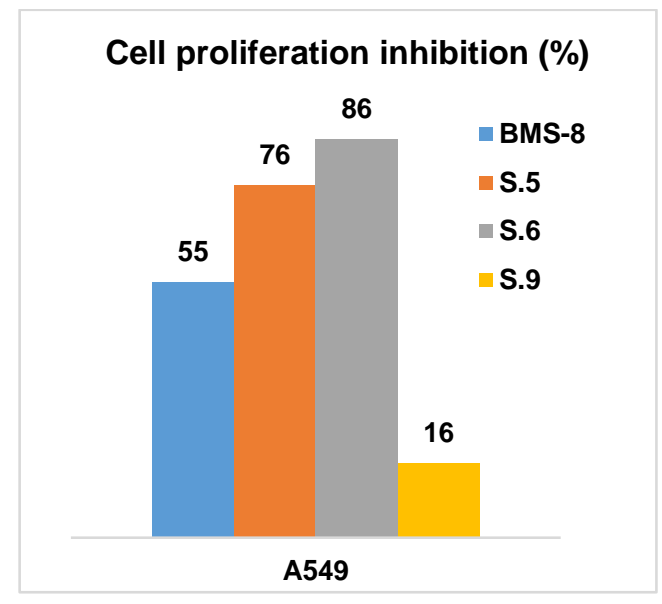

Figure 4.34. Inhibition of cell proliferation (\%) in the presence of Jurkat T cells. Data are representative of three experiments. 
Data provided in figure 4.34 show that $\mathbf{S . 5}$ and $\mathbf{S . 6}$ are able to inhibit A549 cell proliferation in co-culture improving the effect exerted by BMS-8. Thus, it can be affirmed that these derivatives act disturbing PD-1/PD-L1 interaction.

\subsubsection{Conclusions of biological evaluation of family 3}

The biological study of triazolyl-ureas T.1-T.8 has revealed that derivatives with chlorine substitution in meta or para positions are the most active as antiangiogenic and immunomodulator agents. Concretely, T.2 $(m-\mathrm{Cl})$ has offered a great potency in both down-regulation of VEGFR-2 and inhibition of the kinase activity of this receptor, showing better results than the ones obtained by the reference compounds. Regarding immunomodulator properties, T.2 has been the most effective triazolyl-urea in inhibiting cell proliferation in co-cultures, since due to its interaction with PD-L1 it has increased its antiproliferative effect in almost a $50 \%$.

In reference to the second group of ureas that were synthetized to improve the activity of triazolyl-ureas, they have offered antiproliferative and apoptotic activity at lower doses than triazoles. In general, derivatives with trans configuration have been the most active ones improving the biological activity shown by the reference compounds. Concretely, $\mathbf{S . 5}(m-\mathrm{Cl})$ and $\mathbf{S . 6}(p-\mathrm{Cl})$ inhibit tube formation of endothelial cells at $0.1 \mu \mathrm{M}$ and show inhibition of VEGFR-2 tyrosine kinase activity at lower concentrations than triazolyl-ureas and the reference compounds. As regards immunomodulator effect, they are able to improve the effect of BMS-8 in the inhibition of PD-L1 and C-Myc proteins. S.6 has been the most effective urea in inhibiting cell proliferation in co-cultures, since it has increased its antiproliferative effect in more than $80 \%$. These results agree with preliminary docking assays that were carried out on the binding site of PD-L1. In this study, two hydrophobic pockets were identified and placed in a manner that $E$-analogues fits better than Z-ones (see figure 4.35). 

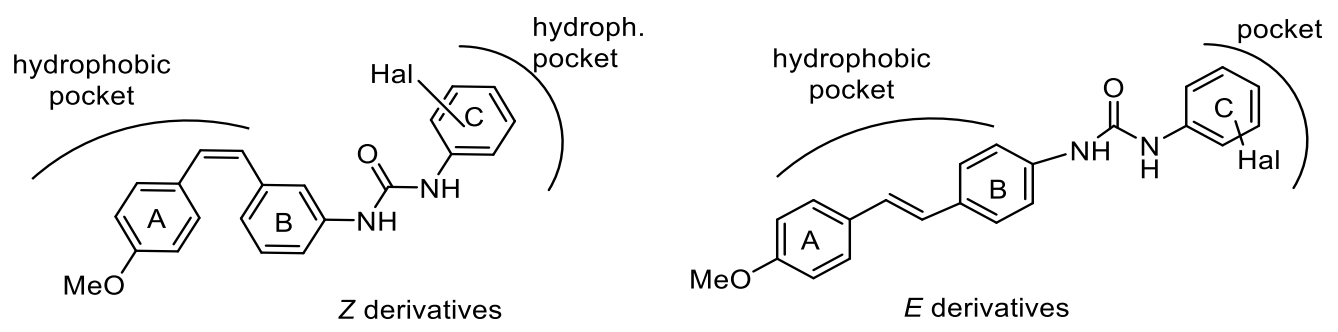

Figure 4.35. Molecular docking comparison between $E$ and $Z$ structures.

In summary, compounds T.2 (triazole, $\mathrm{m}$-Cl), S.5 (E-stilbene, $\mathrm{m}$-Cl) and $\mathbf{S . 6}$ (E-stilbene, $p$-Cl) show the best antiangiogenic and immunomodulator properties for every group of synthetized compounds. Thus, it is possible to assume that the presence of a chlorophenyl urea unit is important for the dual action of these kind of derivatives. Even so, comparing the results obtained for the triazoles with those achieved with the stilbenes, it can be concluded that the presence of the triazole ring in these aryl urea derivatives does not provide an improvement of the biological activity when compared to the reference compounds. However, the substitution of the triazole ring for the $E$-stilbene unit leads to the enhancement of the expected dual biological activity. This is the first example in the literature of the design and synthesis of compounds with both anti-PD-L1 and anti-angiogenic effect, so that a new door is opened to new kind of promising targeted anticancer agents. 


\subsection{In vivo experiments}

In this section it is presented an example of the in vivo evaluation process of a promising candidate, concretely, a colchicine derivative V.6 previously synthetized in our research group (see figure 4.36). First, a summary of the in vitro biological evaluation is provided and, then, the results obtained after in vivo evaluation are discussed. The latter were developed during a stay in the Rega Institute KU Leuven.
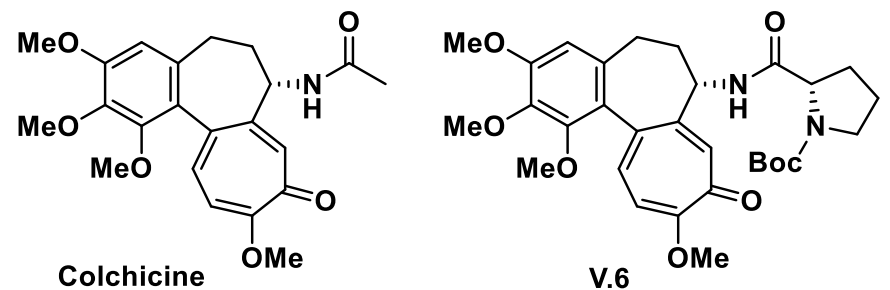

Figure 4.36. Structures of colchicine and V.6.

\subsubsection{Summary of preliminary in vitro studies}

Table 4.26 shows the results obtained in the cell proliferation assay, where $\mathrm{IC}_{50}$ and Selectivity Index (SI) values are presented for different cell lines. SI values are calculated by dividing the $\mathrm{IC}_{50}$ values of the non-tumor cell line (HEK293) by those of the corresonding tumor cell line. This parameter helps to estimate the possible selectivity of compouds for cancer cells in front of non cancer ones. Thus, a higher SI value indicates a higher therapeutic safety margin. V.6 offers $I_{50}$ values in the nanomolar range similar to colchicine, but it greatly improves the therapeutic safety reducing the toxicity properties associated to colchicine.

Table 4.26. $\mathrm{IC}_{50}(\mathrm{nM})$ and SI values for colchicine and V.6.

\begin{tabular}{llllllll}
\hline Compound & HT-29 & MCF-7 & A549 & HEK-293 & SIA $^{a^{a}}$ & SI $^{{ }^{b}}$ & SIC $^{\mathbf{c}}$ \\
\hline Colchicine & $50 \pm 3$ & $12 \pm 7$ & $12.2 \pm 0.7$ & $5 \pm 1$ & 0.1 & 0.4 & 0.4 \\
V.6 & $80 \pm 3$ & $44 \pm 6$ & $38 \pm 3$ & $150 \pm 10$ & 2 & 3 & 4 \\
\hline
\end{tabular}

${ }^{\mathrm{a} S I_{A}}=\mathrm{IC}_{50}(\mathrm{HEK}-293) / \mathrm{IC}_{50}(\mathrm{HT}-29) .{ }^{\mathrm{b}} \mathrm{SI}_{\mathrm{B}}=\mathrm{IC}_{50}(\mathrm{HEK}-293) / \mathrm{IC}_{50}(\mathrm{MCF}-7)$.

${ }^{c} S I_{C}=I C_{50}(H E K-293) / I C_{50}($ A549). Data are the average $( \pm S D)$ of three experiments. 
Table 4.27 shows the tubulin critical concentration $(\mathrm{CrC})$ in the presence of colchicine and V.6. It $t_{50}$ values are also presented, which correspond to the time needed to reach $50 \%$ of the polymerization equilibrium. Thus, although V.6 is less effective than colchicine in inhibiting tubulin polymerization, it is able to delay the nucleation and elongation process.

Table 4.27. $\mathrm{CrC}(\mu \mathrm{M})$ and $\mathrm{It}_{50}(\mathrm{~min})$ values for colchicine and V.6.

\begin{tabular}{lll}
\hline Compound & $\mathbf{C r C}(\boldsymbol{\mu M})$ & It $_{50}(\mathrm{~min})$ \\
\hline Control & $9 \pm 2$ & $16 \pm 1$ \\
Colchicine & $24 \pm 1$ & - \\
V. 6 & $13 \pm 1$ & $22 \pm 1$ \\
\hline Data are the average $( \pm S \mathrm{SD})$ of three experiments.
\end{tabular}

Table 4.28 depicts the cell distribution results obtained after cell cycle evaluation assay. Concretely, V.6 causes interruption of the cell cycle at the $\mathrm{G}_{2} / \mathrm{M}$ phase when tested at $0.1 \mu \mathrm{M}$ concentration provoking the arrest of the half of cell population.

Table 4.28. Cell cycle distribution (\%).

\begin{tabular}{llllll}
\hline Compound & Conc. $(\mu \mathrm{M})$ & Sub G $_{0}$ & $\mathrm{G}_{0} / \mathrm{G}_{1}$ & $\mathbf{S}$ & $\mathrm{G}_{2} / \mathbf{M}$ \\
\hline Control & - & $2 \pm 1$ & $73 \pm 3$ & $15 \pm 6$ & $11 \pm 4$ \\
Colchicine & 0.050 & $3 \pm 1$ & $27 \pm 14$ & $11 \pm 2$ & $59 \pm 17$ \\
V.6 & 0.1 & $3 \pm 1$ & $35 \pm 2$ & $15 \pm 2$ & $47 \pm 5$ \\
\hline \multicolumn{5}{r}{ Data are the average $( \pm \mathrm{SD})$ of three experiments. }
\end{tabular}

Figure 4.37 represents the percentage of gene expression in HT-29 cell line after $48 \mathrm{~h}$ treatment with colchicine and V.6 at their minimal active concentration ( $40 \mathrm{nM}$ and $80 \mathrm{nM}$, respectively). Gene expression was normalized using $\beta$-actin as endogenous gene. Control corresponds to $100 \%$ of gene expression. Interestingly, compound V.6 is able to down-regulate the expression of the three tested genes, improving the effect shown by colchicine in VEGF and hTERT gene expression. 


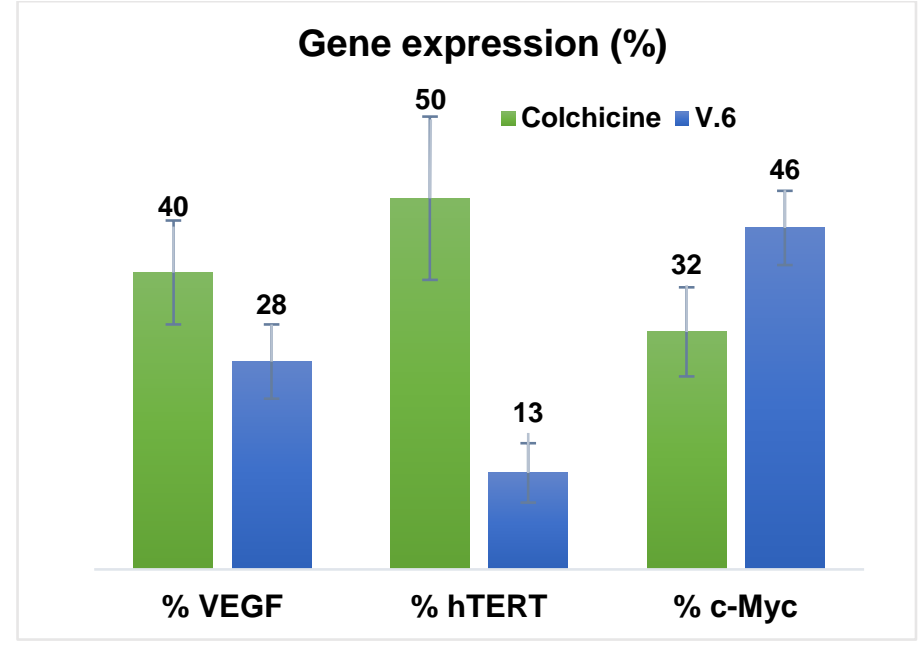

Figure 4.37. Gene expression (\%) of VEGF, hTERT and c-Myc. Data are the average $( \pm S D)$ of three experiments.

V.6 exerts a decrease in the expression of oncogenes involved in tumor aggressiveness at a concentration in which there is no antimitotic effect. Therefore, it can be concluded that compound V.6 could offer the possibility to be used in cancer therapy with lower dosages and less acute toxicity problems than in the case of colchicine.

\subsubsection{In vivo evaluation}

Given the good in vitro results obtained by compound V.6, it was decided to carry out an in vivo study to determine its pharmacological action in more complex biological systems as are SCID (severe combined immunodeficiency) mice. First, $\mathrm{LD}_{50}$ (median lethal dose) was determined for V.6 and compared to colchicine's value. This parameter is used as a general indicator of a substance's acute toxicity, since it refers to the dose required to kill half the members of a tested population after a specific treatment. Thus, a lower $L D_{50}$ is indicative of higher toxicity. The results obtained were very promising since $L_{50}$ for $\mathbf{V} .6$ was $>100 \mathrm{mg} / \mathrm{kg}$ while $L_{50}$ for colchicine was $2.5 \mathrm{mg} / \mathrm{kg}$. Then, it was established a metastatic breast cancer xenograft mouse model employing MDA- 
MB-231 (LM2) cells expressing firefly luciferase. ${ }^{169}$ LM2 cells were orthotopically engrafted in the mammary fat pad of SCID mice and V.6 was injected intraperitoneally (i.p.) at $75 \mathrm{mg} / \mathrm{kg}$ at day 17, when tumours were around 100 $\mathrm{mm}^{3}$ in size, at day 21 and at day 25 (see figure 4.38).

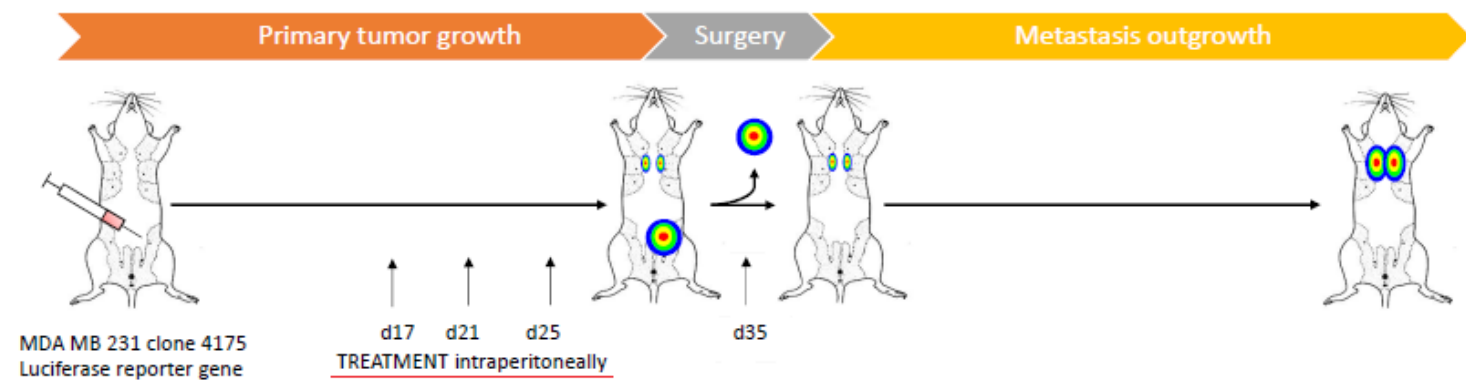

Figure 4.38. In vivo experimental scheme.

Tumor growth was measured by in vivo bioluminescent imaging (BLI) until day 35. By day 18, $24 \mathrm{~h}$ hours after starting the treatment, a dramatic decrease in luminescent signal was noted in the treated group whereas control tumors continued to grow (see figure 4.39.A). By day 20, treated tumors had slightly regrown but were significantly smaller than control tumors. Interestingly, already $2 \mathrm{~h}$ after treatment a significant decrease in BLI was observed, which is typically caused by the shutdown of blood flow after VDA treatment. This decrease was even more pronounced after $24 \mathrm{~h}$, whereas $72 \mathrm{~h}$ after treatment tumors had slightly regrown (see figure 4.39.B).

\footnotetext{
169 Hulpia, F.; Noppen, S. Schols, D.; Andrei, G.; Snoeck, R.; Liekens, S.; Vervaeke, P.;
} Calenbergh, S. Eur. J. Med. Chem. 2018, 157, 248-267. 
A

B
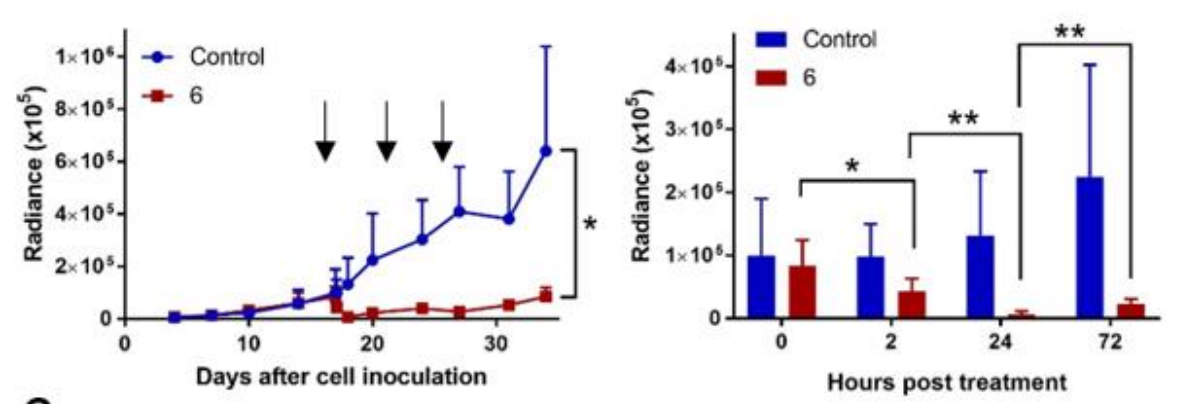

C

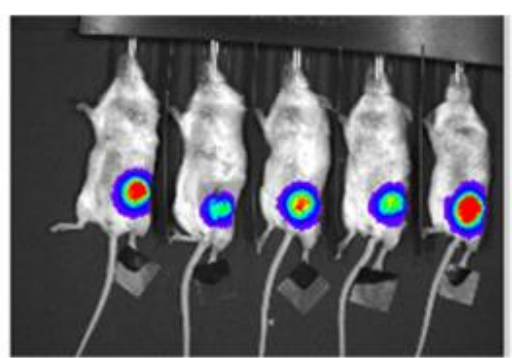

Control

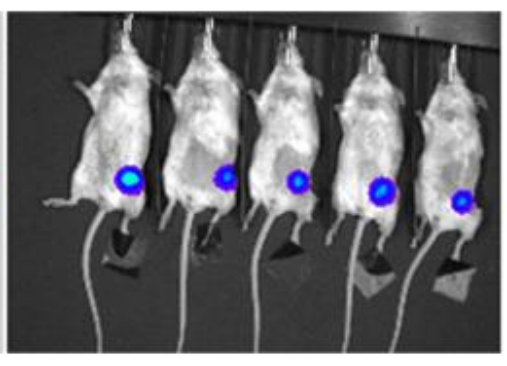

6

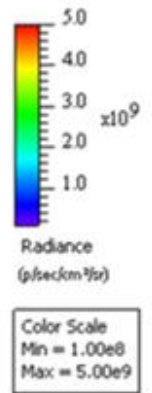

$\operatorname{Max}=5.00 \mathrm{eg}$

Figure 4.39. (A, B) Graphical representation of BLI signal over time emitted from the primary tumor. (C) Representative bioluminescence images at day 34. All data are mean $\pm \mathrm{SD}, \mathrm{n}=5 .{ }^{*} \mathrm{p}<0.05,{ }^{* *} \mathrm{p}<0.01$ (multiple t-test). Arrows indicate compound administration.

Calliper measurements also demonstrate that tumor growth was significantly retarded after treatment with V.6 (see figure 4.40.A). At day 35 after cell inoculation, primary tumors were removed and the measurement of tumor size also revealed a difference between control and treated groups. It was noted that tumors treated with V.6 were smaller and less vascularized (figure 4.40.B).

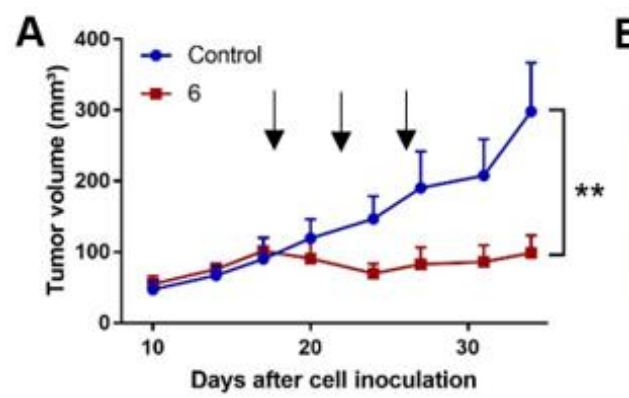

B

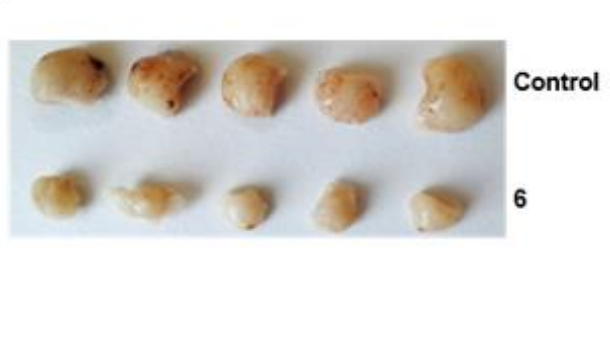

Figure 4.40. (A) Graphical representation of primary tumor volume evolution. (B) Pictures of dissected tumors after mice sacrifice at day 35. All data are mean $\pm S D, n=$ 5. ${ }^{* *} \mathrm{p}<0.01$ (multiple t-test). Arrows indicate compound administration. 
To assess the effect of V.6 on metastasis, primary tumours were covered with black paper and BLI from the lungs was measured (see figure 4.41). No significant difference between control and V.6 was observed at day 34. Thus, compound V.6 has no effect on the dissemination of cancer cells from the primary tumour towards the lungs. Previous reports ${ }^{170}$ already showed that micrometastatic tumours are resistant to antiangiogenic therapy because no tumour vasculature is established yet and tumour cells can benefit from existing vasculature.
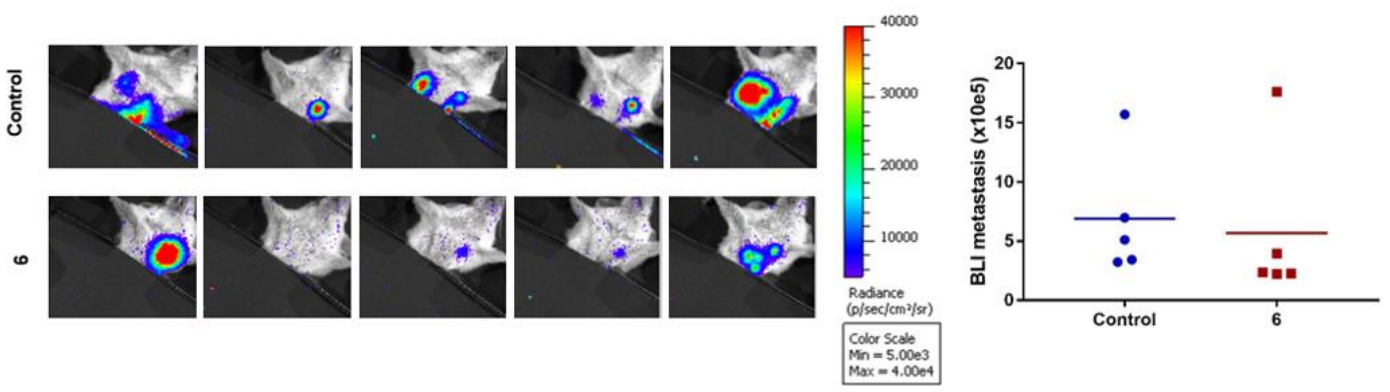

Figure 4.41. Lung metastasis quantification at day 34 after shielding the primary tumor.

In general, results obtained in the in vivo study demonstrate that V.6 impairs tumor growth but not metastasis when administered i.p. in SCID mice. Thus, in an early stage of tumor development, V.6 exerts a potent antitumor effect and low toxicity placing it as promising anticancer agent.

170 Welti, J.; Loges, S.; Dimmeler, S.; Carmeliet, P. J. Clin. Invest. 2013, 123, 3190-3200. 


\subsection{Experimental section}

\subsubsection{Docking studies}

Molecular docking was performed using Autodock 4.2. The crystal structures of VEGFR-2 kinase domain (PDB ID: 3EWH) and PD-L1 (PDB ID: 4ZQK) were used as templates. Ligands were previously removed from the protein structures in order to perform docking simulation. Discovery Studio Visualizer program ${ }^{171}$ was used to build the structures of the ureas U.1-U.26. The cluster was compared on the basis of the free energy of binding. The Lamarckian genetic algorithm (LGA) was employed with the default parameters; g_eval was set to 2500000 (medium) and 100 LGA runs were conducted. Molecular graphics were done with PYMOL (PyMOL Molecular Graphics System, version 1.6).

\subsubsection{Cell culture}

Cell culture media were purchased from Gibco (Grand Island, NY). Fetal bovine serum (FBS) was obtained from Harlan-Seralab (Belton, U.K.). Supplements and other chemicals not listed in this section were obtained from Sigma Chemical Co. (St. Louis, MO). Plastics for cell culture were supplied by Thermo Scientific BioLite. All tested compounds were dissolved in DMSO at a concentration of $10 \mathrm{mM}$ and stored at $-20^{\circ} \mathrm{C}$ until use.

HT-29, MCF-7, HeLa, A549, MDA-MB-231, HL-60 and HEK-293 cell lines were maintained in Dulbecco's modified Eagle's medium (DMEM) containing glucose $(1 \mathrm{~g} / \mathrm{L})$, glutamine $(2 \mathrm{mM})$, penicillin $(50 \mu \mathrm{g} / \mathrm{mL})$, streptomycin $(50$ $\mu \mathrm{g} / \mathrm{mL})$, and amphotericin B $(1.25 \mu \mathrm{g} / \mathrm{mL})$, supplemented with $10 \% \mathrm{FBS}$. HMEC1 cell line was maintained in Dulbecco's modified Eagle's medium (DMEM)/Low glucose containing glutamine $(2 \mathrm{mM})$, penicillin $(50 \mu \mathrm{g} / \mathrm{mL})$, streptomycin $(50$ $\mu \mathrm{g} / \mathrm{mL})$, and amphotericin B $(1.25 \mu \mathrm{g} / \mathrm{mL})$, supplemented with $10 \%$ FBS. For the

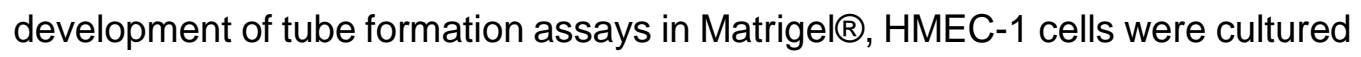
in EGM-2MV Medium supplemented with EGM-2MV SingleQuots.

171 Dassault Systèmes BIOVIA, Discovery Studio Visualizer, v16.1.0.15350, San Diego: Dassault Systèmes, 2015. 


\subsubsection{Cell proliferation assay}

In 96-well plates, $3 \times 10^{3}$ (HeLa, A549, HMEC-1, HEK-293), $5 \times 10^{3}$ (HT-29, MCF-7) or $1 \times 10^{4}$ (HL-60) cells per well were incubated with serial dilutions of the tested compounds in a total volume of $100 \mu \mathrm{L}$ of their respective growth media. The 3-(4,5-dimethylthiazol-2-yl)-2,5-diphenyltetrazolium bromide (MTT; Sigma Chemical Co.) dye reduction assay was used, as previously described ${ }^{172}$. After 2 days of incubation ( $37^{\circ} \mathrm{C}, 5 \% \mathrm{CO}_{2}$ in a humid atmosphere), $10 \mu \mathrm{L}$ of MTT (5 mg/mL in phosphate-buffered saline, PBS) were added to each well, and the plate was incubated for a further $3 \mathrm{~h}\left(37^{\circ} \mathrm{C}\right)$. For adherent cells, the supernatant was discarded and replaced by $100 \mu \mathrm{L}$ of DMSO to dissolve the formazan crystals. For non-adherent cells (HL-60), plates were centrifugated at $800 \mathrm{rpm}$ for $5 \mathrm{~min}$ before discarding the supernatant. The absorbance was then read at $540 \mathrm{~nm}$ by spectrophotometry. Three independent experiments were performed, and the $\mathrm{IC}_{50}$ values were graphically determined using GraphPad Prism 4 software.

In the case of MDA-MB-231 cells, $1 \times 10^{4}$ cells per well were seeded in 48well plates in $1 \mathrm{~mL}$ of growth medium. One day later, 5 -fold dilutions of the compounds were added. After 3 days of incubation, cells were trypsinized and counted in a Coulter counter (Rega Institute for Medical Research, KU Leuven). The $\mathrm{IC}_{50}$ value was determined as the compound concentration required to reduce cell proliferation by $50 \%$.

\subsubsection{Tubulin self-assembly assay}

Purified tubulin ${ }^{173}$ was used for these measurements. In a 96 well-plate, 50 $\mu \mathrm{L}$ of tubulin solution ( $25 \mu \mathrm{M}$ in GAB buffer) were added to $50 \mu \mathrm{L}$ of $27.5 \mu \mathrm{M}$ solution of the corresponding compounds in $\mathrm{GAB}$ buffer $\left(20 \mathrm{mM} \mathrm{Na}_{3} \mathrm{PO}_{4}, 10 \mathrm{mM}\right.$ $\mathrm{MgCl}_{2}, 1 \mathrm{mM}$ EGTA, 30\% glycerol) and $0.1 \mathrm{mM}$ GTP at pH 6.5. Then, the plate

\footnotetext{
172 Rodríguez-Nieto, S.; Medina, M. A.; Quesada, A. R. Anticancer Res. 2011, 21, 3457-3460.

173 See experimental procedure in: Andreu, J. M. Methods Mol. Med. 2007, 137, 17-28.
} 
was incubated at $37^{\circ} \mathrm{C}$ in Multiskan $\AA$ and absorbance was measured at $340 \mathrm{nM}$ and registered every $30 \mathrm{sec}$ during $2 \mathrm{~h}$.

\subsubsection{EBI assay}

MDA-MB-231 cells were seeded in 6 -well plates at $5 \times 10^{5}$ cells per well. After $48 \mathrm{~h}$, compounds were added to the cells for $16 \mathrm{~h}$ before adding $\mathrm{EBI}$ ( $\mathrm{N}$, $\mathrm{N}$ '-ethylene-bis(iodoacetamide)) at $100 \mu \mathrm{M}$. After $1.5 \mathrm{~h}$, cells were harvested and cell extracts were prepared for Western blot analysis. $30 \mu \mathrm{g}$ of proteins were subjected to gel electrophoresis using $0.1 \%$ SDS (85\% purity) and $10 \%$ polyacrylamide gels. After electrophoresis, proteins were transferred to pretreated Hybond-P polyvinylidene difluoride (PVDF) membranes, which were incubated overnight at $4^{\circ} \mathrm{C}$ in blocking buffer $(2.5 \%$ non-fat dry milk in PBS containing $0.1 \%$ Tween) and, subsequently, for $1 \mathrm{~h}$ at $\mathrm{r}$. $\mathrm{t}$. in blocking buffer primary antibody raised against $\beta$-tubulin. After washing, membranes were incubated with the corresponding HRP-conjugated secondary antibody in blocking buffer for 30 min at r. t. Next, membranes were washed extensively and immunoreactive proteins were detected by chemiluminiscence (ECLplus, BioRad).

\subsubsection{Cell cycle analysis}

Progression of the cell cycle was analysed by means of flow cytometry with propidium iodide. After incubation with compounds for $24 \mathrm{~h}$, A549 cells were fixed, treated with RNase and stained with propidium iodide following instructions of BD Cycletest ${ }^{\mathrm{TM}}$ DNA Kit. Analysis was performed with a BD Accuri $^{\mathrm{TM}} \mathrm{C} 6$ flow cytometer.

\subsubsection{Microtubule network study by immunofluorescence}

Immunofluorescent analysis of the microtubule network was performed on the A549 cell line. In this assay, $1.5 \times 10^{5}$ cells were plated on a coverglass and incubated with the different concentrations of selected compounds for $16 \mathrm{~h}$. Cells were then washed with PEMP, permeabilized with PEM-Triton X-100 0.5\% for $90 \mathrm{sec}$ at r. t. and fixed with 3.7\% formaldehyde (in PEM pH 7.4) for $30 \mathrm{~min}$ at $r$. 
t. Direct immunostaining was carried out for $2.5 \mathrm{~h}$ at $37^{\circ} \mathrm{C}$ in darkness with primary FITC-conjugated anti- $\alpha$-tubulin antibody (dilution 1:400 in PBS-BSA 1\% from a $1 \mathrm{mg} / \mathrm{mL}$ solution; monoclonal antibody, clone DM1A, Sigma-Aldrich). Next, cells were washed with PBS and incubated for $30 \mathrm{~min}$ at $r$. t. in darkness with Hoechst $2 \mathrm{mM}$ in water. Then, cells were washed in PBS and coverglasses were mounted with $10 \mu \mathrm{L}$ of Glycine/Glycerol buffer. The cytoskeleton was imaged by a confocal laser scanning microscope (CLSM) Leica SP5 with a Leica inverted microscope, equipped with a Plan-Apochromat $63 \times$ oil immersion objective (NA=1.4). Each image was recorded with the CLSM's spectral mode selecting specific domains of the emission spectrum. The FITC fluorophore was excited at $488 \mathrm{~nm}$ with an argon laser and its fluorescence emission was collected between $496 \mathrm{~nm}$ and $535 \mathrm{~nm}$.

\subsubsection{Apoptosis assay}

Apoptosis was determined by quantifying FITC-Annexin $\mathrm{V}$ translocation by means of flow cytometry. A549 cells were incubated with compounds for $24 \mathrm{~h}$ and then stained following instructions of BD Apoptosis Detection ${ }^{\mathrm{TM}}$ Kit. Analysis was performed with a BD Accuri ${ }^{\mathrm{TM}} \mathrm{C} 6$ flow cytometer.

\subsubsection{Tube destruction assay}

Wells of a 96-well $\mu$-plate for angiogenesis were coated with $12 \mu \mathrm{L}$ of Matrigel ${ }^{\circledR}(10 \mathrm{mg} / \mathrm{mL}, \mathrm{BD}$ Biosciences $)$ at $4^{\circ} \mathrm{C}$. After gelatinization at $37^{\circ} \mathrm{C}$ for 30 min, HMEC- 1 cells were seeded at $2 \times 10^{4}$ cells/well in $35 \mu \mathrm{L}$ of culture medium on top of the Matrigel $\AA^{\circledR}$. After $20 \mathrm{~h}$ of incubation at $37^{\circ} \mathrm{C}$, when tube-like structures were detectable, compounds were added at different concentrations. Next, 4 h later, tube destruction was evaluated by giving a score from 0 to 3 (3: intact tubular network as seen in the control, 2: missing connections and/or dead ends, 1: many separate small tubes that are not connected, 0: no tubes). 


\subsubsection{Tube formation inhibition assay}

Wells of a 96-well $\mu$-plate for angiogenesis were coated with $12 \mu \mathrm{L}$ of Matrigel $(10 \mathrm{mg} / \mathrm{mL}, \mathrm{BD}$ Biosciences $)$ at $4^{\circ} \mathrm{C}$. After gelatinization at $37^{\circ} \mathrm{C}$ for 30 min, HMEC- 1 cells were seeded at $2 \times 10^{4}$ cells/well in $35 \mu \mathrm{L}$ of culture medium on top of the Matrigel ${ }^{\circledR}$ and were incubated 30 min at $37^{\circ} \mathrm{C}$ while are attached. Then, compounds were added dissolved in $35 \mu \mathrm{L}$ of culture medium and after $20 \mathrm{~h}$ of incubation at $37^{\circ} \mathrm{C}$, tube destruction was evaluated by giving a score from 0 to 3 (3: intact tubular network as seen in the control, 2: missing connections and/or dead ends, 1: many separate small tubes that are not connected, 0: no tubes).

\subsubsection{Ligand-VEGFR-2 direct interaction measurement}

Compounds were incubated at $30 \mu \mathrm{M}$ with Lyophilized Human VEGFR-2 Protein Standard (103 pg/ml) for $30 \mathrm{~min}$ at $\mathrm{r}$. t. Then, the amount of free VEGFR2 was quantified following the manufacturer's instructions of Human VEGFR-2 ELISA Kit (Sigma-Aldrich).

\subsubsection{VEGFR-2 quantification by flow cytometry}

VEGFR-2 was determined quantifying Alexa Fluor® 647 Mouse Anti-Human CD309 (VEGFR-2) by means of flow cytometry. To detect membrane VEGFR2 , cells were incubated with compounds for $24 \mathrm{~h}$ and then they were collected, fixed and stained with Alexa Fluor® 647 Mouse Anti-Human CD309 (VEGFR2). For the detection of total VEGFR-2 (membrane and cytosolic), cells were incubated with compounds for $24 \mathrm{~h}$, then lysates were obtained and stained with Alexa Fluor® 647 Mouse Anti-Human CD309 (VEGFR-2).

\subsubsection{VEGFR-2 quantification by immunofluorescence}

A549 cells were plated on a coverglass and incubated with the different compounds for $24 \mathrm{~h}$. Then, they were permeabilized with PEM-Triton-X-100 $0.5 \%$ and fixed with $3.7 \%$ formaldehyde. Direct immunostaining was carried out for $2 \mathrm{~h}$ at $37^{\circ} \mathrm{C}$ in darkness with Alexa Fluor ${ }^{\circledR} 647$ Mouse Anti-Human CD309 
(VEGFR-2). Then, cells were washed in PBS and coverglasses were mounted with $10 \mu \mathrm{L}$ of Glycine/Glycerol buffer.

Images were obtained by a confocal laser scanning microscope (CLSM) Leica SP5 with a Leica inverted microscope, equipped with a Plan-Apochromat $63 x$ oil immersion objective $(N A=1.4)$. Each image was recorded with the CLSM's spectral mode selecting specific domains of the emission spectrum. Alexa Fluor ${ }^{\circledR 6} 67$ fluorophore was excited at $650 \mathrm{~nm}$ with an argon laser and its fluorescence emission was collected at $665 \mathrm{~nm}$.

\subsubsection{4. phospho-VEGFR-2 quantification by ELISA}

For endothelial cells (HMEC-1): $5 \cdot 10^{5}$ cells/well were seeded in 6-well plates and once they were at $80 \%$ of their confluency, they were starved with medium containing $0.1 \%$ of FBS for $24 \mathrm{~h}$. Then, cells were incubated with the corresponding compounds at $10 \mu \mathrm{M}$ for $24 \mathrm{~h}$ and next cells were stimulated with $100 \mathrm{ng} / \mathrm{ml}$ of Recombinant VEGF-165 for $30 \mathrm{~min}$ at $37^{\circ} \mathrm{C}$. After that, lysates were collected, protein quantification was carried out by Bradford test ${ }^{174}$ and, then, phospho-VEGFR-2 was quantified using PathScan ${ }^{\circledR}$ Phospho-VEGFR2(Tyr1175) Sandwich ELISA Kit according to the manufacturer's instructions.

For tumor cells (A549): $3 \cdot 10^{5}$ cells/well were seeded in 6-well plates and were incubated with the corresponding compounds at $10 \mu \mathrm{M}$ for $30 \mathrm{~min}$. After that, lysates were collected, protein quantification was carried out by Bradford test and, then, phospho-VEGFR-2 was quantified using PathScan ${ }^{\circledR}$ PhosphoVEGFR-2(Tyr1175) Sandwich ELISA Kit according to the manufacturer's instructions.

\subsubsection{5. phospho-Erk1/2 quantification by Western blot}

From the lysates extracted in the previous experiment, $80 \mu \mathrm{g}$ of proteins were subjected to gel electrophoresis using Bolt 4-12\% Bis-Tris plus gels. Then, proteins were transferred to Hybond-P polyvinylidene difluoride (PVDF)

174 Bradford, M. M. Anal. Biochem. 1976, 72, 248-254. 
membranes using the iBlot gel transfer system, and the resulting membranes were incubated first for $1 \mathrm{~h}$ at $\mathrm{r}$. t. in blocking buffer (5\% non-fat dry milk in TBS $1 \times$ containing $0.1 \%$ Tween) and subsequently overnight at $4 \stackrel{\circ}{\circ}$ in TBST buffer primary antibody solution (Anti-ERK1+ERK2 phosphoT202+T204). After washing, membranes were incubated with the corresponding HRP-conjugated secondary antibody in blocking buffer for $1 \mathrm{~h}$ at $\mathrm{r}$. t. Next, membranes were washed extensively and immunoreactive proteins were detected by chemiluminescence (ImageQuant LAS500).

\subsubsection{PD-L1 and C-Myc quantification by ELISA}

A549 cells were seeded $\left(3 \cdot 10^{5}\right.$ cells/well) in 6 -well plates and were incubated with the corresponding compounds for $24 \mathrm{~h}$. After that, lysates were collected, protein quantification was carried out by Bradford test and, then, PD-L1 and CMyc were quantified using Human PD-L1 ELISA Kit 28-8 (ab214565) and c-Myc (Total) Human ELISA Kit (KHO2041), respectively, according to the manufacturer's instructions.

\subsubsection{PD-L1 and C-Myc determination by immunofluorescence}

A549 cells were plated on a coverglass and incubated with the different compounds for $24 \mathrm{~h}$. Then, they were permeabilized with PEM-Triton-X-100 $0.5 \%$ and fixed with $3.7 \%$ formaldehyde. Direct immunostaining was carried out for $2 \mathrm{~h}$ at $37^{\circ} \mathrm{C}$ in darkness with Anti-PD-L1-AlexaFluor ${ }^{\circledR 6} 67$ and Anti-C-MycFITC. Then, cells were washed in PBS and coverglasses were mounted with 10 $\mu \mathrm{L}$ of Glycine/Glycerol buffer. Images were obtained by a confocal laser scanning microscope (CLSM) Leica SP5 with a Leica inverted microscope, equipped with a Plan-Apochromat $63 x$ oil immersion objective $(N A=1.4)$. Each image was recorded with the CLSM's spectral mode selecting specific domains of the emission spectrum. Alexa Fluor $\AA_{647}$ fluorophore was excited at $650 \mathrm{~nm}$ with an argon laser and its fluorescence emission was collected at $665 \mathrm{~nm}$. FITC fluorophore was excited at $488 \mathrm{~nm}$ with an argon laser and its fluorescence emission was collected between $496 \mathrm{~nm}$ and $535 \mathrm{~nm}$. 


\subsubsection{Cell proliferation evaluation in co-cultures}

In 6-well plates, $5 \times 10^{4}$ tumor cells/well were seeded and incubated for 24 $\mathrm{h}$ with the tested compounds at $200 \mu \mathrm{M}$ in a total volume of $1.5 \mathrm{~mL}$ in the presence of $2 \times 10^{5}$ Jurkat T cells/well. Then, supernatant was discarded and tumor cells were collected with trypsin and counted using the Neubauer chamber.

\subsubsection{In vivo experiments}

Human metastatic breast cancer xenografts were established as previously described $^{169}$. The luciferase-positive LM2 lung metastatic cell line (MDA-MB-231 clone 4715) was a kind gift of Prof. Massagué ${ }^{175}$. Female severe combined immunodeficient (SCID) mice were used at the age of 8 weeks. $10^{6}$ LM2 cells were suspended in 50\% Matrigelß (BD Biosciences) in PBS and injected in the mammary fat pad of anesthetized SCID mice. When tumors were around 100 $\mathrm{mm}^{3}$ in size, compound V.6 was injected intraperitoneally (i.p.) at $75 \mathrm{mg} / \mathrm{kg}$ in PBS containing 5\% DMSO and $20 \%$ cremophor. Control mice received only $5 \%$ DMSO, 20\% cremophor in PBS. Tumor growth was measured with an IVIS Spectrum imaging system (Caliper Life Sciences, Hopkinton, MA, USA). Before imaging, mice were anesthetized and injected subcutaneously with $150 \mathrm{mg} / \mathrm{kg}$ luciferin. Images were recorded every $2 \mathrm{~min}$ and maximum radiance values (photons/sec) were retained. Lung metastasis was determined after shielding the primary tumor with a black paper. Tumor size was measured using a digital caliper and calculated with the following formula: tumor volume $\left(\mathrm{mm}^{3}\right)=0.5 a b^{2}$, where $a$ is the longest diameter and $b$ is the shortest diameter. All studies were done in compliance with the ethical guidelines for animal welfare of KU Leuven (P277/2015).

\footnotetext{
175 Minn, A. J.; Gupta, G. P.; Siegel, P. M.; Bos, P. D.; Shu, W.; Giri, D. D.; Viale, A.; Olshen, A. B.; Gerald, W. L.; Massagué, J. Nature 2005, 436, 518-524.
} 



\section{GENERAL CONCLUSIONS}





\section{GENERAL CONCLUSIONS}

- Synthesis and characterization of three families of compounds have been carried out based on the general structure of aminocombretastatin. The application of relatively simple synthetic sequences has resulted in the obtention of all the desired compounds with moderate yields. It is interesting to notice that from one unique general structure, it has been possible to design and obtain a wide variety of derivatives acting on different targets.

- Family 1: Compounds C.9 ( $m$-Br), C.10 (p-Br), C.12 (m-OMe) and C.25 (m$\mathrm{Me}, p-\mathrm{Cl})$ have offered the best biological activity as VDAs. They show high antiproliferative activity, good selectivity towards tumor cells, antimitotic properties and apoptotic effect. Concretely, C.12 ( $m$-OMe) has been selected as the most promising carbamate, since it improves AmCA-4 activity and predictions have revealed good bioavailabilty.

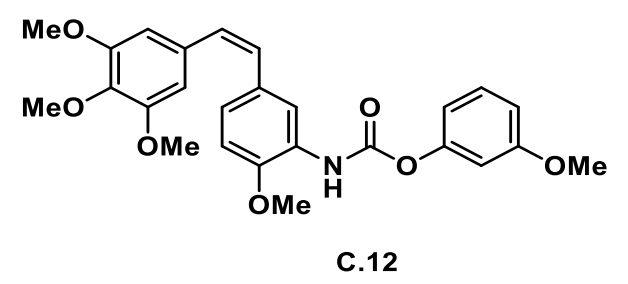

- Family 2: Compounds U.5 (o-Cl) and U.8 (o-Br) seem to be the best antiangiogenic derivatives. They exert antiproliferative activity at submicromolar level, antimitotic and apoptotic effect, inhibition of tubulogenesis and also VEGFR-2 kinase activity, improving the results provided by sorafenib.
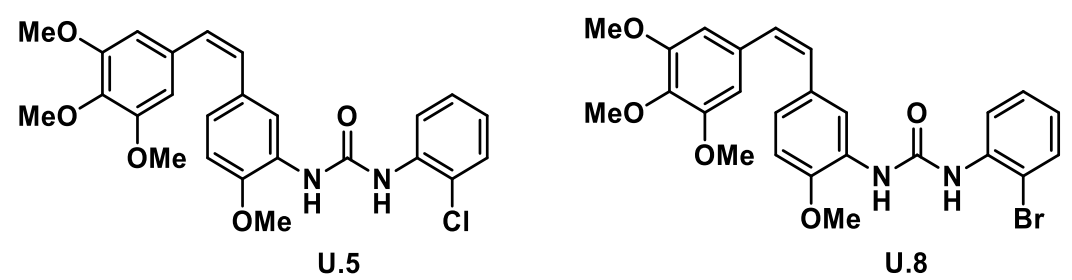
- Family 3: It can be concluded that the presence of chlorophenyl urea unit, as in compounds T.2 and S.6, is important for the dual action of these kind of derivatives as antiangiogenics and immunomodulators. Moreover, Estilbene $\mathbf{S . 6}$ is able to improve the biological effect provided by triazolyl-urea T.2 and reference compounds (sorafenib and BMS-8), since it exerts higher inhibitory effect at lower concentrations.<smiles>COc1ccc(/C=C/c2cccc(NC(=O)Nc3ccc(Cl)cc3)c2)cc1</smiles>

- $\quad$ The results obtained from the in vivo study convert colchicine derivative V.6 in a potential candidate for treatment of tumors in early stage of development. In order to impair also the metastatic process, it could be interesting to study the effect exerted by the antiangiogenics U.5 or U.8. Additionally, in order to avoid resistance phenomena, multitarget inhibitor S.6 could be a promising anti-cancer agent for ongoing in vivo studies. 


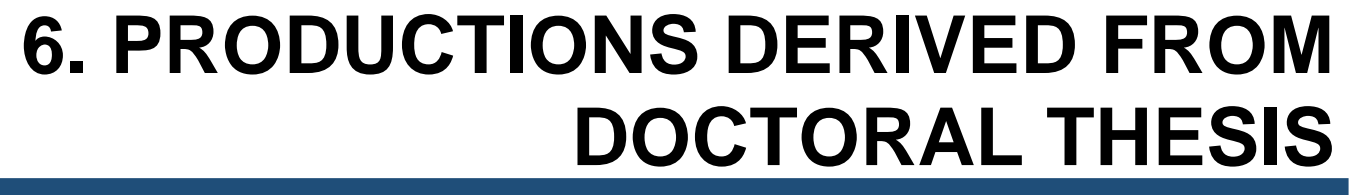





\section{PRODUCTIONS DERIVED FROM DOCTORAL THESIS}

\subsection{Congress communications}

- Poster: "Novel small-molecules showing both antiangiogenic and immunomodulatory properties”, Conesa-Milián, L.; Falomir, E.; Murga, J.; Carda, M.; Marco, J. A.; 54 ${ }^{\text {th }}$ International Conference on Medicinal Chemistry (RICT), Strasbourg (France), July 2018.

- Oral communication: "Synthesis and biological evaluation of novel anticancer drugs", Conesa-Milián, L.; Falomir, E.; Murga, J.; Carda, M.; Marco, J. A.; XXXVI Biennial Meeting of the Spanish Royal Society of Chemistry (RSEQ), Sitges (Spain), June 2017.

- Oral communication: "Biological evaluation of aminocombretastatin derivatives", Conesa-Milián, L.; Falomir, E.; Murga, J.; Carda, M.; Marco, J. A.; IV Symposium of Medicinal Chemistry Young Researchers (SEQT), Barcelona (Spain), May 2017.

- Poster: "Biological evaluation of combretastatin analogues based on sorafenib synthetic drug", Conesa-Milián, L.; Falomir, E.; Murga, J.; Carda, M.; Marco, J. A.; 52 ${ }^{\text {nd }}$ International Conference on Medicinal Chemistry (RICT), Caen (France), July 2016.

- Poster: "Synthesis and biological evaluation of aminocombretastatin derivatives", Conesa-Milián, L.; Agut, R.; Falomir, E.; Murga, J.; Carda, M.; Marco, J. A.; XXVI Biennial Meeting of the Spanish Royal Society of Organic Chemistry (RSEQ), Huelva (Spain), June 2016.

- Poster: "New antineoplastic candidates based on combretastatin natural product and sorafenib synthetic drug", Conesa-Milián, L.; Falomir, E.; Murga, J.; Carda, M.; Marco, J. A.; $3^{\text {rd }}$ Konstanz Symposium Chemical Biology, Konstanz (Germany), October 2015. 
- Poster: "Synthesis and biological evaluation of colchicine and aminocombretastatin derivatives", Agut, R.; Conesa-Milián, L.; MarzoMas, A.; Falomir, E.; Murga, J.; Carda, M.; Marco, J. A.; XXV Biennial Meeting of the Spanish Royal Society of Organic Chemistry (RSEQ), A Coruña (Spain), July 2015.

\subsection{Publications}

- "Synthesis and biological evaluation of carbamates derived from aminocombretastatin A-4 as vascular disrupting agents" Conesa-Milián, L.; Falomir, E.; Murga, J.; Carda, M.; Meyen, E.; Liekens, S.; Marco, J. A. European Journal of Medicinal Chemistry 2018, 147, 183-193.

- "Synthesis and biological evaluation as antiangiogenic agents of ureas derived from 3'-aminocombretastatin A-4" Conesa-Milián, L.; Falomir, E.; Murga, J.; Carda, M.; Marco, J. A. European Journal of Medicinal Chemistry 2019, 162, 781-792.

- "Novel multitarget inhibitors with antiangiogenic and immunomodulator properties" Conesa-Milián, L.; Falomir, E.; Murga, J.; Carda, M.; Marco, J. A. European Journal of Medicinal Chemistry 2019, 170, 87-98.

- "Trick or treat: amino acid conjugated colchicine derivatives as promising anticancer agents" Marzo-Mas, A.; Conesa-Milián, L.; Noppen, S.; Liekens, S.; Falomir, E.; Murga, J.; Carda, M.; Marco, J. A. (sent to Medicinal Chemistry). 
7. SELECTED SPECTRA 

O.1
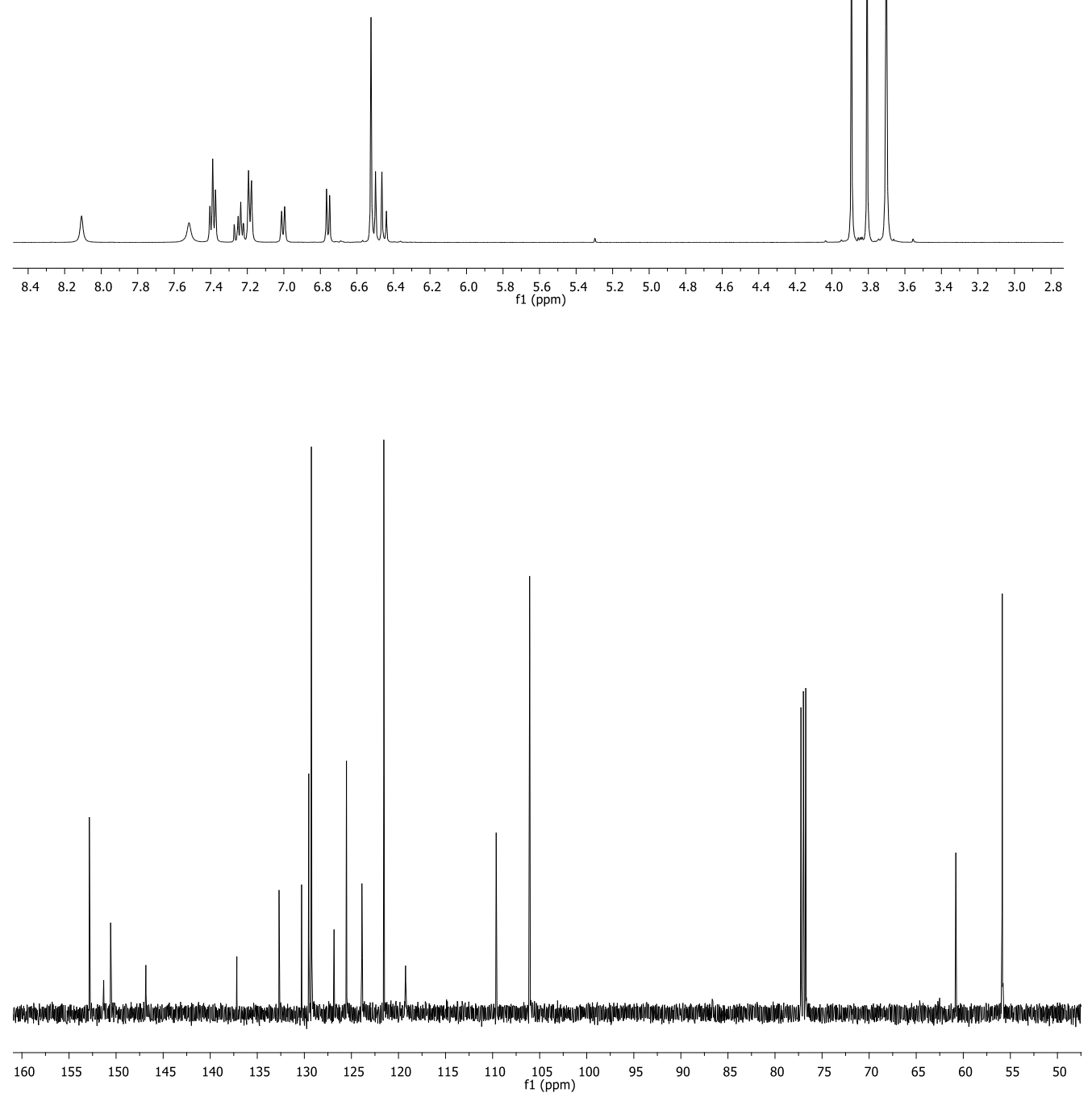
<smiles>COc1ccc(/C=C\c2ccc(OC)c(OC)c2)cc1NC(=O)Oc1ccccc1F</smiles>

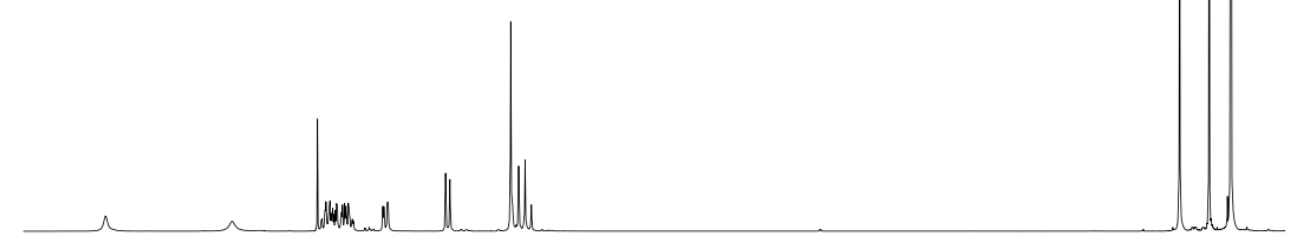

\begin{tabular}{lllllllllllllllllllllllllllllll}
\hline 8.4 & 8.2 & 8.0 & 7.8 & 7.6 & 7.4 & 7.2 & 7.0 & 6.8 & 6.6 & 6.4 & 6.2 & 6.0 & 5.8 & 5.6 & 5.4 & 5.2 & 5.0 & 4.8 & 4.6 & 4.4 & 4.2 & 4.0 & 3.8 & 3.6
\end{tabular}

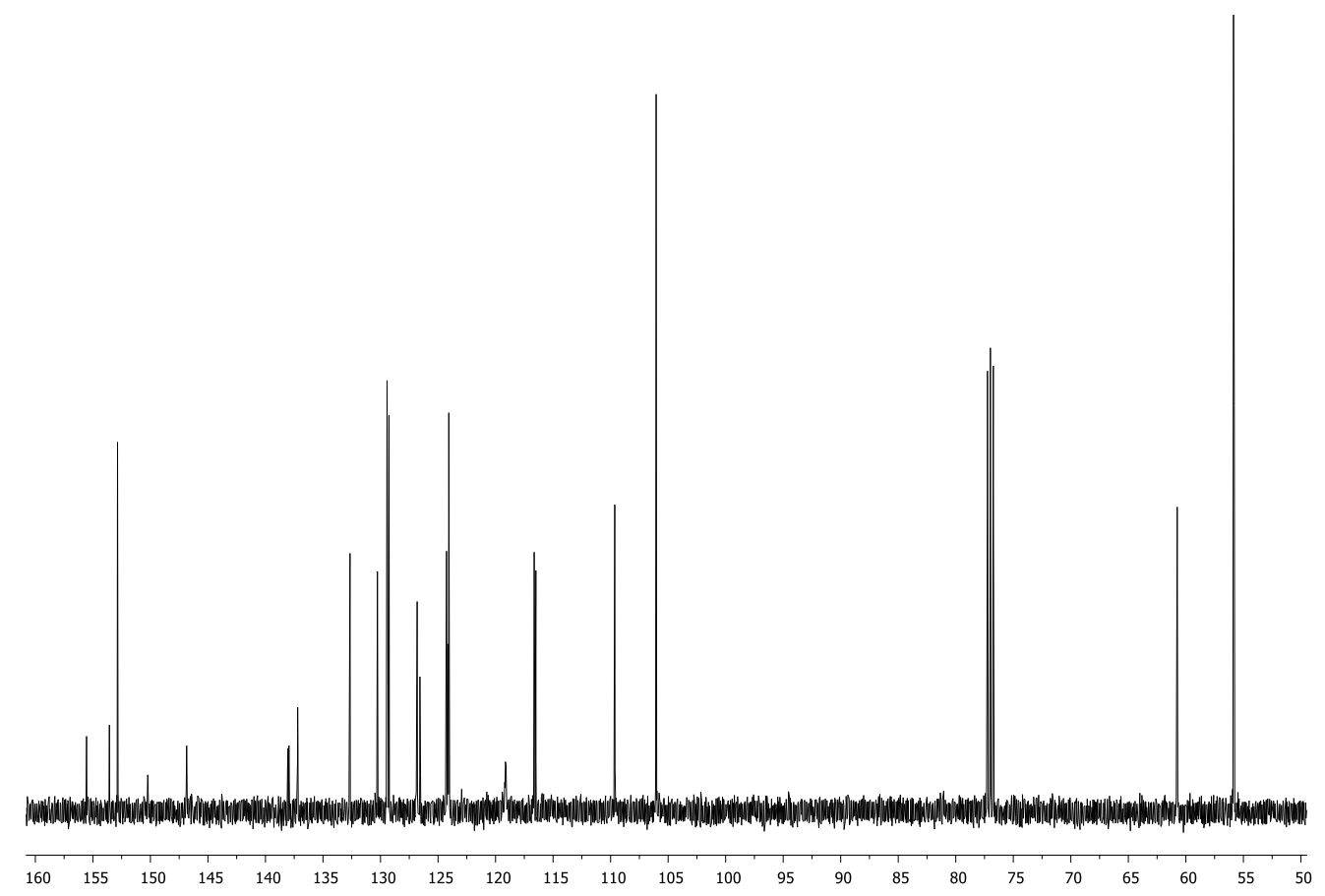



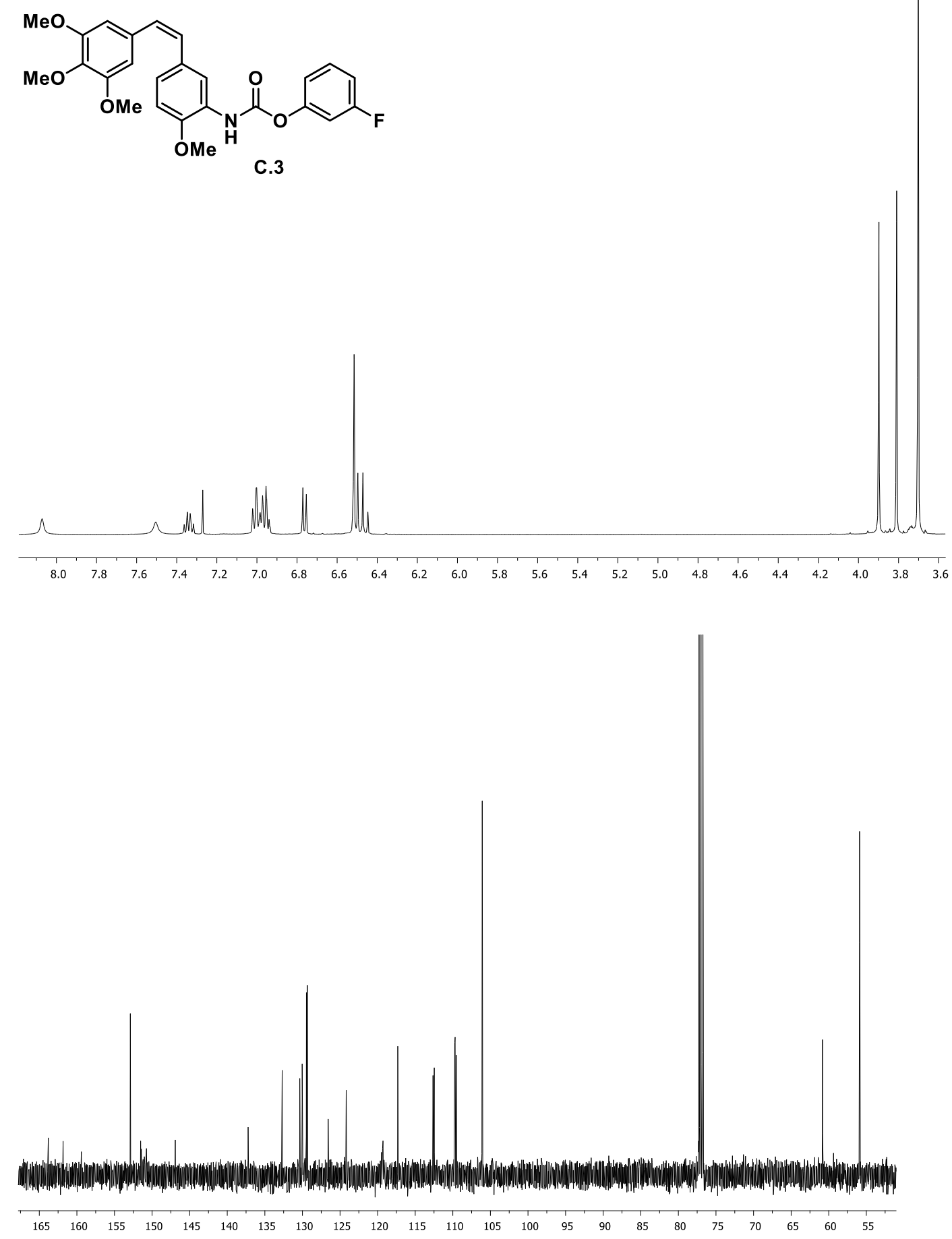
190

Chapter 7
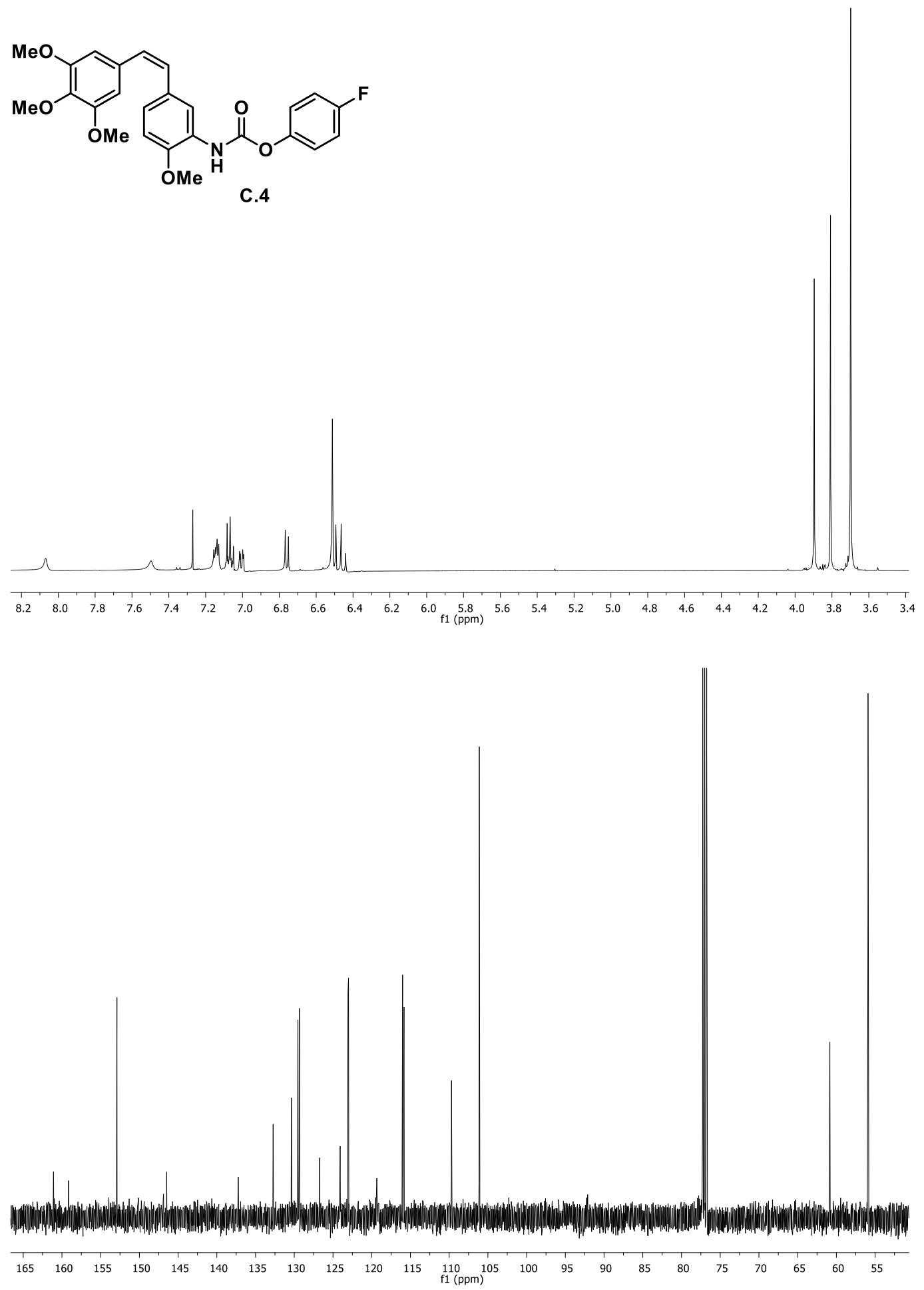

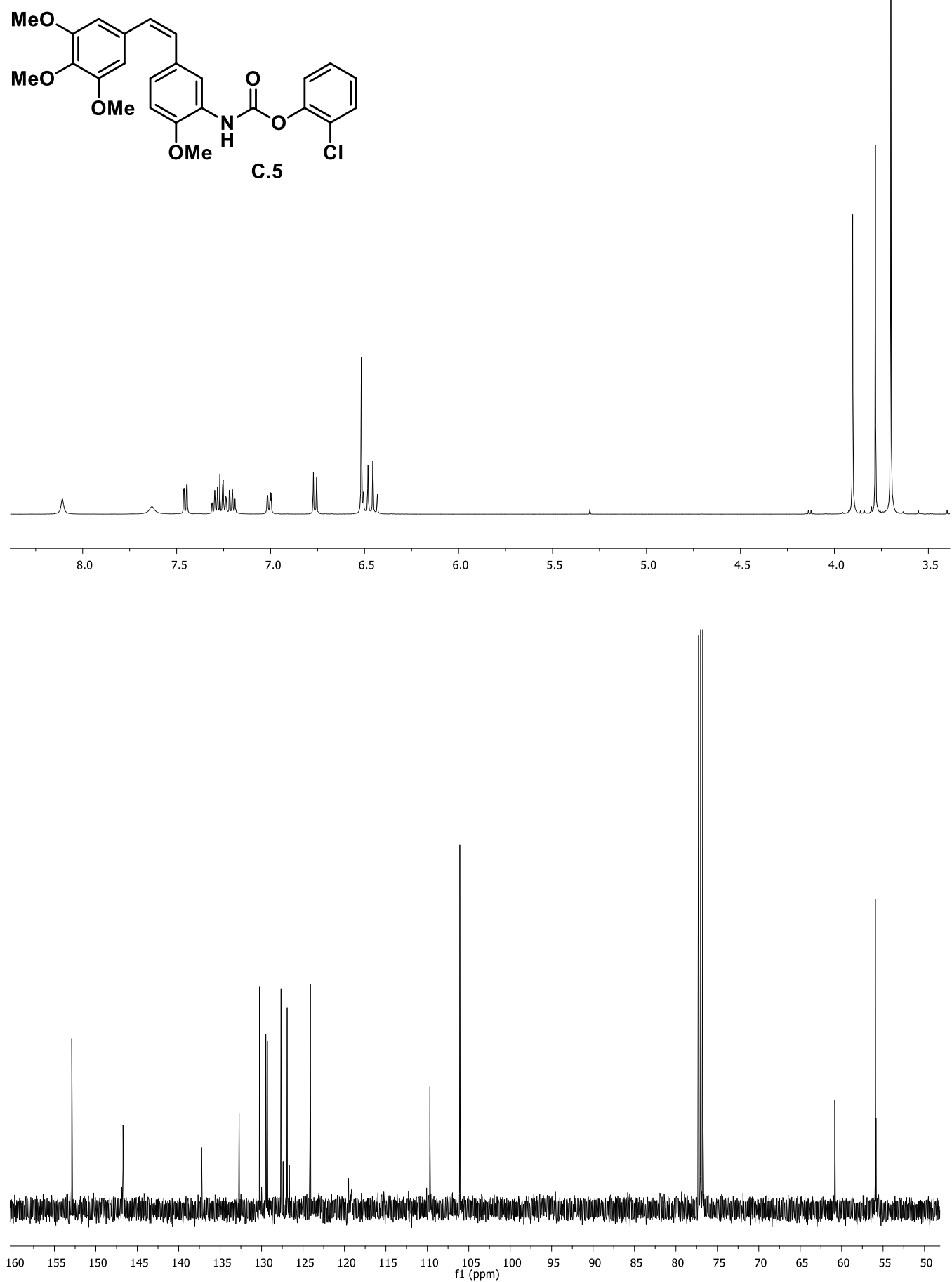

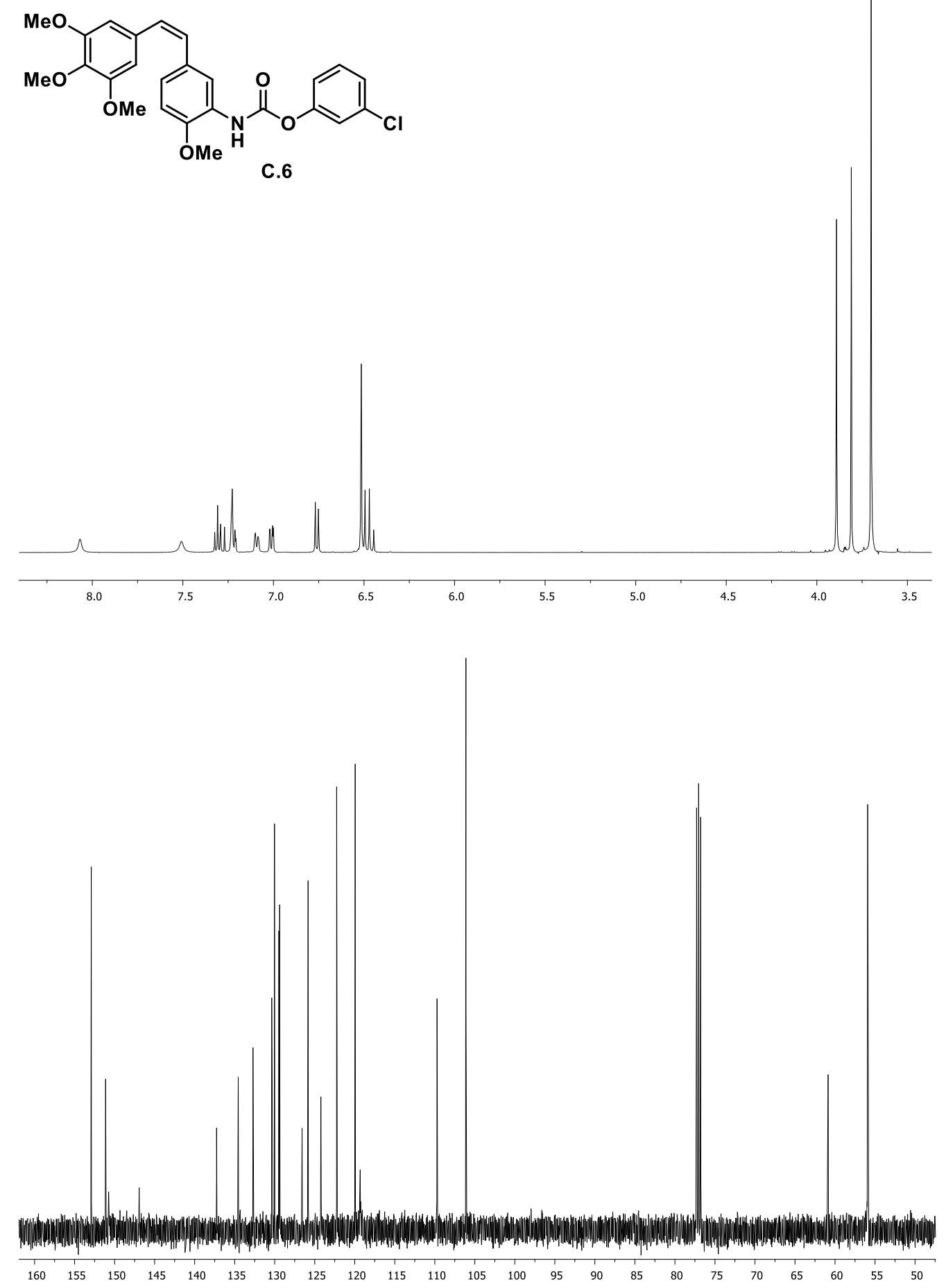

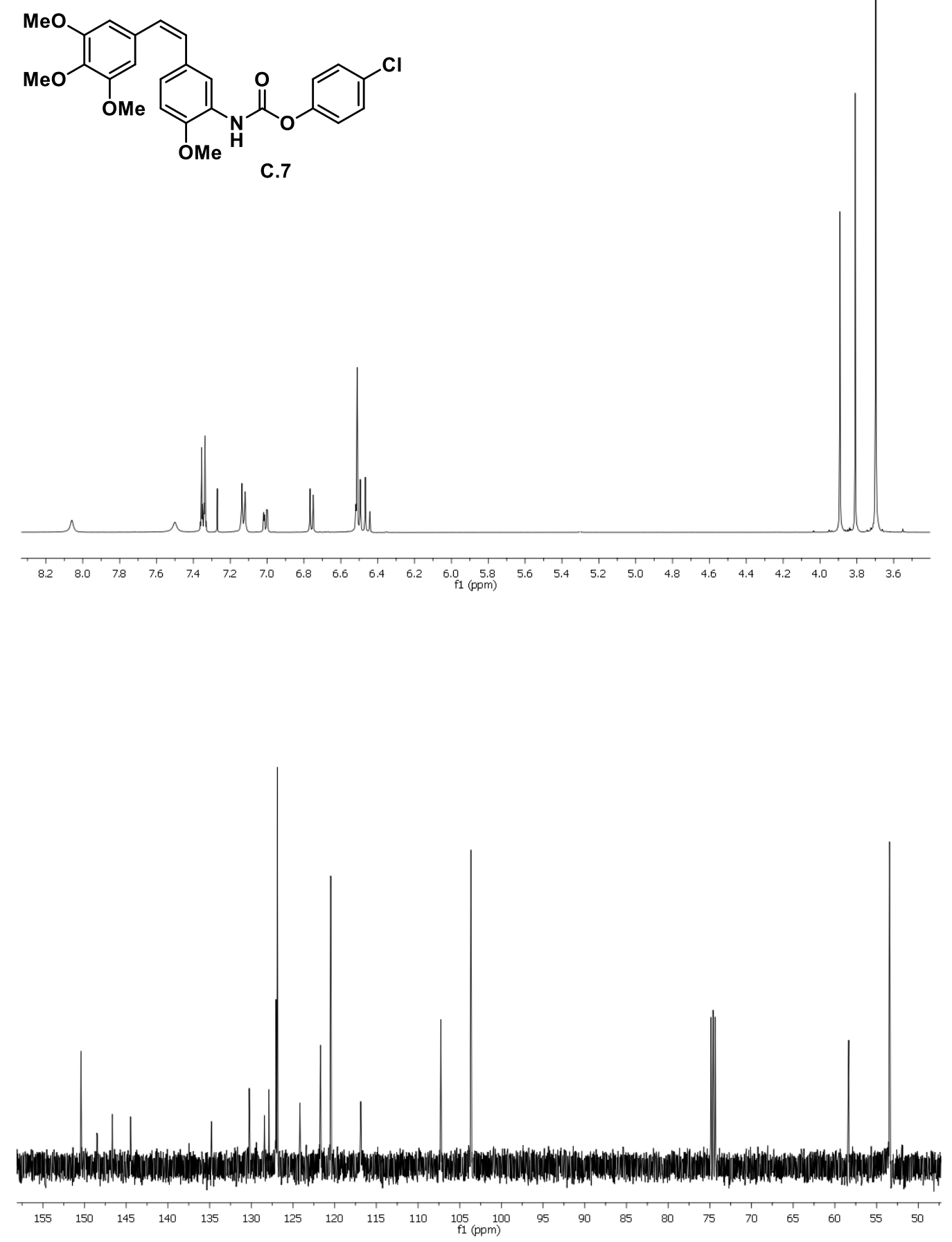
<smiles>COc1ccc(/C=C\c2cc(OC)c(OC)c(OC)c2)cc1NC(=O)Oc1ccccc1Br</smiles>
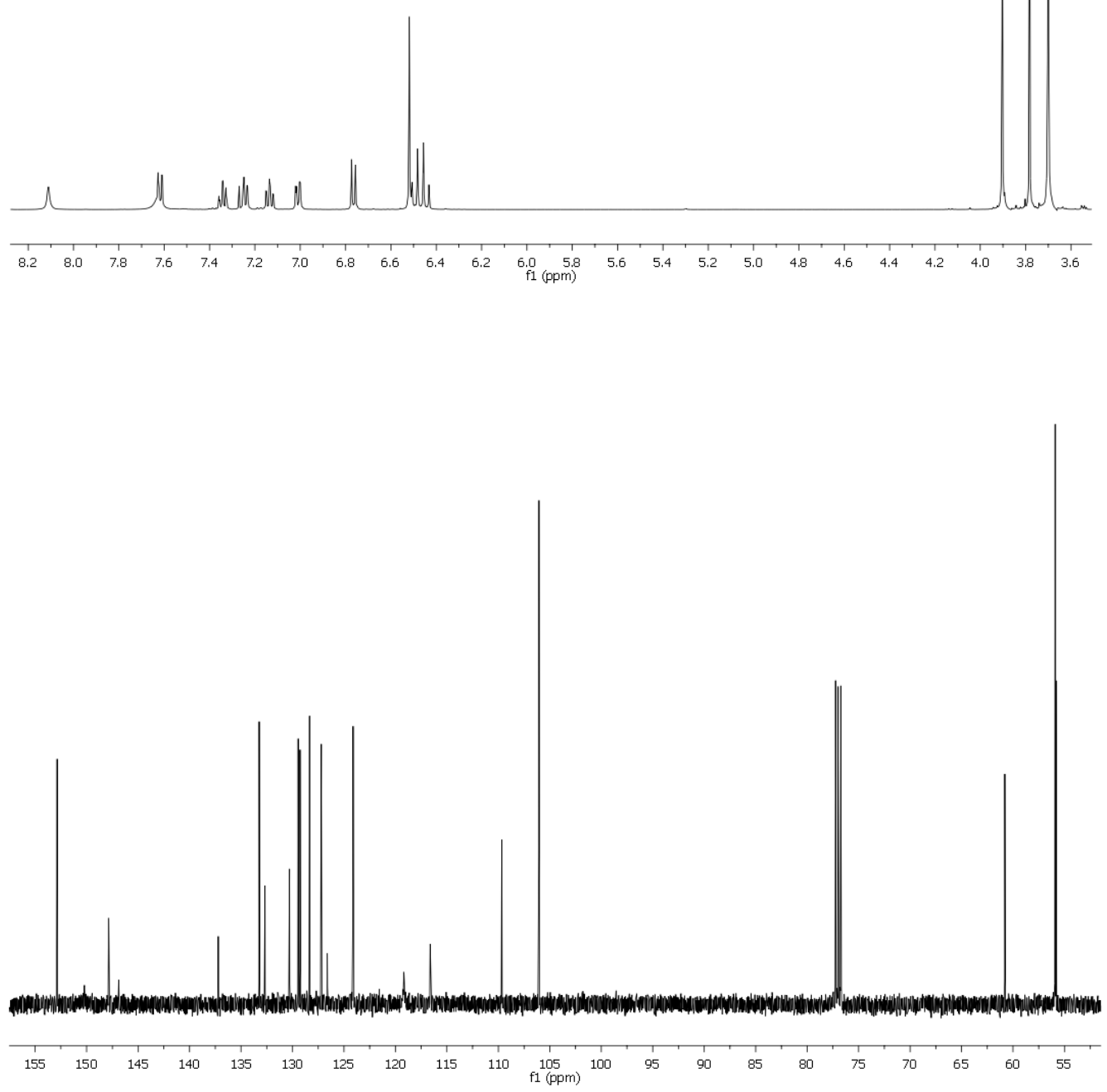

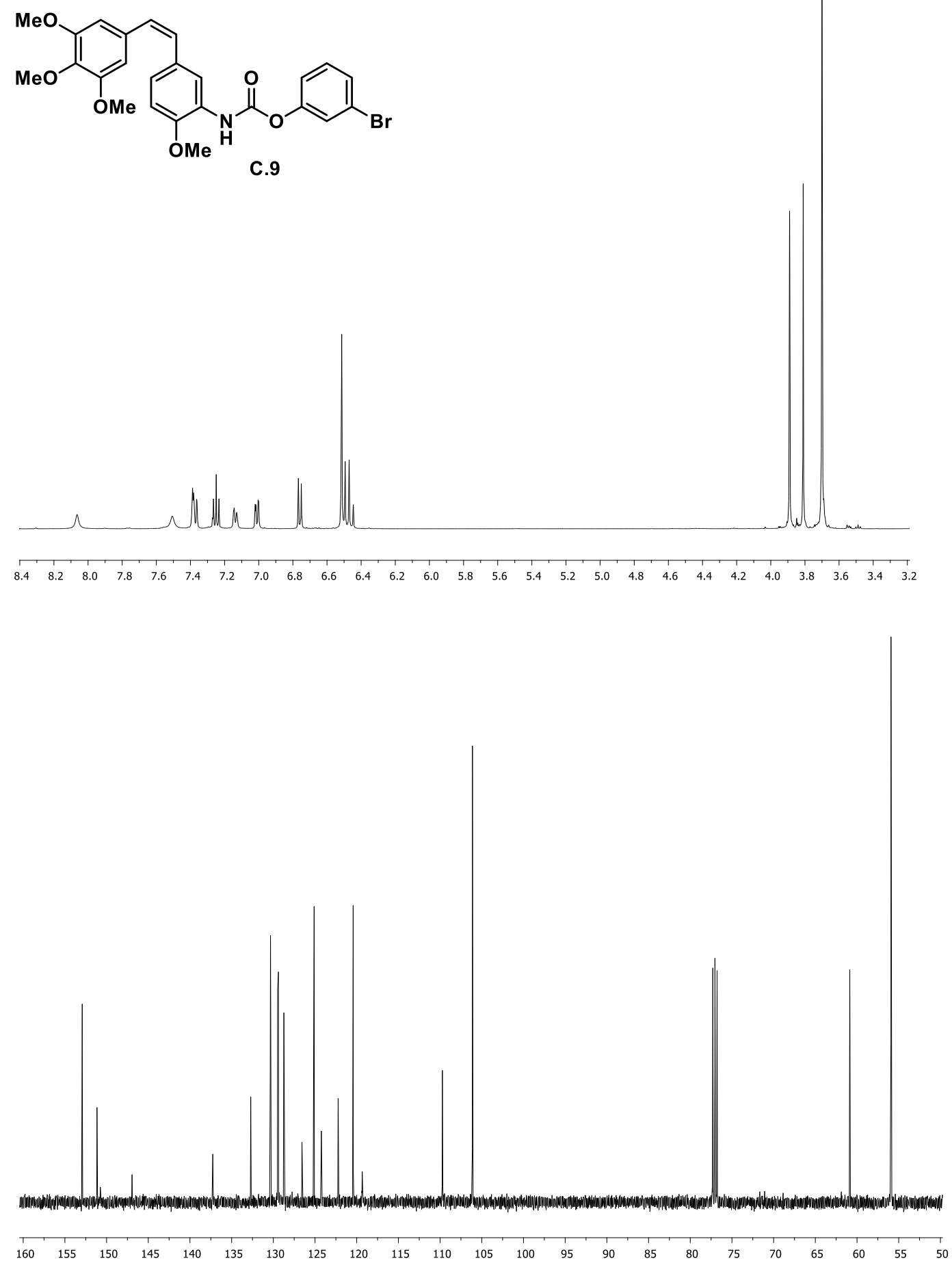
<smiles>COc1ccc(/C=C\c2cc(OC)c(OC)c(OC)c2)cc1NC(=O)Oc1ccc(Br)cc1</smiles>
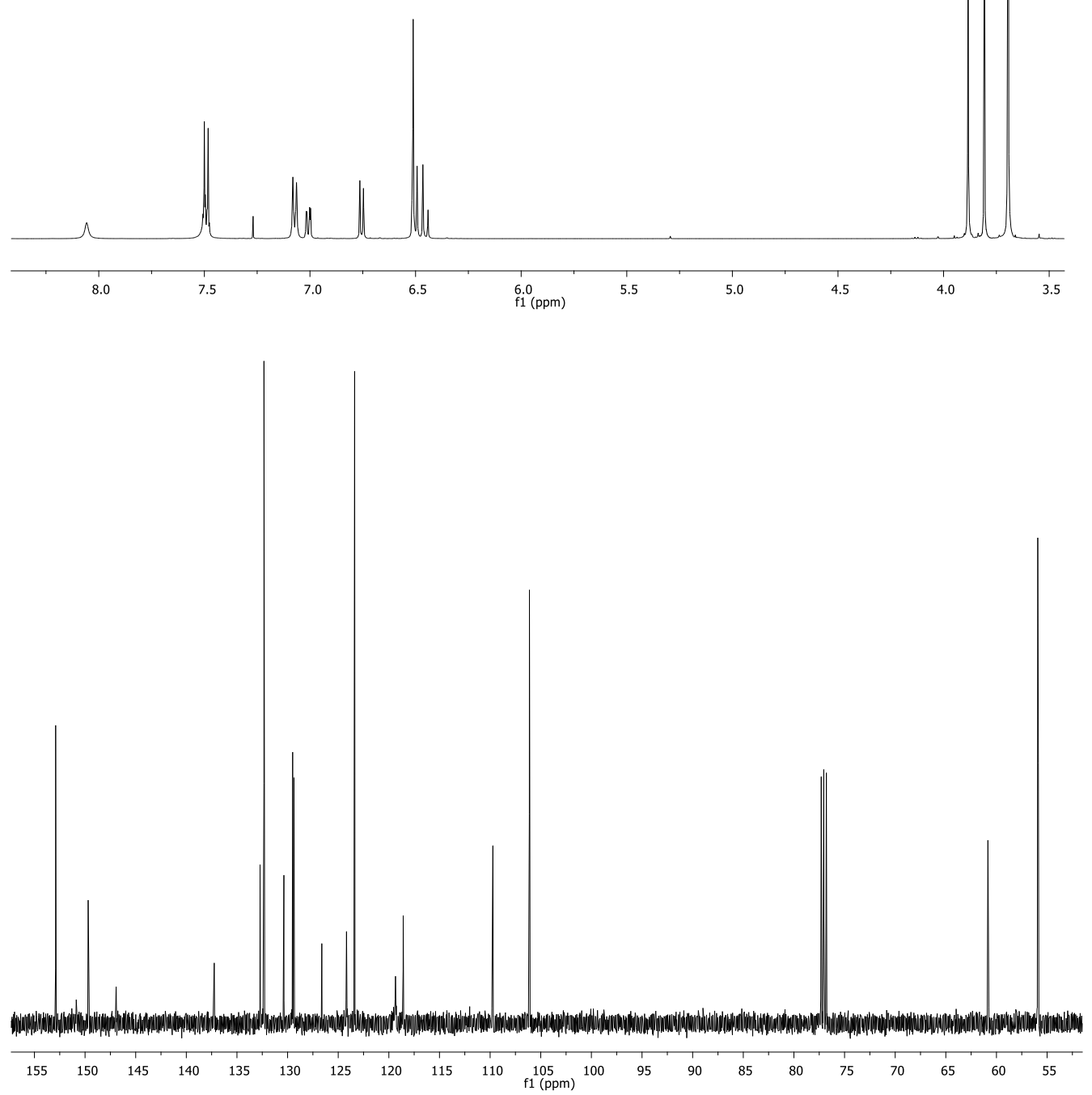

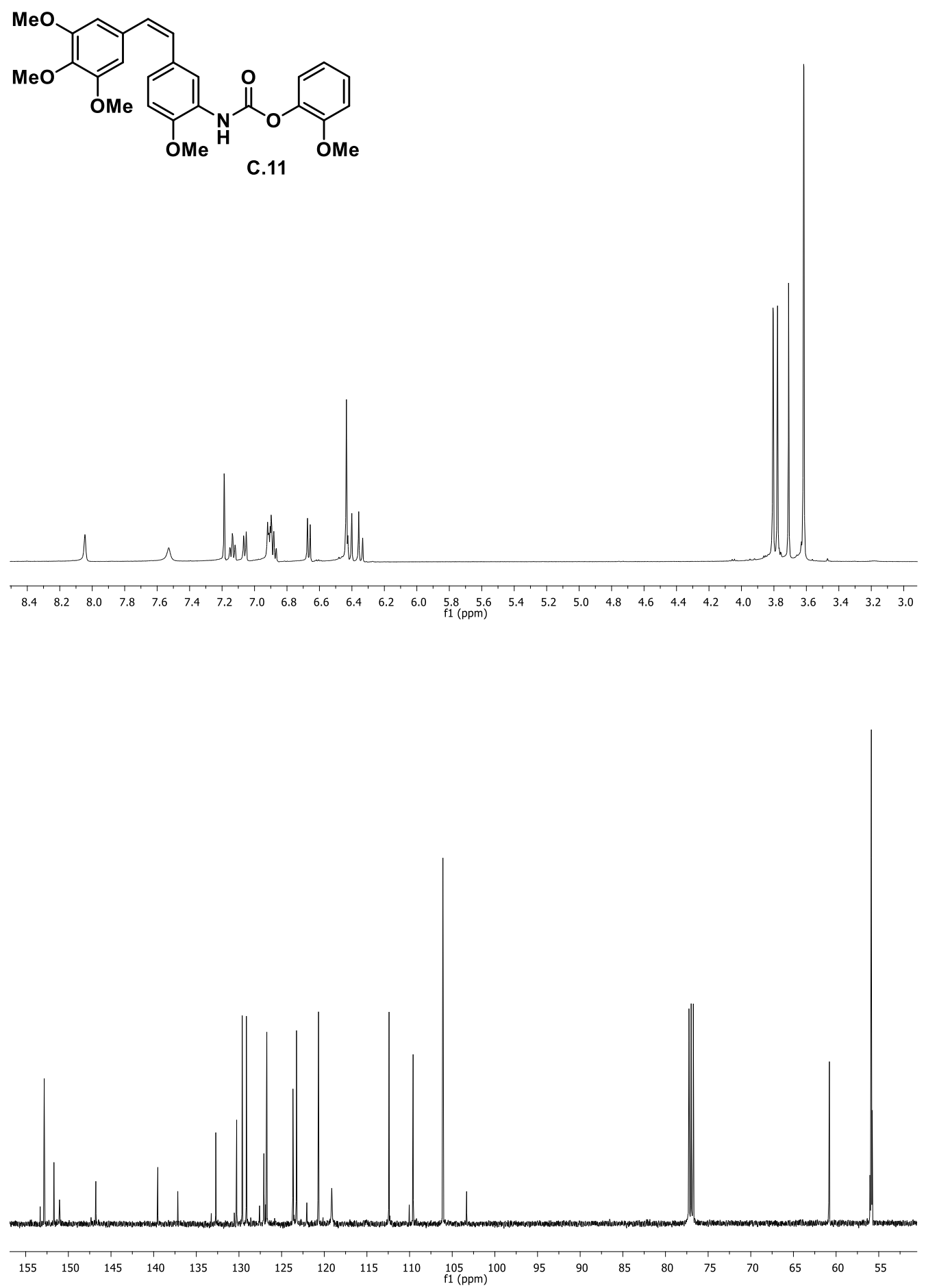

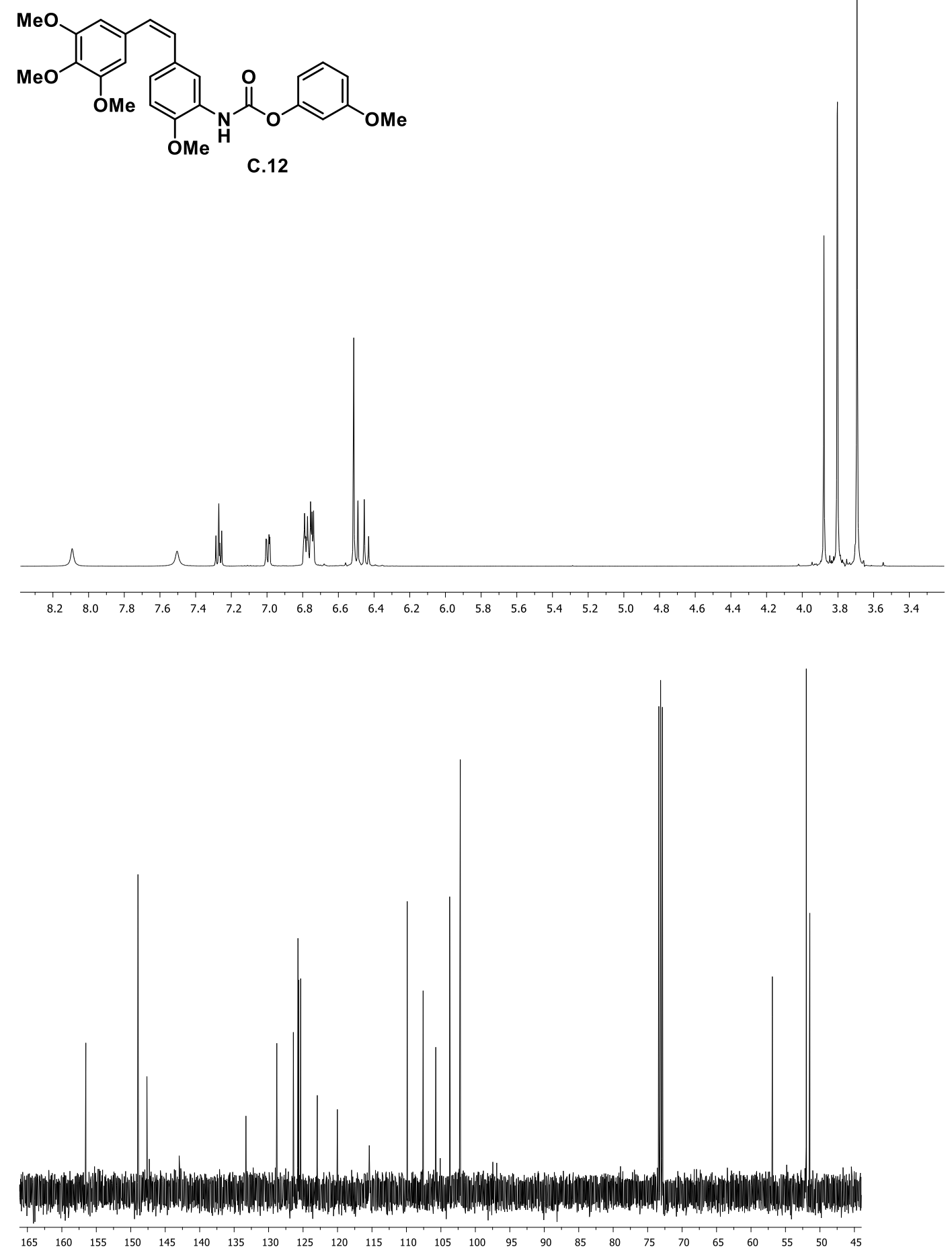
<smiles>COc1ccc(OC(=O)Nc2cc(/C=C\c3ccc(OC)c(OC)c3)ccc2OC)cc1</smiles>
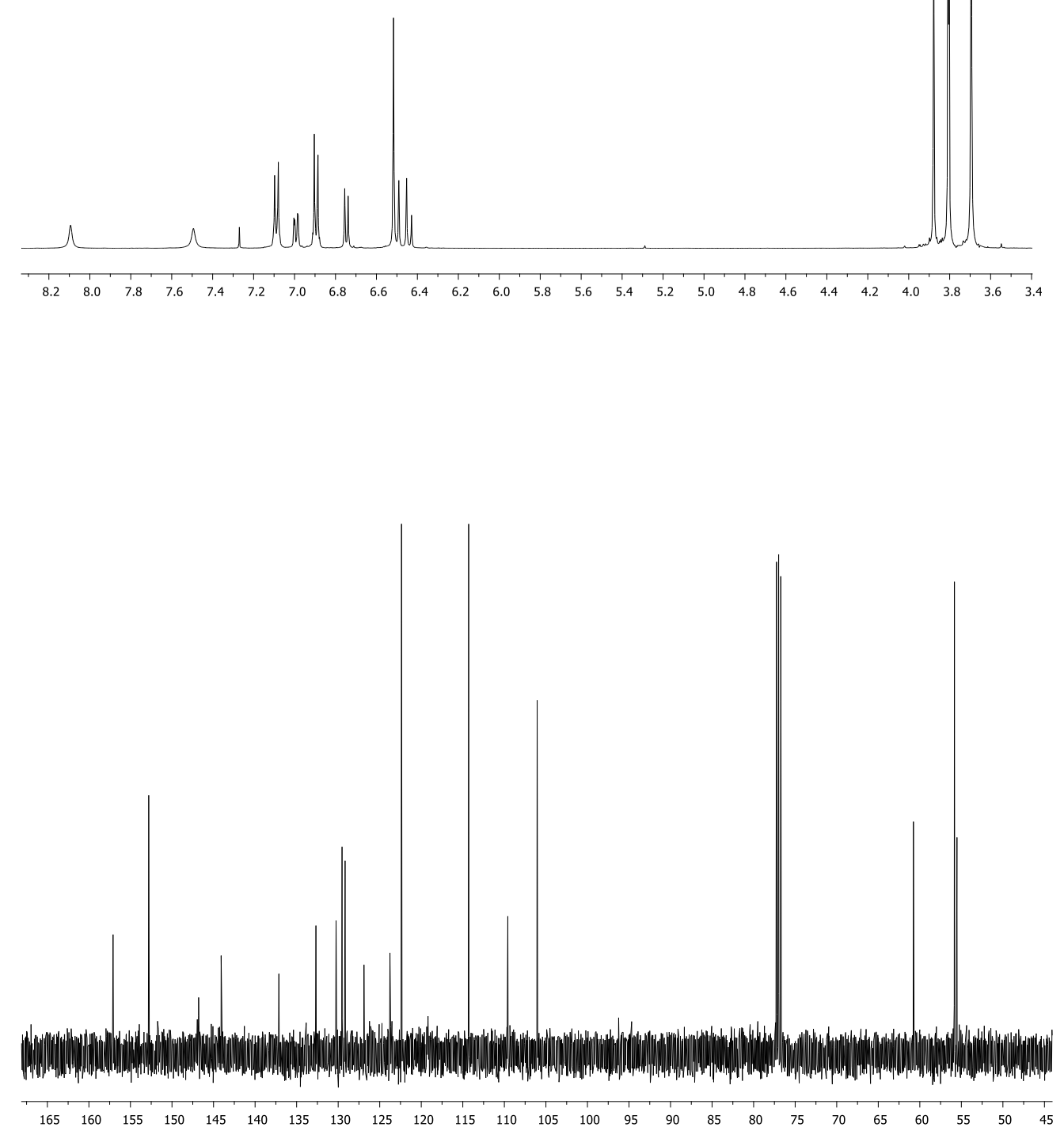

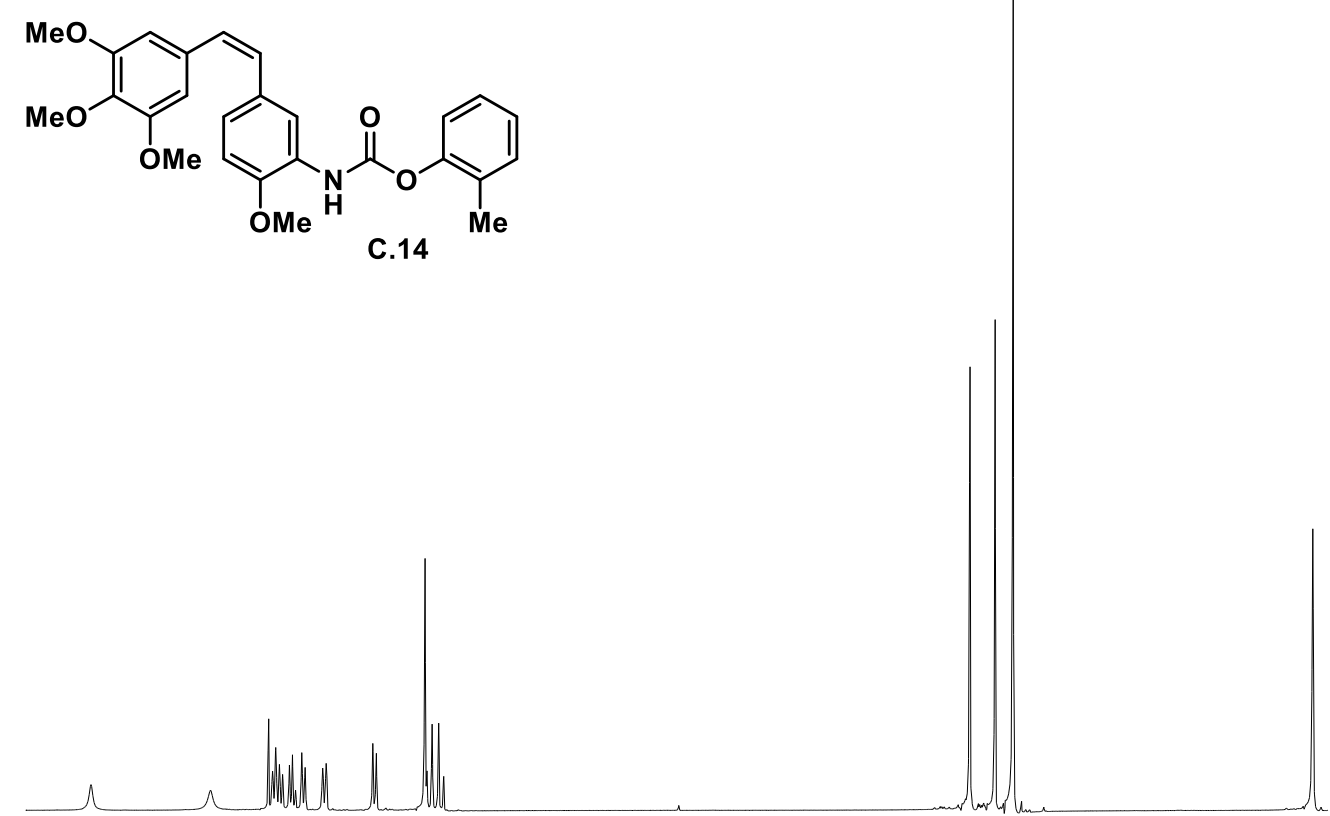

$\begin{array}{llllllllllllllllllllllllllllllllllllllllllll}8.4 & 8.2 & 8.0 & 7.8 & 7.6 & 7.4 & 7.2 & 7.0 & 6.8 & 6.6 & 6.4 & 6.2 & 6.0 & 5.8 & 5.6 & 5.4 & 5.2 & 5.0 & 4.8 & 4.6 & 4.4 & 4.2 & 4.0 & 3.8 & 3.6 & 3.4 & 3.2 & 3.0 & 2.8 & 2.6 & 2.4 & 2.2\end{array}$

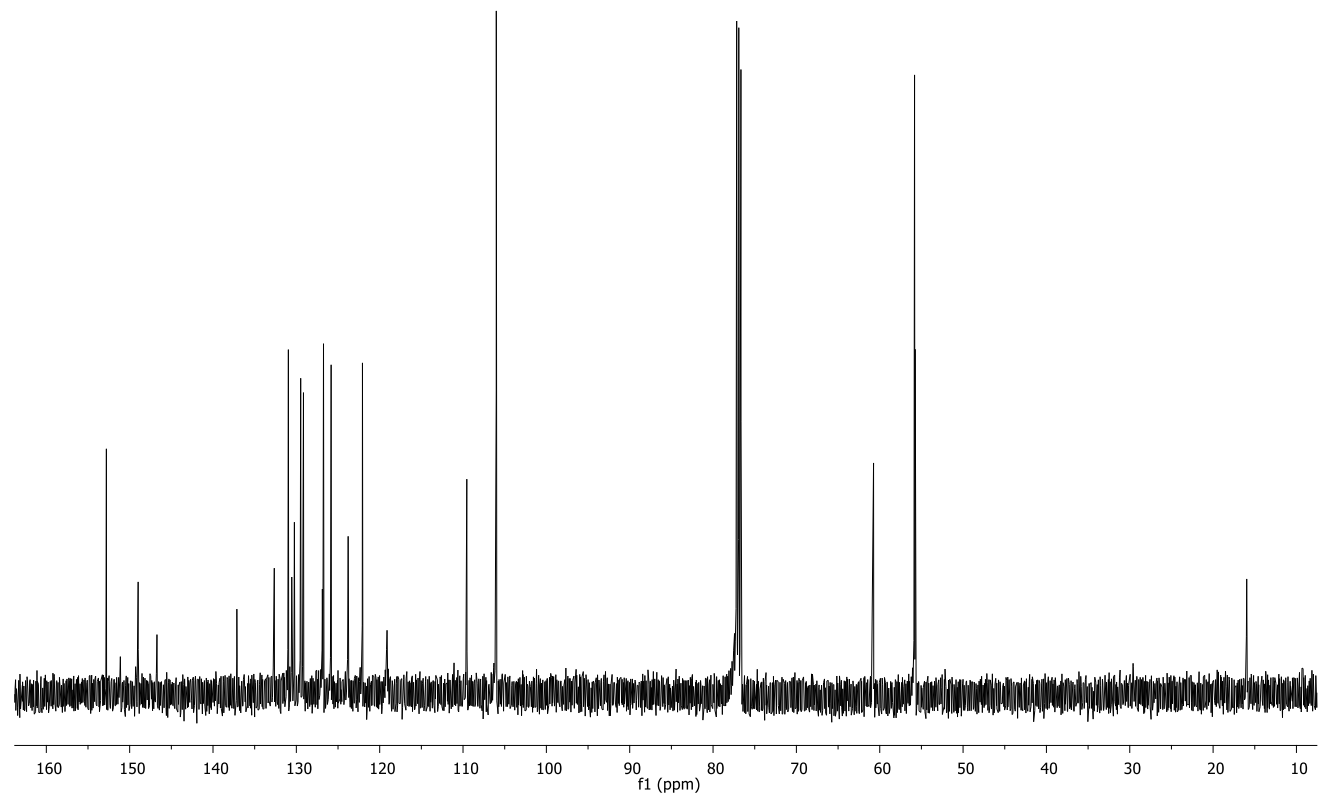



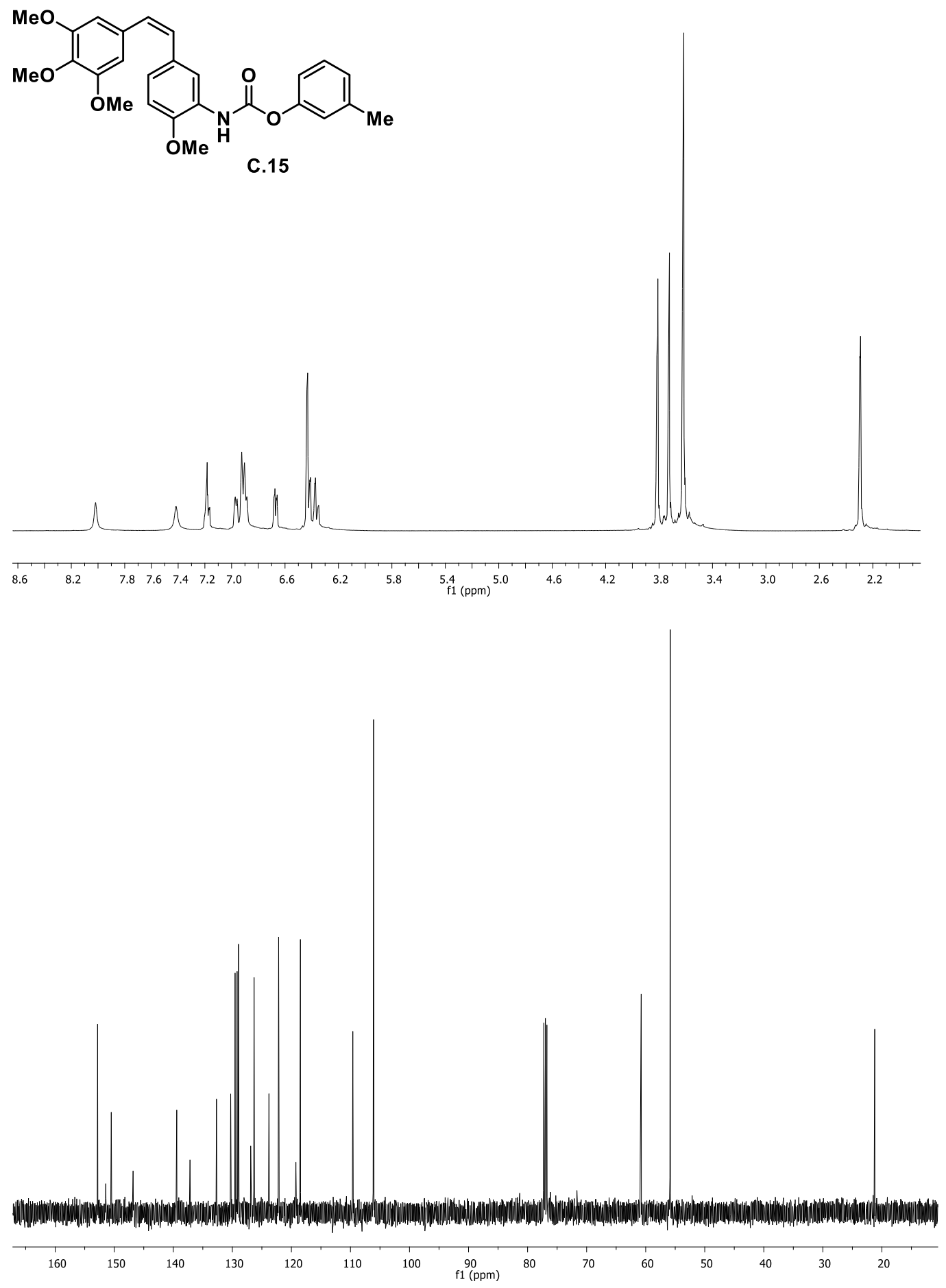


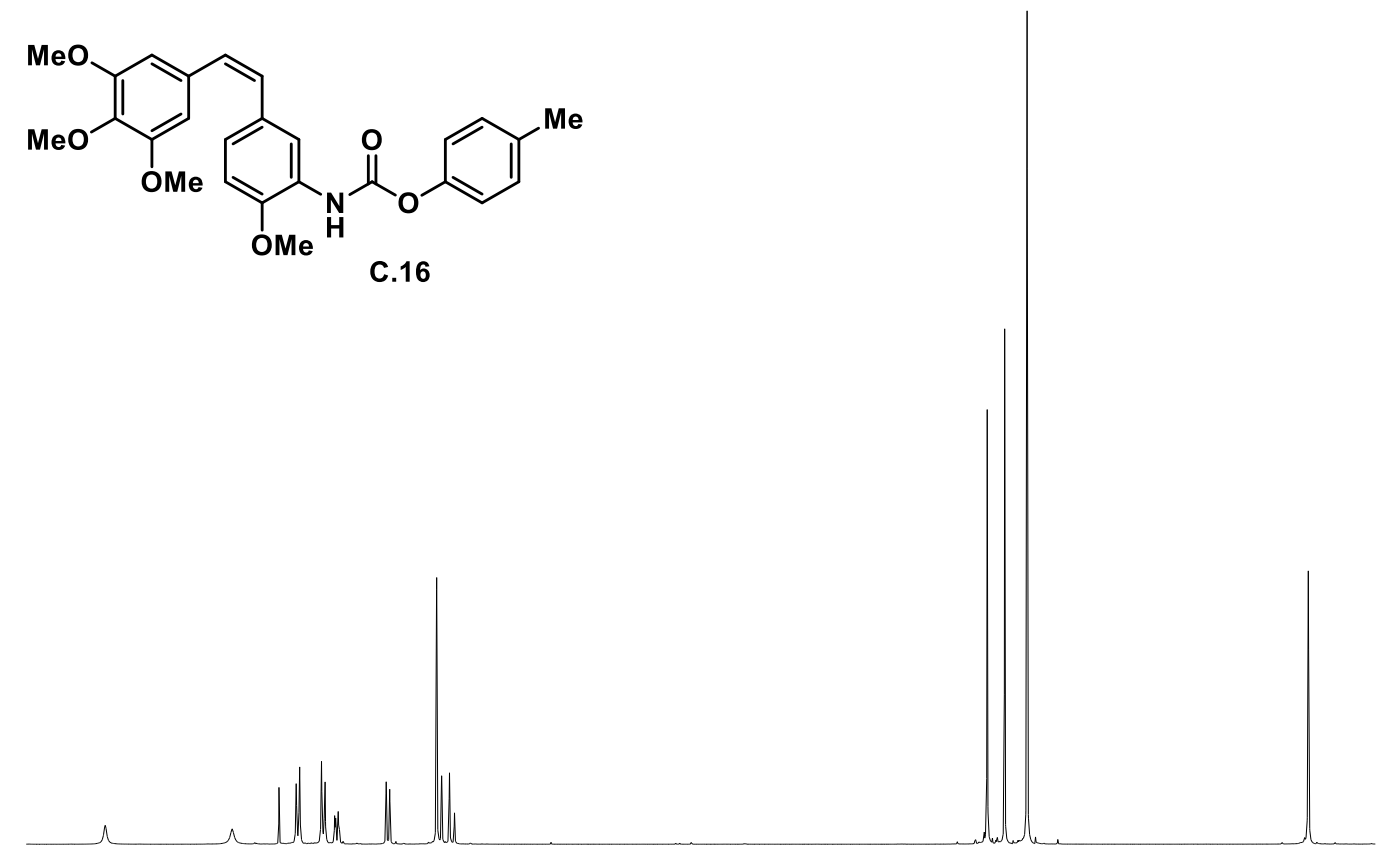

\begin{tabular}{llllllllllllllllllllllllllllllllllllll}
\hline 8.4 & 8.2 & 8.0 & 7.8 & 7.6 & 7.4 & 7.2 & 7.0 & 6.8 & 6.6 & 6.4 & 6.2 & 6.0 & 5.8 & 5.6 & 5.4 & 5.2 & 5.0 & 4.8 & 4.6 & 4.4 & 4.2 & 4.0 & 3.8 & 3.6 & 3.4 & 3.2 & 3.0 & 2.8 & 2.6 & 2.4 & 2.2
\end{tabular}

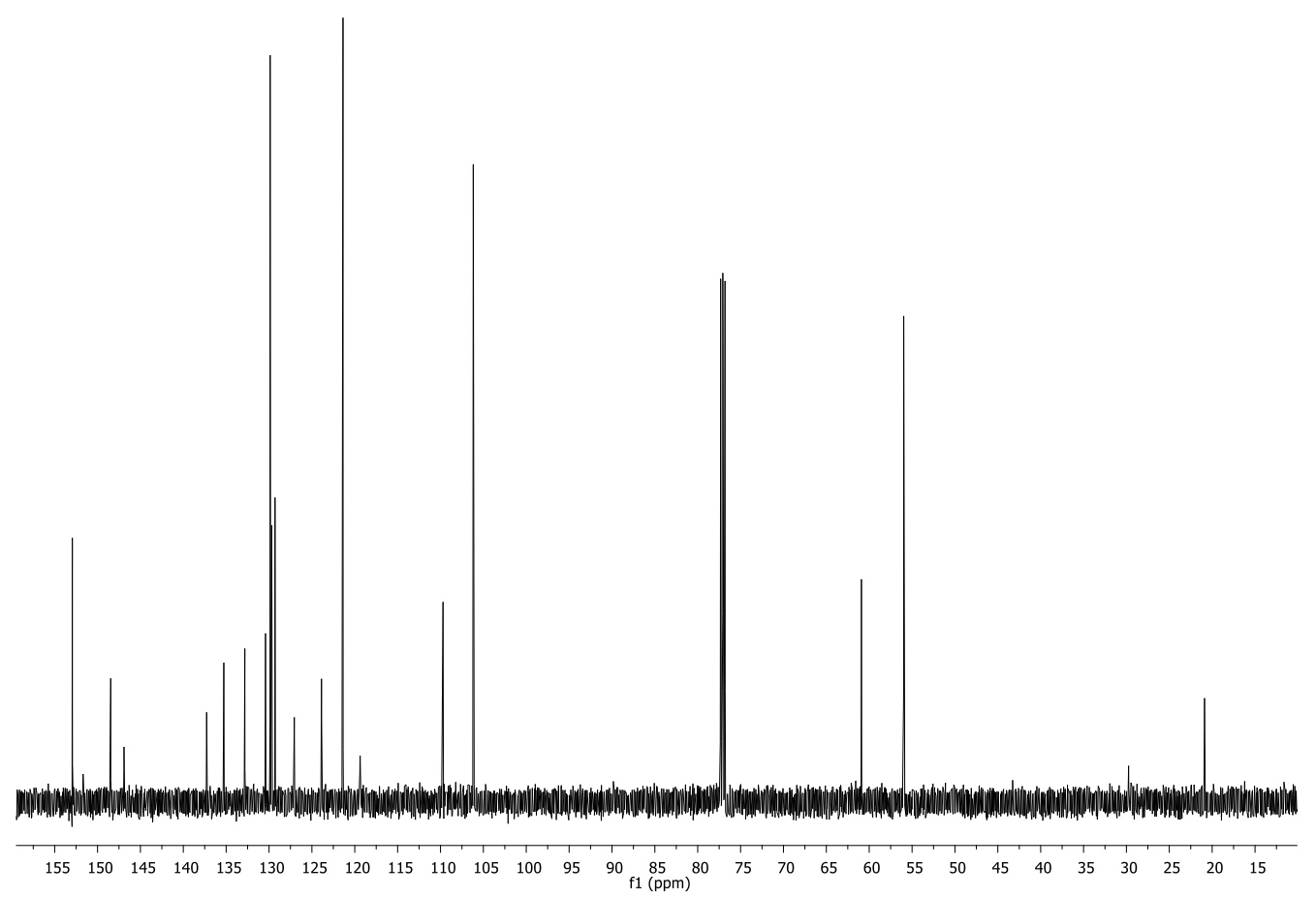



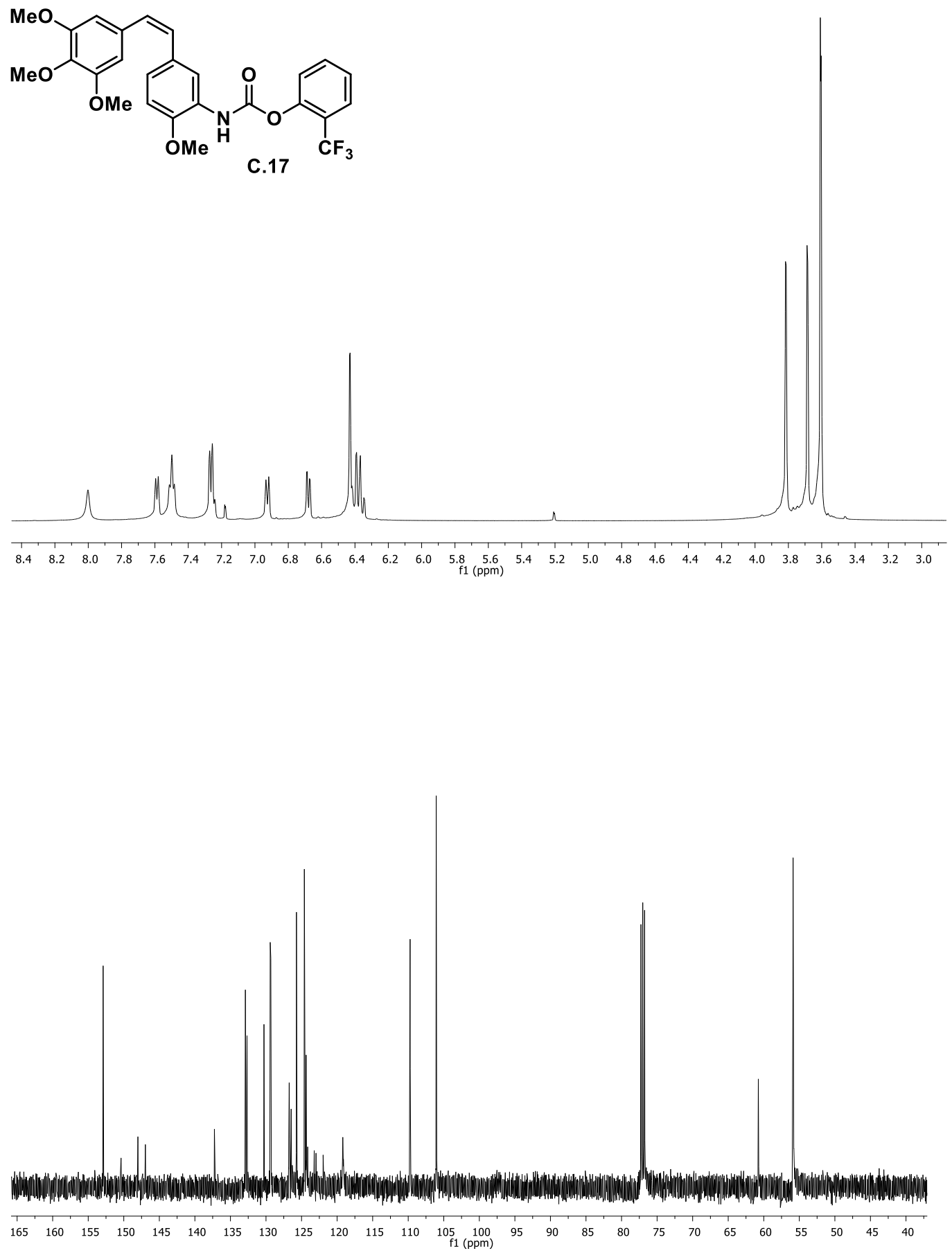
204

Chapter 7

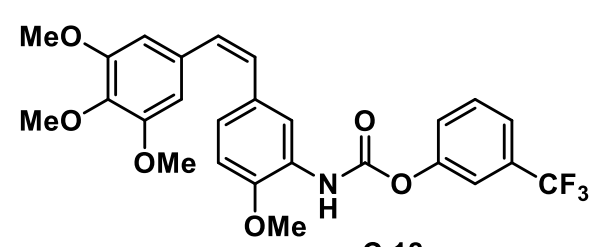

C. 18
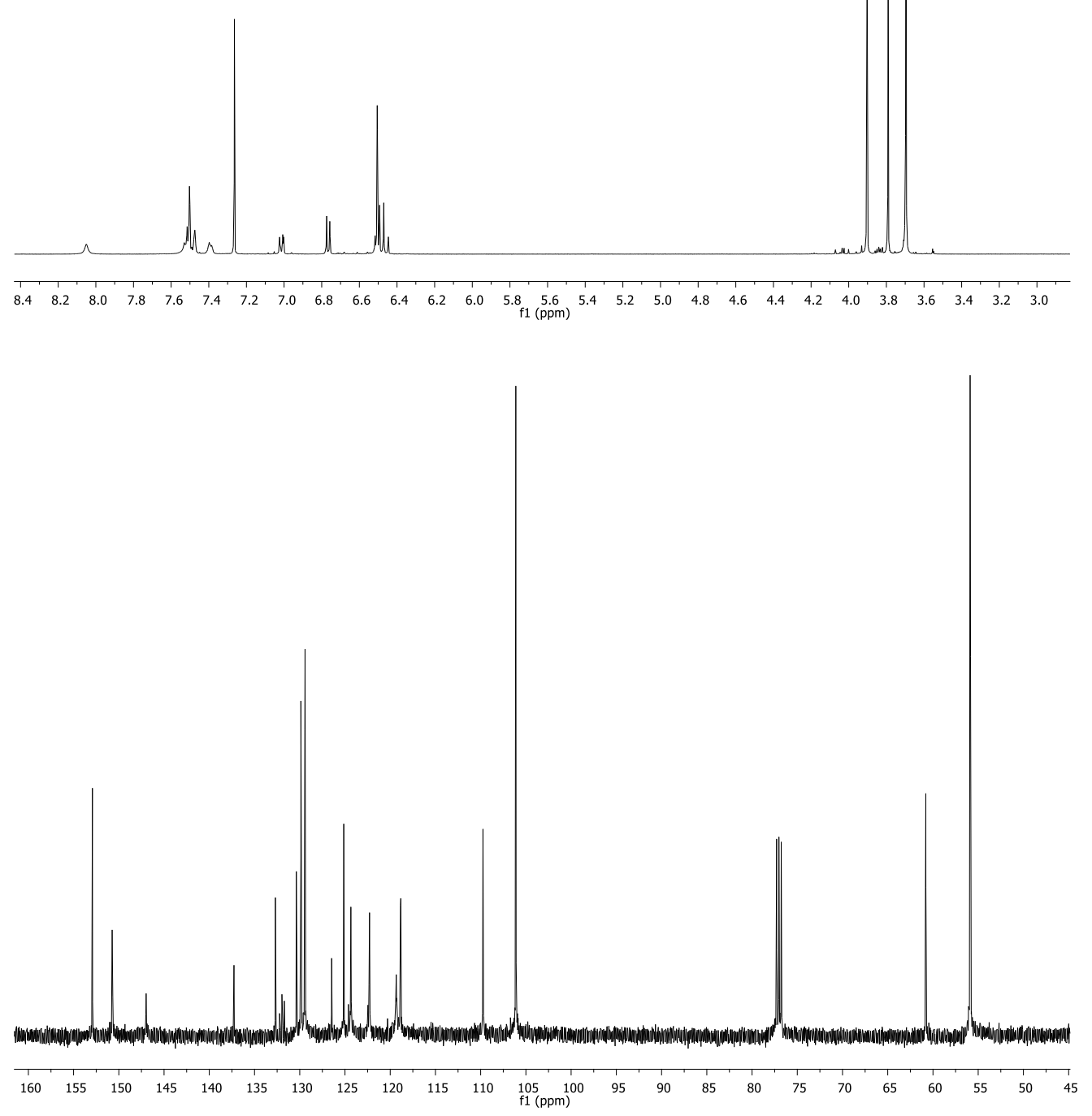
<smiles>COc1ccc(/C=C\c2cc(OC)c(OC)c(OC)c2)cc1NC(=O)Oc1ccc(C(F)(F)F)cc1</smiles>

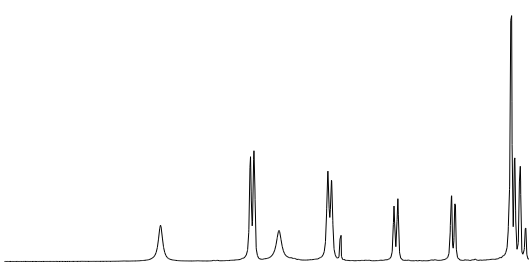

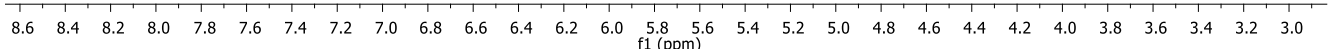

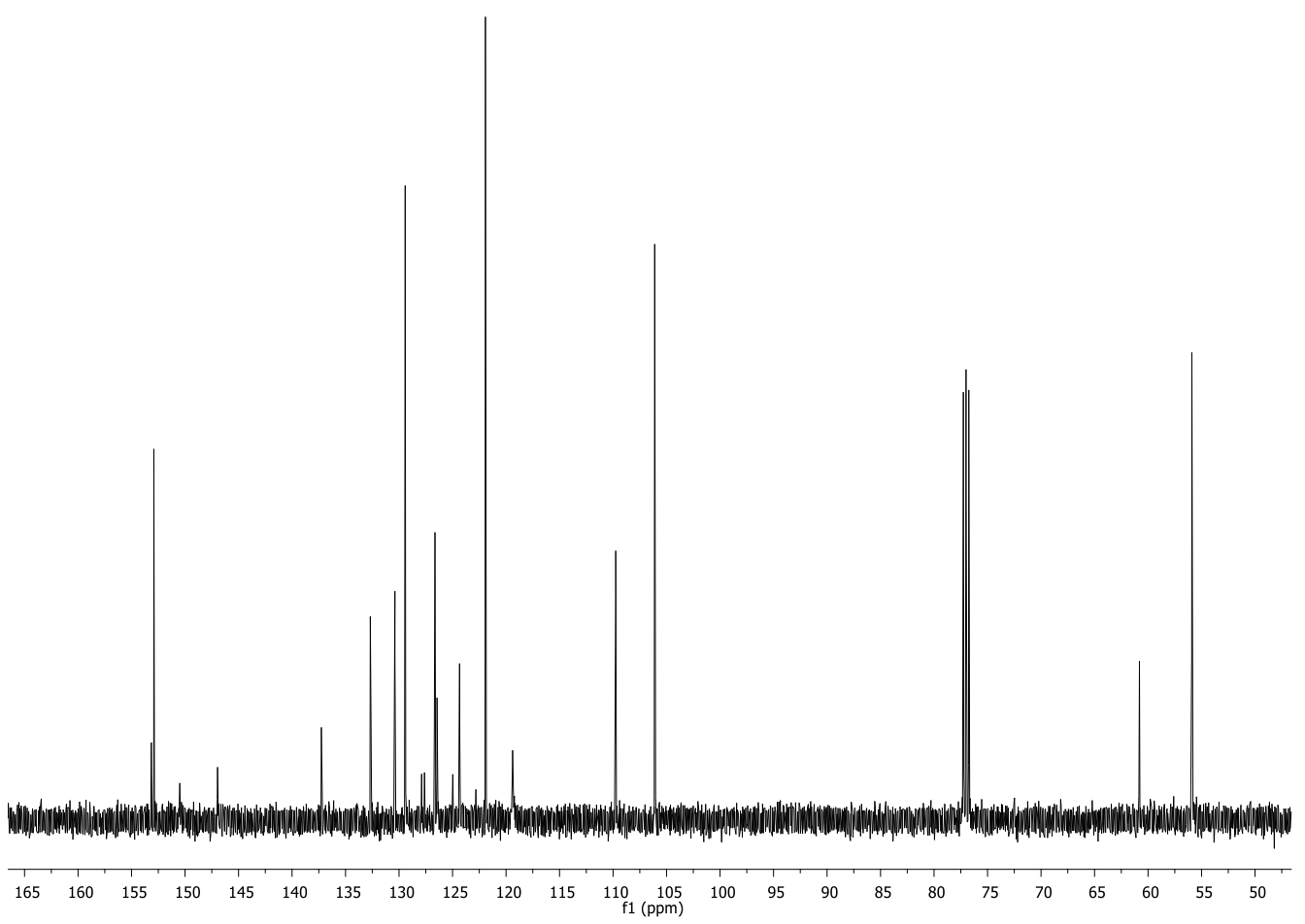



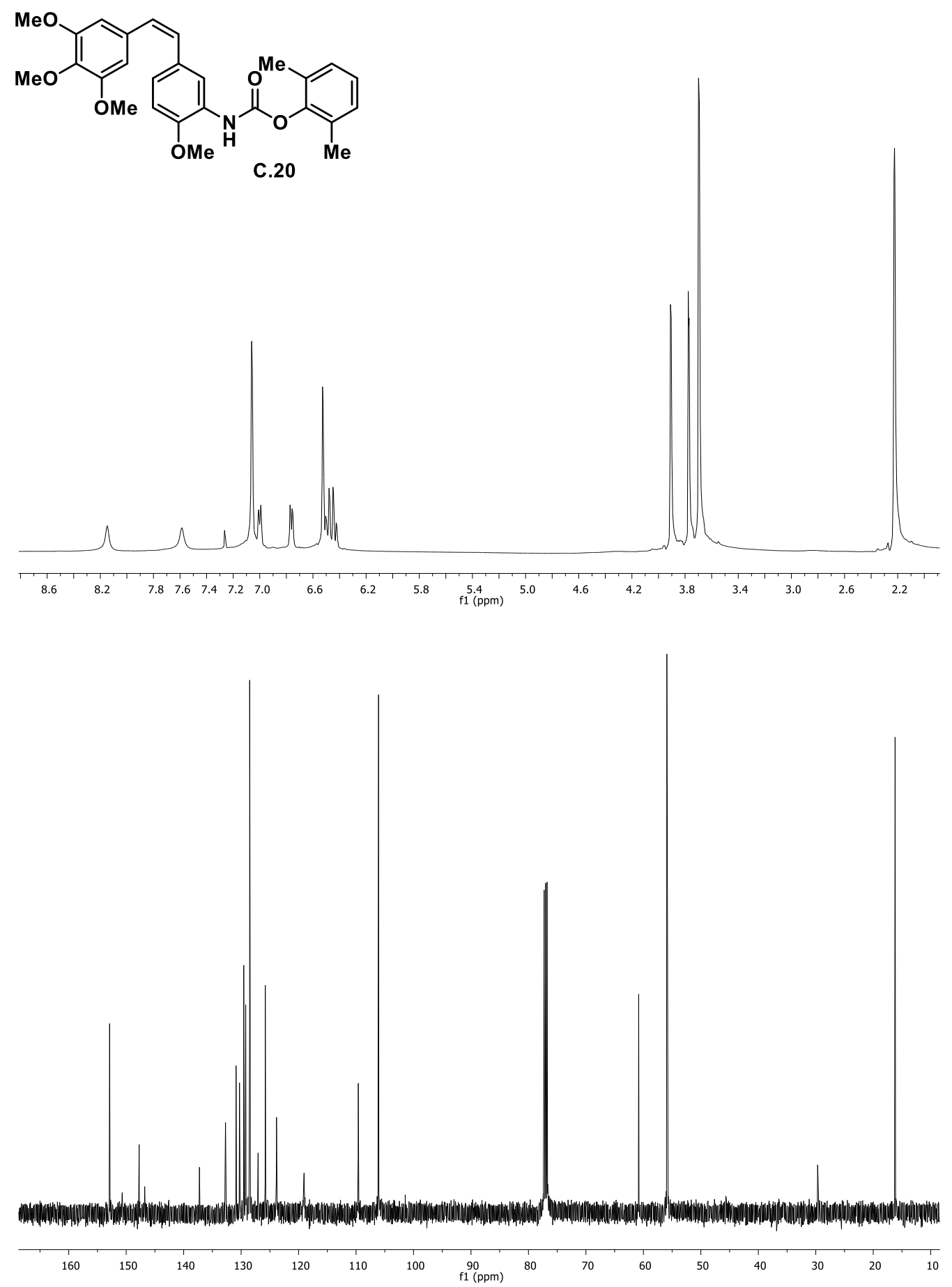
<smiles>COc1ccc(/C=C\c2ccc(OC)c(OC)c2)cc1NC(=O)Oc1cc(C)cc(C(C)(C)C)c1</smiles>
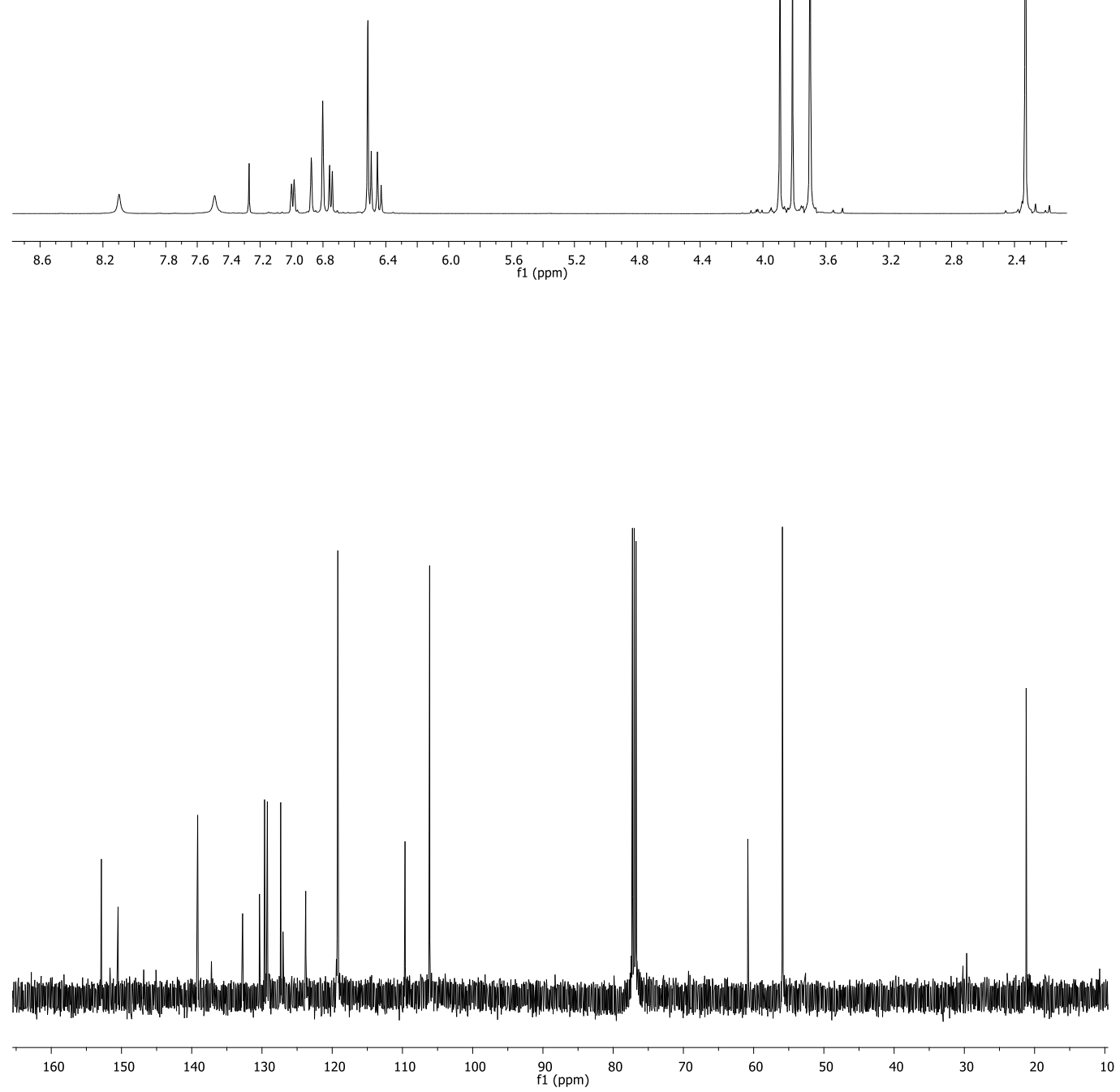

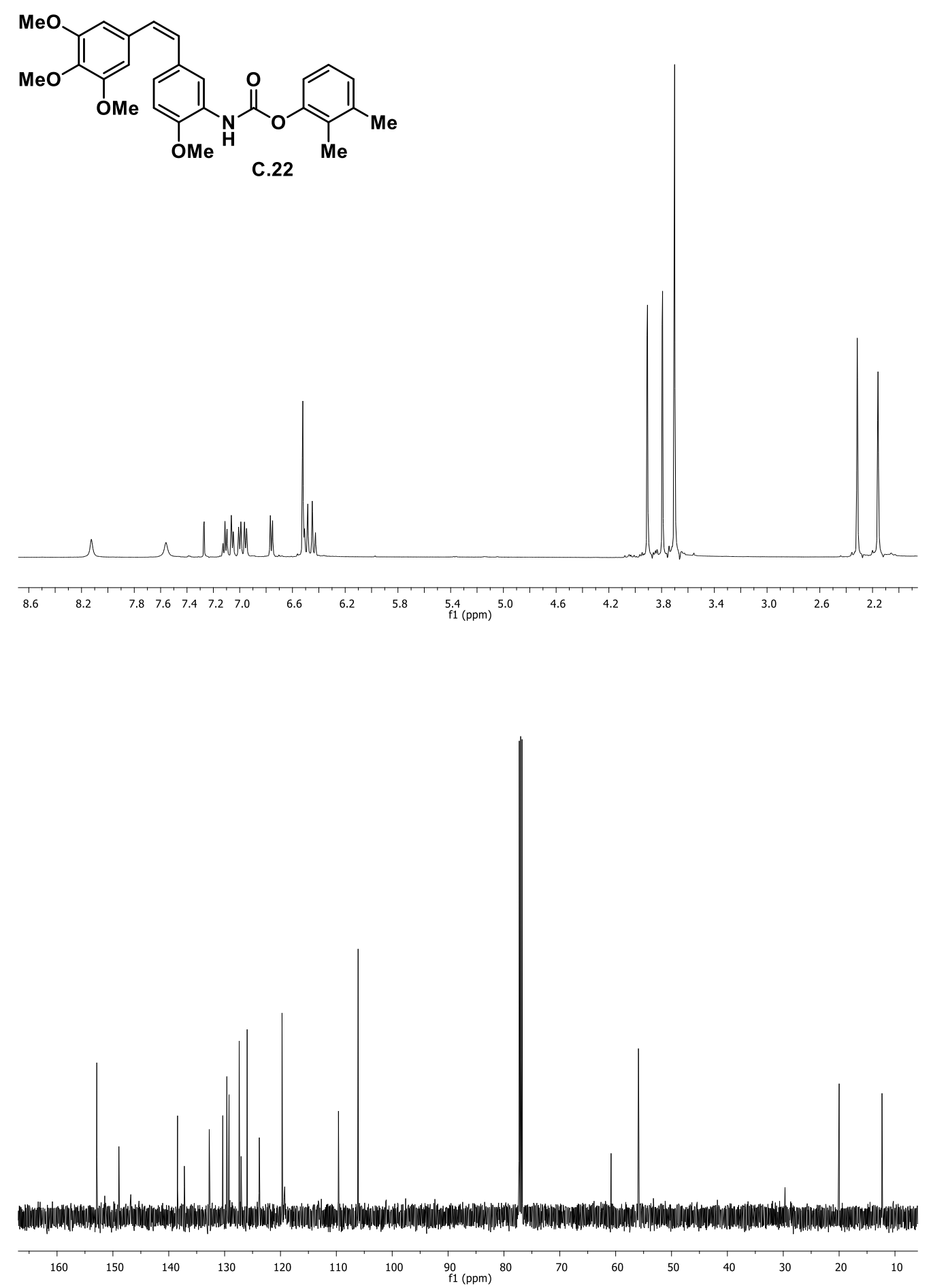
Selected spectra

209

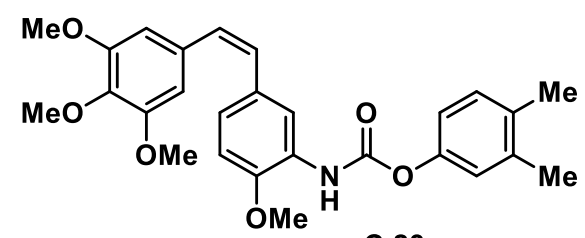

C. 23
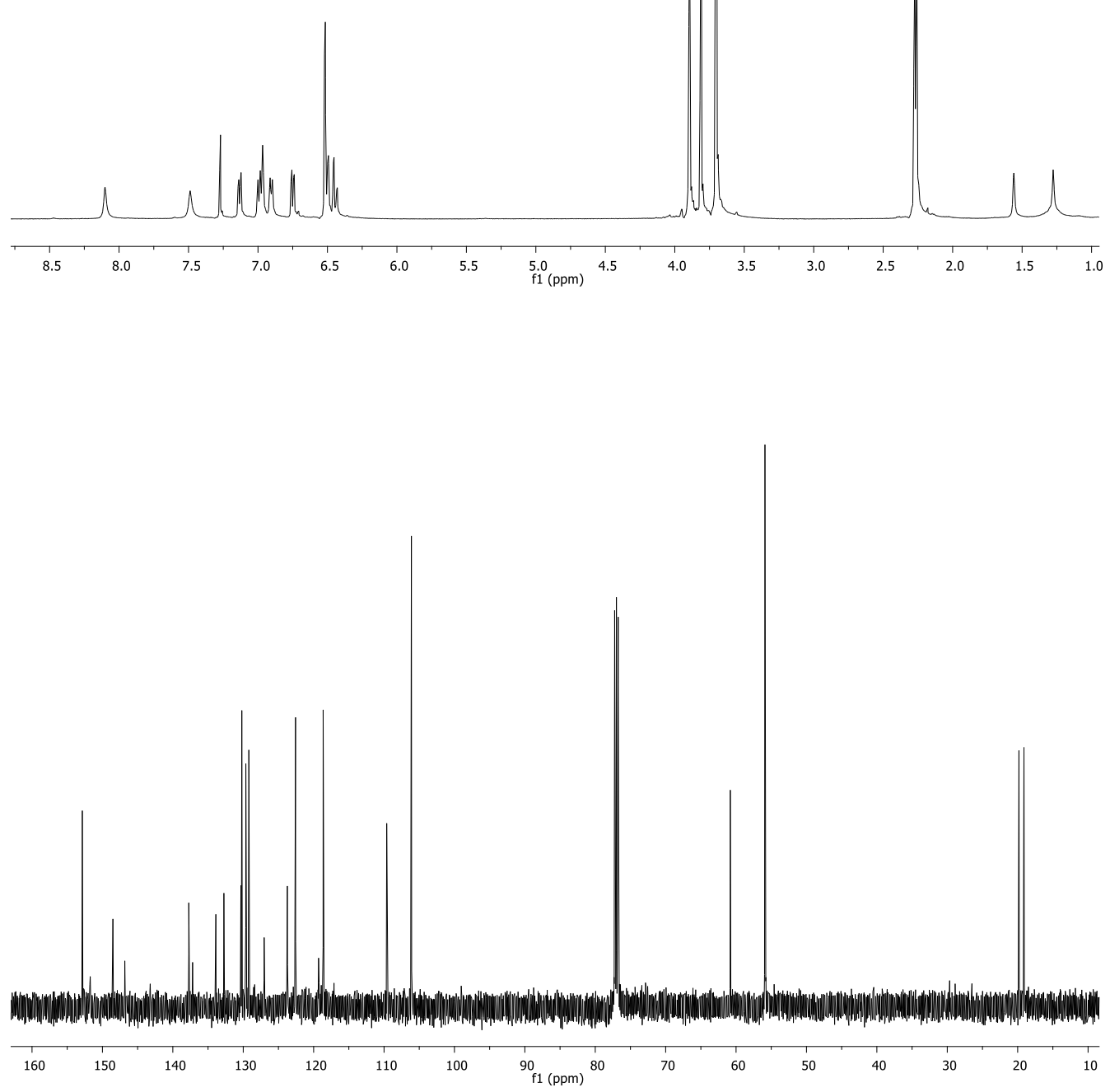

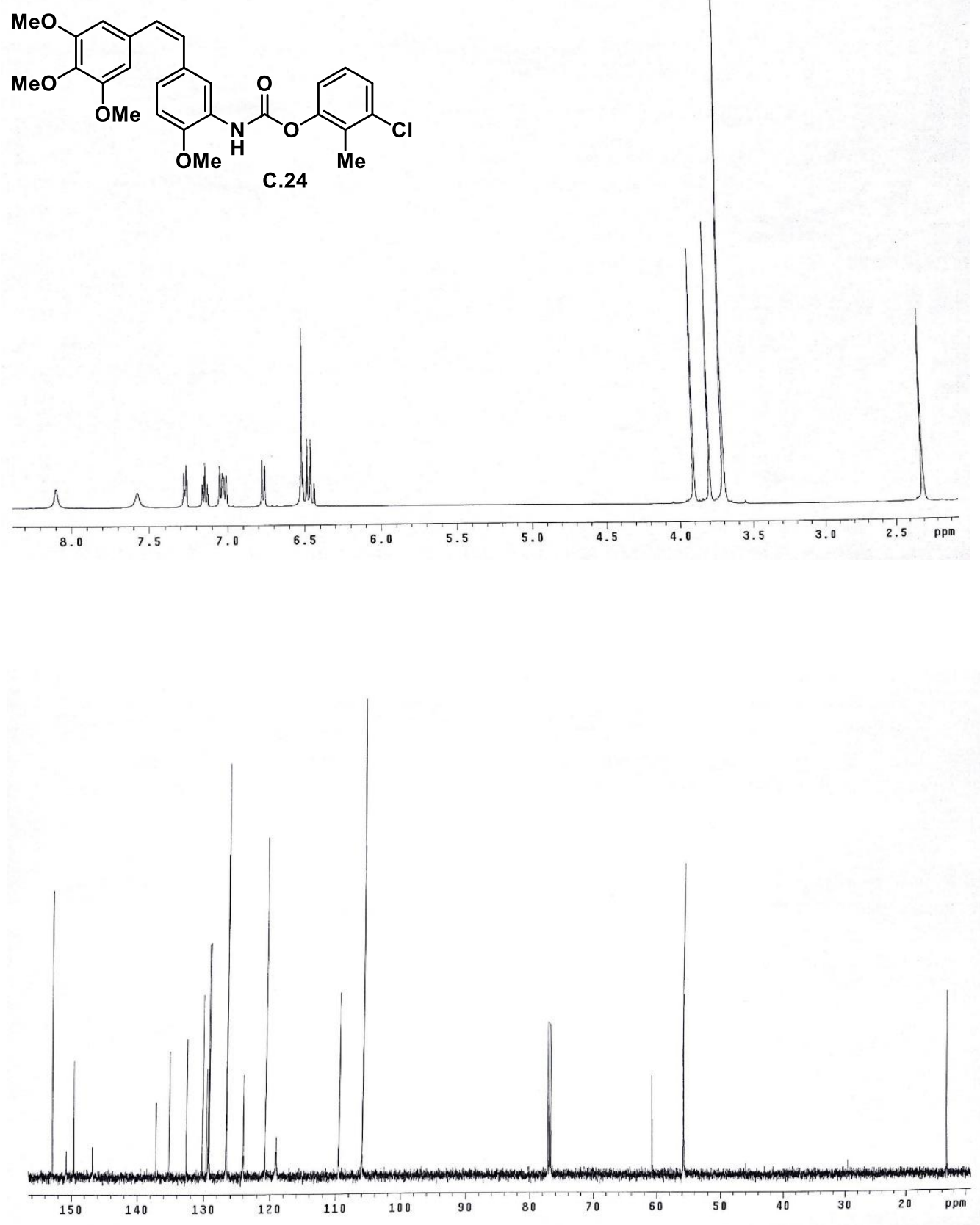

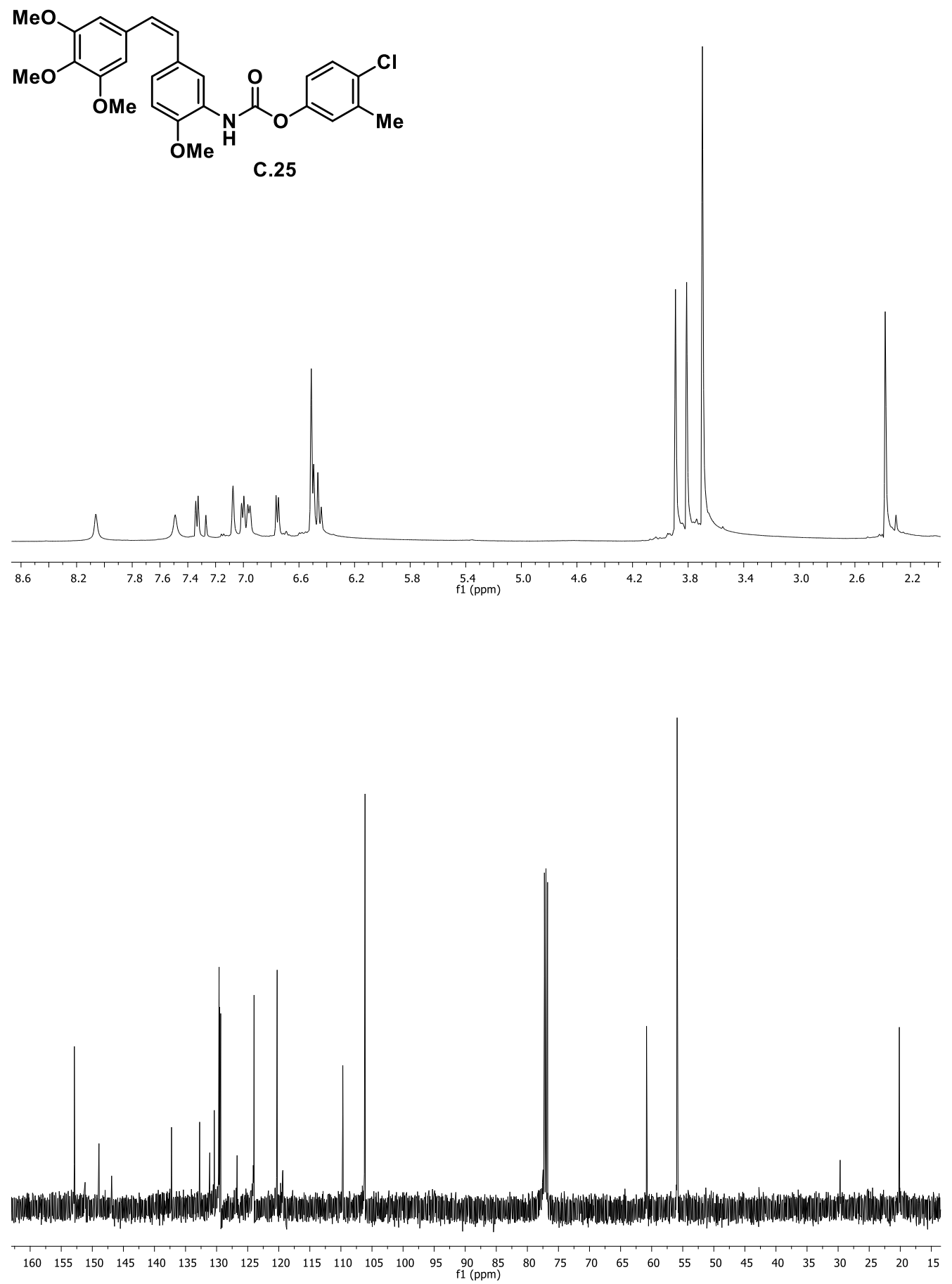
<smiles>COc1ccc(/C=C\c2ccc(OC)c(OC)c2)cc1NC(=O)Oc1ccc(C)c(Cl)c1</smiles>
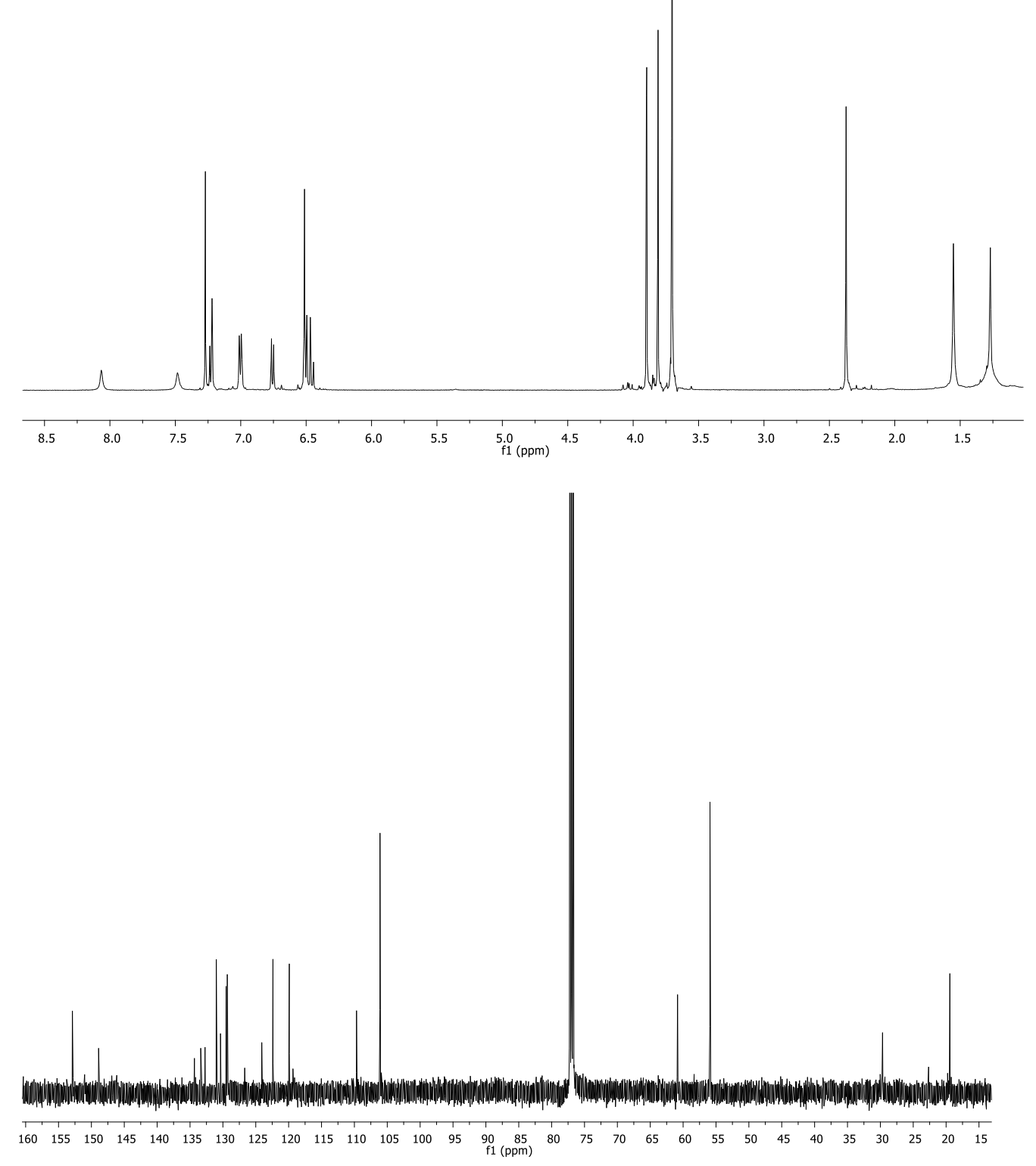
<smiles>COc1ccc(/C=C\c2ccc(OC)c(OC)c2)cc1NC(=O)Nc1ccccc1</smiles>

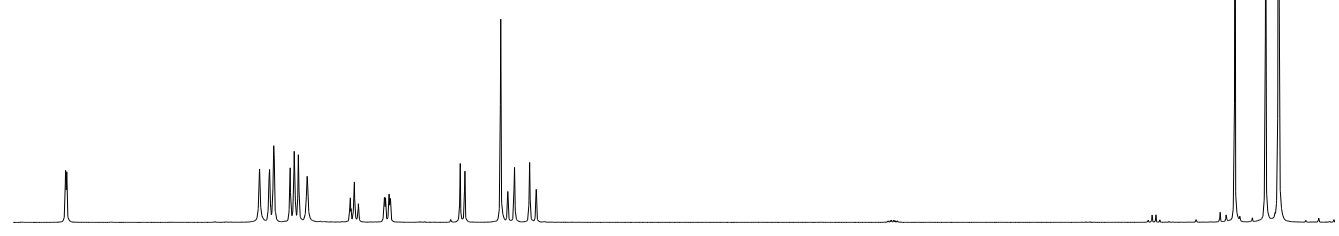

\begin{tabular}{llllllllllllllllllllllllllllllll}
\hline 8.2 & 8.0 & 7.8 & 7.6 & 7.4 & 7.2 & 7.0 & 6.8 & 6.6 & 6.4 & 6.2 & 6.0 & 5.8 & 5.6 & 5.4 & 5.2 & 5.0 & 4.8 & 4.6 & 4.4 & 4.2 & 4.0 & 3.8 & 3.6 & 3.4
\end{tabular}

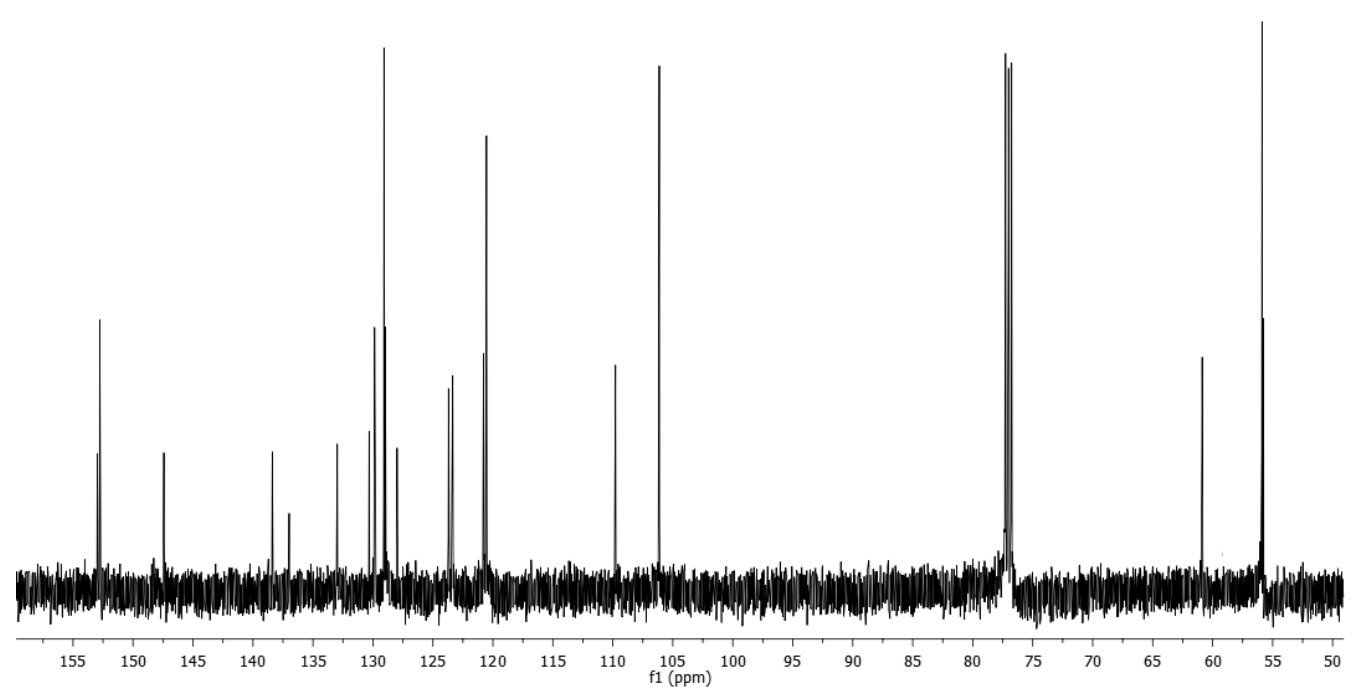


<smiles>COc1ccc(/C=C\c2cc(OC)c(OC)c(OC)c2)cc1NC(=O)Nc1ccccc1F</smiles>
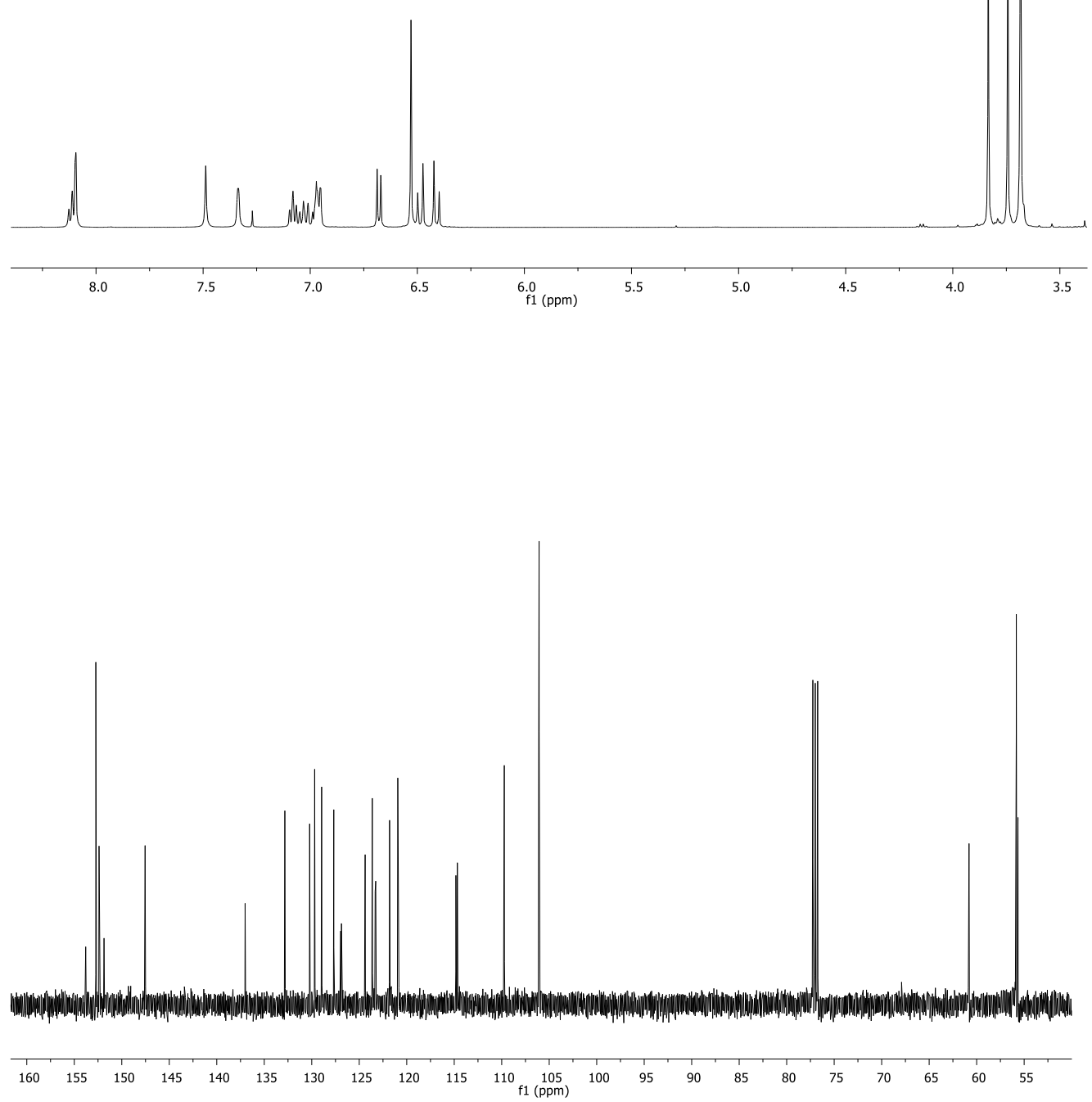

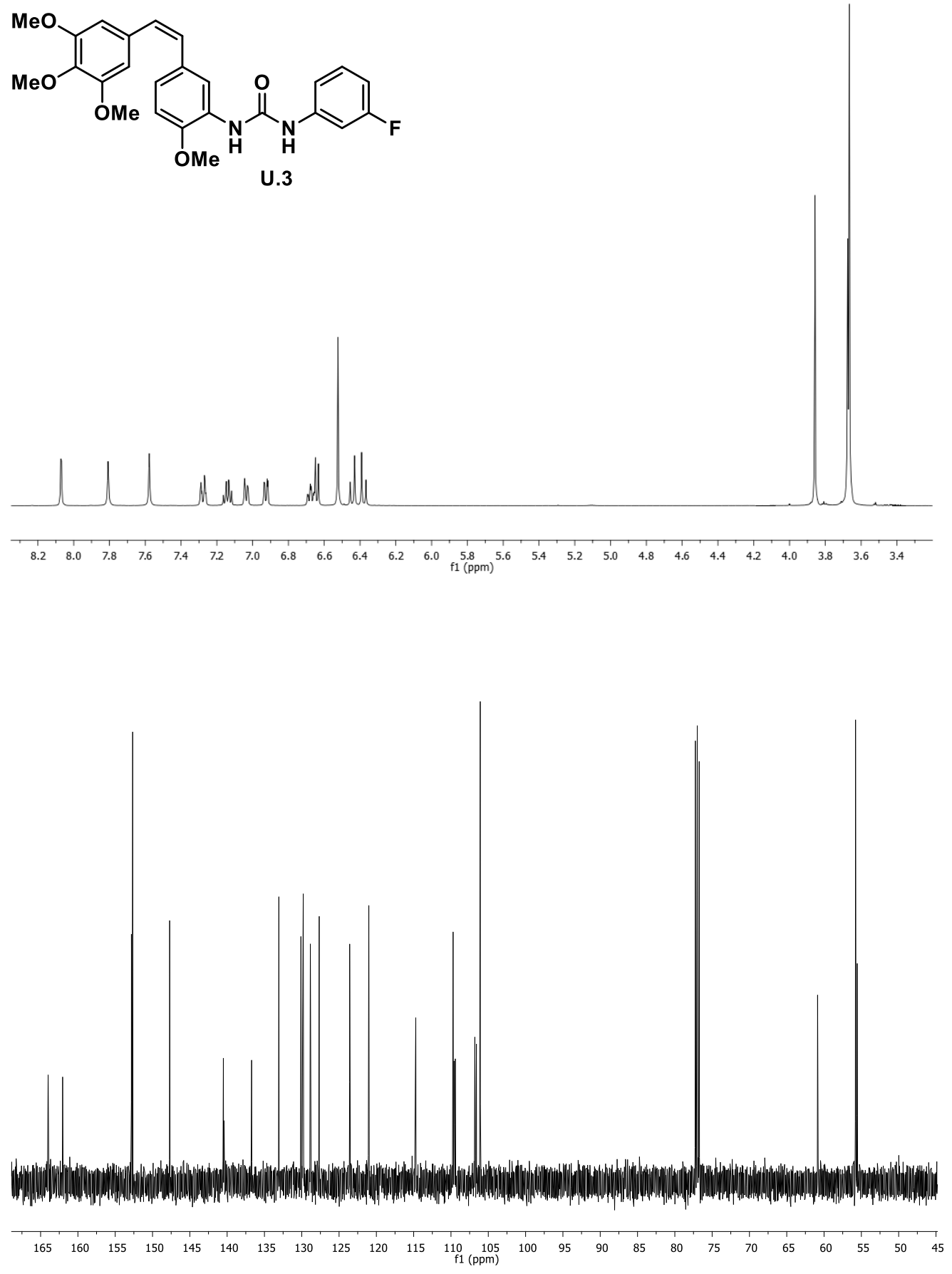
<smiles>COc1ccc(/C=C\c2cc(OC)c(OC)c(OC)c2)cc1NC(=O)Nc1ccc(F)cc1</smiles>
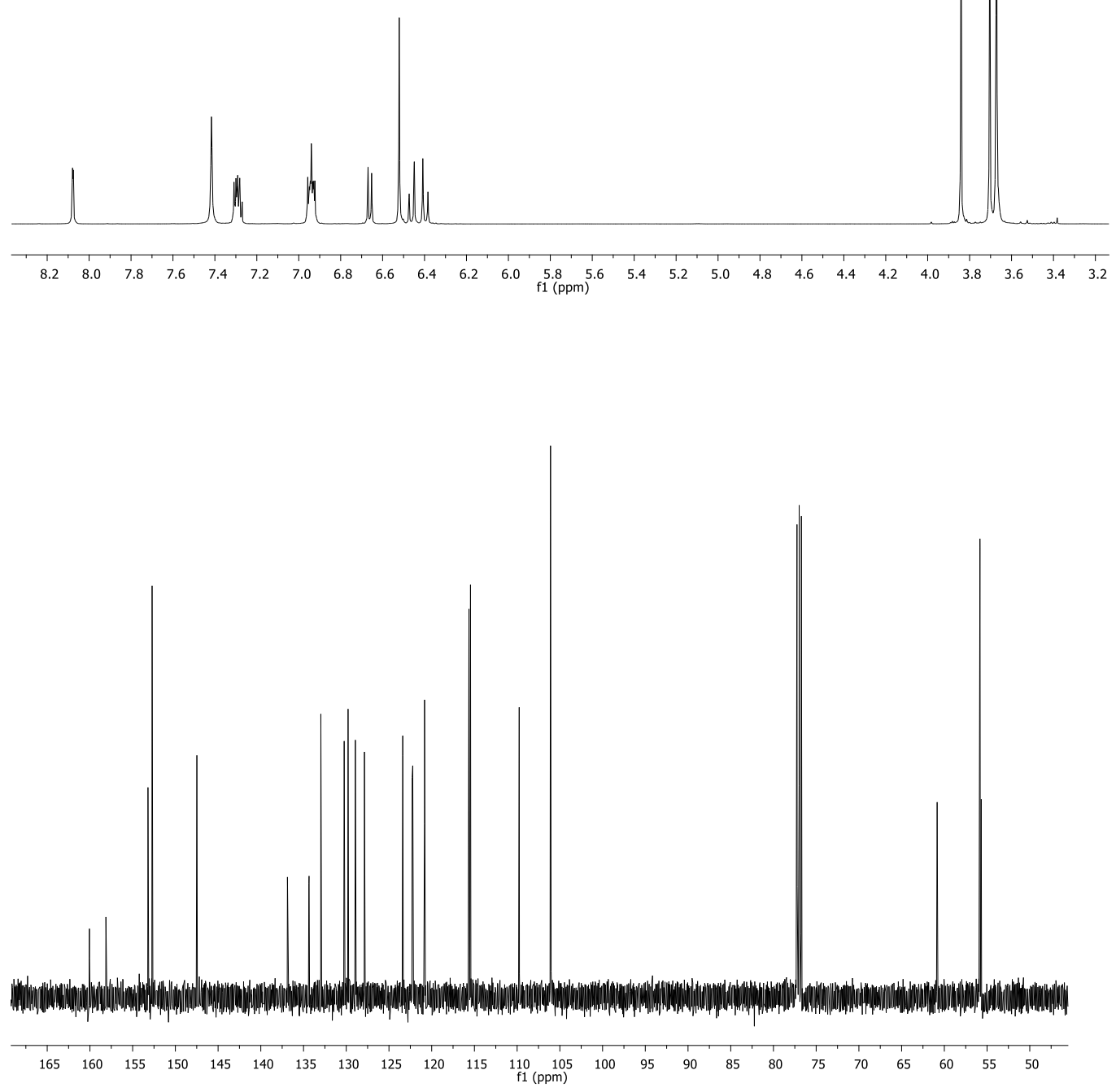

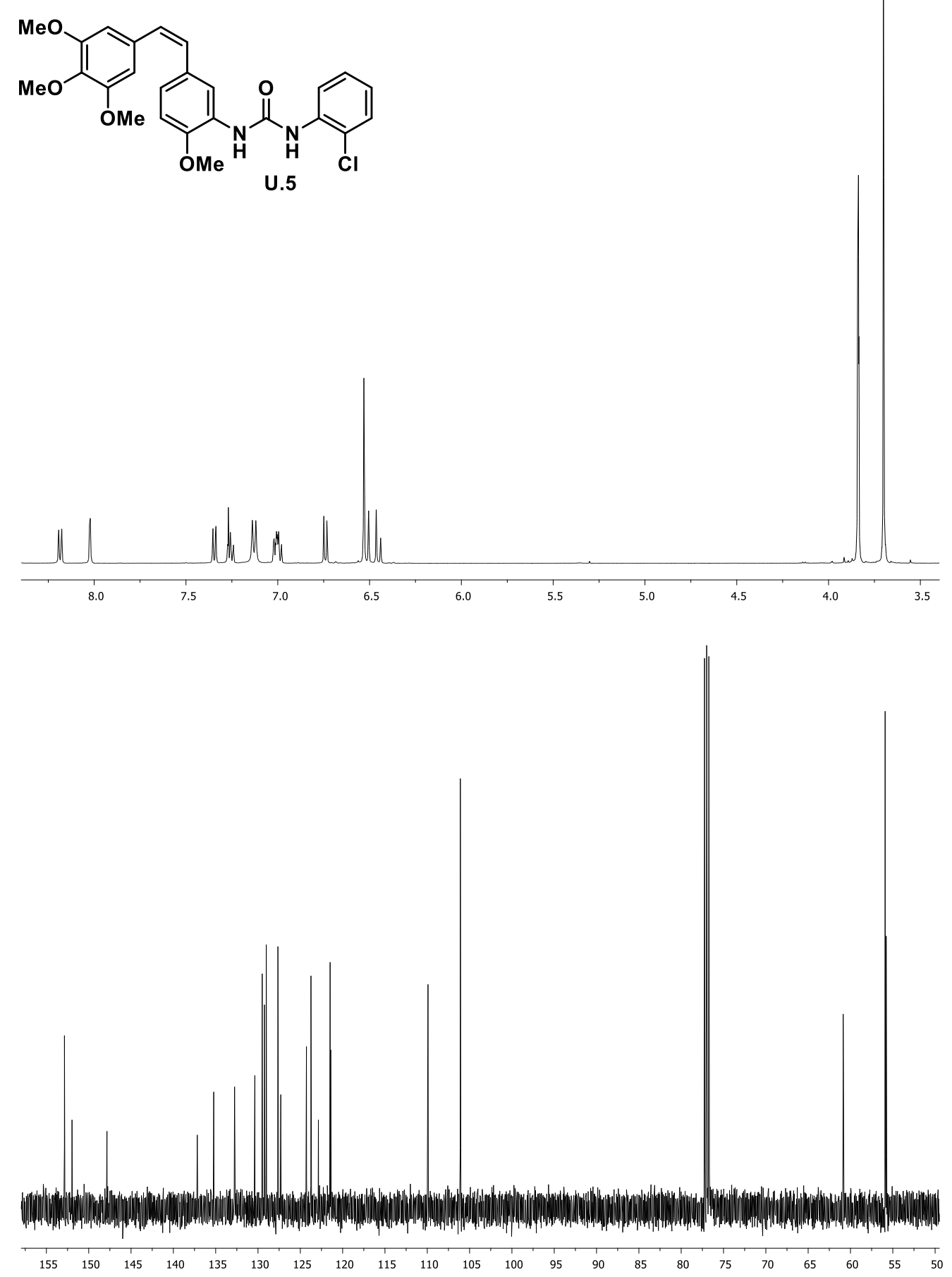
<smiles>COc1ccc(/C=C\c2ccc(OC)c(OC)c2)cc1NC(=O)Nc1cccc(Cl)c1</smiles>
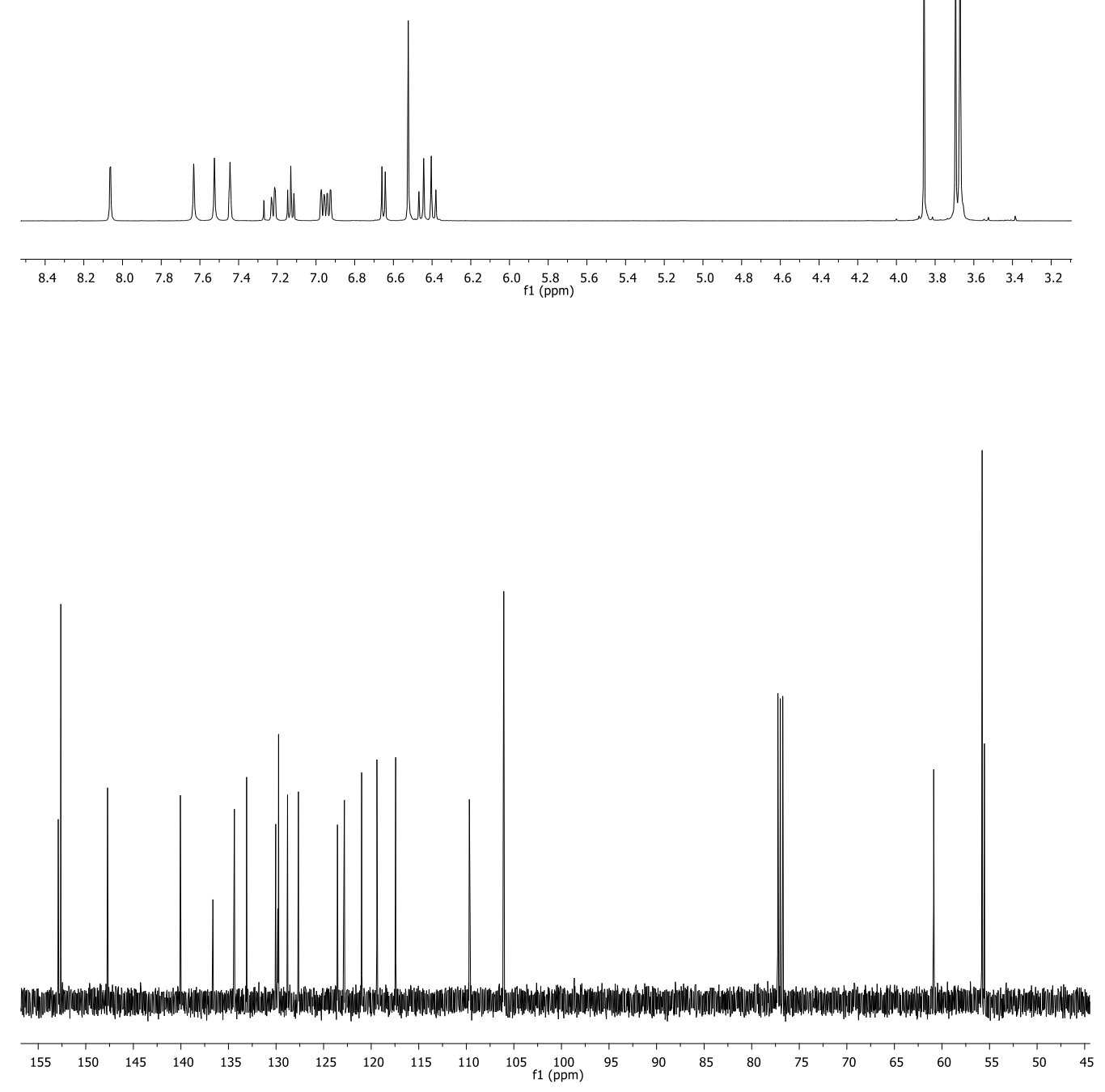
<smiles>COc1ccc(/C=C\c2ccc(OC)c(OC)c2)cc1NC(=O)Nc1ccc(Cl)cc1</smiles>
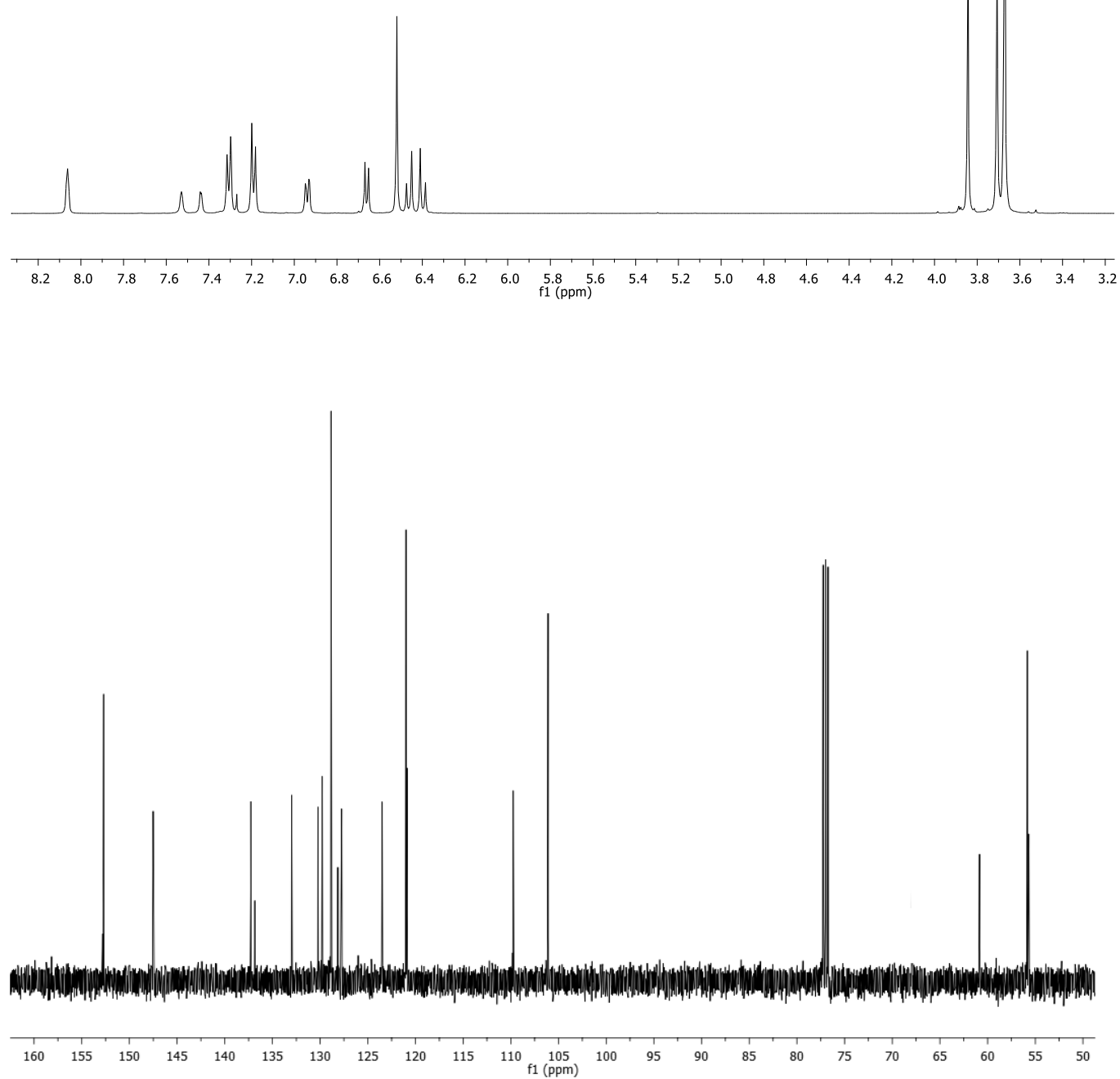

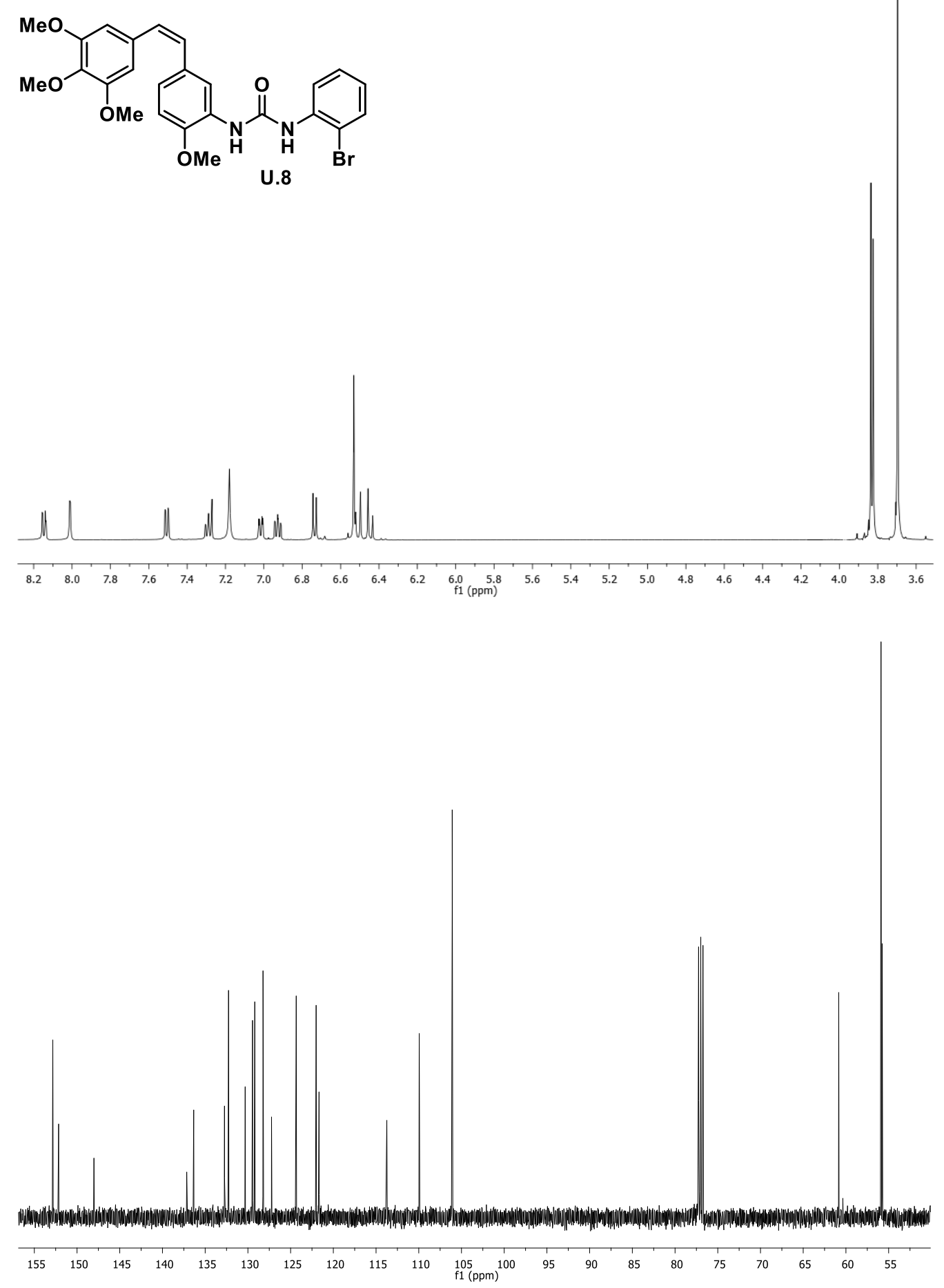

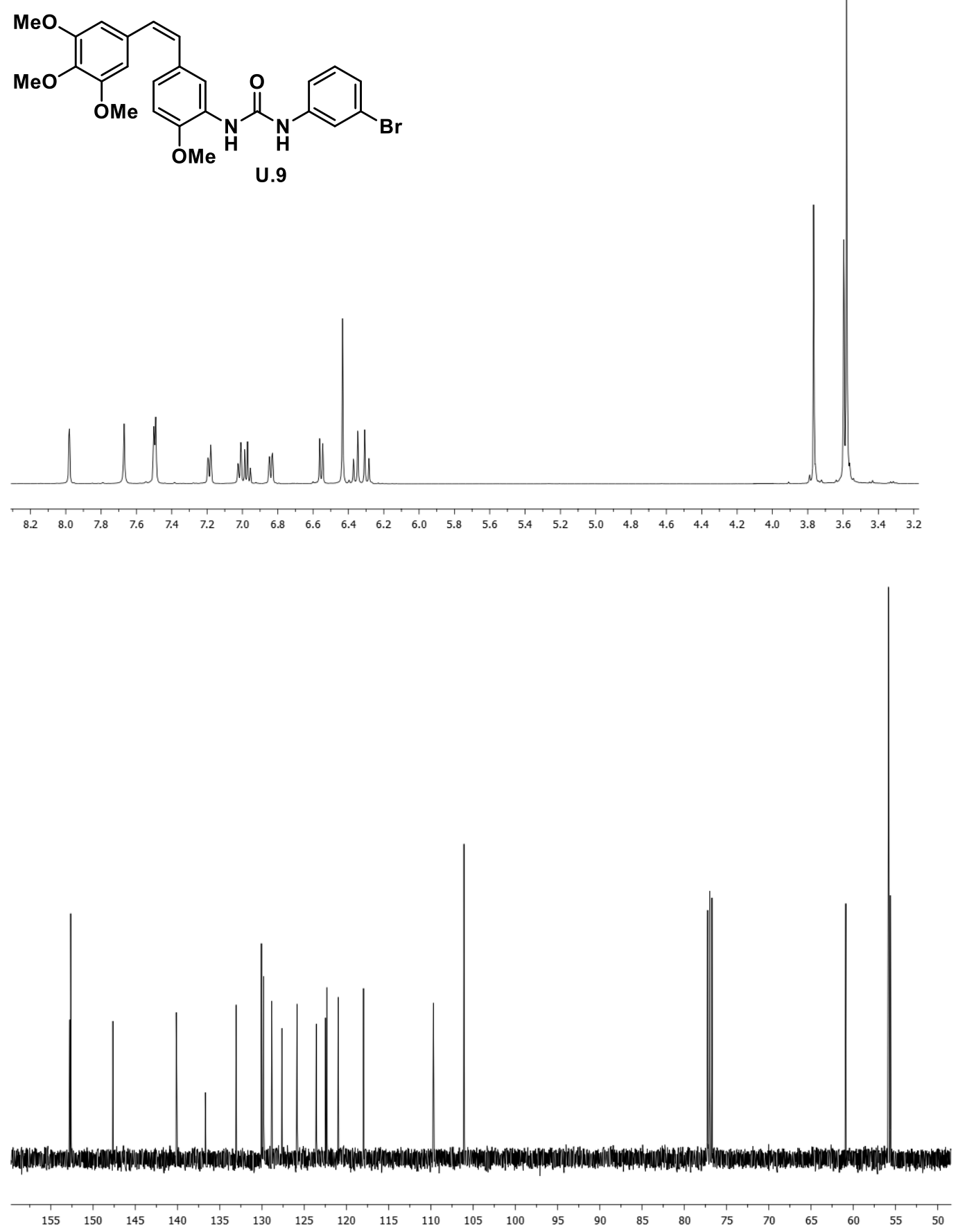
<smiles>COc1ccc(/C=C\c2cc(OC)c(OC)c(OC)c2)cc1NC(=O)Nc1ccc(Br)cc1</smiles>
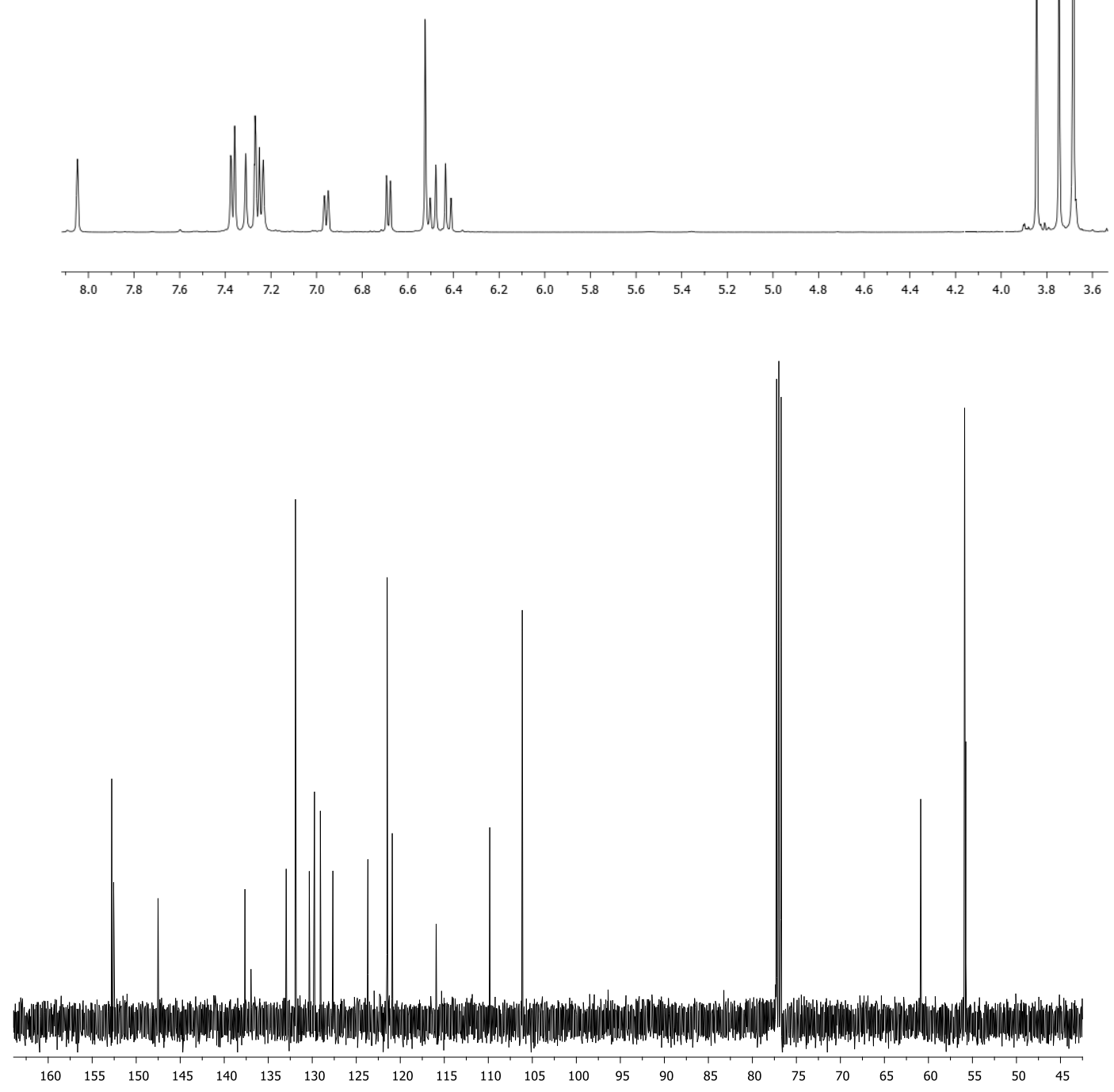

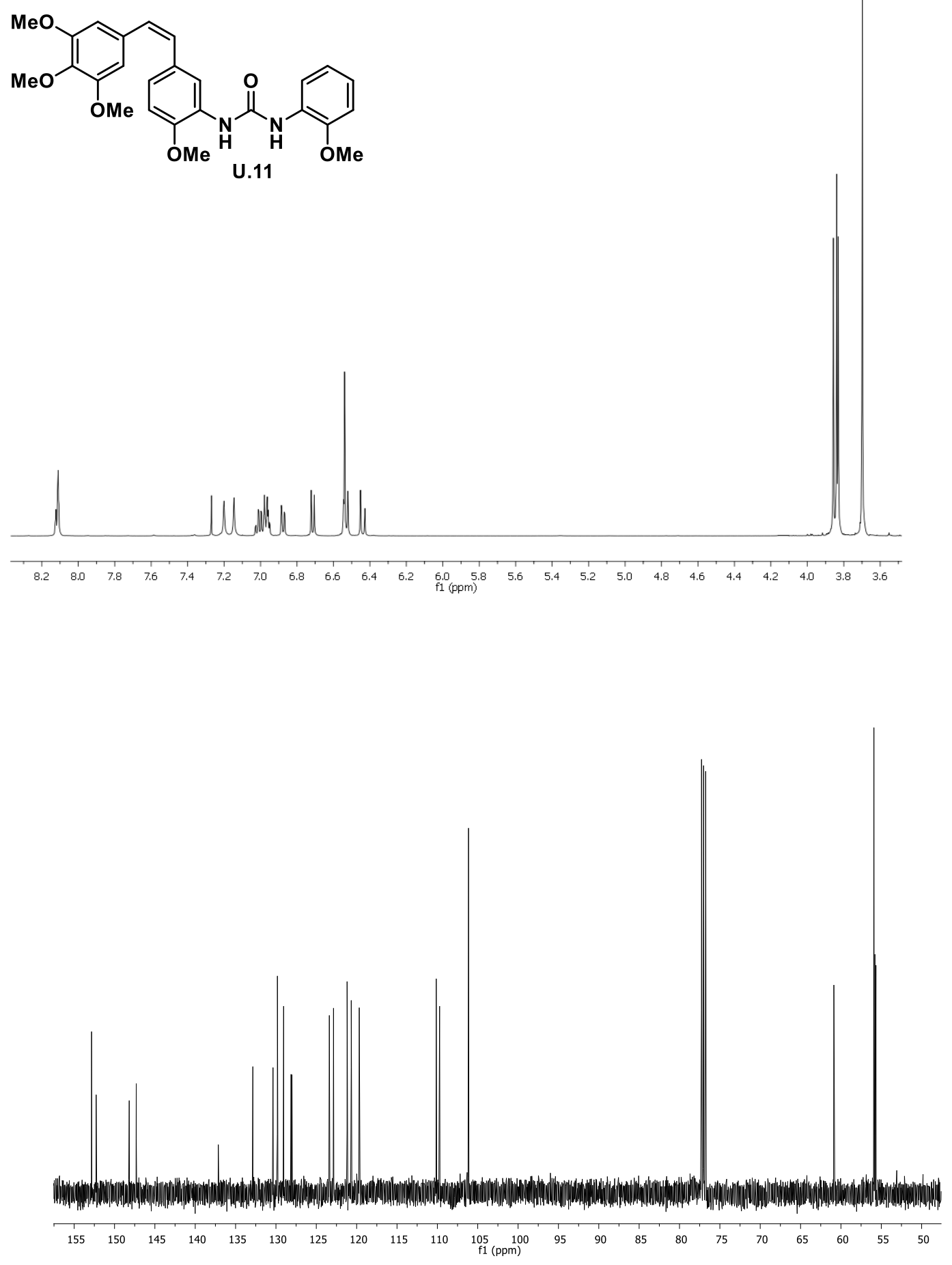

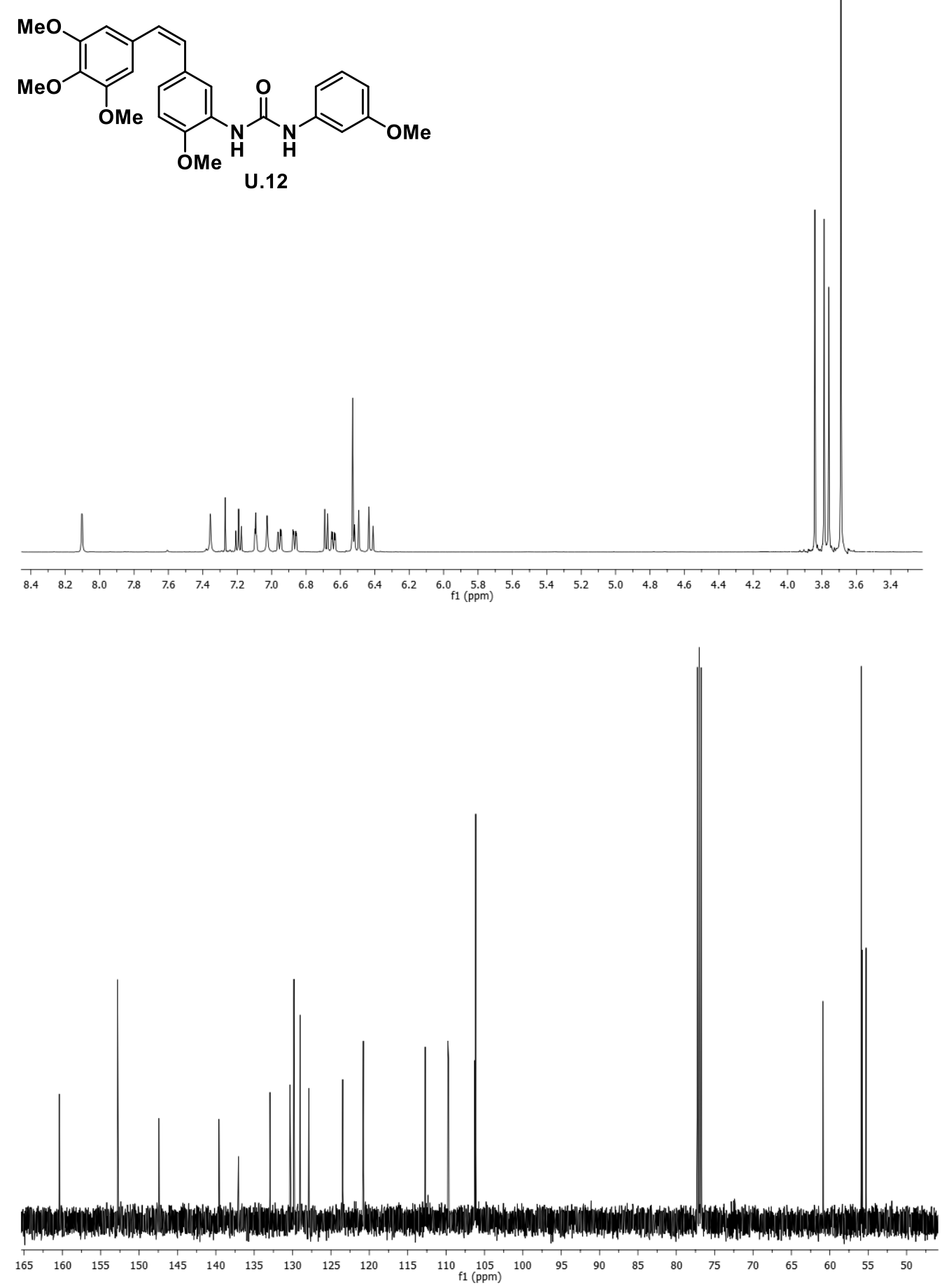
<smiles>COc1ccc(NC(=O)Nc2cc(/C=C\c3cc(OC)c(OC)c(OC)c3)ccc2OC)cc1</smiles>
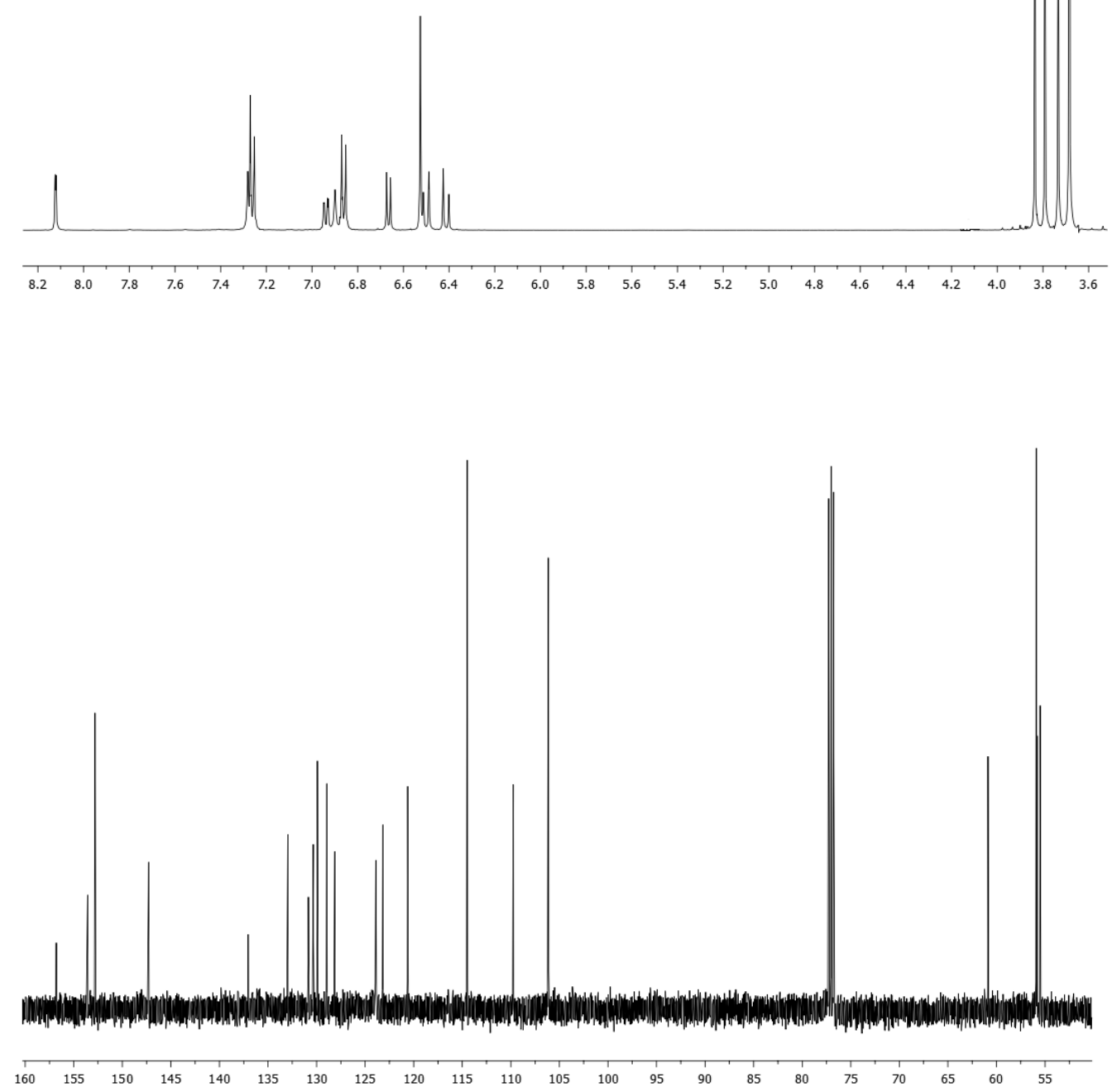

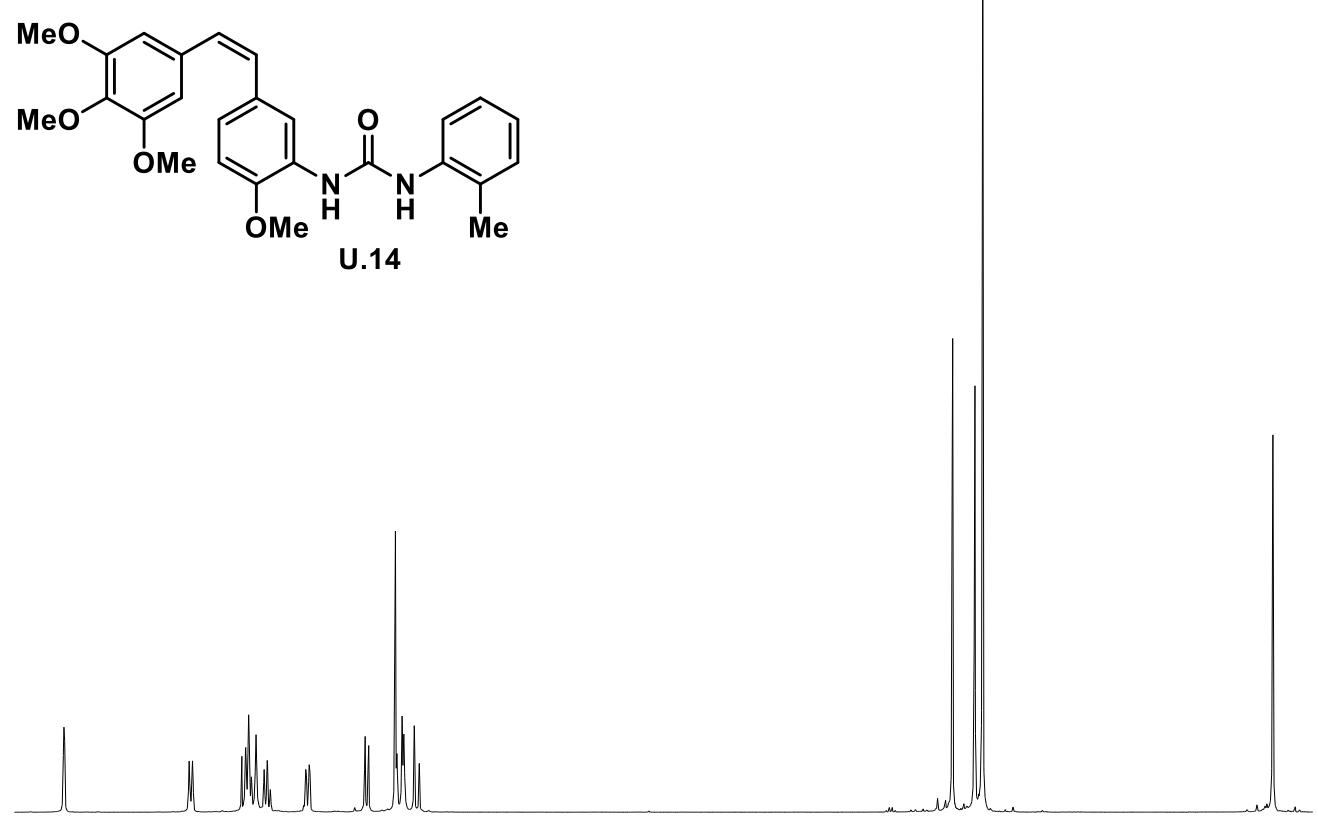

\begin{tabular}{lllllllllllllllllllllllllllllllllllllllll}
8.2 & 8.0 & 7.8 & 7.6 & 7.4 & 7.2 & 7.0 & 6.8 & 6.6 & 6.4 & 6.2 & 1.0 & 5.8 & 5.6 & 5.4 & 5.2 & 5.0 & 4.8 & 4.6 & 4.4 & 4.2 & 4.0 & 3.8 & 1.6 & 1.4 & 1.2 & 1.0 & 1.8 & 1.6 & 1.4 & 1 \\
\hline
\end{tabular}

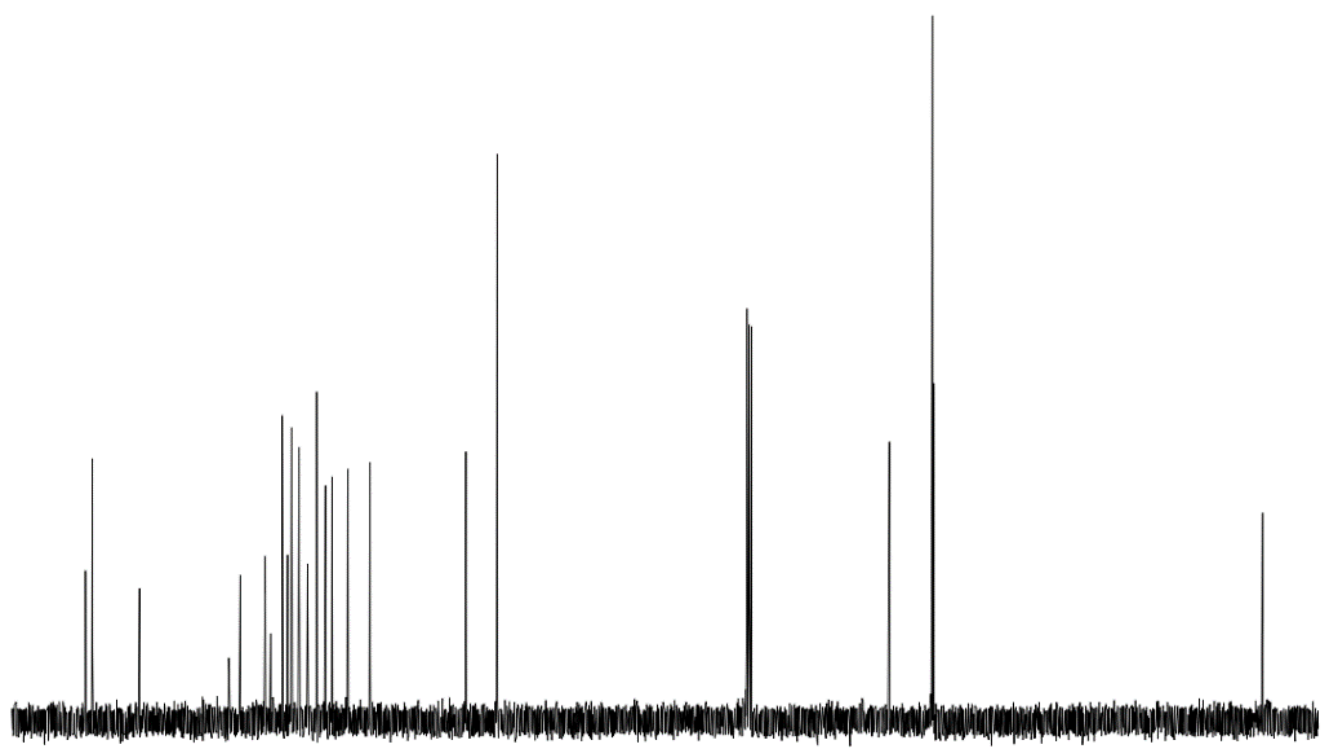

$\begin{array}{lllllllllllllllllllllllllllllllllllll}160 & 155 & 150 & 145 & 140 & 135 & 130 & 125 & 120 & 115 & 110 & 105 & 100 & 95 & 90 & 85 & 80 & 75 & 70 & 65 & 60 & 55 & 50 & 45 & 40 & 35 & 30 & 25 & 20 & 15\end{array}$ 

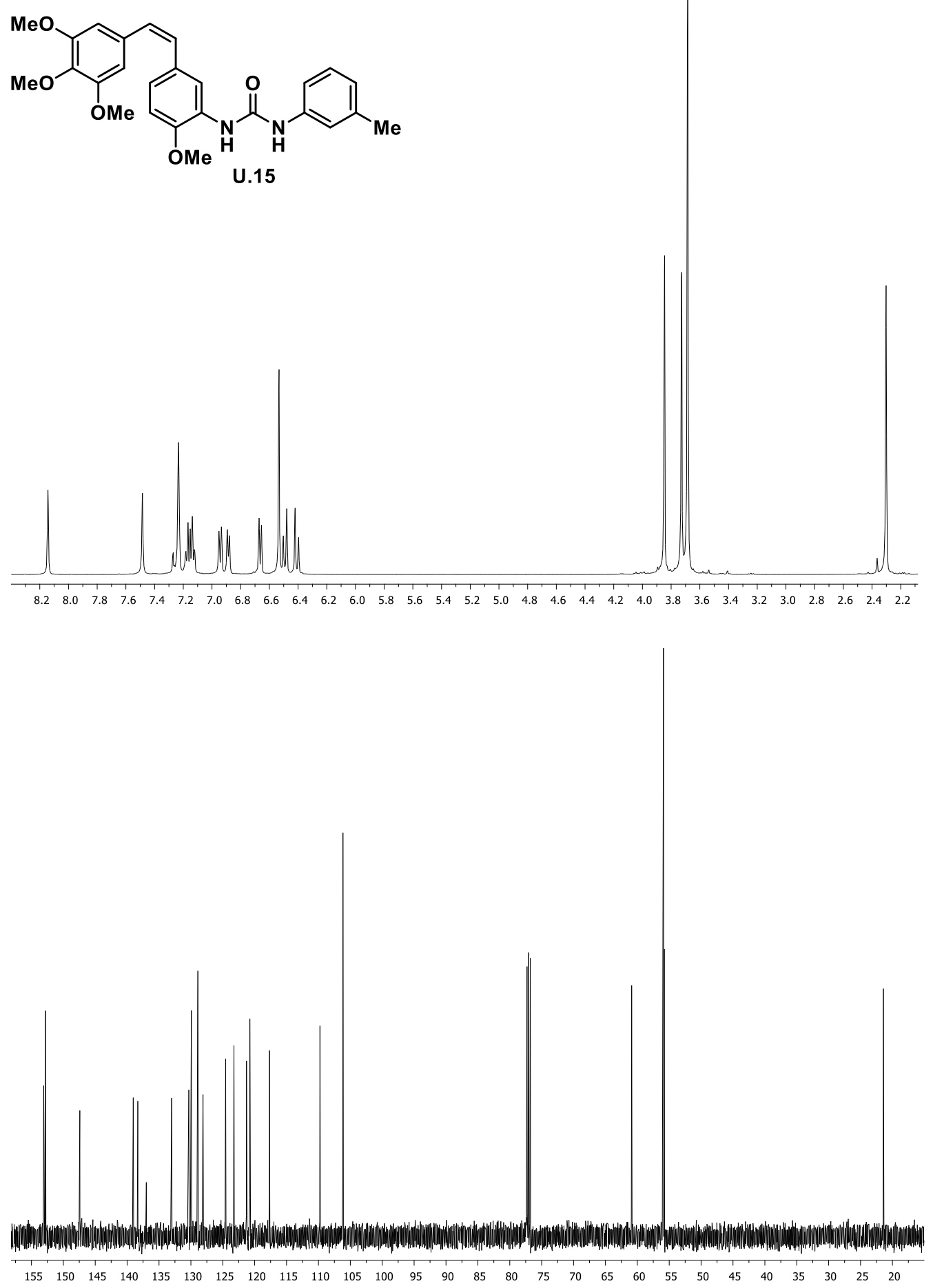
228

Chapter 7
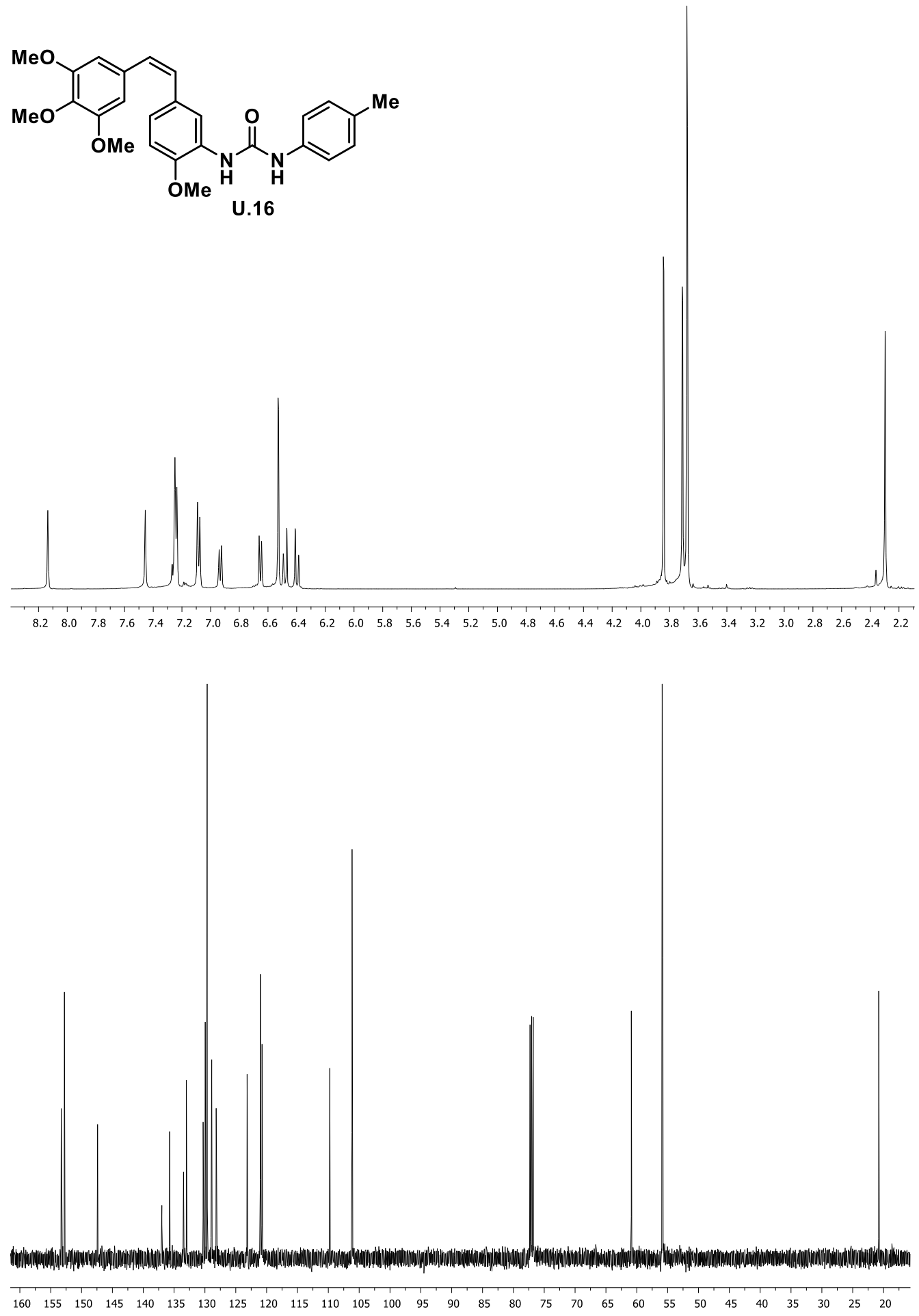
<smiles>COc1ccc(/C=C\c2cc(OC)c(OC)c(OC)c2)cc1NC(=O)Nc1ccccc1C(F)(F)F</smiles>
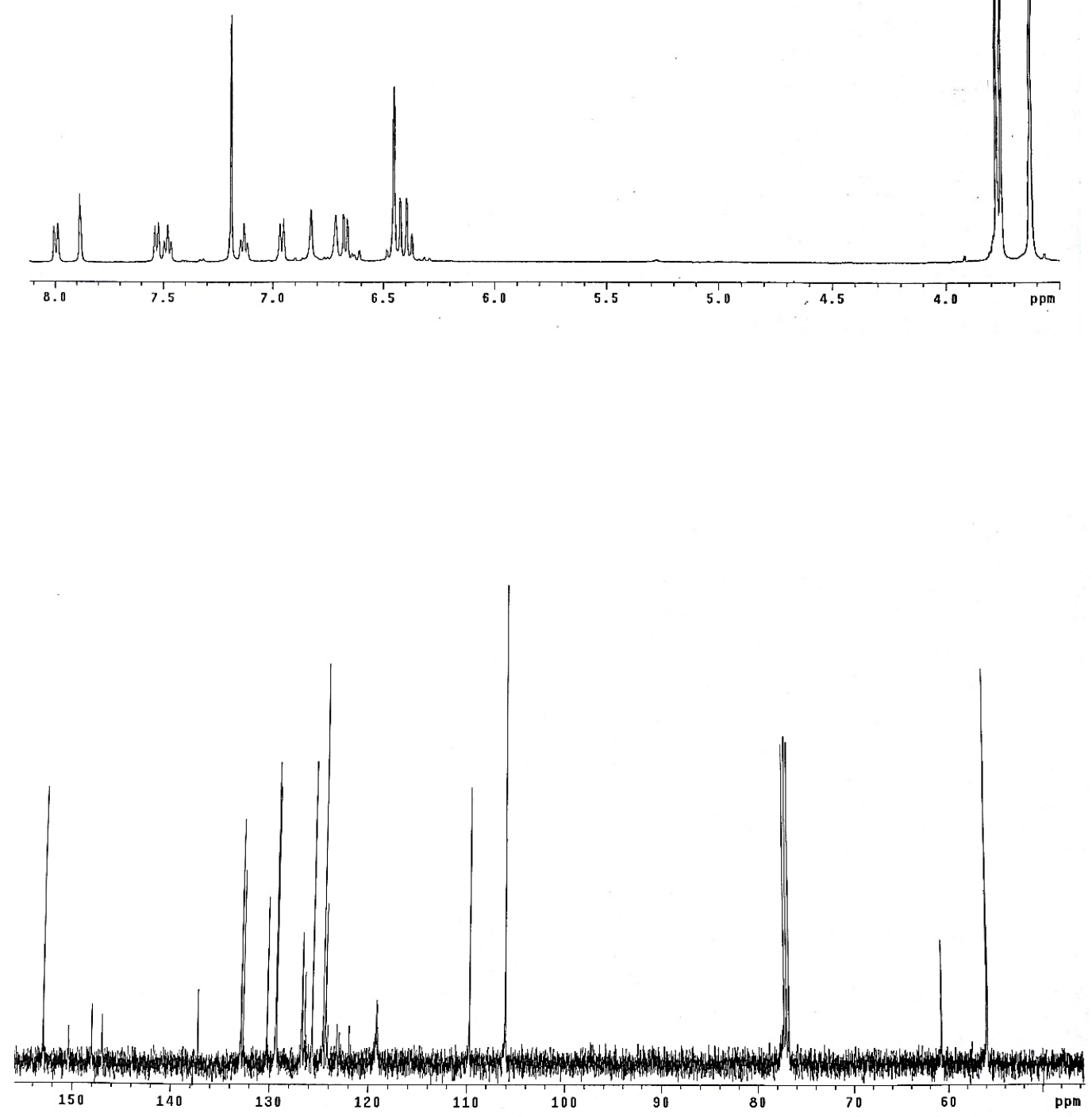
230

Chapter 7
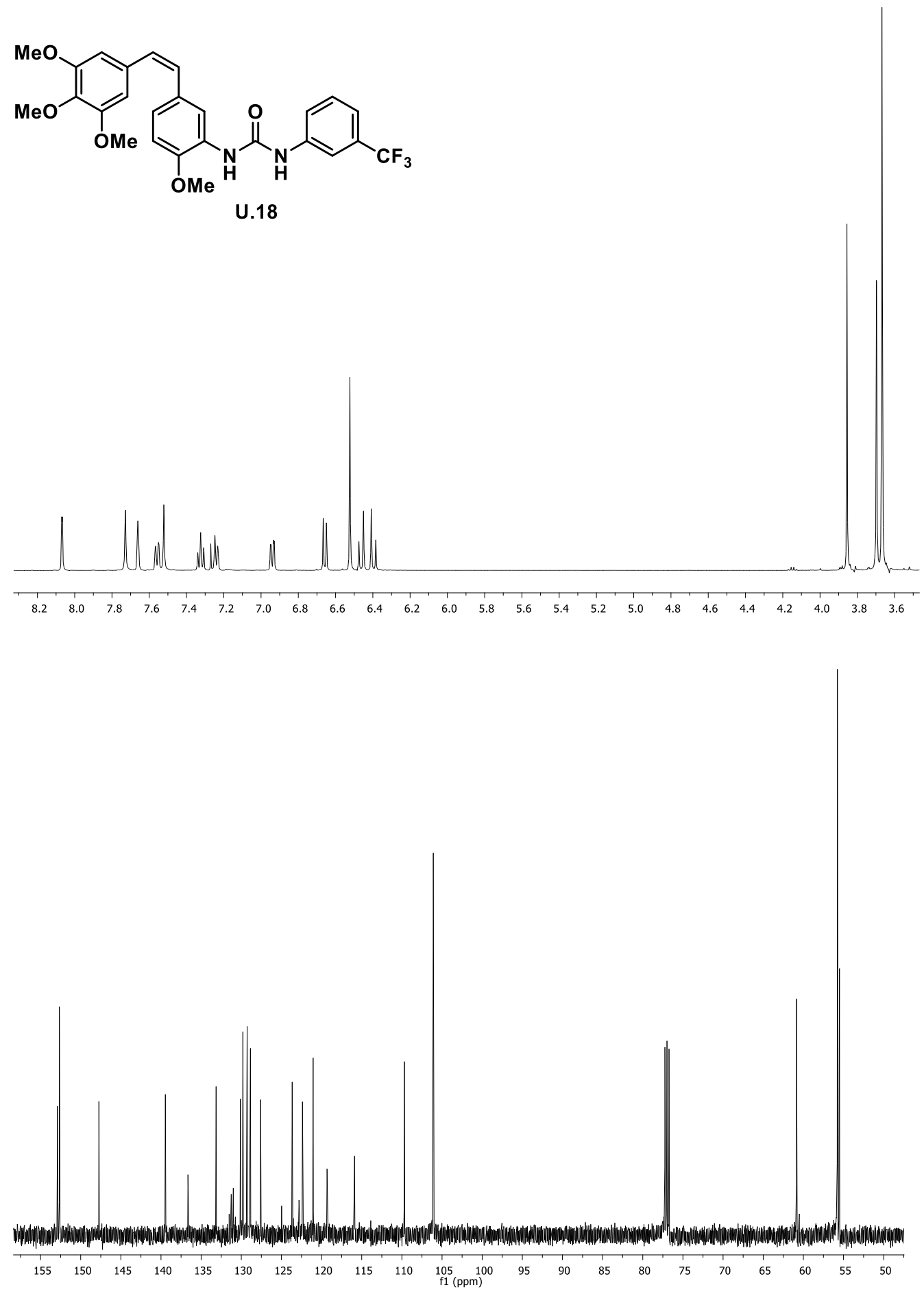

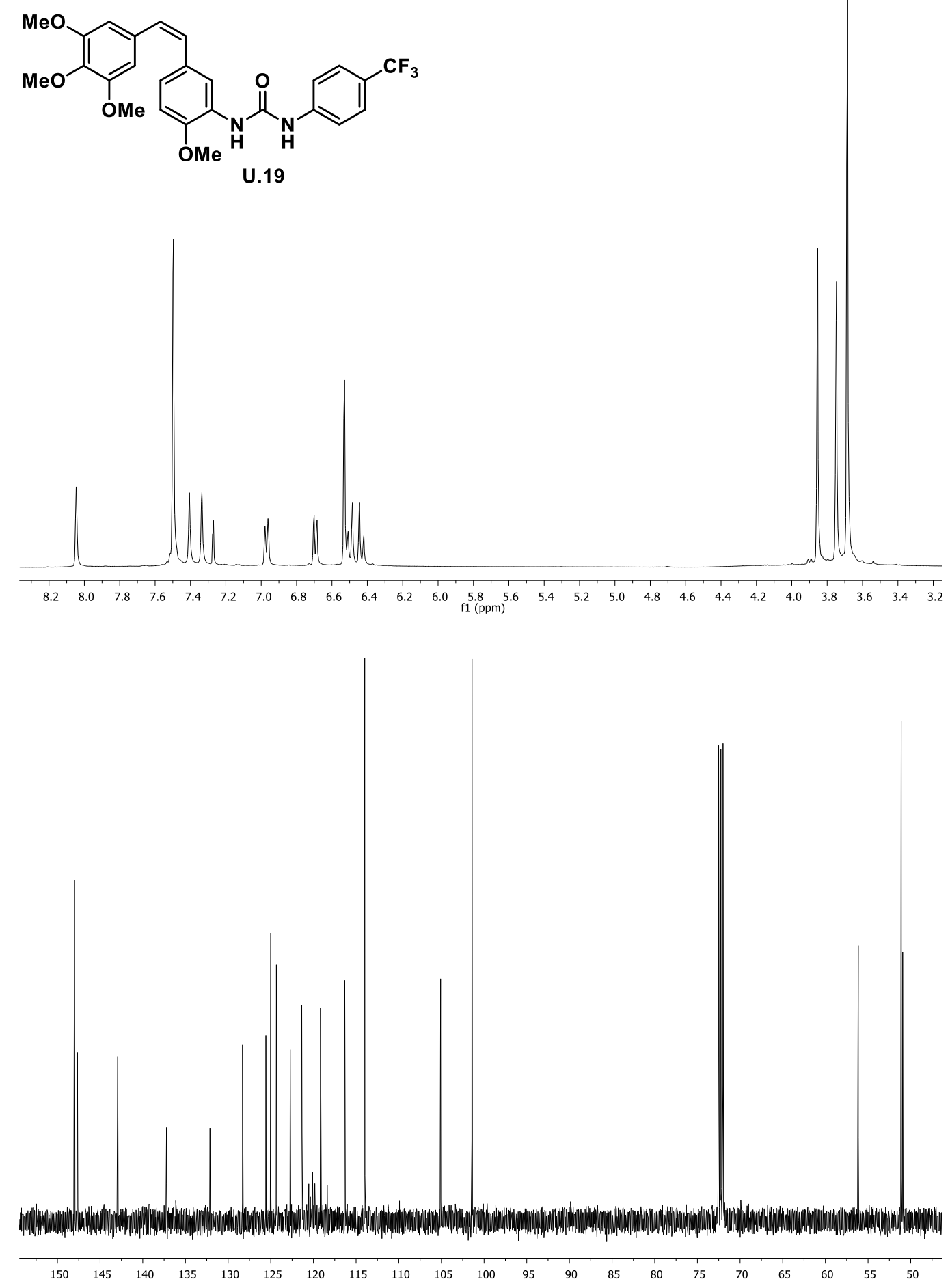

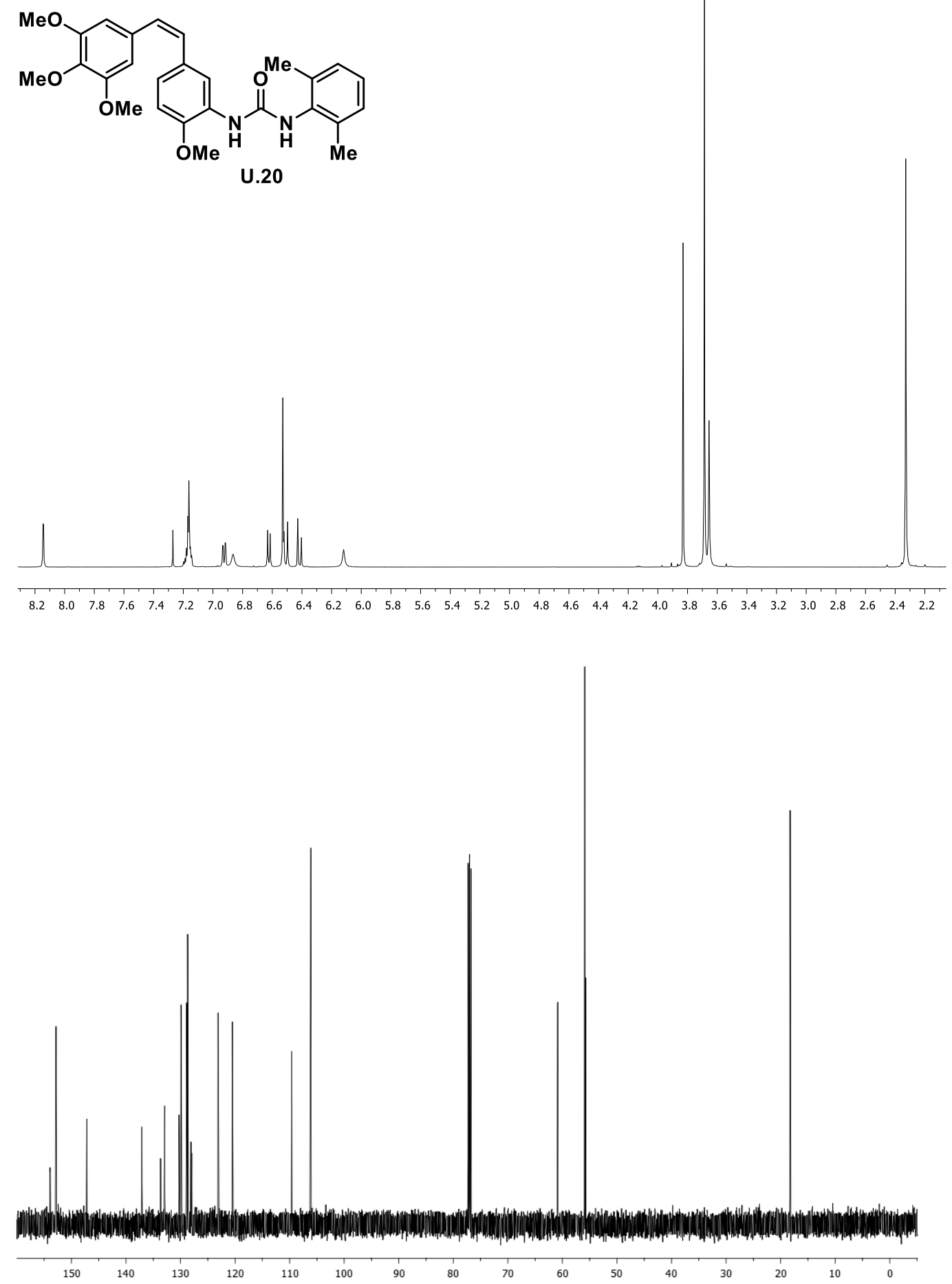

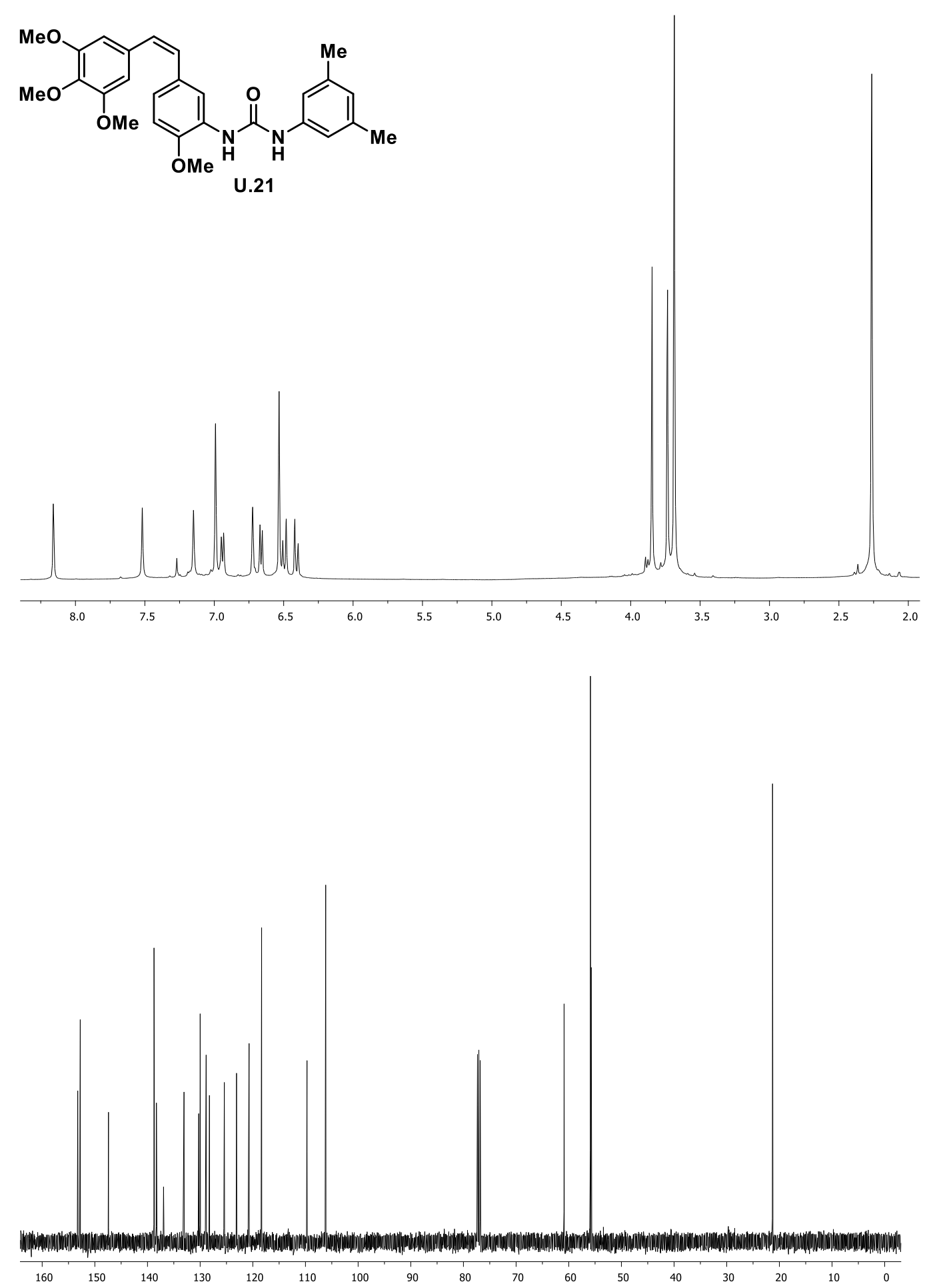

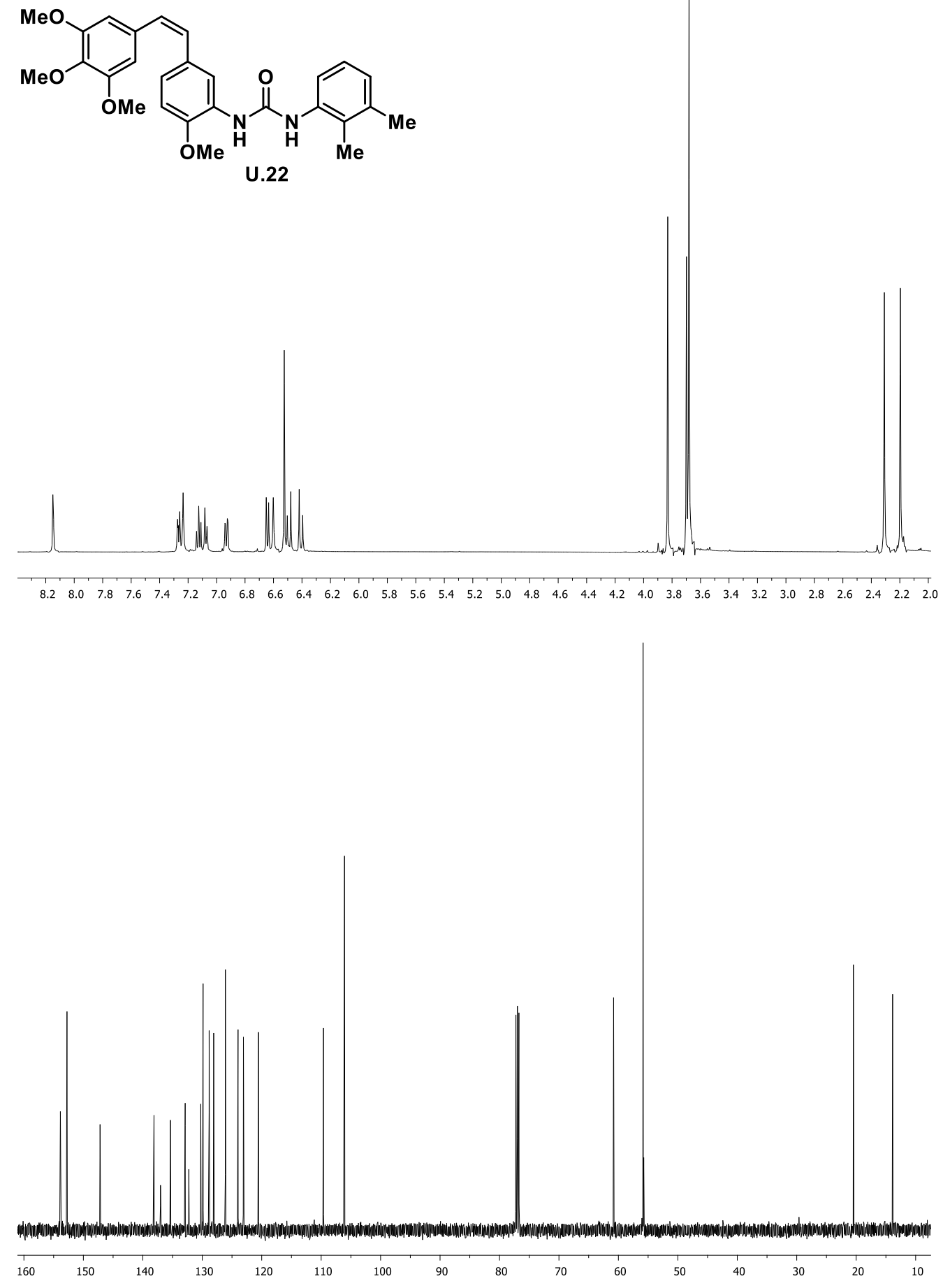
<smiles>COc1ccc(/C=C\c2cc(OC)c(OC)c(OC)c2)cc1NC(=O)Nc1ccc(C)c(C)c1</smiles>

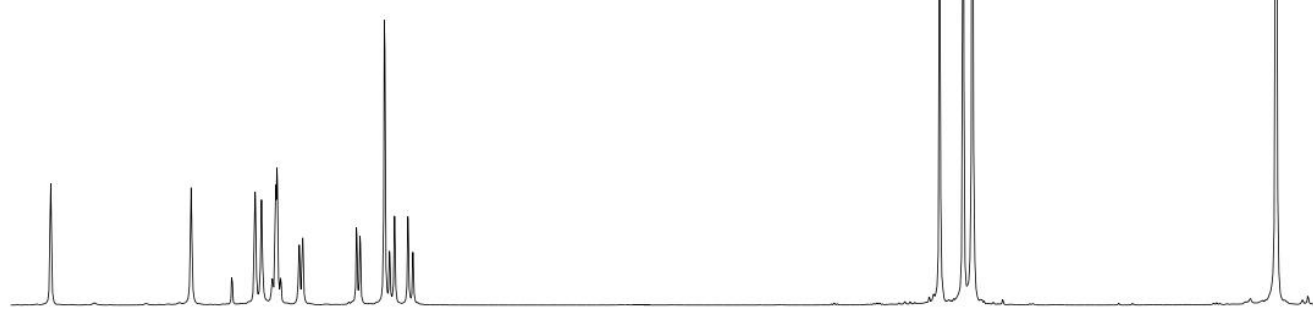

$\begin{array}{lllllllllllllllllllllllllllllllllllllllll}8.2 & 8.0 & 7.8 & 7.6 & 7.4 & 7.2 & 7.0 & 6.8 & 6.6 & 6.4 & 6.2 & 6.0 & 5.8 & 5.6 & 5.4 & 5.2 & 5.0 & 4.8 & 4.6 & 4.4 & 4.2 & 4.0 & 3.8 & 3.6 & 3.4 & 3.2 & 3.0 & 2.8 & 2.6 & 2.4 & 2.2\end{array}$

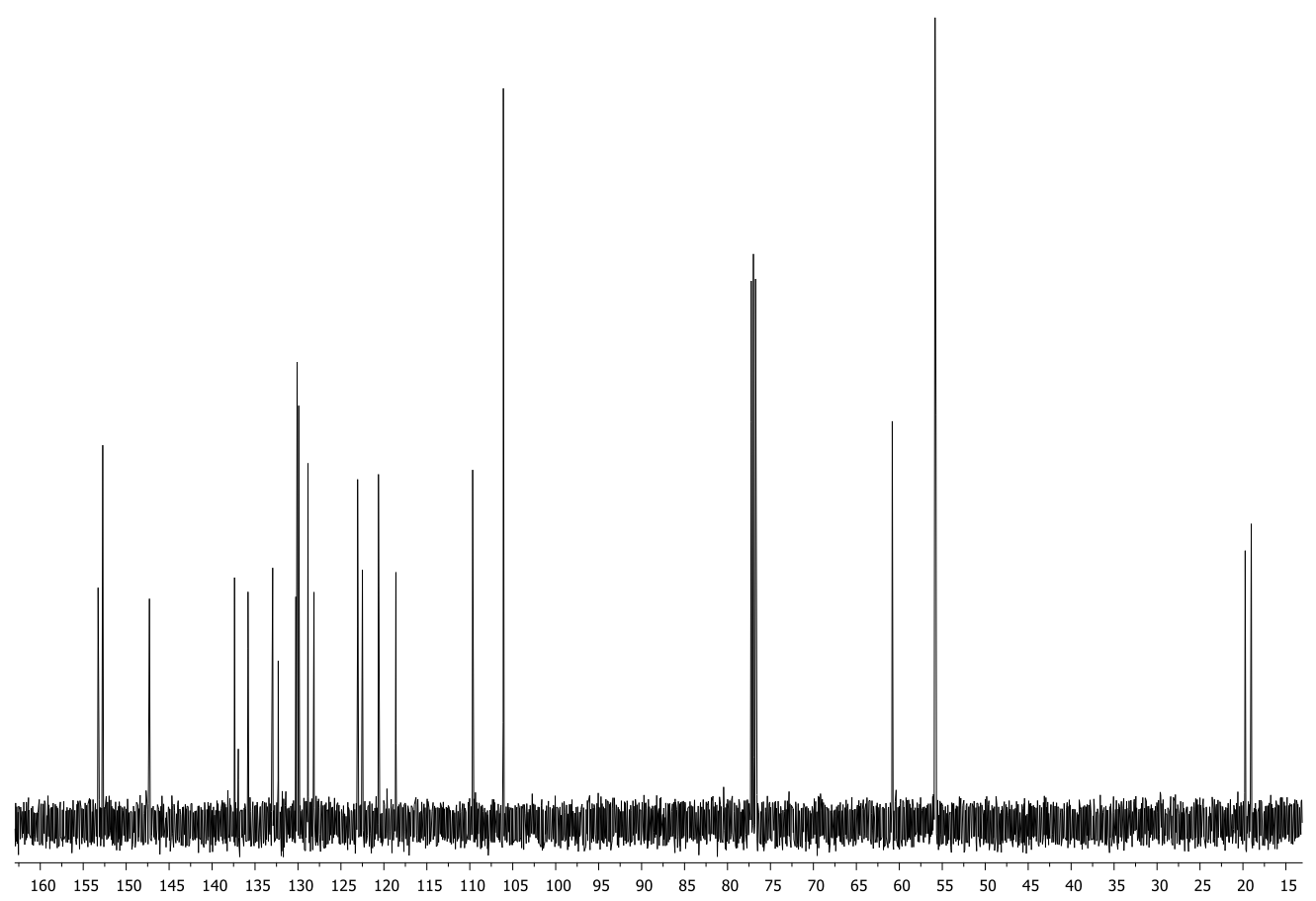



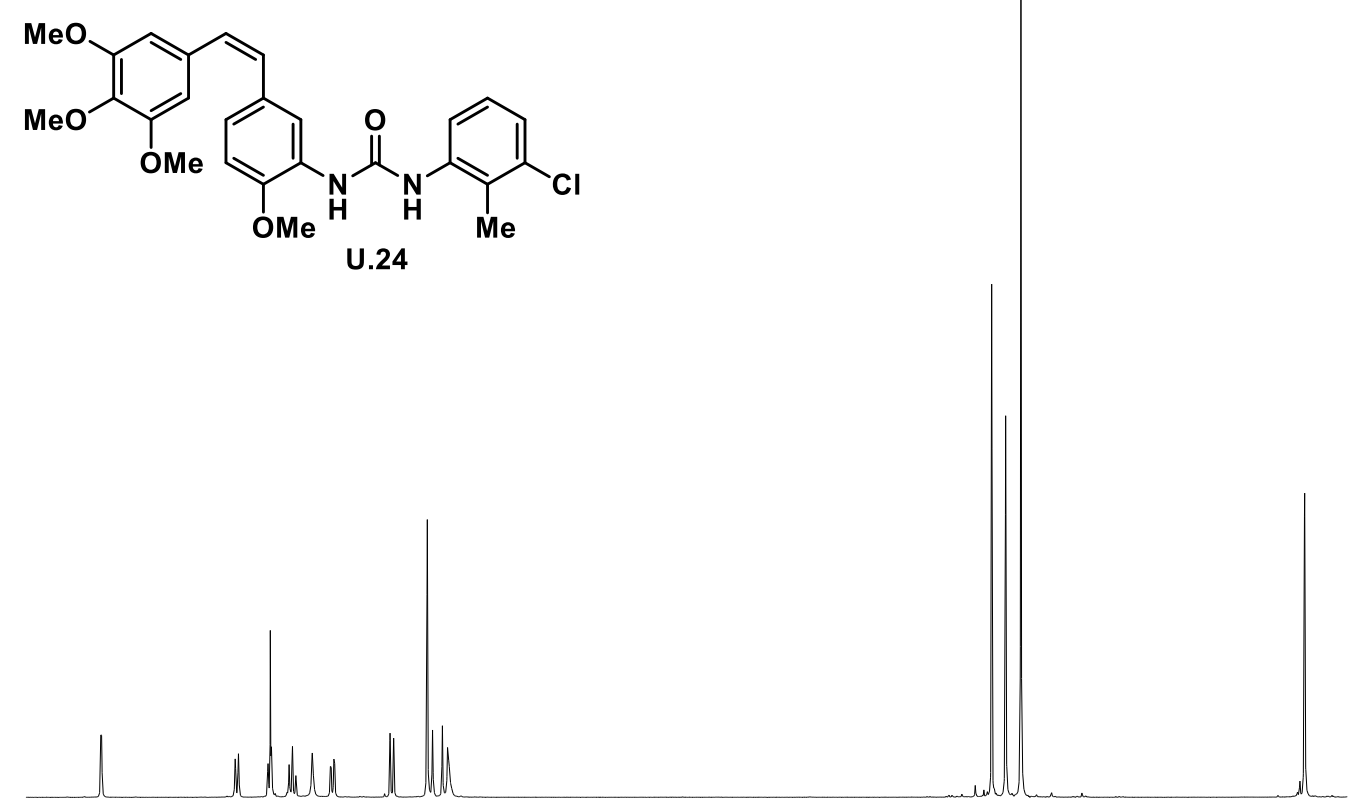

$\begin{array}{llllllllllllllllllllllllllllllllllllll}8.4 & 8.2 & 8.0 & 7.8 & 7.6 & 7.4 & 7.2 & 7.0 & 6.8 & 6.6 & 6.4 & 6.2 & 6.0 & 5.8 & 5.6 & 5.4 & 5.2 & 5.0 & 4.8 & 4.6 & 4.4 & 4.2 & 4.0 & 3.8 & 3.6 & 3.4 & 3.2 & 3.0 & 2.8 & 2.6 & 2.4 & 2.2\end{array}$

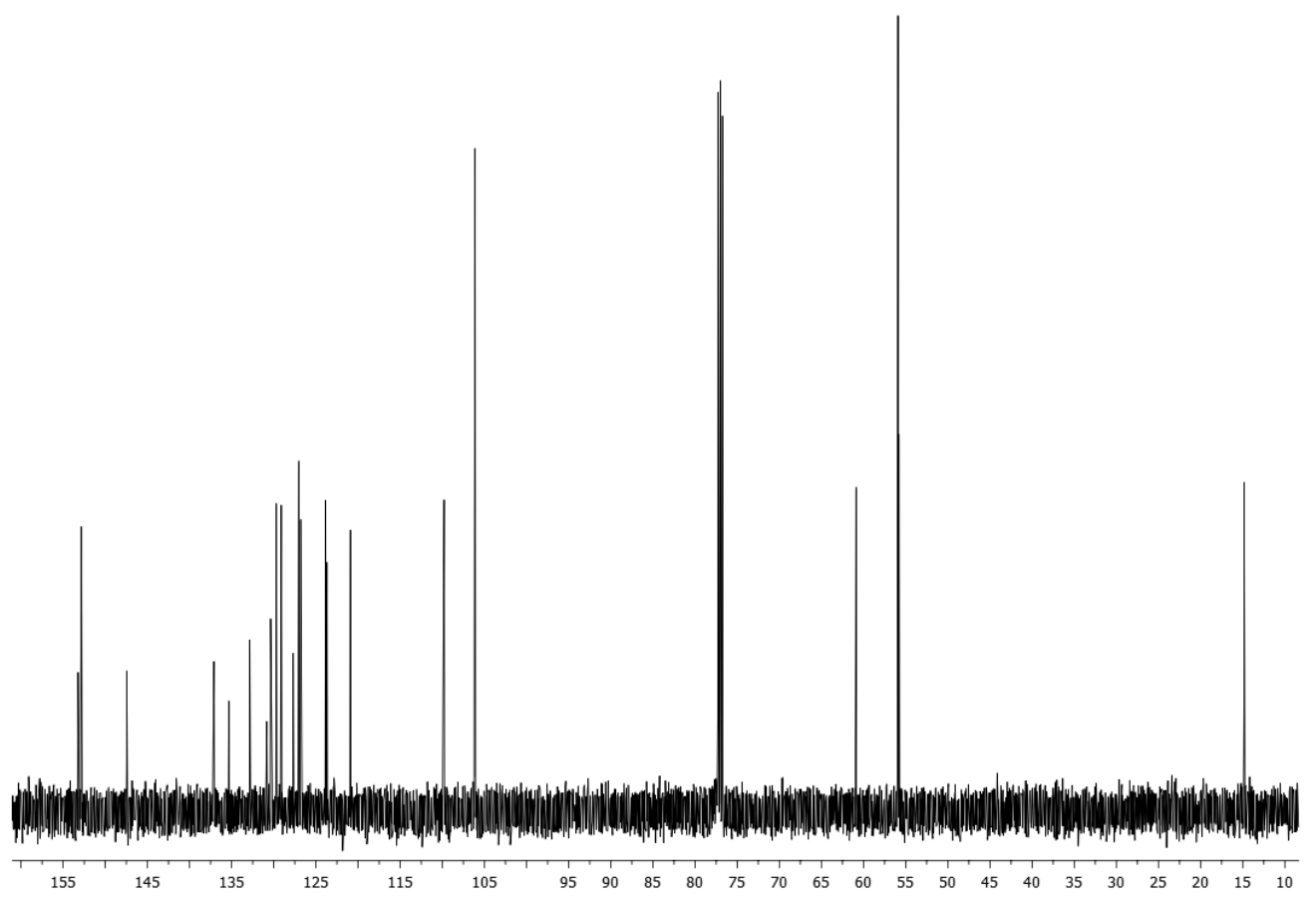


<smiles>COc1ccc(/C=C\c2cc(OC)c(OC)c(OC)c2)cc1NC(=O)Nc1ccc(Cl)c(C)c1</smiles>
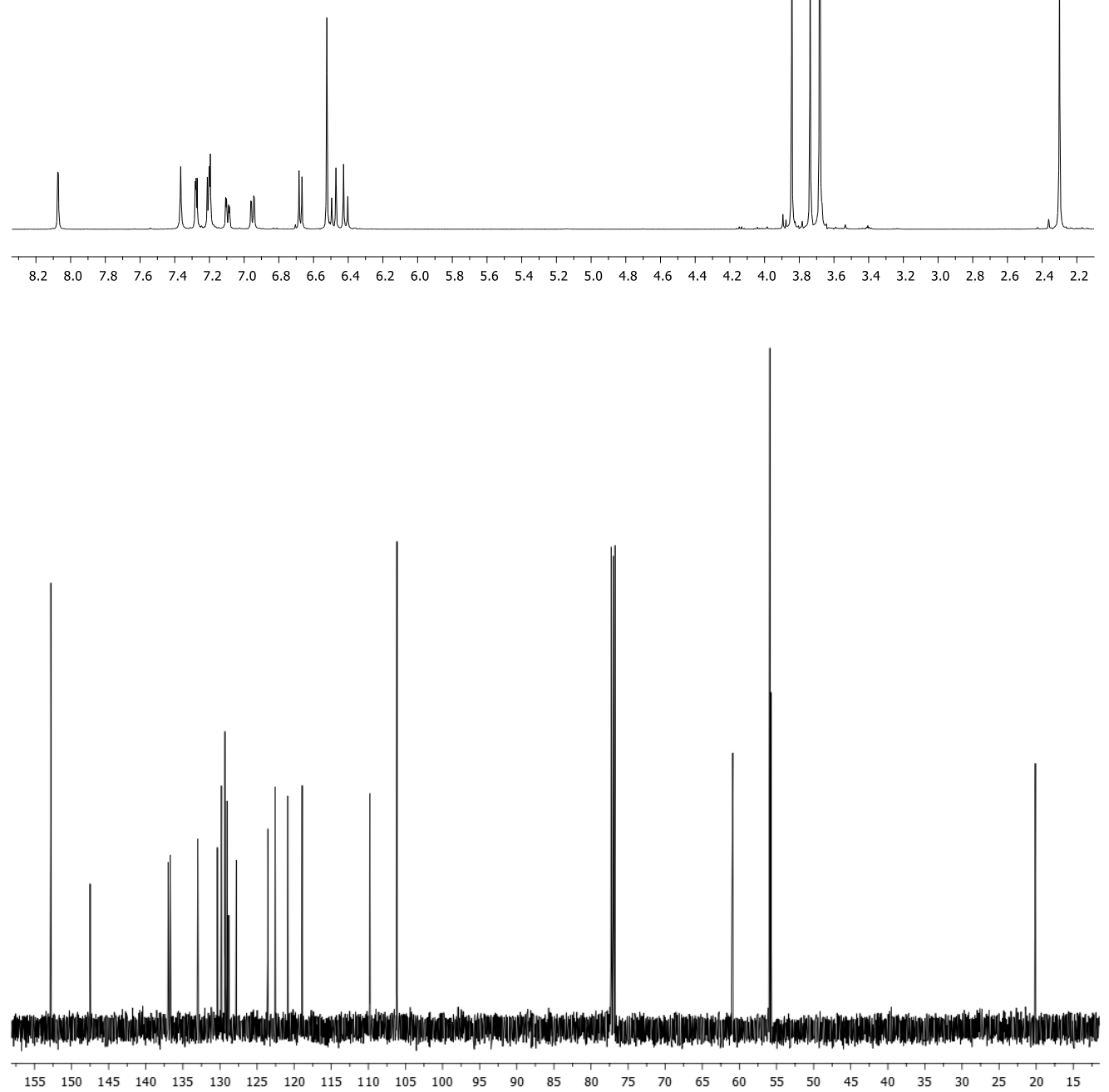

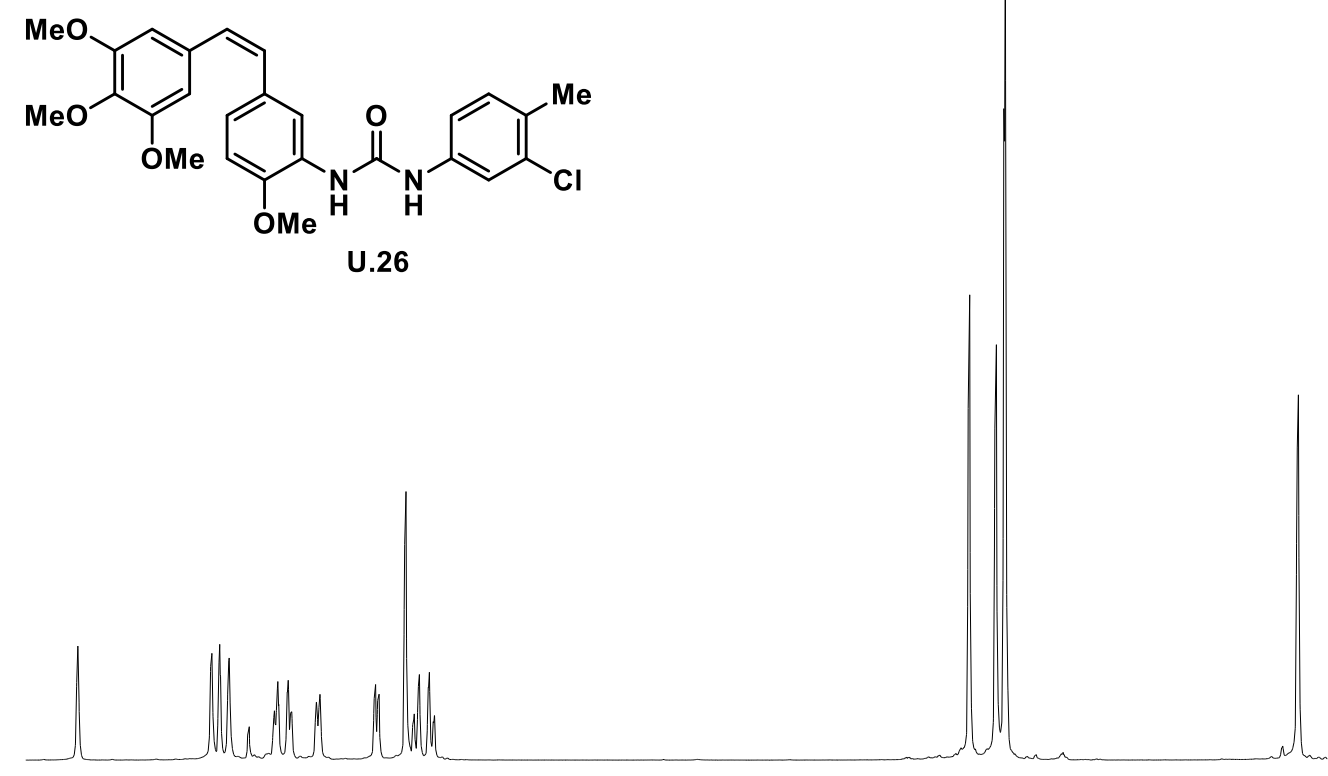

$\begin{array}{llllllllllllllllllllllllllllllllllllllllllllll}8.2 & 8.0 & 7.8 & 7.6 & 7.4 & 7.2 & 7.0 & 6.8 & 6.6 & 6.4 & 6.2 & 6.0 & 5.8 & 5.6 & 5.4 & 5.2 & 5.0 & 4.8 & 4.6 & 4.4 & 4.2 & 4.0 & 3.8 & 3.6 & 3.4 & 3.2 & 3.0 & 2.8 & 2.6 & 2.4 & 2.2\end{array}$

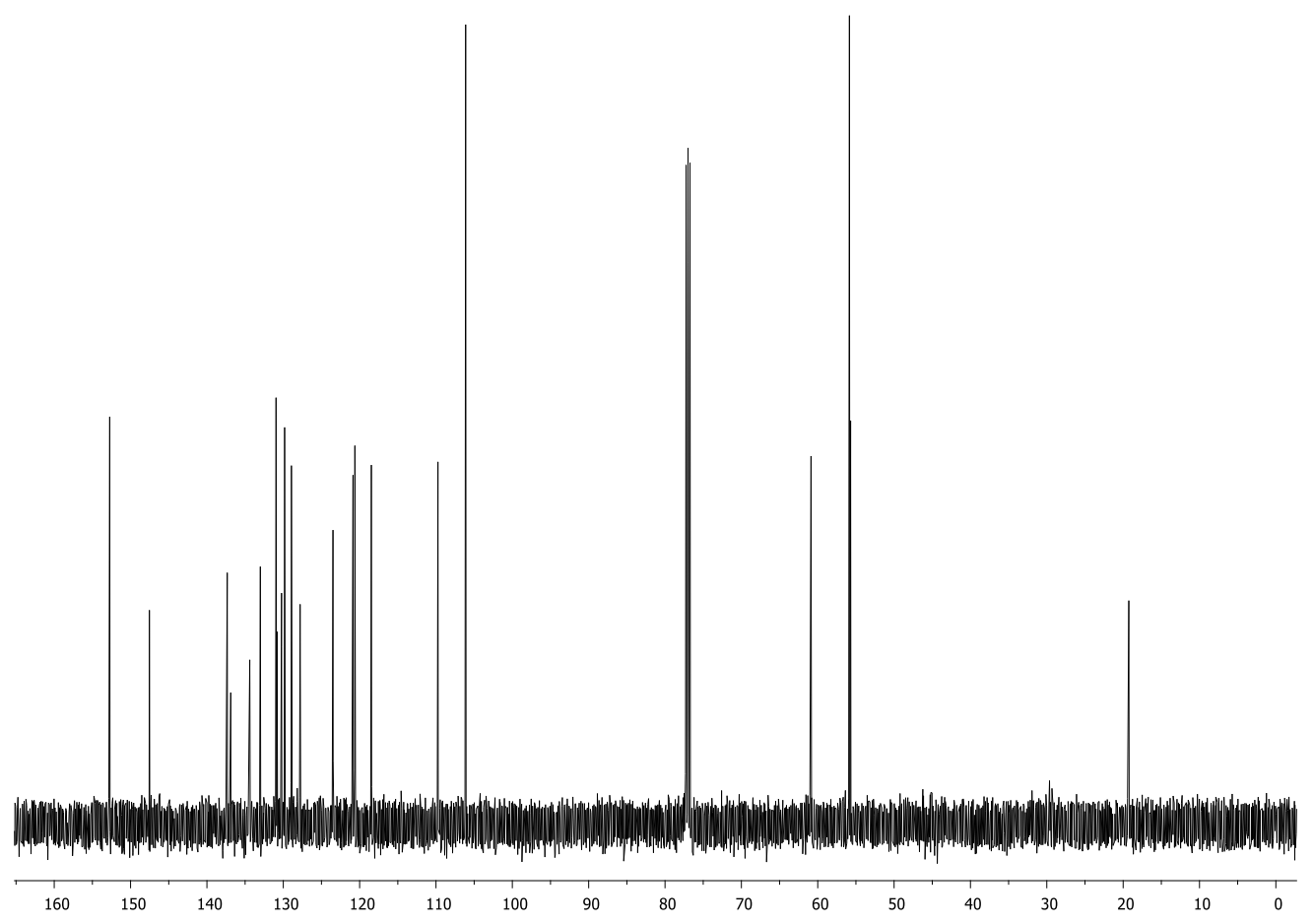


<smiles>COc1ccc(Cc2cn(CCNC(=O)Nc3ccccc3)nn2)cc1</smiles>
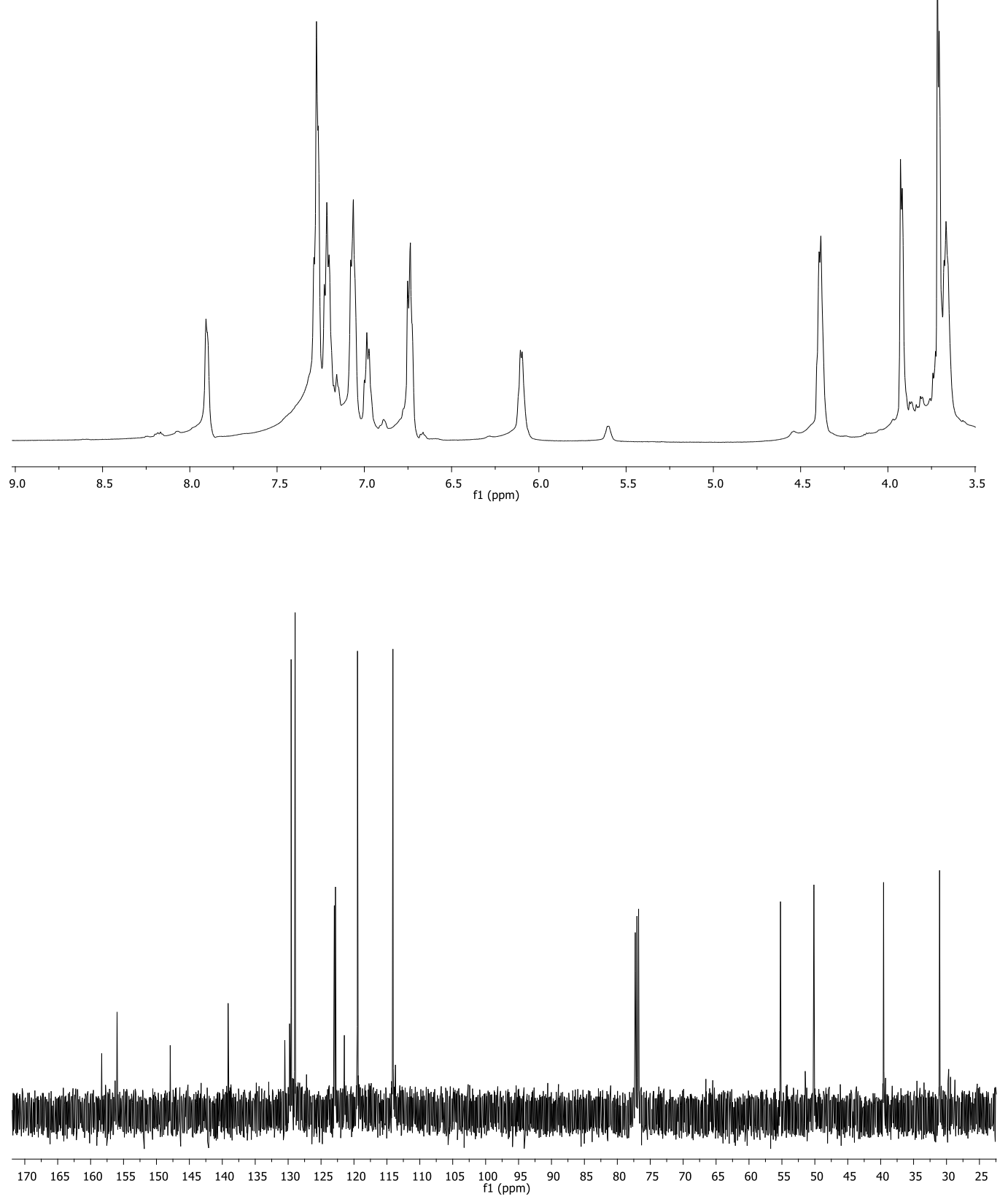
$\overbrace{\mathrm{N}_{\mathrm{T}=\mathrm{N}^{\prime}}^{\mathrm{N}}}^{\mathrm{N}} \overbrace{\mathrm{O}}^{\mathrm{N}}$
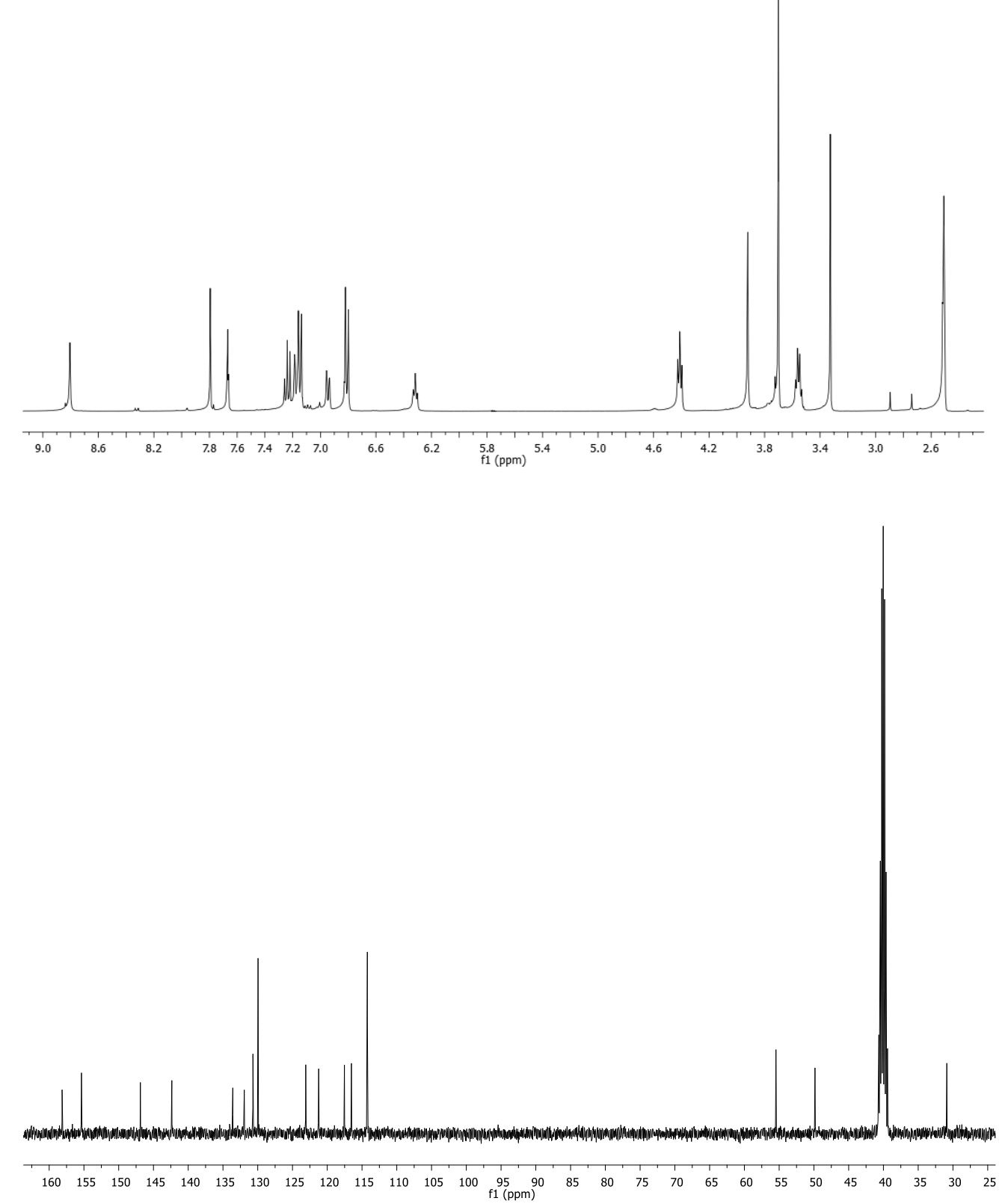
<smiles>COc1ccc(Cc2cn(CCNC(=O)Nc3ccc(Cl)cc3)nn2)cc1</smiles>
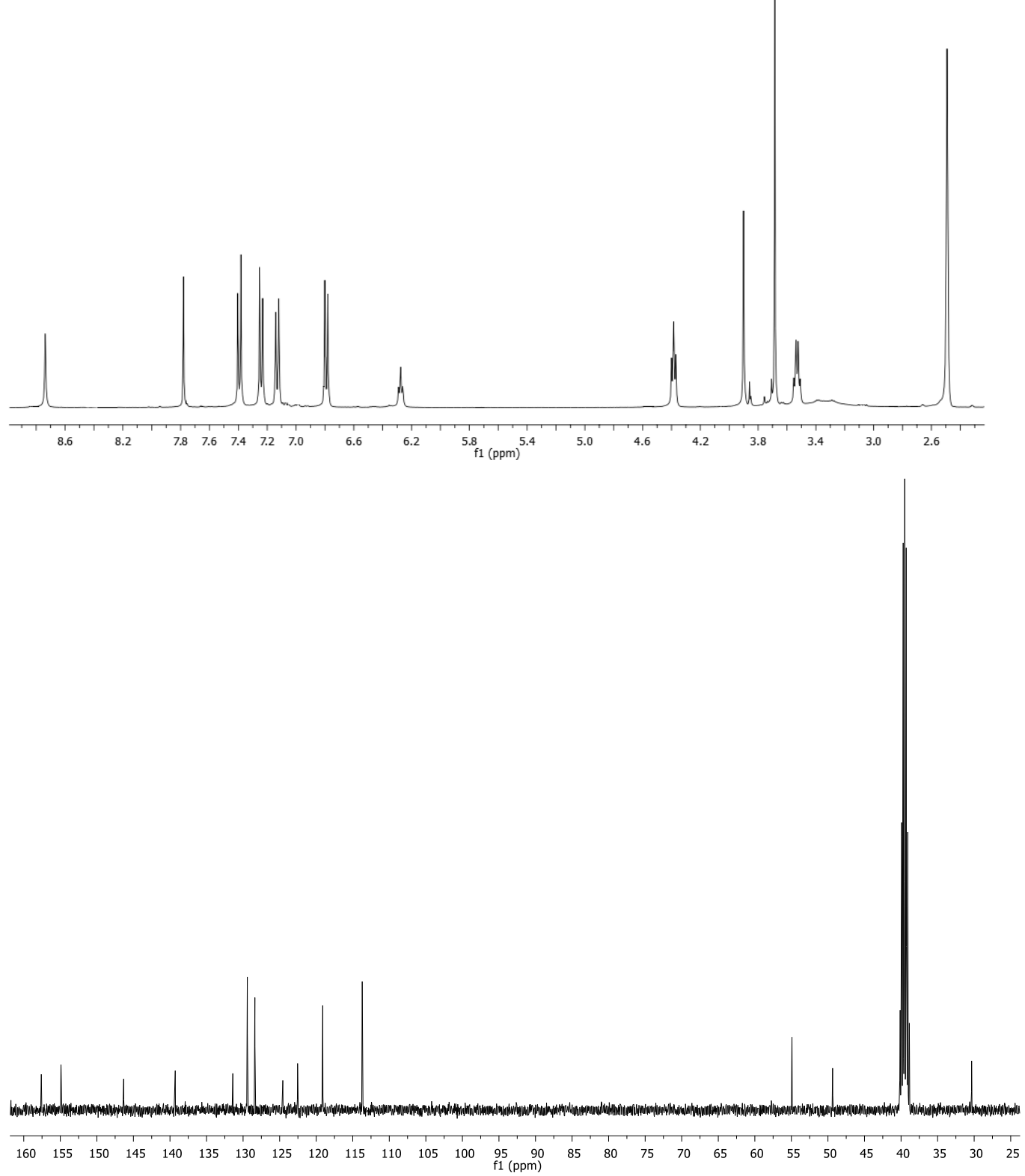


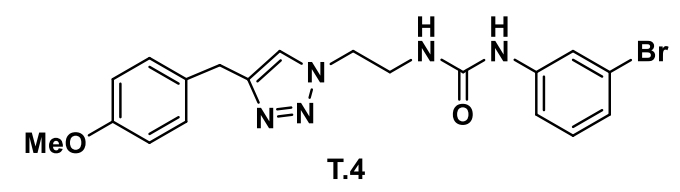

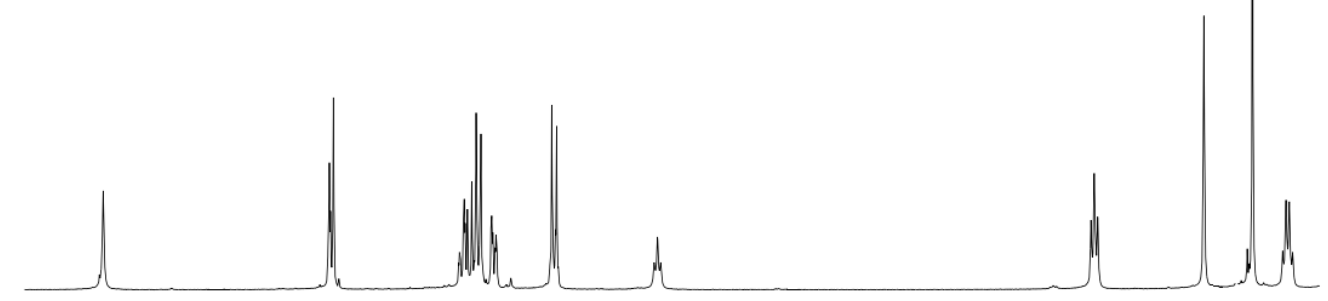

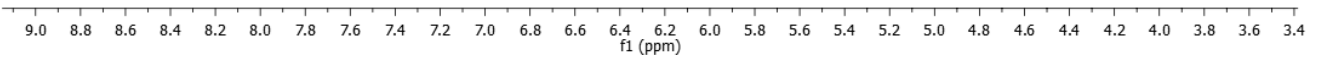

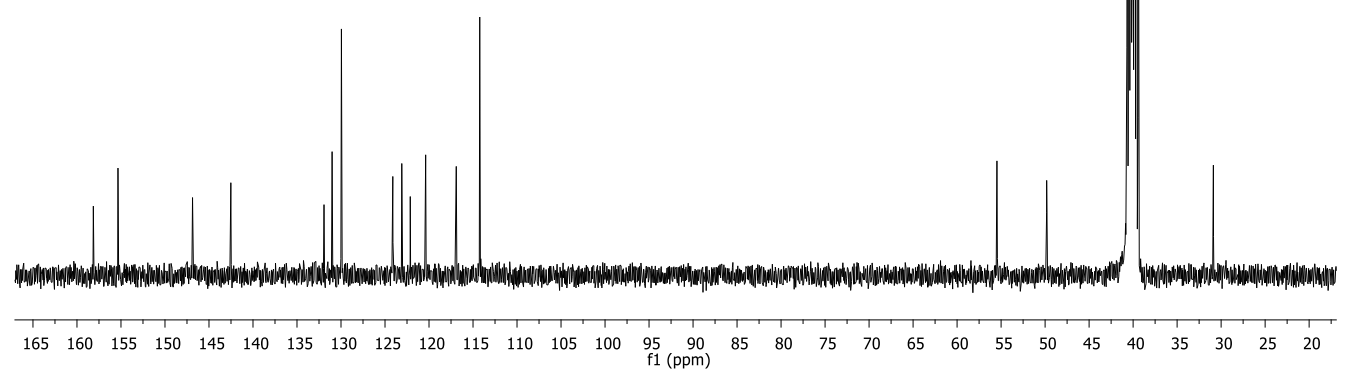


<smiles>COc1ccc(Cc2cn(CCNC(=O)Nc3ccc(Br)cc3)nn2)cc1</smiles>
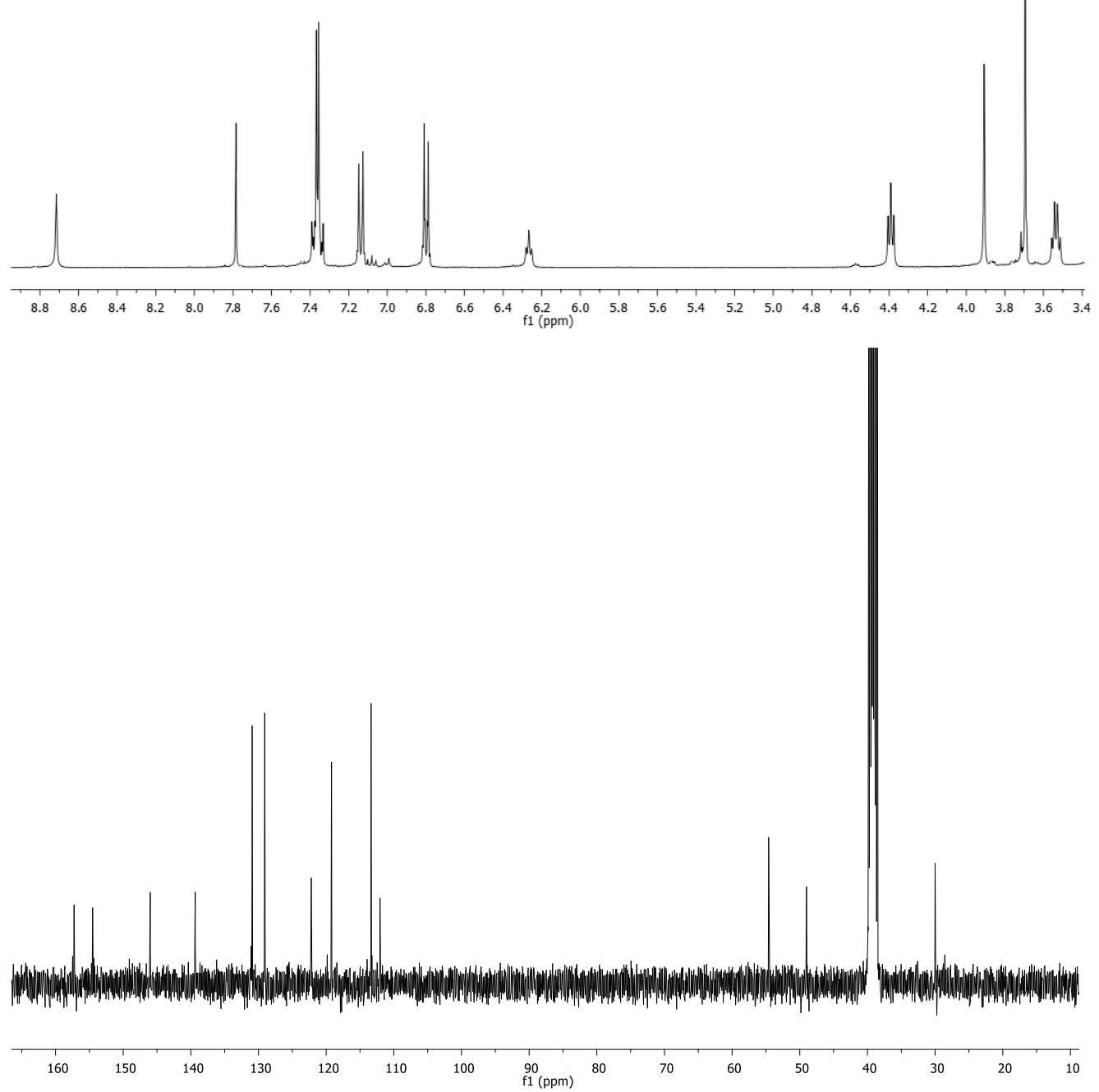
244

Chapter 7
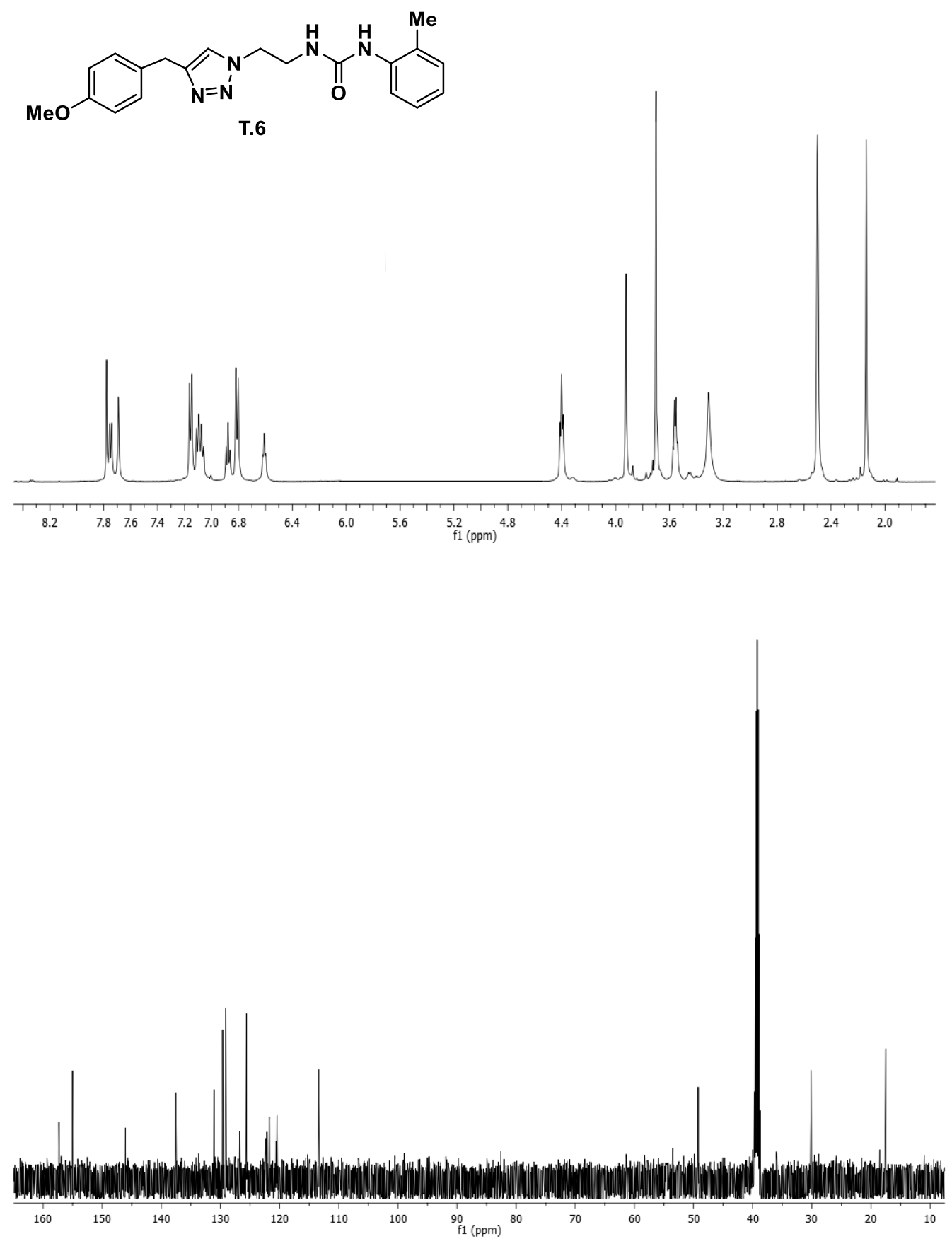
Selected spectra

245
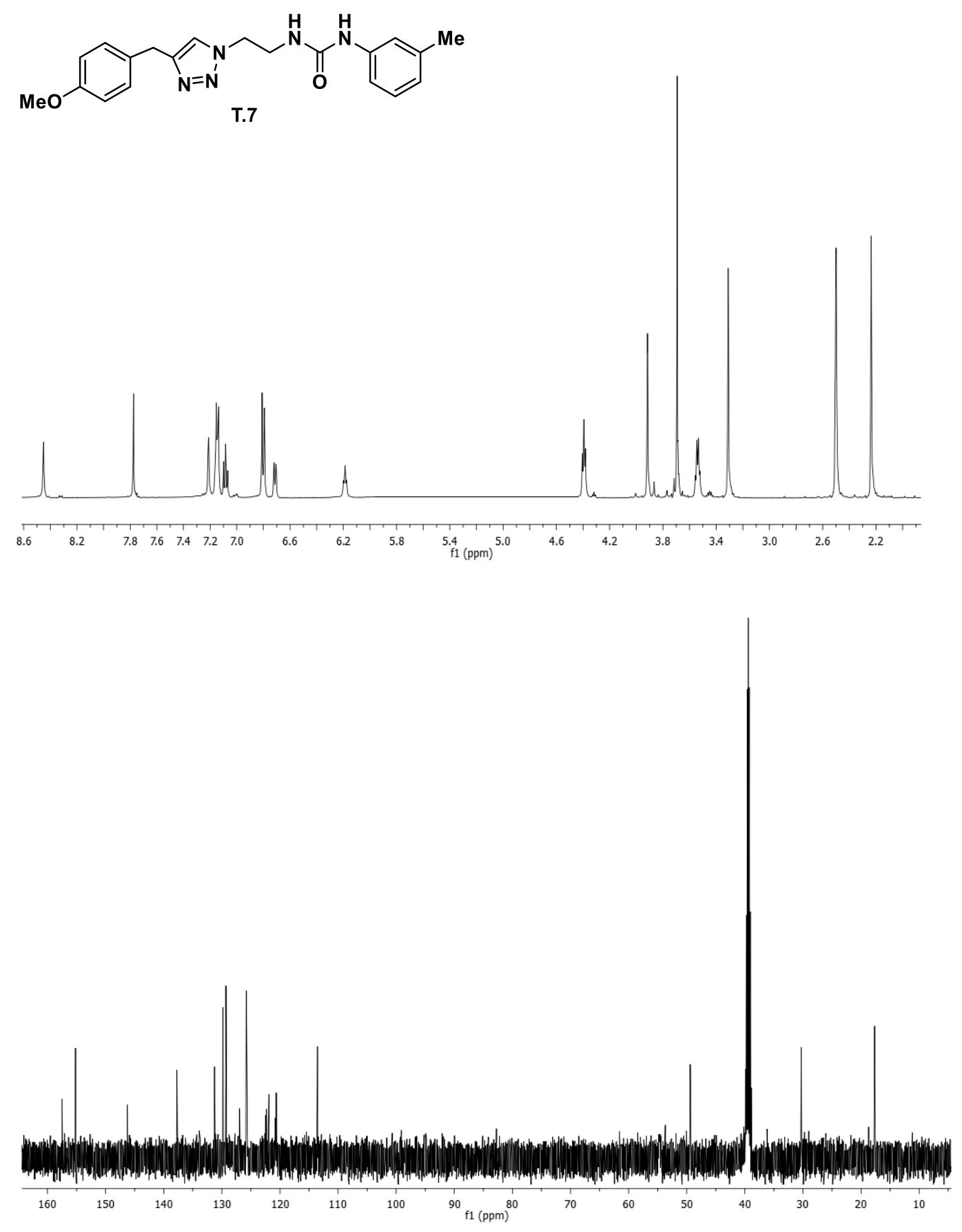

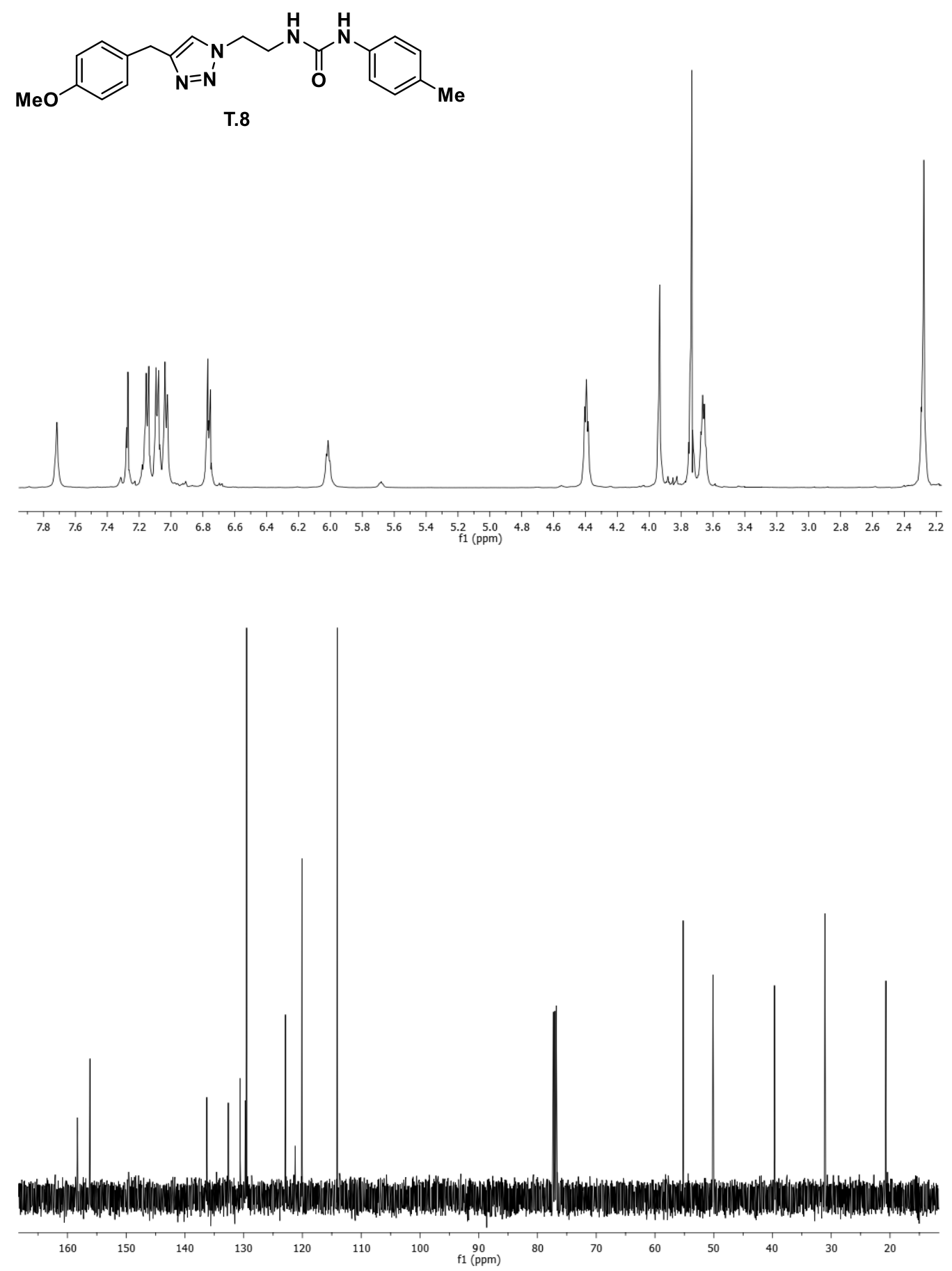
<smiles>COc1ccc(/C=C\c2cccc(NC(=O)Nc3ccccc3Cl)c2)cc1</smiles>

S. 1
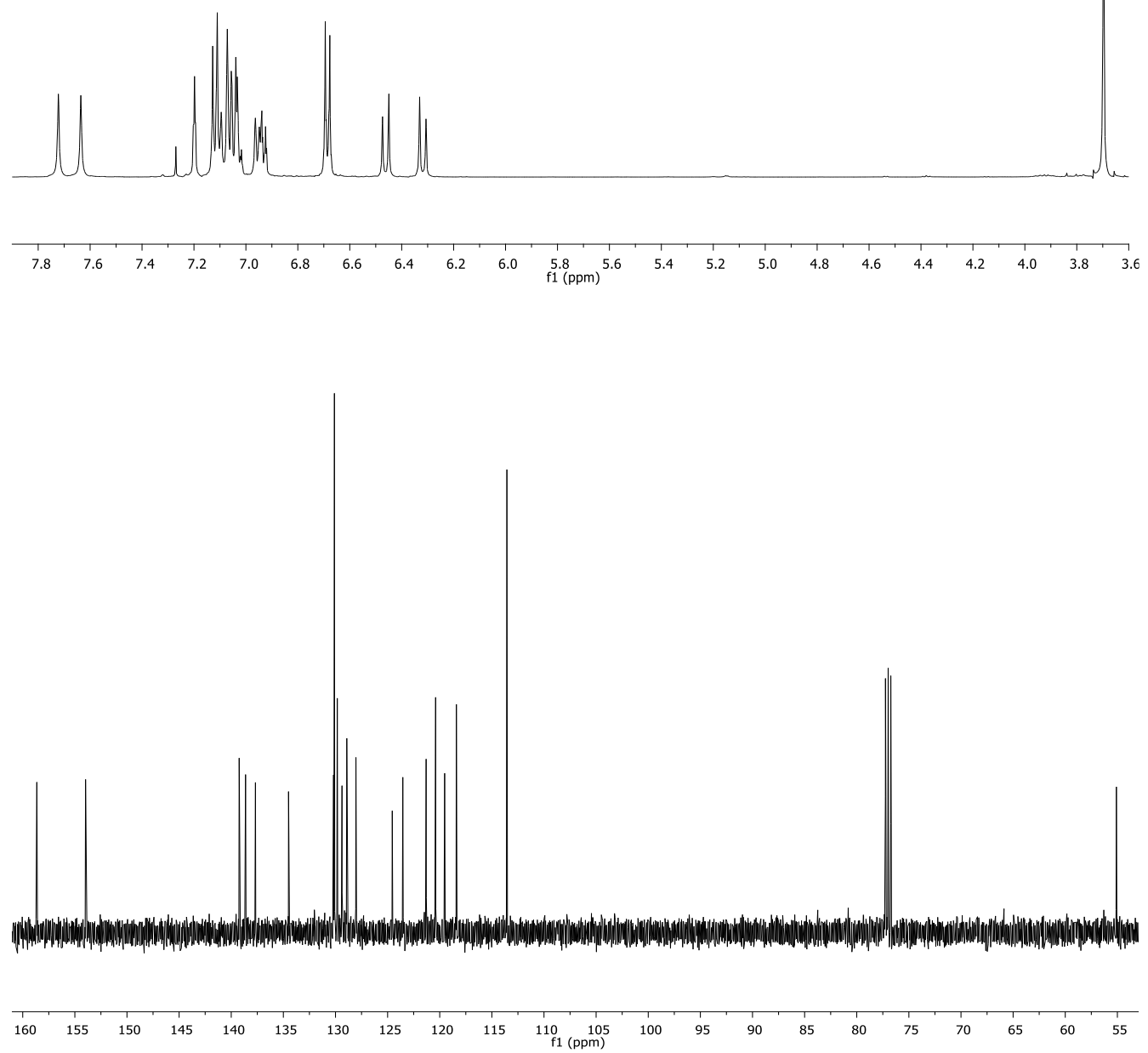
<smiles>COc1ccc(/C=C\c2cccc(NC(=O)Nc3cccc(Cl)c3)c2)cc1</smiles>
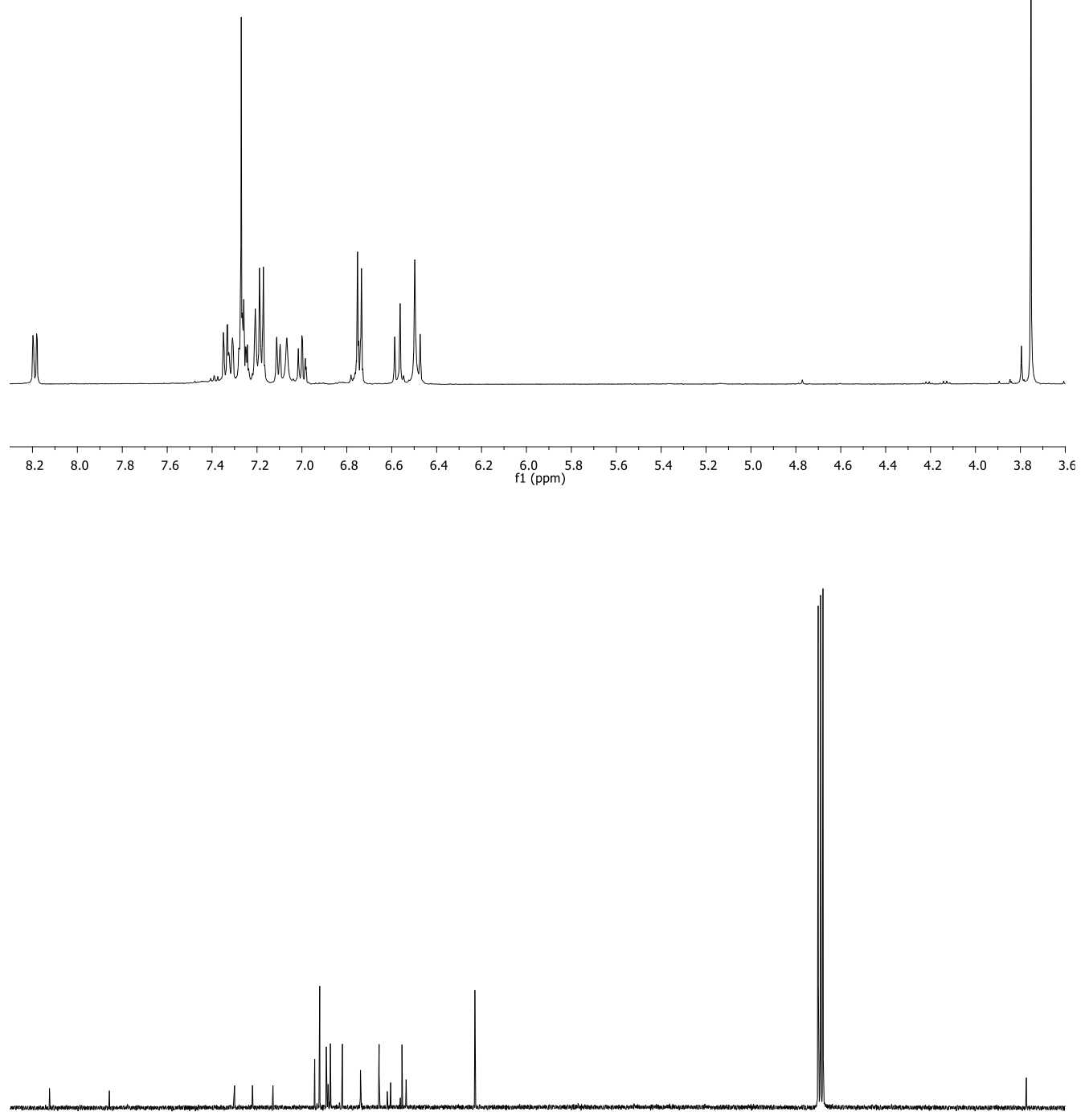

$\begin{array}{llllllllllllllllllllll}160 & 155 & 150 & 145 & 140 & 135 & 130 & 125 & 120 & 115 & \underset{\mathrm{f} 1(\mathrm{ppm})}{110} & 100 & 95 & 90 & 85 & 80 & 75 & 70 & 65 & 60 & 55\end{array}$ 
<smiles>COc1ccc(/C=C\c2cccc(NC(=O)Nc3ccc(Cl)cc3)c2)cc1</smiles>
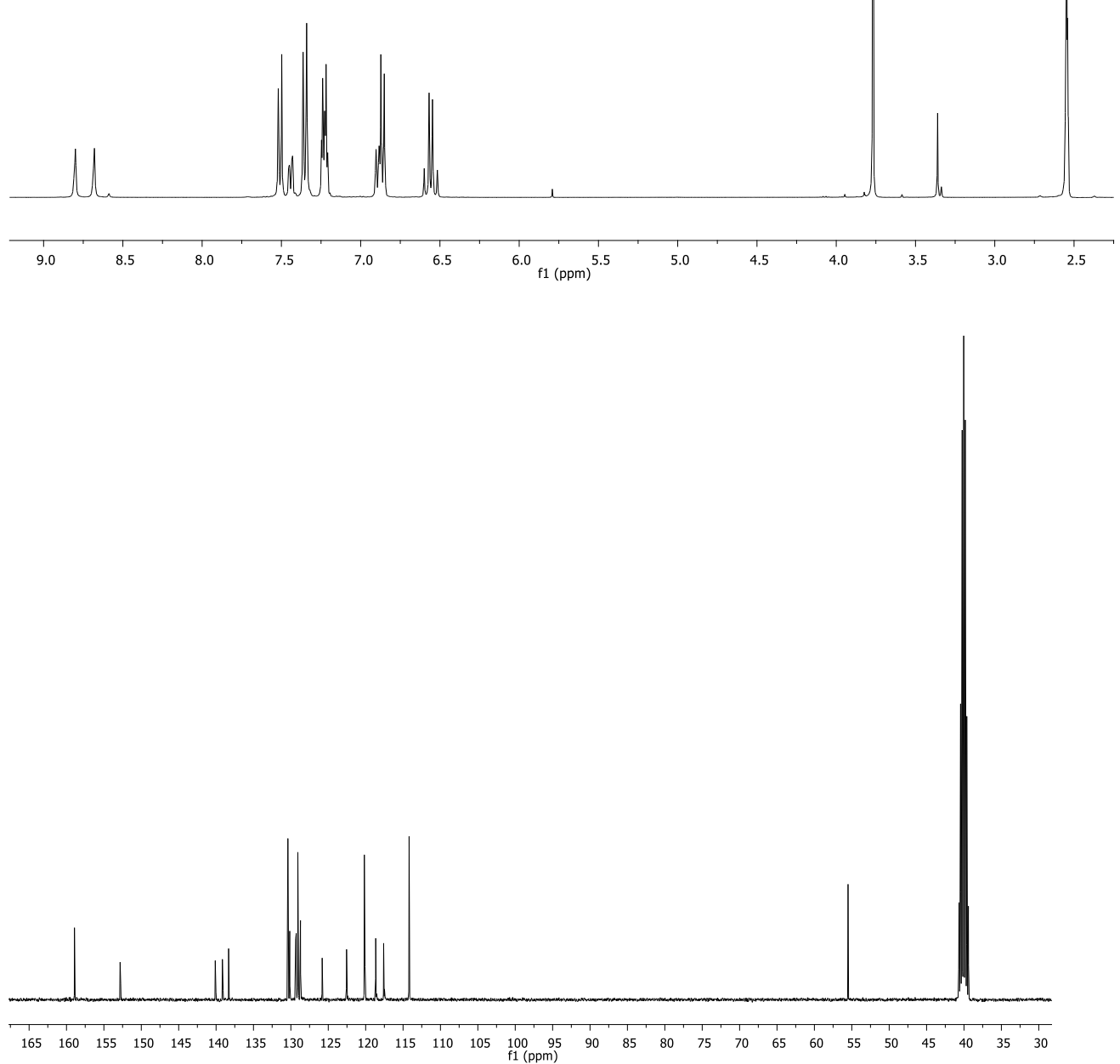
<smiles>COc1ccc(/C=C/c2cccc(NC(=O)Nc3ccccc3Cl)c2)cc1</smiles>
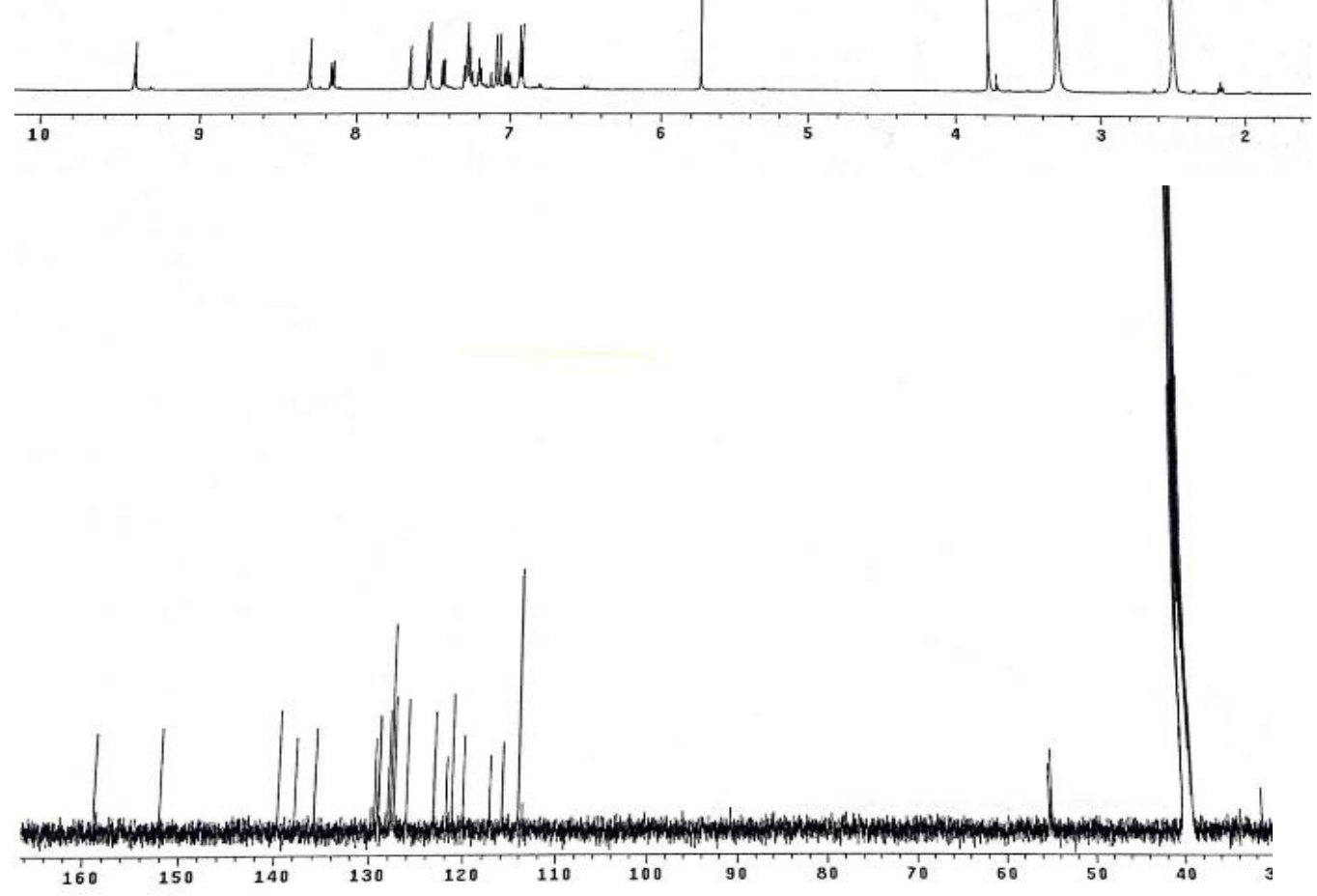
<smiles>COc1ccc(/C=C/c2cccc(NC(=O)Nc3cccc(Cl)c3)c2)cc1</smiles>
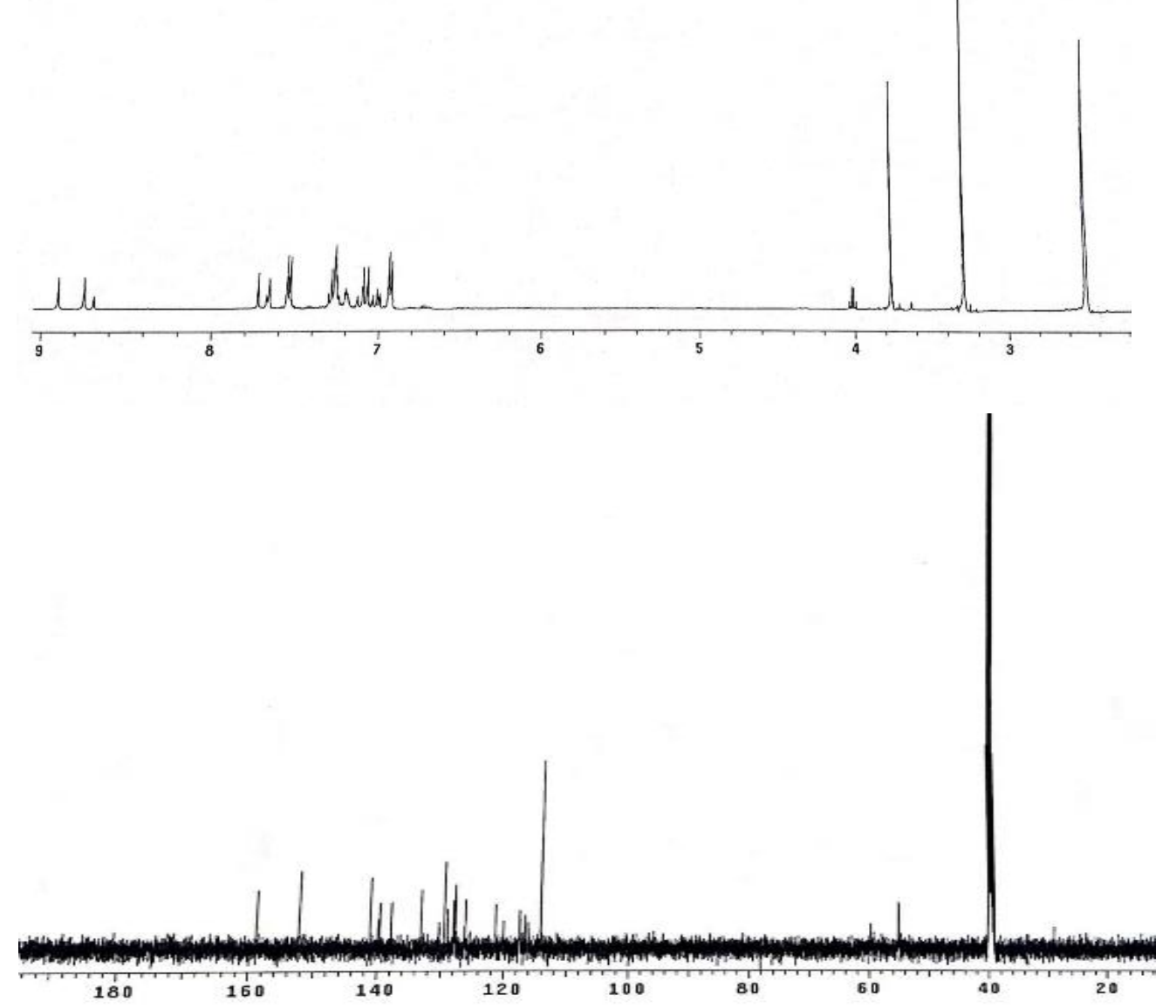

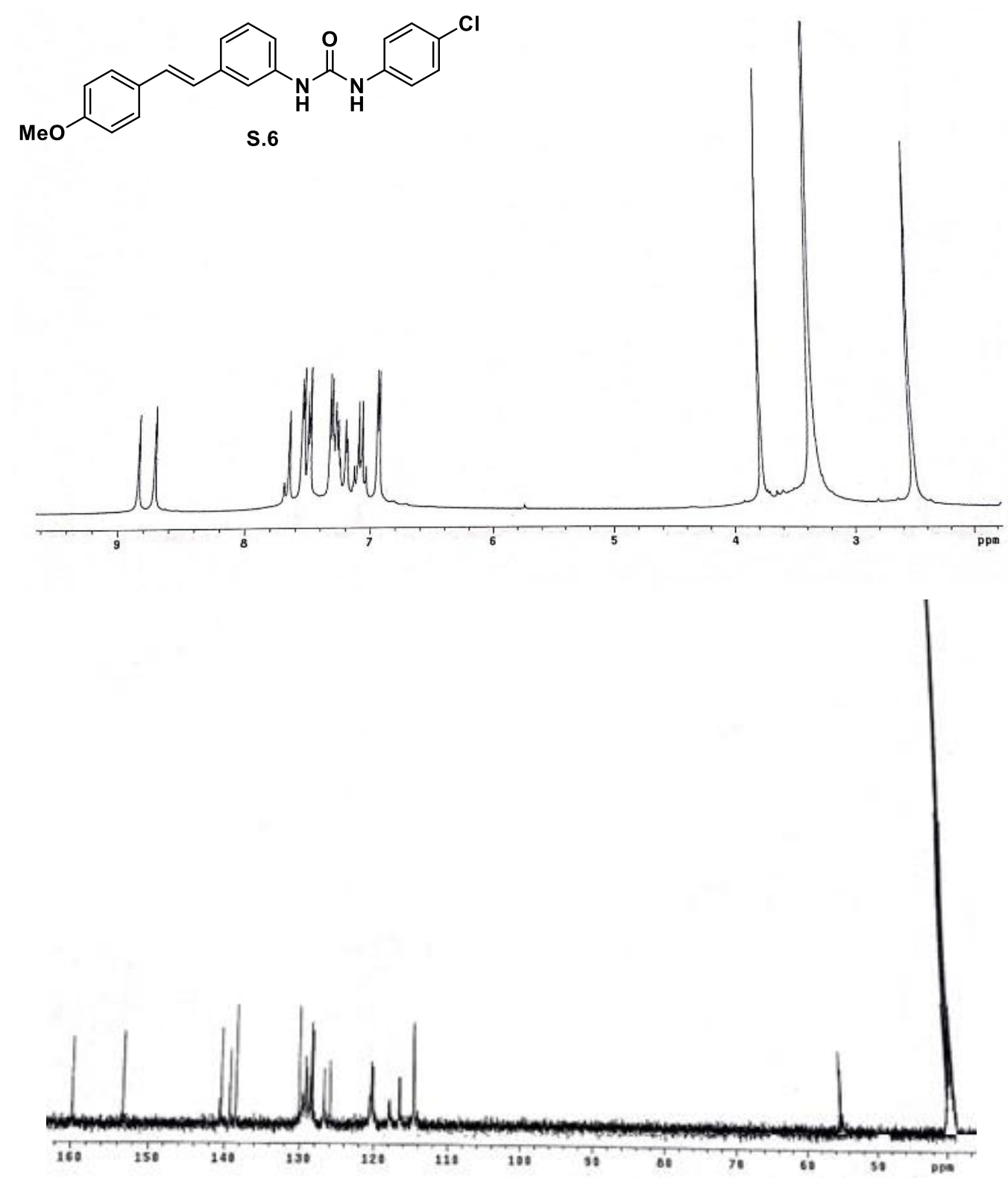
<smiles>COc1ccc(CCc2cccc(NC(=O)Nc3ccccc3Cl)c2)cc1</smiles>
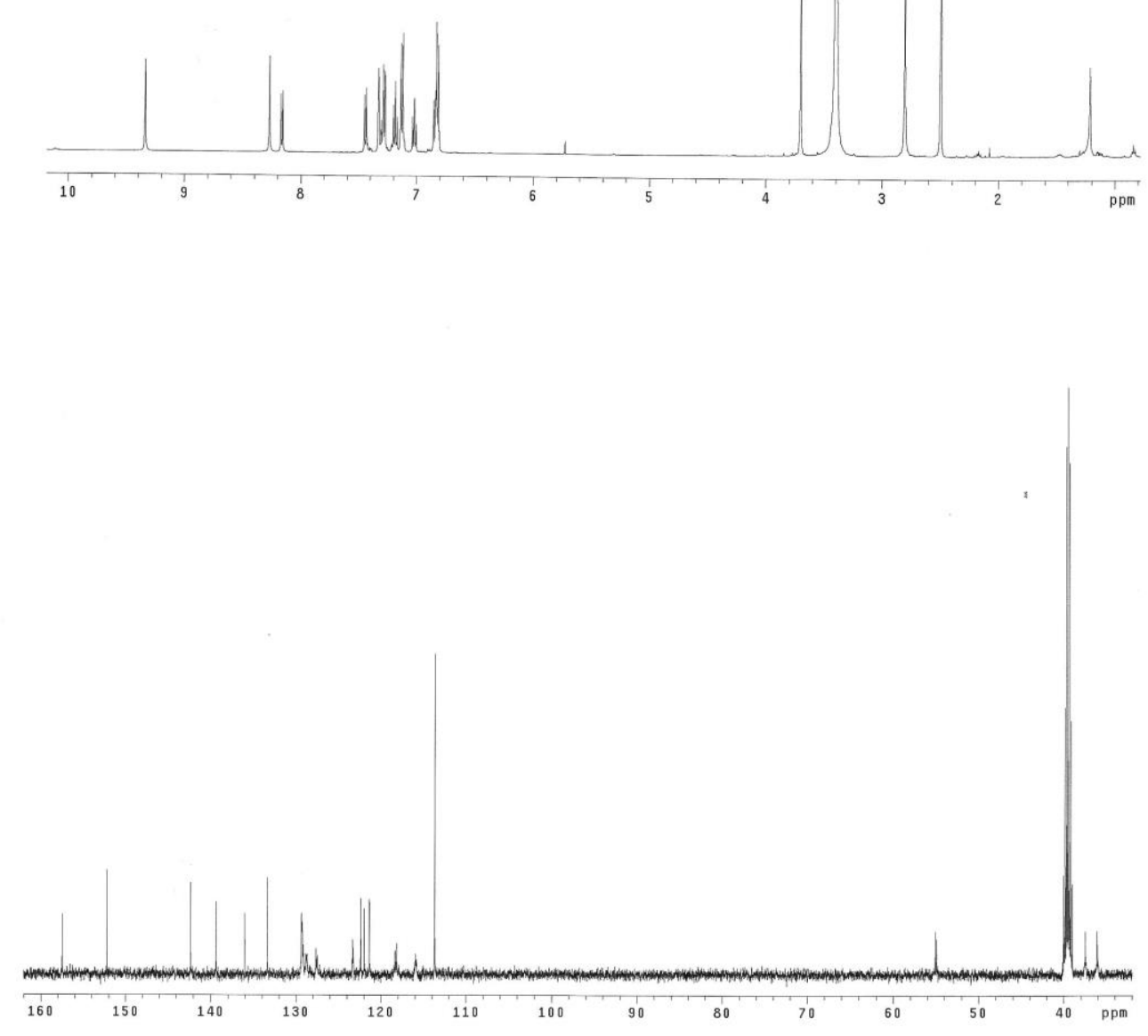

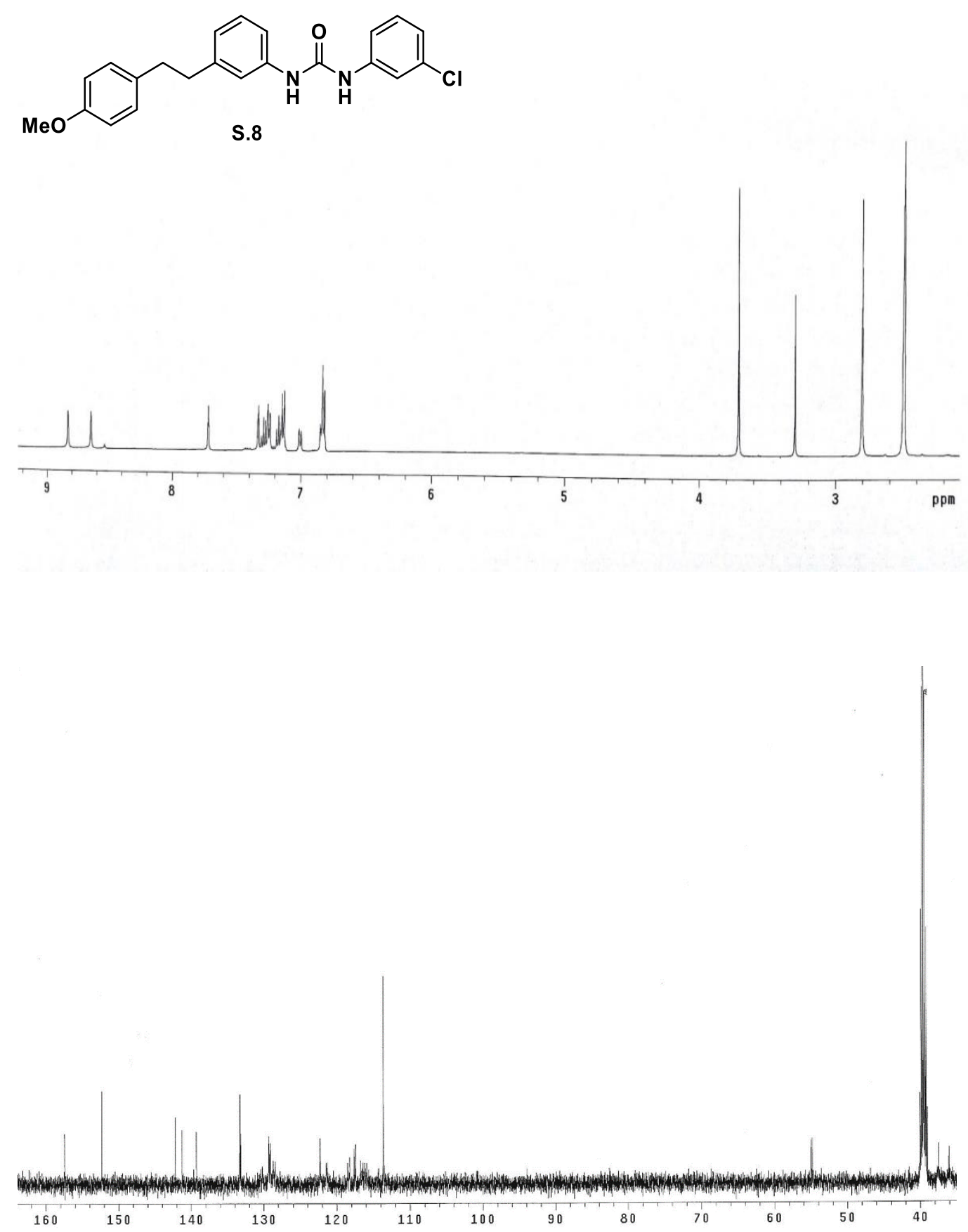
<smiles>COc1ccc(CCc2cccc(NC(=O)Nc3ccc(Cl)cc3)c2)cc1</smiles>
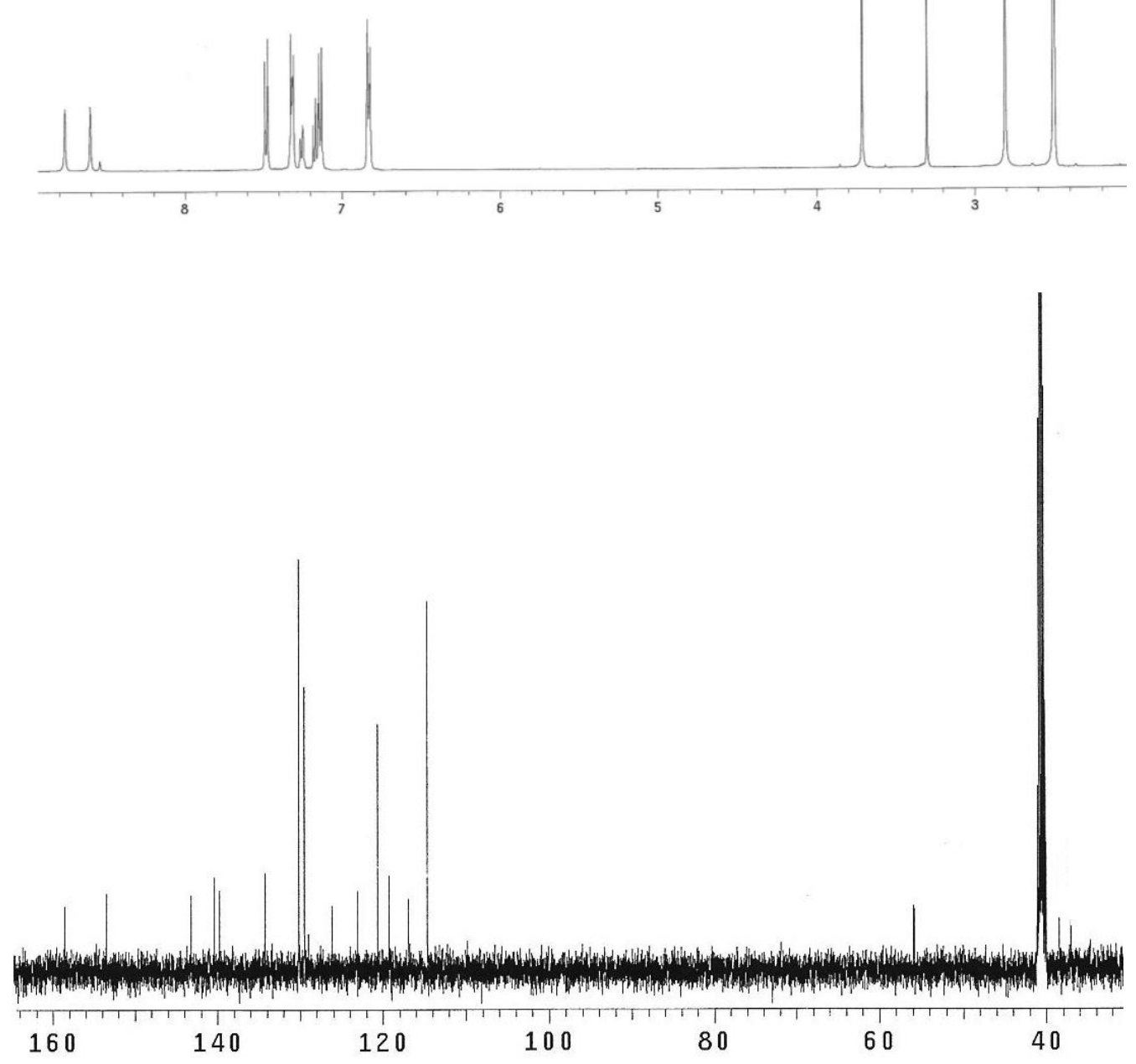

